\title{
HYDROLOGIC DATA FOR URBAN STUDIES IN THE HOUSTON, TEXAS, METROPOLITAN AREA, 1980
}

By Fred Liscum, J.S. Hutchison, J.P. Bruchmiller, and L.S. Walther U.S. GEOLOGICAL SURVEY Open-File Report 83-264

Prepared in cooperation with the CITY of HOUSTON Austin, Texas 


\author{
UNITED STATES DEPARTMENT OF THE INTERIOR \\ JAMES G. WATT, Secretary \\ GEOLOGICAL SURVEY \\ Dallas L. Peck, Director
}

For additional information write to:

District Chief

U.S. Geological Survey

300 East 8th Street

Austin, TX 78701
Copies of this report can be purchased from:

Open-File Services Section Western Distribution Branch Box 25425, Federal Center Denver, C0 80225

Telephone: (303) 234-5888 


\section{CONTENTS}

Introduction- 1

Location and description of the area-

Data-collection methods-_.

Precipitation data-

Runoff data-_-

Water-qual ity data-_. 16

Sel ected references-- 18

Compilation of data- 19

San Jacinto River basin:

South Mayde Creek:

Buffal o Bayou near Addicks, Tex.

Buffalo Bayou at West Belt Drive, Houston, Tex.

Bettina Street Ditch drainage basin

Bettina Street Ditch at Houston, Tex.

Storm of December 12, 1979

Buffalo Bayou at Piney Point, Tex. 36

Buffalo Bayou at Houston, Tex. 37

Whiteoak Bayou:

Whiteoak Bayou drainage basin- 39

Cole Creek drainage basin- 41

Bingle Road Storm Sewer drainage basin-_. 43

Bingle Road Storm Sewer at Houston, Tex.

Storm of June 9, 1980

Storm of Jul y 21,1980

Storm of Sept. 5-7, 1980

Cole Creek at Deihl Road, Houston, Tex. 53

Storm of Jan. 20-24, 1980

Storm of March 27-31, 1980__._.

Brickhouse Gully drainage basin

Brickhouse Gully at Clarblak Street, Houston, Tex. 63

Storm of Oct. 30, 1979

Storm of March 29-30, 1980 65

Brickhouse Gully at Costa Rica Street, Houston, Tex._. 66

Storm of 0ct. 30-31, 1979 70

Storm of March 27-30, 1980

Lazybrook Street Storm Sewer drainage basin-_. 74

Lazybrook Street Storm Sewer at Houston, Tex. 77

Storm of Oct. 30, 1979

Storm of March 27-28, 1980

Storm of April 25, 1980

Whiteoak Bayou at Houston, Tex.

Storm of Oct. 22-23, 1979

Storm of Oct. 30-Nov. 1, 1979

Storm of Jan. 17-19, 1980

Storm of Jan. 20-27, 1980

Storm of March 27-April 2, 1980

$-i-$ 
Compilation of data--Continued

San Jacinto River basin--Continued

Little Whiteoak Bayou drainage basin 97

Little Whiteoak Bayou at Trimble Street at Houston, Tex.

Storm of Oct. 30-31, 1979

Storm of Dec. 12-13, 1979

Storm of Jan. 17-18, 1980

Brays Bayou:

Brays Bayou drainage basin-

Brays Bayou at Al ief, Tex.

Storm of Oct. 30-Nov. 1, 1979

Storm of Dec. 12-14, 1979

Storm of Jan. 20-24, 1980

Keegans Bayou drainage basin

Keegans Bayou at Keegan Road near Houston, Tex......

Keegans Bayou at Roark Road near Houston, Tex.

Storm of Dec. 12-14, 1979

Storm of Jan. 20-25, 1980

Brays Bayou at Gessner Drive, Houston Tex.

Storm of Jan. 20-25, 1980

Bintl iff Ditch drainage basin-

Bintliff Ditch at Bissonnet Street, Houston, Tex.

Storm of Jan. 20-22, 1980

Hummingbird Street Ditch drainage basin

Hummingbird Street Ditch at Houston, Tex....

Brays Bayou at Houston, Tex.

Storm of Dec. 12-14, 1979-_...

Storm of Jan. 20-25, 1980-1

Brays Bayou at Scott Street, Houston, Tex.

Sims Bayou:

Sims Bayou drainage basin-

Sims Bayou at Hiram Clarke Street, Houston, Tex.

Storm of Oct. 30-31, 1979_.........

Storm of Jan. 20-24, 1980

Sims Bayou at Martin Luther King Boulevard, Houston, Tex...........................

Sims Bayou at Houston, Tex.

Storm of Oct. 30-Nov. 1, 1979

Storm of Jan. 20-25, 1980

Berry Bayou drainage basin-

Berry Bayou at Gilpin Street, Houston, Tex.

Storm of Jan. 20-22, $1980 \ldots \ldots$

Berry Bayou at Forest 0aks Street, Houston, Tex.

Storm of 0ct. 30-31, 1979

Vince Bayou:

Vince Bayou drainage basin-

Vince Bayou at Pasadena, Tex.

Hunting Bayou:

Hunting Bayou drainage basin- 


\section{CONTENTS--Continued}

Compilation of data--Continued

San Jacinto River basin:--Continued

Hunting Bayou drainage basin--Continued

Hunting Bayou at Falls Street, Houston, Tex.

Storm of Jan. 20-23, 1980

Storm of April 25-26, 1980

Hunting Bayou at Interstate Highway 610, Houston, Tex.

Storm of Jan. 20-24, 1980

Storm of April 25-27, 1980

Greens Bayou:

Greens Bayou drainage basin-

Greens Bayou at Cutten Road near Houston, Tex.

Storm of Feb. 8-11, 1980

Greens Bayou at U.S. Highway 75 near Houston, Tex.

Storm of Jan. 20-24, 1980

Storm of March 27-31, 1980

Greens Bayou near Houston, Tex.

Storm of Jan. 20-26, 1980

Storm of March 27-April 2, 1980

Halls Bayou drainage basin-.

Halls Bayou at Deertrail Street near Houston, Tex.

Storm of Jan. 20-24, 1980

Storm of Sept. 5-7, 1980

Hall s Bayou at Houston, Tex.

Storm of Nov. 21-24, 1979

Storm of Jan. 20-24, 1980-_.

Greens Bayou at Ley Road, Houston, Tex.

Storm of Jan. 20-26, 1980

Storm of March 27-April 2, 1980-

Clear Creek basin:

Cl ear Creek near Pearland, Tex.

Daily and monthly rainfall summary for gages north of

Buffalo Bayou-c.

Daily and monthly rainfall summary for gages south of

Buffalo Bayou-_.

Monthly rainfall-data summary in the Houston metropolitan

area, National Weather Service Stations, 1980 water year-_...-. 
Figure 1. Map showing locations of data-collection sites

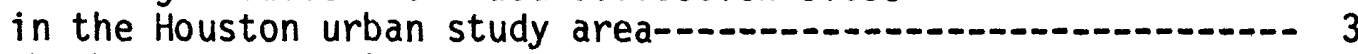

2. Graph showing rainfall at five drainage basins

in the Houston metropolitan area, 1980 water

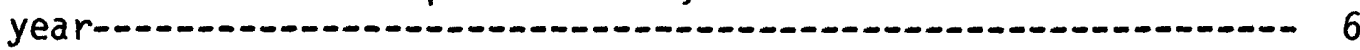

3. Graph showing runoff from six drainage basins

in the Houston metropolitan area, 1980 water

year, and average runoff for the period 1953-70

4-20. Map showing locations of data-collection sites in and near the:

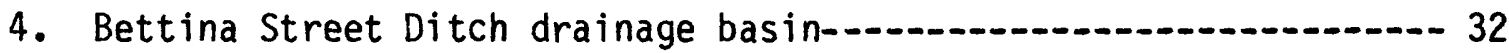

5. Whiteoak Bayou drainage basin-_-...- 40

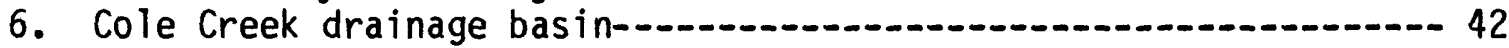

7. Bingle Road Storm Sewer drainage basin-.......................... 44

8. Brickhouse Gully drainage basin-_............................... 61

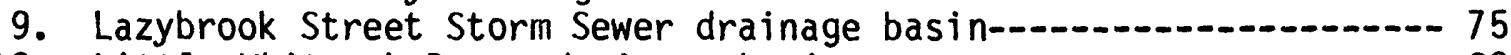

10. Little Whiteoak Bayou drainage basin-_............................. 98

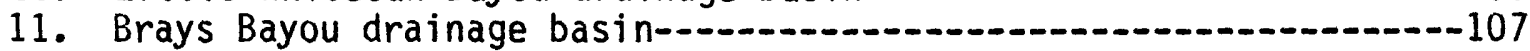

12. Keegans Bayou drainage basin-_._._._._._.

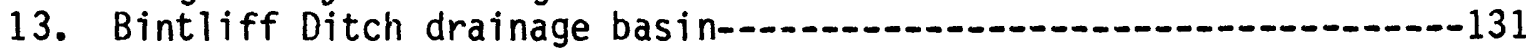

14. Hummingbird Street Ditch drainage basin-_._.....................-137

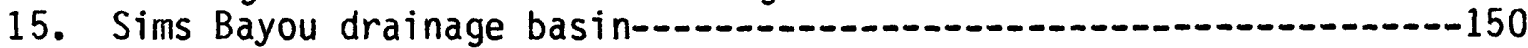

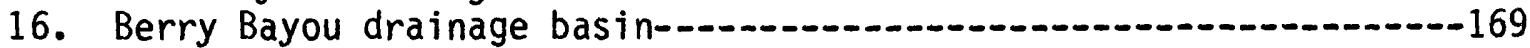

17. Vince Bayou drainage basin-_-_._-_.

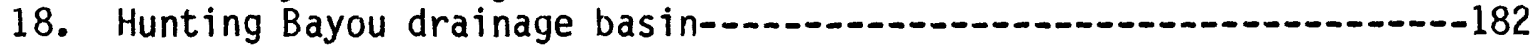

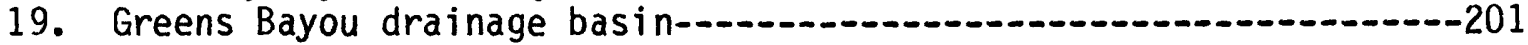

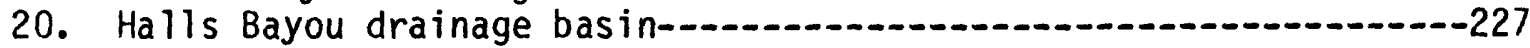




\section{TABLES}

Table 1. Percent increases in development in various drainage areas in the Houston metropolitan area from 1969 to 1976

2. Weighted-mean precipitation factors for drainage basins above stations in the Houston metropolitan a rea---n-

3-17. Storm rainfal1-runoff data, 1980 water year,:

3. Bettina Street Ditch

4. Bingle Road Storm Sewer-a 45

5. Cole Creek-

6. Brickhouse Gully-_an 62

7. Lazybrook St reet Storm Sewer- 76

8. Whiteoak Bayou 83

9. Little Whiteoak Bayou-_ 99

10. Brays Bayou-

11. Keegans Bayou-_-_._-

12. Bintliff Ditch-_._-

13. Sims Bayou-

14. Berry Bayou-_-_._-

15. Hunting Bayou-

16. Greens Bayou-_._.

17. Halls Bayou-

18. Recording and non recording rain gages in the Houston area at sites other than stream-gaging stations-_- 


\section{METRIC CONVERSIONS}

For those readers interested in using the metric system, the inch-pound units of measurements used in this report may be converted to metric units by using the following conversion factors:

\begin{tabular}{|c|c|c|c|c|}
\hline \multicolumn{2}{|c|}{ From } & \multirow[b]{2}{*}{$\begin{array}{l}\text { Multiply } \\
\text { by }\end{array}$} & \multicolumn{2}{|c|}{ To obtain } \\
\hline Unit & $\begin{array}{c}\text { Abbrevia- } \\
\text { tion }\end{array}$ & & Unit & $\begin{array}{c}\text { Abbrevia- } \\
\text { tion } \\
\end{array}$ \\
\hline inch & in & 25.4 & millimeter & $\mathrm{mm}$ \\
\hline foot & $\mathrm{ft}$ & 0.3048 & meter & m \\
\hline mile & mi & 1.609 & kilometer & $\mathrm{km}$ \\
\hline square mile & $m i^{2}$ & 2.590 & square kilometer & $\mathrm{km}^{2}$ \\
\hline $\begin{array}{l}\text { cubic foot } \\
\text { per second }\end{array}$ & $\mathrm{ft}^{3} / \mathrm{s}$ & 0.02832 & cubic meter per second & $\mathrm{m}^{3} / \mathrm{s}$ \\
\hline foot per mile & $\mathrm{ft} / \mathrm{mi}$ & 0.189 & meter per kilometer & $\mathrm{m} / \mathrm{km}$ \\
\hline acre-foot & -- & 1233 & cubic meter & $\mathrm{m}^{3}$ \\
\hline & & 0.001233 & cubic hectometer & $\mathrm{hm}^{3}$ \\
\hline
\end{tabular}


HYDROLOGIC DATA FOR URBAN STUDIES IN THE

HOUSTON, TEXAS, METROPOLITAN AREA

1980

By

Fred Liscum, J. S. Hutchison, J. P. Bruchmiller, and L. S. Walther

INTRODUCTION

Hydrologic investiaations of urban watersheds in Texas were begun by the 1J.S. Geological Survey in 1954. Studies are now in progress in Austin, and Houston. Studies have been completed in the ?allas-Fort Worth and San Antonio areas.

The U.S. Geological Survey, in cooperation with the city of Houston, began studies in the Houston metropolitan area in 1964. The program was expanded in 1968 to include collection of water-quality data. The objectives of the Houston urban-hydrology study are as follows:

1. To determine, on the basis of historical data and hydrologic analyses, the magnitude and frequency of flood peaks and flood volumes.

2. To determine the effect of urban development on flood peaks and volumes.

3. To ascertain the variation in water quality for different flow conditions and different seasons.

This report, the seventeenth in a series of reports to be published annually, is primarily applicable to objective ?. The report presents hydrologic data collected in the Houston urban area for the 1980 water year (0ctober 1,1979 to September 30, 1980).

A report by Johnson and Sayre (1973) utilized records collected from 1965 to 1969 to study the effects of urbanization on flonds in the Houston area. The report also summarizes various basin parameters. A report by Waddell, Massey, and Jennings (1979) presents data on runoff from the Houston area and computed concentrations and loads of selected water-quality constituents discharged to Galveston Bay. The study utilized a variation of the "STORM" model developed by the Hydrologic Enqineering Center of the U.S. Army Corps of Engineers. A report prepared by Liscum and Massey (1980) presents a technique for estimating the magnitude and frequency of floods in the Houston area from drainage areas, bank-full conveyance, and percentage of urban development. 
A definition of terms related to streamflow, water quality, and other hydrologic data, as used in this report, are defined in "I.S. Geological Survey, Water-resources data for Texas, volume 2, 1980."

To facilitate the publication and distribution of this report some material has been included that does not conform to the formal publications standards of the U.S. Geological Survey.

\section{I_OSATION AND DESCRIPTION OF THE AREA}

The Houston study area, which is located about 45 miles from the Gulf of Mexico, is on an almost level plain. The land surface in the area increases in altitude from 35 feet above the National Geodetic Vertical Datum of 1929 (NGVD) in the southeast to 135 feet in the northwest.

Records show that the entire Houston urban study area is being developed rapidly. Percent increases in development in various drainage-basin areas in the Houston metropolitan area from 1969 to 1976 are given in table 1.

Soils in the area are predominately clay, clay loams, and fine sandy loams of low permeability.

The major stream draining the area is Buffalo Bayou, a tributary of the San Jacinto River. Buffalo Rayou is regulated by the Barker and Addicks flood-detention reservoirs near the western limits of the area. From these reservoirs, Buffalo Bayou meanders east and is fed by five major tributaries: Whiteoak, Brays, Sims, Hunting, and Greens Bayous. The drainage area of Buffalo Rayou, excluding the area above the flood-detention reservoirs, is about 810 square miles.

The climate of the Houston area is characterized by short mild winters, long hot summers, high relative humidity, and prevailing southeasterly winds. The mean annual temperature $(1941-70)$ is $68.9^{\circ} \mathrm{F}\left(20.5^{\circ} \mathrm{C}\right)$; the lowest temperature recorded was $5^{\circ} \mathrm{F}\left(-15^{\circ} \mathrm{C}\right)$ in 1930; and the maximum recorded was $108^{\circ} \mathrm{F}$ $\left(42^{\circ} \mathrm{C}\right)$ in 1909.

The 30-year average (1941-70) annaual rainfall for Houston is 48.19 inches, which is distributed uniformly throughout the year. The maximum annual rainfall was 72.86 inches in 1900; and the minimum was 17.55 inches in 1917.

\section{DATA-COLLECTION METHODS}

The drainage basins and locations of hydrologic-instrument installations and water-quality sampling sites in the Houston urban study area are shown on figure 1. The locations of hydrologic instruinents and data-collection sites in the individual basins are shown later on figures 4-20. 


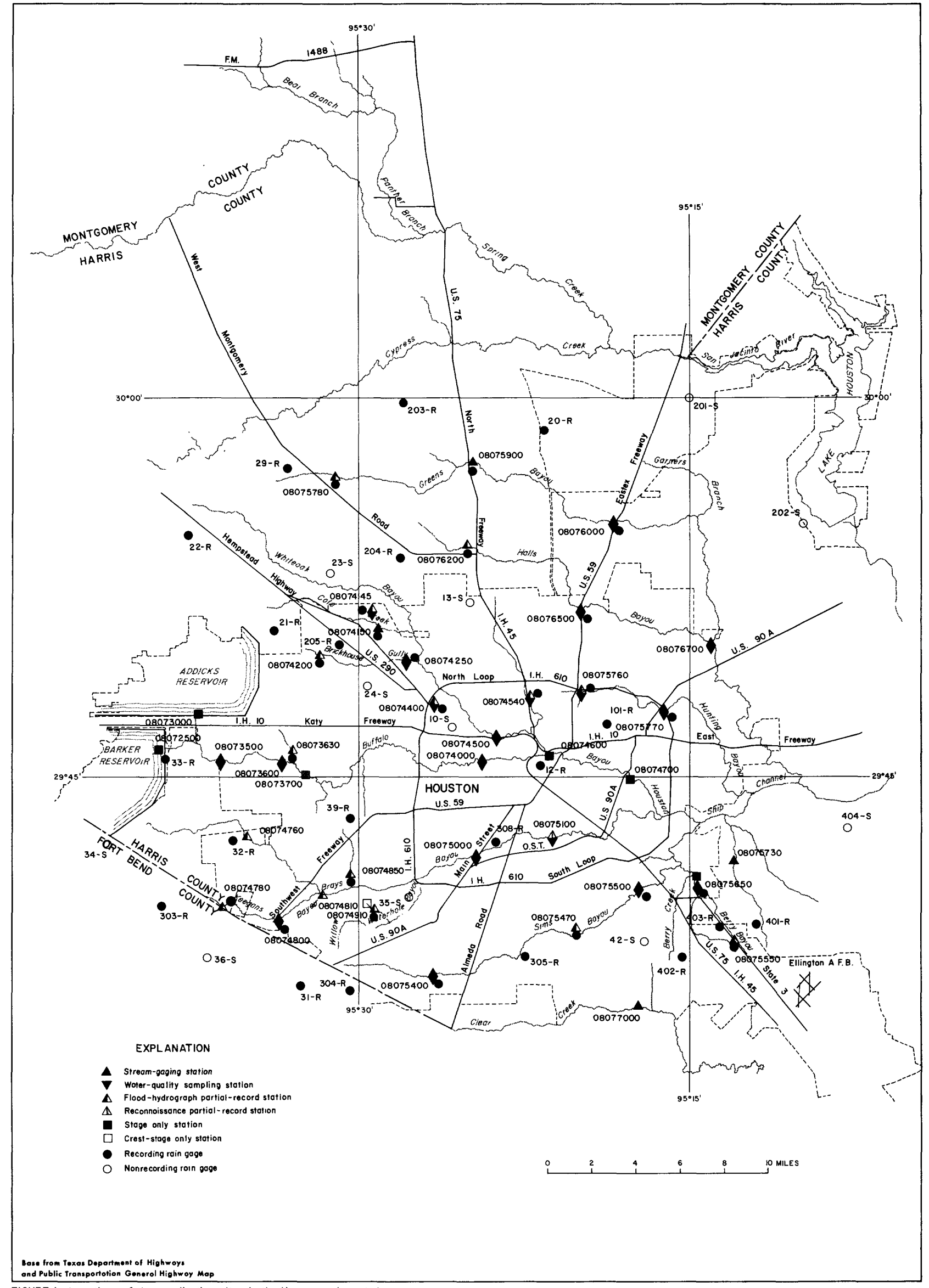

FIGURE I.-Locations of data-collection sites in the Houston urban study area 
Table 1.---Percent increases in development in various drainage areas above stream gaging stations in the Houston metropolitan area from 1969 to 1976

\begin{tabular}{|c|c|c|c|c|}
\hline $\begin{array}{l}\text { Station } \\
\text { no. }\end{array}$ & Station name & $\begin{array}{c}1969 \\
\text { a } /\end{array}$ & $\begin{array}{l}1976 \\
\text { b/ }\end{array}$ & $\begin{array}{l}\text { Percent } \\
\text { increase }\end{array}$ \\
\hline 08074150 & Cole Creek at Diehl Road & 34.3 & 54.0 & 19.7 \\
\hline 08074200 & Brickhouse Gully at Clarblak Street & 34.6 & 54.7 & 20.1 \\
\hline 08074250 & Brickhouse Gully at Costa Rica Street & 61.0 & 77.5 & 16.5 \\
\hline 08074500 & Whiteoak Bayou at Houston & 45.2 & 57.7 & 12.5 \\
\hline 08074780 & Keegans Bayou at Keegan Road & 21.0 & 44.9 & 23.9 \\
\hline 08074800 & Keegans Bayou at Roark Road & 26.3 & 55.7 & 29.4 \\
\hline 08075000 & Brays Bayou at Houston & 44.5 & 64.4 & 19.8 \\
\hline 08075400 & Sims Bayou at Hiram Clarke Street & 40.4 & 69.3 & 28.9 \\
\hline 08075500 & Sims Bayou at Houston & 50.2 & 73.7 & 23.5 \\
\hline 08075550 & Berry Bayou at Gilpin Street & 58.0 & 71.8 & 13.8 \\
\hline 08075650 & Berry Bayou at Forest Daks Street & 72.9 & 85.3 & 12.4 \\
\hline 08075760 & Hunting Bayou at Falls Street & 95.9 & 98.9 & 3.0 \\
\hline 08075770 & $\begin{array}{l}\text { Hunting Bayou at Interstate } \\
\text { Highway } 610\end{array}$ & 83.3 & 95.0 & 11.7 \\
\hline 08075780 & Greens Bayou at Cutten Road & 24.4 & 47.2 & 22.8 \\
\hline 08076000 & Greens Bayou near Houston & 26.3 & 43.9 & 17.6 \\
\hline 08076200 & Halls Bayou at Deertrail Street & 30.4 & 52.8 & 22.4 \\
\hline 08076500 & Halls Bayou at Houston & 60.3 & 74.1 & 13.8 \\
\hline
\end{tabular}

af Johnson and Sayre, 1973.

b/ Liscum and Massey, 1980. 


\section{Precipitation Data}

Precipitation data are based on 36 recording rain gages maintained by the U.S. Geological Survey in the Houston metropolitan area. The gages are distributed throughout the drainage basins to measure total precipitation and to define rainfall intensities.

Additional rainfall data are available from rain-gage networks operated by the National Weather Service and are given in the section "Compilation of Data". Locations of recording and nonrecording rain gages at sites other than stream-gaging stations are given later in table 18.

Precipitation at individual gages and weighted precipitation in each study basin is given in the section "Compilation of data." Daily and monthly rainfall amounts are also given in the section "Compilation of data."

Weighted-mean precipitation factors for drainage basins in the Houston area are given in table 2. Weighted-mean precipitation for a study area is determined by the Thiessen method as described by Linsley, Kohler, and Paulhus (1949). All of the rain gages, recording and non recording, are used to compute the monthly and annual rainfall amounts. Only the functioning recording gages are used to compute storm rainfall amounts. For example, the monthly and annual weighted-mean precipitation for the drainage basin upstream from the Cole Creek at the Deihl Road gaging station could be computed as follows: Multiply the recorded precipitation at the rain gage at station 08074150 by 0.25 ; to that value add the recorded precipitation at the rain gage at station $205 R$ multiplied by 0.15 ; to that value add the recorded precipitation at the rain gage at station $23 \mathrm{~S}$ multiplied by 0.15 ; and to that value add the recorded precipitation at the rain gage at station $21 R$ multiplied by 0.45 .

Rainfall for the current year was unevenly distributed over the area. Individual station totals ranged from 34.49 inches at the Fort Bend County rain gage in Stafford (station $31 R$ ) to 50.82 inches at the National Weather Service rain gage at the San Jacinto River dam (station 202S). Figure 2 shows the comparison of accumulated monthly rainfall for the 1980 water year over five widely separated drainage basins with the 30-year rainfall average (194170) of 48.19 inches for Houston. This fiqure illustrates the deficiency of rainfall in 1980 for the entire metropolitan area. Note that the rains of January and September were not sufficient to overcome the exceedingly dry periods of November and December, and May through August.

There were only eight storms occurring during the 1980 water year that produced rainfall totals of over 2.0 inches. Several of these were confined to only a few drainage basins. The three major storms, in terms of total rainfall and areal coverage, occurred on October 30, January 20-22, and March 27-29. The storm of 0ctober 30 produced rainfall amounts ranging from more than 4.0 inches in the Hunting, Berry, and Vince Bayou drainage basins to about 1.5 inches in the Keegans and upper Rrays Bayou drainage basins. The storin of January 20-22 produced rainfall ranging from more than 6.0 inches in the Sims and Berry Bayou drainage basins to more than 3.0 inches throughout the metropolitan area. The storm of March 27 consistently produced rainfall 


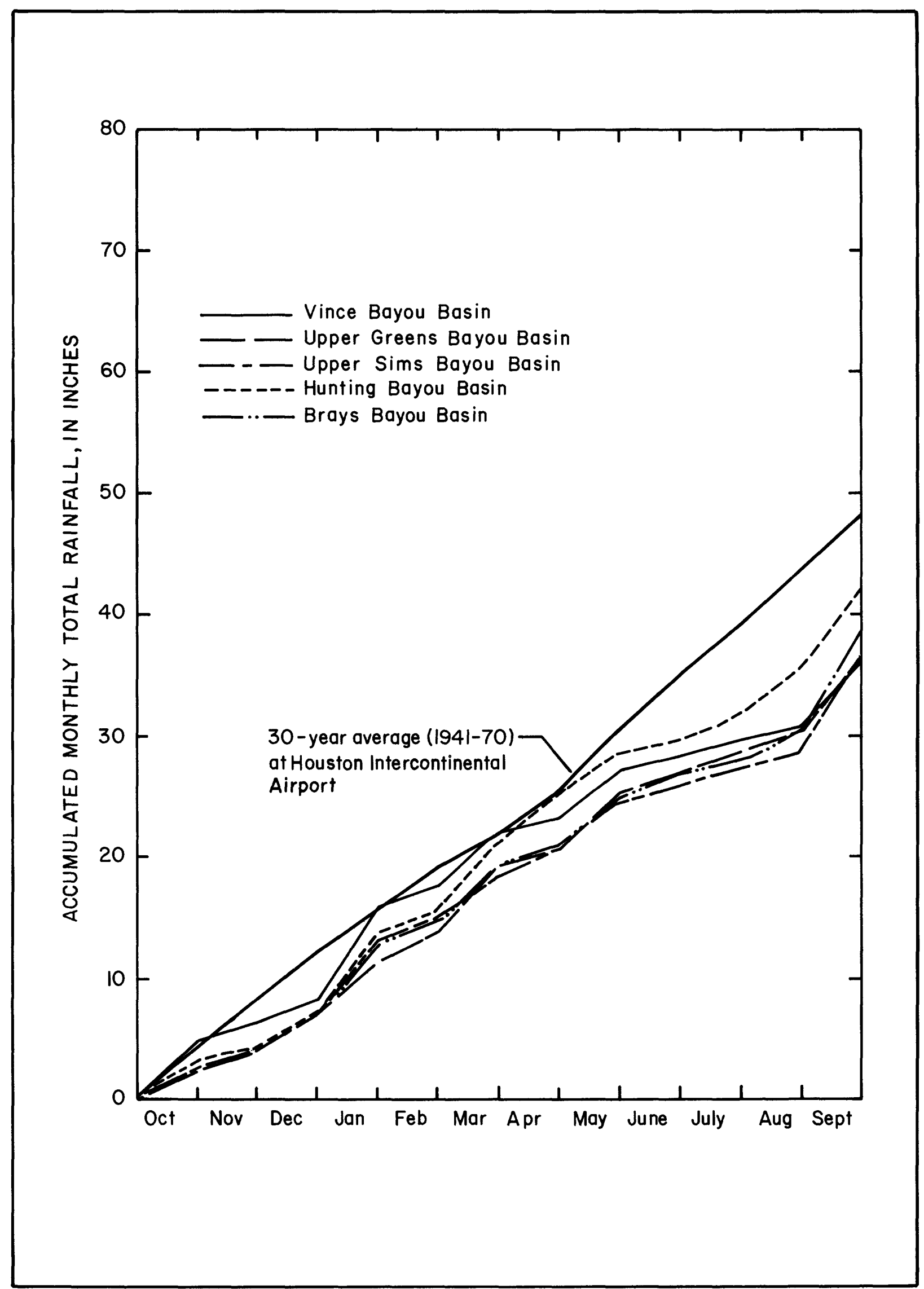

Figure 2.- Rainfall at five drainage basins in the Houston metropolitan area, 1980 water year 
Table 2.--Weighted-mean precipitation factors for drainage basins above stations in the Houston metropolitan area

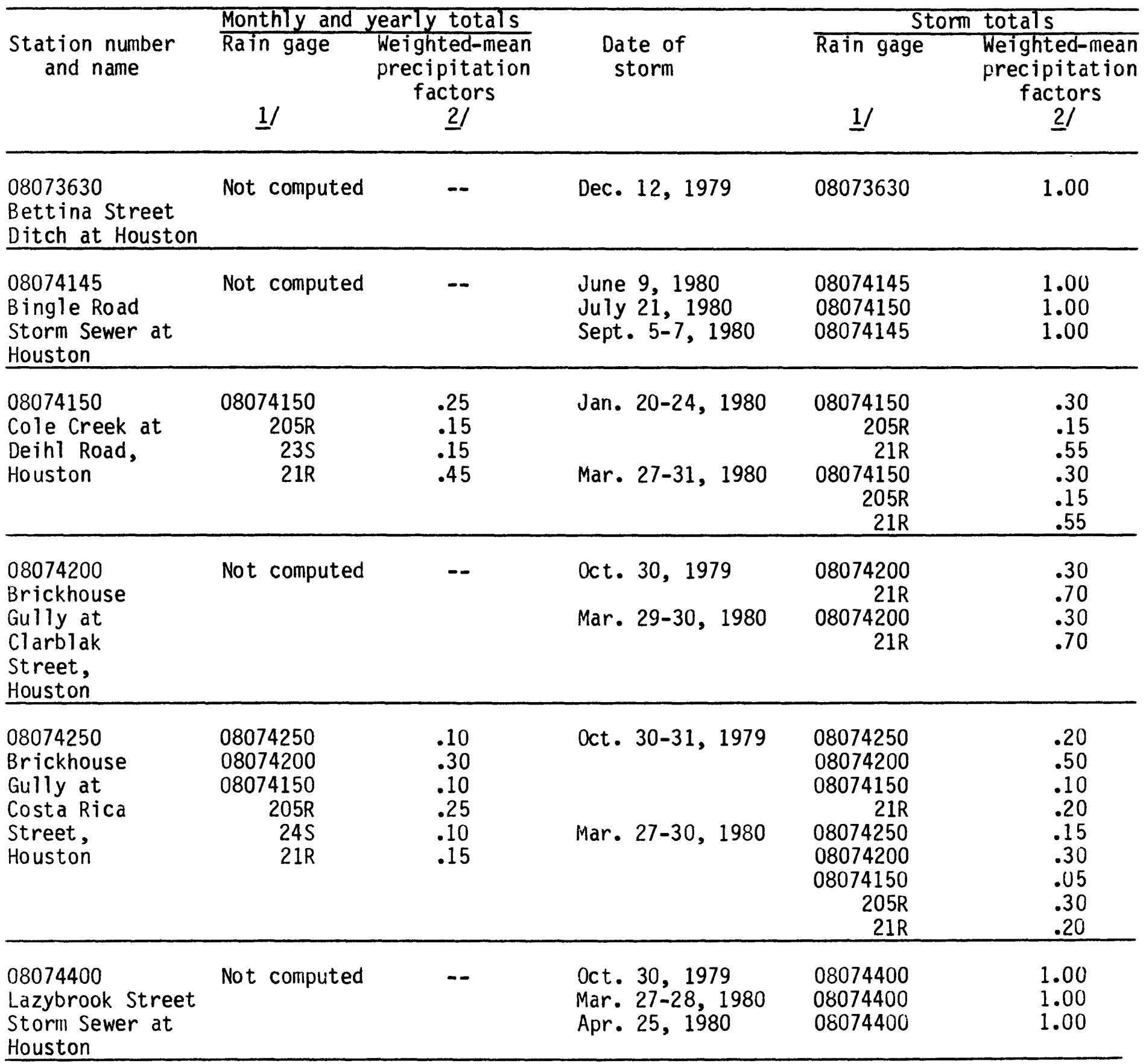

See footnotes at end of table. 
Table 2.--Weighted-mean precipitation factors for drainage basins above stations in the Houston metropolitan area--Continued

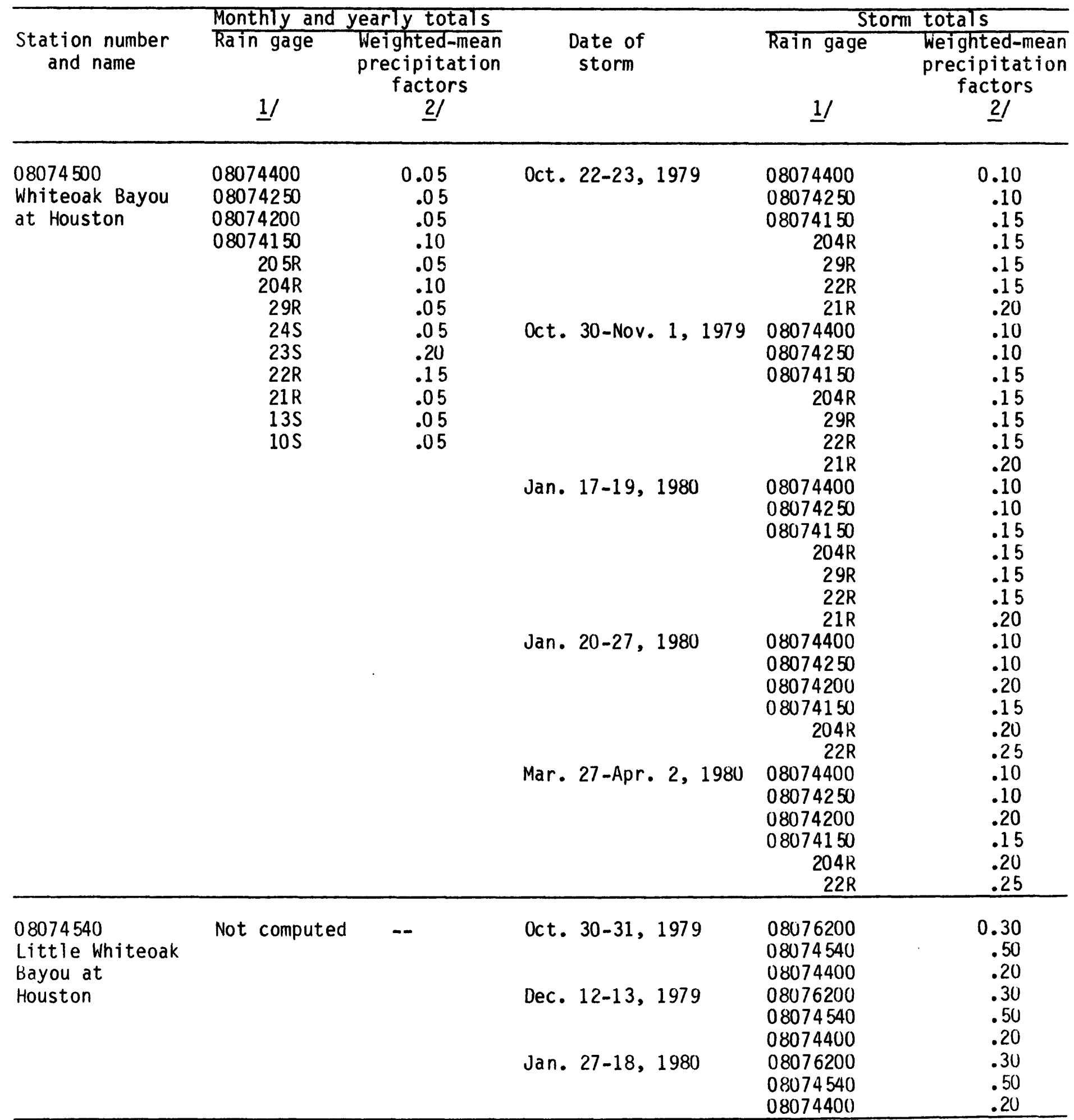

See footnotes at end of table. 
Table 2.--Weighted-mean precipitation factors for drainage basins above stations in the Houston metropolitan area--Continued

\begin{tabular}{|c|c|c|c|c|c|}
\hline & Monthाy and & yearly totals & & Storm & totals \\
\hline $\begin{array}{l}\text { Station number } \\
\text { and name }\end{array}$ & $\begin{array}{c}\text { Rain gage } \\
1 /\end{array}$ & $\begin{array}{c}\text { Weighted-mean } \\
\text { precipitation } \\
\text { factors } \\
\text { 2/ }\end{array}$ & $\begin{array}{l}\text { Date of } \\
\text { storm }\end{array}$ & $\begin{array}{c}\text { Rain gage } \\
\text { 1/ }\end{array}$ & $\begin{array}{c}\text { Weighted-mean } \\
\text { precipitation } \\
\text { factors } \\
2 \text { I }\end{array}$ \\
\hline $\begin{array}{l}08074760 \\
\text { Brays Bayou } \\
\text { at Al ief }\end{array}$ & Not computed & -- & $\begin{array}{l}\text { Oct. } 30-\text { Nov. } 1,1979 \\
\text { Dec. } 12-14,1979 \\
\text { Jan. } 20-24,1980\end{array}$ & $\begin{array}{l}33 R \\
32 R \\
33 R \\
32 R \\
33 R \\
32 R \\
\end{array}$ & $\begin{array}{r}0.30 \\
.70 \\
.30 \\
.70 \\
.30 \\
.70 \\
\end{array}$ \\
\hline $\begin{array}{l}08074780 \\
\text { Keegans Bayou } \\
\text { at Keegan } \\
\text { Road, Houston }\end{array}$ & Not computed & -- & No storms published & -- & -- \\
\hline $\begin{array}{l}08074800 \\
\text { Keegans Bayou } \\
\text { at Roark Road, } \\
\text { Houston }\end{array}$ & $\begin{array}{r}08074800 \\
08074780 \\
303 R \\
34 S\end{array}$ & $\begin{array}{r}0.10 \\
.45 \\
.35 \\
.10\end{array}$ & $\begin{array}{l}\text { Dec. } 12-14,1979 \\
\text { Jan. } 20-25,1980\end{array}$ & $\begin{array}{r}08074800 \\
08074780 \\
303 \mathrm{R} \\
08074800 \\
303 \mathrm{R} \\
\end{array}$ & $\begin{array}{l}.10 \\
.45 \\
.45 \\
.40 \\
.60 \\
\end{array}$ \\
\hline $\begin{array}{l}08074810 \\
\text { Brays Bayou } \\
\text { at Gessner } \\
\text { Drive, } \\
\text { Houston } \\
\end{array}$ & Not computed & -- & Jan. 20-25, 1980 & $\begin{array}{r}08074850 \\
08074800 \\
303 R \\
33 \mathrm{R} \\
32 \mathrm{R} \\
\end{array}$ & $\begin{array}{l}.05 \\
.30 \\
.20 \\
.05 \\
.40 \\
\end{array}$ \\
\hline $\begin{array}{l}08074850 \\
\text { Bintliff Ditch } \\
\text { at Bissonnet } \\
\text { Street, } \\
\text { Houston } \\
\end{array}$ & Not computed & -- & Jan. $20-22,1980$ & 0074850 & 1.00 \\
\hline $\begin{array}{l}08074910 \\
\text { Hummingbird } \\
\text { Street Ditch } \\
\text { at Houston } \\
\end{array}$ & Not computed & -- & No storms published & -- & - \\
\hline
\end{tabular}

See footnotes at end of table. 
Table 2.--Weighted-mean precipitation factors for drainage basins above stations in the Houston metropolitan area--Continued

\begin{tabular}{|c|c|c|c|c|c|}
\hline $\begin{array}{l}\text { Station number } \\
\text { and name }\end{array}$ & $\begin{array}{l}\text { Monthly and } \\
\text { Rain gage } \\
1 /\end{array}$ & $\begin{array}{c}\text { yearly totals } \\
\text { Weighted-mean } \\
\text { precipitation } \\
\text { factors } \\
\text { 2/ }\end{array}$ & $\begin{array}{l}\text { Date of } \\
\text { storm }\end{array}$ & $\begin{array}{c}\text { Storm } \\
\text { Rain gage } \\
1 /\end{array}$ & $\begin{array}{l}\text { totals } \\
\text { Weighted-mean } \\
\text { precipitation } \\
\text { factors } \\
2 /\end{array}$ \\
\hline $\begin{array}{l}08075000 \\
\text { Brays Bayou at } \\
\text { Houston }\end{array}$ & $\begin{array}{r}08074910 \\
08074800 \\
08074780 \\
308 R \\
303 R \\
39 R \\
35 \mathrm{R} \\
34 \mathrm{~S} \\
33 \mathrm{R} \\
32 \mathrm{R} \\
31 \mathrm{R} \\
\end{array}$ & $\begin{array}{l}0.10 \\
.10 \\
.10 \\
.05 \\
.05 \\
.10 \\
.10 \\
.10 \\
.05 \\
.20 \\
.05 \\
\end{array}$ & $\begin{array}{l}\text { Dec. } 12-14,1979 \\
\text { Jan. } 20-25,1980\end{array}$ & $\begin{array}{r}08074850 \\
08074800 \\
308 R \\
303 R \\
32 R \\
08074850 \\
08074800 \\
308 R \\
303 R \\
32 R\end{array}$ & $\begin{array}{l}0.30 \\
.20 \\
.10 \\
.15 \\
.25 \\
.30 \\
.20 \\
.10 \\
.15 \\
.25\end{array}$ \\
\hline $\begin{array}{l}08075400 \\
\text { Sims Bayou at } \\
\text { Hi ram Clarke } \\
\text { Street, } \\
\text { Houston } \\
\end{array}$ & $\begin{array}{r}08075400 \\
304 R\end{array}$ & $\begin{array}{l}.40 \\
.60\end{array}$ & $\begin{array}{l}\text { Oct. } 30-31,1979 \\
\text { Jan. } 20-24,1980\end{array}$ & $\begin{array}{r}08075400 \\
304 R \\
08075400\end{array}$ & $\begin{array}{r}.40 \\
.60 \\
1.00\end{array}$ \\
\hline $\begin{array}{l}08075470 \\
\text { Sims Bayou at } \\
\text { Martin Luther } \\
\text { King Blvd., } \\
\text { Houston } \\
\end{array}$ & Not computed & -- & No storms published & - & -- \\
\hline $\begin{array}{l}08075500 \\
\text { Sims Bayou at } \\
\text { Houston }\end{array}$ & $\begin{array}{r}08075500 \\
08075400 \\
08075470 \\
305 R \\
304 R \\
42 S\end{array}$ & $\begin{array}{l}.05 \\
.25 \\
.20 \\
.25 \\
.20 \\
.05\end{array}$ & $\begin{array}{l}\text { Oct. 30-Nov. 1, } 1979 \\
\text { Jan. } 20-25,1980\end{array}$ & $\begin{array}{r}08075500 \\
08075400 \\
305 R \\
304 R \\
08075500 \\
08075470 \\
08075400 \\
305 R \\
31 R\end{array}$ & $\begin{array}{l}.15 \\
.30 \\
.35 \\
.20 \\
.10 \\
.20 \\
.40 \\
.20 \\
.10\end{array}$ \\
\hline
\end{tabular}

See footnotes at end of table. 
Table 2.--Weighted-mean precipitation factors for drainage basins above stations in the Houston metropolitan area--Continued

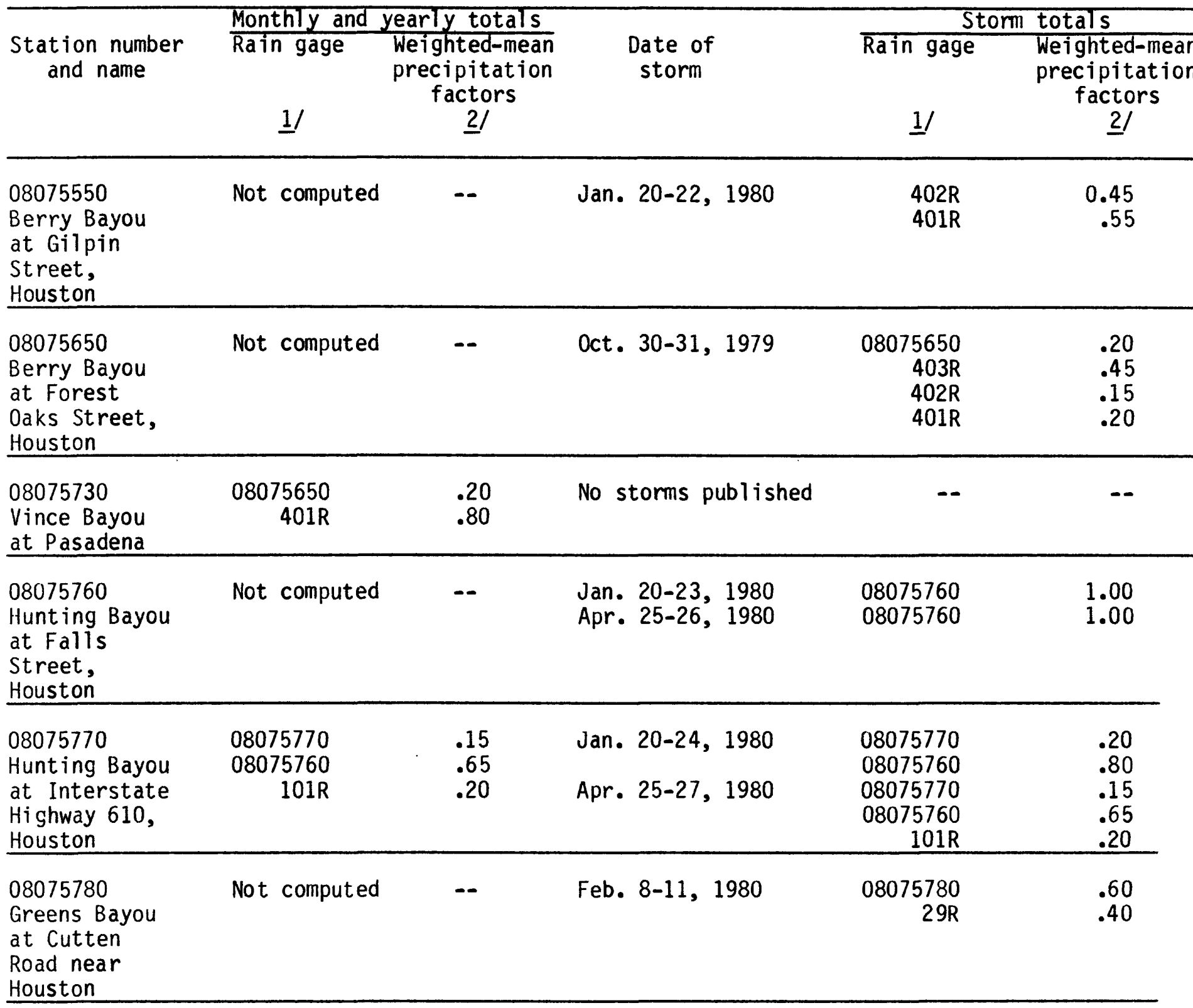

See footnotes at end of table. 
Table 2.--Weighted-mean precipitation factors for drainage basins above stations in the Houston metropolitan area--Continued

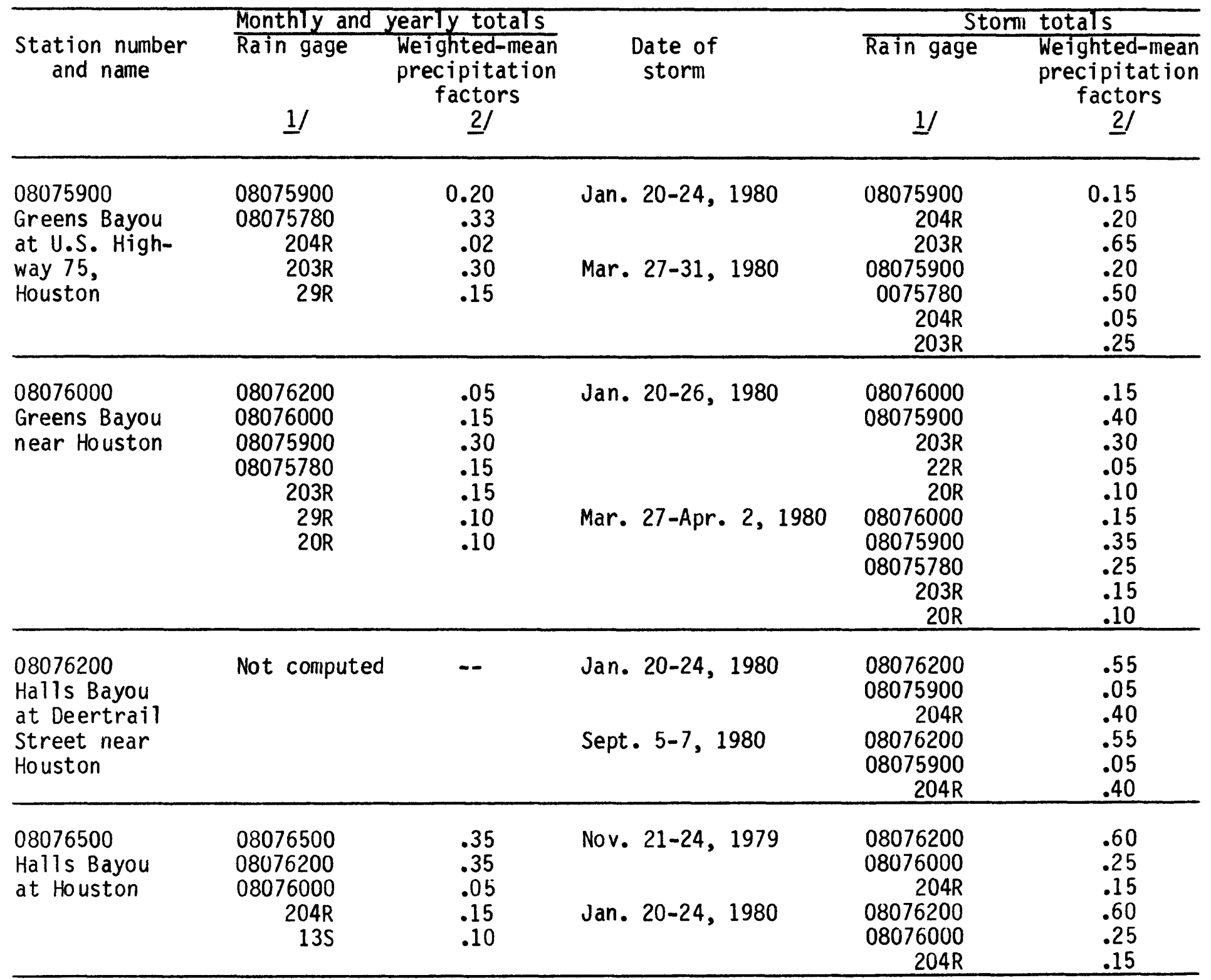

See footnotes at end of table. 
Table 2.--Weighted-mean precipitation factors for drainage basins above stations in the Houston metropolitan area--Continued

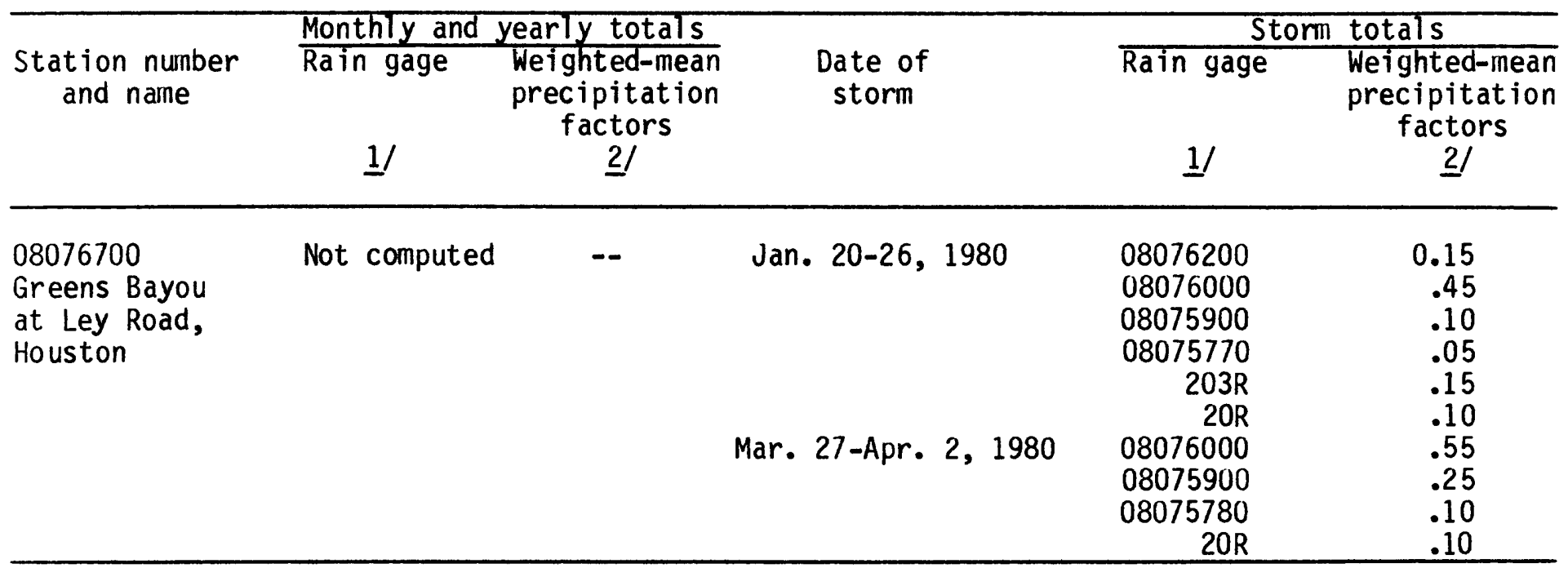

1/ See table 18 for locations of stations other than stream-gaging stations.

2/ See section on "Precipitation Data" for explanation of use of weighted-mean precipitation factors. 
greater than 2.0 inches. This storm combined with a storm on March 29 to produce a total rainfall ranging from more than 4.0 inches in the Whiteoak Bayou drainage basin to more than 2.5 inches throughout the metropolitan area.

The storm of January 20-22 was analyzed for all stations except those where rainfall distribution was suspect, where the quality of recorded data was poor, or where the stage-discharge relationship was poorly defined. The storms of 0ctober 30 and March 27-29 were analyzed for a selected number of stations based on the quality of the recorded data. Other storms were selected for analysis based on discharge, quality of recorded data, distribution of rainfall, and availability of water-quality data.

\section{Runoff Data}

Runoff data are based on discharge measurements and stage records at 18 continuous-record stream-gaging stations, and 16 flood-hydrograph partial-record stations.

Annual records of either daily discharge or maximum gage height at continuous-record stream-gaging stations, and maximum discharge at flood-hydrograph partial-record stations are given in the section "Compilation of data." Tables of storm runoff data, including accumulated rainfall totals, are also given for selected storms in the section "Compilation of data."

Figure 3 shows the accumulated monthly runoff from six basins for the 1980 water year and the average runoff for the period 1953-70. The average annual rainfall for the $1953-70$ period was 46 inches or approximately equal to the 30-year (1941-70) rainfall average of 48.19 inches at Houston. Figure 3 shows that runoff for the 1980 water year is appreciably greater than the average runoff for the period 1953-70 even through rainfall for the selected basins averaged at least 15 percent less during 1980 than the 46 -inch average during 1953-70. This high ratio of runoff to rainfall is one of the effects of the continual urban development in the metropolitan area. The figure also illustrates the effects of the January, March, and September rains on total runoff.

The most significant storms of the 1980 water year were those of October 30, January 20-22, and March 27-29. Data published in the section "Compilation of data" show computed storm runoff in excess of 2.5 inches from the storn of January 20-22 in all major drainage basins in the metropolitan area with the exception of the Whiteoak Bayou drainage basin. This storm was the inajor producer of the annual peak discharge for the 1980 water year. The storin of March 27-29, which was primarily confined to the Whiteoak Bayou and fireens Bayou drainage basins, also produced annual peak discharges at several sites. Computed runoff for this storm ranged from 1.5 to 2.8 inches. The runoff for the storm of 0ctober 30 ranged from 0.5 to 2.5 inches.

The ratio of runoff to rainfall was determined for all storms selected for analysis. The range of this ratio was 0.2 to 0.6 for the storm of 0ctober 30 . The storm of January 20-22 resulted in high ratio values with a range of 0.5 to 0.9. For this storm the ratio exceeded 0.75 for 11 sites, ranging from 0.76 to 0.92. The storm of March 27-29 al so resulted in high runoff to rainfall ratio 


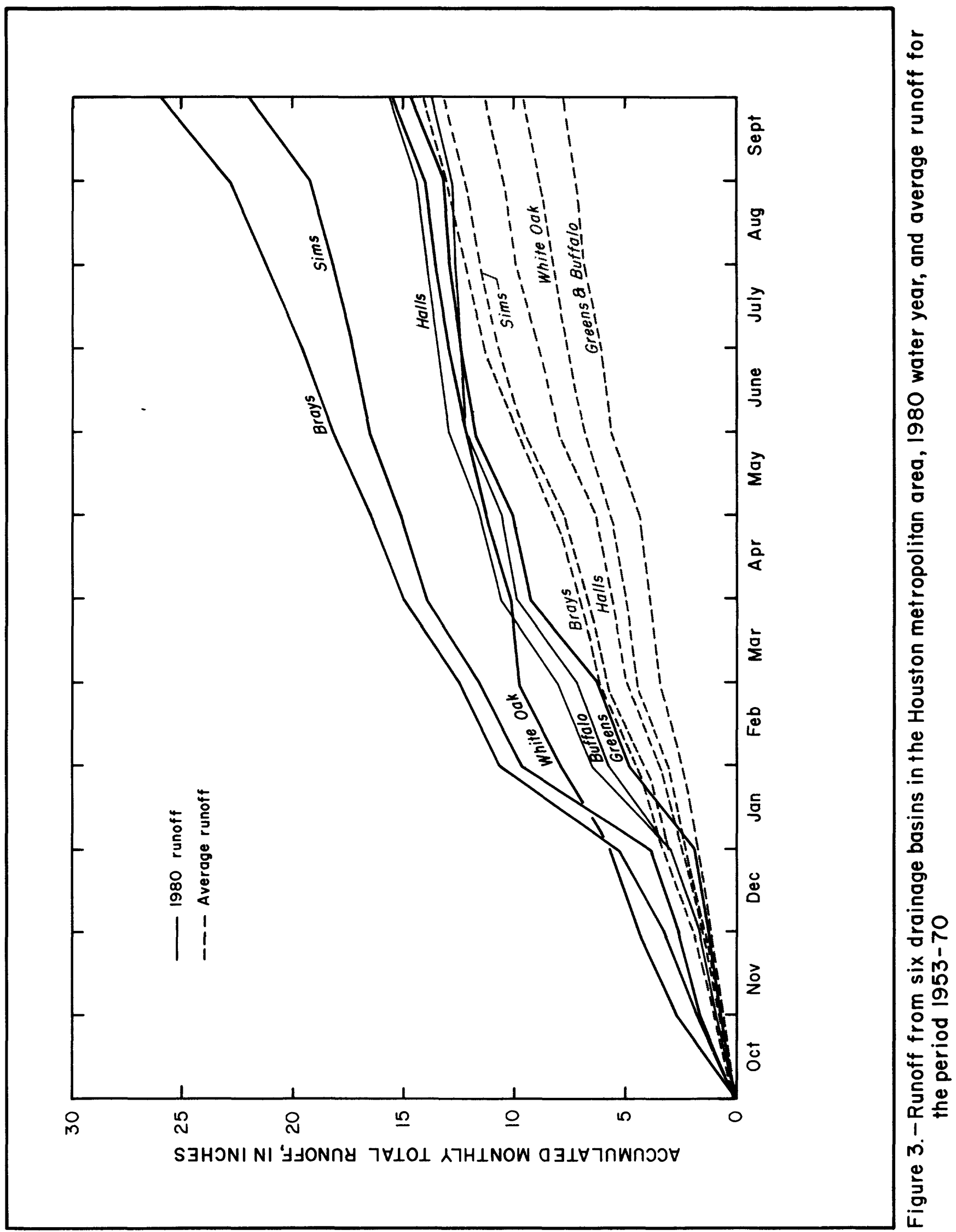


values ranging from 0.5 to 0.75 . A high ratio of runoff to rainfall may result from saturated soil moisture conditions, high intenstiy rainfall, and long duration rainfall in conjunction with highly developed drainage basins which include a large portion of impervious land cover and efficient storm drainage systems. However, caution is urged in the use of these computed values as the accuracies of the ratios may be adversely effected by inadequate rain gage coverage, indeterminate drainage area boundaries, basin exchange, and indefinite stage-discharge relationships.

Values for total storm runoff, storm peak discharge, ratio of runoff to rainfall, and other pertinent data for all storms analyzed in the 1980 water year are given in tables 3-17. A total of 12 storms have been analyzed for the 1980 water year resulting in a total of 51 separate storm-data 1istings. The storm dates and the number of stream-gaging stations for which data are published in the section "Compilation of data" are:

\begin{tabular}{rlc}
\hline No. & Storm Date & $\begin{array}{c}\text { Number of stations for } \\
\text { which data is published }\end{array}$ \\
1 & October 22 & 1 \\
2 & October 30 & 9 \\
3 & November 21-22 & 1 \\
4 & December 12-13 & 5 \\
5 & January 17 & 2 \\
6 & January 20-22 & 17 \\
7 & February 8-9 & 1 \\
8 & March 27-29 & 8 \\
9 & Apri1 25 & 3 \\
10 & June 9 & 1 \\
11 & Ju1y 21 & 1 \\
12 & September 5-7 & 2 \\
\hline
\end{tabular}

Water-Quality nata

Water-quality data were collected at 19 locations in the study area during the 1980 water year. The locations of the water-quality data collection sites are shown on figure 1. Water-quality data and streamflow data are presented in downstream order in the section "Compilation of data." Time and discharge values for water-quality data may vary slightly from those published in the Water Resources Data for Texas, 1980, report, due to the correction of some previously published values.

Water-quality data are collected from a wide range of discharge representing various flow and seasonal conditions, and include determinations for physical, chemical, and biological parameters. Physical determinations include measurements of temperature, $\mathrm{pH}$, turbidity, suspended and volatile solids, and color. Chemical analyses include specific conductance, dissolved oxygen, standard inorganic chemical (major ions), selected nutrient determinations of total organic carbon, nitrogen, and phosphorus. Chemical analyses of trace substances include minor elements, and pesticides. Biological analyses include measurements of BOD (biochemical oxygen demand) and bacterioloqical analyses for total coliform, fecal coliform, and fecal streptococci. 
Water samples were also collected during selected storms to determine the quality of storm runoff in the Houston metropolitan area. Storm dates and stations where at least three water-quality samples were collected during the storms are:

\begin{tabular}{|c|c|c|}
\hline $\begin{array}{l}\text { Station } \\
\text { no. }\end{array}$ & Station name & Date of storm \\
\hline 08074145 & Bingle Road Storm Sewer at Houston, Tex. & $\begin{array}{l}\text { June } 9,1980 \\
\text { July } 21,1980\end{array}$ \\
\hline 08074250 & $\begin{array}{l}\text { Brickhouse Gully at Costa Rica St., } \\
\text { Houston, Tex. }\end{array}$ & October 30, 1979 \\
\hline 08074400 & $\begin{array}{l}\text { Lazybrook Street Storm Sewer at Houston, } \\
\text { Tex. }\end{array}$ & April 25, 1980 \\
\hline 08074500 & Whiteoak Bayou at Houston, Tex. & $\begin{array}{l}\text { October } 22,1979 \\
\text { January } 17,1980\end{array}$ \\
\hline 08074540 & Little Whiteoak Bayou at Houston, Tex. & $\begin{array}{l}\text { December } 12-13,1979 \\
\text { January } 17,1980\end{array}$ \\
\hline 08074800 & $\begin{array}{l}\text { Keegans Bayou at Roark Road near } \\
\text { Houston, Tex. }\end{array}$ & December 12-13, 1979 \\
\hline 08075000 & Brays Rayou at Houston, Tex. & necemher 12-13, 1979 \\
\hline 08075760 & $\begin{array}{l}\text { Hunting Bayou at Falls Street, } \\
\text { Houston, Tex. }\end{array}$ & April 25, 1980 \\
\hline 08075770 & $\begin{array}{l}\text { Hunting Bayou at Interstate Hiqhway } \\
610 \text {, Houston, Tex. }\end{array}$ & April 2.5, 1980 \\
\hline 08076000 & Greens Rayou near Houston, Tex. & January 20-22, 1980 \\
\hline 08076500 & Halls Bayou at Houston, Tex. & January 20-22, 1980 \\
\hline 08076700 & Greens Bayou at Ley Road, Houston, Tex. & January 20-22, 1980 \\
\hline
\end{tabular}




\section{SELECTED REFERENCES}

Johnson, S. L., and Sayre, D. M., 1973, Effects of urbanization on floods in the Houston, Texas, metropolitan area: U.S. Geological Survey WaterResources Investigations 3-73, $50 \mathrm{p}$.

Linsley, R. K., Kohler, M. A., and Paulhus, J. L. H., 1949, Applied hydrology: New York, McGraw-Hill Book Company, Inc., 689 p.

Liscum, Fred, and Massey, B. C., 1980, Technique for estimating the magnitude and frequency of floods in the Houston, Texas, metropolitan area: U.S. Geological Survey Water-Resources Investigations 80-17, $40 \mathrm{p}$.

U.S. Department of Commerce, Clinatography of the United States No. 81 (by State), Monthly normals of temperature, precipitation, and heating and coolinq degree days, 1941-70, Texas: National 0ceanic and Atmospheric Administration Environmental Data Service, U.S. Department of Commerce publication.

U.S. Geological Survey, 1980, Water resources data for Texas, volume 2: U.S. Geological Survey Water-Data Report, TX-80-2, 472 p.

Wadde 11 , Kidd M., Massey, Bernard C., and Jennings, Marshall E., 1979, Use of the STORM model for estimating the quantity and quality of runoff from the metropolitan area of Houston, Texas: U.S. Geological Survey Water Resources Investigations $79-74,29 \mathrm{p}$. 
COMPILATION OF DATA

$-19-$ 
SAN JACINTO RIVER BASIN

08073500 BUFFALO BAYOU NEAR ADDICKS, TX

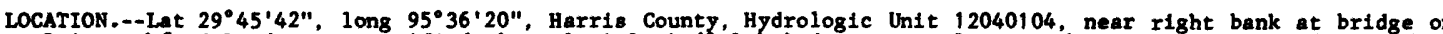
Dairy-Ashford Road over rectifled channel, $1.8 \mathrm{mi}(2.9 \mathrm{~km})$ downstream from South Mayde Creek, and $2.6 \mathrm{mi}$ (4.2 $\mathrm{km}$ ) Boutheast of Addicks.

DRAINAGE AREA.--293 $\mathrm{mi}^{2}\left(759 \mathrm{~km}^{2}\right)$, unadjusted for basin boundary changes.

WATER-DISCHARGE RECORDS

PERIOD OF RECORD.--August 1945 to current year.

REVISED RECORDS.--WSP 1922: Drainage area.

GAGE.--Water-stage recorder and crest-stage gages. Datum of gage $1 \mathrm{~s} 1.40 \mathrm{ft}$ (0.427 m) below National Geodetic Vertical Datum of 1929, 1973 adjustment; records unadjusted to land-surface subsidence. Prior to Feb. 2 , 1948 water-atage recorder at bridge on natural channel $1,200 \mathrm{ft}(370 \mathrm{~m})$ to right at $\mathrm{same}$ datum. Feb. 2 to May 21, 1948, nonrecording gage at present site and datum.

REMARKS.--Water-discharge records fair. Floodflow regulated by Barker and Addicks Reservoirs (stations 08072500 and 08073000$) 3.2$ and $3.0 \mathrm{mi}(5.1$ and $4.8 \mathrm{~km})$ upstream, respectively, total capacity $315,900 \mathrm{acre}-\mathrm{ft}(390 \mathrm{hm})^{2}$. Extreme low flow is sustained by drainage from irrigated lands.

AVERAGE DISCHARGE.--35 years, $208 \mathrm{ft}^{3} / \mathrm{s}\left(5.891 \mathrm{~m}^{3} / \mathrm{s}\right), 150,700 \mathrm{acre}-\mathrm{ft} / \mathrm{yr}\left(186 \mathrm{hm}^{3} / \mathrm{yr}^{\mathrm{r}}\right)$.

EXTREMES FOR PERIOD OF RECORD.--Maximum discharge, 11,200 $\mathrm{ft}^{3} / \mathrm{s}\left(317 \mathrm{~m}^{3} / \mathrm{s}\right)$ Aug. 29, 1945, gage helght, 81.23 ft $(24.759 \mathrm{~m})$, former site; no flow at times.

EXTREMES OUTSIDE PERIOD OF RECORD.--Maximum stage since at least 1896, $85.6 \mathrm{ft}$ (26.09 m) in December 1935 , adjusted to former site from floodmark $0.5 \mathrm{mi}(0.8 \mathrm{~km})$ downstream, on basis of lope of flood of Aug. 29 , ig45, from information by local resident.

EXTREMES FOR CURREIT YEAR.--Maximum discharge, $1,800 \mathrm{ft}^{3} / \mathrm{s}\left(51.0 \mathrm{~m}^{3} / \mathrm{s}\right)$ Jan. 25 at 1200 hours, gage height, 63.23 ft $(19.273 \mathrm{~m})$; maximum gage height, $63.35 \mathrm{ft}(19.309 \mathrm{~m})$ Oct. 1 at 1130 hours; minimum daily discharge, il ft; $\left(0.31 \mathrm{~m}^{3} / \mathrm{s}\right) \mathrm{Jan} .14-16$.

DISCHARGE, IN CUBIC FEET PER SECOND, WATER YEAR OCTOBER 1979 TO SEPTEMBER 1980

\begin{tabular}{|c|c|c|c|c|c|c|c|c|c|c|c|c|}
\hline DAY & OCT & NOV & DEC & JAN & FEB & MAR & APR & MAY & Jus & JUL & AUG & SEP \\
\hline $\begin{array}{l}1 \\
2 \\
3 \\
4 \\
5\end{array}$ & $\begin{array}{l}1630 \\
1610 \\
1600 \\
1580 \\
1560\end{array}$ & $\begin{array}{l}140 \\
350 \\
630 \\
594 \\
536\end{array}$ & $\begin{array}{l}52 \\
43 \\
35 \\
27 \\
24\end{array}$ & $\begin{array}{r}124 \\
64 \\
350 \\
508 \\
399\end{array}$ & $\begin{array}{l}1460 \\
1430 \\
1370 \\
1290 \\
1130\end{array}$ & $\begin{array}{l}42 \\
29 \\
24 \\
26 \\
27\end{array}$ & $\begin{array}{r}857 \\
1210 \\
1270 \\
1470 \\
1410\end{array}$ & $\begin{array}{l}26 \\
52 \\
32 \\
22 \\
18\end{array}$ & $\begin{array}{l}28 \\
27 \\
25 \\
25 \\
26\end{array}$ & $\begin{array}{l}26 \\
26 \\
24 \\
25 \\
24\end{array}$ & $\begin{array}{l}94 \\
73 \\
58 \\
52 \\
41\end{array}$ & $\begin{array}{l}33 \\
37 \\
34 \\
31 \\
66\end{array}$ \\
\hline $\begin{array}{r}6 \\
7 \\
8 \\
9 \\
10\end{array}$ & $\begin{array}{l}1540 \\
1520 \\
1500 \\
1460 \\
1430\end{array}$ & $\begin{array}{r}324 \\
49 \\
35 \\
34 \\
35\end{array}$ & $\begin{array}{l}22 \\
20 \\
19 \\
17 \\
16\end{array}$ & $\begin{array}{r}172 \\
63 \\
36 \\
22 \\
18\end{array}$ & $\begin{array}{r}399 \\
39 \\
231 \\
681 \\
804\end{array}$ & $\begin{array}{l}24 \\
22 \\
24 \\
22 \\
21\end{array}$ & $\begin{array}{r}1260 \\
504 \\
37 \\
25 \\
22\end{array}$ & $\begin{array}{l}16 \\
29 \\
30 \\
33 \\
25\end{array}$ & $\begin{array}{r}26 \\
23 \\
22 \\
216 \\
420\end{array}$ & $\begin{array}{l}24 \\
25 \\
26 \\
25 \\
25\end{array}$ & $\begin{array}{l}33 \\
88 \\
73 \\
35 \\
29\end{array}$ & $\begin{array}{r}334 \\
224 \\
540 \\
1000 \\
1080\end{array}$ \\
\hline $\begin{array}{l}11 \\
12 \\
13 \\
14 \\
15\end{array}$ & $\begin{array}{r}1360 \\
1160 \\
943 \\
740 \\
501\end{array}$ & $\begin{array}{l}31 \\
28 \\
25 \\
21 \\
17\end{array}$ & $\begin{array}{r}16 \\
480 \\
489 \\
1120 \\
1480\end{array}$ & $\begin{array}{l}16 \\
14 \\
13 \\
11 \\
11\end{array}$ & $\begin{array}{l}794 \\
771 \\
746 \\
737 \\
690\end{array}$ & $\begin{array}{l}22 \\
22 \\
21 \\
18 \\
18\end{array}$ & $\begin{array}{l}20 \\
18 \\
44 \\
25 \\
20\end{array}$ & $\begin{array}{l}21 \\
21 \\
22 \\
49 \\
93\end{array}$ & $\begin{array}{r}311 \\
86 \\
53 \\
44 \\
41\end{array}$ & $\begin{array}{l}28 \\
33 \\
35 \\
34 \\
31\end{array}$ & $\begin{array}{l}27 \\
25 \\
23 \\
21 \\
58\end{array}$ & $\begin{array}{r}888 \\
651 \\
232 \\
81 \\
61\end{array}$ \\
\hline $\begin{array}{l}16 \\
17 \\
18 \\
19 \\
20\end{array}$ & $\begin{array}{r}150 \\
60 \\
47 \\
57 \\
50\end{array}$ & $\begin{array}{r}16 \\
16 \\
16 \\
19 \\
286\end{array}$ & $\begin{array}{r}1430 \\
1360 \\
1190 \\
782 \\
412\end{array}$ & $\begin{array}{r}11 \\
110 \\
315 \\
285 \\
297\end{array}$ & $\begin{array}{r}598 \\
437 \\
216 \\
81 \\
59\end{array}$ & $\begin{array}{l}19 \\
23 \\
24 \\
21 \\
44\end{array}$ & $\begin{array}{l}18 \\
17 \\
16 \\
16 \\
15\end{array}$ & $\begin{array}{l}357 \\
428 \\
347 \\
386 \\
710\end{array}$ & $\begin{array}{l}37 \\
35 \\
34 \\
32 \\
32\end{array}$ & $\begin{array}{l}47 \\
43 \\
36 \\
40 \\
44\end{array}$ & $\begin{array}{l}46 \\
38 \\
30 \\
25 \\
24\end{array}$ & $\begin{array}{l}52 \\
44 \\
41 \\
42 \\
42\end{array}$ \\
\hline $\begin{array}{l}21 \\
22 \\
23 \\
24 \\
25\end{array}$ & $\begin{array}{l}47 \\
60 \\
50 \\
33 \\
30\end{array}$ & $\begin{array}{r}633 \\
716 \\
846 \\
1170 \\
1380\end{array}$ & $\begin{array}{r}67 \\
48 \\
138 \\
125 \\
140\end{array}$ & $\begin{array}{r}1080 \\
764 \\
676 \\
1370 \\
1770\end{array}$ & $\begin{array}{l}48 \\
42 \\
37 \\
32 \\
28\end{array}$ & $\begin{array}{l}42 \\
24 \\
21 \\
24 \\
21\end{array}$ & $\begin{array}{l}15 \\
15 \\
15 \\
15 \\
60\end{array}$ & $\begin{array}{r}702 \\
488 \\
1090 \\
691 \\
609\end{array}$ & $\begin{array}{r}30 \\
142 \\
46 \\
36 \\
30\end{array}$ & $\begin{array}{l}81 \\
84 \\
78 \\
74 \\
79\end{array}$ & $\begin{array}{l}25 \\
24 \\
25 \\
28 \\
29\end{array}$ & $\begin{array}{r}40 \\
44 \\
40 \\
47 \\
104\end{array}$ \\
\hline $\begin{array}{l}26 \\
27 \\
28 \\
29 \\
30 \\
31\end{array}$ & $\begin{array}{r}32 \\
33 \\
39 \\
38 \\
300 \\
450\end{array}$ & $\begin{array}{r}1360 \\
1300 \\
1190 \\
683 \\
70 \\
-\end{array}$ & $\begin{array}{r}66 \\
44 \\
36 \\
353 \\
459 \\
359\end{array}$ & $\begin{array}{l}1680 \\
1650 \\
1600 \\
1570 \\
1540 \\
1510\end{array}$ & $\begin{array}{r}25 \\
25 \\
25 \\
25 \\
-- \\
--\end{array}$ & $\begin{array}{r}21 \\
610 \\
616 \\
773 \\
300 \\
355\end{array}$ & $\begin{array}{r}30 \\
20 \\
18 \\
16 \\
16 \\
---\end{array}$ & $\begin{array}{r}444 \\
42 \\
35 \\
36 \\
55 \\
39\end{array}$ & $\begin{array}{r}27 \\
26 \\
27 \\
27 \\
25 \\
-\end{array}$ & $\begin{array}{r}89 \\
104 \\
123 \\
201 \\
208 \\
139\end{array}$ & $\begin{array}{l}25 \\
41 \\
27 \\
40 \\
51 \\
40\end{array}$ & $\begin{array}{r}100 \\
110 \\
100 \\
81 \\
304 \\
-\end{array}$ \\
\hline $\begin{array}{l}\text { TOTAL } \\
\text { MEAN } \\
\text { MAX } \\
\text { MIN } \\
\text { AC-FT }\end{array}$ & $\begin{array}{r}21610 \\
697 \\
1630 \\
30 \\
42860\end{array}$ & $\begin{array}{r}12550 \\
418 \\
1380 \\
16 \\
24890\end{array}$ & $\begin{array}{r}10869 \\
351 \\
1480 \\
16 \\
21560\end{array}$ & $\begin{array}{r}18049 \\
582 \\
1770 \\
11 \\
35800\end{array}$ & $\begin{array}{r}14250 \\
491 \\
1460 \\
25 \\
28260\end{array}$ & $\begin{array}{r}3300 \\
106 \\
773 \\
18 \\
6550\end{array}$ & $\begin{array}{r}8494 \\
283 \\
1470 \\
15 \\
16850\end{array}$ & $\begin{array}{r}6948 \\
224 \\
1090 \\
16 \\
13780\end{array}$ & $\begin{array}{r}1959 \\
65.3 \\
420 \\
22 \\
3890\end{array}$ & $\begin{array}{r}1881 \\
60.7 \\
208 \\
24 \\
3730\end{array}$ & $\begin{array}{r}1248 \\
40.3 \\
94 \\
21 \\
2480\end{array}$ & $\begin{array}{r}6483 \\
216 \\
1080 \\
31 \\
12860\end{array}$ \\
\hline
\end{tabular}

$\begin{array}{llllllllll}\text { CAL YR } 1979 & \text { TOTAL } 152939 & \text { MEAN } 419 & \text { MAX } & 1740 & \text { MIN } 16 & \text { AC-FT } & 303400\end{array}$ 
SAN JACINTO RIVER BASIN

08073500 BUFFALO BAYOU NEAR ADDICKS, TX--Continued

WATER-QUALITY RECORDS

PERIOD OF RECORD.--Chemical, blochemical, and pesticide analyses: August 1970 to current year.

WATER QUALITY DATA, WATER YEAR OCTOBER 1979 TO SEPTEMBER 1980

\begin{tabular}{|c|c|c|c|c|c|c|c|c|c|c|}
\hline DATE & T IME & $\begin{array}{l}\text { STREAM- } \\
\text { FLOW, } \\
\text { INSTAN- } \\
\text { TANEOUS } \\
\text { (CFS) }\end{array}$ & $\begin{array}{l}\text { SPE- } \\
\text { CIFIC } \\
\text { CON- } \\
\text { DUCT- } \\
\text { ANCE } \\
\text { (MICRO- } \\
\text { MHOS) }\end{array}$ & $\begin{array}{c}\text { PH } \\
\text { FIELD } \\
\text { (UNITS) }\end{array}$ & $\begin{array}{c}\text { TEMPER- } \\
\text { ATURE, } \\
\text { WATER } \\
\text { (DEG C) }\end{array}$ & $\begin{array}{l}\text { COLOR } \\
\text { (PLAT- } \\
\text { INUM } \\
\text { COBALT } \\
\text { UNITS) }\end{array}$ & $\begin{array}{l}\text { TUR- } \\
\text { BID- } \\
\text { ITY } \\
\text { (NTU) }\end{array}$ & $\begin{array}{c}\text { OXYGEN, } \\
\text { DIS - } \\
\text { SOLVED } \\
(M G / L)\end{array}$ & $\begin{array}{c}\text { OXYGEN, } \\
\text { DIS- } \\
\text { SOLVED } \\
\text { (PER- } \\
\text { CENT } \\
\text { SATUR- } \\
\text { ATION) }\end{array}$ & $\begin{array}{c}\text { OXYGEN } \\
\text { DEMAND, } \\
\text { BIOCHEM } \\
\text { UNINHI B } \\
5 \text { DAY } \\
(\mathrm{MG} / \mathrm{L})\end{array}$ \\
\hline $\begin{array}{l}\text { OcT } \\
04 \ldots \\
11 \ldots \\
15 \ldots \\
\text { Nov }\end{array}$ & $\begin{array}{l}0900 \\
0935 \\
1005\end{array}$ & $\begin{array}{r}1800 \\
1550 \\
496\end{array}$ & $\begin{array}{l}112 \\
165 \\
251\end{array}$ & $\begin{array}{l}6.8 \\
6.9 \\
7.9\end{array}$ & $\begin{array}{l}25.0 \\
22.0 \\
21.0\end{array}$ & $\begin{array}{r}70 \\
100 \\
110\end{array}$ & $\begin{array}{r}12 \\
4.3 \\
3.8\end{array}$ & $\begin{array}{l}3.1 \\
2.9 \\
6.8\end{array}$ & $\begin{array}{l}37 \\
33 \\
76\end{array}$ & $\begin{array}{c}6.2 \\
14 \\
4.0\end{array}$ \\
\hline${ }_{J A N}^{28} \ldots$ & 1055 & 1200 & 139 & 6.9 & 14.0 & 160 & 50 & 9.1 & 86 & 4.5 \\
\hline${ }_{\text {MAY }}^{29} \ldots$ & 1255 & 1570 & 96 & 6.7 & 12.0 & 140 & 96 & 7.0 & 64 & 3.2 \\
\hline $\begin{array}{r}19 \ldots \\
21 \ldots \\
\text { AUG }\end{array}$ & $\begin{array}{l}1130 \\
0950\end{array}$ & $\begin{array}{r}489 \\
1150\end{array}$ & $\begin{array}{l}200 \\
110\end{array}$ & $\begin{array}{l}6.7 \\
6.7\end{array}$ & $\begin{array}{l}23.0 \\
24.0\end{array}$ & 160 & $160^{--}$ & $\begin{array}{l}7.0 \\
5.6\end{array}$ & $\begin{array}{l}80 \\
66\end{array}$ & $\begin{array}{l}6.5 \\
5.8\end{array}$ \\
\hline & 1140 & 52 & 600 & 7.6 & 28.0 & 30 & 39 & 6.5 & 82 & 4.5 \\
\hline $09 \ldots$ & 1130 & 985 & 190 & 7.1 & 26.0 & 86 & 72 & 7.3 & 89 & 3.5 \\
\hline
\end{tabular}

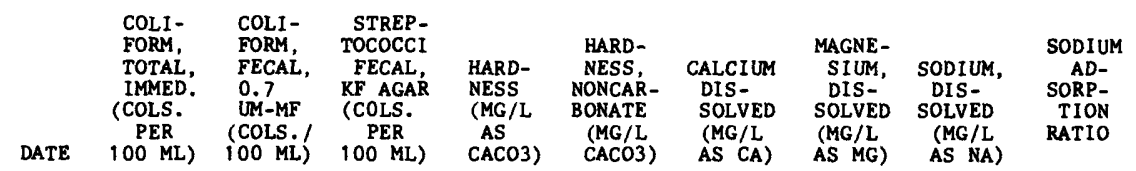

\begin{tabular}{|c|c|c|c|c|c|c|c|c|c|}
\hline \multicolumn{10}{|l|}{ OCT } \\
\hline $\begin{array}{l}04 \ldots \\
11 \ldots\end{array}$ & $\begin{array}{l}10000 \\
12000\end{array}$ & $\begin{array}{r}40 \\
\times 14\end{array}$ & $\begin{array}{r}40 \\
350\end{array}$ & $\begin{array}{l}38 \\
52\end{array}$ & $\begin{array}{l}0 \\
0\end{array}$ & $\begin{array}{l}11 \\
15\end{array}$ & $\begin{array}{l}2.5 \\
3.6\end{array}$ & $\begin{array}{l}6.3 \\
9.2\end{array}$ & $\begin{array}{l}.4 \\
.6\end{array}$ \\
\hline NOV & 3100 & 40 & 60 & - & $\therefore$ & $=$ & $=$ & $\therefore$ & - \\
\hline$\underset{\text { JAN }}{28} \ldots$ & 6700 & 240 & 620 & - & -- & -. & -- & -- & - \\
\hline MAY & 2000 & 190 & 240 & - & - & -- & - & - & - \\
\hline $\begin{array}{l}19 \ldots \\
21 \ldots \\
\text { AUG }\end{array}$ & $\begin{array}{r}31000 \\
6700\end{array}$ & $\begin{array}{l}5200 \\
1400\end{array}$ & $\begin{array}{r}8100 \\
820\end{array}$ & $\begin{array}{l}58 \\
--\end{array}$ & 2 & 19 & $\begin{array}{r}2.6 \\
--\end{array}$ & 14 & .8 \\
\hline $\begin{array}{l}04 . \cdots \\
\text { SEP }\end{array}$ & 13000 & 820 & 98 & 150 & 0 & 48 & 7.6 & 65 & 2.3 \\
\hline $09 \ldots$ & 15000 & 1000 & 1300 & -- & -- & -- & -- & -- & -- \\
\hline DAT & $\begin{array}{l}\text { POTAS- } \\
\text { SIUM, } \\
\text { DIS- } \\
\text { SOLVED } \\
\text { (MG / L } \\
\text { AS K) }\end{array}$ & $\begin{array}{c}\text { BICAR- } \\
\text { BONATE } \\
\text { (MG /L } \\
\text { AS } \\
\text { HCO3) }\end{array}$ & $\begin{array}{c}\text { CAR- } \\
\text { BONATE } \\
(\text { MG } / \mathrm{L} \\
\text { AS CO3) }\end{array}$ & $\begin{array}{l}\text { SULFATE } \\
\text { DIS- } \\
\text { SOLVED } \\
\text { (MG/L } \\
\text { AS SO4) }\end{array}$ & $\begin{array}{l}\text { CHLO- } \\
\text { RIDE, } \\
\text { DIS- } \\
\text { SOLVED } \\
\text { (MG/L } \\
\text { AS CL) }\end{array}$ & $\begin{array}{l}\text { FLUO- } \\
\text { RIDE, } \\
\text { DIS- } \\
\text { SOLVED } \\
(M G / L \\
\text { AS F) }\end{array}$ & $\begin{array}{l}\text { SILICA, } \\
\text { DIS- } \\
\text { SOLVED } \\
\text { (MG/L } \\
\text { AS } \\
\text { SIO2) }\end{array}$ & $\begin{array}{c}\text { SOLIDS, } \\
\text { SUM OF } \\
\text { CONSTI - } \\
\text { TUENTS, } \\
\text { DIS- } \\
\text { SOLVED } \\
\text { (MG/L) }\end{array}$ & $\begin{array}{l}\text { SOLIDS, } \\
\text { RESIDUE } \\
\text { AT IOS } \\
\text { DEG. C, } \\
\text { SUS - } \\
\text { PENDED } \\
\text { (MG /L) }\end{array}$ \\
\hline
\end{tabular}

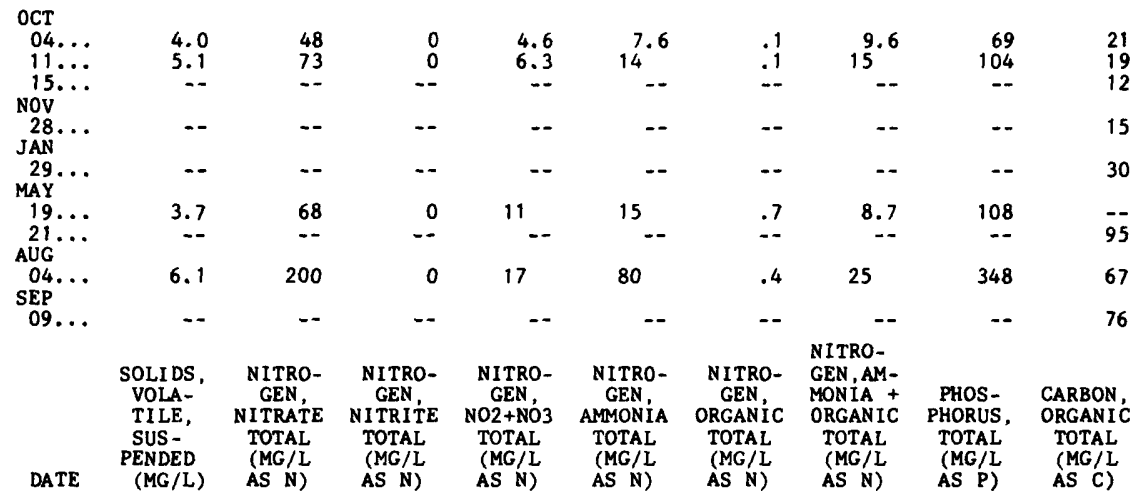

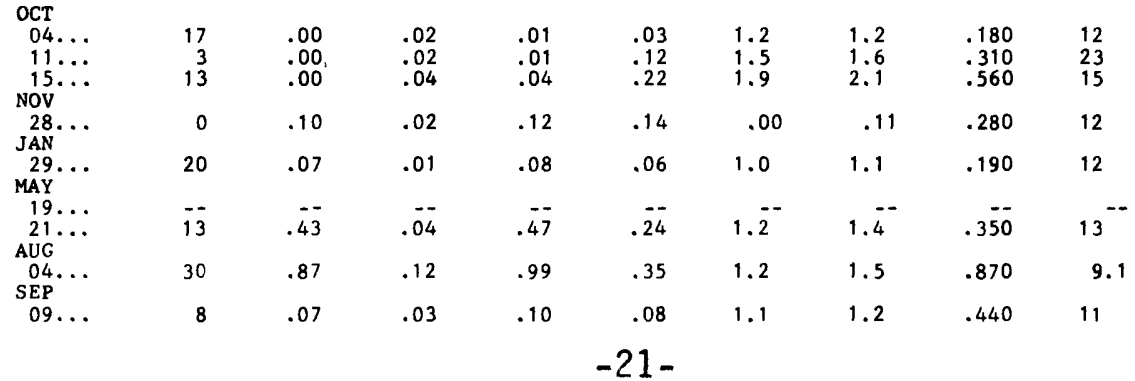


SAN JACINTO RIVER BASIN

08073500 BUFFALO BAYOU NEAR ADDICKS, TX--Continued

WATER QUALITY DATA, WATER YEAR OCTOBER 1979 TO SEPTEMBER 1980

\begin{tabular}{|c|c|c|c|c|c|c|c|}
\hline DATE & TIME & $\begin{array}{c}\text { ARSENIC } \\
\text { DIS- } \\
\text { SOLVED } \\
\text { (UG /L } \\
\text { AS AS) }\end{array}$ & $\begin{array}{l}\text { BARIUM, } \\
\text { DIS- } \\
\text { SOLVED } \\
\text { (UG/L } \\
\text { AS BA) }\end{array}$ & $\begin{array}{c}\text { CADMIUM } \\
\text { DIS- } \\
\text { SOLVED } \\
\text { (UG/L } \\
\text { AS CD) }\end{array}$ & $\begin{array}{l}\text { CHRO- } \\
\text { MIUM, } \\
\text { DIS- } \\
\text { SOLVED } \\
\text { (UG/L } \\
\text { AS CR) }\end{array}$ & $\begin{array}{c}\text { COPPER, } \\
\text { DIS- } \\
\text { SOLVED } \\
\text { (UG/L } \\
\text { AS CU) }\end{array}$ & $\begin{array}{l}\text { IRON, } \\
\text { DIS- } \\
\text { SOLVED } \\
\text { (UG/L } \\
\text { AS FE) }\end{array}$ \\
\hline $\begin{array}{l}\text { MAY } \\
19 . . . \\
\text { AUG }\end{array}$ & 1130 & 3 & 90 & $<1$ & 0 & 4 & 80 \\
\hline $04 \ldots$ & 1140 & 5 & 200 & $<1$ & 10 & 1 & 20 \\
\hline
\end{tabular}

\begin{tabular}{|c|c|c|c|c|c|c|}
\hline DATE & $\begin{array}{l}\text { LEAD, } \\
\text { DIS- } \\
\text { SOLVED } \\
\text { (UG/L } \\
\text { AS PB) }\end{array}$ & $\begin{array}{l}\text { MANGA- } \\
\text { NESE, } \\
\text { DIS- } \\
\text { SOLVED } \\
\text { (UG/L } \\
\text { AS MN) }\end{array}$ & $\begin{array}{c}\text { MERCURY } \\
\text { DIS- } \\
\text { SOLVED } \\
\text { (UG/L } \\
\text { AS HG) }\end{array}$ & $\begin{array}{l}\text { SELE- } \\
\text { NIUM, } \\
\text { DIS- } \\
\text { SOLVED } \\
\text { (UG/L } \\
\text { AS SE) }\end{array}$ & $\begin{array}{c}\text { SILVER, } \\
\text { DIS- } \\
\text { SOLVED } \\
\text { (UG/L } \\
\text { AS AG) }\end{array}$ & $\begin{array}{l}\text { ZINC, } \\
\text { DIS- } \\
\text { SOLVED } \\
\text { (UG/L } \\
\text { AS ZN) }\end{array}$ \\
\hline $\begin{array}{l}\text { MAY } \\
19 . \ldots \\
\text { AUG }\end{array}$ & 0 & 6 & .1 & 0 & 0 & 10 \\
\hline & 0 & $<1$ & .0 & 0 & 0 & $<3$ \\
\hline
\end{tabular}

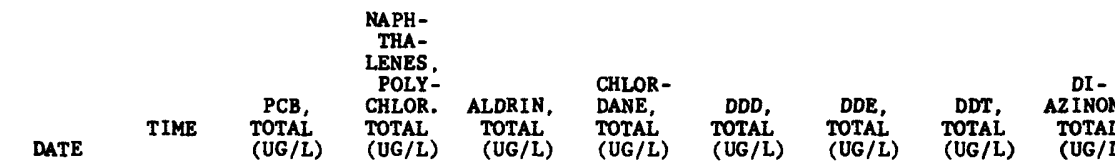

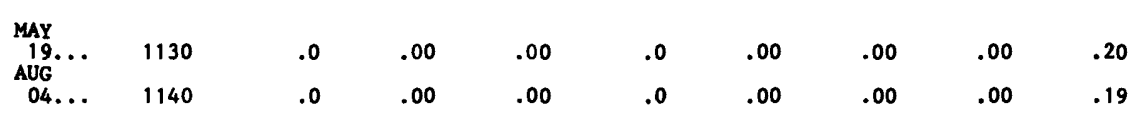

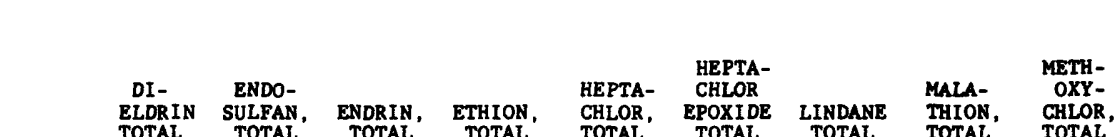

$\begin{array}{cccccccccc} & \text { ELDRIN } & \text { SULFAN, } & \text { ENDRIN, } & \text { ETHION, } & \text { CHLOR, } & \text { EPOXIDE } & \text { LINDANE } & \text { THION, } & \text { CHLOR, } \\ \text { DATE } & \text { TOTAL } & \text { TOTAL } & \text { TOTAL } & \text { TOTAL } & \text { TOTAL } & \text { TOTAL } & \text { TOTAL } & \text { TOTAL } & \text { TOTAL } \\ \text { (UG } / L) & (U G / L) & \text { (UG/L) } & \text { (UG/L) } & \text { (UG/L) } & \text { (UG/L) } & \text { (UG/L) } & \text { (UG/L) }\end{array}$

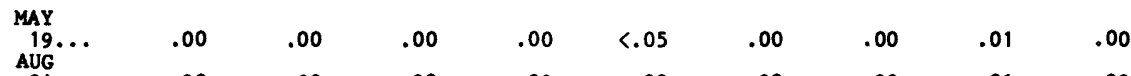

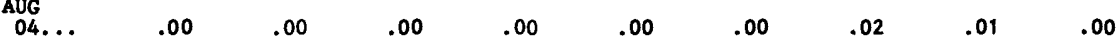

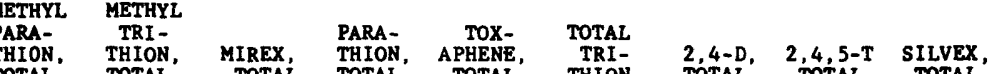

THION, THION, MIREX, THION, APHENE, TRI- 2,4-D, 2,4,5-T SILVEX,

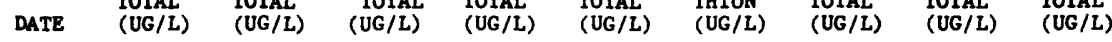

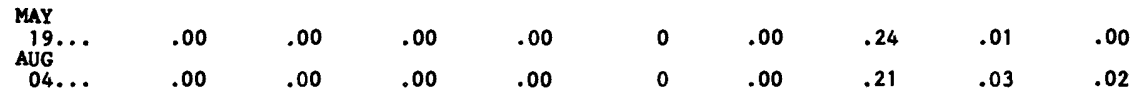


SAN JACINTO RIVER BASIN

08073600 BUfFALO BAYOU AT WEST BELT DRIVE, HOUSTON, TX

LOCATION.--Lat 29 $45^{\prime} 43^{\prime \prime}$, long $95^{\circ} 33^{\prime} 27^{\prime \prime}$, Harris County, Hydrologic Unit 12040104, at downstrean side of bridge on West Belt Drive in west Houston, $100 \mathrm{ft}$ (30 m) downstream from Rummel Creek, $3.5 \mathrm{mi}$ (5.6 km) downstream from station 08073500 , and $3.7 \mathrm{mi}(6.0 \mathrm{~km})$ upstream from station 08073700 .

DRAINAGE AREA. - -307 $\mathrm{mi}^{2}\left(795 \mathrm{~km}^{2}\right)$, unadjusted for basin boundary changes.

WATER-DISCHARGE RECORDS

PERIOD OF RECORD.---September 1971 to current year.

GAGE.--Water-stage recorders and crest-stage gage. Datum of gage 180.67 ft (0.204 m) below National Geodetic Verticsl Datum of 1929,1973 adjustment.

REMARKS.--Records falr. Floodflow regulated by Barker and Addlcks Reservolrs (statlons 08072500 and 08073000 ) 10.1 and $10.3 \mathrm{ml}(16.3$ and $16.6 \mathrm{~km})$ upstream, respectively. Low flow is sustalned by sewage effluent from Houston suburbs. The Corps of Engineers has a gage-helght telemeter at station.

AVERAGE DISCHARGE.--9 years, $322 \mathrm{ft}^{3} / \mathrm{s}\left(9.119 \mathrm{~m}^{3} / \mathrm{s}\right), 233,300 \mathrm{acre}-\mathrm{ft} / \mathrm{yr}$ (288 has/yr).

EXTREMES FOR PERIOD OF RECORD.--Maximum discharge, 3,770 $\mathrm{ft}^{3} / \mathrm{s}\left(107 \mathrm{~m}^{\mathrm{s}} / \mathrm{s}\right) \mathrm{Mar}$. 20, 1972, gage helght, 62.15 ft $(18.943 \mathrm{~m})$; minlmum dally, $25 \mathrm{ft}^{3} / \mathrm{s}\left(0.71 \mathrm{~m}^{3} / \mathrm{s}\right)$ Nov. $21,1971$.

EXTREMES FOR CURRENT YEAR.--Maxlmum discharge, $1,810 \mathrm{ft}^{3} / \mathrm{s}\left(51.3 \mathrm{~m}^{3} / \mathrm{s}\right)$ June 22 at $1830 \mathrm{hours}$, gage helght, $53.72 \mathrm{ft}$ $(16.374 \mathrm{~m}) ; \mathrm{minimum}$ dally, $36 \mathrm{ft}^{3 / \mathrm{s}}\left(1.02 \mathrm{~m}^{3} / \mathrm{s}\right) \mathrm{Jan} .14-16$.

DISCHARGE, IN CUBIC FEET PER SECOND, WATER YEAR OCTOBER 1979 TO SEPTEMBER 1980

\begin{tabular}{|c|c|c|c|c|c|c|c|c|c|c|c|c|}
\hline DAY & OCT & NOV & DEC & JAN & FEB & MAR & APK & MAY & JUN & JUL & AUG & SEP \\
\hline $\begin{array}{l}1 \\
2 \\
3 \\
4 \\
5\end{array}$ & $\begin{array}{l}1590 \\
1580 \\
1570 \\
1560 \\
1550\end{array}$ & $\begin{array}{l}178 \\
500 \\
698 \\
669 \\
615\end{array}$ & $\begin{array}{l}85 \\
69 \\
59 \\
54 \\
50\end{array}$ & $\begin{array}{r}166 \\
99 \\
369 \\
465 \\
396\end{array}$ & $\begin{array}{l}1450 \\
1420 \\
1360 \\
1280 \\
1140\end{array}$ & $\begin{array}{r}88 \\
67 \\
56 \\
62 \\
61\end{array}$ & $\begin{array}{r}754 \\
1160 \\
1220 \\
1440 \\
1400\end{array}$ & $\begin{array}{l}72 \\
95 \\
72 \\
58 \\
52\end{array}$ & $\begin{array}{l}51 \\
51 \\
48 \\
48 \\
49\end{array}$ & $\begin{array}{l}48 \\
47 \\
45 \\
46 \\
44\end{array}$ & $\begin{array}{r}114 \\
97 \\
81 \\
75 \\
68\end{array}$ & $\begin{array}{r}60 \\
66 \\
62 \\
58 \\
113\end{array}$ \\
\hline $\begin{array}{r}6 \\
7 \\
8 \\
9 \\
10\end{array}$ & $\begin{array}{l}1540 \\
1530 \\
1510 \\
1480 \\
1450\end{array}$ & $\begin{array}{r}374 \\
89 \\
70 \\
66 \\
70\end{array}$ & $\begin{array}{l}46 \\
45 \\
44 \\
42 \\
39\end{array}$ & $\begin{array}{r}209 \\
110 \\
79 \\
52 \\
44\end{array}$ & $\begin{array}{r}504 \\
82 \\
342 \\
678 \\
763\end{array}$ & $\begin{array}{l}54 \\
53 \\
56 \\
54 \\
51\end{array}$ & $\begin{array}{r}1270 \\
542 \\
79 \\
64 \\
58\end{array}$ & $\begin{array}{l}47 \\
79 \\
79 \\
76 \\
61\end{array}$ & $\begin{array}{r}46 \\
44 \\
43 \\
307 \\
436\end{array}$ & $\begin{array}{l}44 \\
46 \\
47 \\
45 \\
46\end{array}$ & $\begin{array}{r}56 \\
137 \\
105 \\
63 \\
52\end{array}$ & $\begin{array}{r}486 \\
255 \\
411 \\
958 \\
1090\end{array}$ \\
\hline $\begin{array}{l}11 \\
12 \\
13 \\
14 \\
15\end{array}$ & $\begin{array}{r}1400 \\
1240 \\
1030 \\
896 \\
566\end{array}$ & $\begin{array}{l}63 \\
62 \\
56 \\
55 \\
48\end{array}$ & $\begin{array}{r}41 \\
735 \\
472 \\
979 \\
1440\end{array}$ & $\begin{array}{l}42 \\
40 \\
38 \\
36 \\
36\end{array}$ & $\begin{array}{l}764 \\
740 \\
714 \\
737 \\
702\end{array}$ & $\begin{array}{l}51 \\
53 \\
51 \\
47 \\
49\end{array}$ & $\begin{array}{r}56 \\
57 \\
104 \\
65 \\
56\end{array}$ & $\begin{array}{r}52 \\
53 \\
64 \\
119 \\
124\end{array}$ & $\begin{array}{r}331 \\
107 \\
64 \\
55 \\
51\end{array}$ & $\begin{array}{l}49 \\
55 \\
58 \\
57 \\
49\end{array}$ & $\begin{array}{r}50 \\
46 \\
43 \\
41 \\
137\end{array}$ & $\begin{array}{r}967 \\
746 \\
360 \\
115 \\
90\end{array}$ \\
\hline $\begin{array}{l}16 \\
17 \\
18 \\
19 \\
20\end{array}$ & $\begin{array}{r}323 \\
88 \\
81 \\
86 \\
79\end{array}$ & $\begin{array}{r}51 \\
51 \\
51 \\
54 \\
275\end{array}$ & $\begin{array}{r}1420 \\
1350 \\
1230 \\
823 \\
437\end{array}$ & $\begin{array}{r}36 \\
201 \\
293 \\
314 \\
393\end{array}$ & $\begin{array}{l}628 \\
438 \\
261 \\
132 \\
107\end{array}$ & $\begin{array}{l}53 \\
73 \\
58 \\
53 \\
92\end{array}$ & $\begin{array}{l}51 \\
47 \\
51 \\
49 \\
44\end{array}$ & $\begin{array}{l}398 \\
489 \\
341 \\
536 \\
668\end{array}$ & $\begin{array}{l}48 \\
44 \\
44 \\
42 \\
41\end{array}$ & $\begin{array}{l}69 \\
66 \\
56 \\
59 \\
66\end{array}$ & $\begin{array}{l}79 \\
63 \\
53 \\
47 \\
43\end{array}$ & $\begin{array}{l}76 \\
69\end{array}$ \\
\hline $\begin{array}{l}21 \\
22 \\
23 \\
24 \\
25\end{array}$ & $\begin{array}{l}77 \\
81 \\
76 \\
68 \\
62\end{array}$ & $\begin{array}{r}711 \\
850 \\
880 \\
1120 \\
1340\end{array}$ & $\begin{array}{r}125 \\
90 \\
191 \\
168 \\
165\end{array}$ & $\begin{array}{r}1370 \\
1150 \\
642 \\
1250 \\
1740\end{array}$ & $\begin{array}{l}94 \\
84 \\
77 \\
70 \\
64\end{array}$ & $\begin{array}{l}76 \\
49 \\
49 \\
52 \\
44\end{array}$ & $\begin{array}{r}47 \\
48 \\
47 \\
51 \\
168\end{array}$ & $\begin{array}{r}810 \\
456 \\
1020 \\
716 \\
630\end{array}$ & $\begin{array}{r}60 \\
459 \\
169 \\
68 \\
57\end{array}$ & $\begin{array}{r}202 \\
109 \\
97 \\
91 \\
93\end{array}$ & $\begin{array}{l}45 \\
44 \\
43 \\
45 \\
48\end{array}$ & $\begin{array}{r}70 \\
134\end{array}$ \\
\hline $\begin{array}{l}26 \\
27 \\
28 \\
29 \\
30 \\
31\end{array}$ & $\begin{array}{r}64 \\
63 \\
73 \\
70 \\
290 \\
367\end{array}$ & $\begin{array}{r}1370 \\
1310 \\
1210 \\
848 \\
126 \\
---\end{array}$ & $\begin{array}{r}104 \\
77 \\
64 \\
353 \\
406 \\
357\end{array}$ & $\begin{array}{l}1710 \\
1660 \\
1610 \\
1570 \\
1550 \\
1500\end{array}$ & $\begin{array}{r}58 \\
59 \\
61 \\
59 \\
--- \\
---\end{array}$ & $\begin{array}{r}52 \\
935 \\
697 \\
1160 \\
374 \\
302\end{array}$ & $\begin{array}{r}87 \\
65 \\
55 \\
50 \\
50 \\
--\end{array}$ & $\begin{array}{r}421 \\
69 \\
62 \\
63 \\
78 \\
64\end{array}$ & $\begin{array}{r}53 \\
50 \\
50 \\
49 \\
48 \\
---\end{array}$ & $\begin{array}{l}103 \\
112 \\
165 \\
262 \\
225 \\
156\end{array}$ & $\begin{array}{l}45 \\
62 \\
47 \\
87 \\
96 \\
77\end{array}$ & $\begin{array}{l}166 \\
144 \\
127 \\
108 \\
423 \\
--.\end{array}$ \\
\hline $\begin{array}{l}\text { TOTAL } \\
\text { MEAN } \\
\text { MAX } \\
\text { MIN } \\
\text { AC-FT }\end{array}$ & $\begin{array}{r}22440 \\
724 \\
1590 \\
62 \\
44510\end{array}$ & $\begin{array}{r}13860 \\
462 \\
1370 \\
48 \\
27490\end{array}$ & $\begin{array}{r}11560 \\
373 \\
1440 \\
39 \\
22930\end{array}$ & $\begin{array}{r}19170 \\
618 \\
1740 \\
36 \\
38020\end{array}$ & $\begin{array}{r}14868 \\
513 \\
1450 \\
58 \\
29490\end{array}$ & $\begin{array}{r}4972 \\
160 \\
1160 \\
44 \\
9860\end{array}$ & $\begin{array}{r}9235 \\
308 \\
1440 \\
44 \\
18320\end{array}$ & $\begin{array}{r}7924 \\
256 \\
1020 \\
47 \\
15720\end{array}$ & $\begin{array}{r}3013 \\
100 \\
459 \\
41 \\
5980\end{array}$ & $\begin{array}{r}2657 \\
85.7 \\
262 \\
44 \\
5270\end{array}$ & $\begin{array}{r}2089 \\
67.4 \\
137 \\
41 \\
4140\end{array}$ & $\begin{array}{r}7556 \\
252 \\
1090 \\
58 \\
14990\end{array}$ \\
\hline
\end{tabular}

$\begin{array}{lllllllllll}\text { CAL YR } 1979 & \text { TOTAL } & 167000 & \text { MEAN } & 458 & \text { MAX } & 2410 & \text { MIN } & 39 & \text { AC-FT } & 331200 \\ \text { WTR YR } & 1980 & \text { TOTAL } & 119344 & \text { MEAN } 326 & \text { MAX } & 1740 & \text { MIN } & 36 & \text { AC-FT } & 236700\end{array}$ 
SAN JACINTO RIVER BASIN

08073600 BUFTALO BAYOU AT WEST BELT DRIVE, hOUSTON, TX - Continued

WATER-QUALITY RECORDS

PERIOD OF RECORD.--Chemical and biochemical analyes: December 1978 to current year.

PERIOD OF DAILY RECORD.--

SPECIFIC CONDUCTANCE: June 1979 to current year.

WATER TEMPERATURES: Jine 1979 to current year.

REMARKS.--Mean monthl and annual concentretions and loads for selected chemical constituents have been computed using the deily (or continuous) records of specific conductance and regression relationships between each chemical constituent and apecific conductance. Regression equations developed for this station may be obtained from the Geological Survey District office upon request.

EXTREMES FOR PERIOD OF DAILY RECORD.--

SPECIFIC CONDUCTANCE: Maximum deily, 922 micromhos June 25, 1979 ; minimum daily, 90 micromhos Jen. $25,1980$. WATER TEMPERATURES: Maximum dally, $30.5^{\circ} \mathrm{C}$ July 1, 1978; minimum daily, 9.0 ${ }^{\circ} \mathrm{C}$ Feb. $11,12,1980$.

EXTREMES FOR CURRENT YEAR.--

SPECIFIC CONDUCTANCE: Maximum daily, 865 micromhos Mar. 15; minimum dally, 90 micromhos Jan. 25.

WATER QUALITY DATA, WATER YEAR OCTOBER 1979 TO SEPTEMBER 1980

\begin{tabular}{|c|c|c|c|c|c|c|c|c|c|c|c|c|}
\hline DATE & TIME & $\begin{array}{l}\text { STREAM- } \\
\text { FLOW, } \\
\text { INSTAN- } \\
\text { TANEOUS } \\
\text { (CFS) }\end{array}$ & $\begin{array}{l}\text { SPE- } \\
\text { CIFIC } \\
\text { CON- } \\
\text { DUCT- } \\
\text { ANCE } \\
\text { (MICRO- } \\
\text { MHOS) }\end{array}$ & $\begin{array}{c}\text { PH } \\
\text { FIELD } \\
\text { (UNITS) }\end{array}$ & $\begin{array}{c}\text { TEMPER- } \\
\text { ATURE, } \\
\text { WATER } \\
\text { (DEG C) }\end{array}$ & $\begin{array}{l}\text { COLOR } \\
\text { (PLAT- } \\
\text { INUM } \\
\text { COBALT } \\
\text { UNITS) }\end{array}$ & $\begin{array}{c}\text { TUR- } \\
\text { BID- } \\
\text { ITY } \\
\text { (NTU) }\end{array}$ & $\begin{array}{c}\text { OXYGEN, } \\
\text { DIS- } \\
\text { SOLVED } \\
(M G / L)\end{array}$ & $\begin{array}{c}\text { OXYGEN, } \\
\text { DIS- } \\
\text { SOLVED } \\
\text { (PER- } \\
\text { CENT } \\
\text { SATUR- } \\
\text { ATION) }\end{array}$ & $\begin{array}{c}\text { OXYGEN } \\
\text { DEMAND, } \\
\text { BIOCHEM } \\
\text { UNINHIB } \\
5 \text { DAY } \\
\text { (MG/L) }\end{array}$ & $\begin{array}{c}\text { COLI- } \\
\text { FORM, } \\
\text { TOTAL, } \\
\text { IMMED. } \\
\text { (COLS. } \\
\text { PER } \\
100 \mathrm{ML} \text { ) }\end{array}$ & $\begin{array}{l}\text { COLI- } \\
\text { FORM, } \\
\text { FECAL, } \\
0.7 \\
\text { UM-MF } \\
\text { (COLS.' } \\
100 \mathrm{ML} \text { ) }\end{array}$ \\
\hline $\begin{array}{l}\text { OCT } \\
15 \ldots \\
17 \ldots \\
\text { NOV }\end{array}$ & $\begin{array}{l}1100 \\
1230\end{array}$ & $\begin{array}{r}582 \\
93\end{array}$ & $\begin{array}{l}284 \\
620\end{array}$ & $\begin{array}{l}7.2 \\
7.5\end{array}$ & $\begin{array}{l}21.5 \\
26.0\end{array}$ & $\begin{array}{r}110 \\
30\end{array}$ & $32^{8.1}$ & $\begin{array}{l}6.5 \\
8.7\end{array}$ & $\begin{array}{r}73 \\
106\end{array}$ & $\begin{array}{l}9.0 \\
8.0\end{array}$ & $\begin{array}{r}4400 \\
-.\end{array}$ & $\begin{array}{r}84 \\
270\end{array}$ \\
\hline $\begin{array}{l}13 \ldots \\
28 \ldots\end{array}$ & $\begin{array}{l}1200 \\
1235\end{array}$ & $\begin{array}{r}62 \\
1210\end{array}$ & $\begin{array}{l}820 \\
153\end{array}$ & $\begin{array}{l}7.5 \\
6.8\end{array}$ & $\begin{array}{l}22.0 \\
14.0\end{array}$ & $1 \overline{20}$ & $\begin{array}{r}200 \\
42\end{array}$ & $\begin{array}{l}4.3 \\
9.1\end{array}$ & $\begin{array}{l}48 \\
86\end{array}$ & $\begin{array}{l}8.1 \\
4.9\end{array}$ & 4100 & $\begin{array}{r}20000 \\
140\end{array}$ \\
\hline JAN $13 . \cdots$ & 1005 & 216 & 230 & 7.4 & 12.0 & -- & 140 & 11.8 & 106 & 4.7 & $\cdots$ & 620 \\
\hline $\begin{array}{l}08 \ldots \\
29 \ldots \\
\text { FEB }\end{array}$ & $\begin{array}{l}1145 \\
1330\end{array}$ & $\begin{array}{r}94 \\
1570\end{array}$ & $\begin{array}{l}500 \\
104\end{array}$ & $\begin{array}{l}8.0 \\
6.8\end{array}$ & $\begin{array}{l}15.0 \\
13.0\end{array}$ & $1 \ddot{20}$ & $\begin{array}{r}57 \\
110\end{array}$ & $\begin{array}{r}10.2 \\
7.7\end{array}$ & $\begin{array}{r}100 \\
72\end{array}$ & $\begin{array}{l}8.0 \\
4.0\end{array}$ & 7000 & $\begin{array}{r}88 \\
250\end{array}$ \\
\hline$\underset{\text { MAR }}{05} \cdots$ & 1130 & 1150 & 157 & 7.1 & 11.5 & -- & 71 & 9.8 & 88 & 1.9 & -- & 94 \\
\hline$\underset{A P R}{04 \ldots}$ & 1150 & 66 & 800 & 7.8 & 18.0 & -- & 26 & 8.3 & 87 & 5.8 & -- & K1 \\
\hline$\underset{\text { MAY }}{01} \cdots$ & 1255 & 734 & 148 & 7.3 & 18.0 & -- & 180 & 8.8 & 93 & 4.4 & -- & K6 \\
\hline $\begin{array}{c}06 \ldots \\
19 . \ldots \\
\text { JUN }\end{array}$ & $\begin{array}{l}1045 \\
1245\end{array}$ & $\begin{array}{r}53 \\
708\end{array}$ & $\begin{array}{l}780 \\
230\end{array}$ & $\begin{array}{l}7.7 \\
6.8\end{array}$ & $\begin{array}{l}24.5 \\
23.0\end{array}$ & $\begin{array}{r}10 \\
160\end{array}$ & $\begin{array}{r}14 \\
200\end{array}$ & $\begin{array}{l}6.0 \\
6.5\end{array}$ & $\begin{array}{l}71 \\
75\end{array}$ & $14^{7.1}$ & 16000 & $\begin{array}{r}\text { K3 } \\
4600\end{array}$ \\
\hline$\underset{\text { JUL }}{10 . .}$ & 1110 & 450 & 275 & 7.3 & 25.0 & 30 & 350 & 6.7 & 80 & 15 & - & 80 \\
\hline$\underset{\text { AUG }}{08}$ & 1145 & 50 & 800 & 7.7 & 29.5 & 15 & 4.7 & 5.2 & 68 & 2.4 & -- & 130 \\
\hline $\begin{array}{l}04 \ldots \\
19 \ldots \\
\text { sEP }\end{array}$ & $\begin{array}{l}1240 \\
1025\end{array}$ & $\begin{array}{l}81 \\
47\end{array}$ & $\begin{array}{l}700 \\
820\end{array}$ & $\begin{array}{l}7.6 \\
8.0\end{array}$ & $\begin{array}{l}29.0 \\
28.0\end{array}$ & $\begin{array}{l}25 \\
--\end{array}$ & $\begin{array}{l}22 \\
15\end{array}$ & $\begin{array}{l}5.9 \\
4.7\end{array}$ & $\begin{array}{l}77 \\
59\end{array}$ & $\begin{array}{r}5.2 \\
.7\end{array}$ & $\begin{array}{r}190 \\
--\end{array}$ & $\begin{array}{r}\mathrm{K} 18 \\
\mathrm{~K} 2\end{array}$ \\
\hline $04 \ldots$ & $\begin{array}{l}1035 \\
1235\end{array}$ & $\begin{array}{r}58 \\
934\end{array}$ & $\begin{array}{l}730 \\
213\end{array}$ & $\begin{array}{l}7.8 \\
7.3\end{array}$ & $\begin{array}{l}27.5 \\
27.0\end{array}$ & $\begin{array}{l}30 \\
76\end{array}$ & $\begin{array}{l}34 \\
92\end{array}$ & $\begin{array}{l}5.5 \\
6.9\end{array}$ & $\begin{array}{l}69 \\
85\end{array}$ & $\begin{array}{l}3.7 \\
4.8\end{array}$ & $32 \overline{0}$ & $\begin{array}{r}\mathbf{K 1} \\
290\end{array}$ \\
\hline
\end{tabular}

\begin{tabular}{|c|c|c|c|c|c|c|c|c|c|c|c|c|}
\hline & $\begin{array}{c}\text { STREP- } \\
\text { TOCOCCI } \\
\text { FECAL, } \\
\text { KF AGAR } \\
\text { (COLS. } \\
\text { PER } \\
100 \mathrm{ML} \text { ) }\end{array}$ & $\begin{array}{l}\text { HARD- } \\
\text { NESS } \\
\text { (MG /L } \\
\text { AS } \\
\text { CACO3) }\end{array}$ & $\begin{array}{c}\text { HARD- } \\
\text { NESS, } \\
\text { NONCAR- } \\
\text { BONATE } \\
\text { (MG/L } \\
\text { CACO3) }\end{array}$ & $\begin{array}{l}\text { CALCIUM } \\
\text { DIS- } \\
\text { SOLVED } \\
\text { (MG/L } \\
\text { AS CA) }\end{array}$ & $\begin{array}{l}\text { MAGNE- } \\
\text { SIUM, } \\
\text { DIS- } \\
\text { SOLVED } \\
\text { (MG/L } \\
\text { AS MG) }\end{array}$ & $\begin{array}{c}\text { SODIUM, } \\
\text { DIS- } \\
\text { SOLVED } \\
\text { (MG/L } \\
\text { AS NA) }\end{array}$ & $\begin{array}{c}\text { SODIUM } \\
\text { AD- } \\
\text { SORP- } \\
\text { TION } \\
\text { RATIO }\end{array}$ & $\begin{array}{l}\text { POTAS- } \\
\text { SIUM, } \\
\text { DIS - } \\
\text { SOLVED } \\
\text { (MG/L } \\
\text { AS K) }\end{array}$ & $\begin{array}{c}\text { BICAR- } \\
\text { BONATE } \\
\text { (MG/L } \\
\text { AS } \\
\text { HCO3) }\end{array}$ & $\begin{array}{c}\text { CAR- } \\
\text { BONATE } \\
\text { (MG/L } \\
\text { AS CO3) }\end{array}$ & $\begin{array}{c}\text { SULFATE } \\
\text { DIS- } \\
\text { SOLVED } \\
\text { (MG/L } \\
\text { AS S04) }\end{array}$ & $\begin{array}{l}\text { CHLO- } \\
\text { RIDE, } \\
\text { DIS- } \\
\text { SOLVED } \\
\text { (MG/L } \\
\text { AS CL) }\end{array}$ \\
\hline
\end{tabular}

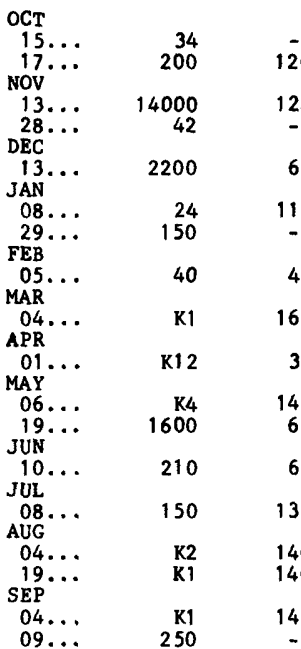

$\begin{array}{rcc}120 & -- & 35 \\ 120 & 0 & 37 \\ -- & -- & - \\ 63 & 7 & 19 \\ 110 & 0 & 32 \\ -- & -- & - \\ 43 & 0 & 13 \\ 160 & 0 & 50 \\ 36 & 0 & 11 \\ 140 & 0 & 43 \\ 60 & 0 & 19 \\ 62 & 0 & 20 \\ 130 & 0 & 41 \\ 140 & 0 & 43 \\ 140 & 0 & 42 \\ 140 & 0 & 42 \\ -- & -- & -\end{array}$

\begin{tabular}{|c|c|}
\hline $6 . \overline{7}$ & $77^{--}$ \\
\hline $\begin{array}{r}7.2 \\
-.-\end{array}$ & 120 \\
\hline 3.8 & 22 \\
\hline 6.3 & 58 \\
\hline 2.6 & 13 \\
\hline 9.3 & 96 \\
\hline 2.1 & 13 \\
\hline $\begin{array}{l}7.9 \\
3.0\end{array}$ & $\begin{array}{r}120 \\
17\end{array}$ \\
\hline 3.0 & 28 \\
\hline 7.7 & 120 \\
\hline $\begin{array}{l}7.2 \\
7.6\end{array}$ & $\begin{array}{r}93 \\
100\end{array}$ \\
\hline $\begin{array}{r}7.7 \\
-\end{array}$ & 92 \\
\hline
\end{tabular}

3.1
4.7
--
1.2
2.5
--
.9
3.3
.9
4.4
1.0
1.5
4.5
3.5
3.7
3.4
.-

$5 . \overline{8} \quad 200$

8.0290

5.1

69

$6.1 \quad 180$

3.2

-.

6.4

280

3.4

$\begin{array}{rr}6.9 & 280 \\ 3.8 & 76\end{array}$

$6.1 \quad 77$

$7.5 \quad 290$

$\begin{array}{ll}6.5 & 250 \\ 9.2 & 270\end{array}$

$-$

(s 504 )

As $\mathrm{CL}$ ) 
SAN JACINTO RIVER BASIN

08073600 BUFFALO BAYOU AT WEST BELT DRIVE, HOUSTON, TX--Continued

WATER QUALITY DATA, WATER YEAR OCTOBER 1979 TO SEPTEMBER 1980

\begin{tabular}{|c|c|c|c|c|c|c|c|c|c|c|c|c|}
\hline DATE & $\begin{array}{l}\text { FLUO- } \\
\text { RIDE, } \\
\text { DIS- } \\
\text { SOLVED } \\
\text { (HG/L } \\
\text { AS F) }\end{array}$ & $\begin{array}{l}\text { SILICA, } \\
\text { DIS- } \\
\text { SOLVED } \\
\text { (HG/L } \\
\text { AS } \\
\text { SIO2) }\end{array}$ & $\begin{array}{l}\text { SOLIDS, } \\
\text { RESIDUE } \\
\text { AT } 180 \\
\text { DEG. C } \\
\text { DIS- } \\
\text { SOLVED } \\
(M G / L)\end{array}$ & $\begin{array}{l}\text { SOLIDS, } \\
\text { SUM OF } \\
\text { CONSTI- } \\
\text { TUENTS, } \\
\text { DIS- } \\
\text { SOLVED } \\
\text { (MG/L) }\end{array}$ & $\begin{array}{l}\text { SOLIDS, } \\
\text { RESIDUE } \\
\text { AT } 105 \\
\text { DEG. C, } \\
\text { SUS- } \\
\text { PENDED } \\
\text { (MG/L) }\end{array}$ & $\begin{array}{l}\text { SOLIDS, } \\
\text { VOLA- } \\
\text { TILE, } \\
\text { SUS- } \\
\text { PENDED } \\
\text { (MG/L) }\end{array}$ & $\begin{array}{l}\text { NITRO- } \\
\text { GEN, } \\
\text { NITRATE } \\
\text { TOTAL } \\
\text { (MG/L } \\
\text { AS N) }\end{array}$ & $\begin{array}{c}\text { NITRO- } \\
\text { GEN, } \\
\text { NITRITE } \\
\text { TOTAL } \\
\text { (MG/L } \\
\text { AS N) }\end{array}$ & $\begin{array}{c}\text { NITRO- } \\
\text { GEN, } \\
\text { NO2+NO3 } \\
\text { TOTAL } \\
\text { (MG/L } \\
\text { AS N) }\end{array}$ & $\begin{array}{c}\text { NITRO- } \\
\text { GEN, } \\
\text { NO2+NO3 } \\
\text { DIS- } \\
\text { SOLVED } \\
\text { (MG/L } \\
\text { AS N) }\end{array}$ & $\begin{array}{l}\text { NITRO- } \\
\text { GEN, } \\
\text { AMMONIA } \\
\text { TOTAL } \\
\text { (MG/L } \\
\text { AS N) }\end{array}$ & $\begin{array}{l}\text { NITRO- } \\
\text { GEN, } \\
\text { AMENONIA } \\
\text { DIS- } \\
\text { SOLVED } \\
\text { (MG/L } \\
\text { AS N) }\end{array}$ \\
\hline $\begin{array}{l}\text { oct } \\
15 \ldots \\
17 \ldots \\
\text { Nov }\end{array}$ & .4 & $21^{--}$ & 350 & $3 \overline{35}$ & $\begin{array}{l}17 \\
--\end{array}$ & $\begin{array}{l}16 \\
--\end{array}$ & .00 & $\begin{array}{r}.100 \\
--\end{array}$ & $\begin{array}{r}.05 \\
.18\end{array}$ &.$\overline{62}$ & $\begin{array}{r}.400 \\
7.200\end{array}$ & 5.100 \\
\hline $\begin{array}{c}13 \ldots \\
28 . \ldots\end{array}$ & .4 & 26 & $\begin{array}{r}406 \\
--\end{array}$ & $\begin{array}{r}466 \\
=-\end{array}$ & $-\overline{8}$ & $\overline{0}$ &.$\overline{10}$ & $.0 \overline{0}$ & $\begin{array}{l}.66 \\
.15\end{array}$ & .15 & $\begin{array}{r}16.000 \\
.500\end{array}$ & $\begin{array}{r}9.700 \\
-\end{array}$ \\
\hline $\operatorname{JAN}^{13} \cdots$ & .2 & 11 & 168 & 141 & -- & -- & -- & -- & .84 & .68 & .660 & .640 \\
\hline $\begin{array}{r}08 \ldots \\
29 \ldots\end{array}$ & .3 & 14 & $\begin{array}{c}280 \\
--\end{array}$ & $\begin{array}{r}285 \\
--\end{array}$ & $\overline{36}$ & $-\overline{2}$ & .07 & $.0 \overline{10}$ & $\begin{array}{l}.50 \\
.08\end{array}$ & .37 & $\begin{array}{r}3.000 \\
.240\end{array}$ & $\begin{array}{r}2.400 \\
--\end{array}$ \\
\hline$\underset{\text { MAR }}{05}$ & .1 & 7.0 & 95 & 86 & -- & -- & -- & -- & .20 & .20 & .460 & .460 \\
\hline$\underset{\mathrm{APR}}{04} \cdots$ & .4 & 19 & 412 & 439 & -- & -- & -- & - & .91 & .46 & 7.200 & 6.600 \\
\hline$\underset{\text { MAY }}{01} \cdots$ & .2 & .1 & 97 & 78 & $\cdots$ & - & -- & $\cdots$ & .52 & .47 & .950 & .860 \\
\hline $\begin{array}{c}06 \ldots \\
19 . . \\
\text { JUN }\end{array}$ & $\begin{array}{l}.4 \\
.2\end{array}$ & $\begin{array}{r}22 \\
9.0\end{array}$ & $\begin{array}{r}457 \\
--\end{array}$ & $\begin{array}{l}464 \\
122\end{array}$ & $\begin{array}{r}29 \\
244\end{array}$ & $\begin{array}{r}0 \\
22\end{array}$ & .60 & .140 & $\begin{array}{l}.47 \\
.74\end{array}$ & 1.5 & $\begin{array}{r}2.500 \\
.780\end{array}$ & $\begin{array}{r}5.400 \\
--\end{array}$ \\
\hline $\begin{array}{c}10 \ldots \\
\text { JUL }\end{array}$ & .4 & 7.8 & 166 & 156 & 408 & 48 & -- & -- & .94 & .94 & .550 & .550 \\
\hline $\begin{array}{c}08 \\
\text { AUG }\end{array}$ & .7 & 23 & 455 & 486 & 3 & 1 & -- & -- & 1.6 & 1.6 & 5.500 & 5.600 \\
\hline $\begin{array}{c}04 \ldots . \\
19 . \ldots \\
\text { SEP }\end{array}$ & $\begin{array}{l}.4 \\
.5\end{array}$ & $\begin{array}{l}26 \\
24\end{array}$ & $4 \overline{34}$ & $\begin{array}{l}413 \\
449\end{array}$ & 25 & $\begin{array}{l}28 \\
--\end{array}$ & .53 & $\begin{array}{r}.440 \\
-\end{array}$ & 1.97 & $1.1^{--}$ & $\begin{array}{l}3.400 \\
6.300\end{array}$ & 6.300 \\
\hline $\begin{array}{l}04 \ldots \\
09 . . .\end{array}$ & .5 & 24 & $\begin{array}{r}351 \\
--\end{array}$ & $\begin{array}{r}410 \\
--\end{array}$ & $\begin{array}{l}36 \\
84\end{array}$ & $\begin{array}{l}2 \\
6\end{array}$ &.$\overline{9}$ & .030 & $\begin{array}{l}1.1 \\
.12\end{array}$ & 1.2 & $\begin{array}{r}4.600 \\
.380\end{array}$ & $\begin{array}{r}4.500 \\
--\end{array}$ \\
\hline DATE & $\begin{array}{l}\text { NITRO- } \\
\text { GEN, } \\
\text { ORGANIC } \\
\text { TOTAL } \\
\text { (MG/L } \\
\text { AS N) }\end{array}$ & $\begin{array}{c}\text { NITRO- } \\
\text { GEN, } \\
\text { ORGANIC } \\
\text { DIS- } \\
\text { SOLVED } \\
\text { (MG/L } \\
\text { AS N) }\end{array}$ & $\begin{array}{l}\text { NITRO- } \\
\text { GEN,AM- } \\
\text { MONIA + } \\
\text { ORGANIC } \\
\text { TOTAL } \\
\text { (MG/L } \\
\text { AS N) }\end{array}$ & $\begin{array}{l}\text { NITRO- } \\
\text { GEN,AM- } \\
\text { MONIA + } \\
\text { ORGANIC } \\
\text { DIS. } \\
\text { (MG/L } \\
\text { AS N) }\end{array}$ & $\begin{array}{c}\text { PHOS- } \\
\text { PHORUS, } \\
\text { TOTAL } \\
\text { (MG /L } \\
\text { AS P) }\end{array}$ & $\begin{array}{l}\text { PHOS- } \\
\text { PHORUS, } \\
\text { DIS- } \\
\text { SOLVED } \\
\text { (MG/L } \\
\text { AS P) }\end{array}$ & $\begin{array}{c}\text { CARBON, } \\
\text { ORGANIC } \\
\text { TOTAL } \\
\text { (MG/L } \\
\text { AS C) }\end{array}$ & $\begin{array}{l}\text { CARBON, } \\
\text { ORGANIC } \\
\text { DIS- } \\
\text { SOLVED } \\
\text { (MG/L } \\
\text { AS C) }\end{array}$ & $\begin{array}{l}\text { CARBON, } \\
\text { ORGANIC } \\
\text { SUS- } \\
\text { PENDED } \\
\text { (MG/L } \\
\text { AS C) }\end{array}$ & $\begin{array}{l}\text { SEDI - } \\
\text { MENT, } \\
\text { SUS- } \\
\text { PENDED } \\
\text { (MG /L) }\end{array}$ & $\begin{array}{l}\text { SEDI - } \\
\text { MENT } \\
\text { DIS- } \\
\text { CHARGE, } \\
\text { SUS- } \\
\text { PENDED } \\
\text { (T/DAY) }\end{array}$ & $\begin{array}{c}\text { SED. } \\
\text { SUSP. } \\
\text { SIEVE } \\
\text { DIAM. } \\
\times \text { FINER } \\
\text { THAN } \\
.062 \text { MPA }\end{array}$ \\
\hline $\begin{array}{l}\text { OCT } \\
15 \ldots \\
17 \ldots \\
\text { Nov }\end{array}$ & $\begin{array}{c}1.5 \\
.00\end{array}$ &.$\overline{00}$ & $\begin{array}{l}1.9 \\
4.0\end{array}$ &.$\overline{65}$ & $\begin{array}{l}.750 \\
.070\end{array}$ & .180 & $\begin{array}{l}14 \\
27\end{array}$ & $=$ & -- & $\overline{72}$ & $18^{--}$ & 75 \\
\hline $\begin{array}{c}13 \ldots \\
28 \ldots \\
\text { DEC }\end{array}$ & ${ }^{36} 1.1$ & 38 & $\stackrel{52}{1.6}$ & 48 & $\begin{array}{r}.040 \\
.360\end{array}$ & $\begin{array}{r}.040 \\
--\end{array}$ & $\begin{array}{r}260 \\
12\end{array}$ & $\ddot{--}$ & 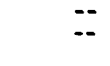 & $\begin{array}{r}313 \\
--\end{array}$ & $52 \ldots$ & 93 \\
\hline${ }_{\text {JAN }}^{13} \cdots$ & 1.6 & 1.2 & 2.3 & 1.8 & .750 & .510 & - & 9.9 & 1.1 & 205 & 120 & 90 \\
\hline $\begin{array}{c}08 . \cdots \\
29 . \cdots\end{array}$ & $\begin{array}{l}5.8 \\
1.2\end{array}$ & $\begin{array}{c}1.8 \\
--\end{array}$ & $\begin{array}{l}8.8 \\
1.4\end{array}$ & $\begin{array}{r}4.2 \\
-.\end{array}$ & $\begin{array}{r}2.300 \\
.240\end{array}$ & 1.700 & $\begin{array}{l}24 \\
14\end{array}$ & $=-$ & $=$ & 118 & 30 & 98 \\
\hline MAR & 1.2 & .45 & 1.7 & .91 & .350 & .210 & 14 & -- & -- & 58 & 180 & 66 \\
\hline$\underset{A P R}{04} \cdots$ & 4.8 & 3.3 & 12 & 9.9 & 2.800 & 2.400 & - & 15 & 3.3 & 42 & 7.5 & 91 \\
\hline$\underset{\text { MAY }}{01} \cdots$ & 2.8 & 1.2 & 3.7 & 2.1 & .560 & .410 & 12 & -- & - & 184 & 365 & 88 \\
\hline $\begin{array}{c}06 \ldots \\
19 . .\end{array}$ & $\begin{array}{l}5.7 \\
1.8\end{array}$ & -- & $\begin{array}{l}8.2 \\
2.6\end{array}$ & $=$ & $\begin{array}{r}2.600 \\
.790\end{array}$ & $\begin{array}{r}1.600 \\
\ldots\end{array}$ & $\begin{array}{l}26 \\
19\end{array}$ & $=-$ & $=$ & $\begin{array}{l}23 \\
--\end{array}$ & 3.3 & $\begin{array}{l}96 \\
--\end{array}$ \\
\hline $\begin{array}{c}10 . . \\
\text { JUL }\end{array}$ & 2.4 & 2.0 & 2.9 & 2.5 & 1.400 & .780 & -- & 13 & -- & 595 & 723 & 99 \\
\hline AUG 08 & 1.8 & .20 & 7.3 & 5.8 & 3.800 & 2.500 & 13 & -- & $\cdots$ & 7 & .94 & 93 \\
\hline $\begin{array}{c}04 \ldots \\
19 \ldots \\
\text { SEP }\end{array}$ & $\begin{array}{l}1.2 \\
3.6\end{array}$ & $2.3^{-}$ & $\begin{array}{l}4.6 \\
9.9\end{array}$ & $8.6^{--}$ & $\begin{array}{l}1.200 \\
2.500\end{array}$ & 2.200 & $\begin{array}{l}12 \\
16\end{array}$ & $=$ & $=$ & $\overline{16}$ & $2.0^{--}$ & $\ddot{88}$ \\
\hline $\begin{array}{l}04 \ldots \\
09 . .\end{array}$ & $\begin{array}{l}9.4 \\
1.2\end{array}$ & 1.8 & $\begin{array}{l}14 \\
1.6\end{array}$ & $\begin{array}{c}6.3 \\
--\end{array}$ & $\begin{array}{r}2.600 \\
.560\end{array}$ & 2.700 & $28^{--}$ & 13 & .8 & $\begin{array}{l}45 \\
--\end{array}$ & $\begin{array}{l}7.0 \\
-\end{array}$ & $\begin{array}{l}97 \\
--\end{array}$ \\
\hline
\end{tabular}


SAN JACINTO RIVER BASIN

08073600 BUFFALO BAYOU AT WEST BELT DRIVE, HOUSTON, TX--Continued

WATER QUALITY DATA, HATER YEAR OCTOBER 1979 TO SEPTEMBER 1980

\begin{tabular}{|c|c|c|c|c|c|c|c|c|c|c|c|}
\hline DATE & TIME & $\begin{array}{c}\text { ARSENIC } \\
\text { TOTAL } \\
\text { (UG/L } \\
\text { AS AS) }\end{array}$ & $\begin{array}{c}\text { ARSENIC } \\
\text { SUS- } \\
\text { PENDED } \\
\text { TOTAL } \\
\text { (UG/L } \\
\text { AS AS) }\end{array}$ & $\begin{array}{c}\text { ARSENIC } \\
\text { DIS- } \\
\text { SOLVED } \\
\text { (UG/L } \\
\text { AS AS) }\end{array}$ & $\begin{array}{l}\text { BARIUM, } \\
\text { TOTAL } \\
\text { RECOV- } \\
\text { ERABLE } \\
\text { (UG/L } \\
\text { AS BA) }\end{array}$ & $\begin{array}{l}\text { BARIUM, } \\
\text { SUS- } \\
\text { PENDED } \\
\text { RECOV- } \\
\text { ERABLE } \\
\text { (UG/L } \\
\text { AS BA) }\end{array}$ & $\begin{array}{c}\text { BARIUM, } \\
\text { DIS- } \\
\text { SOLVED } \\
\text { (UG/L } \\
\text { AS BA) }\end{array}$ & $\begin{array}{l}\text { CADMIUM } \\
\text { TOTAL } \\
\text { RECOV- } \\
\text { ERABLF } \\
\text { (UG/L } \\
\text { AS CD) }\end{array}$ & $\begin{array}{c}\text { CADMIUM } \\
\text { SUS- } \\
\text { PENDED } \\
\text { RECOV- } \\
\text { ERABLE } \\
\text { (UG/L } \\
\text { AS CD) }\end{array}$ & $\begin{array}{c}\text { CADMIUM } \\
\text { DIS- } \\
\text { SOLVED } \\
\text { (UG /L } \\
\text { AS CD) }\end{array}$ & $\begin{array}{l}\text { CHRO- } \\
\text { MIUM, } \\
\text { TOTAL } \\
\text { RECOV- } \\
\text { ERABLE } \\
\text { (UG/L } \\
\text { AS CR) }\end{array}$ \\
\hline Nov & & & & & & & & & & & \\
\hline $13 \ldots$ & 1200 & -- & -- & -- & -- & -- & -- & -- & -- & -- & -- \\
\hline FEB & 1005 & 4 & 1 & 3 & 300 & 200 & 70 & 2 & 1 & $<1$ & 24 \\
\hline $05 \ldots$ & 1130 & -- & -- & -- & -- & -- & -- & -- & -- & -- & -- \\
\hline $\begin{array}{l}\text { MAR } \\
\text { MAY }\end{array}$ & 1150 & 4 & 1 & 3 & 300 & 100 & 200 & 6 & 5 & $<1$ & 10 \\
\hline $06 . .$. & 1045 & -- & -- & -- & -- & -- & -- & -- & -- & -- & - \\
\hline$\underset{\text { JUN }}{19} \cdot \ldots$ & 1245 & -- & -- & 4 & -- & -- & 80 & -- & -- & $<1$ & $-\infty$ \\
\hline $\begin{array}{c}10 . \ldots \\
\text { AUG }\end{array}$ & 1110 & 5 & 0 & 5 & 200 & 100 & 100 & 0 & -- & $<1$ & 10 \\
\hline $04 \ldots$ & 1240 & -- & 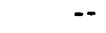 & 4 & -- & -- & 200 & -- & -- & $<1$ & -- \\
\hline SEP & 1025 & -- & -- & -- & -- & -- & -- & - & -- & $-\infty$ & $\cdots$ \\
\hline $04 \ldots$ & 1035 & 7 & 2 & 5 & 200 & 0 & 200 & 3 & -- & $<1$ & 10 \\
\hline DATE & $\begin{array}{l}\text { CHRO- } \\
\text { MIUM, } \\
\text { SUS- } \\
\text { PENDED } \\
\text { RECOV. } \\
\text { (UG/L } \\
\text { AS CR) }\end{array}$ & $\begin{array}{l}\text { CHRO- } \\
\text { MIUM, } \\
\text { DIS- } \\
\text { SOLVED } \\
\text { (UG / L } \\
\text { AS CR) }\end{array}$ & $\begin{array}{l}\text { COBALT, } \\
\text { TOTAL } \\
\text { RECOV- } \\
\text { ERABLE } \\
\text { (UG/L } \\
\text { AS CO) }\end{array}$ & $\begin{array}{l}\text { COBALT, } \\
\text { SUS- } \\
\text { PENDED } \\
\text { RECOV- } \\
\text { ERABLE } \\
\text { (UG/L } \\
\text { AS CO) }\end{array}$ & $\begin{array}{l}\text { COBALT, } \\
\text { DIS- } \\
\text { SOLVED } \\
\text { (UG/L } \\
\text { AS CO) }\end{array}$ & $\begin{array}{l}\text { COPPER, } \\
\text { TOTAL } \\
\text { RECOV- } \\
\text { ERABLE } \\
\text { (UG /L } \\
\text { AS CU) }\end{array}$ & $\begin{array}{l}\text { COPPER, } \\
\text { SUS-- } \\
\text { PENDED } \\
\text { RECOV- } \\
\text { ERABLE } \\
\text { (UG/L } \\
\text { AS CU) }\end{array}$ & $\begin{array}{l}\text { COPPER, } \\
\text { DIS- } \\
\text { SOLVED } \\
\text { (UG/L } \\
\text { AS CU) }\end{array}$ & $\begin{array}{l}\text { IRON, } \\
\text { TOTAL } \\
\text { RECOV- } \\
\text { ERABLE } \\
\text { (UG / L } \\
\text { AS FE) }\end{array}$ & $\begin{array}{l}\text { IRON, } \\
\text { SUS- } \\
\text { PENDED } \\
\text { RECOV- } \\
\text { ERABLE } \\
\text { (UG/L } \\
\text { AS FE) }\end{array}$ & $\begin{array}{l}\text { IRON, } \\
\text { DIS- } \\
\text { SOLVED } \\
\text { (UG/L } \\
\text { AS FE) }\end{array}$ \\
\hline nov & & & & & & & & & & & \\
\hline${ }_{D E C}^{13} \cdots$ & -- & -- & -- & -- & -- & -- & -- & -- & -- & -- & -- \\
\hline FEB & 24 & 0 & 2 & 0 & $<3$ & 15 & 15 & 40 & 3800 & 3500 & 270 \\
\hline$\underset{\text { MAR }}{05 \ldots}$ & - & -- & -- & -- & -- & -- & -- & -- & - & -- & - \\
\hline$\underset{\text { MAY }}{04 .}$ & 10 & 0 & 0 & 0 & $<3$ & 7 & 7 & 0 & 650 & 630 & 20 \\
\hline $06 \ldots$ & - & -- & -- & -- & -- & -- & -- & $-\bar{c}$ & -- & -- & $=$ \\
\hline JUN & -- & 0 & -- & -- & -- & -- & $\cdots$ & 5 & -- & -- & 70 \\
\hline AUG & 10 & 0 & 3 & -- & $<3$ & 12 & 7 & 5 & 9100 & 9000 & 70 \\
\hline $04 \ldots$ & -- & 0 & -- & -- & -- & -- & -- & 2 & -- & -- & 10 \\
\hline $\begin{array}{l}19 . \ldots \\
\text { SEP }\end{array}$ & -- & -- & -- & -- & -- & -- & -- & -- & -- & $\cdots$ & -- \\
\hline $04 \ldots$ & 10 & 0 & 1 & -- & $<3$ & 5 & 1 & 4 & 680 & 650 & 30 \\
\hline DAT & $\begin{array}{l}\text { LEAD, } \\
\text { TOTAL } \\
\text { RECOV- } \\
\text { ERABLE } \\
\text { (UG/L } \\
\text { AS PB) }\end{array}$ & $\begin{array}{l}\text { LEAD, } \\
\text { SUS- } \\
\text { PENDED } \\
\text { RECOV- } \\
\text { ERABLE } \\
\text { (UG/L } \\
\text { AS PB) }\end{array}$ & $\begin{array}{l}\text { LEAD, } \\
\text { DIS- } \\
\text { SOLVED } \\
\text { (UG/L } \\
\text { AS PB) }\end{array}$ & $\begin{array}{l}\text { MANGA- } \\
\text { NESE, } \\
\text { TOTAL } \\
\text { RECOV- } \\
\text { ERABLE } \\
\text { (UG/L } \\
\text { AS MN) }\end{array}$ & $\begin{array}{l}\text { MANGA- } \\
\text { NESE, } \\
\text { SUS- } \\
\text { PENDED } \\
\text { RECOV. } \\
\text { (UG/L } \\
\text { AS MN) }\end{array}$ & $\begin{array}{l}\text { MANGA- } \\
\text { NESE, } \\
\text { DIS- } \\
\text { SOLVED } \\
\text { (UG/L } \\
\text { AS MN) }\end{array}$ & $\begin{array}{l}\text { MERCURY } \\
\text { TOTAL } \\
\text { RECOV- } \\
\text { ERABLE } \\
\text { (UG/L } \\
\text { AS HG) }\end{array}$ & $\begin{array}{c}\text { MERCURY } \\
\text { SUS- } \\
\text { PENDED } \\
\text { RECOV- } \\
\text { ERABLE } \\
\text { (UG/L } \\
\text { AS HG) }\end{array}$ & $\begin{array}{c}\text { MERCURY } \\
\text { DIS- } \\
\text { SOLVED } \\
\text { (UG/L } \\
\text { AS HG) }\end{array}$ & $\begin{array}{l}\text { NICKEL, } \\
\text { TOTAL } \\
\text { RECOV- } \\
\text { ERABLE } \\
\text { (UG/L } \\
\text { AS NI) }\end{array}$ & $\begin{array}{c}\text { NICKEL, } \\
\text { SUS- } \\
\text { PENDED } \\
\text { RECOV- } \\
\text { ERABLE } \\
\text { (UG/L } \\
\text { AS NI) }\end{array}$ \\
\hline
\end{tabular}

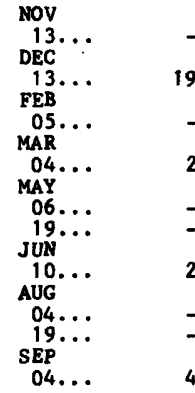

--
90
--
--
29
--
-

--
190
--
23
--
--
20
--
--
28

$\begin{array}{rr}-- & -- \\ 160 & 130 \\ -- & -- \\ 110 & 20 \\ -- & -- \\ -- & -- \\ 240 & 240 \\ -- & -- \\ -- & -- \\ 70 & 40\end{array}$ 
SAN JACINTO RIVER BASIN

08073600 BUFFALO BAYOU AT WEST BELT DRIVE, HOUSTON, TX--Continued

WATER QUALITY DATA, WATER YEAR OCTOBER 1979 TO SEPTEMBER 1980

\begin{tabular}{|c|c|c|c|c|c|c|c|c|c|c|}
\hline ATE & $\begin{array}{l}\text { NICKEL, } \\
\text { DIS- } \\
\text { SOLVED } \\
\text { (UG/L } \\
\text { AS NI) }\end{array}$ & $\begin{array}{l}\text { SELE- } \\
\text { NIUM, } \\
\text { TOTAL } \\
\text { (UG/L } \\
\text { AS SE) }\end{array}$ & $\begin{array}{l}\text { SELE- } \\
\text { NIUM, } \\
\text { SUS- } \\
\text { PENDED } \\
\text { TOTAL } \\
\text { (UG/L } \\
\text { AS SE) }\end{array}$ & $\begin{array}{l}\text { SELE- } \\
\text { NIUM, } \\
\text { DIS- } \\
\text { SOLVED } \\
\text { (UG/L } \\
\text { AS SE) }\end{array}$ & $\begin{array}{c}\text { SILVER, } \\
\text { TOTAL } \\
\text { RECOV- } \\
\text { ERABLE } \\
\text { (UG/L } \\
\text { AS AG) }\end{array}$ & $\begin{array}{c}\text { SILVER, } \\
\text { SUS- } \\
\text { PENDED } \\
\text { RECOV- } \\
\text { ERABLE } \\
\text { (UG / L } \\
\text { AS AG) }\end{array}$ & $\begin{array}{c}\text { SILVER, } \\
\text { DIS-- } \\
\text { SOLVED } \\
\text { (UG /L } \\
\text { AS AG) }\end{array}$ & $\begin{array}{l}\text { ZINC, } \\
\text { TOTAL } \\
\text { RECOV- } \\
\text { ERABLE } \\
\text { (UG/L } \\
\text { AS ZN) }\end{array}$ & $\begin{array}{l}\text { ZINC, } \\
\text { SUS- } \\
\text { PENDED } \\
\text { RECOV- } \\
\text { ERABLE } \\
\text { (UG/L } \\
\text { AS ZN) }\end{array}$ & $\begin{array}{l}\text { ZINC, } \\
\text { DIS- } \\
\text { SOLVED } \\
\text { (UG / } \\
\text { AS ZN) }\end{array}$ \\
\hline
\end{tabular}

NOV
$13 \ldots$
DEC $\ldots$
$13 \ldots$
FEB $\ldots$
$05 \ldots$
MAR $\ldots$
$04 \ldots$
MAY $\ldots$
$06 \ldots$
$19 \ldots$
JUN $\ldots$
$10 \ldots$
AUG $\ldots$
$04 \ldots$
$19 \ldots$
SEP $\ldots$
$04 \ldots$

$$
\begin{array}{ccc}
-- & -- & -- \\
4 & 0 & 0 \\
-- & -- & -- \\
0 & 0 & 0 \\
-\therefore & -- & -- \\
-- & -- & \\
5 & 1 & 1 \\
-- & -- & - \\
2 & -- & -
\end{array}
$$

-
0
$\cdots$
0
-
0
0
0
--
0

$$
\begin{array}{lll}
3 & \cdots & - \\
1 & 0 & -
\end{array}
$$

$\begin{array}{ccc}-- & -- & -- \\ 160 & 140 & 20 \\ -- & -- & -- \\ 30 & 10 & 20 \\ -- & -- & -- \\ -- & -- & 7 \\ 80 & -- & <3 \\ -- & -- & 5 \\ -- & -- & -- \\ 30 & 20 & 7\end{array}$

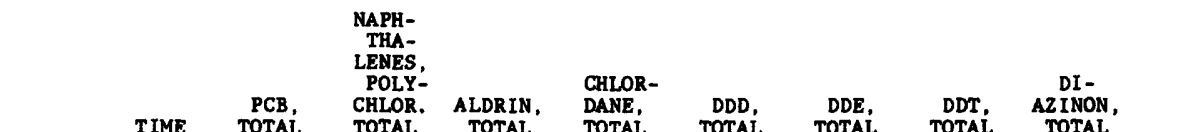


SAN JACINTO RIVER BASIN

08073600 BUfFalO BAYOU AT WEST BELT DRIVE, hOUSTON, TX--Continued

PHYTOPLANKTON ANALYSES, OCTOBER 1979 TO SEPTEMBER 1980

\section{DATE}

TOTAL CELLS/ML

DIVERSITY : DIVISION . CLASS . OFAMILY .....GENUS

ORGANISM

CHLOROPHYTA (GREEN ALGAE)

- CHLOROPHYCEAE

. CHLOROCOCCALES

...MICRACTINIACEAE

.... MI CRACTINIUM

... OOCYSTACEAE

.... ANKISTRODESMUS

.... CHLORELILA

.... DICTYOSPHAERI UN

.... KIRCHNERIELLA

.... TETRAEDRON

$\because$ TCENEBARIA

$\because$...SCENEDESMUS

…SCENEDESMUS

$\because$ VOLVTRACALES

... CHLAMYDOMONADACEAE

.... CHLAMYDOMONAS

CHRYSOPHYTA

- BACILLARI OPHYCEAE

. C. CONTRALES

.... CYCLOTELLLA

....MELOSIRA

....sTEPHANODISCUS

PENhales

...ACHNANTHACEAE

.... A CHNANTHES

… COCCONE IS

... CYMBELLACEA

.... CYMBELLA

... FRAGI LAR IACEAE

.... SYNEDRA

.. gOMPHONEMATACEAE

.... GOMPHONEMA

...nAVICUIACEA

.... NAVICUIA

... NITZSCHIACEAE

....NITZSCHIA

MAR 4.
1150
1100
1.4
1.4
2.0
2.8
3.2

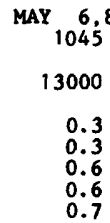

JUN 110,80

JUL 8,80

Aug 19,80

19.80
1025
5000
0.4
0.4
0.8
0.8
0.8

$\operatorname{SEP}_{1035}^{4,80}$

6300

1400

1.5
1.5
2.0
2.1
2.1

990

1.0
1.0
1.5
1.6
1.6

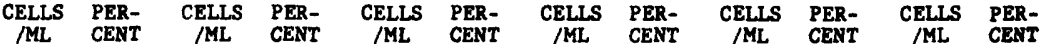

\begin{tabular}{|c|c|c|c|c|c|c|c|c|c|c|}
\hline 6 & 1 & -- & - & -- & - & -- & - & -- & - & -. \\
\hline 67 & 6 & * & 0 & -- & - & $350 t$ & 25 & -- & - & 13 \\
\hline 6 & 1 & $\because$ & $\overline{-}$ & $\because$ & - & $\overline{-}$ & $\overline{-}$ & $\overline{26}$ & $i$ & $\ddot{-}$ \\
\hline 12 & 1 & * & 0 & -- & - & -- & - & - & - & - \\
\hline $\begin{array}{l}12 \\
--\end{array}$ & 1 & * & $\begin{array}{l}0 \\
0\end{array}$ & $=$ & - & -- & $\overline{-}$ & 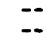 & $\overline{-}$ & =- \\
\hline 73 & 6 & 210 & 2 & 430 & 7 & -- & - & 100 & 2 & 26 \\
\hline 49 & 4 & -- & - & -- & - & -- & - & -- & - & $\cdots$ \\
\hline- & - & * & 0 & -- & - & -- & - & -- & - & -- \\
\hline
\end{tabular}

$\begin{array}{cccccccccccc}230 * & 20 & 90 & 1 & 360 & 6 & 39 & 3 & * & 0 & 13 & 1 \\ 12 & 1 & -- & - & -- & - & -- & - & -- & - & -- & - \\ -- & - & -- & - & 72 & 1 & -- & - & -- & - & -- & - \\ -- & - & -- & - & 72 & 1 & -- & - & -- & - & -- & - \\ 6 & 1 & -- & - & -- & - & -- & - & -- & - & -- & - \\ 6 & 1 & -- & - & -- & - & -- & - & -- & - & -- & - \\ 31 & 3 & -- & - & -- & - & -- & - & -- & - & -- & - \\ 18 & 2 & -- & - & -- & - & -- & - & -- & - & -- & - \\ 86 & 8 & -- & - & -- & - & 13 & 1 & 39 & 1 & -- & - \\ 3704 & 32 & 90 & 1 & 500 & 8 & 64 & 5 & 64 & 1 & 64 & 6\end{array}$

CRYPTOPHYTA (CRYPTOMONADS)

. CRYPTOPHYCEAE

CRYPTOMHADAL

...CRYPTOMONADACEAE

..... CRYPTOMONAS

CYANOPHYTA (BLUE-GREEN ALGAE)

. CYANOPHYCEAE

C. CHROOCOCCALES

a. CHROOCOCCACEAE

… AGMENELLUM

.....AAMACYSTIS

... ANACYSTIS

... HORMOGONALES

.... A PHANI Z OMENON

...OSCILLATORIACEAE

..... LYNGBYA

.... OSCILLATORIA

…sCHIZOTHRIX

..... SPIRULINA

EUGLENOPHYTA (EUGLENOIDS)

- EUGLENOPHYCEA

.. EUGLENALES

... EUGLENACEAE

.... EUGLENA

.... TRACHELomonas

PYRRHOPHYTA (FIRE ALGAE)

. DINOPHYCEAE

. PERIDINIALES

... GLENODIN IACEAE

.... GLENODINIUM

NOTE: - DOMINANT ORGANISM; EQUAL TO OR GREATER THAN 15x

* - OBSERVEd ORGANISM, MAY NOT HAVE BEEN COUNTED; LESS THAN $1 / 2 x$ 
SAN JACINTO RIVER BASIN

08073600 BUFFALO BAYOU AT WEST BELT DRIVE, hOUSTON, TX--Continued

MONTHLY AND ANNUAL MEANS AND LOADS FOR OCTOBER 1979 TO SEPTEMBER 1980

\begin{tabular}{|c|c|c|c|c|c|c|c|c|c|c|}
\hline MONTH & YEAR & $\begin{array}{l}\text { DISCHARGE } \\
\text { (CFS-DAYS) }\end{array}$ & $\begin{array}{c}\text { SPECIFIC } \\
\text { CONDUCT- } \\
\text { ANCE } \\
\text { (MICRO- } \\
\text { MHOS) }\end{array}$ & $\begin{array}{l}\text { DIS- } \\
\text { SOLVED } \\
\text { SOLIDS } \\
\text { (MG/L) }\end{array}$ & $\begin{array}{l}\text { DIS- } \\
\text { SOLVED } \\
\text { SOLIDS } \\
\text { (TONS) }\end{array}$ & $\begin{array}{c}\text { DIS- } \\
\text { SOLVED } \\
\text { CHLORIDE } \\
\text { (MG/L) }\end{array}$ & $\begin{array}{l}\text { DIS- } \\
\text { SOLVED } \\
\text { CHLORIDE } \\
\text { (TONS) }\end{array}$ & $\begin{array}{l}\text { DIS- } \\
\text { SOLVED } \\
\text { SULFATE } \\
(M G / L)\end{array}$ & $\begin{array}{l}\text { DIS- } \\
\text { SOLVED } \\
\text { SULFATE } \\
\text { (TONS) }\end{array}$ & $\begin{array}{c}\text { HARDNESS } \\
(\mathrm{CA}, \mathrm{MG}) \\
(\mathrm{MG} / \mathrm{L})\end{array}$ \\
\hline OCT. & 1979 & 22440 & 178 & 100 & 6040 & 16 & 983 & 11 & 658 & 45 \\
\hline Nov. & 1979 & 13860 & 179 & 100 & 3750 & 16 & 615 & 11 & 404 & 45 \\
\hline DEC. & 1979 & 11560 & 179 & 100 & 3120 & 16 & 508 & 11 & 338 & 45 \\
\hline JAN. & 1980 & 19170 & 148 & 83 & 4280 & 13 & 690 & 9.2 & 474 & 38 \\
\hline FEB. & 1980 & 14868 & 180 & 101 & 4030 & 16 & 655 & 11 & 441 & 45 \\
\hline MAR. & 1980 & 4972 & 336 & 187 & 2510 & 34 & 452 & 16 & 221 & 69 \\
\hline APR. & 1980 & 9235 & 210 & 117 & 2920 & 20 & 503 & 11 & 284 & 47 \\
\hline MAY & 1980 & 7924 & 264 & 147 & 3140 & 25 & 536 & 15 & 313 & 61 \\
\hline JUNE & 1980 & 3013 & 477 & 265 & 2160 & 47 & 386 & 24 & 194 & 100 \\
\hline JULY & 1980 & 2657 & 612 & 339 & 2440 & 63 & 450 & 28 & 202 & 120 \\
\hline AUG. & 1980 & 2089 & 576 & 320 & 1800 & 58 & 330 & 27 & 154 & 120 \\
\hline SEPT & 1980 & 7556 & 285 & 159 & 3240 & 27 & 547 & 16 & 330 & 67 \\
\hline \multicolumn{2}{|c|}{ TOTAL } & 119344 & $\star \star *$ & $\star \star$ & 39400 & ** & 6650 & $\star *$ & 4010 & $\star \star$ \\
\hline \multicolumn{2}{|c|}{ WTD. AVG. } & 326 & 219 & 122 & $\star \star$ & 21 & $* *$ & 12 & ** & 52 \\
\hline
\end{tabular}

SPECIFIC CONDUCTANCE (MICROMHOS/CM AT 25 DEG. C), WATER YEAR OCTOBER 1979 TO SEPTEMBER 1980

\begin{tabular}{|c|c|c|c|c|c|c|c|c|c|c|c|c|}
\hline DAY & OCT & NOV & DEC & JAN & FEB & MAR & APR & MAY & JUN & JUL & AUG & SEP \\
\hline $\begin{array}{l}1 \\
2 \\
3 \\
4 \\
5\end{array}$ & $\begin{array}{l}114 \\
110 \\
115 \\
123 \\
129\end{array}$ & $\begin{array}{l}333 \\
178 \\
150 \\
160 \\
188\end{array}$ & $\begin{array}{l}325 \\
380 \\
435 \\
485 \\
520\end{array}$ & $\begin{array}{l}225 \\
323 \\
244 \\
167 \\
178\end{array}$ & $\begin{array}{l}111 \\
123 \\
130 \\
137 \\
144\end{array}$ & $\begin{array}{l}535 \\
545 \\
668 \\
733 \\
737\end{array}$ & $\begin{array}{l}131 \\
115 \\
109 \\
108 \\
110\end{array}$ & $\begin{array}{l}781 \\
570 \\
650 \\
700 \\
716\end{array}$ & $\begin{array}{l}624 \\
640 \\
665 \\
702 \\
718\end{array}$ & $\begin{array}{l}748 \\
752 \\
760 \\
750 \\
753\end{array}$ & $\begin{array}{l}558 \\
575 \\
588 \\
649 \\
675\end{array}$ & $\begin{array}{l}450 \\
560 \\
638 \\
693 \\
642\end{array}$ \\
\hline $\begin{array}{r}6 \\
7 \\
8 \\
9 \\
10\end{array}$ & $\begin{array}{l}127 \\
128 \\
151 \\
159 \\
164\end{array}$ & $\begin{array}{l}213 \\
398 \\
549 \\
586 \\
638\end{array}$ & $\begin{array}{l}576 \\
649 \\
675 \\
690 \\
682\end{array}$ & $\begin{array}{l}250 \\
307 \\
394 \\
528 \\
597\end{array}$ & $\begin{array}{l}209 \\
495 \\
350 \\
217 \\
175\end{array}$ & $\begin{array}{l}740 \\
767 \\
800 \\
814 \\
775\end{array}$ & $\begin{array}{l}127 \\
205 \\
513 \\
612 \\
662\end{array}$ & $\begin{array}{l}742 \\
667 \\
666 \\
645 \\
633\end{array}$ & $\begin{array}{l}708 \\
733 \\
735 \\
425 \\
300\end{array}$ & $\begin{array}{l}752 \\
748 \\
757 \\
751 \\
764\end{array}$ & $\begin{array}{l}694 \\
460 \\
496 \\
563 \\
620\end{array}$ & $\begin{array}{l}200 \\
235 \\
220 \\
200 \\
175\end{array}$ \\
\hline $\begin{array}{l}11 \\
12 \\
13 \\
14 \\
15\end{array}$ & $\begin{array}{l}183 \\
207 \\
203 \\
204 \\
273\end{array}$ & $\begin{array}{l}640 \\
681 \\
693 \\
712 \\
745\end{array}$ & $\begin{array}{l}690 \\
200 \\
264 \\
127 \\
106\end{array}$ & $\begin{array}{l}640 \\
661 \\
663 \\
695 \\
736\end{array}$ & $\begin{array}{l}135 \\
136 \\
143 \\
137 \\
176\end{array}$ & $\begin{array}{l}826 \\
820 \\
821 \\
836 \\
865\end{array}$ & $\begin{array}{l}685 \\
703 \\
600 \\
627 \\
720\end{array}$ & $\begin{array}{l}690 \\
703 \\
732 \\
403 \\
475\end{array}$ & $\begin{array}{l}374 \\
400 \\
472 \\
520 \\
550\end{array}$ & $\begin{array}{l}750 \\
724 \\
745 \\
789 \\
729\end{array}$ & $\begin{array}{l}657 \\
701 \\
707 \\
737 \\
400\end{array}$ & $\begin{array}{l}208 \\
226 \\
235 \\
253 \\
475\end{array}$ \\
\hline $\begin{array}{l}16 \\
17 \\
18 \\
19 \\
20\end{array}$ & $\begin{array}{l}370 \\
569 \\
635 \\
629 \\
638\end{array}$ & $\begin{array}{l}728 \\
740 \\
753 \\
797 \\
291\end{array}$ & $\begin{array}{l}103 \\
112 \\
123 \\
148 \\
167\end{array}$ & $\begin{array}{l}742 \\
500 \\
319 \\
340 \\
388\end{array}$ & $\begin{array}{l}215 \\
209 \\
264 \\
324 \\
394\end{array}$ & $\begin{array}{l}864 \\
858 \\
861 \\
851 \\
600\end{array}$ & $\begin{array}{l}733 \\
788 \\
814 \\
803 \\
804\end{array}$ & $\begin{array}{l}296 \\
190 \\
207 \\
199 \\
176\end{array}$ & $\begin{array}{l}637 \\
666 \\
677 \\
694 \\
710\end{array}$ & $\begin{array}{l}701 \\
673 \\
676 \\
671 \\
664\end{array}$ & $\begin{array}{l}388 \\
500 \\
647 \\
725 \\
697\end{array}$ & $\begin{array}{l}510 \\
544 \\
578 \\
668 \\
650\end{array}$ \\
\hline $\begin{array}{l}21 \\
22 \\
23 \\
24 \\
25\end{array}$ & $\begin{array}{l}635 \\
534 \\
576 \\
631 \\
646\end{array}$ & $\begin{array}{l}165 \\
150 \\
144 \\
130 \\
124\end{array}$ & $\begin{array}{l}296 \\
390 \\
350 \\
325 \\
330\end{array}$ & $\begin{array}{r}142 \\
158 \\
170 \\
108 \\
90\end{array}$ & $\begin{array}{l}450 \\
495 \\
529 \\
531 \\
609\end{array}$ & $\begin{array}{l}612 \\
631 \\
632 \\
714 \\
793\end{array}$ & $\begin{array}{l}822 \\
825 \\
827 \\
834 \\
600\end{array}$ & $\begin{array}{l}118 \\
294 \\
141 \\
150 \\
163\end{array}$ & $\begin{array}{l}731 \\
320 \\
389 \\
584 \\
650\end{array}$ & $\begin{array}{l}510 \\
528 \\
558 \\
616 \\
650\end{array}$ & $\begin{array}{l}685 \\
692 \\
700 \\
702 \\
729\end{array}$ & $\begin{array}{l}640 \\
550 \\
570 \\
565 \\
450\end{array}$ \\
\hline $\begin{array}{l}26 \\
27 \\
28 \\
29 \\
30 \\
31\end{array}$ & $\begin{array}{l}651 \\
699 \\
704 \\
596 \\
400 \\
230\end{array}$ & $\begin{array}{l}120 \\
125 \\
137 \\
154 \\
272 \\
---\end{array}$ & $\begin{array}{l}375 \\
406 \\
472 \\
286 \\
150 \\
175\end{array}$ & $\begin{array}{r}95 \\
92 \\
100 \\
106 \\
105 \\
109\end{array}$ & \begin{tabular}{l}
662 \\
704 \\
693 \\
701 \\
\hdashline.- \\
$-\cdots$
\end{tabular} & $\begin{array}{l}768 \\
175 \\
193 \\
120 \\
144 \\
236\end{array}$ & $\begin{array}{l}617 \\
614 \\
656 \\
700 \\
739 \\
---\end{array}$ & $\begin{array}{l}275 \\
426 \\
569 \\
615 \\
654 \\
623\end{array}$ & $\begin{array}{l}697 \\
702 \\
725 \\
724 \\
736 \\
-\cdots\end{array}$ & $\begin{array}{l}638 \\
637 \\
570 \\
450 \\
472 \\
508\end{array}$ & $\begin{array}{l}715 \\
650 \\
705 \\
517 \\
411 \\
399\end{array}$ & $\begin{array}{l}384 \\
400 \\
415 \\
455 \\
323 \\
-\end{array}$ \\
\hline MEAN & 372 & 390 & 355 & 328 & 331 & 656 & 573 & 480 & 607 & 671 & 611 & 437 \\
\hline
\end{tabular}


SAN JACINTO RIVER BASIN

08073600 BUFFALO BAYOU AT WEST BELT DRIVE, hOUSTON, TX--Continued

TEMPERATURE, WATER (DEG. C), WATER YEAR OCTOBER' 1979 TO SEPTEMBER 1980

\begin{tabular}{|c|c|c|c|c|c|c|c|c|c|c|c|c|}
\hline DAY & OCT & HOV & DEC & JAN & FEB & MAR & APR & MAY & JUN & JUL & AUG & SEP \\
\hline $\begin{array}{l}1 \\
2 \\
3 \\
4 \\
5\end{array}$ & $\begin{array}{l}25.5 \\
25.0 \\
24.0 \\
24.5 \\
23.5\end{array}$ & $\begin{array}{l}18.0 \\
16.0 \\
18.0 \\
18.5 \\
21.0\end{array}$ & $\begin{array}{r}--- \\
22.0 \\
22.5 \\
22.5\end{array}$ & $\begin{array}{r}18.0 \\
18.0 \\
18.0 \\
14.5\end{array}$ & $\begin{array}{r}12.0 \\
12.0 \\
\cdots \\
\cdots\end{array}$ & $\begin{array}{r}14.5 \\
16.0 \\
16.0 \\
15.0 \\
-\end{array}$ & $\begin{array}{l}17.0 \\
18.5 \\
19.0 \\
19.0 \\
18.0\end{array}$ & $\begin{array}{r}22.0 \\
22.5 \\
23.0 \\
22.5\end{array}$ & $\begin{array}{r}27.0 \\
26.5 \\
-1.0 \\
25.0 \\
26.0\end{array}$ & $\begin{array}{l}27.5 \\
28.0 \\
27.5 \\
27.5\end{array}$ & $\begin{array}{l}27.0 \\
27.0 \\
28.0 \\
28.0 \\
28.5\end{array}$ & $\begin{array}{l}27.5 \\
27.0 \\
26.5 \\
22.0 \\
26.5\end{array}$ \\
\hline $\begin{array}{r}6 \\
7 \\
8 \\
9 \\
10\end{array}$ & $\begin{array}{l}22.5 \\
24.5 \\
24.5 \\
25.0 \\
23.0\end{array}$ & $\begin{array}{l}22.5 \\
22.0 \\
23.0 \\
24.5 \\
24.0\end{array}$ & $\begin{array}{l}23.0 \\
23.5 \\
25.0 \\
26.5 \\
24.0\end{array}$ & $\begin{array}{l}15.0 \\
17.5 \\
17.0 \\
18.0 \\
19.0\end{array}$ & $\begin{array}{l}12.0 \\
16.0 \\
16.5 \\
12.0 \\
13.0\end{array}$ & $\begin{array}{r}16.5 \\
18.0 \\
22.0 \\
20.0\end{array}$ & $\begin{array}{l}18.5 \\
19.0 \\
20.5 \\
19.5 \\
19.5\end{array}$ & $\begin{array}{l}23.0 \\
24.0 \\
24.5 \\
21.5 \\
23.0\end{array}$ & $\begin{array}{r}26.5 \\
27.0 \\
27.0 \\
27.0 \\
-.-\end{array}$ & $\begin{array}{l}28.0 \\
27.5 \\
28.0 \\
28.0 \\
28.0\end{array}$ & $\begin{array}{l}28.0 \\
28.0 \\
27.0 \\
28.5 \\
27.5\end{array}$ & $\begin{array}{l}25.5 \\
26.0 \\
26.0 \\
26.5 \\
26.5\end{array}$ \\
\hline $\begin{array}{l}11 \\
12 \\
13 \\
14 \\
15\end{array}$ & $\begin{array}{l}22.5 \\
22.0 \\
22.5 \\
22.5\end{array}$ & $\begin{array}{l}23.5 \\
22.0 \\
22.0 \\
21.5 \\
22.0\end{array}$ & $\begin{array}{l}24.0 \\
22.0 \\
19.5 \\
17.0 \\
17.5\end{array}$ & $\begin{array}{l}20.5 \\
18.5 \\
19.0 \\
20.0 \\
22.0\end{array}$ & $\begin{array}{r}9.0 \\
9.0 \\
10.0 \\
11.0 \\
13.5\end{array}$ & $\begin{array}{l}21.5 \\
21.0 \\
19.5 \\
16.0 \\
17.0\end{array}$ & $\begin{array}{l}20.5 \\
20.5 \\
21.0 \\
14.0 \\
16.5\end{array}$ & $\begin{array}{l}25.0 \\
24.5 \\
25.0 \\
23.0 \\
23.5\end{array}$ & $\begin{array}{l}26.5 \\
25.0 \\
25.5 \\
25.0 \\
26.0\end{array}$ & $\begin{array}{r}28.0 \\
29.0 \\
29.0\end{array}$ & $\begin{array}{l}26.0 \\
27.5 \\
28.0 \\
27.5 \\
28.0\end{array}$ & $\begin{array}{l}27.0 \\
27.0 \\
26.5 \\
27.0 \\
27.0\end{array}$ \\
\hline $\begin{array}{l}16 \\
17 \\
18 \\
19 \\
20\end{array}$ & $\begin{array}{l}23.0 \\
26.0 \\
24.5 \\
24.5 \\
25.0\end{array}$ & $\begin{array}{l}23.0 \\
23.0 \\
22.5 \\
25.0 \\
24.0\end{array}$ & $\begin{array}{r}18.0 \\
15.5 \\
16.0 \\
17.0\end{array}$ & $\begin{array}{l}22.0 \\
22.0 \\
19.0 \\
19.5 \\
19.5\end{array}$ & $\begin{array}{l}12.0 \\
12.5 \\
10.0 \\
12.0 \\
15.0\end{array}$ & $\begin{array}{l}18.5 \\
16.0 \\
10.5 \\
20.0 \\
19.0\end{array}$ & $\begin{array}{l}19.0 \\
19.5 \\
20.0 \\
19.5 \\
20.0\end{array}$ & $\begin{array}{r}22.5 \\
22.0 \\
21.0 \\
22.0\end{array}$ & $\begin{array}{r}27.0 \\
26.5 \\
27.0 \\
27.5 \\
-.-\end{array}$ & $\begin{array}{l}29.0 \\
28.0 \\
28.5 \\
28.5 \\
29.0\end{array}$ & $\begin{array}{r}27.5 \\
27.5 \\
27.0 \\
27.5\end{array}$ & $\begin{array}{r}-1.5 \\
27.5 \\
28.0 \\
\ldots\end{array}$ \\
\hline $\begin{array}{l}21 \\
22 \\
23 \\
24 \\
25\end{array}$ & $\begin{array}{l}26.0 \\
25.0 \\
25.0 \\
22.0 \\
22.5\end{array}$ & $\begin{array}{r}24.0 \\
19.5 \\
17.5 \\
19.5\end{array}$ & $\begin{array}{l}18.0 \\
24.0 \\
21.5 \\
16.5 \\
16.0\end{array}$ & $\begin{array}{l}13.0 \\
17.0 \\
12.0 \\
14.5 \\
13.0\end{array}$ & $\begin{array}{l}18.0 \\
13.0 \\
18.5 \\
19.0 \\
12.5\end{array}$ & $\begin{array}{l}17.0 \\
17.5 \\
18.0 \\
19.0 \\
19.0\end{array}$ & $\begin{array}{l}21.0 \\
21.0 \\
22.0 \\
22.0 \\
23.5\end{array}$ & $\begin{array}{l}24.0 \\
24.0 \\
24.5 \\
25.0 \\
25.5\end{array}$ & $\begin{array}{l}26.5 \\
25.0 \\
24.5 \\
27.0 \\
28.0\end{array}$ & $\begin{array}{l}28.5 \\
28.5 \\
22.0 \\
27.5 \\
28.0\end{array}$ & $\begin{array}{l}27.0 \\
27.0 \\
27.5 \\
28.5 \\
27.5\end{array}$ & $\begin{array}{r}27.5 \\
27.0\end{array}$ \\
\hline $\begin{array}{l}26 \\
27 \\
28 \\
29 \\
30 \\
31\end{array}$ & $\begin{array}{l}25.5 \\
25.5 \\
25.0 \\
25.5 \\
23.5 \\
18.5\end{array}$ & $\begin{array}{l}15.5 \\
18.5 \\
19.0 \\
17.0 \\
16.0 \\
-\end{array}$ & $\begin{array}{r}12.5 \\
17.0 \\
19.5 \\
17.5 \\
17.0\end{array}$ & $\begin{array}{l}15.0 \\
15.5 \\
14.5 \\
14.5 \\
14.0 \\
13.5\end{array}$ & $\begin{array}{r}15.0 \\
13.5 \\
16.0 \\
19.0 \\
\ldots-0\end{array}$ & $\begin{array}{l}18.5 \\
16.5 \\
15.0 \\
17.5 \\
18.0 \\
16.0\end{array}$ & $\begin{array}{r}20.0 \\
20.5 \\
20.5 \\
21.0 \\
\ldots\end{array}$ & $\begin{array}{l}27.0 \\
26.5 \\
26.5 \\
26.0 \\
26.0 \\
26.5\end{array}$ & $\begin{array}{r}25.5 \\
27.0 \\
25.5 \\
27.0 \\
26.5 \\
\ldots\end{array}$ & $\begin{array}{r}27.0 \\
28.0 \\
25.5 \\
26.0 \\
27.5\end{array}$ & $\begin{array}{l}27.5 \\
27.0 \\
26.5 \\
26.0 \\
27.5 \\
28.0\end{array}$ & $\begin{array}{r}26.0 \\
24.0 \\
25.5 \\
26.5 \\
25.0 \\
-\end{array}$ \\
\hline MEAN & 24.0 & 21.0 & 20.0 & 17.0 & 13.5 & 17.5 & 19.5 & 24.0 & 26.5 & 27.5 & 27.5 & 26.5 \\
\hline
\end{tabular}




\section{BETTINA STREET OITCH DRAINAGE BASIN}

The locations of data-collection sites in the Bettina Street Ditch drainage basin are shown in figure 4 .

Weighted-mean rainfall for the 1980 water year was not determined.

The storm of December 12, 1979 was selected for analys is at station 08073630 , Bettina Street Ditch at Houston. 


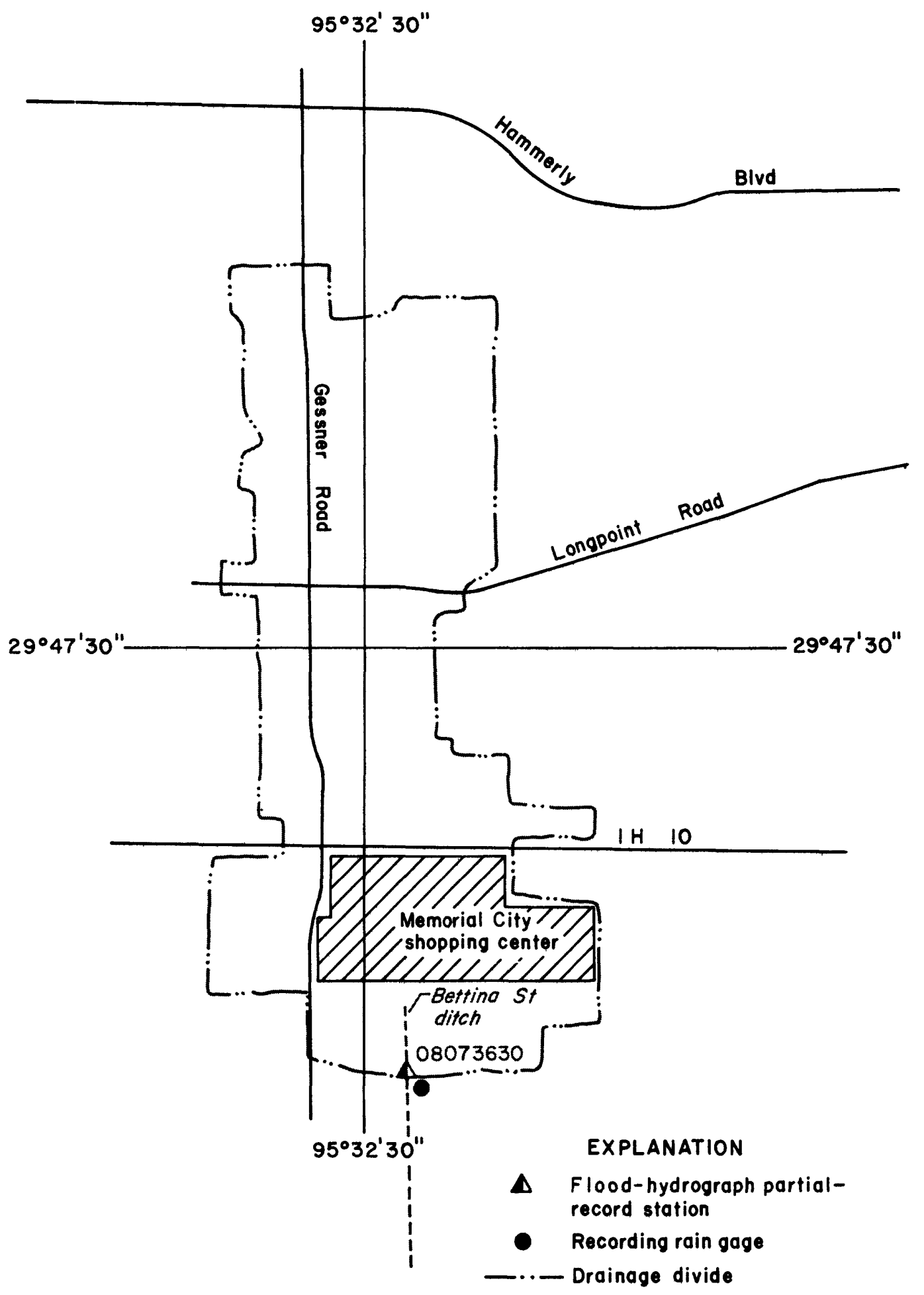

0 I MILE

Base from USGS Topographic

Quadrangle

Figure 4.-Locations of data-collection sites in and near Bettina Street Ditch drainage basin 


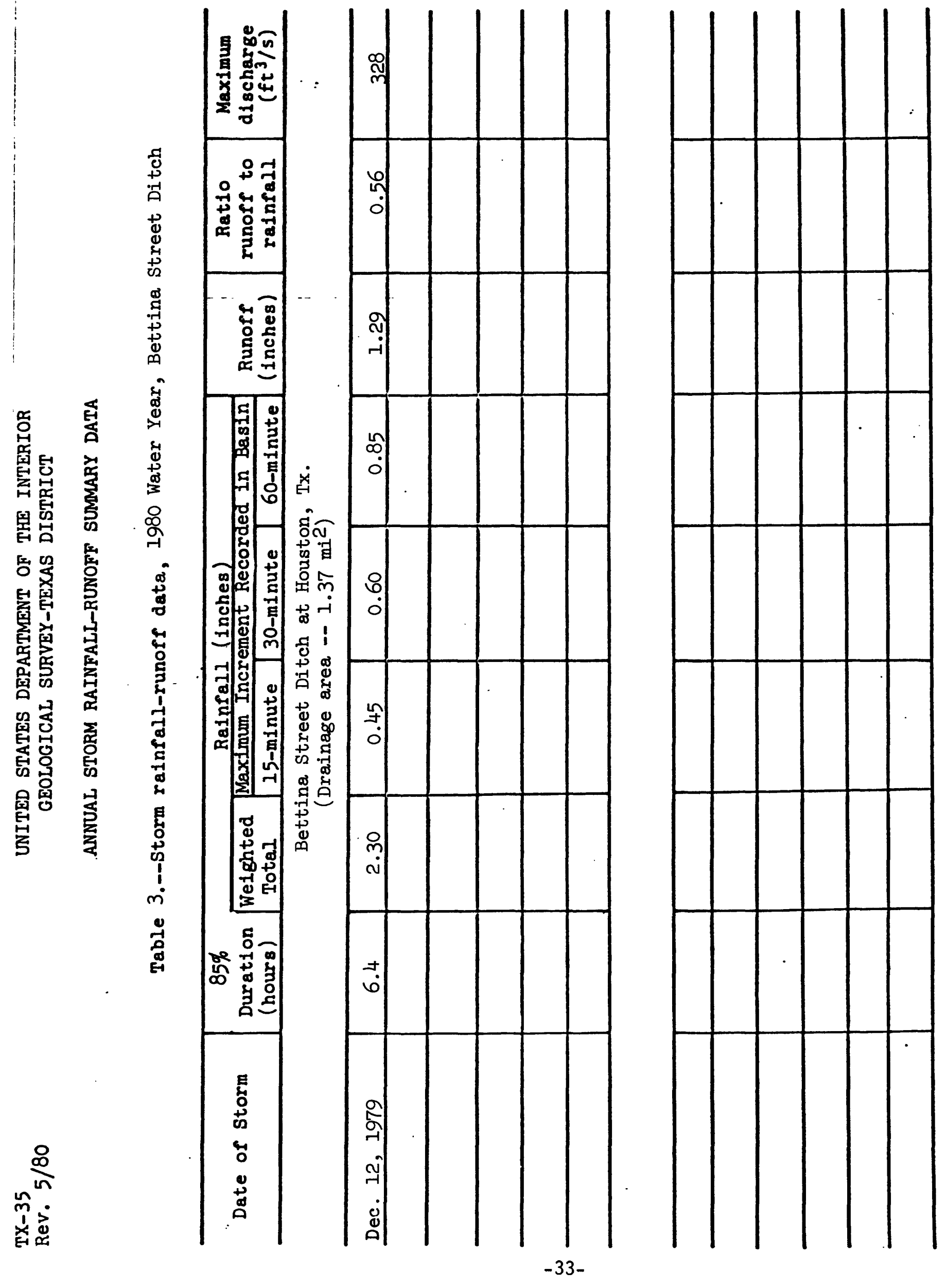


08073630 BETTINA STREET DITCH AT HOUSTON, TEX.

(Flood-hydrograph partial-record station)

LOCATION.--Lat $29^{\circ} 46^{\prime} 32^{\prime \prime}$, Iong $95^{\circ} 32^{\prime} 23^{\prime \prime}$, Harris County, Hydrologic Unit 12040104, at downstream side of bridge at intersection of Bettina Street Ditch and Kimberly Street in west Houston.

DRAINAGE AREA.--1.37 $\mathrm{mi}^{2}$.

PERIOD OF RECORD.--Nov. 3, 1978 to current year.

GAGE.--Digital flood-hydrograph and rainfall recorders and crest-stage gage. Datum of gage is National Geodetic Vertical Datum of 1929, 1964 adjustment, unadjusted for land-surface subsidence.

REMARKS.--Records fair.

EXTREMES FOR PERIOD OF RECORN.--Maximum discharqe $510 \mathrm{ft}^{3} / \mathrm{s}$, Sept. 19, 1979, (gage-height $81.25 \mathrm{ft}$.); minimum not determined.

EXTREMES FOR CURRENT YEAR.--Peak discharge above base of $200 \mathrm{ft}^{3} / \mathrm{s}$ and maximum $(*)$ :

DATE

0ct. 30

Dec. 12

Mar. 29

Apr. 25

July 29
TIME

1705

1120

1005

1010

1625
DISCHAPGE.

$\left(\mathrm{ft}^{3} / \mathrm{s}\right)$

387

328

$\star 470$

236

333
GAGE HEIGHT

(ft.)

80.16

79.55

80.91

78.37

79.61

Minimum discharge not determined. 


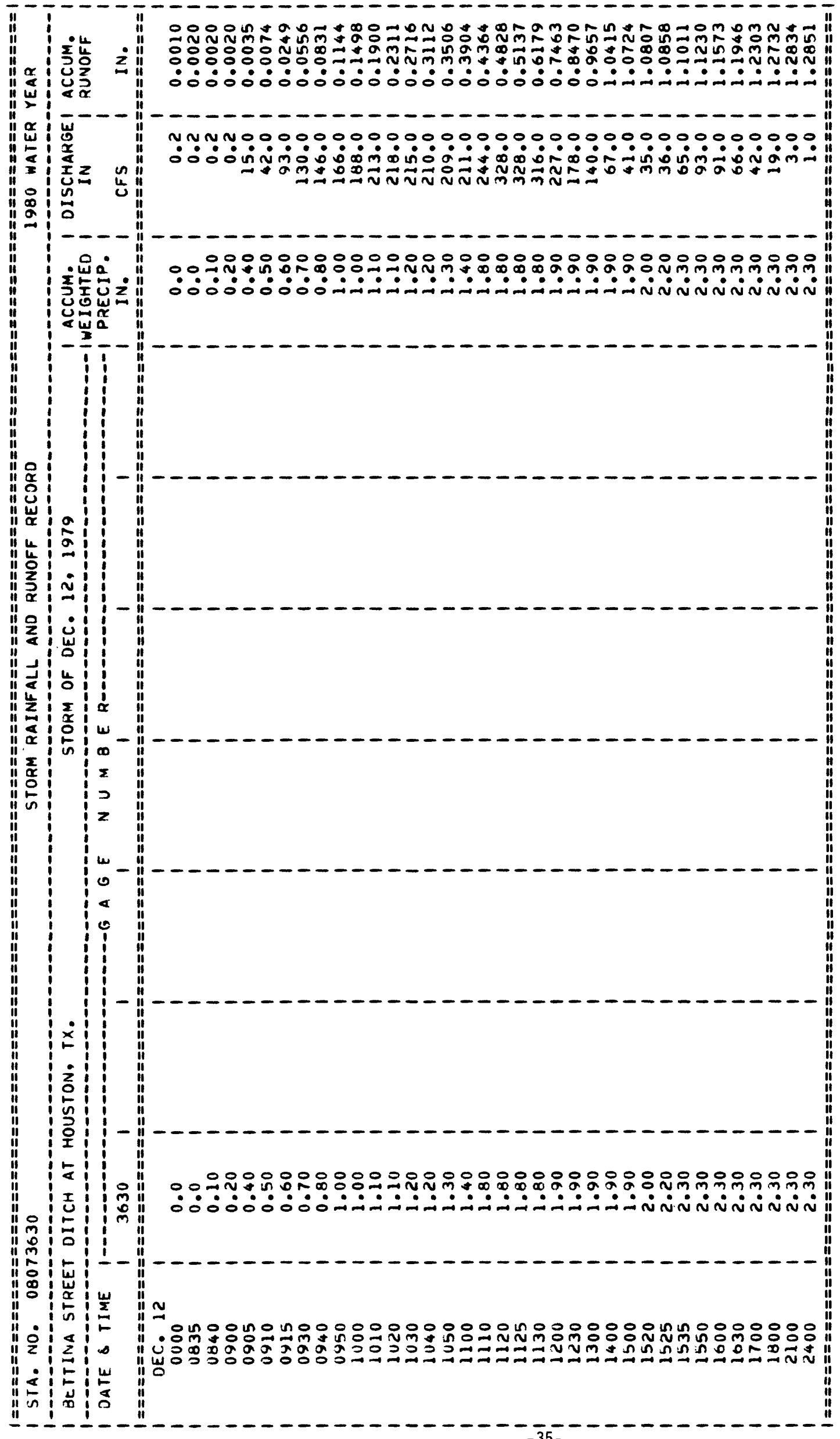


SAN JACINI'U RIVER BASIN

08073700 BUFFALO BAYOU A'T PINEY POIN', TX

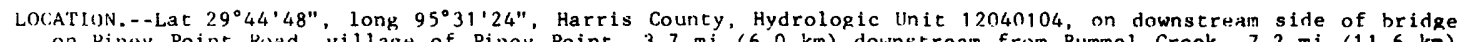
on Piney Point Road, village of Piney Point, $3.7 \mathrm{mi}(6.0 \mathrm{~km})$ downstream from Rummel Creek, $7.2 \mathrm{mi}(11.6 \mathrm{~km})$
downstreain from gage near Addicks (station 08073500$)$, and $12.5 \mathrm{mi}(20.1 \mathrm{~km}$ ) upstream from page at Houston (station 08074000).

DRAINAGE AREA. $--317 \mathrm{mi}^{2}\left(821 \mathrm{kin}^{2}\right)$.

PER[OD OF RECORD.--Octuber 1963 to September 1976, October 1976 to current year (gage heights only).

GAGE.--Water-stage recorder. Datun of gage is $1.35 \mathrm{ft}(0.412 \mathrm{~m})$ below National (eodetic Vertical Datun of 1929 , 1973 ddjustment.

REMARKS.--Station is operated for the purpose of gate regulations at Rarker and Addicks Reservoirs (stations 08072500 and 08073000$)$, located 14.0 and $13.8 \mathrm{mi}(22.5$ and $22.2 \mathrm{~km}$ ) upstream, respectively. Iow flow is partly sustained by sewage effluent from Houston suburbs. Corps of Engineers gage-height telemeter at station.

AVERAGE DISCHARGE.--13 years (water years 1963-76), $265 \mathrm{ft}^{3} / \mathrm{s}\left(7.505 \mathrm{~m}^{3} / \mathrm{s}\right), 192,000 \mathrm{acre-ft} / \mathrm{yr}(237 \mathrm{hm} / \mathrm{yr})$. EXIREMES FOR PEKIOD OF RECORD.--Maximum discharge, $4,470 \mathrm{ft}^{3} / \mathrm{s}\left(127 \mathrm{~m}^{3} / \mathrm{s}\right)$ June 13 , 1973, gage height, $54.98 \mathrm{ft}$ $(16.758 \mathrm{~m})$; maximum gage height, $55.15 \mathrm{ft}(16.810 \mathrm{~m})$ Sept. 19, 1979; minimum daily discharge, $6.0 \mathrm{ft} / \mathrm{s}(0.17$ in $/ s$ ) Dec. 6, 7,1964 .

EXTRESES MOR CURRENT YEAR.--Maximum gage height, $45.63 \mathrm{ft}(13.908 \mathrm{~m})$ June 22 at 2100 hours; ininimum, 32.65 ft $(9.952 \mathrm{~m})$ Aug. 14, 15.

GAGE HEICH'T, IN FEET, WATER YLAK OCTUBEK 1979 TU SEYTEMBEK 1980 Madiriun VALUtS

\begin{tabular}{|c|c|c|c|c|c|c|c|c|c|c|c|c|}
\hline DAY & vC'T & Nov & UEC & JAN & res & IAKK & $A P K$ & WAY & JUN & JUL & AUG & Str \\
\hline $\begin{array}{l}1 \\
2 \\
3 \\
4 \\
5\end{array}$ & $\begin{array}{l}44.72 \\
44.72 \\
44.70 \\
44.67 \\
44.65\end{array}$ & \begin{tabular}{c}
36.85 \\
$\therefore-$ \\
\hdashline- \\
$\therefore-$
\end{tabular} & $\begin{array}{l}34.50 \\
34.13 \\
33.85 \\
33.64 \\
33.37\end{array}$ & $\begin{array}{l}36.30 \\
36.74 \\
39.78 \\
38.17 \\
38.17\end{array}$ & $\begin{array}{l}43.73 \\
43.45 \\
43.16 \\
42.82 \\
42.38\end{array}$ & $\begin{array}{l}34.60 \\
34.02 \\
33.51 \\
33.80 \\
33.63\end{array}$ & $\begin{array}{l}41.34 \\
42.25 \\
43.57 \\
44.07 \\
43.94\end{array}$ & $\begin{array}{l}34.77 \\
34.34 \\
34.04 \\
33.60 \\
33.30\end{array}$ & $\begin{array}{r}53.40 \\
33.30 \\
33.30 \\
33.30\end{array}$ & $\begin{array}{l}33.15 \\
33.14 \\
33.10 \\
33.15 \\
33.17\end{array}$ & $\begin{array}{l}34.04 \\
34.18 \\
33.97 \\
33.74 \\
33.48\end{array}$ & $\begin{array}{l}33.82 \\
33.70 \\
33.63 \\
33.38 \\
38.53\end{array}$ \\
\hline $\begin{array}{r}6 \\
7 \\
8 \\
9 \\
10\end{array}$ & $\begin{array}{l}44.58 \\
44.48 \\
44.38 \\
44.28 \\
44.20\end{array}$ & $\begin{array}{l}\cdots \\
\cdots \\
\cdots \\
\cdots\end{array}$ & $\begin{array}{r}33 .+23 \\
31.442 \\
330.413\end{array}$ & $\begin{array}{l}36.89 \\
34.95 \\
34.33 \\
13.54 \\
31.42\end{array}$ & $\begin{array}{l}41.48 \\
34.55 \\
40.93 \\
40.50 \\
39.94\end{array}$ & $\begin{array}{l}35.58 \\
39.65 \\
13.00 \\
31.55\end{array}$ & $\begin{array}{l}43.60 \\
42.88 \\
34.40 \\
33.10 \\
33.60\end{array}$ & $\begin{array}{l}33.28 \\
34.88 \\
34.37 \\
33.98 \\
33.70\end{array}$ & $\begin{array}{r}13.30 \\
0 . . \\
34.80 \\
38.80\end{array}$ & $\begin{array}{l}53.17 \\
33.08 \\
33.12 \\
33.03 \\
33.03\end{array}$ & $\begin{array}{l}13.33 \\
36.76 \\
34.79 \\
13.04 \\
13.34\end{array}$ & $\begin{array}{l}39.36 \\
37.33 \\
40.13 \\
41.70 \\
41.98\end{array}$ \\
\hline $\begin{array}{l}11 \\
12 \\
13 \\
14 \\
15\end{array}$ & $\begin{array}{l}44.05 \\
49.60 \\
42.60 \\
41.00 \\
40.13\end{array}$ & $\begin{array}{l}--. \\
\cdots \\
\cdots \\
\cdots \\
\cdots\end{array}$ & $\begin{array}{l}44.18 \\
42.015 \\
42.35 \\
43.56\end{array}$ & $\begin{array}{l}-\cdots \\
-\cdots \\
-- \\
--\end{array}$ & $\begin{array}{l}34.41 \\
3 y .78 \\
14.00 \\
+10.70 \\
40.31\end{array}$ & $\begin{array}{l}13.47 \\
19.5+4 \\
13.44 \\
19 .+3 \\
13.00\end{array}$ & $\begin{array}{r}31.30 \\
\ldots . \\
31.70 \\
13.50\end{array}$ & $\begin{array}{l}33.30 \\
31.20 \\
35.30 \\
35.44 \\
36.30\end{array}$ & $\begin{array}{l}37.75 \\
35.30 \\
34.07 \\
33.62 \\
33.51\end{array}$ & $\begin{array}{l}33.06 \\
33.26 \\
33.32 \\
39.32 \\
19.13\end{array}$ & $\begin{array}{l}33.23 \\
33.13 \\
33.0 \% \\
32.49 \\
37.03\end{array}$ & $\begin{array}{l}41.01 \\
40.01 \\
39.14 \\
34.08 \\
14.12\end{array}$ \\
\hline $\begin{array}{l}10 \\
17 \\
18 \\
19 \\
20\end{array}$ & $\begin{array}{r}38.13 \\
3+.73 \\
\ldots \ldots \\
\ldots . .\end{array}$ & $\begin{array}{c}\cdots \\
\cdots \\
\cdots \\
30.30\end{array}$ & $\begin{array}{l}43.35 \\
41.35 \\
42.48 \\
41.92 \\
39 .+0\end{array}$ & $\begin{array}{l}-- \\
-- \\
-- \\
--\end{array}$ & $\begin{array}{l}3 y .+4 y \\
30.3 y \\
37.44 \\
35.111 \\
34.31\end{array}$ & $\begin{array}{l}13.67 \\
34.80 \\
33.63 \\
13.15 \\
14.62\end{array}$ & $\begin{array}{r}13.40 \\
13.40 \\
13 .+0 \\
=\ldots \\
=.2\end{array}$ & $\begin{array}{l}17.04 \\
3 y .00 \\
17.27 \\
41.37 \\
41.02\end{array}$ & $\begin{array}{l}33.33 \\
33.34 \\
33.34 \\
33.23 \\
33.24\end{array}$ & $\begin{array}{r}\text { 13.00 } \\
13.53 \\
33.34 \\
\ldots\end{array}$ & $\begin{array}{l}15.47 \\
35.57 \\
35.56 \\
33.17 \\
31.08\end{array}$ & $\begin{array}{l}33.80 \\
33.37 \\
33.74 \\
33.47 \\
33.30\end{array}$ \\
\hline $\begin{array}{l}21 \\
21 \\
23 \\
24 \\
\angle 3\end{array}$ & $\begin{array}{l}--- \\
--- \\
--- \\
--- \\
--\end{array}$ & $\begin{array}{l}41.30 \\
40.01 \\
41.40 \\
42.77 \\
45.37\end{array}$ & $\begin{array}{l}30.92 \\
34.30 \\
30.114 \\
30.52 \\
35.43\end{array}$ & $\begin{array}{l}-\cdots \\
\cdots- \\
\cdots- \\
\cdots-\end{array}$ & $\begin{array}{l}14.00 \\
3+.100 \\
13.90 \\
33.71 \\
33.62\end{array}$ & $\begin{array}{l}34.36 \\
33.61 \\
33.54 \\
33.74 \\
33.44\end{array}$ & $\begin{array}{l}35.413 \\
33.35 \\
35.31 \\
33.48 \\
39.15\end{array}$ & $\begin{array}{l}42.11 \\
40.40 \\
42.011 \\
41.04 \\
34.40\end{array}$ & $\begin{array}{l}10.40 \\
+5.613 \\
44.13 \\
33.80 \\
13.45\end{array}$ & $\begin{array}{r}38 . \% \\
33.90 \\
33.90 \\
13.90\end{array}$ & $\begin{array}{l}33.14 \\
13.13 \\
33.38 \\
33.28 \\
33.27\end{array}$ & $\begin{array}{l}33.35 \\
33.37 \\
33.40 \\
33.63 \\
36.01\end{array}$ \\
\hline $\begin{array}{l}26 \\
27 \\
28 \\
29 \\
34 \\
31\end{array}$ & $\begin{array}{r}-.- \\
\cdots \\
\cdots- \\
-- \\
42.9 y \\
42.9 y\end{array}$ & $\begin{array}{r}+3.51 \\
\ldots .0 \\
42.30 \\
31.80 \\
. .-\end{array}$ & $\begin{array}{l}34.92 \\
3+.24 \\
33.91 \\
30.35 \\
31.76 \\
31.09\end{array}$ & $\begin{array}{r}-.- \\
\ldots \\
44.10 \\
+4.11 \\
+1.48\end{array}$ & $\begin{array}{r}33.41 \\
\text { 33.5y } \\
35.24 \\
31.511 \\
\ldots .\end{array}$ & $\begin{array}{l}3.8 y \\
+4.30 \\
+3.60 \\
43.2 y \\
41.85 \\
14.1, y\end{array}$ & $\begin{array}{r}34.45 \\
34.11 \\
33.02 \\
39 .+7 \\
39.33 \\
\ldots . .\end{array}$ & $\begin{array}{r}38.03 \\
35.011 \\
33.30 \\
33.813 \\
33.40 \\
. .-\end{array}$ & $\begin{array}{r}33.30 \\
33.23 \\
13.23 \\
33.23 \\
33.18 \\
\ldots\end{array}$ & $\begin{array}{l}30 \\
30.83 \\
36.02 \\
37.05 \\
15.17\end{array}$ & $\begin{array}{l}34.38 \\
31.38 \\
36.40 \\
35.73 \\
34.17\end{array}$ & $\begin{array}{r}36.18 \\
15.71 \\
35.06 \\
35.17 \\
34.86 \\
\ldots\end{array}$ \\
\hline $\begin{array}{l}\text { VAX } \\
\text { VIIV }\end{array}$ & $\cdots$ & $\cdots$ & $\ldots$ & -.. & $\begin{array}{l}3.13 \\
31 .+1\end{array}$ & --- & $\cdots$ & $\cdots$ & - & -- &.- & $\begin{array}{l}41.48 \\
39.38\end{array}$ \\
\hline
\end{tabular}




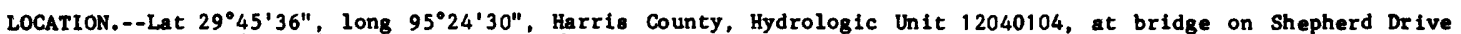
in bouston and $0.8 \mathrm{mi}(1.3 \mathrm{~km})$ upstream from Waugh Drive.

DRAINAGE AREA.---358 $\mathrm{mi}^{2}\left(927 \mathrm{~km}^{2}\right)$, unadjusted for bas $1 \mathrm{n}$ boundary changes.

\section{WATER-DISCHARGE RECORDS}

PERIOD OF RECORD.--May 1936 to September 1957, October 1957 to December 1961 (high-water records and discharge measurements), January 1962 to September 1975, October 1975 to current year (high-water records and discharge messurements):

REVISED RECORDS.--WSP 1732: Drainage area (former site).

GAGE.--Water-stage recorder and crest-stege gages. Datum of gage is 1.36 ft (0.414 m) below National Geodetic Vertical Datum of 1929, 1973 adjustment; records unadjusted for land-surface subsidence. Prior to June 19 1936, nonrecording gage, and June 19, 1936, to Jan. 16, 1962, water-stage recorder at site $0.8 \mathrm{mi}$ ( $1.3 \mathrm{~km}$ ) downstreem at 4.08-foot $(1.244 \mathrm{~m})$ lower datum. Jan. 17,1962 , to Sept. 30, 1973, auxillary water-stage recorder $0.8 \mathrm{mi}(1.3 \mathrm{~km})$ downstream. Water-stage recorder at Main Street (station 08074600) used as auxiliary gage after Sept. $30,1973$.

REMARKS.--Water-discharge records fair. Although floodflows are regulated by Barker and Addicks Reservoirs (stations 08072500 and 08073000 ) located 26.3 and $26.8 \mathrm{mi}(42.3$ and $42.6 \mathrm{~km}$ ) upstream, respectively, flood peaks frow the urbanized areas below these reservoirs are often independent of the regulation. Discharge is computed using a stage-fali-discharge relationship for all storms which produce peak discharges above 1,500 $\mathrm{ft}^{3} / \mathrm{s}\left(42.5 \mathrm{~m}^{3} / \mathrm{s}\right)$. Discharges below $1,000 \mathrm{ft}^{3} / \mathrm{s}$ are computed or estimated following designated storm periods only. Low flow is mostly sustained by sewage effluent from Houston suburbs. Gage heights are affected by
tides, backwater from Whiteoak Bayou, and other streams. Corps of Engineers gage-height telemeter at station.

AVERAGE DISCHARGE.--8 years (water years 1936-44) unregulated, $272 \mathrm{ft}^{3} / \mathrm{s}\left(7.703 \mathrm{~m}^{3} / \mathrm{s}\right), 197,100 \mathrm{acre-ft} / \mathrm{yr}(243$ $\left.\mathrm{hm}^{3} / \mathrm{yr}\right) ; 26$ years (water years 1944-57, 1962-75) regulated, $274 \mathrm{ft}^{3} / \mathrm{s}\left(7.760 \mathrm{w}^{\circ} / \mathrm{s}\right) ; 198,500 \mathrm{acre-ft/yr}(245$ $\left.\mathrm{hm}^{3} / \mathrm{yr}\right)$.

EXTREMES FOR PERIOD OF RECORD.--Maximum discharge, $10,900 \mathrm{ft}^{3} / \mathrm{s}\left(309 \mathrm{~m}^{3} / \mathrm{s}\right)$ Aug. 30, 1945, gage height, $28.82 \mathrm{ft}$ $(8.784 \mathrm{~m})$, at site $0.8 \mathrm{ml}(1.3 \mathrm{~km})$ downstream at present datum; minimum daily, $1.3 \mathrm{ft} / \mathrm{s}^{3}(0.037 \mathrm{~m} / \mathrm{s}) \mathrm{May} 24$, 1939, Nov. 5,1950 .

EXTREMES OUTSIDE PERIOD OF RECORD,--A1 1 flood data at site $0.8 \mathrm{~m} 1$ (1.3 $\mathrm{km})$ downstream at present datum. Maximum gage helght ince at least $1835,49.0 \mathrm{ft}(14.94 \mathrm{~m})$ Dec. 9,1935 , discharge $40.000 \mathrm{ft}^{3} / \mathrm{s}\left(1,130 \mathrm{~m}^{3} / \mathrm{s}\right) ; \mathrm{furn}^{\mathrm{s}} \mathrm{shed}$ by engineer for Harris County. Flood of May 31, 1929, reached a gage height of $43.5 \mathrm{ft}$ (13.26 m), discharge $19,000 \mathrm{ft}^{3 / \mathrm{s}}\left(538 \mathrm{~m}^{3} / \mathrm{s}\right)$, at bridge on Capitol Avenue affected by bridge; furnished by city of Houston.

EXTREMES FOR CURRENT YEAR.--Maxmum discharge, 3,630 $\mathrm{ft}^{2} / \mathrm{s}\left(103 \mathrm{~m}^{2} / \mathrm{s}\right)$ Jan. 22 at 1300 hours, gage helght, $16.08 \mathrm{ft}$ $(4.901 \mathrm{~m})$; minimum discharge not determined (affected by tides).

DISCHARGE, IN CUBIC FEET PEK SECOND, WATER YEAR OCTOBER 1979 TO SEPTEMBER 1980

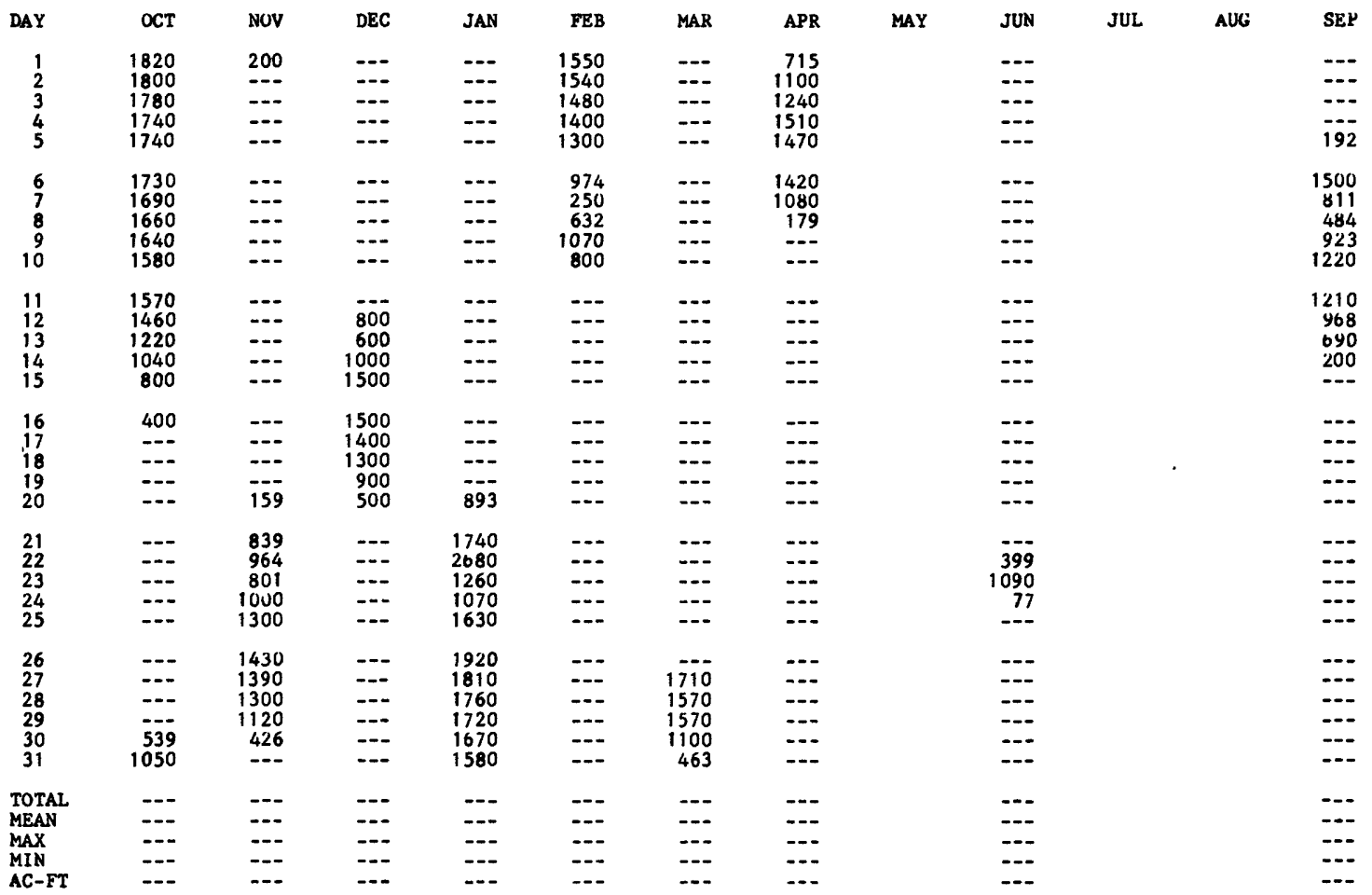

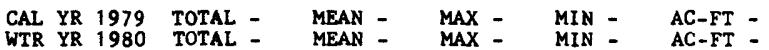


SAN JACINTO RIVER BASIN

08074000 BUFFALO BAYOU AT HOUSTON, TX--Continued

WATER-QUALITY RECORDS

PERIOD OF RECORD.--Chemical, biochemical, and pesticide anslyses: October 1968 to current year.

WATER QUALITY DATA, WATER YEAR OCTOBER 1979 TO SEPTEMBER 1980

\begin{tabular}{|c|c|c|c|c|c|c|c|c|c|c|c|c|}
\hline DATE & TIME & $\begin{array}{l}\text { STREAM- } \\
\text { FLOW. } \\
\text { INSTAN- } \\
\text { TANEOUS } \\
\text { (CFS) }\end{array}$ & $\begin{array}{l}\text { SPE- } \\
\text { CIFIC } \\
\text { CON- } \\
\text { DUCT- } \\
\text { ANCE } \\
\text { (MICRO- } \\
\text { MHOS) }\end{array}$ & $\begin{array}{c}\text { PH } \\
\text { FIELD } \\
\text { (UNITS) }\end{array}$ & $\begin{array}{c}\text { TEMPER - } \\
\text { ATURE, } \\
\text { WATER } \\
\text { (DEG C) }\end{array}$ & $\begin{array}{l}\text { COLOR } \\
\text { (PLAT- } \\
\text { INUM } \\
\text { COBALT } \\
\text { UNITS) }\end{array}$ & $\begin{array}{l}\text { TUR- } \\
\text { BID- } \\
\text { ITY } \\
\text { (NTU) }\end{array}$ & $\begin{array}{c}\text { OXYGEN, } \\
\text { DIS- } \\
\text { SOLVED } \\
(M G / L)\end{array}$ & $\begin{array}{l}\text { OXYGEN, } \\
\text { DIS- } \\
\text { SOLVED } \\
\text { (PER- } \\
\text { CENT } \\
\text { SATUR- } \\
\text { ATION) }\end{array}$ & $\begin{array}{l}\text { OXYGEN } \\
\text { DEMAND } \\
\text { B IOCHEM } \\
\text { UNINHIB } \\
5 \text { DAY } \\
(M G / L)\end{array}$ & $\begin{array}{c}\text { COLI - } \\
\text { FORH, } \\
\text { TOTAL, } \\
\text { IMMED. } \\
\text { (COLS, } \\
\text { PER } \\
100 \mathrm{ML} \text { ) }\end{array}$ & $\begin{array}{l}\text { COLI- } \\
\text { FORM, } \\
\text { FECAL, } \\
0.7 \\
\text { UH-MF } \\
\text { (COLS.' } \\
\text { IOO ML) }\end{array}$ \\
\hline $\begin{array}{l}\text { OCT } \\
\text { I5 } \\
\text { JAN }\end{array}$ & 1225 & 801 & 269 & 7.1 & 22.5 & 50 & 58 & 4.8 & 54 & 11 & 30000 & 6700 \\
\hline $29 .$. & 1025 & 1710 & 120 & 6.6 & 13.0 & 160 & 110 & 8.0 & 75 & 4.7 & 28000 & 7000 \\
\hline & 1125 & 60 & 780 & 7.3 & 29.0 & 25 & 2.1 & 3.0 & 38 & 34 & 1200000 & 460000 \\
\hline & 1340 & 1040 & 218 & 7.5 & 27.0 & 80 & 160 & 6.5 & 80 & 14 & 84000 & 40000 \\
\hline
\end{tabular}

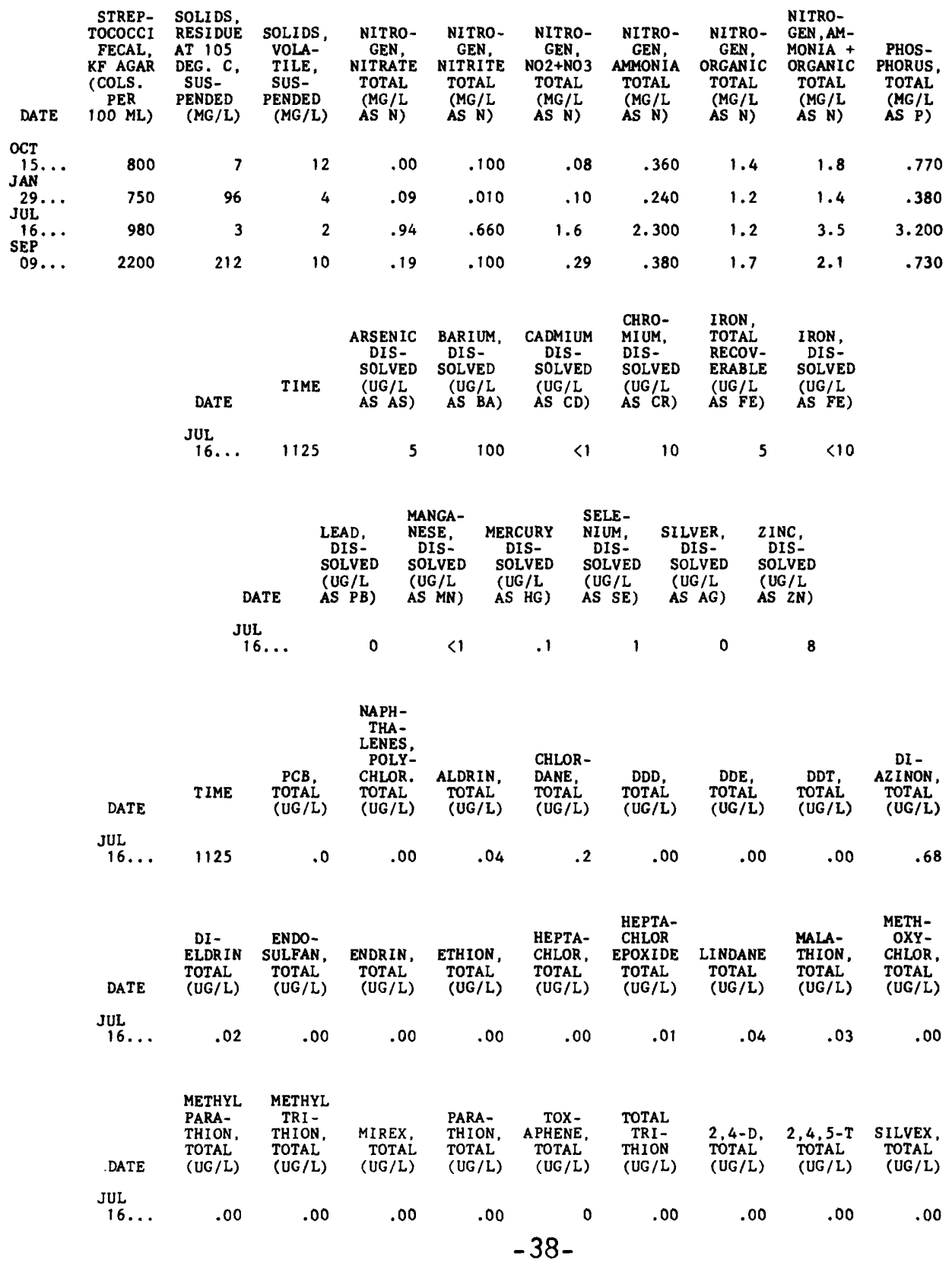




\section{WHITEOAK BAYOU DRAINAGE BASIN}

The locations of data-collection sites in and near the Whiteoak Bayou drainage basin are shown in figure 5 .

Cole Creek (including Bingle Road Storm Sewer), Brickhouse Gully, Lazybrook Street Storm Sewer, and Little Whiteoak Bayou are shown as separate drainage basins within the Whiteoak Bayou section.

Weighted-mean rainfall in the drainage basins, based on thirteen rain gages, for the 1980 water year was 39.06 inches or 9.13 inches less than the 30 -year (1941-70) average of 48.19 inches for Houston.

The storms of Oct. 22-23, Oct. 30 to Nov. 1, Jan. 17-19, Jan. 20-27, and March 27 to April 2 were selected for analysis at the Whiteoak Bayou at Houston (08074500) gaging station. 


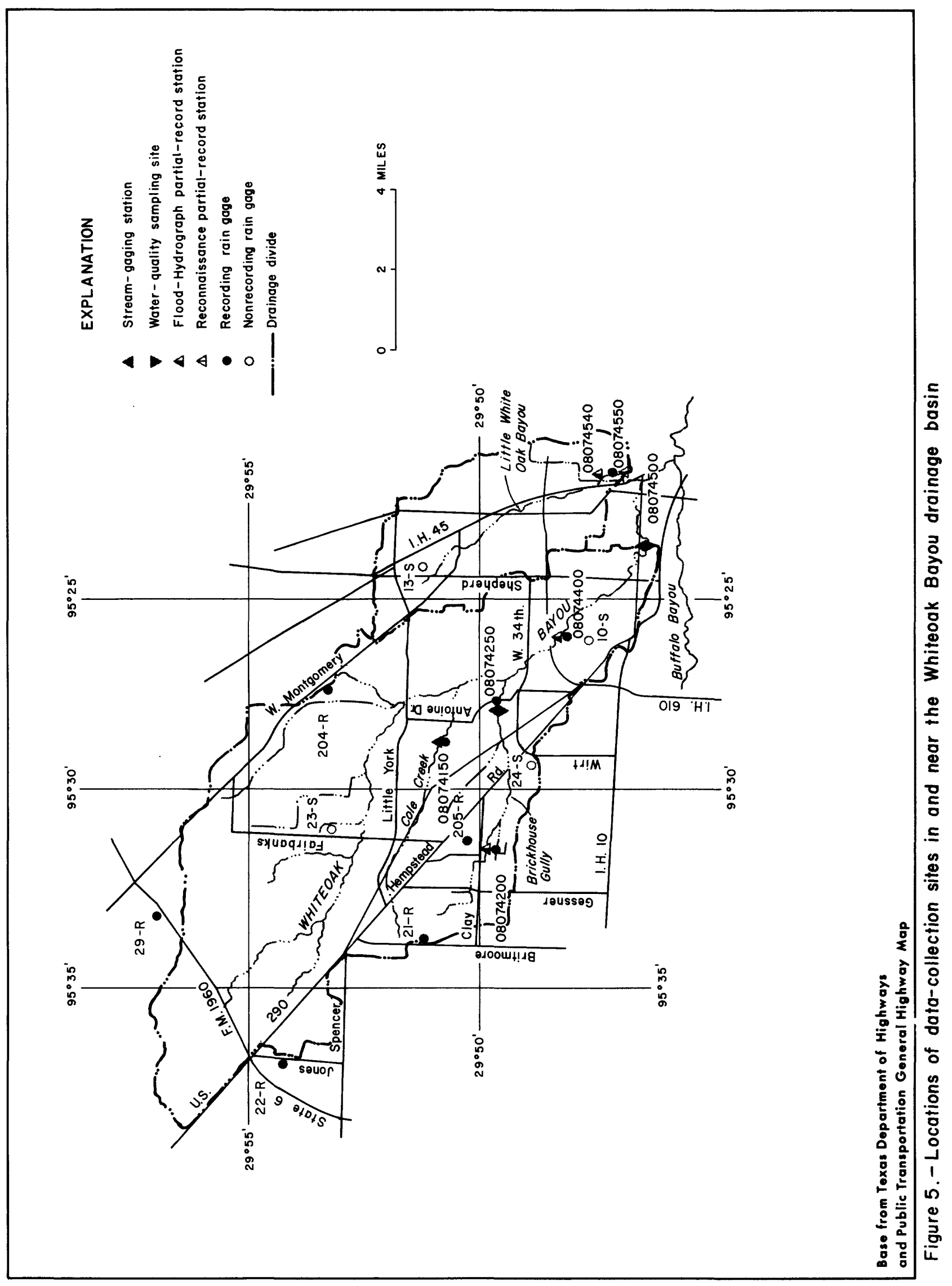




\section{COLE CREEK DRAINAGE BASIN}

The locations of data-collection sites in and near the Cole Creek drainage basin are shown in figure 6 .

Bingle Road Storm Sewer is shown as a separate drainage basin within the Cole Creek section.

Weighted-mean rainfall in the drainage basins, based on four rain gages, for the 1980 water year was 38.41 inches, or 9.78 inches less than the 30-year (1941-70) average of 48.19 inches for Houston.

The storms of Jan. 20-24 and Mar. 27-30 were selected for analysis at station 08074150, Cole Creek at Deihl Road. 

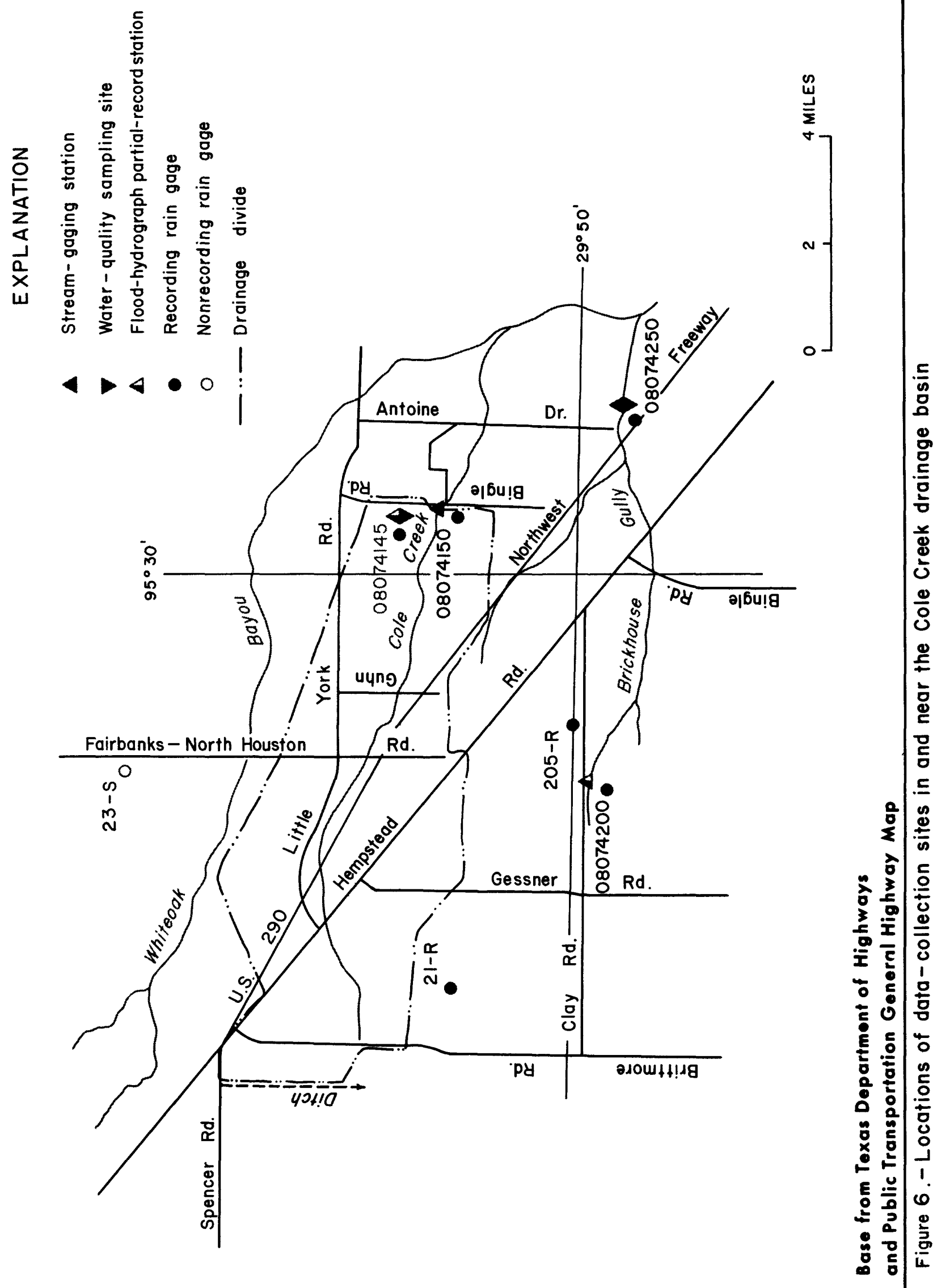


\section{BINGLE ROAD STORM SEWER DRAINAGE BASIN}

The location of data-collection sites in and near the Bingle Road Storm Sewer drainage basin are shown in figure 7.

Flood-hydrograph partial-record station 08074145, Bingle Road Storm Sewer at Houston, Tex. was put into operation on May 16, 1980 by the U.S. Geological Survey.

Weighted-mean rainfall for the 1980 water year was not determined.

The storms of June 9, July 21, and Sept. 5-7 were selected for analysis at station 08074145, Bingle Road Storm Sewer at Houston, Tex. 


\section{EXPLANATION}
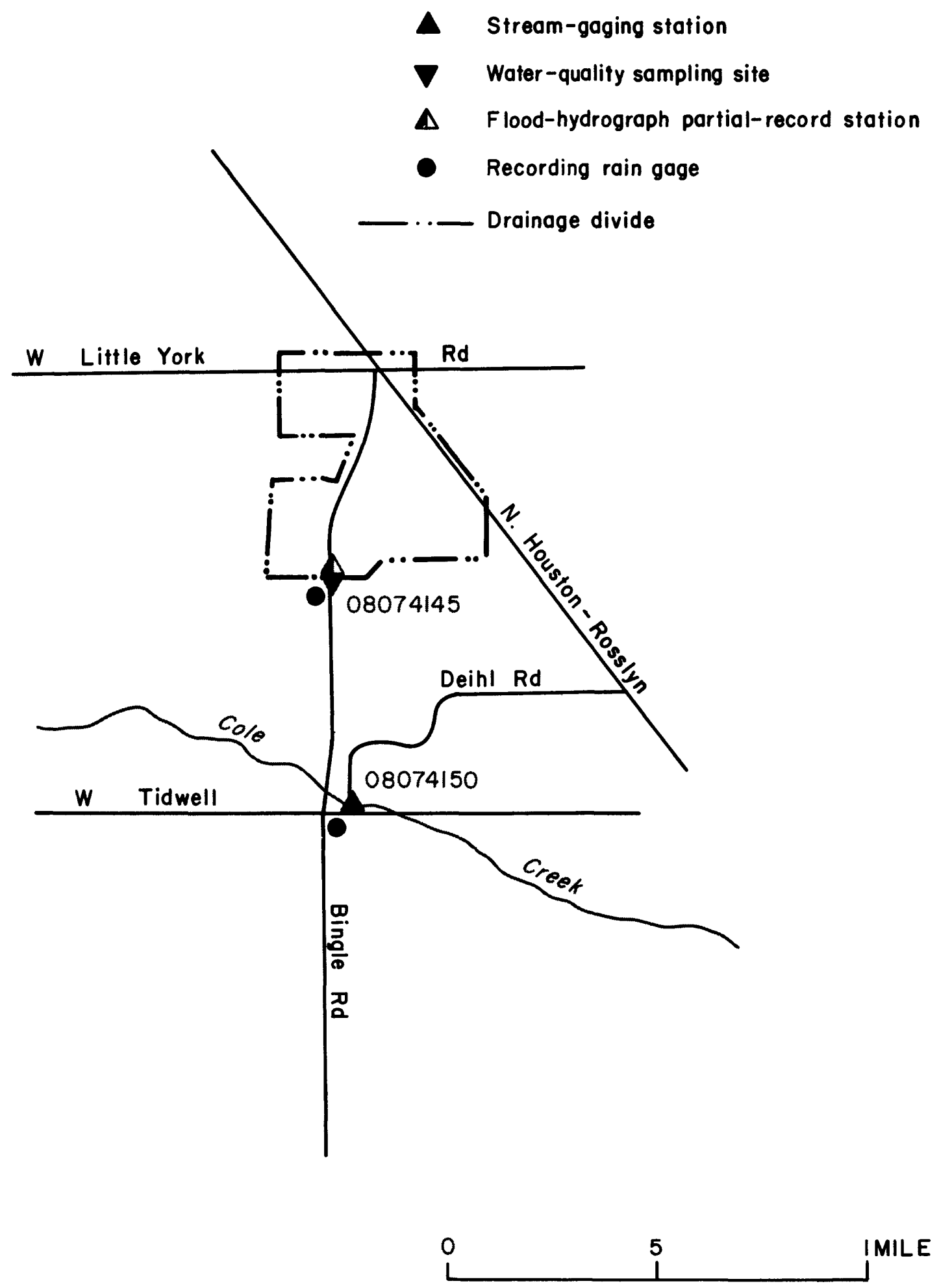

Base from USGS Topographic

Quadrangle

Figure 7.-Locations of data-collection sites in and near the Bingle Road storm sewer drainage basin 


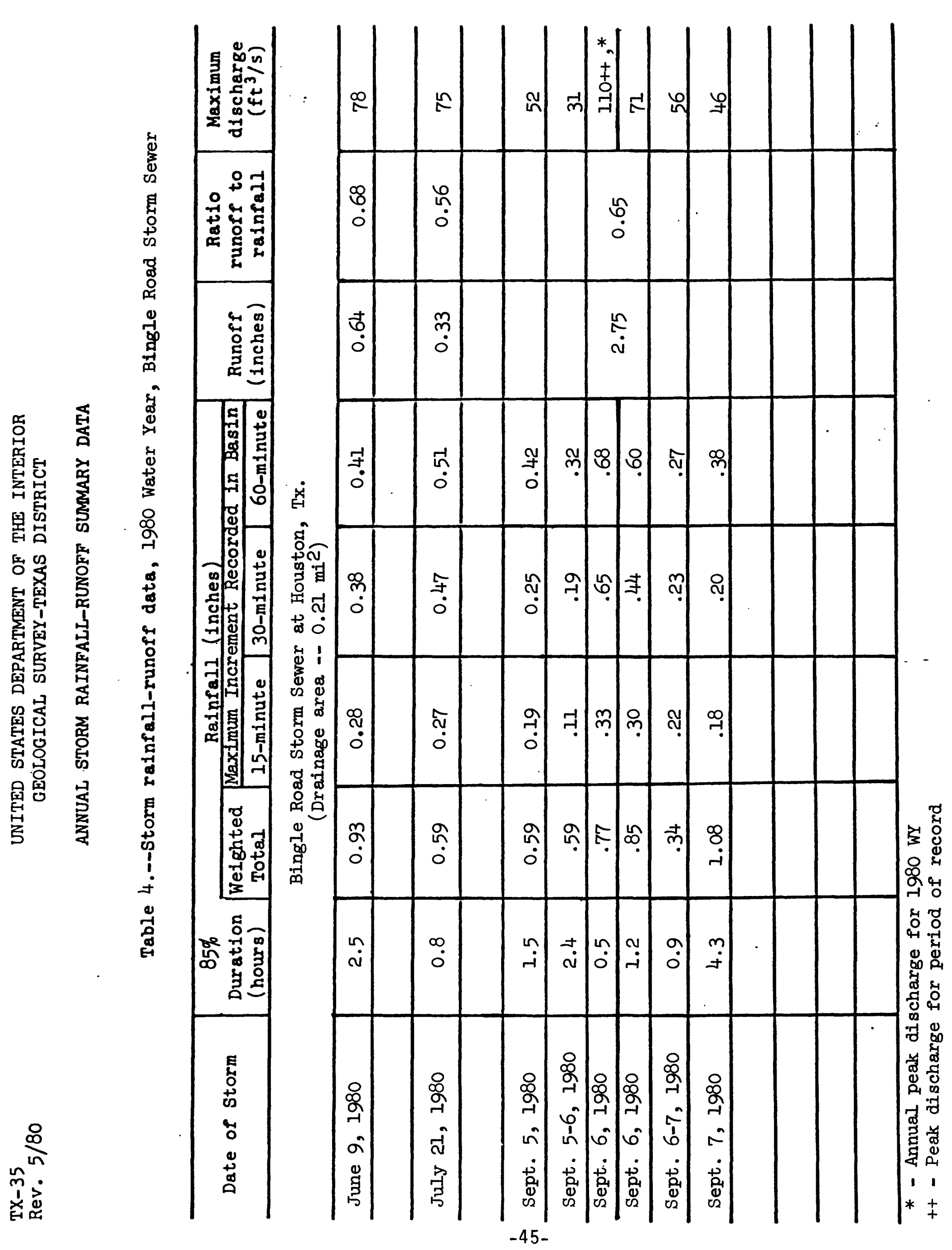


SAN JACINTO RIVER BASIN

08074145 BINGLE ROAD STORM SEWER AT HOUSTON, TX (Flood-hydrograph partial-record station)

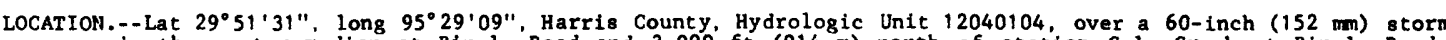
sewer in the center median at Bingle Road and $3,000^{\circ} \mathrm{ft}(914 \mathrm{~m})$ north of station Cole Creek at Bingle Road, Houston $(08074150)$.

DRAINAGE AREA. $-0.21 \mathrm{mi}^{2} \cdot\left(0.54 \mathrm{~km}^{2}\right)$.

PERIOD OF RECORD, --May to current year.

GAGE.--Flood-hydrograph and rainfall recorder and crest-stage gage. Datum of gage is arbitrary.

REMARKS.--Additional storm rainfall-runoff data for the period after May 16, 1980, can be obtained from the report "Hydrologic Data for Urban Studies in the Houston, Texas Metropolitan Area, 1980".

EXTREMES FOR PERIOD OF RECORD.--Maximum discharge, $110 \mathrm{ft}^{3} / \mathrm{s}\left(3.12 \mathrm{~m}^{3} / \mathrm{s}\right)$, Sept. 6,1980 , elevation, $8.71 \mathrm{ft}(2.655 \mathrm{~m})$. EXTREMES FOR CURRENT YEAR.--Peak discharges for period May to September above base of $75 \mathrm{ft}^{3} / \mathrm{s}(2.12 \mathrm{~m} / \mathrm{s})$ and $\operatorname{maximum}(*)$

\begin{tabular}{|c|c|c|c|c|c|c|}
\hline Date & & Time & $\begin{array}{r}\text { Dis } \\
\left(\mathrm{ft}^{3} / \mathrm{s}\right.\end{array}$ & $\begin{array}{l}\mathrm{rge} \\
\left(\mathrm{m}^{3} / \mathrm{s}\right)\end{array}$ & $\begin{array}{l}\text { Gage } \\
(\mathrm{ft})\end{array}$ & $\underset{(m)}{e i g h t}$ \\
\hline $\begin{array}{l}\text { June } \\
\text { aJuly } \\
\text { Sept. }\end{array}$ & $\begin{array}{r}9 \\
21 \\
6\end{array}$ & $\begin{array}{l}0950 \\
1100 \\
0700\end{array}$ & $\begin{array}{r}78 \\
75 \\
\star 110\end{array}$ & $\begin{array}{l}2.21 \\
2.12 \\
3.12\end{array}$ & $\begin{array}{l}8.10 \\
8.05 \\
8.71\end{array}$ & $\begin{array}{l}2.469 \\
2.454 \\
2.655\end{array}$ \\
\hline
\end{tabular}

a Water-quality smples were obtained during the runoff event.

WATER-QUALITY RECORDS

PERIOD OF RECORD.--Chemical, biochemical, and pesticide analyses: May to September 1980.

WATER QUALITY DATA, WATER YEAR OCTOBER 1979 TO SEPTEMBER 1980

\begin{tabular}{|c|c|c|c|c|c|c|c|c|c|c|}
\hline DATE & TIME & $\begin{array}{l}\text { STREAM- } \\
\text { FLOW, } \\
\text { INSTAN- } \\
\text { TANEOUS } \\
\text { (CFS) }\end{array}$ & $\begin{array}{l}\text { SPE- } \\
\text { CIFIC } \\
\text { CON- } \\
\text { DUCT- } \\
\text { ANCE } \\
\text { (MICRO- } \\
\text { MHOS) }\end{array}$ & $\begin{array}{l}\text { COLOR } \\
\text { (PLAT- } \\
\text { INUM } \\
\text { COBALT } \\
\text { UNITS) }\end{array}$ & $\begin{array}{c}\text { TUR- } \\
\text { BID- } \\
\text { ITY } \\
\text { (NTU) }\end{array}$ & $\begin{array}{c}\text { OXYGEN } \\
\text { DEMAND, } \\
\text { BIOCHEM } \\
\text { UNINH I } \\
5 \text { DAY } \\
(M G / L)\end{array}$ & $\begin{array}{c}\text { COLI- } \\
\text { FORM, } \\
\text { TOTAL, } \\
\text { IMMED. } \\
\text { (COLS. } \\
\text { PER } \\
100 \mathrm{ML} \text { ) }\end{array}$ & $\begin{array}{l}\text { COLI- } \\
\text { FORM, } \\
\text { FECAL, } \\
0.7 \\
\text { UM-MF } \\
\text { (COLS.' } \\
\text { 100 ML) }\end{array}$ & $\begin{array}{c}\text { STREP- } \\
\text { TOCOCCI } \\
\text { FECAL, } \\
\text { KF AGAR } \\
\text { (COLS. } \\
\text { PER } \\
100 \mathrm{ML} \text { ) }\end{array}$ & $\begin{array}{l}\text { SOLIDS, } \\
\text { RESIDUE } \\
\text { AT 105 } \\
\text { DEG. C, } \\
\text { SUS- } \\
\text { PENDED } \\
\text { (MG/L) }\end{array}$ \\
\hline \multicolumn{11}{|l|}{ JUN } \\
\hline $09 \ldots$ & 0912 & 5.1 & 163 & 50 & 190 & (2) & 480000 & 12000 & 11000 & 462 \\
\hline $09 \ldots$ & 0921 & 29 & $=$ & -- & \multirow{2}{*}{ 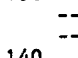 } & $>24$ & $\overline{--}$ & $=$ & $=$ & \multirow{2}{*}{488} \\
\hline $\begin{array}{l}09 \ldots \\
09 \ldots\end{array}$ & $\begin{array}{l}0940 \\
0949\end{array}$ & $\begin{array}{l}63 \\
76\end{array}$ & 60 & 15 & & \multirow{2}{*}{$12^{-}$} & 51000 & 7300 & 25000 & \\
\hline $09 \ldots$ & 1007 & 30 & -- & $\ldots$ & \multirow{2}{*}{0} & & & 1000 & & - \\
\hline $09 \ldots$ & 1017 & 16 & 75 & 30 & & -- & 25000 & 6700 & 15000 & 271 \\
\hline 2 & 0938 & 6. & $\cdots$ & -- & - & 4.7 & 6700 & 3000 & 60 & -- \\
\hline & 095 & & -- & -- & 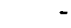 & 5.0 & 26 & 12 & 360 & -- \\
\hline & & 6 & $-\cdot$ & -- & - & 9.9 & 56000 & 44000 & 580 & - \\
\hline
\end{tabular}

\begin{tabular}{|c|c|c|c|c|c|c|c|c|c|}
\hline DATE & $\begin{array}{l}\text { SOLIDS, } \\
\text { VOLA- } \\
\text { TILE, } \\
\text { SUS- } \\
\text { PENDED } \\
\text { (MG/L) }\end{array}$ & $\begin{array}{c}\text { NITRO- } \\
\text { GEN, } \\
\text { NITRATE } \\
\text { TOTAL } \\
\text { (MG/L } \\
\text { AS N) }\end{array}$ & $\begin{array}{l}\text { NITRO- } \\
\text { GEN } \\
\text { NITRITE } \\
\text { TOTAL } \\
\text { (MG/L } \\
\text { AS N) }\end{array}$ & $\begin{array}{c}\text { NITRO- } \\
\text { GEN, } \\
\text { NO2+NO3 } \\
\text { TOTAL } \\
\text { (MG/L } \\
\text { AS N) }\end{array}$ & $\begin{array}{l}\text { NITRO- } \\
\text { GEH, } \\
\text { ANIMOHIA } \\
\text { TOTAL } \\
\text { (MG/L } \\
\text { AS NI) }\end{array}$ & $\begin{array}{l}\text { HITRO- } \\
\text { GEN, } \\
\text { ORGANIC } \\
\text { TOTAL } \\
\text { (HG/L } \\
\text { AS N) }\end{array}$ & $\begin{array}{l}\text { NITRO- } \\
\text { GEN,AM- } \\
\text { MOHIA + } \\
\text { ORGANIC } \\
\text { TOTAL } \\
\text { (MG/L } \\
\text { AS N) }\end{array}$ & $\begin{array}{c}\text { PHOS- } \\
\text { PHORUS, } \\
\text { TOTAL } \\
\text { (MG/L } \\
\text { AS P) }\end{array}$ & $\begin{array}{l}\text { CARBON } \\
\text { ORGANIC } \\
\text { TOTAL } \\
\text { (MG/L } \\
\text { AS C) }\end{array}$ \\
\hline \multicolumn{10}{|l|}{ JUN } \\
\hline $09 \ldots$ & 68 & .81 & .060 & .87 & .470 & 2.8 & 3.3 & .710 & 54 \\
\hline $09 \ldots$ & -- & -- & -- & -- & $\cdots$ & -- & -- & -- & $\cdots$ \\
\hline $09 . .$. & $\overline{148}$ & $\overline{23}$ & $0 \overline{30}$ & 26 & $\overline{0}$ & $8^{-}$ & $0^{-}$ & 150 & $20^{--}$ \\
\hline $09 \ldots$ & -- & - & - & -. & . & & & - & \\
\hline $09 \ldots$ & 31 & .25 & .030 & .28 & .150 & 1.4 & 1.5 & .180 & 20 \\
\hline JUL & & & & & & & & & \\
\hline 21 & $\because$ & 1.8 & .010 & 1.8 & .060 & 1.3 & 1.4 & .280 & - \\
\hline 2 & $=$ & $\begin{array}{r}2.1 \\
.77\end{array}$ & .060 & .83 & .030 & $\begin{array}{l}2.3 \\
1.6\end{array}$ & $\begin{array}{l}2.3 \\
1.7\end{array}$ & .190 & $\overline{-}$ \\
\hline
\end{tabular}




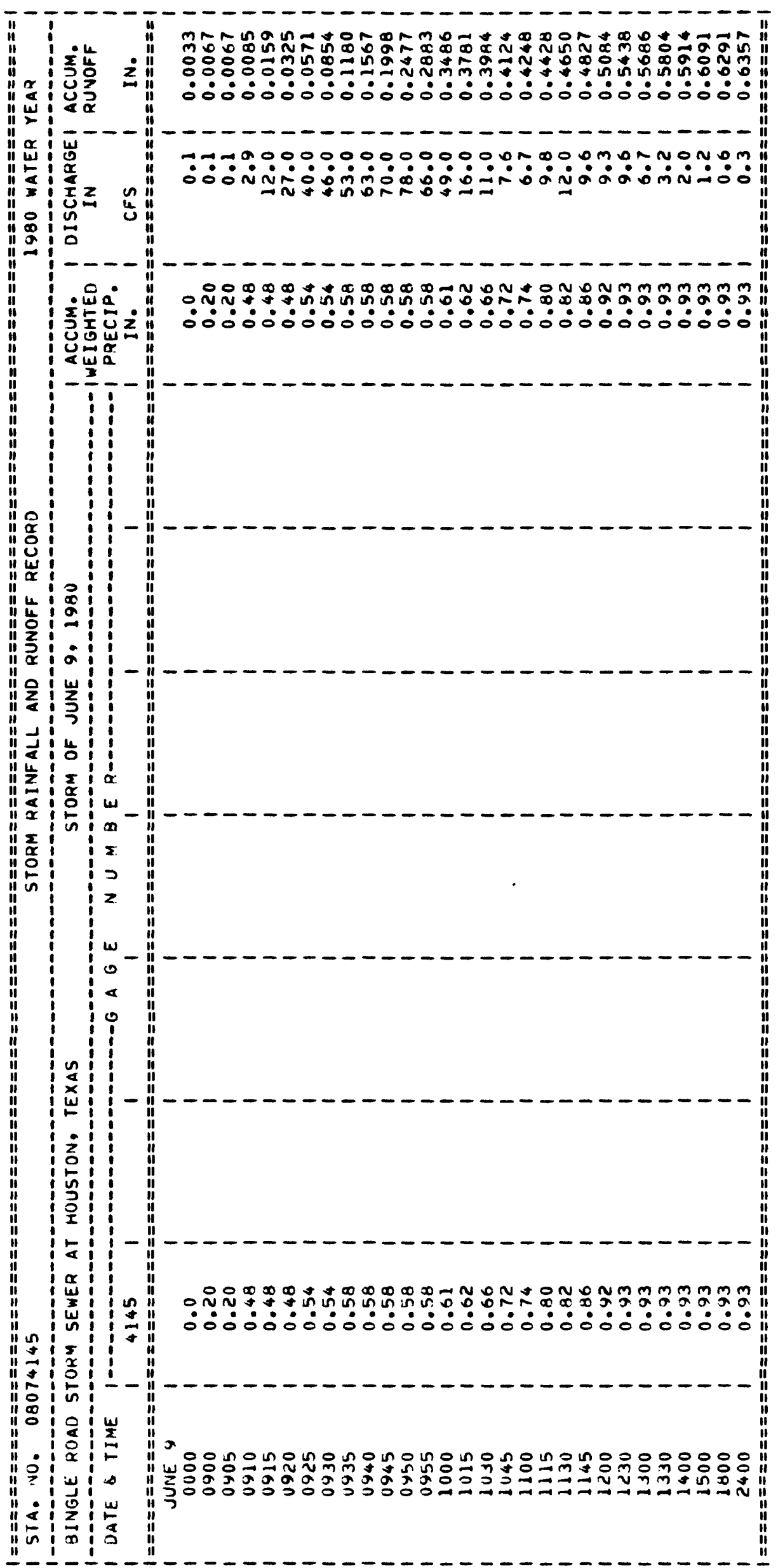




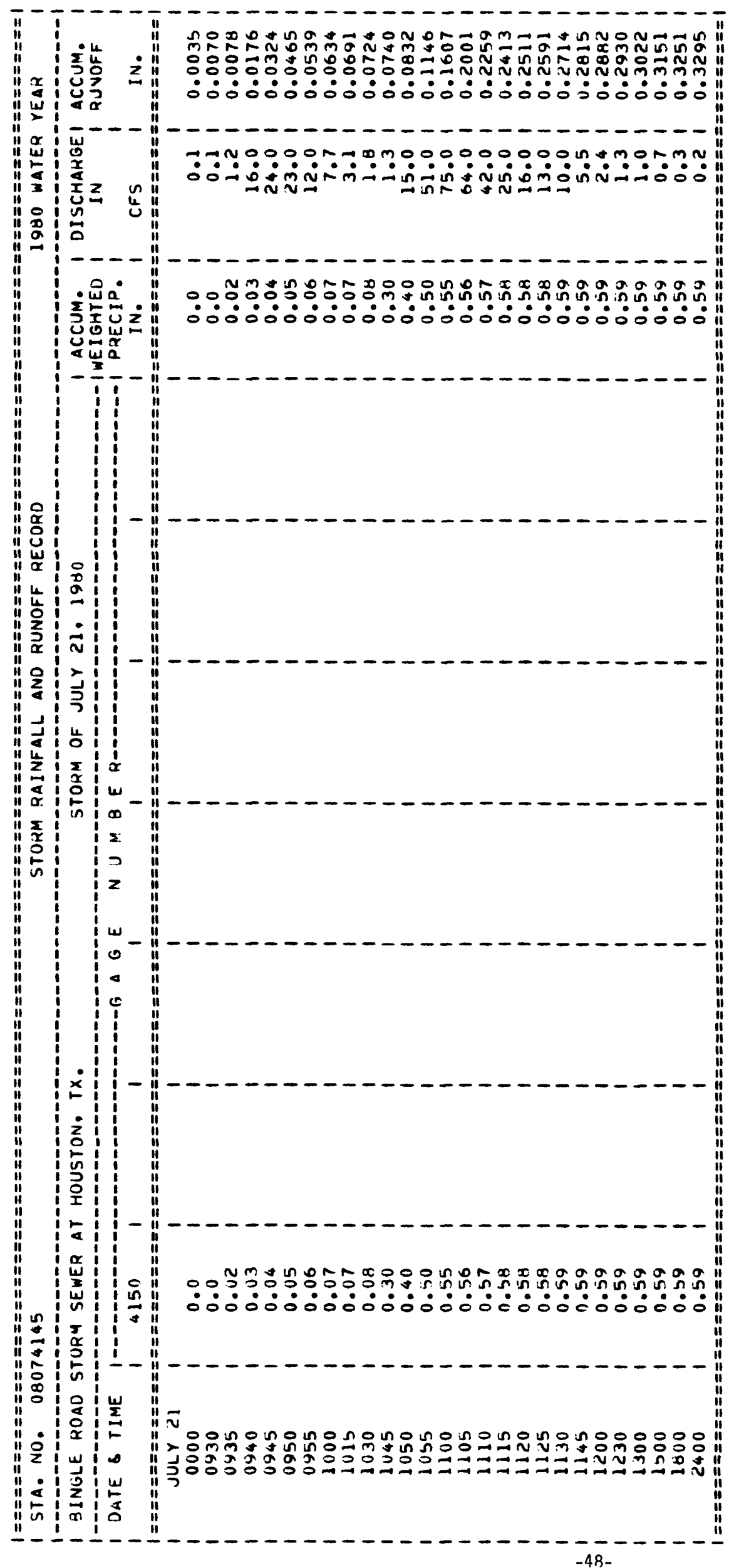




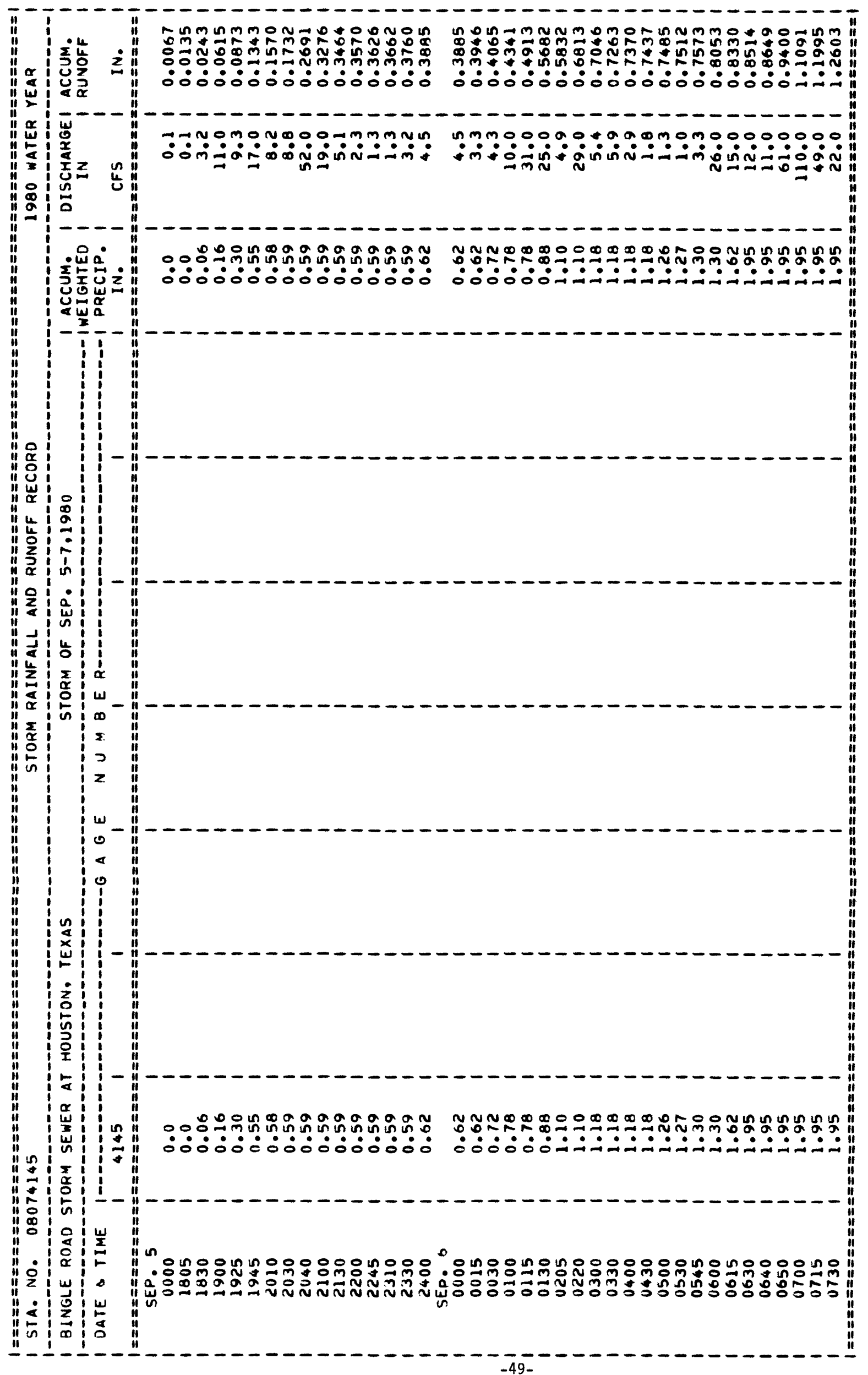




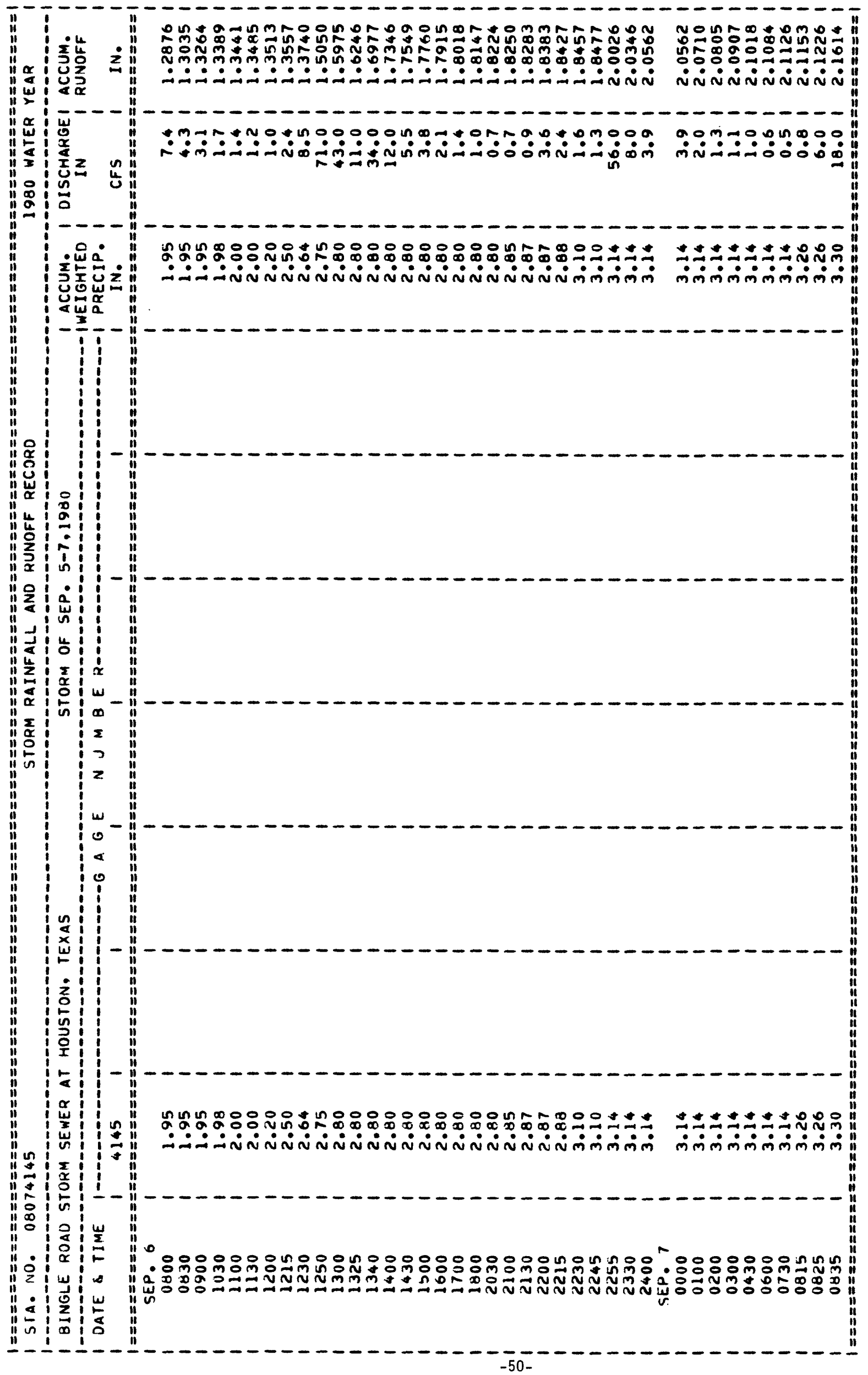




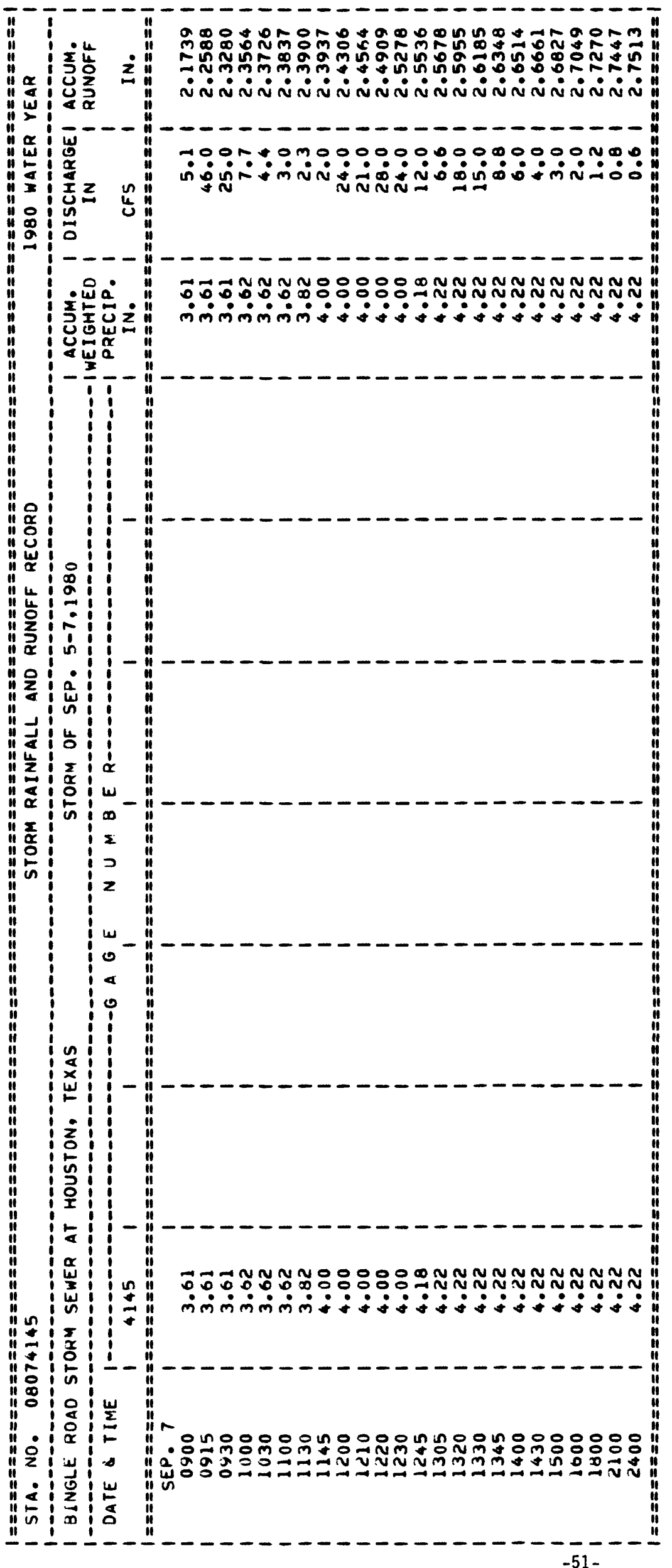




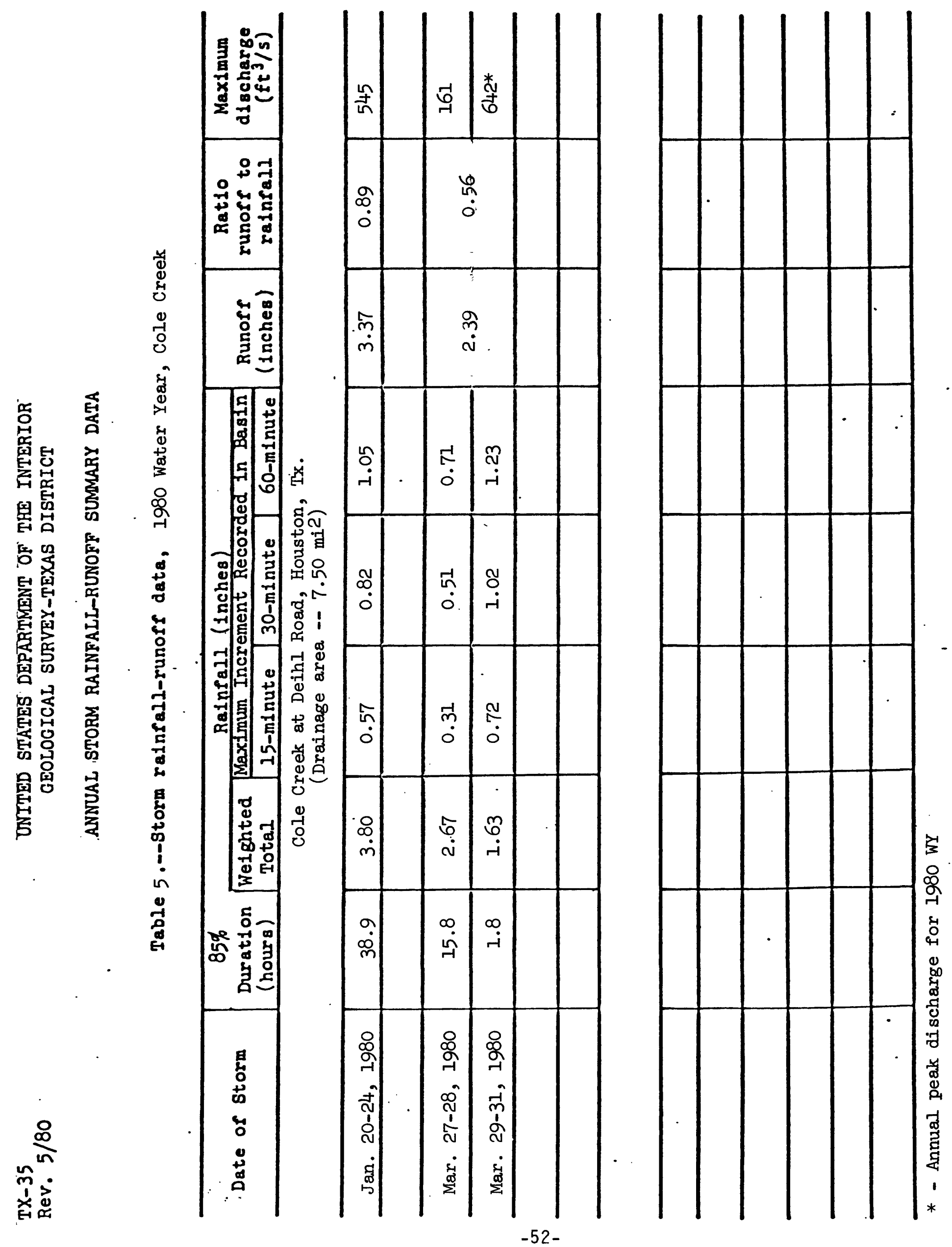




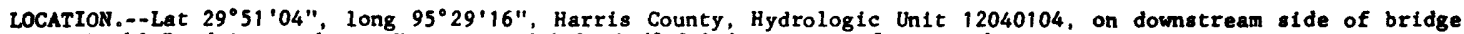
at Deih1 Road in northwest Houston and $1.8 \mathrm{mi}(2.9 \mathrm{~km})$ upstream from mouth.

DRAIMAge AREA (revised).$-7.50 \mathrm{mi}^{2}\left(19.42 \mathrm{~km}^{2}\right)$. Prior to Oct. 1, 1976, 8.05 $\mathrm{mi}^{2}$ (20.85 $\left.\mathrm{km}^{2}\right)$. Prior to 0ct. 1, 1979, $7.33 \mathrm{mi}^{2}\left(18.98 \mathrm{~km}^{2}\right)$. Drainage area changes are the result of drainage ditch relocations and extensions.

PERIOD OF RECORD.--April 1964 to current year. Gage at temporary location $1.0 \mathrm{mi}$ (1.6 km) downstream at Antoine Drive May 18, 1965, to Sept. 1, 1966, due to bridge construction and channel rectification.

REVISED RECORDS.--WRD TX-74-1: Drainage area.

GAGE.--Water-stage recorder and crest-stage gage. Datum of gage is National Geodetic Vertical Datum of 1929 , 1957 adjustment; unadjusted for land-surface subsidence.

REMARKS.--Records fair. No diversion above station. Low flow is partly sustained by sewage effluent from Houston suburbs. Recording rain gage at station. Several observations of water temperature were made during the year. AVERAGE DISCHARGE. --16 years, $7.63 \mathrm{ft}^{3} / \mathrm{s}\left(0.216 \mathrm{~m}^{3} / \mathrm{s}\right), 5.530 \mathrm{acre-ft} / \mathrm{yr}\left(6.82 \mathrm{hm}^{3} / \mathrm{yr}^{\mathrm{r}}\right)$.

EXTREMES FOR PERIOD OF RECORD.--Maximum discharge, 2,020 $\mathrm{ft}^{3} / \mathrm{s}\left(57.2 \mathrm{~m}^{3} / \mathrm{s}\right)$ Mar. 20, 1972, elevation, 78.60 ft $(23.957 \mathrm{~m})$; no flow at times.

EXTREMES FOR CURRENT YEAR.--Peak discharges above base of $400 \mathrm{ft}^{3} / \mathrm{s}\left(11.3 \mathrm{~m}^{3} / \mathrm{s}\right)$ and maximum ( $(*)$ :

\begin{tabular}{|c|c|c|c|c|c|}
\hline Date & Time & $\begin{array}{r}\text { Dis } \\
\left(\mathrm{ft}^{3} / \mathrm{s}\right.\end{array}$ & $\begin{array}{l}\mathrm{rge} \\
\left(\mathrm{m}^{3} / \mathrm{s}\right)\end{array}$ & $\begin{array}{l}\text { Elev } \\
(\mathrm{ft})\end{array}$ & ation \\
\hline $\begin{array}{l}\text { Jan. } 21 \\
\text { Mar. } 29\end{array}$ & $\begin{array}{l}0500 \\
1200\end{array}$ & $\begin{array}{r}545 \\
\star 642\end{array}$ & $\begin{array}{l}15.4 \\
18.2\end{array}$ & $\begin{array}{l}75.11 \\
76.13\end{array}$ & $\begin{array}{l}22.894 \\
23.204\end{array}$ \\
\hline
\end{tabular}

Minimum dafly discharge, $0.08 \mathrm{ft}^{3} / \mathrm{s}\left(0.002 \mathrm{~m}^{3} / \mathrm{s}\right)$ July $16,19$.

DISCHARGE, IN CUBIC FEET PER SECOND, WATER YEAR OCTOBER 1979 TO SEPTEMBER 1980

\begin{tabular}{|c|c|c|c|c|c|c|c|c|c|c|c|c|}
\hline DAY & OCT & NOV & DEC & JAN & FEB & MAR & APR & MAY & JUN & JUL & AUG & SEP \\
\hline $\begin{array}{l}1 \\
2 \\
3 \\
4 \\
5\end{array}$ & $\begin{array}{l}.66 \\
.51 \\
.40 \\
.35 \\
.35\end{array}$ & $\begin{array}{c}10 \\
3.0 \\
1.5 \\
.80 \\
.50\end{array}$ & $\begin{array}{l}.35 \\
.35 \\
.31 \\
.38 \\
.38\end{array}$ & $\begin{array}{r}2.2 \\
1.9 \\
12 \\
5.7 \\
3.4\end{array}$ & $\begin{array}{l}1.6 \\
3.9 \\
3.9 \\
2.7 \\
1.8\end{array}$ & $\begin{array}{l}5.5 \\
.83 \\
.72 \\
.95 \\
.73\end{array}$ & $\begin{array}{l}6.7 \\
4.2 \\
2.8 \\
1.7 \\
1.4\end{array}$ & $\begin{array}{l}2.6 \\
3.0 \\
1.0 \\
.69 \\
.69\end{array}$ & $\begin{array}{l}.45 \\
.43 \\
.44 \\
.46 \\
.42\end{array}$ & $\begin{array}{l}.24 \\
.36 \\
.40 \\
.29 \\
.22\end{array}$ & $\begin{array}{r}.18 \\
.17 \\
.13 \\
.12 \\
1.0\end{array}$ & $\begin{array}{l}.44 \\
.94 \\
.98 \\
1.4 \\
7.5\end{array}$ \\
\hline $\begin{array}{r}6 \\
7 \\
8 \\
9 \\
10\end{array}$ & $\begin{array}{l}.78 \\
.62 \\
.41 \\
.28 \\
.28\end{array}$ & $\begin{array}{l}.40 \\
.35 \\
.30 \\
.60 \\
.45\end{array}$ & $\begin{array}{l}.57 \\
.65 \\
.65 \\
.61 \\
.69\end{array}$ & $\begin{array}{l}2.6 \\
2.2 \\
2.1 \\
2.1 \\
1.9\end{array}$ & $\begin{array}{l}1.5 \\
1.4 \\
51 \\
47 \\
13\end{array}$ & $\begin{array}{l}.55 \\
.57 \\
.60 \\
.74 \\
.71\end{array}$ & $\begin{array}{l}2.2 \\
1.9 \\
1.1 \\
.92 \\
.77\end{array}$ & $\begin{array}{l}.78 \\
2.2 \\
1.1 \\
.88 \\
.69\end{array}$ & $\begin{array}{r}.41 \\
.39 \\
14.37 \\
2.3\end{array}$ & $\begin{array}{l}.27 \\
.32 \\
.47 \\
.46 \\
.84\end{array}$ & $\begin{array}{l}.23 \\
1.2 \\
1.3 \\
.39 \\
.20\end{array}$ & $\begin{array}{l}78 \\
28 \\
6.4 \\
2.2 \\
.69\end{array}$ \\
\hline $\begin{array}{l}11 \\
12 \\
13 \\
14 \\
15\end{array}$ & $\begin{array}{l}.28 \\
.45 \\
.40 \\
.36 \\
.39\end{array}$ & $\begin{array}{l}.40 \\
.35 \\
.32 \\
.30 \\
.30\end{array}$ & $\begin{array}{c}125^{.78} \\
23 \\
5.7 \\
2.9\end{array}$ & $\begin{array}{l}1.5 \\
.93 \\
.83 \\
.74 \\
.65\end{array}$ & $\begin{array}{l}5.9 \\
3.8 \\
2.8 \\
3.1 \\
3.6\end{array}$ & $\begin{array}{l}.65 \\
.94 \\
.72 \\
.59 \\
.77\end{array}$ & $\begin{array}{l}.83 \\
.88 \\
6.0 \\
1.6 \\
1.1\end{array}$ & $\begin{array}{l}.65 \\
.53 \\
1.0 \\
11\end{array}$ & $\begin{array}{l}.40 \\
.21 \\
.17 \\
.21 \\
.19\end{array}$ & $\begin{array}{l}.26 \\
.16 \\
.14 \\
.12 \\
.09\end{array}$ & $\begin{array}{r}.16 \\
.26 \\
.23 \\
.17 \\
2.9\end{array}$ & $\begin{array}{l}3.0 \\
.46 \\
.28 \\
.21 \\
.31\end{array}$ \\
\hline $\begin{array}{l}16 \\
17 \\
18 \\
19 \\
20\end{array}$ & $\begin{array}{l}.42 \\
.35 \\
.30 \\
.25 \\
.20\end{array}$ & $\begin{array}{l}.30 \\
.30 \\
.30 \\
.35 \\
.38\end{array}$ & $\begin{array}{l}1.9 \\
1.9 \\
1.9 \\
1.5 \\
1.4\end{array}$ & $\begin{array}{l}67^{.61} \\
16 \\
3.4\end{array}$ & $\begin{array}{l}7.5 \\
4.2 \\
2.7 \\
2.3 \\
1.9\end{array}$ & $\begin{array}{c}1.0 \\
1.6 \\
.71 \\
.82 \\
8.2\end{array}$ & $\begin{array}{l}1.0 \\
1.1 \\
.98 \\
.98 \\
.83\end{array}$ & $\begin{array}{c}24 \\
14 \\
2.9 \\
49 \\
9.2\end{array}$ & $\begin{array}{l}.19 \\
.22 \\
.23 \\
.20 \\
.16\end{array}$ & $\begin{array}{l}.08 \\
.11 \\
.11 \\
.08 \\
1.0\end{array}$ & $\begin{array}{l}5.2 \\
.77 \\
.36 \\
.49 \\
.30\end{array}$ & $\begin{array}{l}.20 \\
.17 \\
.20 \\
.46 \\
.51\end{array}$ \\
\hline $\begin{array}{l}21 \\
22 \\
23 \\
24 \\
25\end{array}$ & $\begin{array}{l}.17 \\
8.0 \\
4.0 \\
2.0 \\
1.0\end{array}$ & $\begin{array}{c}12 \\
3.6 \\
2.2 \\
.78 \\
.53\end{array}$ & $\begin{array}{l}1.4 \\
1.6 \\
9.0 \\
9.5 \\
3.4\end{array}$ & $\begin{array}{r}340 \\
210 \\
38 \\
11 \\
6.9\end{array}$ & $\begin{array}{l}1.6 \\
1.4 \\
1.3 \\
1.0 \\
.85\end{array}$ & $\begin{array}{l}1.7 \\
.78 \\
.99 \\
.82 \\
.55\end{array}$ & $\begin{array}{r}.78 \\
.78 \\
.93 \\
.88\end{array}$ & $\begin{array}{l}2.8 \\
1.4 \\
.99 \\
.76 \\
.65\end{array}$ & $\begin{array}{c}10^{.17} \\
5.3 \\
.66 \\
.33\end{array}$ & $\begin{array}{c}13 \\
5.1 \\
.74 \\
.36 \\
.20\end{array}$ & $\begin{array}{l}.18 \\
.15 \\
.34 \\
.14 \\
.10\end{array}$ & $\begin{array}{l}.52 \\
.51 \\
.37 \\
.58 \\
.44\end{array}$ \\
\hline $\begin{array}{l}26 \\
27 \\
28 \\
29 \\
30 \\
31\end{array}$ & $\begin{array}{r}.70 \\
.50 \\
.40 \\
50.35 \\
30\end{array}$ & $\begin{array}{l}.38 \\
.38 \\
.28 \\
.28 \\
.31 \\
. .-\end{array}$ & $\begin{array}{r}2.1 \\
1.6 \\
1.5 \\
16 \\
5.7 \\
3.1\end{array}$ & $\begin{array}{l}5.0 \\
3.4 \\
2.7 \\
2.6 \\
2.5 \\
2.2\end{array}$ & $\begin{array}{l}.82 \\
.81 \\
.84 \\
.86 \\
-.- \\
-.-\end{array}$ & $\begin{array}{c}2.1 \\
90 \\
110 \\
220 \\
50 \\
12\end{array}$ & $\begin{array}{c}3.5 \\
1.2 \\
.88 \\
.83 \\
1.2 \\
-.-\end{array}$ & $\begin{array}{l}.60 \\
.56 \\
.77 \\
.70 \\
.55 \\
.47\end{array}$ & $\begin{array}{l}.26 \\
.25 \\
.21 \\
.22 \\
.24 \\
-.-\end{array}$ & $\begin{array}{l}.19 \\
1.4 \\
5.2 \\
1.7 \\
.45 \\
.22\end{array}$ & $\begin{array}{c}.25 \\
.22 \\
.19 \\
1.1 \\
4.3 \\
.80\end{array}$ & $\begin{array}{r}.85 \\
.86 \\
.44 \\
.23 \\
12 \\
\ldots-\end{array}$ \\
\hline $\begin{array}{l}\text { TOTAL } \\
\text { MEAN } \\
\text { MAX } \\
\text { MIN } \\
\text { AC-FT } \\
(\dagger t)\end{array}$ & $\begin{array}{r}105.16 \\
3.39 \\
50 \\
.17 \\
209 \\
2.81\end{array}$ & $\begin{array}{r}41.94 \\
1.40 \\
12 \\
.28 \\
83 \\
1.26\end{array}$ & $\begin{array}{r}225.82 \\
7.28 \\
125 \\
.31 \\
448 \\
3.81\end{array}$ & $\begin{array}{r}832.06 \\
26.8 \\
340 \\
.61 \\
1650 \\
5.52\end{array}$ & $\begin{array}{r}175.08 \\
6.04 \\
51 \\
.81 \\
347 \\
1.95\end{array}$ & $\begin{array}{r}516.84 \\
16.7 \\
220 \\
.55 \\
1030 \\
5.97\end{array}$ & $\begin{array}{r}85.97 \\
2.87 \\
36 \\
.77 \\
177 \\
1.95\end{array}$ & $\begin{array}{r}156.16 \\
5.04 \\
49 \\
.47 \\
310 \\
3.92\end{array}$ & $\begin{array}{r}39.49 \\
1.32 \\
14 \\
.16 \\
78 \\
1.72\end{array}$ & $\begin{array}{r}34.58 \\
1.12 \\
13 \\
.08 \\
69 \\
2.49\end{array}$ & $\begin{array}{r}23.53 \\
.76 \\
5.2 \\
.10 \\
47 \\
2.00\end{array}$ & $\begin{array}{r}149.15 \\
4.97 \\
78 \\
.17 \\
296 \\
5.01\end{array}$ \\
\hline
\end{tabular}

$\begin{array}{lllllllllll}\text { CAL YR } 1979 & \text { TOTAL } & 3455.45 & \text { MEAN } 9.47 & \text { MAX } 388 & \text { MIN } & .17 & \text { AC-FT } 6850 & t \dagger & 53.07 \\ \text { WTR YR } & 1980 & \text { TOTAL } & 2385.78 & \text { MEAN } 6.52 & \text { MAX } 340 & \text { MIN } & .08 & \text { AC-FT } 4730 & \text { t† } & 38.41\end{array}$

tt Weighted-mean rainfall, in inches, based on four rain gages.

NOTE. - No gage-height record Oc. 18 to Nov. 19 and Mar. 27-30. 


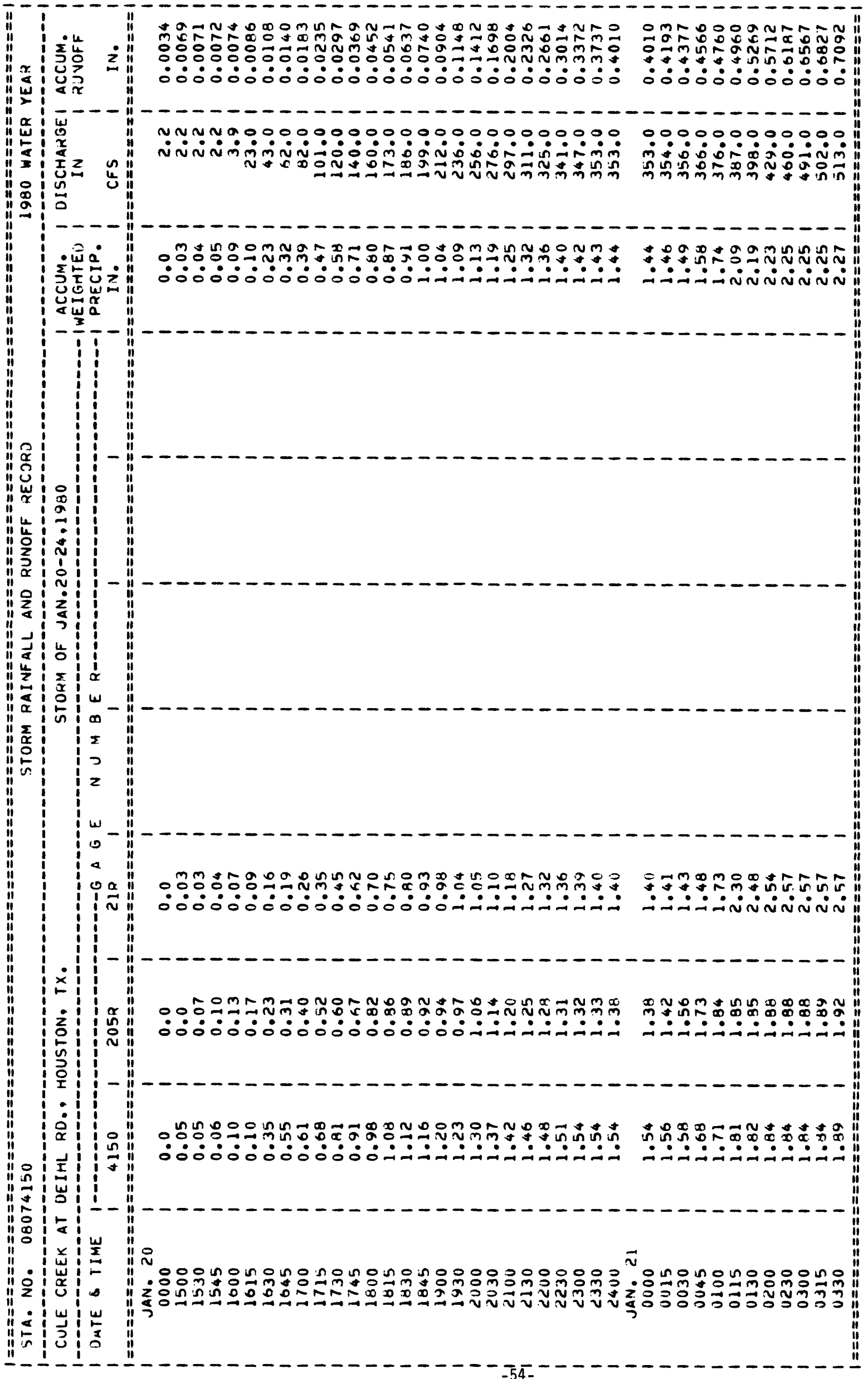




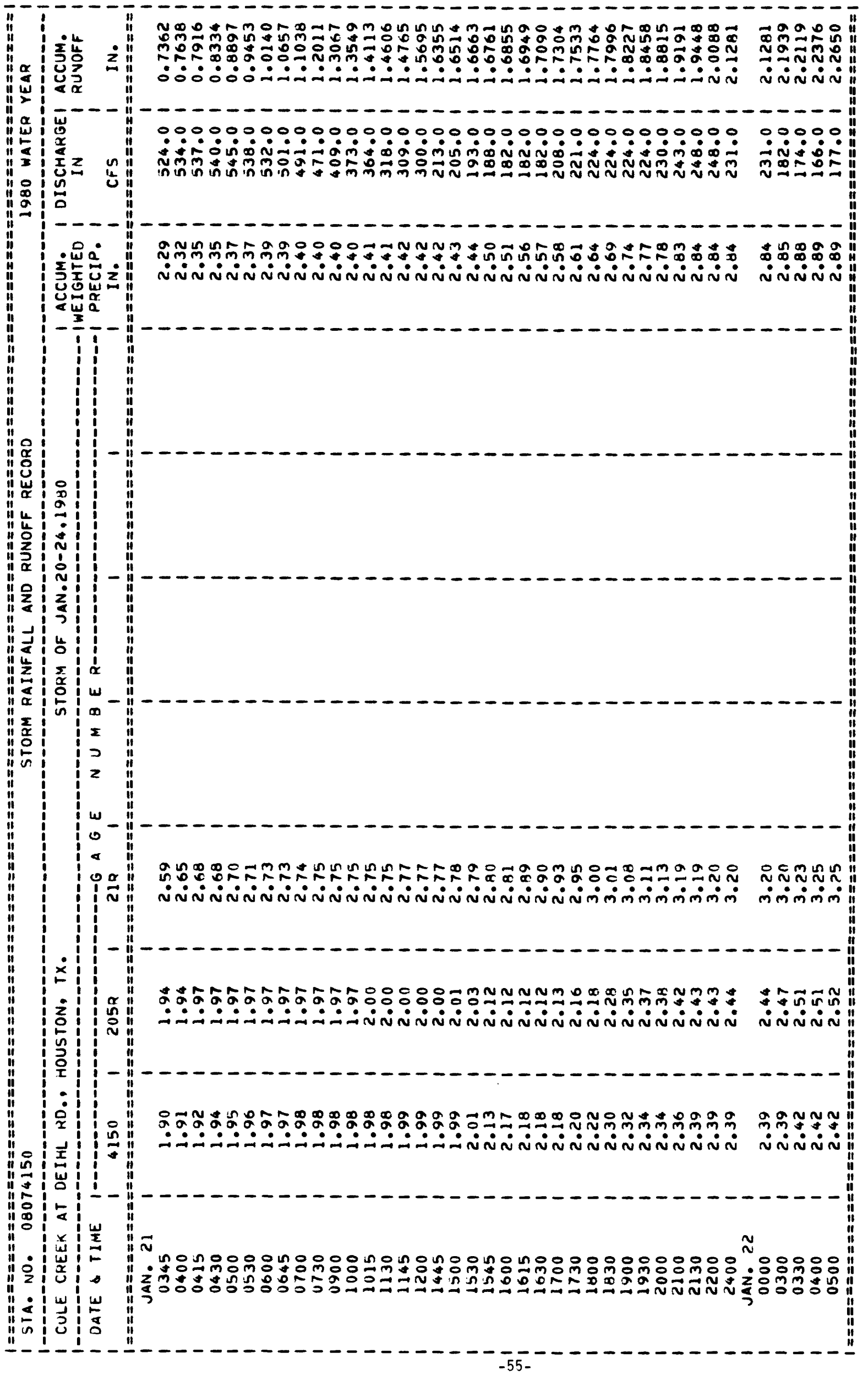




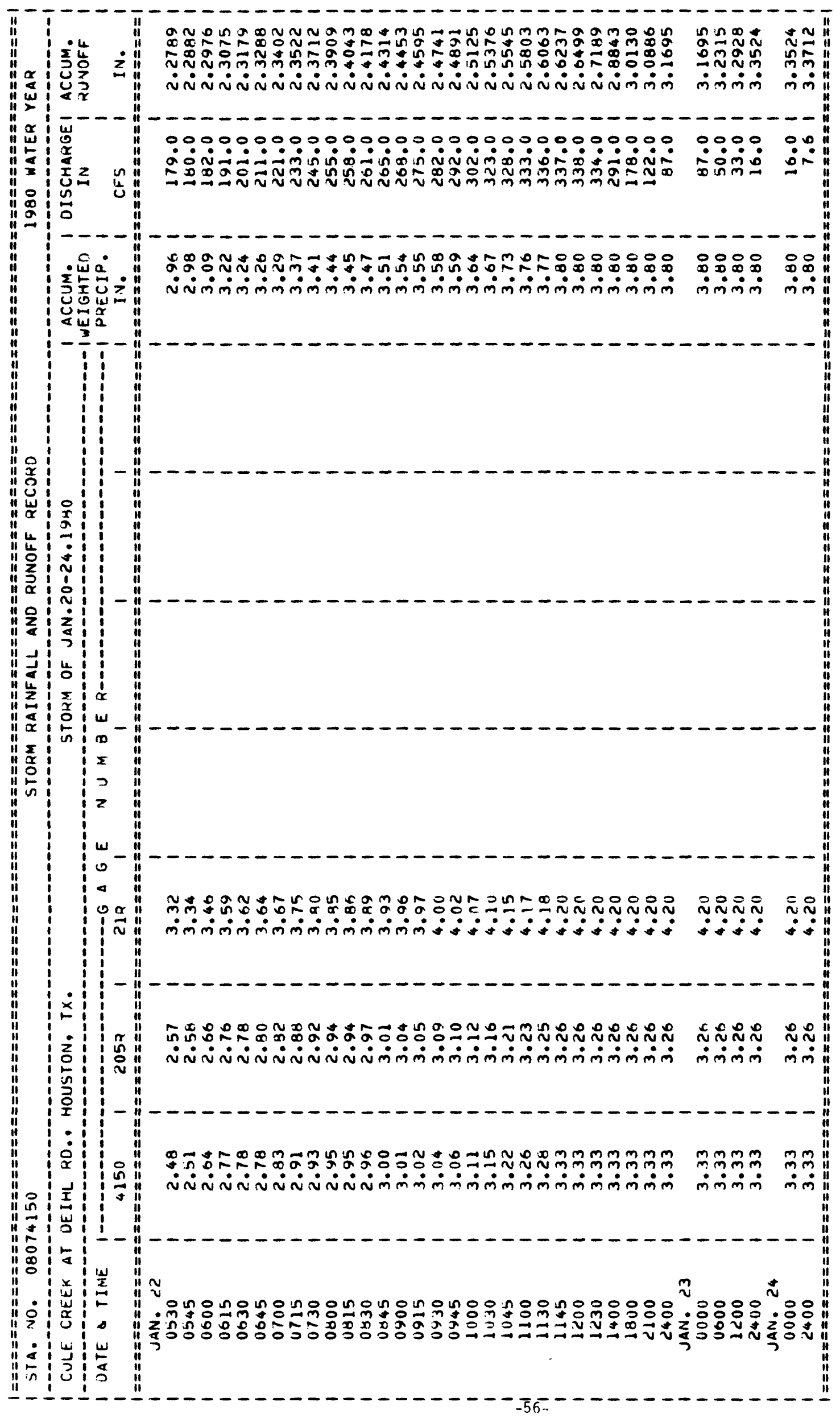




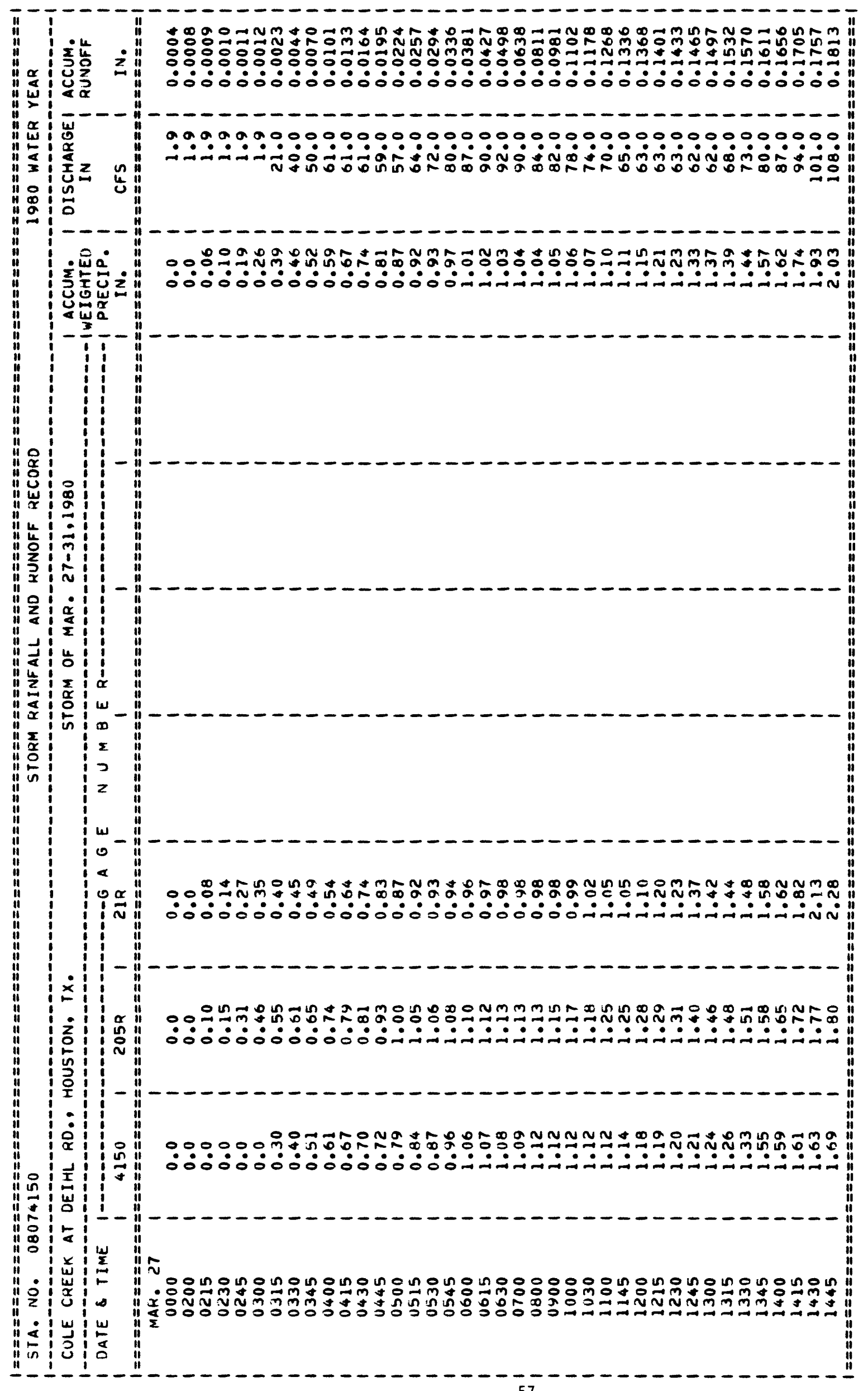

$-57-$ 


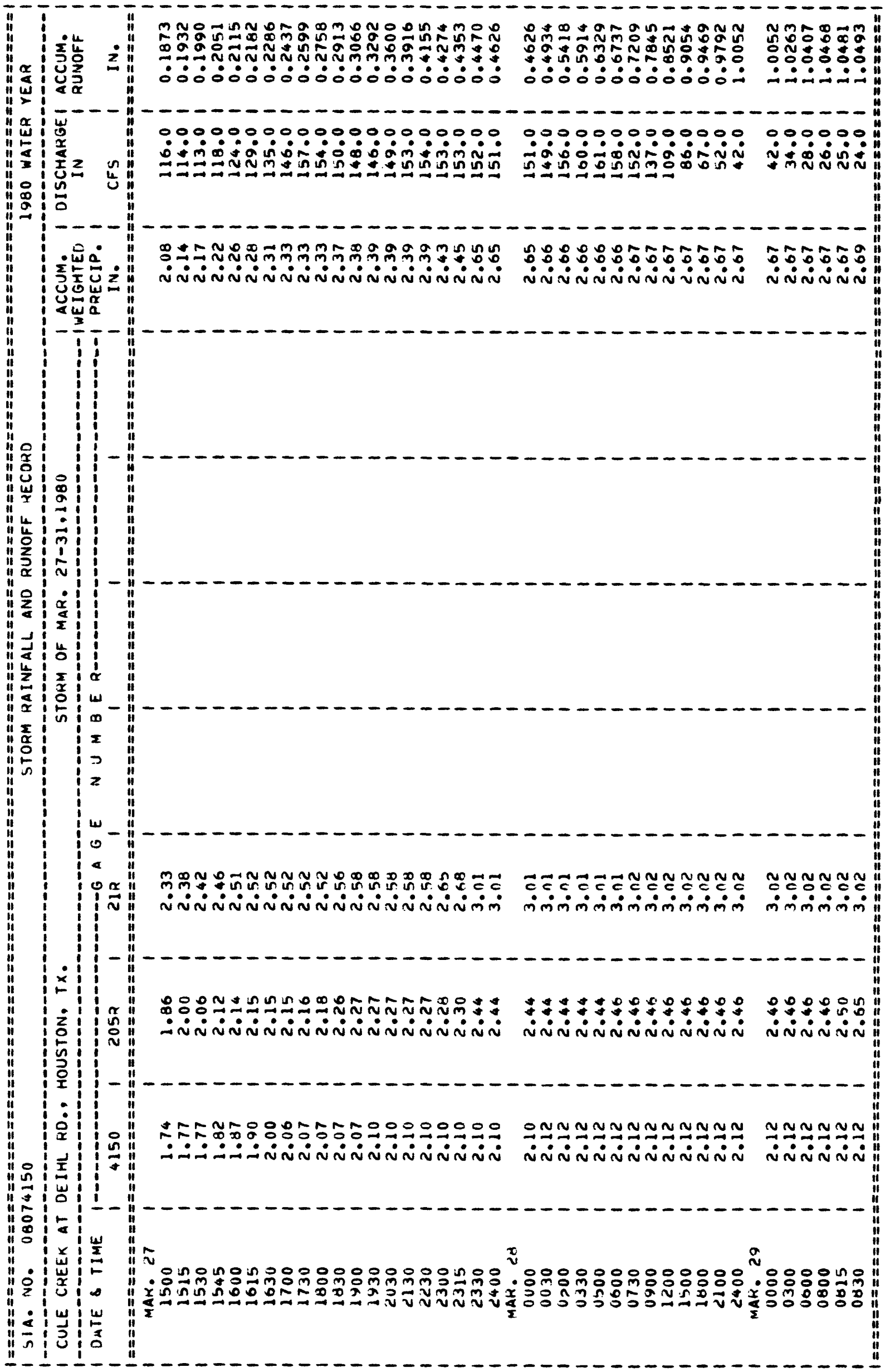




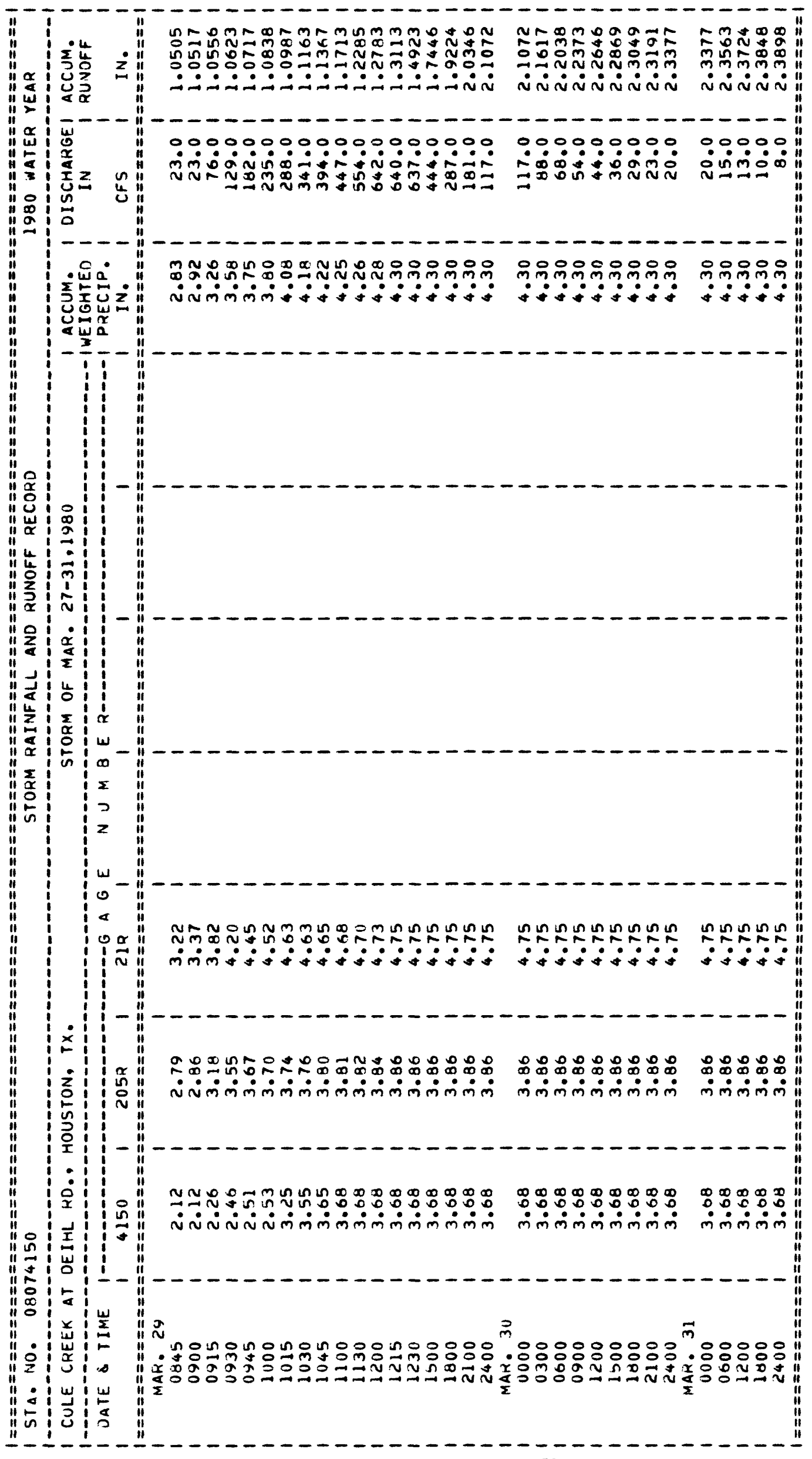




\section{BRICKHOUSE GULLY DRAINAGE BASIN}

The location of data-collection sites in and near the Brickhouse Gully drainage basin are shown in figure 8 .

Weighted-mean rainfall in the drainage basin based on six rain gages for the 1980 water year was 36.84 inches or 11.35 inches less than the 30 -year (1941-70) average of 48.19 inches for Houston.

The storms of Oct. 30 and Mar. 29-30 were selected for analys is at station 08074200, Brickhouse Gully at Clarblak Street, and station 08074250 , Brickhouse Gully at Costa Rica Street. The storm of Mar. 27-28 was also selected for analysis at the latter station. 


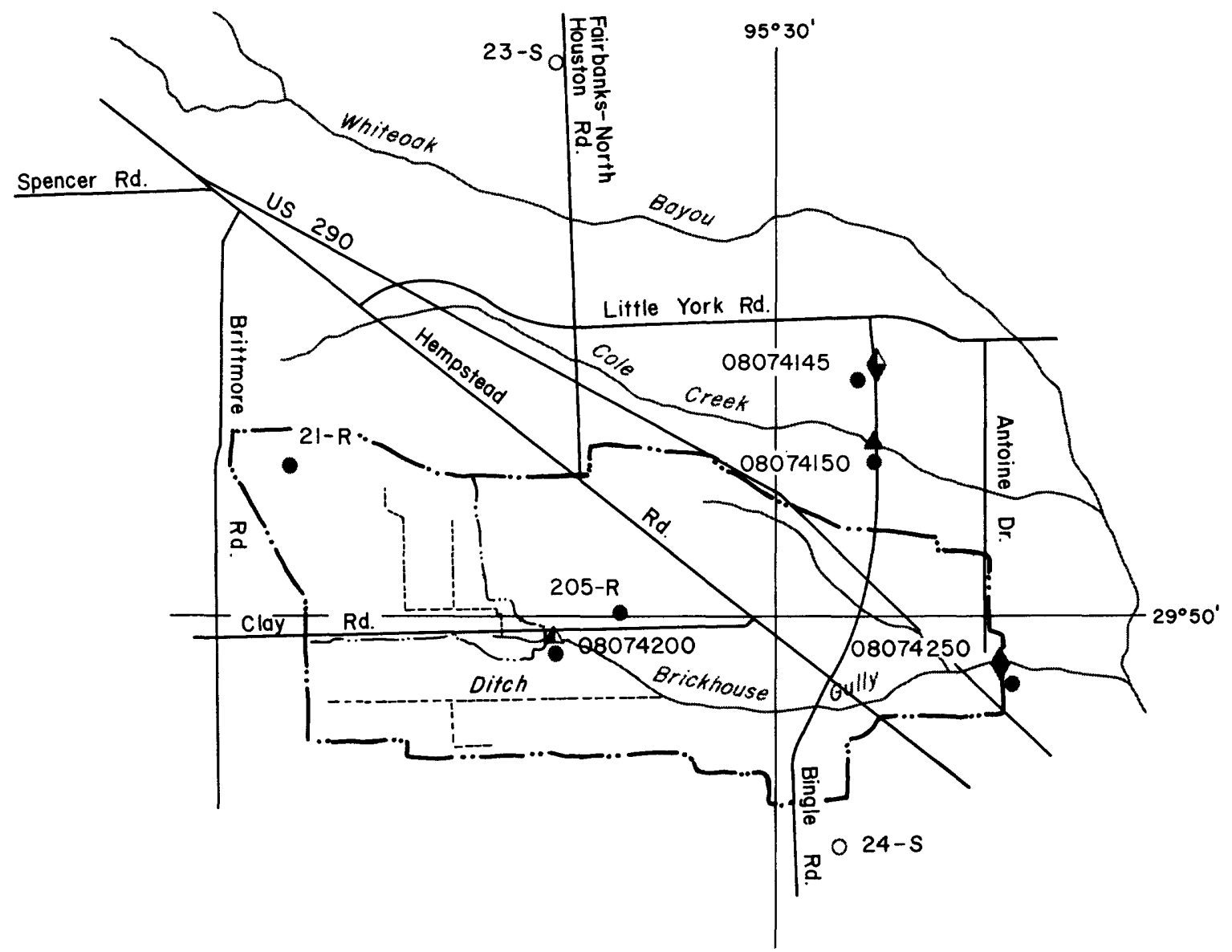

EXPLANATION
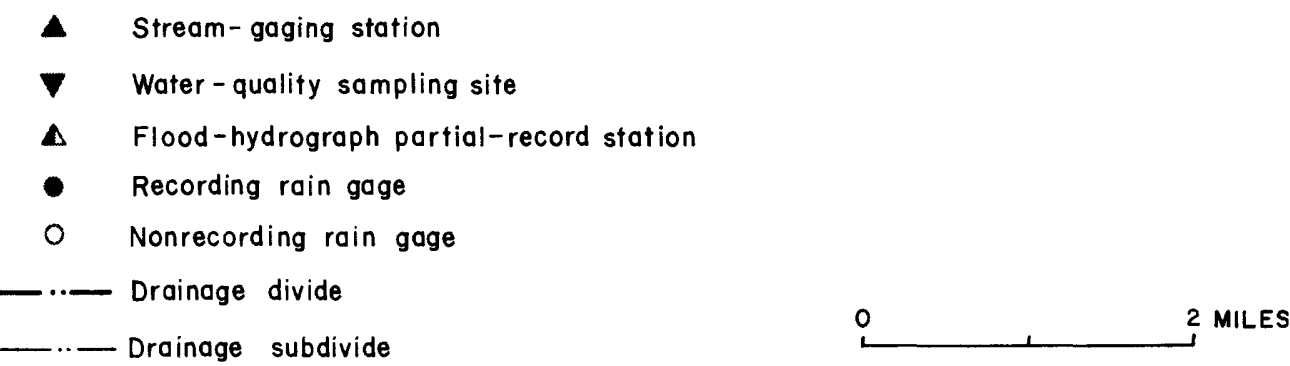

Base from Texas Department of Highways and Public Transportation General Highway Map

Figure 8.- Locations of data-collection sites in and near the Brickhouse Gully drainage basin

$$
-61-
$$




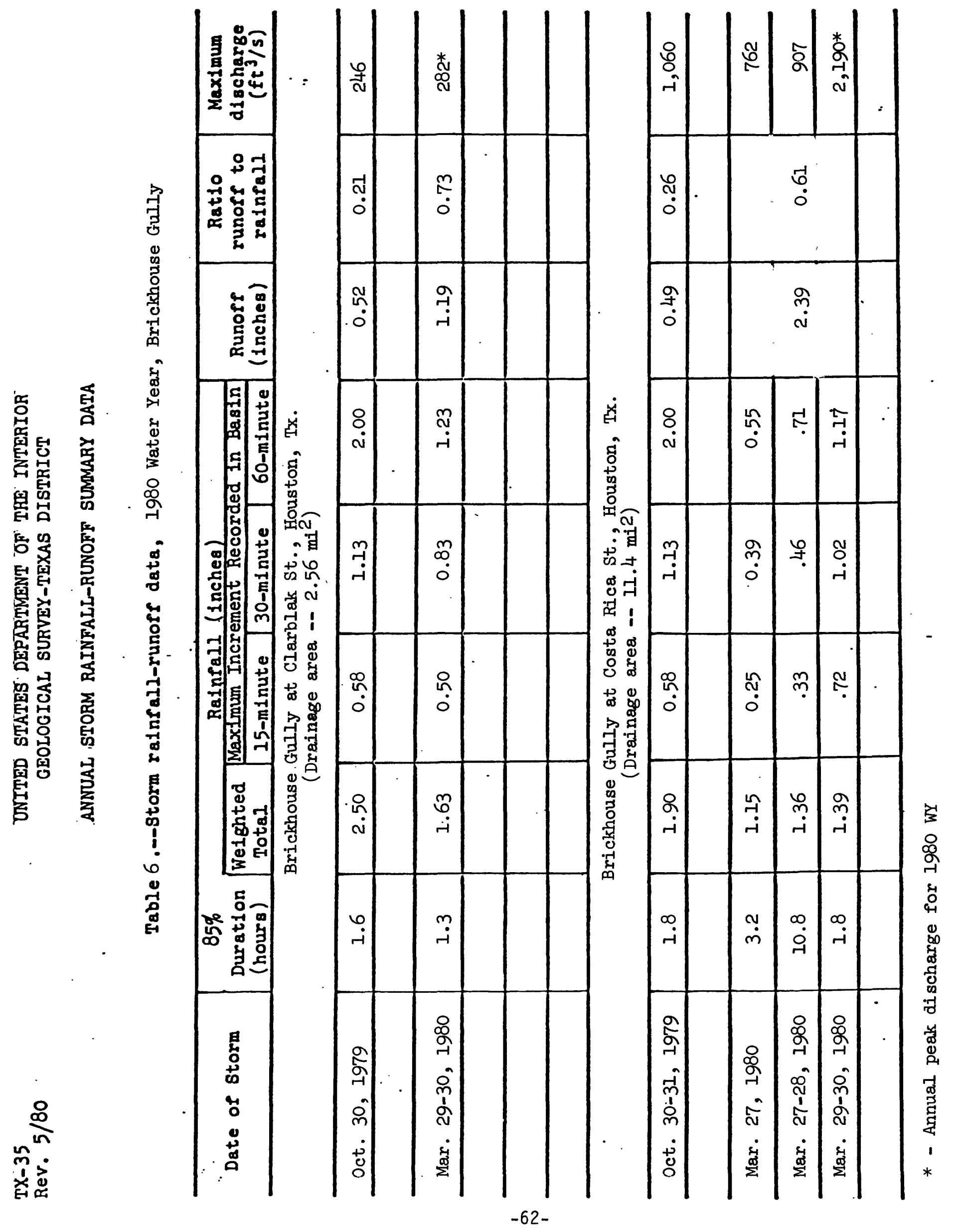


08074200 Brickhouse Gully at Clarblak Street, Houston, Tex. (Flood-hydrograph partial-record station)

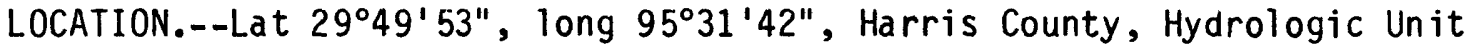
12040104 at bridge on $\mathrm{Clarblak}$ Street, in northwest Houston, and 4.0 miles upstream from station at Costa Rica Street.

DRAINAGE AREA.--2.56 $\mathrm{mi}^{2}$. Drainage area, effective for period, April 1964 to current year. The boundary of the basin is poorly defined due to flat ground slopes.

PERIOD OF RECORD--April 1964 to July 6, 1976, Jan. 26, 1977 to current year.

GAGE.--Digital Tlood-hydrograph and rainfall recorders and crest-stage gage. Prior to April 7, 1978, a flood-hydrograph rainfall recorder (type SR) and crest-stage gage. Datum of gage is National Geodetic Vertical Datum of 1929, 1957 adjustment, unadjusted for land-surface subsidence.

REMARKS.--Records fair.

EXTREMES FOR PERIOD OF RECORD.--Maximum discharge, $296 \mathrm{ft}^{3} / \mathrm{s}$, Sept. 19, 1979 (elevation $87.94 \mathrm{ft}$ ) after concrete lining of channel. Maximum discharge, $399 \mathrm{ft}^{3} / \mathrm{s}$ March 20, 1972 (elevation $94.28 \mathrm{ft}$ ) prior to concrete lining (July 1976).

EXTREMES FOR CURRENT YEAR.--Peak discharges above base of $200 \mathrm{ft}^{3} / \mathrm{s}$ and maximum $(*)$ :

$\begin{array}{lccr}\text { DATE } & \text { TIME } & \begin{array}{c}\text { DISCHARGE } \\ \left(\mathrm{ft}^{3} / \mathrm{s}\right)\end{array} & \begin{array}{c}\text { GAGE HEIGHT } \\ (\mathrm{ft})\end{array} \\ \text { Oct. } 30 & 1745 & & 87.17 \\ \text { Dec. } 12 & 1215 & 246 & 86.82 \\ \text { Dec. } 23 & 1300 & 223 & 86.53 \\ \text { Jan. } 17 & 1445 & 205 & 87.39 \\ \text { Jan. } 21 & 0130 & 260 & 87.52 \\ \text { Feb. } 8 & 1645 & 268 & 86.79 \\ \text { Mar. 29 } 21 & 0945 & * 282 & 87.73 \\ \text { July } 21 & 1330 & 258 & 87.36\end{array}$

Minimum discharge not dețermined. 


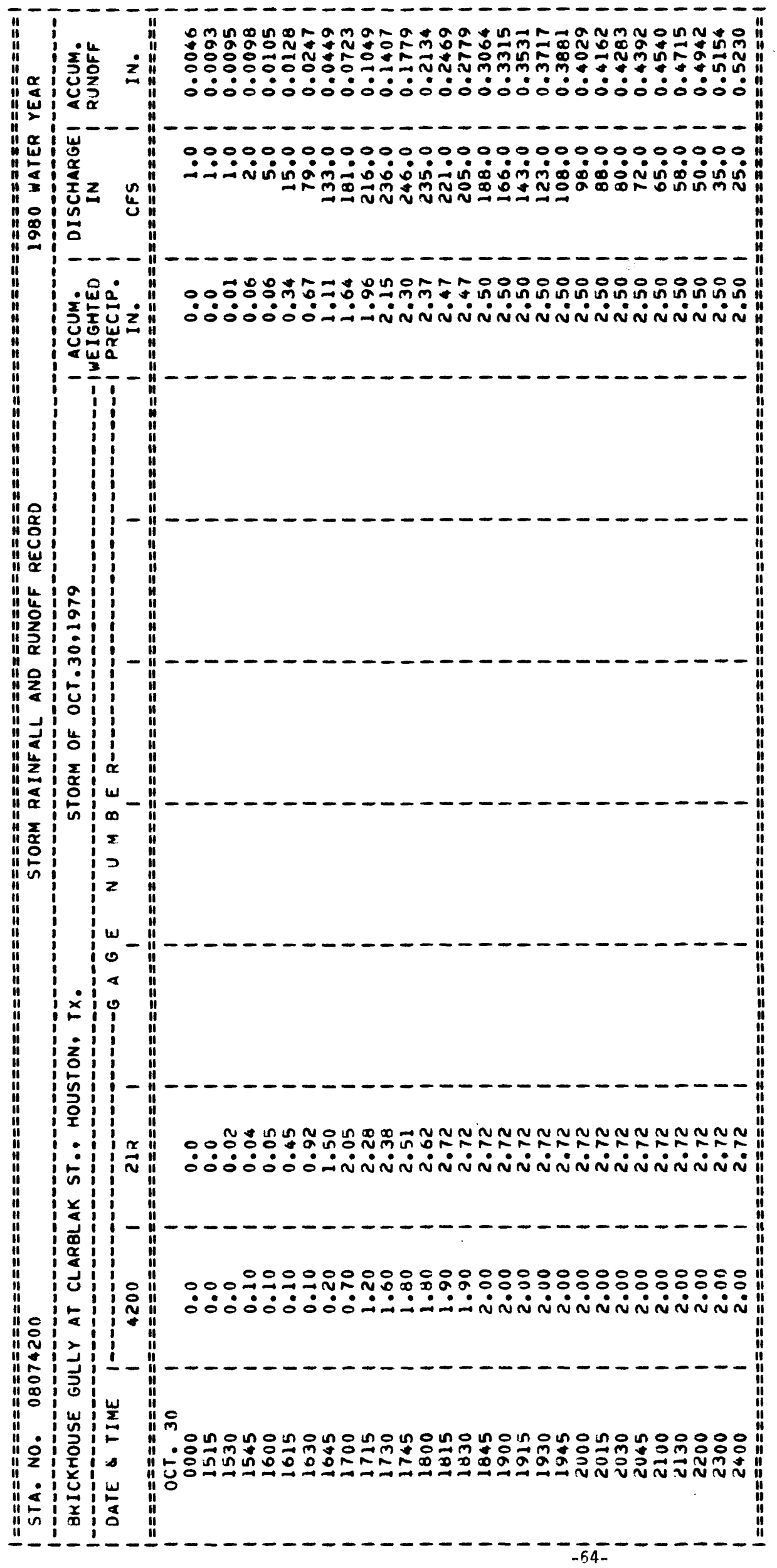




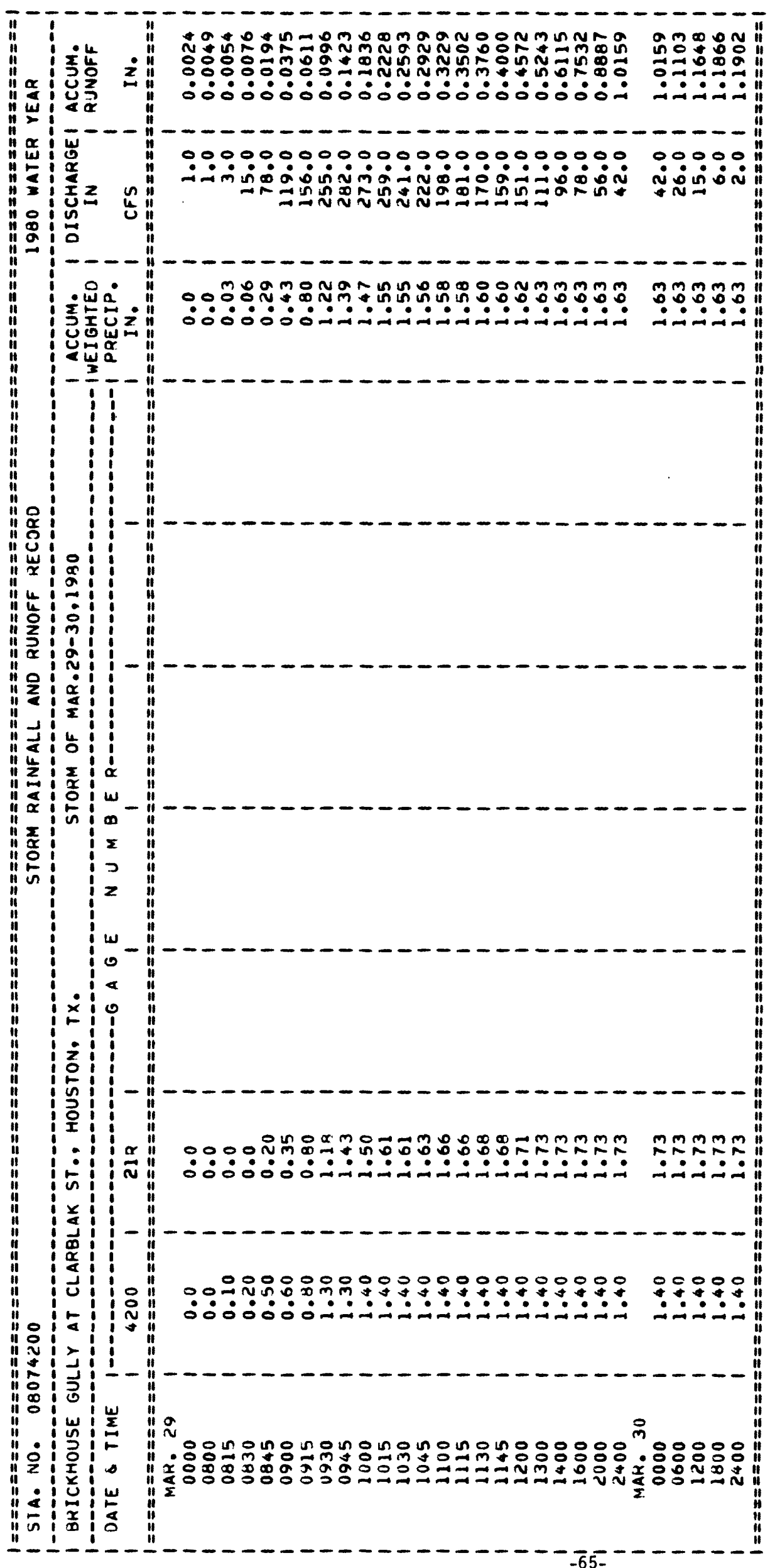


SAN JACINTO KIVEK BASIN

08074250 BRICKHOUSE GULLY AT COSTA RICA STKEET, HOUSTON, TX

LOCATION. - $29^{\circ} 49^{\prime} 40^{\prime \prime}$, long $95^{\circ} 28^{\prime} 09^{\prime \prime}$, Harris County, Hydrologic Unit 12040104, at downstream side of bridge at Costa Rica Street in northwest Houston and $1.0 \mathrm{mi}(1.6 \mathrm{~km})$ upstream from Whiteoak Bayou.

DRAINAGE AREA. $-11.4 \mathrm{mi}^{2}\left(29.5 \mathrm{~km}^{2}\right)$. Prior to Oct. $1,1973,11.6 \mathrm{mi}^{2}\left(30.0 \mathrm{~km}^{2}\right)$.

WATER-DISCHARGE RECORDS

PERIOD OF RECORD. - -August 1964 to current year.

REVISED RECORDS.--WRD TX-74-1: Drainage area.

GAGE. - Water-stage recorder and crest-stage gage, Low-water concrete control since Dec. 9, 1970. Datum of gage is National Geodetic Vertical Datum of 1929, 1957 adjustment; unadjusted for land-surface subsidence. REMARKS. - Water-discharge records good. Low flow is partially sustained by sewage effluent. No know diversion above station. Recording rain gage at station.

AVERAGE DISCHARGE. --16 years, $13.8 \mathrm{ft}^{3} / \mathrm{s}\left(0.391 \mathrm{~m}^{3} / \mathrm{s}\right), 10,000 \mathrm{acre}-\mathrm{ft} / \mathrm{yr}\left(12.3 \mathrm{hm}^{3} / \mathrm{yr}^{2}\right)$.

EXTREMES FOR PERIOD OF RECORD.--Maximum discharge, 5,800 $\mathrm{ft}^{3} / \mathrm{s}\left(164 \mathrm{~m}^{3} / \mathrm{s}\right)$ Mar. 20, 1972, elevation, 6y.20 ft $(21.092 \mathrm{~m})$; no flow at times.

EXTREMES FOR CURRENT YEAR. --Peak discharges above base of $1,500 \mathrm{ft}^{3} / \mathrm{s}\left(42.5 \mathrm{~m}^{3} / 8\right.$ ) and maximum (*):

\begin{tabular}{ccccccc} 
Date & Time & \multicolumn{2}{c}{ Discharge } & \multicolumn{2}{c}{ Elevation } \\
& & & $\left(\mathrm{ft}^{3} / \mathrm{s}\right)$ & $\left(\mathrm{m}^{3} / \mathrm{s}\right)$ & $(\mathrm{ft})$ & $(\mathrm{m})$ \\
aOct. & 30 & 1800 & 1,060 & 30.0 & 59.88 & 18.251 \\
Mar. 29 & 1100 & $* 2,190$ & 62.0 & 62.98 & 19.196 \\
aSept. 6 & 0730 & 1,200 & 34.0 & 60.30 & 18.379
\end{tabular}

a Water-quality samples were obtained during this runoff event.

Minimum daily discharge, $0.43 \mathrm{ft}^{3} / \mathrm{s}\left(0.012 \mathrm{~m}^{3} / \mathrm{s}\right)$ Oct. 21 .

DISCHAKGE, IN CUBIC FEET PER SECOND, WATEK YEAR OCTOBER 1979 TO SEPTEMBER 1980

\begin{tabular}{|c|c|c|c|c|c|c|c|c|c|c|c|c|}
\hline DAY & OCT & NOV & DEC & JAN & FEB & MAR & APR & MAY & JUN & JUL. & AUG & SEP \\
\hline $\begin{array}{l}1 \\
2 \\
3 \\
4 \\
5\end{array}$ & $\begin{array}{l}2.1 \\
1.7 \\
1.6 \\
1.2 \\
1.5\end{array}$ & $\begin{array}{l}4.2 \\
4.1 \\
2.7 \\
3.1 \\
4.4\end{array}$ & $\begin{array}{r}12 \\
8.3 \\
7.2 \\
4.2 \\
4.2\end{array}$ & $\begin{array}{r}3.7 \\
3.9 \\
38 \\
4.8 \\
3.5\end{array}$ & $\begin{array}{l}3.4 \\
7.4 \\
4.8 \\
3.3 \\
4.1\end{array}$ & $\begin{array}{l}28 \\
2.3 \\
2.9 \\
4.0 \\
2.3\end{array}$ & $\begin{array}{l}12 \\
10 \\
7.3 \\
4.8 \\
4.4\end{array}$ & $\begin{array}{r}14 \\
9.3 \\
3.1 \\
3.3 \\
3.7\end{array}$ & $\begin{array}{l}2.5 \\
2.7 \\
3.3 \\
4.1 \\
3.0\end{array}$ & $\begin{array}{l}1.8 \\
2.0 \\
1.6 \\
1.6 \\
1.8\end{array}$ & $\begin{array}{l}2.0 \\
1.7 \\
1.6 \\
1.8 \\
4.8\end{array}$ & $\begin{array}{r}5.1 \\
8.3 \\
7.0 \\
4.3 \\
42\end{array}$ \\
\hline $\begin{array}{r}6 \\
7 \\
8 \\
9 \\
10\end{array}$ & $\begin{array}{l}2.0 \\
1.9 \\
1.5 \\
1.4 \\
1.3\end{array}$ & $\begin{array}{l}4.0 \\
4.4 \\
4.0 \\
4.9 \\
3.4\end{array}$ & $\begin{array}{l}5.9 \\
6.3 \\
4.2 \\
5.5 \\
5.2\end{array}$ & $\begin{array}{l}2.5 \\
2.3 \\
2.6 \\
2.3 \\
3.2\end{array}$ & $\begin{array}{c}4.5 \\
4.3 \\
140 \\
37 \\
10\end{array}$ & $\begin{array}{l}2.3 \\
5.8 \\
5.8 \\
2.2 \\
1.6\end{array}$ & $\begin{array}{l}7.2 \\
5.2 \\
3.7 \\
3.3 \\
3.2\end{array}$ & $\begin{array}{c}5.0 \\
14 \\
9.3 \\
4.2 \\
3.3\end{array}$ & $\begin{array}{r}3.7 \\
3.7 \\
3.7 \\
56 \\
7.7\end{array}$ & $\begin{array}{r}1.8 \\
2.0 \\
2.0 \\
15 \\
4.9\end{array}$ & $\begin{array}{l}2.2 \\
9.9 \\
5.4 \\
2.7 \\
2.8\end{array}$ & $\begin{array}{c}196 \\
69 \\
18 \\
8.1 \\
3.7\end{array}$ \\
\hline $\begin{array}{l}11 \\
12 \\
13 \\
14 \\
15\end{array}$ & $\begin{array}{l}1.5 \\
1.4 \\
1.3 \\
1.92 \\
1.1\end{array}$ & $\begin{array}{l}3.2 \\
2.3 \\
3.2 \\
2.1 \\
3.4\end{array}$ & $\begin{array}{c}237 \\
18 \\
8.5 \\
6.8\end{array}$ & $\begin{array}{l}3.5 \\
2.1 \\
2.3 \\
2.0 \\
5.9\end{array}$ & $\begin{array}{c}6.2 \\
4.7 \\
5.3 \\
14 \\
9.9\end{array}$ & $\begin{array}{l}1.9 \\
3.1 \\
3.2 \\
6.8 \\
8.9\end{array}$ & $\begin{array}{r}3.4 \\
4.2 \\
27 \\
3.1 \\
4.0\end{array}$ & $\begin{array}{l}3.0 \\
3.1 \\
28 \\
26 \\
21\end{array}$ & $\begin{array}{l}3.0 \\
3.7 \\
2.7 \\
2.5 \\
2.7\end{array}$ & $\begin{array}{l}1.8 \\
1.6 \\
1.6 \\
1.0 \\
1.3\end{array}$ & $\begin{array}{r}3.9 \\
7.2 \\
3.0 \\
2.7 \\
23\end{array}$ & $\begin{array}{l}4.1 \\
3.0 \\
3.0 \\
2.9 \\
3.0\end{array}$ \\
\hline $\begin{array}{l}16 \\
17 \\
18 \\
19 \\
20\end{array}$ & $\begin{array}{l}6.8 \\
2.1 \\
1.2 \\
1.0 \\
1.2\end{array}$ & $\begin{array}{l}3.9 \\
4.4 \\
4.2 \\
5.2 \\
4.2\end{array}$ & $\begin{array}{l}5.9 \\
5.2 \\
3.7 \\
3.4 \\
3.2\end{array}$ & $\begin{array}{c}3.4 \\
138 \\
16 \\
6.5 \\
140\end{array}$ & $\begin{array}{r}24 \\
6.9 \\
4.3 \\
4.9 \\
5.8\end{array}$ & $\begin{array}{c}10 \\
19 \\
5.3 \\
6.0 \\
21\end{array}$ & $\begin{array}{l}4.2 \\
4.2 \\
5.6 \\
7.0 \\
6.9\end{array}$ & $\begin{array}{c}54 \\
30 \\
6.3 \\
94 \\
9.8\end{array}$ & $\begin{array}{l}2.2 \\
3.3 \\
4.1 \\
3.3 \\
4.5\end{array}$ & $\begin{array}{l}1.3 \\
1.3 \\
1.5 \\
1.3 \\
2.2\end{array}$ & $\begin{array}{l}12 \\
3.5 \\
3.1 \\
2.7 \\
2.4\end{array}$ & $\begin{array}{l}2.3 \\
2.7 \\
3.5 \\
3.5 \\
2.5\end{array}$ \\
\hline $\begin{array}{l}21 \\
22 \\
23 \\
24 \\
25\end{array}$ & $\begin{array}{c}23^{.43} \\
2.8 \\
2.1 \\
1.6\end{array}$ & $\begin{array}{l}57 \\
12 \\
6.8 \\
3.4 \\
1.8\end{array}$ & $\begin{array}{r}3.2 \\
2.9 \\
29 \\
6.8 \\
3.4\end{array}$ & $\begin{array}{r}211 \\
265 \\
38 \\
18 \\
12\end{array}$ & $\begin{array}{c}6.8 \\
9.7 \\
10 \\
11 \\
9.2\end{array}$ & $\begin{array}{l}5.1 \\
3.6 \\
6.4 \\
6.0 \\
4.7\end{array}$ & $\begin{array}{r}9.1 \\
13 \\
13 \\
13 \\
125\end{array}$ & $\begin{array}{l}4.9 \\
4.9 \\
4.7 \\
3.7 \\
3.0\end{array}$ & $\begin{array}{c}3.3 \\
96 \\
12 \\
2.7 \\
1.8\end{array}$ & $\begin{array}{r}43 \\
8.0 \\
2.7 \\
2.0 \\
1.2\end{array}$ & $\begin{array}{l}3.8 \\
3.4 \\
3.8 \\
4.5 \\
3.9\end{array}$ & $\begin{array}{l}2.0 \\
2.1 \\
1.8 \\
6.9 \\
1.7\end{array}$ \\
\hline $\begin{array}{l}26 \\
27 \\
28 \\
29 \\
30 \\
31\end{array}$ & $\begin{array}{c}1.8 \\
1.4 \\
1.1 \\
1.8 \\
134 \\
16\end{array}$ & $\begin{array}{l}3.2 \\
3.9 \\
4.8 \\
5.9 \\
6.3 \\
-.-\end{array}$ & $\begin{array}{r}3.2 \\
3.7 \\
3.7 \\
52 \\
4.2 \\
3.4\end{array}$ & $\begin{array}{l}8.6 \\
7.2 \\
6.0 \\
6.3 \\
5.4 \\
4.5\end{array}$ & \begin{tabular}{l}
4.0 \\
3.9 \\
5.4 \\
4.6 \\
\hdashline.- \\
.-
\end{tabular} & $\begin{array}{r}14 \\
332 \\
74 \\
288 \\
40 \\
19\end{array}$ & $\begin{array}{l}7.0 \\
3.3 \\
2.2 \\
2.9 \\
2.4 \\
-.-\end{array}$ & $\begin{array}{l}3.3 \\
3.3 \\
3.7 \\
4.1 \\
3.3 \\
3.0\end{array}$ & $\begin{array}{l}2.2 \\
2.0 \\
1.2 \\
1.2 \\
1.8 \\
-.-\end{array}$ & $\begin{array}{l}1.3 \\
15 \\
32 \\
8.1 \\
4.3 \\
1.9\end{array}$ & $\begin{array}{r}4.4 \\
5.9 \\
6.5 \\
11 \\
12 \\
5.9\end{array}$ & $\begin{array}{l}7.8 \\
6.4 \\
3.2 \\
3.4 \\
55 \\
-\end{array}$ \\
\hline $\begin{array}{l}\text { TOTAL } \\
\text { MEAN } \\
\text { MAX } \\
\text { MIN } \\
\text { AC-FT } \\
(++)\end{array}$ & $\begin{array}{r}220.75 \\
7.12 \\
134 \\
.43 \\
438 \\
2.54\end{array}$ & $\begin{array}{r}180.4 \\
6.01 \\
57 \\
1.8 \\
358 \\
1.29\end{array}$ & $\begin{array}{r}475.5 \\
15.3 \\
237 \\
2.9 \\
943 \\
3.46\end{array}$ & $\begin{array}{r}968.5 \\
31.2 \\
265 \\
2.0 \\
1920 \\
5.27\end{array}$ & $\begin{array}{r}369.4 \\
12.7 \\
140 \\
3.3 \\
73 . \\
2.07\end{array}$ & $\begin{array}{r}935.2 \\
30.2 \\
332 \\
1.6 \\
1850 \\
5.58\end{array}$ & $\begin{array}{r}321.6 \\
10.7 \\
125 \\
2.2 \\
638 \\
1.65\end{array}$ & $\begin{array}{r}386.3 \\
12.5 \\
94 \\
3.0 \\
766 \\
3.53\end{array}$ & $\begin{array}{r}246.6 \\
8.22 \\
96 \\
1.2 \\
489 \\
2.20\end{array}$ & $\begin{array}{r}168.9 \\
5.45 \\
43 \\
1.0 \\
335 \\
2.25\end{array}$ & $\begin{array}{r}163.5 \\
5.27 \\
23 \\
1.6 \\
324 \\
1.72\end{array}$ & $\begin{array}{r}482.3 \\
16.1 \\
196 \\
1.7 \\
957 \\
5.28\end{array}$ \\
\hline
\end{tabular}

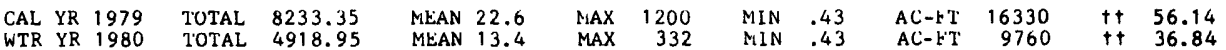

t† Weighted-mean rainfall, in inches, based on six rain gages. 


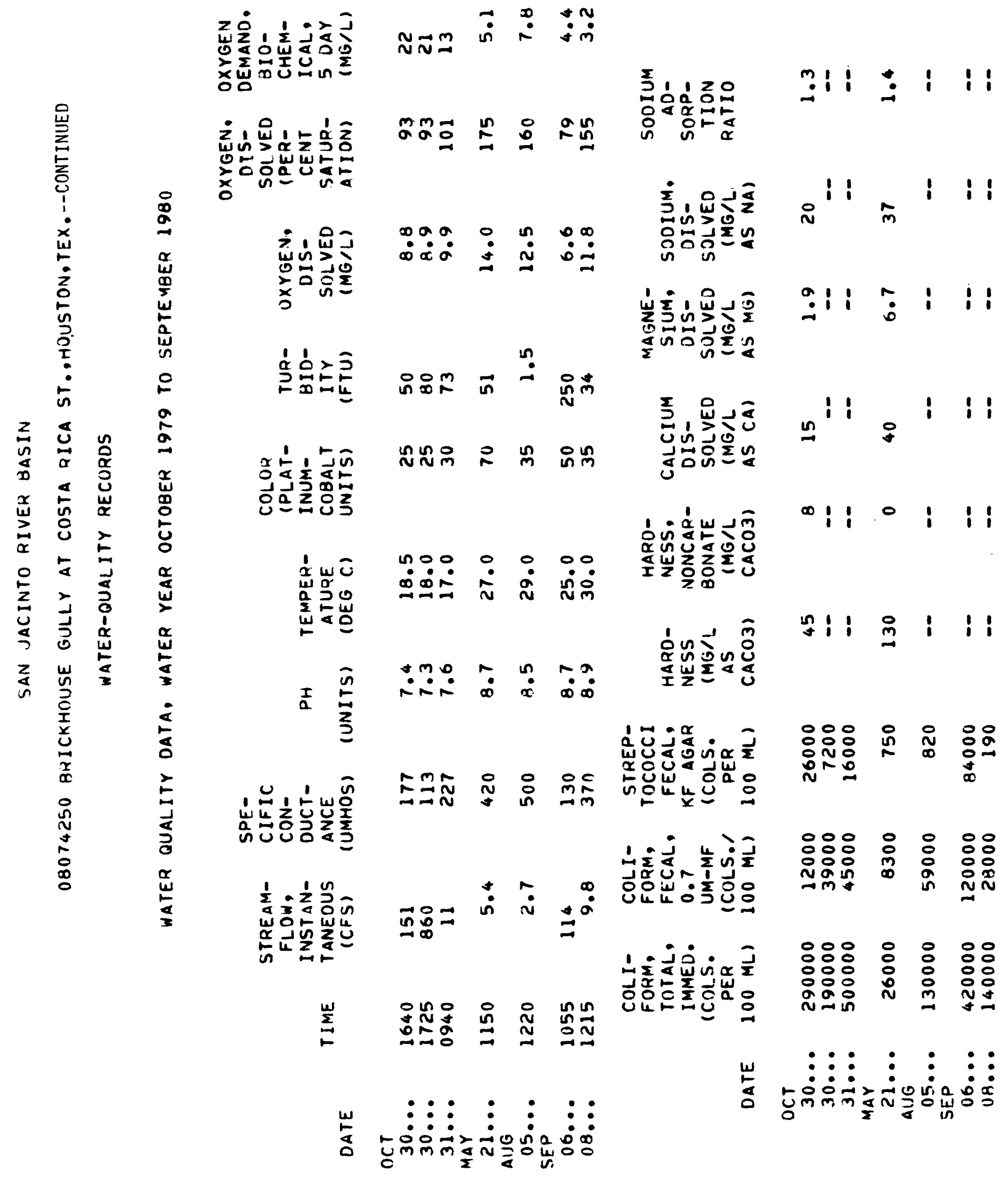




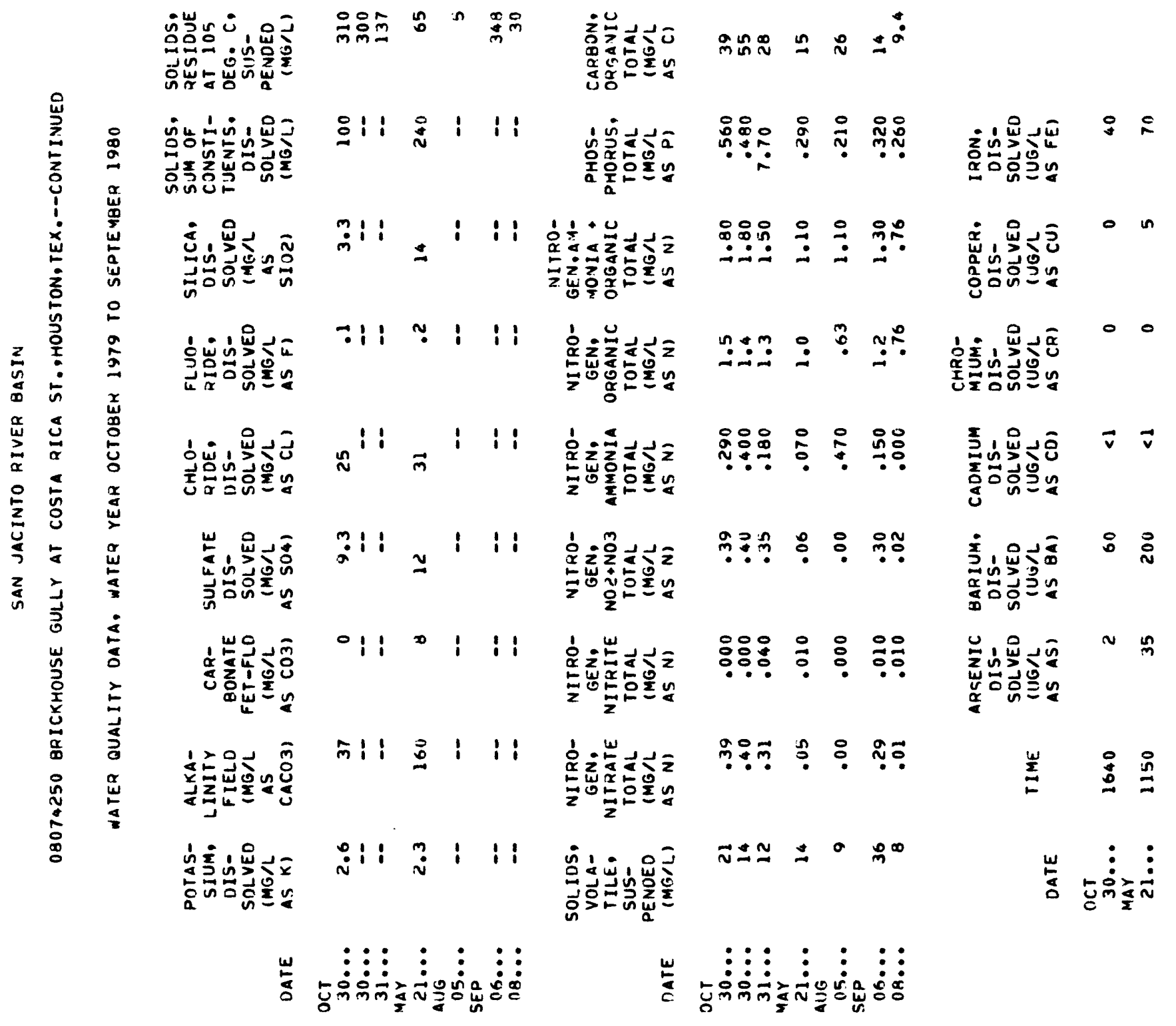




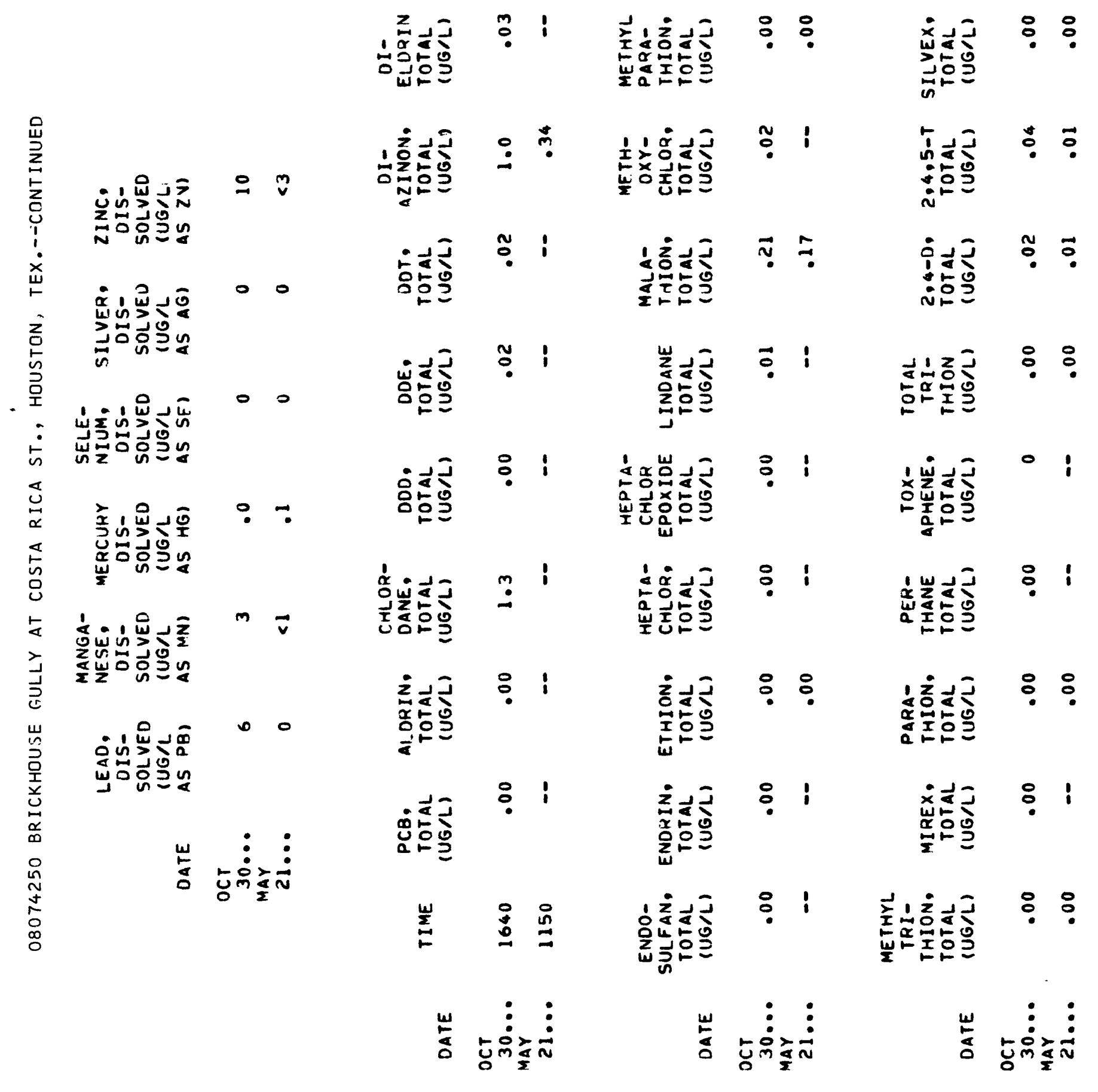




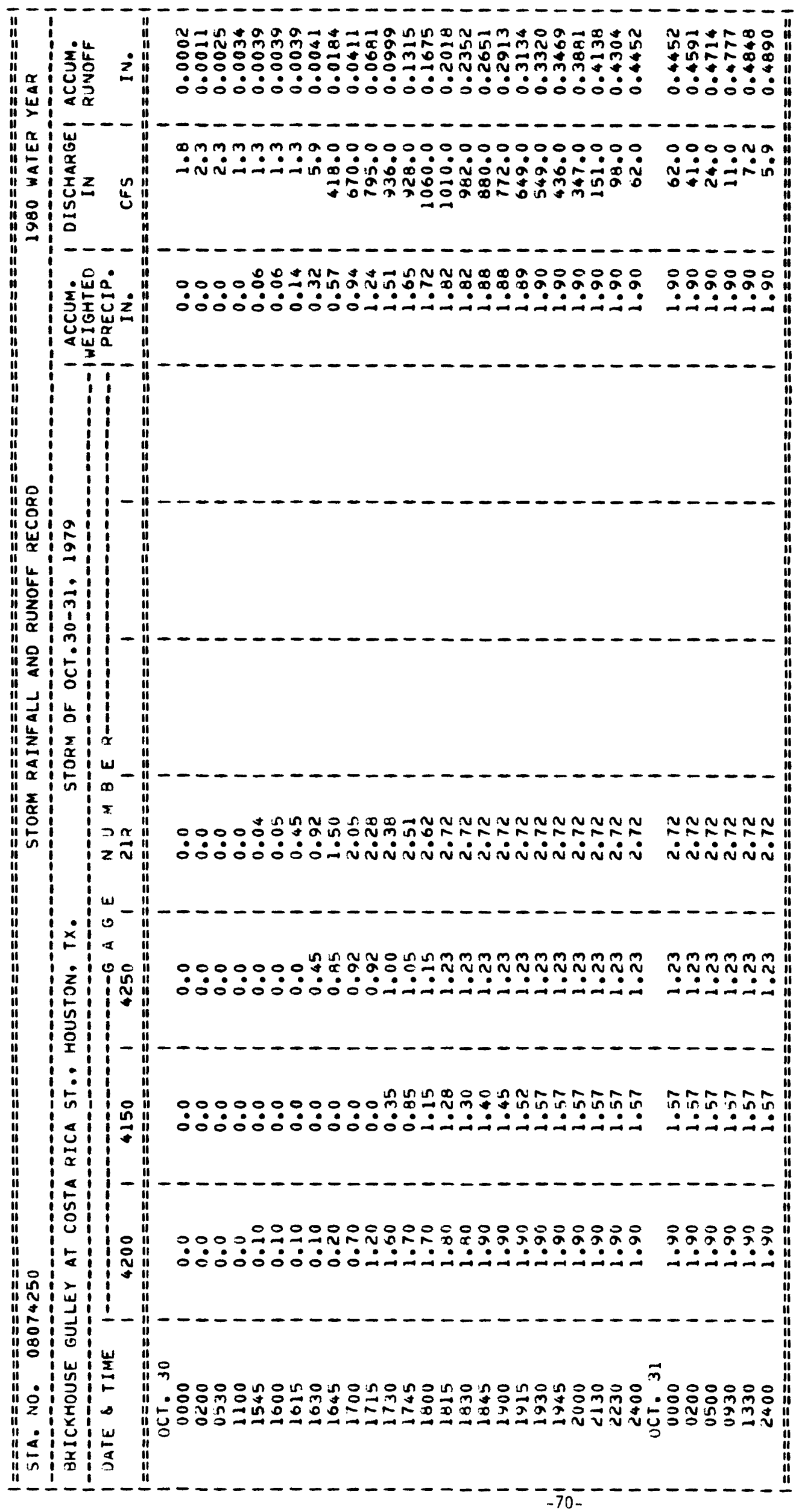




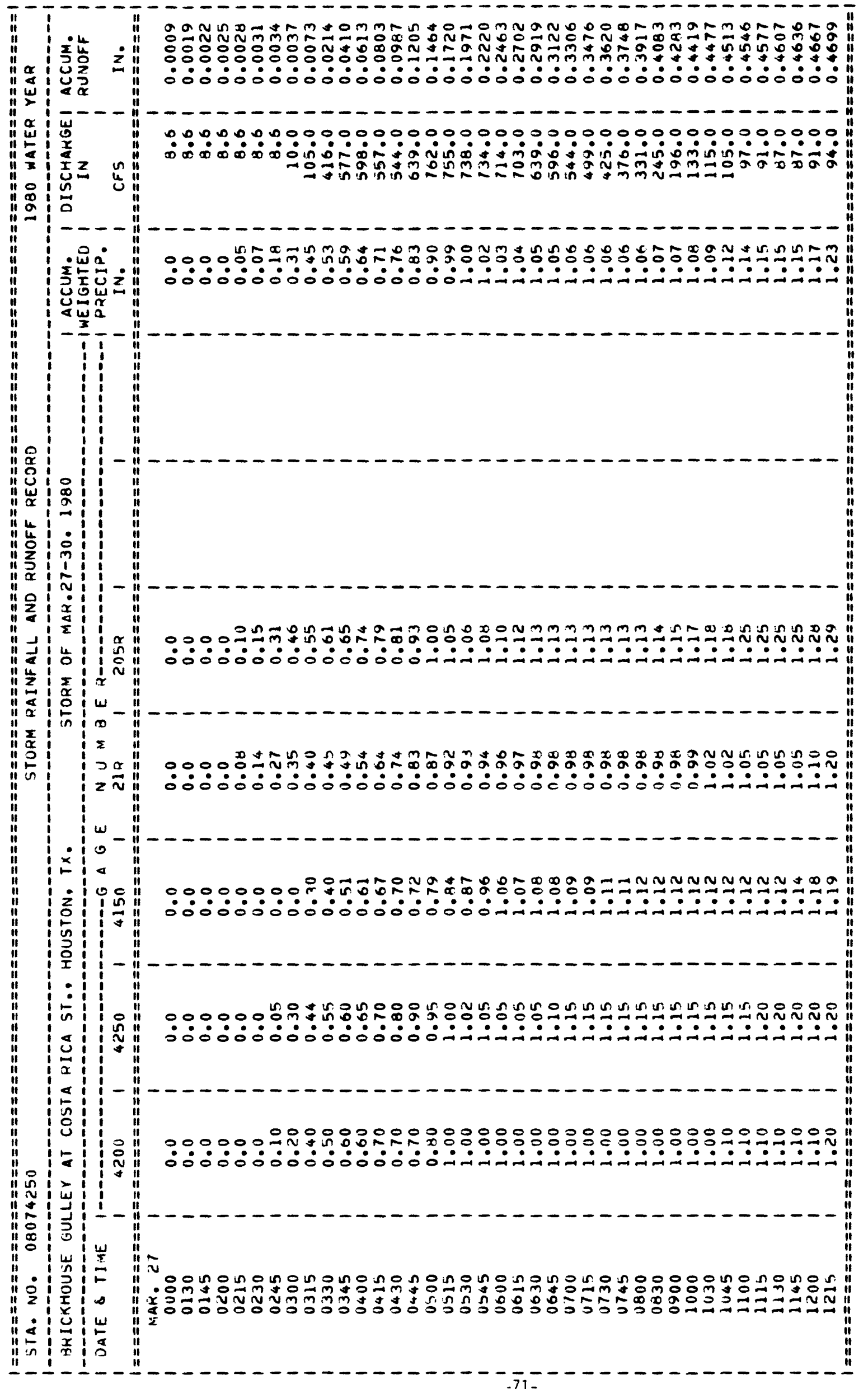




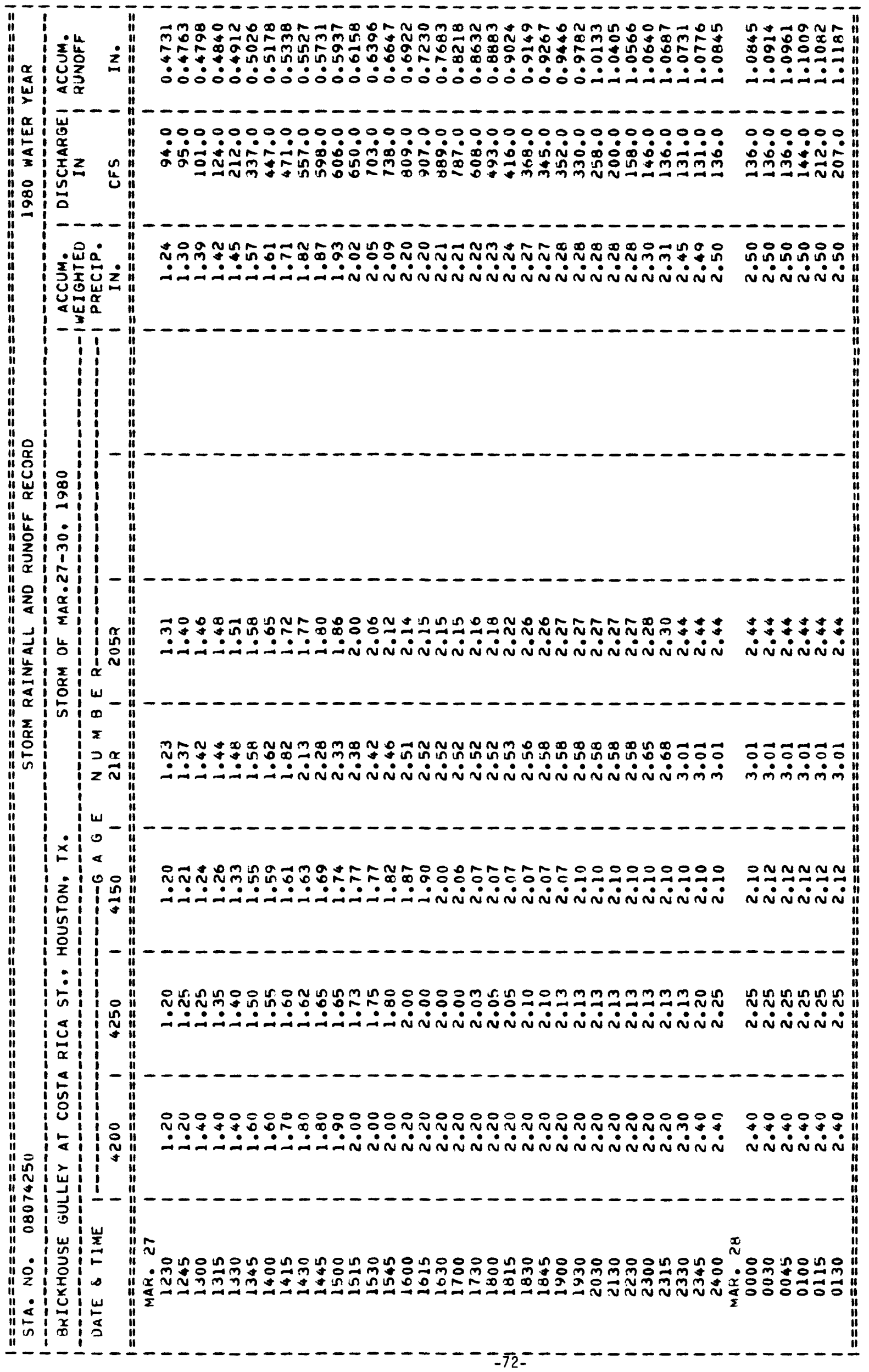




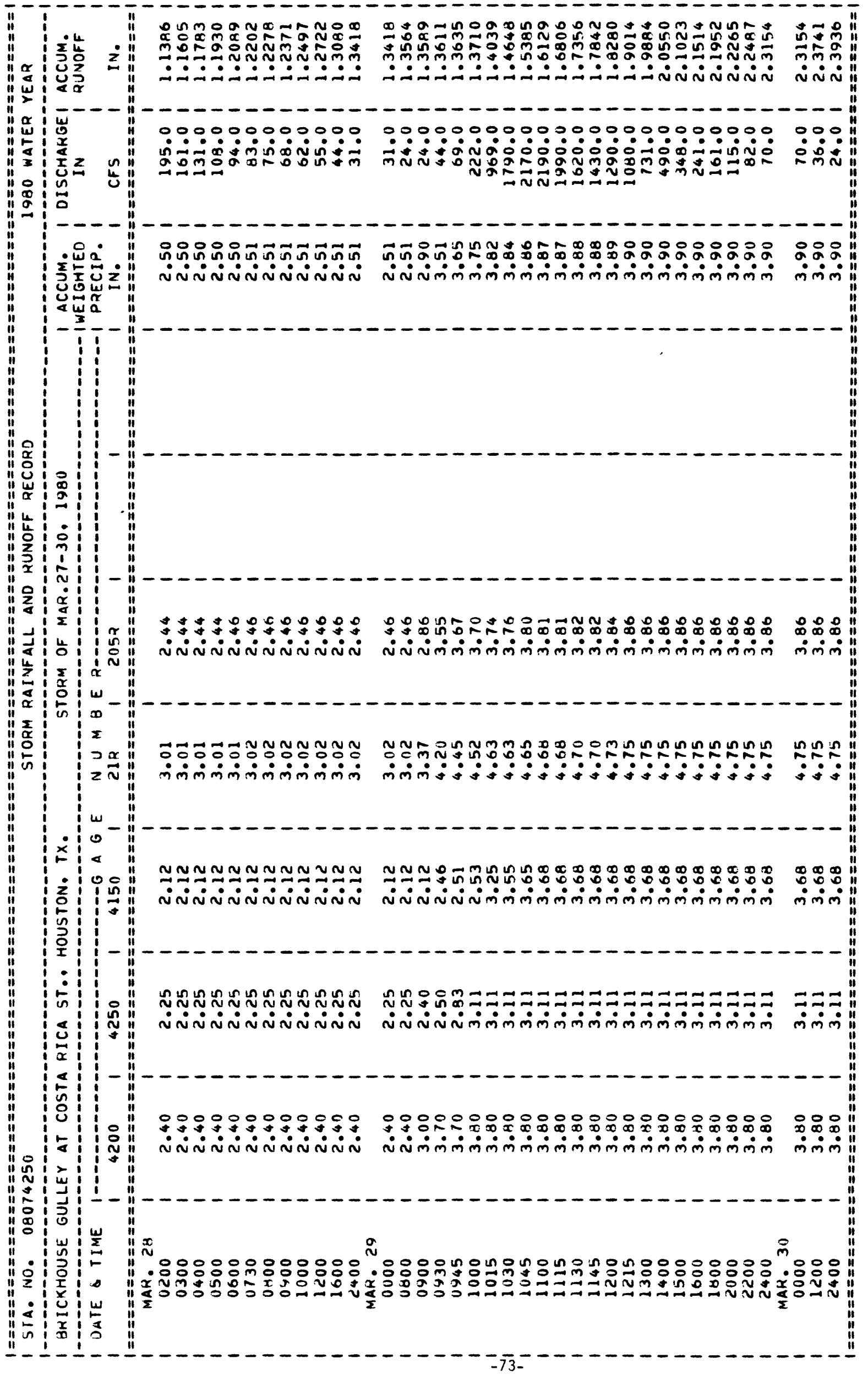




\section{LAZYBROOK STREET STORM SEWER DRAINAGE BASIN}

The locations of data-collection sites in the Lazybrook Street Storm Sewer drainage basin are shown in figure 9.

Weighted-mean rainfall for the 1980 water year was not determined.

The storms of Oct. 30, Mar. 27-28, and April 25 were selected for analysis at station 08074400 , Lazybrook Street Storm Sewer at Houston. 


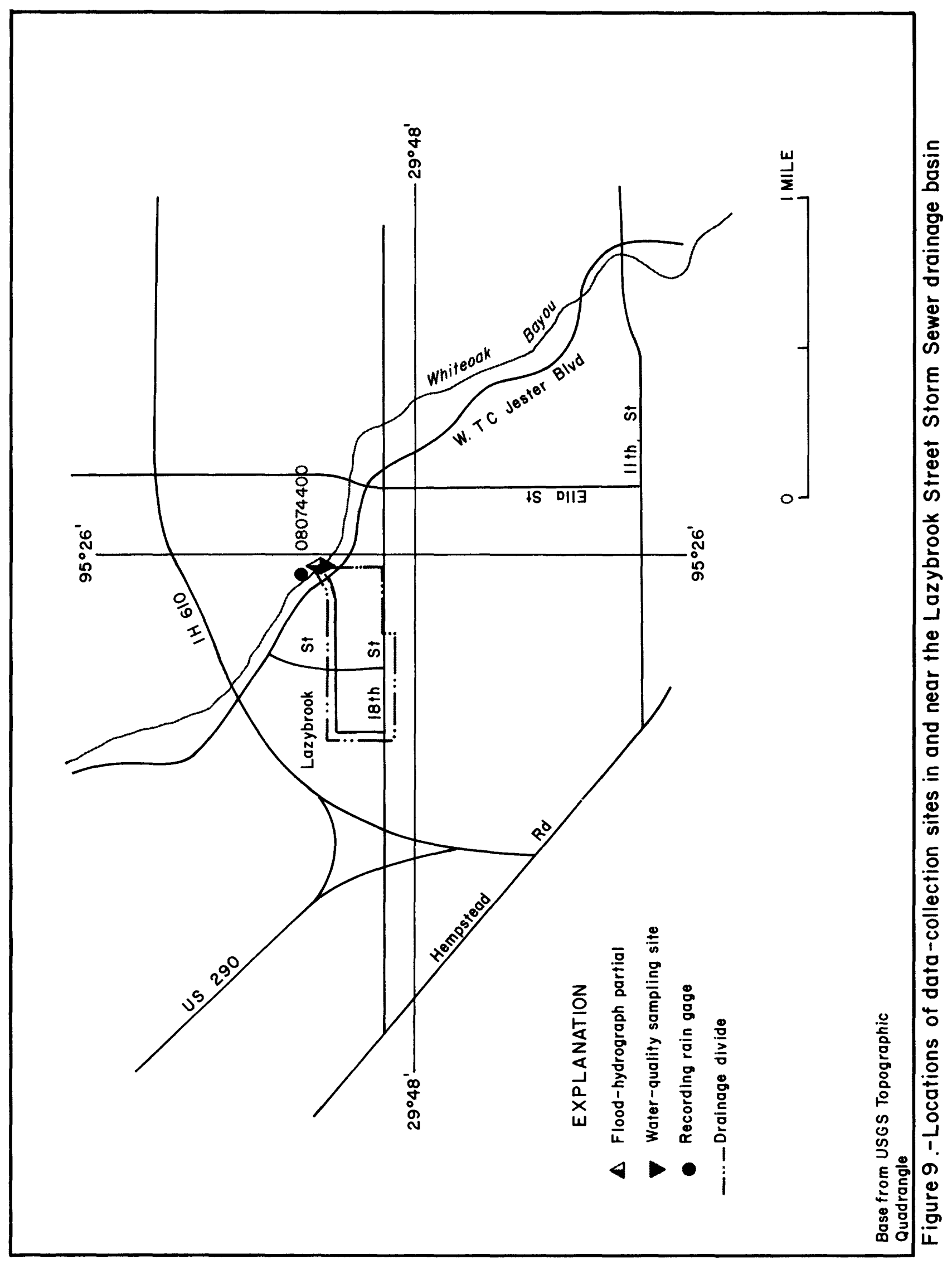




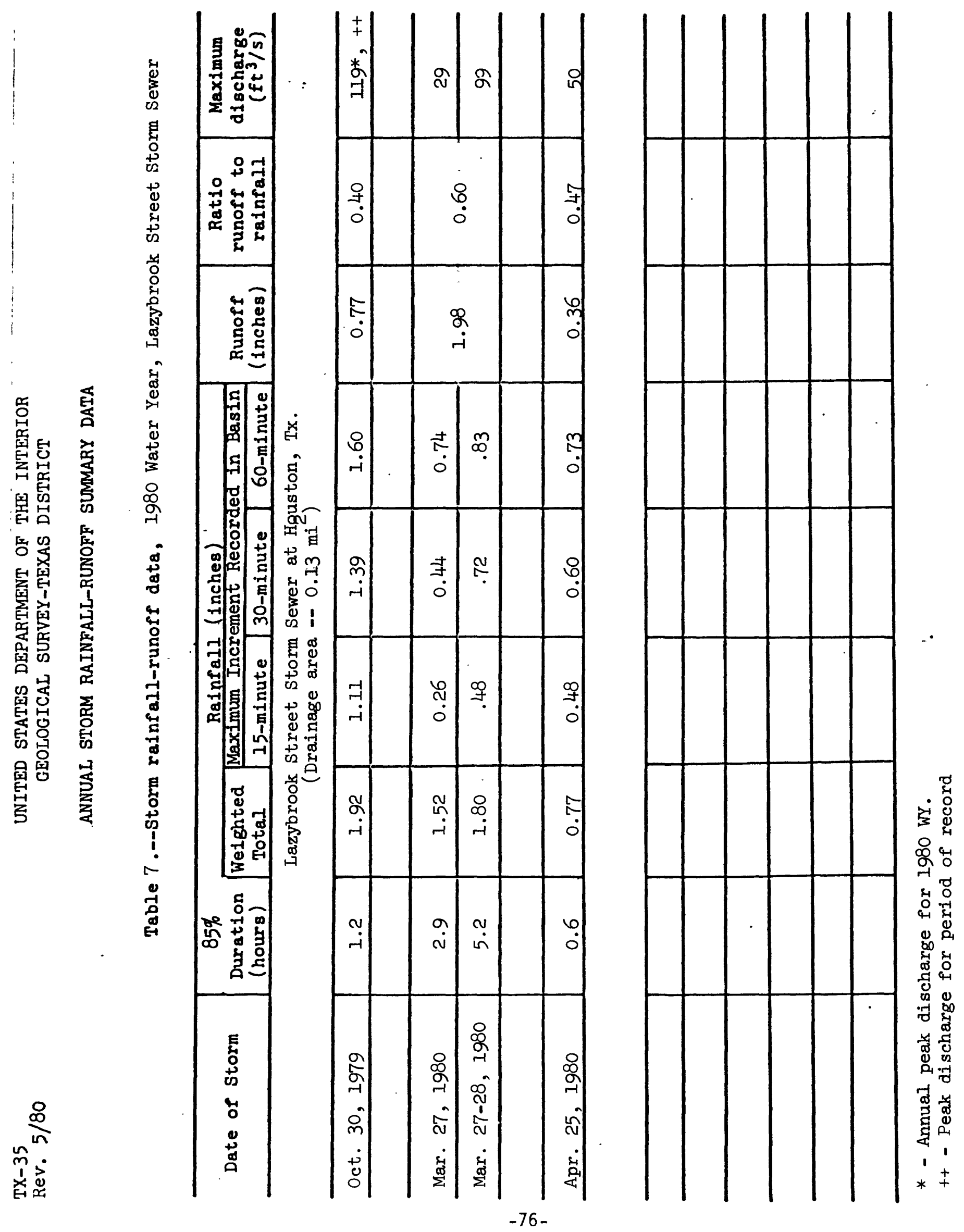




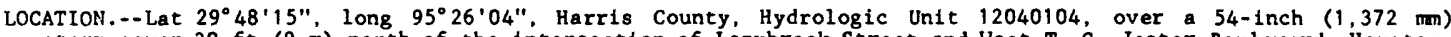
storm sewer $30 \mathrm{ft}(9 \mathrm{~m})$ north of the intersection of Lazybrook Street and West $T$. C. Jester Boulevard, Houston. DRAINAGE AREA. $--0.13 \mathrm{mi}^{2}\left(0.34 \mathrm{~km}^{2}\right)$.

WATER-DISCHARGE RECORDS

PERIOD OF RECORD.--October 1978 to current year.

GAGE.--Flood-hydrograph and rainfall recorder. Datum of gage is $-0.10 \mathrm{ft}(0.030 \mathrm{~m})$ National Geodetic Vertical Datum of 1929, 1973 adjustment.

REMARKS.--Additional storm ralnfall-runoff data for this site can be obtained from the report "Hydrologic Data for Urban Studies in the Houston. Texas Metropolitan Area, 1980".

EXTREMES FOR PERIOD OF RECORD.--Maximum discharge, $119 \mathrm{ft}^{3} / \mathrm{s}\left(3.37 \mathrm{~m}^{3} / \mathrm{s}\right)$ Apr. 19, Aug. 19, and Oct. 30, 1979, gage height, $58.09 \mathrm{ft}(17.706 \mathrm{~m})$.

EXTREMES FOR CURRENT YEAR.--Peak discharges above base of $55 \mathrm{ft}^{3} / \mathrm{s}\left(1.56 \mathrm{~m}^{3} / \mathrm{s}\right.$ ) and maximum ( $*$ ):

\begin{tabular}{|c|c|c|c|c|c|c|c|c|c|c|}
\hline Date & Time & $\begin{array}{r}D 18 \mathrm{c} \\
(\mathrm{ft} / \mathrm{s})\end{array}$ & $\underset{\left(\mathrm{m}^{3} / \mathrm{s}\right)}{\mathrm{rge}}$ & $\begin{array}{l}\text { Gage } \\
(\mathrm{ft})\end{array}$ & $\begin{array}{r}\text { height } \\
\text { (m) }\end{array}$ & Date & Time & $\begin{array}{c}\text { Discharge } \\
\left(\mathrm{ft}^{3} / \mathrm{s}\right)\left(\mathrm{m}^{3} / \mathrm{s}\right)\end{array}$ & $\begin{array}{l}\text { Gage } \\
(\mathrm{ft})\end{array}$ & $\begin{array}{l}\text { hel ght } \\
\text { (m) }\end{array}$ \\
\hline $\begin{aligned} \text { Oct. } & 30 \\
\text { Jan. } & 22 \\
\text { aMar. } & 26\end{aligned}$ & $\begin{array}{l}1650 \\
0945 \\
0235\end{array}$ & $\begin{array}{r}* 119 \\
55 \\
.90\end{array}$ & $\begin{array}{l}3.37 \\
1.56 \\
.025\end{array}$ & $\begin{array}{l}58.09 \\
56.85 \\
54.43\end{array}$ & $\begin{array}{l}17.706 \\
17.328 \\
16.590\end{array}$ & $\begin{aligned} \text { Mar. } 27 \\
\text { aApr. } 25\end{aligned}$ & $\begin{array}{l}1540 \\
1100\end{array}$ & $\begin{array}{l}2.80 \\
1.42\end{array}$ & $\begin{array}{l}57.75 \\
56.74\end{array}$ & $\begin{array}{l}17.602 \\
17.294\end{array}$ \\
\hline
\end{tabular}

a Water-quality samples were obtained during this runoff event.

WATER-QUALITY RECORDS

PERIOD OF RECORD.--Chemical, biochemical, and pesticide analyses: March to September 1980.

WATER QUALITY DATA, WATER YEAR OCTOBER 1979 TO SEPTEMBER 1980

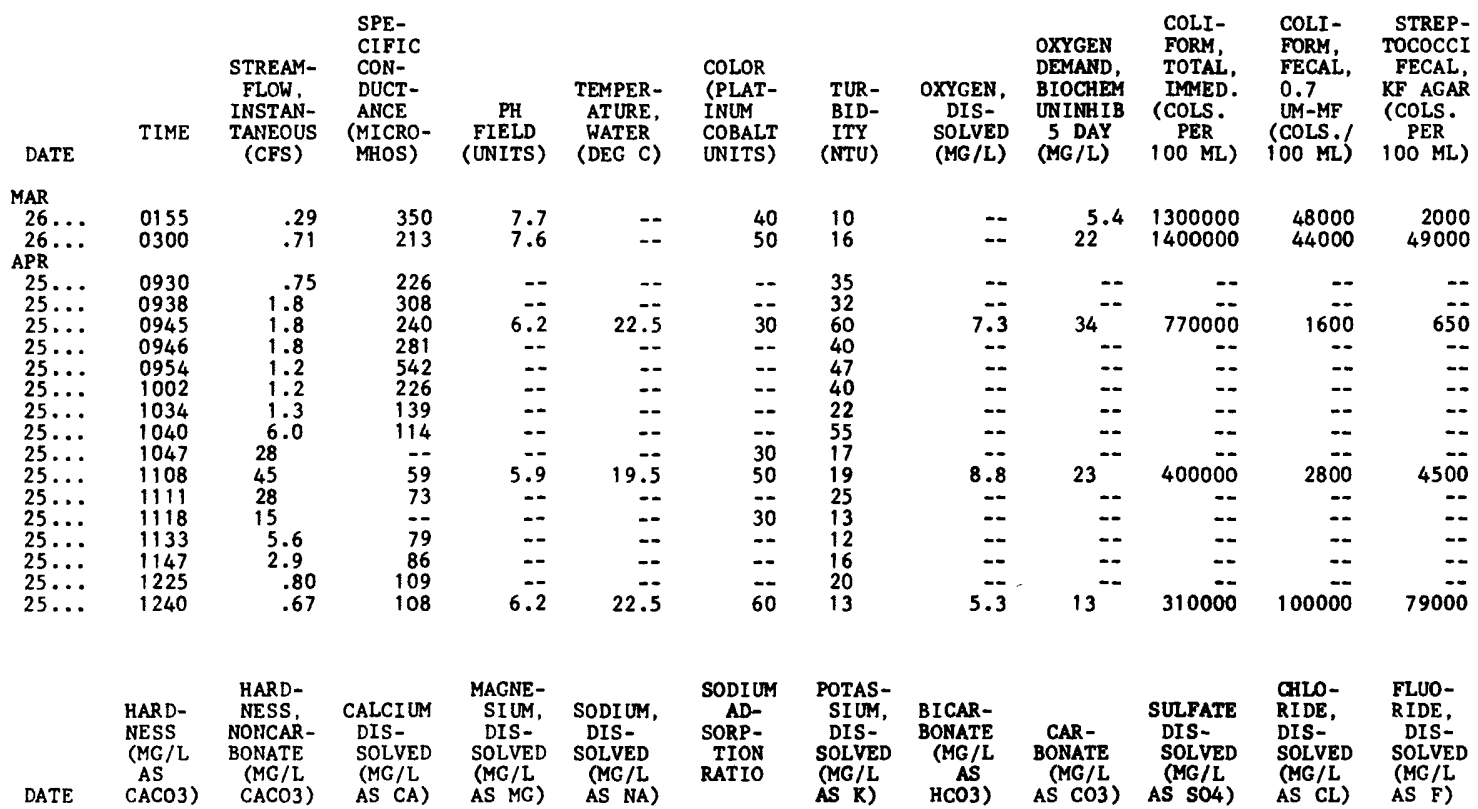

$\begin{array}{rrr}\text { MAR } & & \\ 26 \ldots & 76 & 0 \\ 26 \ldots & 42 & 2 \\ \text { APR } & & \\ 25 \ldots & -- & - \\ 25 \ldots & - & - \\ 25 \ldots & 47 & 6 \\ 25 \ldots & -- & -- \\ 25 \ldots & -- & -- \\ 25 \ldots & -- & -- \\ 25 \ldots & -- & -- \\ 25 \ldots & -- & - \\ 25 \ldots & -- & - \\ 25 \ldots & 12 & 1 \\ 25 \ldots & -- & -- \\ 25 \ldots & -- & -- \\ 25 \ldots & -- & -- \\ 25 \ldots & -- & -- \\ 25 \ldots & -- & -- \\ 25 \ldots & 27 & 2\end{array}$

\begin{tabular}{rrrr}
26 & 2.8 & 40 & 2.0 \\
15 & 1.2 & 23 & 1.5 \\
-- & -- & -- & -- \\
16 & -- & -- & $-\overline{-}$ \\
-- & 1.6 & 21 & 1.3 \\
-- & -- & -- & -- \\
-- & -- & -- & -- \\
-- & -- & -- & -- \\
-- & -- & -- & -- \\
4.4 & -3 & 2.4 & - \\
-- & -- & -- & -- \\
-- & -- & -- & -- \\
-- & -- & -- & -- \\
-- & -- & -- & -- \\
\hline- & -- & -- & - \\
8.9 & 1.1 & 6.6 & .6
\end{tabular}

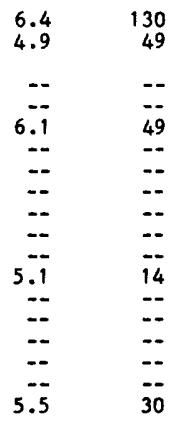

\begin{tabular}{c}
0 \\
0 \\
-- \\
- \\
\hline 0 \\
-- \\
-- \\
-- \\
-- \\
\hline 0 \\
-- \\
-- \\
-- \\
- \\
0
\end{tabular}

19
20
--
27
--
--
-
-
7.7
--
--
--
13

27
22

.3
.2 
SAN JACINTO RIVER BASIN

08074400 LAZYBROOK STREET STORM SEWER AT HOUSTON, TX--Continued

WATER QUALITY DATA, WATER YEAR OCTOBER 1979 TO SEPTEMBER 1980

\begin{tabular}{|c|c|c|c|c|c|c|c|c|c|c|c|c|}
\hline DATE & $\begin{array}{l}\text { SILICA, } \\
\text { DIS- } \\
\text { SOLVED } \\
\text { (MG /L } \\
\text { AS } \\
\text { SIO2) }\end{array}$ & $\begin{array}{l}\text { SOLIDS, } \\
\text { SUM OF } \\
\text { CONSTI- } \\
\text { TUENTS, } \\
\text { DIS- } \\
\text { SOLVED } \\
\text { (MG /L) }\end{array}$ & $\begin{array}{l}\text { SOLIDS, } \\
\text { RESIDUE } \\
\text { AT 105 } \\
\text { DEG. C. } \\
\text { SUS- } \\
\text { PENDED } \\
\text { (MG/L) }\end{array}$ & $\begin{array}{l}\text { SOLIDS, } \\
\text { VOLA- } \\
\text { TILE, } \\
\text { SUS- } \\
\text { PENDED } \\
(M C / L)\end{array}$ & $\begin{array}{l}\text { NITRO- } \\
\text { GEN, } \\
\text { IITRATE } \\
\text { TOTAL } \\
\text { (MG/L } \\
\text { AS N) }\end{array}$ & $\begin{array}{l}\text { NITRO- } \\
\text { GEN, } \\
\text { NITRITE } \\
\text { TOTAL } \\
\text { (MG /L } \\
\text { AS N) }\end{array}$ & $\begin{array}{l}\text { NITRO- } \\
\text { GEN, } \\
\text { NO2+NO3 } \\
\text { TOTAL } \\
\text { (MC/L } \\
\text { AS N) }\end{array}$ & $\begin{array}{l}\text { NITRO- } \\
\text { GEN, } \\
\text { AMMONIA } \\
\text { TOTAL } \\
\text { (MG/L } \\
\text { AS N) }\end{array}$ & $\begin{array}{l}\text { NITRO- } \\
\text { GEN, } \\
\text { ORGANIC } \\
\text { TOTAL } \\
\text { (MC/L } \\
\text { AS II) }\end{array}$ & $\begin{array}{l}\text { NITRO- } \\
\text { GEN, AM- } \\
\text { MONIA + } \\
\text { ORGANIC } \\
\text { TOTAL } \\
\text { (MG/L } \\
\text { AS N) }\end{array}$ & $\begin{array}{l}\text { PHOS- } \\
\text { PHORUS, } \\
\text { TOTAL } \\
\text { (MG/L } \\
\text { AS P) }\end{array}$ & $\begin{array}{l}\text { CARBON, } \\
\text { ORGANIC } \\
\text { TOTAL } \\
\text { (MG/L } \\
\text { AS C) }\end{array}$ \\
\hline $\begin{array}{l}\text { MAR } \\
26 \ldots \\
26 \ldots \\
\text { APR }\end{array}$ & $\begin{array}{l}13 \\
4.9\end{array}$ & $\begin{array}{l}199 \\
115\end{array}$ & $\begin{array}{r}5 \\
35\end{array}$ & $\begin{array}{r}5 \\
19\end{array}$ & $1.1^{.39}$ & $\begin{array}{l}.060 \\
.130\end{array}$ & $=$ & $\begin{array}{r}1.000 \\
.940\end{array}$ & $\because$ & $=$ & $\begin{array}{r}1.100 \\
.890\end{array}$ & $\begin{array}{l}13 \\
18\end{array}$ \\
\hline $\begin{array}{l}25 \ldots \\
25 \ldots\end{array}$ & 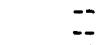 & $=$ & $\overline{-}$ & $=$ & $\because$ & $=$ & $=$ & $=$ & $\because$ & $=$ & $=$ & $=$ \\
\hline & 3.3 & 122 & 218 & 34 & 1.3 & .090 & 1.4 & 2.700 & 7.3 & 10 & 2.100 & 47 \\
\hline $25 \ldots$ & 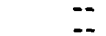 & $=$ & $=$ & 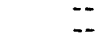 & 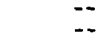 & $=$ & $=-$ & $\because$ & $=$ & $\because$ & $\because$ & $\because$ \\
\hline & - & - & -- & - & $\therefore$ & - & - & $\cdots$ & - & $=$ & $=$ & - \\
\hline & -- & -- & -- & $-\cdot$ & - & -- & -- & - & -- & $\ldots$ & -- & - \\
\hline & -. & -- & $=-$ & $\because-$ & $=$ & - & $=$ & $=$ & - & - & & - \\
\hline & $\overline{1.2}$ & $\overline{3 i}$ & $\begin{array}{r}492 \\
42\end{array}$ & $\begin{array}{r}132 \\
9\end{array}$ & $\begin{array}{l}.53 \\
.35\end{array}$ & $\begin{array}{l}.030 \\
.010\end{array}$ & $\begin{array}{r}.56 \\
.36\end{array}$ & $\begin{array}{r}.990 \\
.730\end{array}$ & $\begin{array}{l}3.1 \\
2.3\end{array}$ & $\begin{array}{l}4.1 \\
3.0\end{array}$ & $\begin{array}{l}.710 \\
.890\end{array}$ & $\begin{array}{l}27 \\
13\end{array}$ \\
\hline & & 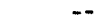 & -- & -- & - & $=$ & $=$ & & & $=-$ & -- & \\
\hline & -- & -- & 40 & 24 & .63 & .020 & .65 & 1.200 & 1.2 & 2.4 & .910 & 11 \\
\hline & 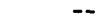 & -- & -- & $\cdots$ & -- & -- & $\cdots$ & $-\cdots$ & - & -- & -- & - \\
\hline & 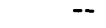 & -- & -- & $\cdots$ & -- & -- & -- & -- & -- & - & -- & -- \\
\hline & $\ddot{2}$ & $=-$ & $\overline{2}$ & $\ddot{10}$ & , & $\bar{s}$ & 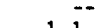 & $\overline{0}$ & $=-$ & $\overline{0}$ & $s=$ & $0^{--}$ \\
\hline & 3.3 & 59 & 22 & 19 & 1.1 & .050 & 1.1 & .980 & 2.0 & 3.0 & 1.500 & 18 \\
\hline
\end{tabular}

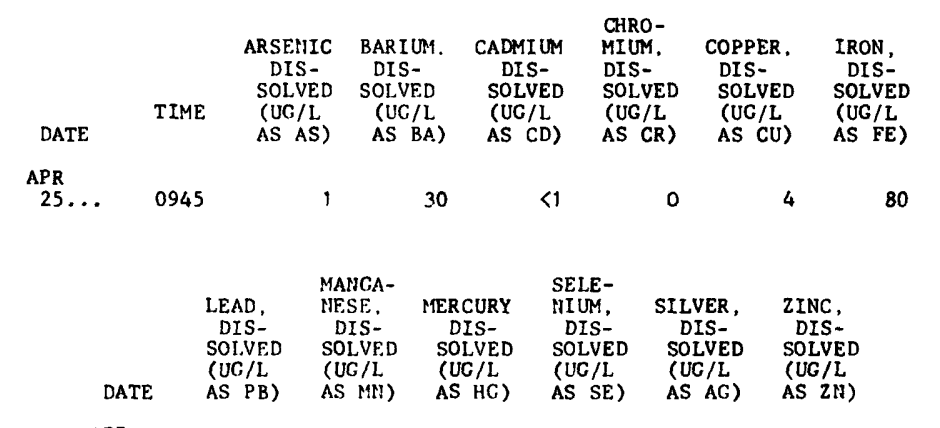

$\begin{array}{rrrrrrr}\text { APR } & & & & & & \\ 25 \ldots & 25 & 30 & .2 & 1 & 0 & 50\end{array}$ 


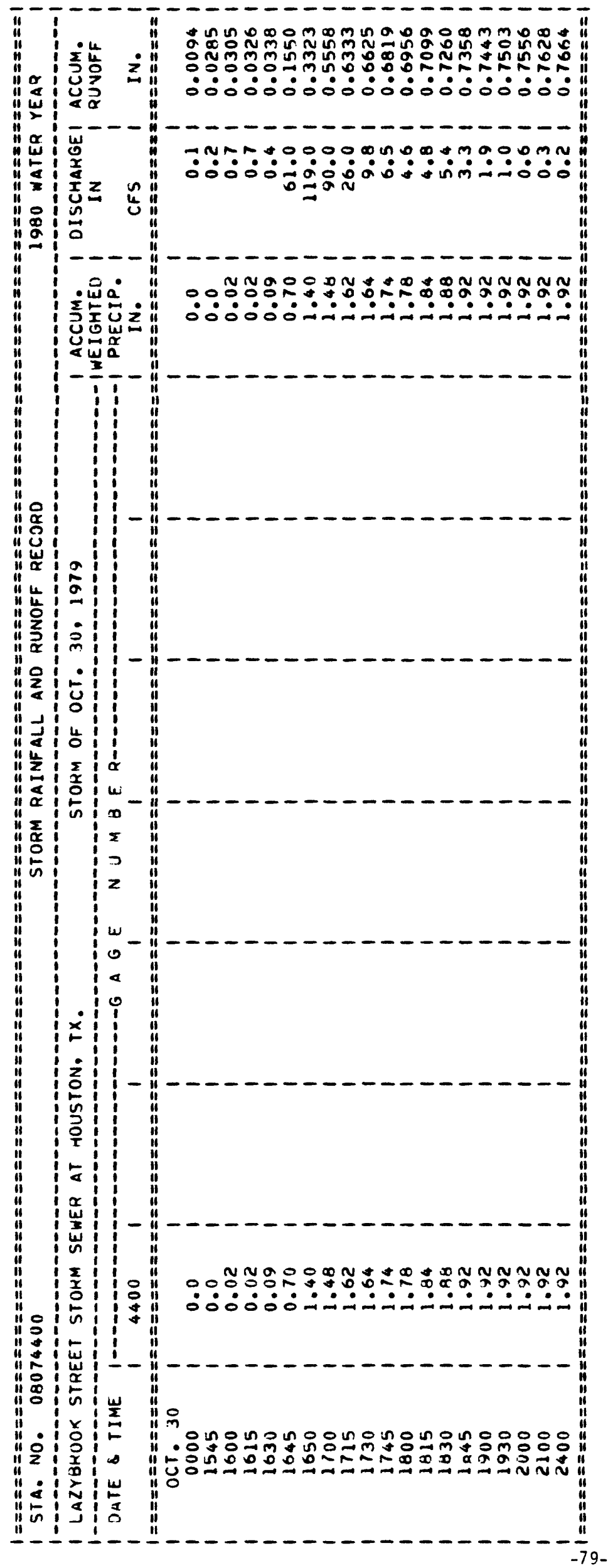




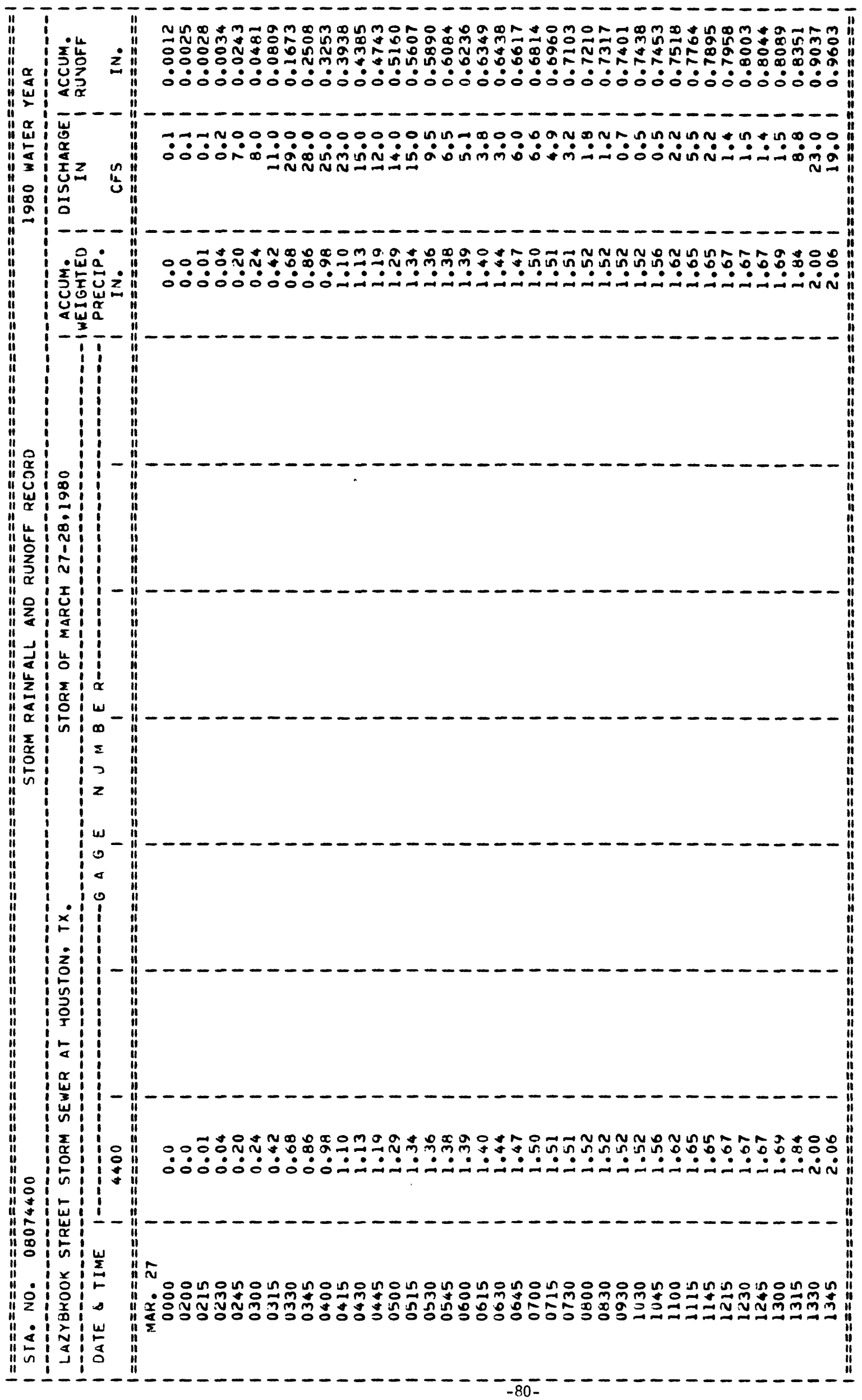




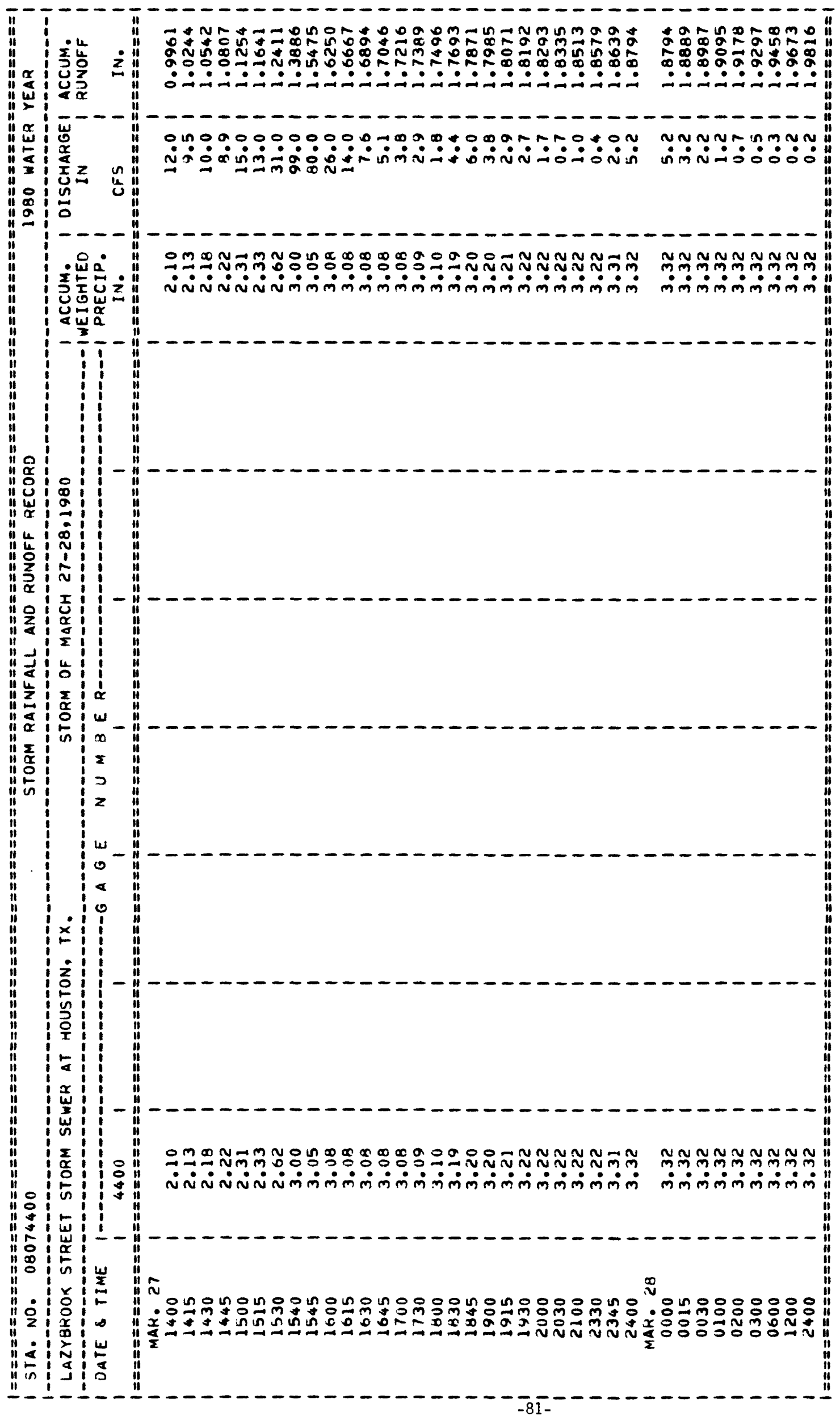




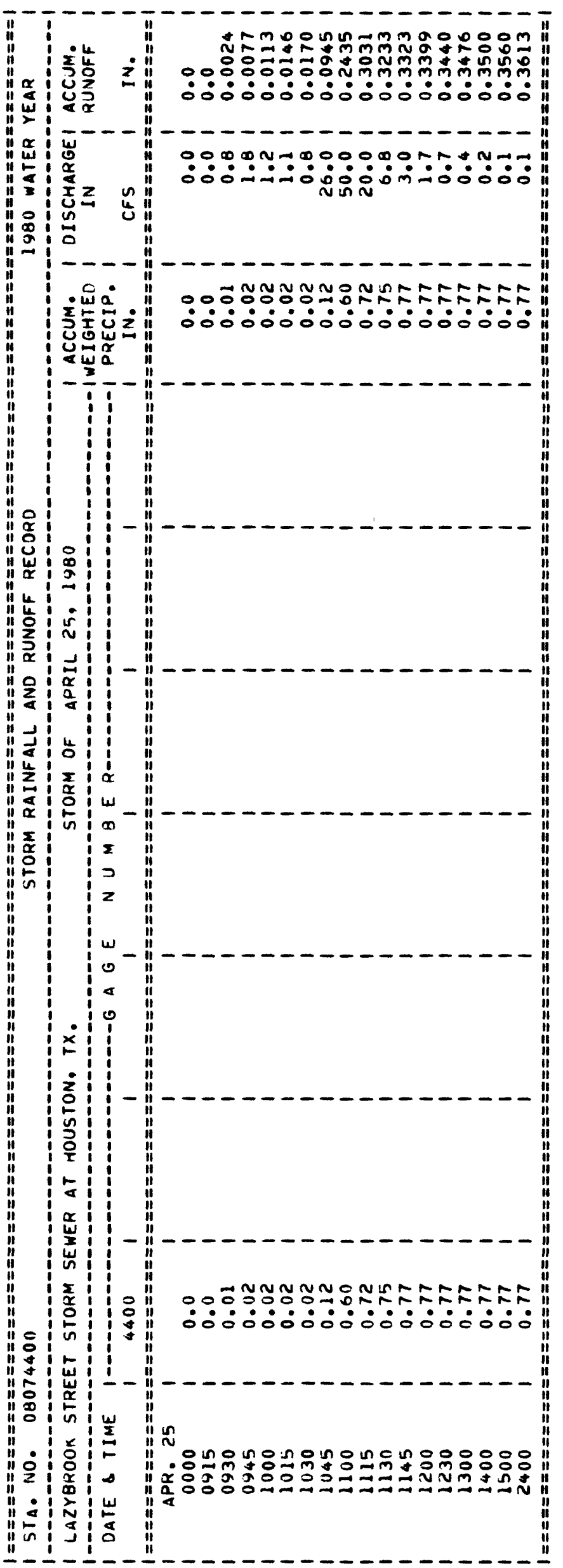




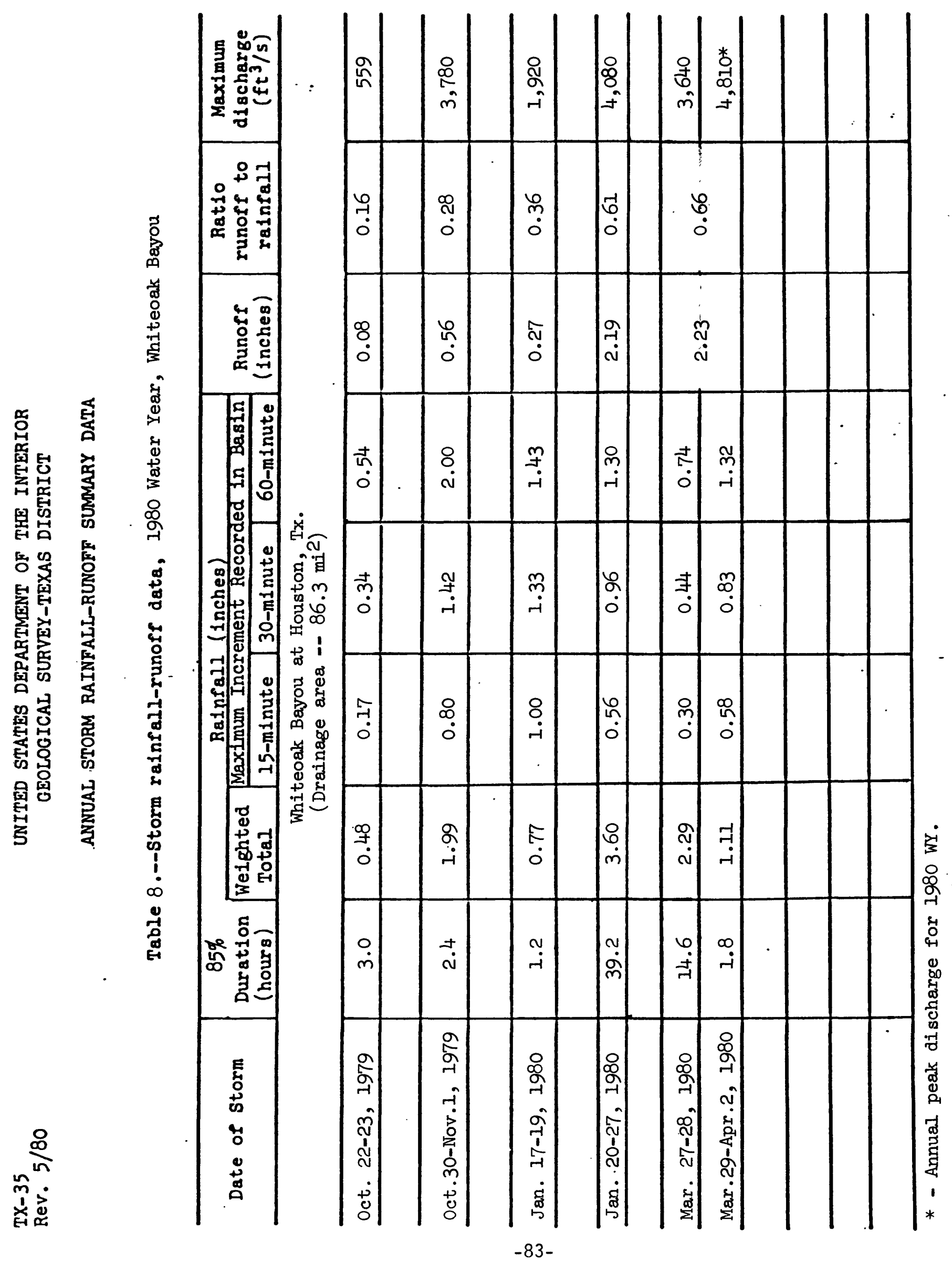


SAN JACINTO RIVER BASIN

08074500 WHITEOAK BAYOU AT HOUSTON, TX

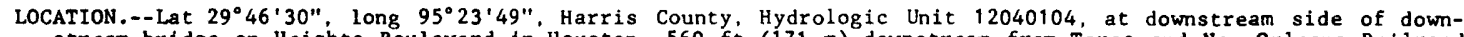
stream bridge on Heights Boulevard in Houston, $560 \mathrm{ft}(171 \mathrm{~m})$ downstream from Texas and New Orleans Railroad
Co. bridge, $2.4 \mathrm{mi}(3.9 \mathrm{~km})$ upstream from Little whiteoak Bayou, and $4.0 \mathrm{mi}$ (6.4 km) upstream from mouth.

DRAINAGE AREA.--86.3 $\mathrm{mi}^{2}\left(223.5 \mathrm{~km}^{2}\right)$. Prior to Oct. $1,1976,84.7 \mathrm{mi}^{2}\left(219.4 \mathrm{~km}^{2}\right)$.

WATER-DISCHARGE RECORDS

PERIOD OF RECORD.--May 1936 to current year (October 1965 to September 1966, monthly discharge only).

REVISED RECORDS.--WSP 1732: Drainage area.

GAGE.-Water-stage recorder and crest-stage gage. Datum of gage is $7.35 \mathrm{ft}(2.240 \mathrm{~m})$ below National Geodetic Vertical Datum of 1929; unadjusted for land-surface subsidence. Prior to June 17, 1936, nonrecording gage, and June 17, 1936, to Apr. 28, 1965, water-stage recorder at site $480 \mathrm{ft}$ (146 m) upstream at same datum. REMARKS.--Water-discharge records fair. Low flow is partly sustained by industrial waste. No diversion above
station

AVERAGE DISCHARGE. --44 years, $79.2 \mathrm{ft}^{3} / \mathrm{s}\left(2.243 \mathrm{~m}^{3} / \mathrm{s}\right), 57,380$ acre- $\mathrm{ft} / \mathrm{yr}(70.7 \mathrm{hm} / \mathrm{yr})$. EXTREMES FOR PERIOD OF RECORD.--Maximum discharge, $17,300 \mathrm{ft}^{3} / \mathrm{s}\left(490 \mathrm{~m}^{3} / \mathrm{s}\right)$ Mar. 20,1972 , gage height, 43.50
$\mathrm{ft}(13.259 \mathrm{~m}) ;$ maximum gage height, $43.60 \mathrm{ft}(13.289 \mathrm{~m})$ Nov. 13, 1961; no flow for many days during 1965 water year (result of construction dams).

EXTREMES OUTSIDE PERIOD OF RECORD.--Maximum stage since at least 1919, $51.5 \mathrm{ft}$ (15.70 m) Dec. 9, 1935, prior to channel rectification, present site and datum, discharge $14,750 \mathrm{ft} 3 / \mathrm{s}\left(418 \mathrm{~m}^{3} / \mathrm{s}\right)$, furnished by the engineer for Harris County. The flood of May 31, 1929, reached a stage of $47.0+0.5 \mathrm{ft}(14.33+0.15 \mathrm{~m})$, prior to channel rectification, present site and datum, discharge $9,360 \mathrm{ft}^{3} / \mathrm{s}\left(265 \mathrm{~m}^{3} / \mathrm{s}\right)$, computed on basis of currentmeter measurement at stage $1.0 \mathrm{ft}(0.30 \mathrm{~m})$ below crest, furnished by city of Houston.

EXTREMES FOR CURRENT YEAR.-. Peak discharges above base of $4,000 \mathrm{ft}^{3} / \mathrm{s}\left(113 \mathrm{~m}^{3} / \mathrm{s}\right)$ and maximum (*):

\begin{tabular}{|c|c|c|c|c|c|c|c|c|c|c|c|}
\hline Date & Time & $\begin{array}{r}\text { Disc } \\
\left(\mathrm{ft}^{3} / \mathrm{s}\right)\end{array}$ & $\underset{\left(\mathrm{m}^{3} / \mathrm{s}\right)}{\operatorname{arge}}$ & $\begin{array}{l}\text { Gage } \\
(\mathrm{ft})\end{array}$ & $\begin{array}{l}\text { eight } \\
\text { (m) }\end{array}$ & Date & Time & $\begin{array}{r}\text { Disc } \\
\left(\mathrm{ft}^{3} / \mathrm{s}\right)\end{array}$ & $\stackrel{\operatorname{arge}}{\left(\mathrm{m}^{3} / \mathrm{s}\right)}$ & $\begin{array}{l}\text { Gage } \\
(\mathrm{ft})\end{array}$ & $\begin{array}{l}\text { eight } \\
\text { (m) }\end{array}$ \\
\hline $\begin{array}{l}0 \mathrm{ct} .22 \\
\text { Jan. } 17 \\
\text { Jan. } 22\end{array}$ & $\begin{array}{l}0845 \\
1600 \\
1230\end{array}$ & $\begin{array}{r}559 \\
1,920 \\
4,080\end{array}$ & $\begin{array}{r}15.8 \\
54.4 \\
116\end{array}$ & $\begin{array}{l}19.10 \\
22.68 \\
26.73\end{array}$ & $\begin{array}{l}5.822 \\
6.913 \\
8.147\end{array}$ & $\begin{array}{c}\text { Mar. } 29 \\
\text { aMay } 19 \\
\text { aSept. } 6\end{array}$ & $\begin{array}{l}1300 \\
0700 \\
1400\end{array}$ & $\begin{array}{r}* 4,810 \\
1,350 \\
2,210\end{array}$ & $\begin{array}{r}136 \\
38.2 \\
62.6\end{array}$ & $\begin{array}{l}27.91 \\
21.36 \\
23.29\end{array}$ & $\begin{array}{l}8.507 \\
6.511 \\
7.099\end{array}$ \\
\hline
\end{tabular}

a Water-quality samples were obtained during this runoff event.

Minimum daily discharge, $20 \mathrm{ft}^{3} / \mathrm{s}\left(0.57 \mathrm{~m}^{3} / \mathrm{s}\right) 0 \mathrm{ct} .13$.

DISCHARGE, IN CUBIC FEET PER SECOND, WATER YEAR OCTOBER 1979 TO SEPTEMBER 1980

\begin{tabular}{|c|c|c|c|c|c|c|c|c|c|c|c|c|}
\hline DAY & $\mathrm{OCT}$ & Nov & DEC & JAN & FEB & MAR & APR & MAY & JUN & JUL & AUG & SEP \\
\hline $\begin{array}{l}1 \\
2 \\
3 \\
4 \\
5\end{array}$ & $\begin{array}{l}31 \\
29 \\
29 \\
30 \\
24\end{array}$ & $\begin{array}{l}98 \\
40 \\
28 \\
25 \\
28\end{array}$ & $\begin{array}{l}35 \\
36 \\
32 \\
26 \\
25\end{array}$ & $\begin{array}{r}32 \\
30 \\
300 \\
35 \\
32\end{array}$ & $\begin{array}{l}41 \\
56 \\
44 \\
36 \\
35\end{array}$ & $\begin{array}{r}144 \\
38 \\
34 \\
42 \\
35\end{array}$ & $\begin{array}{r}115 \\
76 \\
60 \\
47 \\
44\end{array}$ & $\begin{array}{r}80 \\
102 \\
40 \\
34 \\
34\end{array}$ & $\begin{array}{l}31 \\
28 \\
29 \\
28 \\
28\end{array}$ & $\begin{array}{l}33 \\
29 \\
27 \\
26 \\
26\end{array}$ & $\begin{array}{l}23 \\
21 \\
21 \\
24 \\
86\end{array}$ & $\begin{array}{r}26 \\
33 \\
26 \\
25 \\
219\end{array}$ \\
\hline $\begin{array}{r}6 \\
7 \\
8 \\
9 \\
10\end{array}$ & $\begin{array}{l}30 \\
46 \\
37 \\
32 \\
27\end{array}$ & $\begin{array}{l}25 \\
26 \\
30 \\
45 \\
27\end{array}$ & $\begin{array}{l}26 \\
31 \\
26 \\
28 \\
27\end{array}$ & $\begin{array}{l}30 \\
40 \\
35 \\
32 \\
30\end{array}$ & $\begin{array}{r}37 \\
32 \\
594 \\
772 \\
236\end{array}$ & $\begin{array}{l}32 \\
34 \\
36 \\
32 \\
29\end{array}$ & $\begin{array}{l}57 \\
50 \\
38 \\
34 \\
32\end{array}$ & $\begin{array}{l}34 \\
90 \\
64 \\
51 \\
34\end{array}$ & $\begin{array}{r}30 \\
29 \\
31 \\
419 \\
212\end{array}$ & $\begin{array}{l}25 \\
26 \\
36 \\
47 \\
37\end{array}$ & $\begin{array}{l}35 \\
45 \\
33 \\
26 \\
24\end{array}$ & $\begin{array}{r}1220 \\
722 \\
185 \\
88 \\
42\end{array}$ \\
\hline $\begin{array}{l}11 \\
12 \\
13 \\
14 \\
15\end{array}$ & $\begin{array}{l}26 \\
26 \\
20 \\
21 \\
27\end{array}$ & $\begin{array}{l}27 \\
26 \\
26 \\
23 \\
24\end{array}$ & $\begin{array}{r}28 \\
929 \\
403 \\
99 \\
56\end{array}$ & $\begin{array}{l}30 \\
30 \\
30 \\
30 \\
30\end{array}$ & $\begin{array}{r}138 \\
113 \\
65 \\
110 \\
93\end{array}$ & $\begin{array}{l}32 \\
36 \\
30 \\
30 \\
37\end{array}$ & $\begin{array}{r}31 \\
34 \\
151 \\
40 \\
33\end{array}$ & $\begin{array}{r}31 \\
31 \\
61 \\
330 \\
93\end{array}$ & $\begin{array}{l}48 \\
37 \\
32 \\
29 \\
28\end{array}$ & $\begin{array}{l}39 \\
27 \\
27 \\
28 \\
28\end{array}$ & $\begin{array}{r}22 \\
47 \\
26 \\
23 \\
109\end{array}$ & $\begin{array}{l}35 \\
32 \\
33 \\
31 \\
29\end{array}$ \\
\hline $\begin{array}{l}16 \\
17 \\
18 \\
19 \\
20\end{array}$ & $\begin{array}{l}52 \\
27 \\
29 \\
29 \\
24\end{array}$ & $\begin{array}{l}26 \\
30 \\
34 \\
38 \\
40\end{array}$ & $\begin{array}{l}38 \\
41 \\
32 \\
30 \\
27\end{array}$ & $\begin{array}{r}30 \\
474 \\
138 \\
41 \\
403\end{array}$ & $\begin{array}{r}175 \\
102 \\
56 \\
47 \\
44\end{array}$ & $\begin{array}{r}44 \\
127 \\
35 \\
37 \\
137\end{array}$ & $\begin{array}{l}31 \\
30 \\
30 \\
30 \\
30\end{array}$ & $\begin{array}{l}467 \\
292 \\
107 \\
706 \\
240\end{array}$ & $\begin{array}{l}28 \\
31 \\
30 \\
29 \\
35\end{array}$ & $\begin{array}{l}26 \\
26 \\
25 \\
25 \\
37\end{array}$ & $\begin{array}{r}112 \\
28 \\
23 \\
24 \\
23\end{array}$ & $\begin{array}{l}25 \\
25 \\
25 \\
29 \\
26\end{array}$ \\
\hline $\begin{array}{l}21 \\
22 \\
23 \\
24 \\
25\end{array}$ & $\begin{array}{r}21 \\
145 \\
30 \\
27 \\
28\end{array}$ & $\begin{array}{r}421 \\
188 \\
119 \\
48 \\
30\end{array}$ & $\begin{array}{r}27 \\
34 \\
209 \\
103 \\
40\end{array}$ & $\begin{array}{r}1650 \\
1910 \\
544 \\
216 \\
149\end{array}$ & $\begin{array}{l}43 \\
44 \\
44 \\
46 \\
44\end{array}$ & $\begin{array}{l}57 \\
34 \\
41 \\
37 \\
29\end{array}$ & $\begin{array}{r}29 \\
31 \\
30 \\
30 \\
436\end{array}$ & $\begin{array}{l}92 \\
60 \\
44 \\
39 \\
35\end{array}$ & $\begin{array}{r}27 \\
238 \\
114 \\
29 \\
24\end{array}$ & $\begin{array}{r}150 \\
96 \\
29 \\
24 \\
23\end{array}$ & $\begin{array}{l}24 \\
22 \\
24 \\
29 \\
21\end{array}$ & $\begin{array}{l}24 \\
23 \\
21 \\
51 \\
25\end{array}$ \\
\hline $\begin{array}{l}26 \\
27 \\
28 \\
29 \\
30 \\
31\end{array}$ & $\begin{array}{r}29 \\
26 \\
22 \\
21 \\
734 \\
433\end{array}$ & $\begin{array}{r}31 \\
32 \\
29 \\
26 \\
27 \\
--\end{array}$ & $\begin{array}{r}35 \\
30 \\
30 \\
400 \\
50 \\
35\end{array}$ & $\begin{array}{r}102 \\
78 \\
60 \\
61 \\
52 \\
43\end{array}$ & $\begin{array}{r}38 \\
39 \\
40 \\
41 \\
- \\
-\end{array}$ & $\begin{array}{r}65 \\
1610 \\
1140 \\
1490 \\
497 \\
182\end{array}$ & $\begin{array}{r}94 \\
43 \\
37 \\
35 \\
34 \\
---\end{array}$ & $\begin{array}{r}36 \\
36 \\
40 \\
180 \\
63 \\
37\end{array}$ & $\begin{array}{r}24 \\
23 \\
25 \\
26 \\
28 \\
--\end{array}$ & $\begin{array}{r}23 \\
92 \\
179 \\
73 \\
30 \\
23\end{array}$ & $\begin{array}{l}23 \\
27 \\
25 \\
51 \\
87 \\
37\end{array}$ & $\begin{array}{r}47 \\
39 \\
27 \\
24 \\
376 \\
-\end{array}$ \\
\hline $\begin{array}{l}\text { TOTAL } \\
\text { MEAN } \\
\text { MAX } \\
\text { MIN } \\
\text { AC-FT } \\
(\dagger \dagger)\end{array}$ & $\begin{array}{r}2112 \\
68.1 \\
734 \\
20 \\
4190 \\
2.60\end{array}$ & $\begin{array}{r}1617 \\
53.9 \\
421 \\
23 \\
3210 \\
1.54\end{array}$ & $\begin{array}{r}2968 \\
95.7 \\
929 \\
25 \\
5890 \\
3.68\end{array}$ & $\begin{array}{r}6697 \\
216 \\
1910 \\
30 \\
13280 \\
5.08\end{array}$ & $\begin{array}{r}3205 \\
111 \\
772 \\
32 \\
6360 \\
2.23\end{array}$ & $\begin{array}{r}6183 \\
199 \\
1610 \\
29 \\
12260 \\
5.52\end{array}$ & $\begin{array}{r}1792 \\
59.7 \\
436 \\
29 \\
3550 \\
1.83\end{array}$ & $\begin{array}{r}3583 \\
116 \\
706 \\
31 \\
7110 \\
4.69\end{array}$ & $\begin{array}{r}1750 \\
58.3 \\
419 \\
23 \\
3470 \\
2.18\end{array}$ & $\begin{array}{r}1342 \\
43.3 \\
179 \\
23 \\
2660 \\
1.99\end{array}$ & $\begin{array}{r}1145 \\
36.9 \\
112 \\
21 \\
2270 \\
1.76\end{array}$ & $\begin{array}{r}3533 \\
118 \\
1220 \\
21 \\
7010 \\
5.96\end{array}$ \\
\hline
\end{tabular}

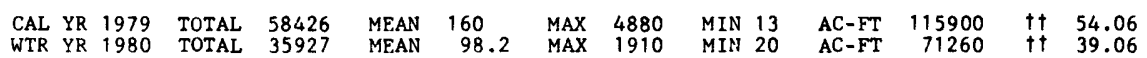

$\dagger^{\dagger}$ Weighted-mean rainfall in inches, based on thirteen rain gages. 


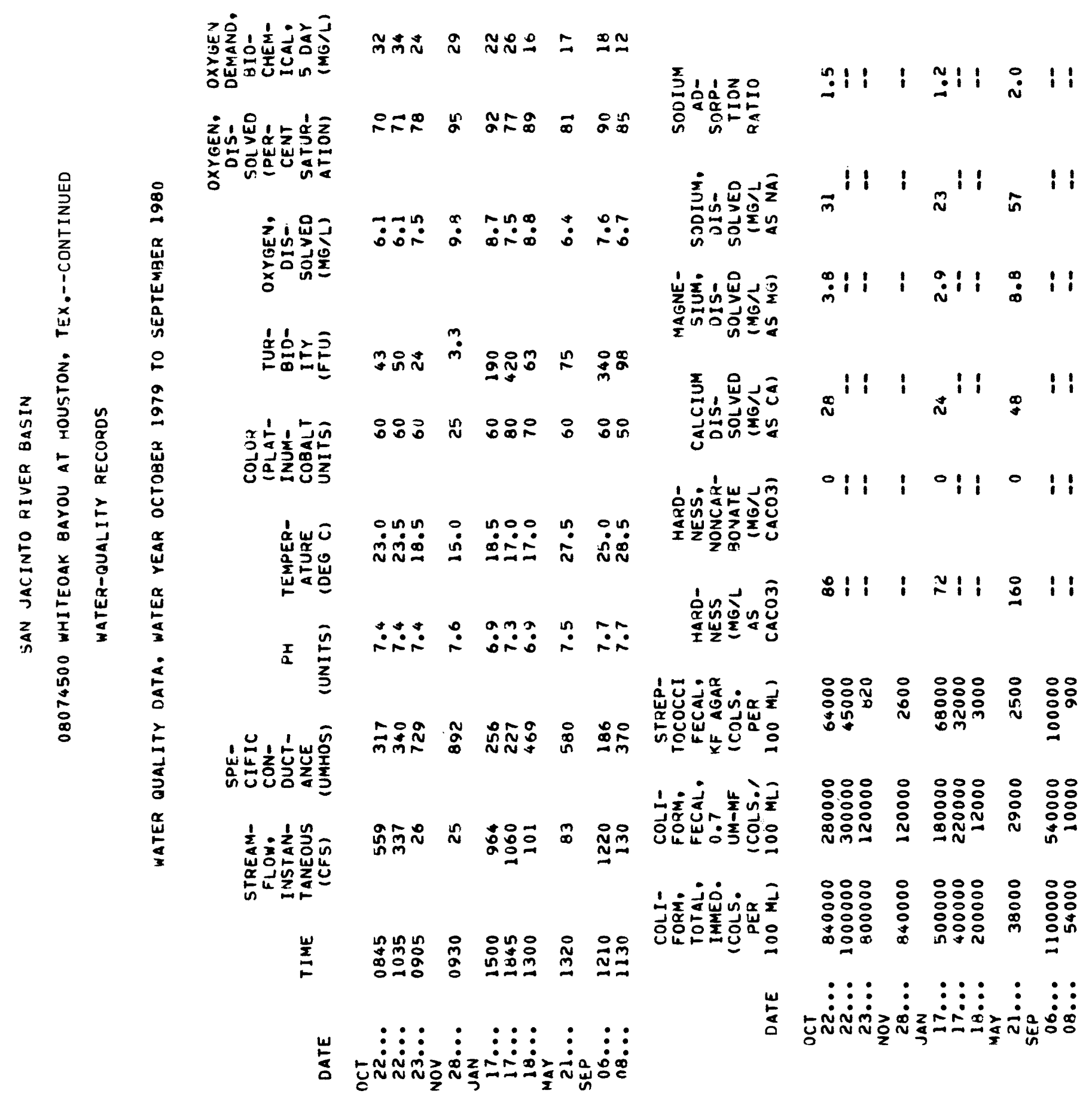




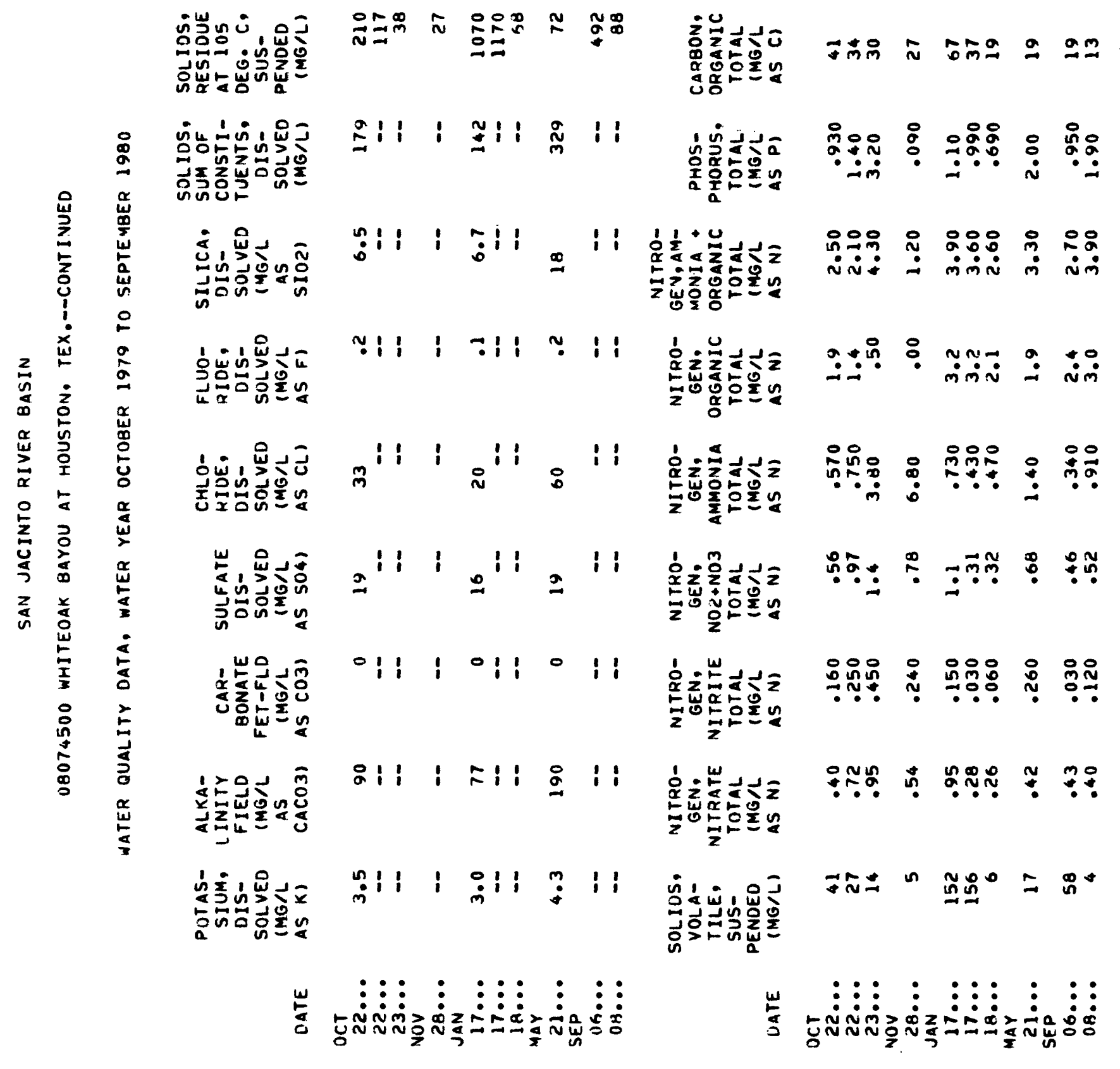




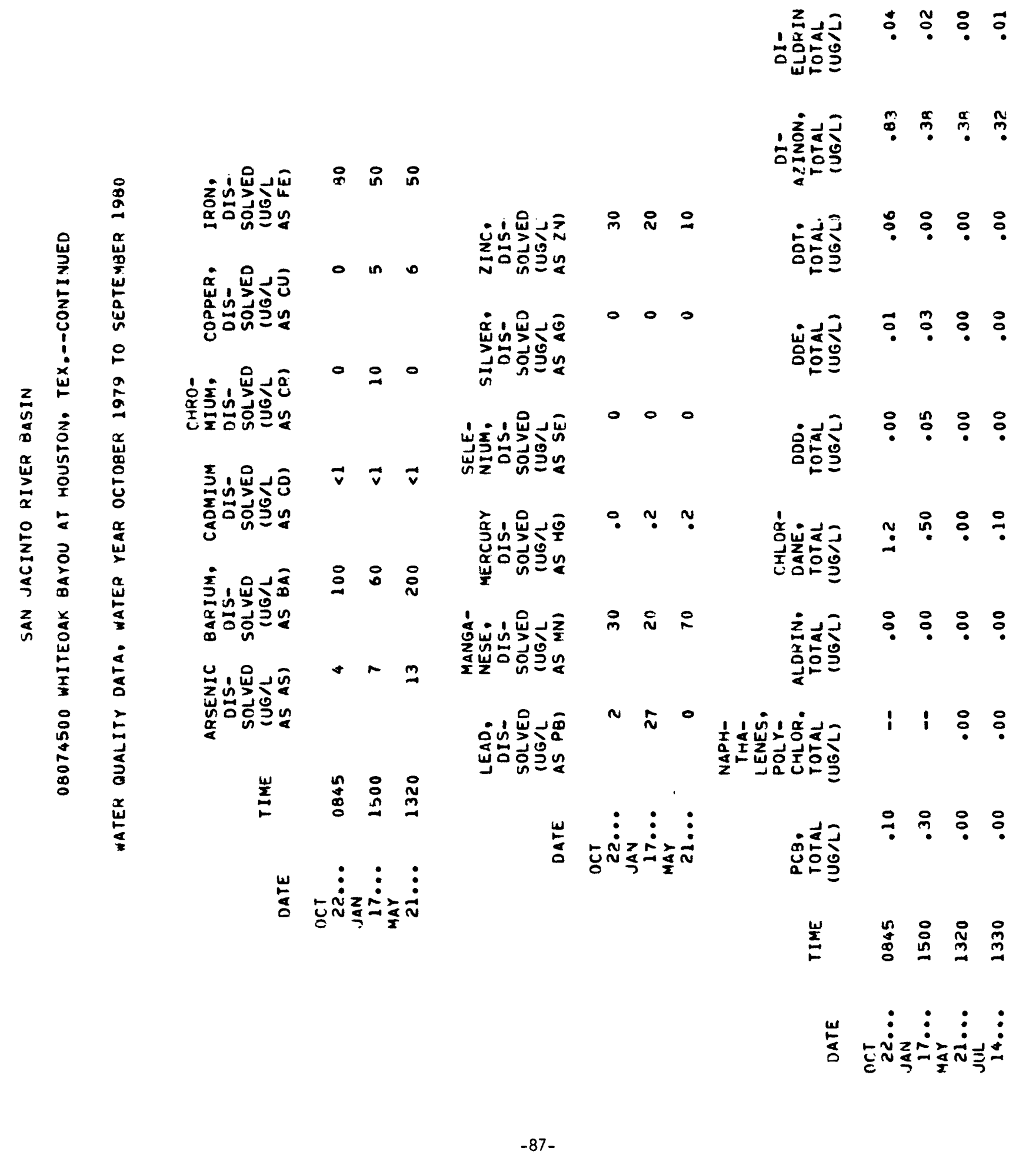




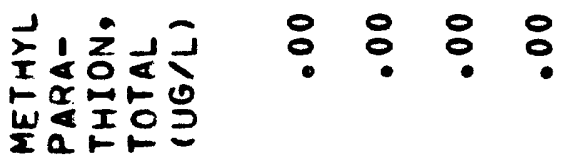

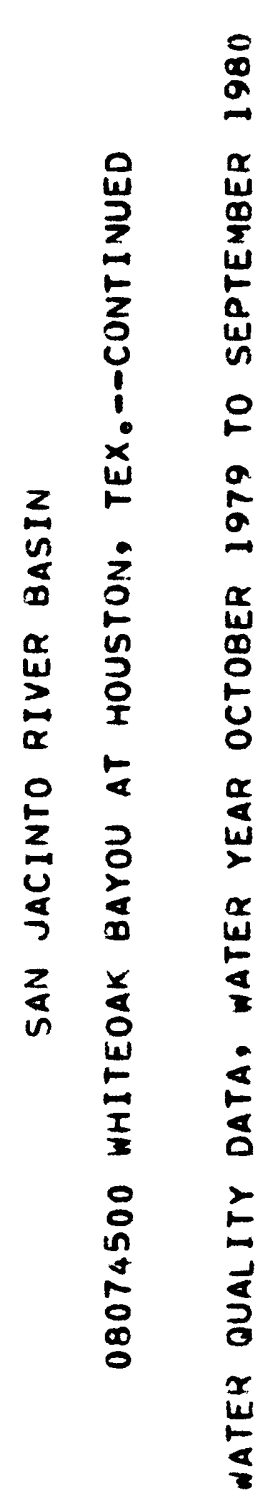

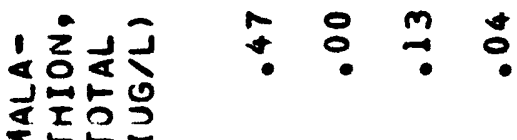

IF

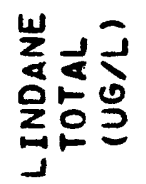

$\stackrel{0}{0} \stackrel{0}{0}$

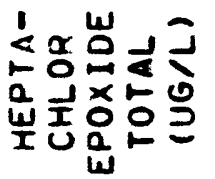

잉ㅇㅇㅇ

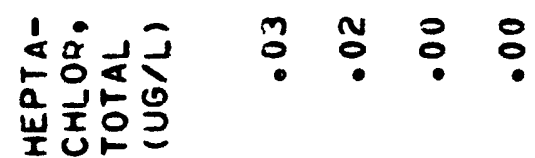

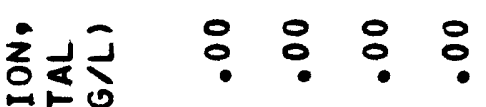

두의

w.

$\sum_{1} \leq j$
$x \leq 0$
$\sum_{u} \leq 0$

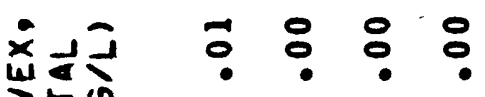
다의

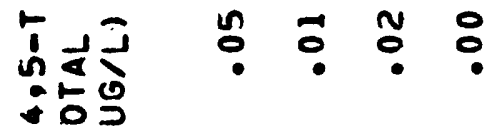

$\therefore$ 는

$0_{1}^{0}+3$

$-65$

NF-

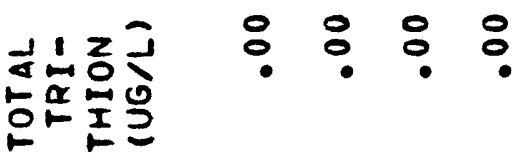

1 $0 \underline{0} \div 0$

- $\frac{1}{2} 02$

웅

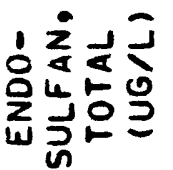

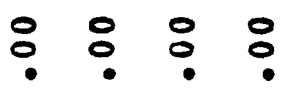

눙

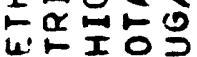

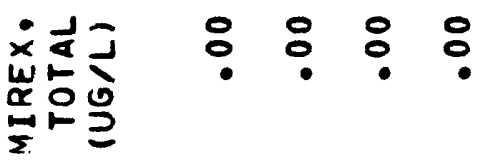

崖 


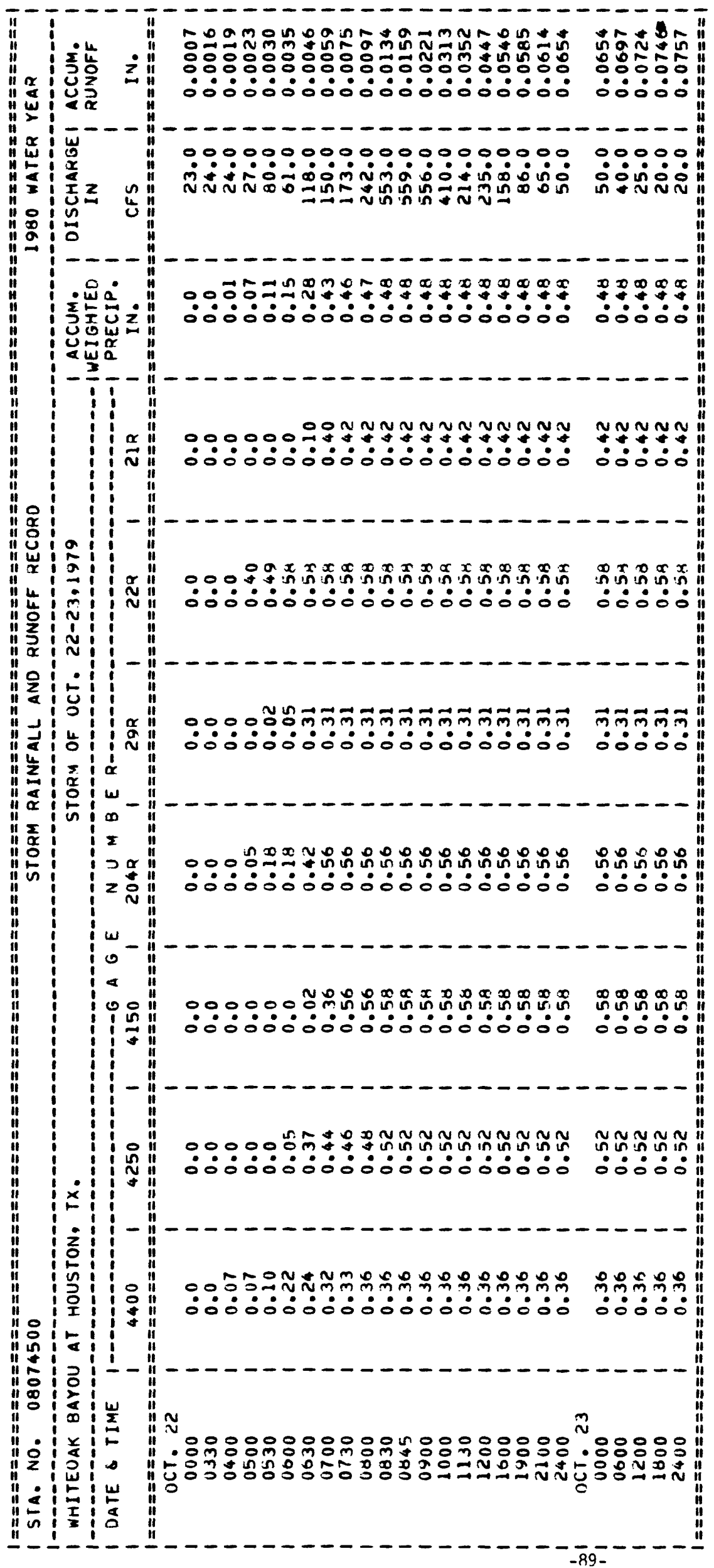




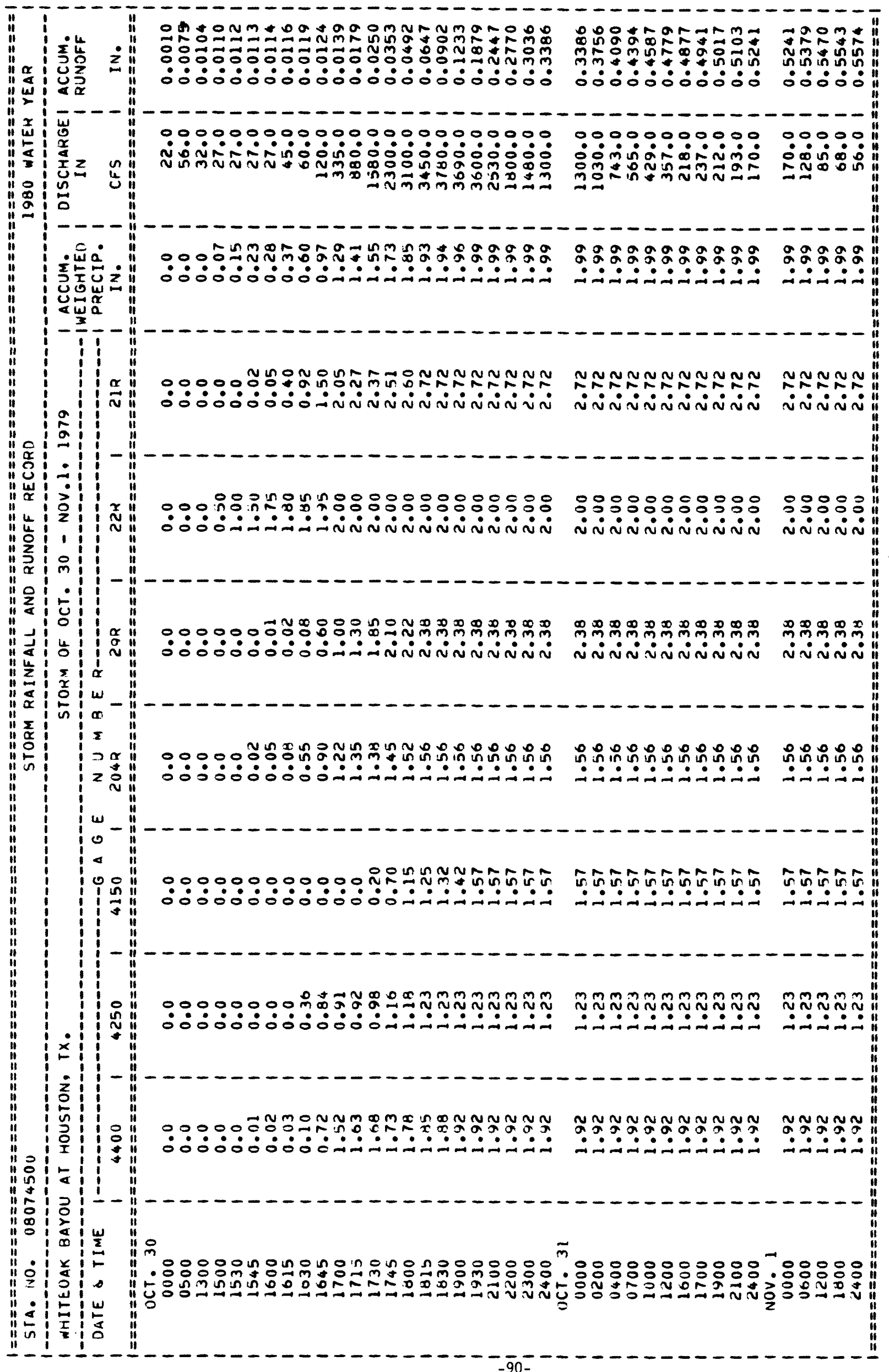




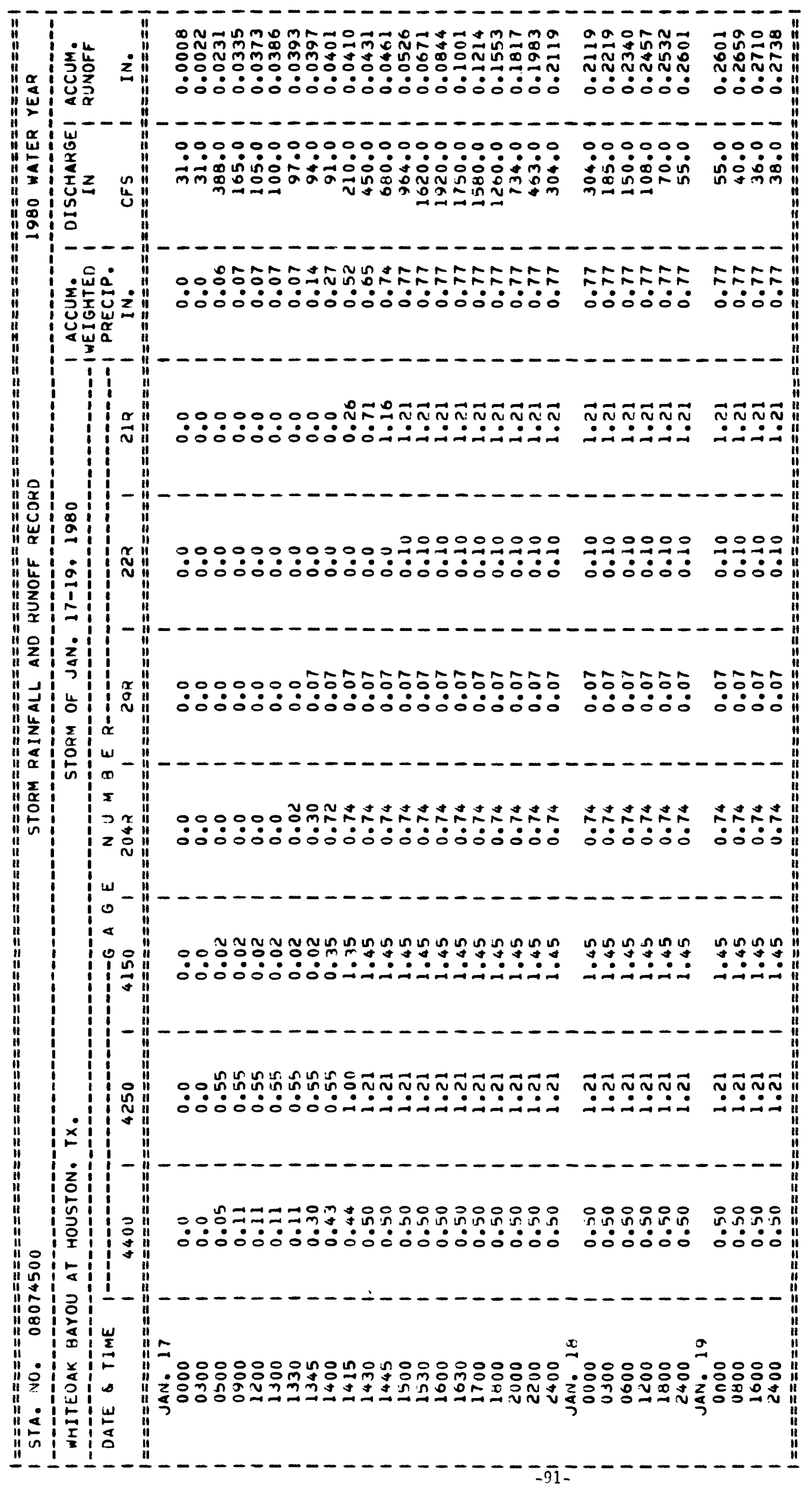




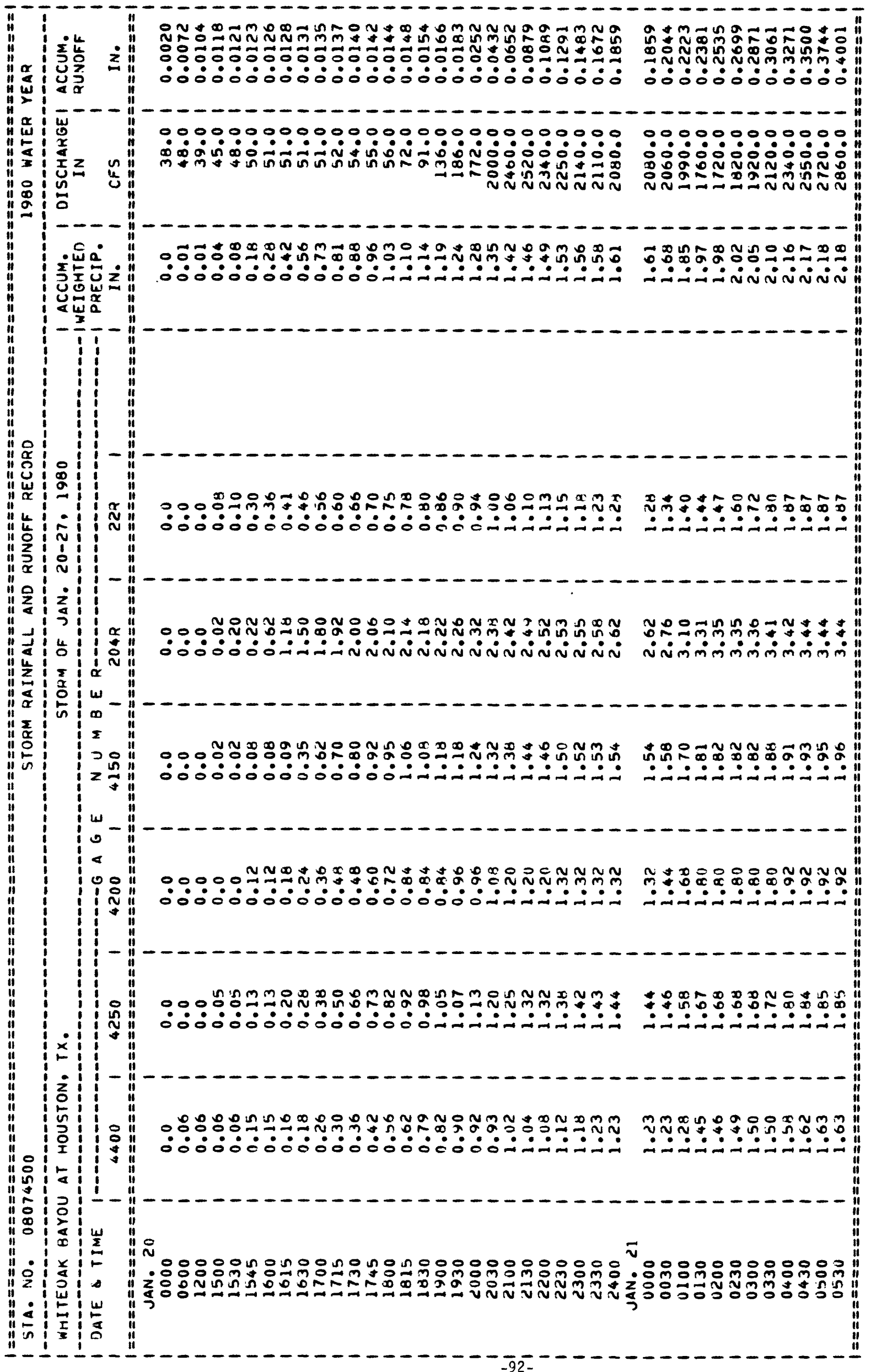




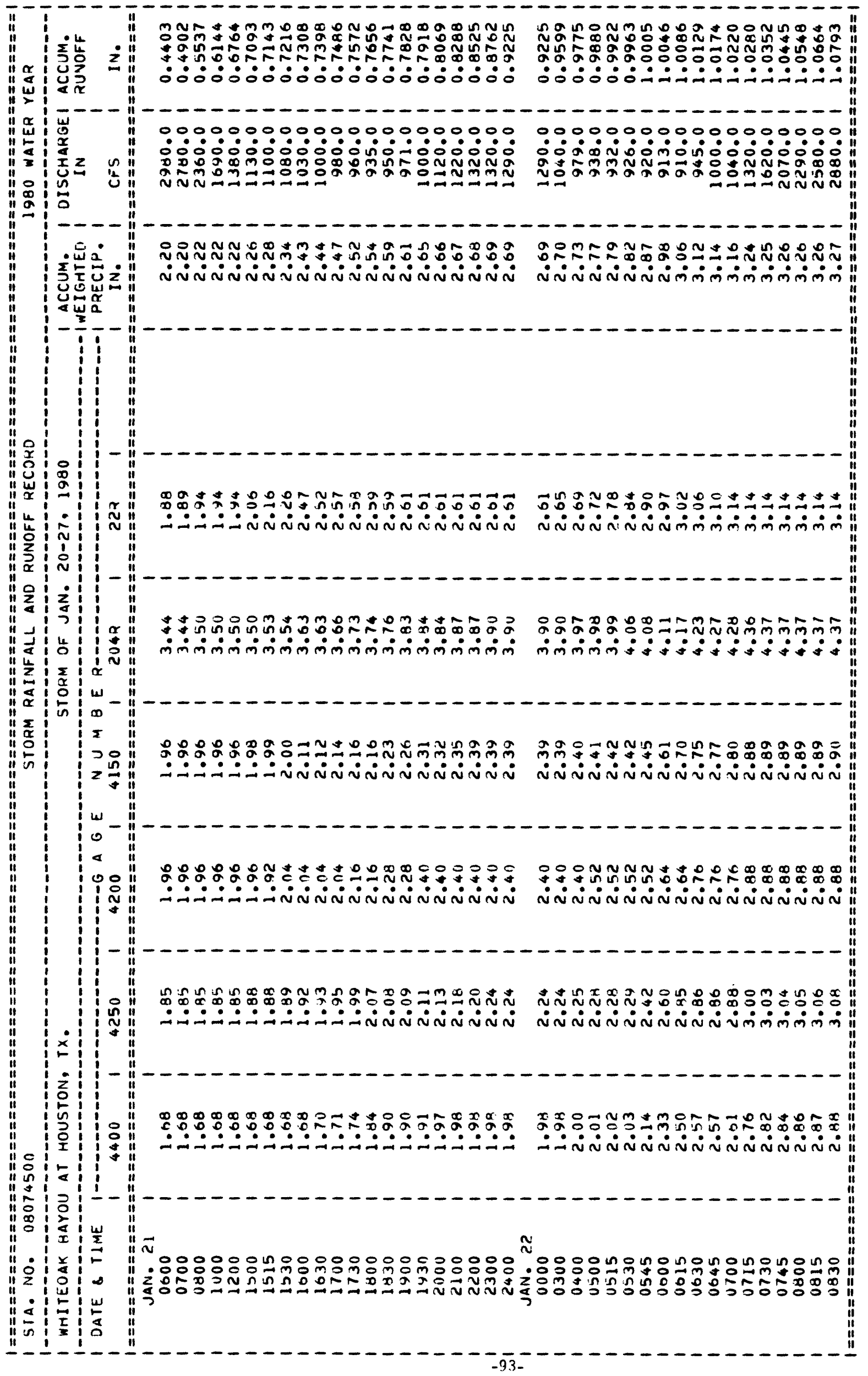




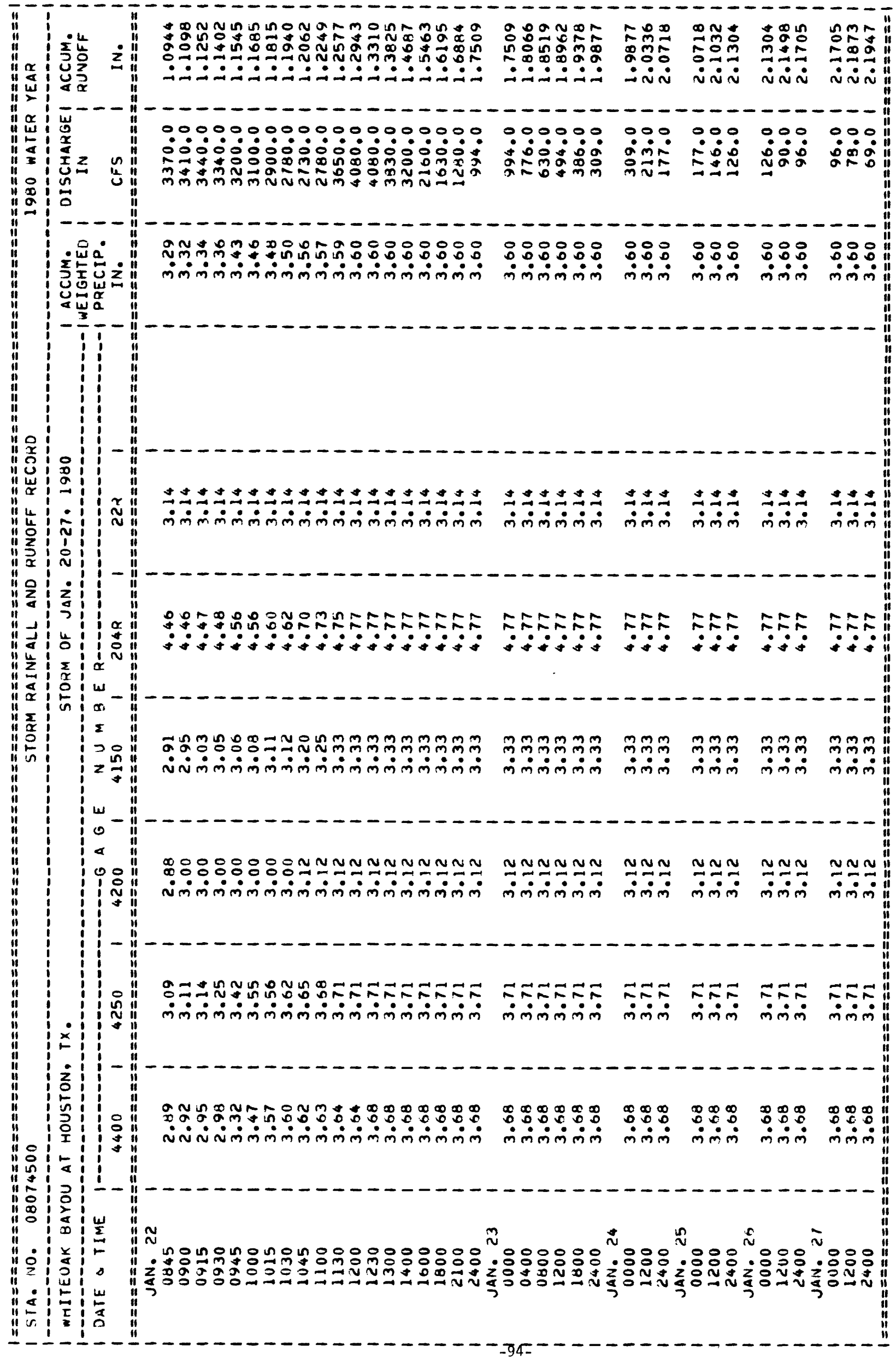




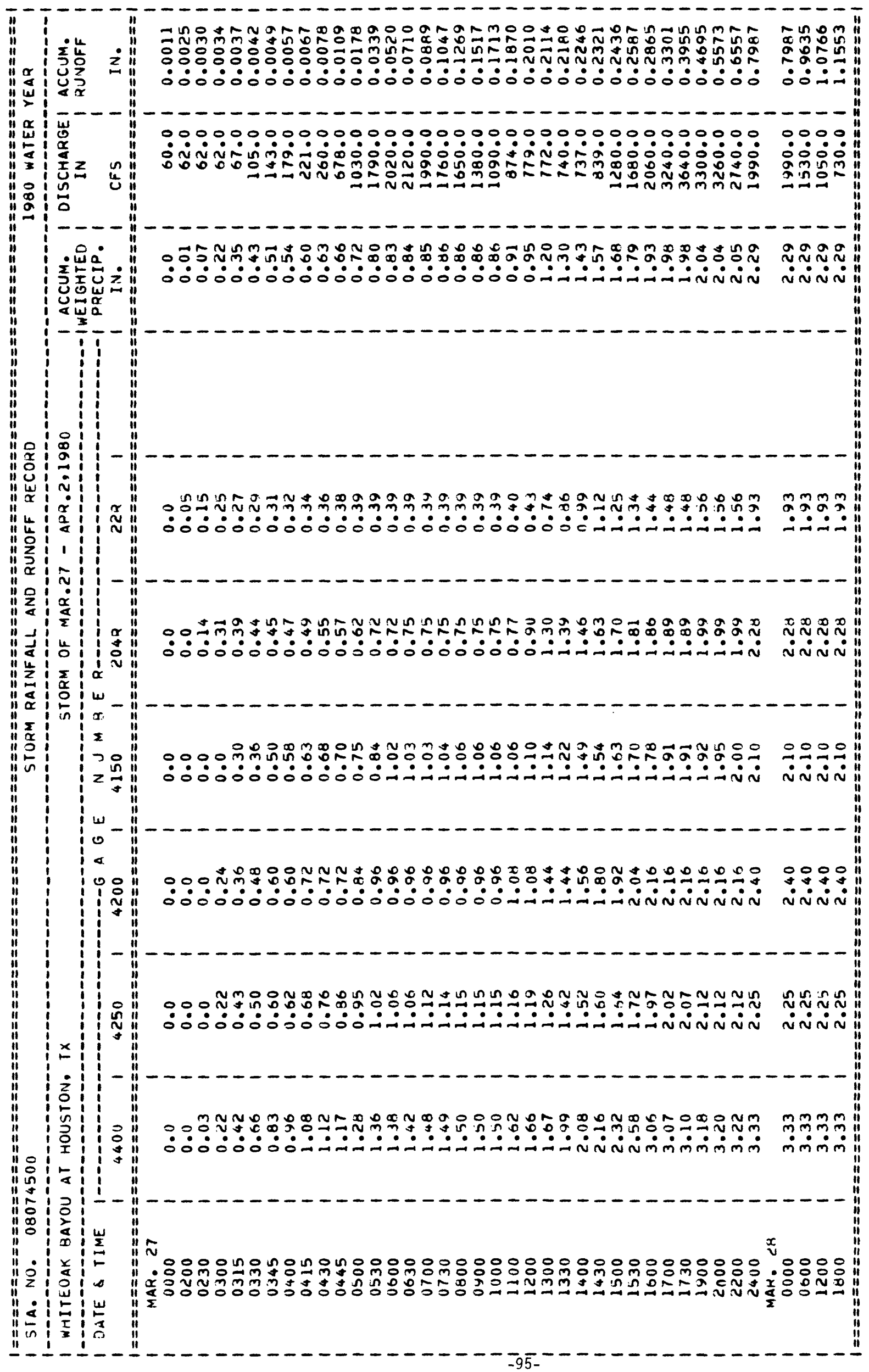




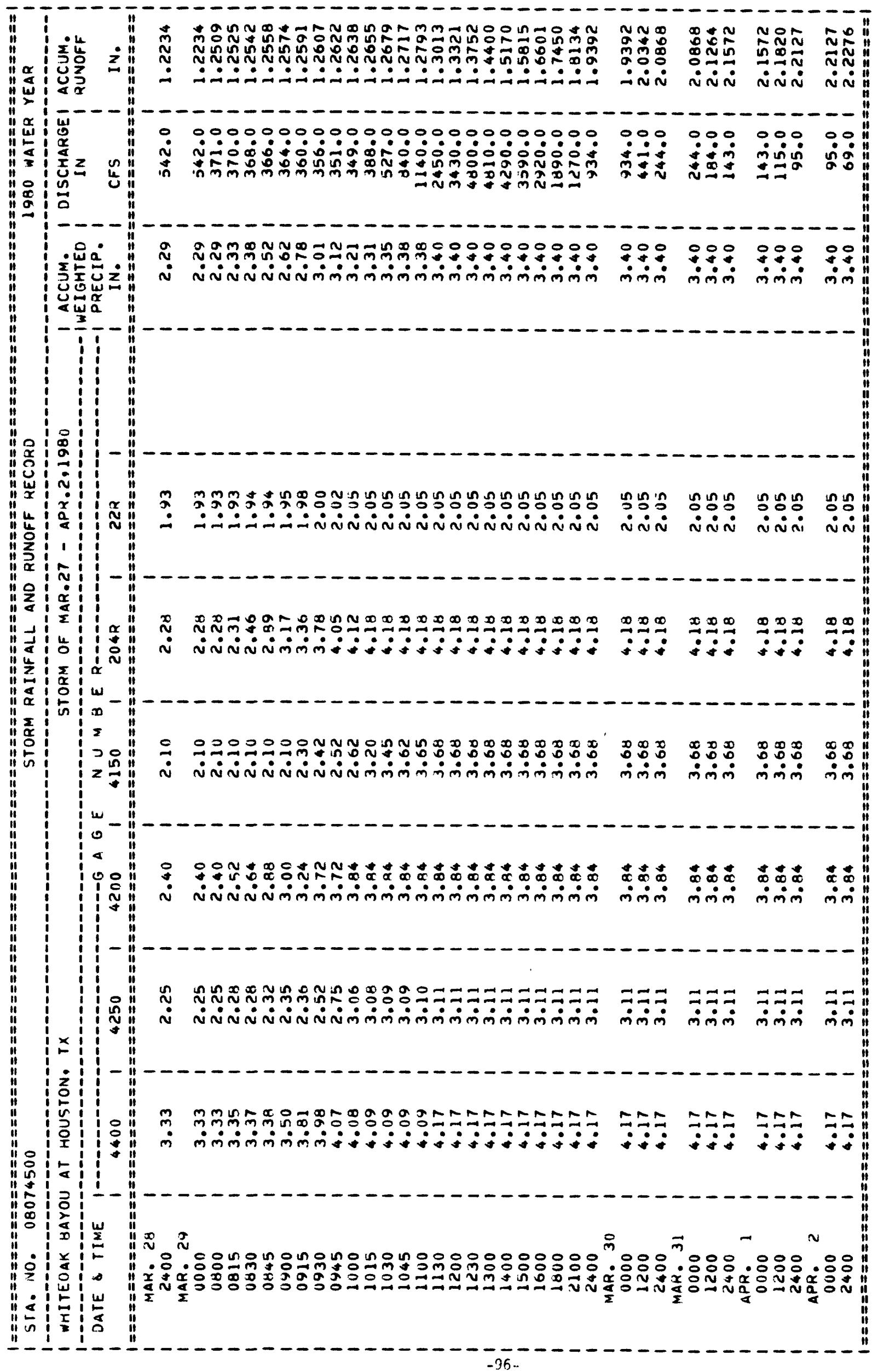




\section{LITTLE WHITEOAK BAYOU DRAINAGE BASIN}

The locations of data-collection sites in and near the Little Whiteoak Bayou drainage basin are shown in figure 10.

Weighted-mean rainfall for the 1980 water year was not determined.

The storm of Oct. 30-31, Dec. 12-13, and Jan. 17-18 were selected for analysis at station 08074540 , Little Whiteoak Bayou at Houston. 


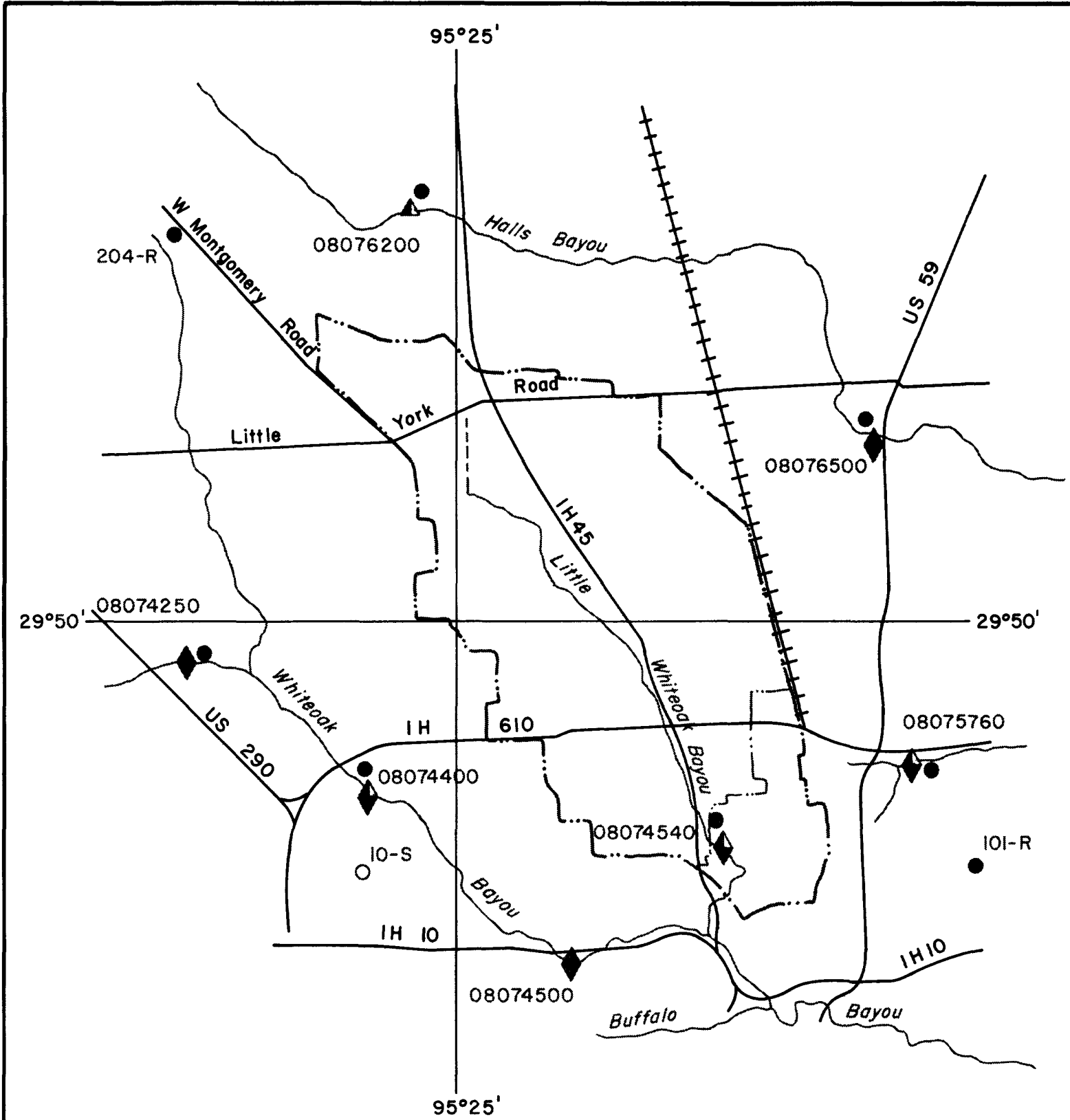

EXPLANATION

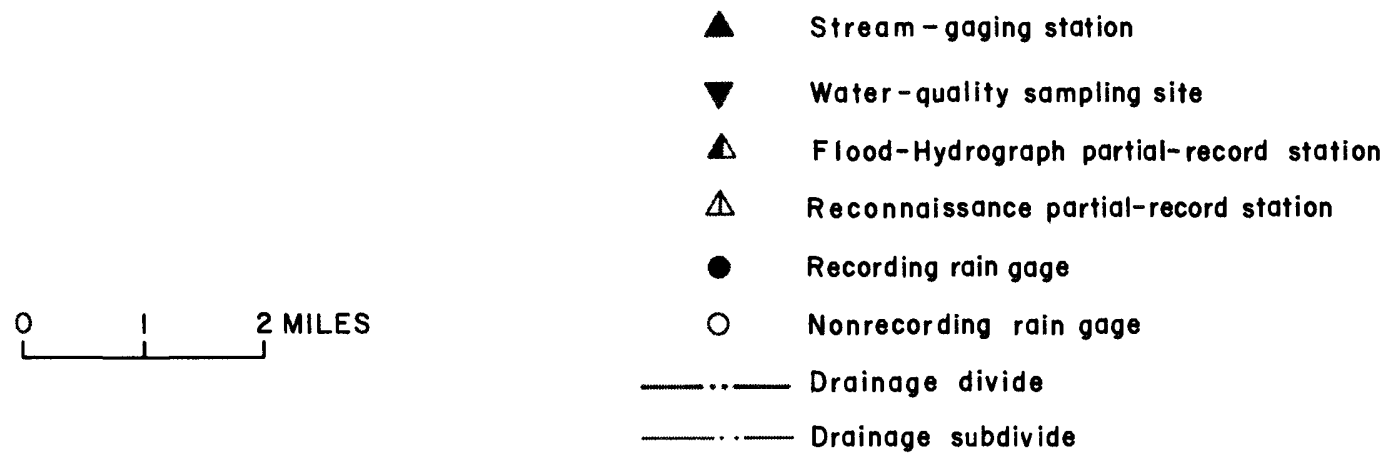

Base from Texas Department of Highways

and Public Transportation General Highway Map

Figure 10.-Locations of data-collection sites in and near the Little Whiteoak Bayou drainage basin 


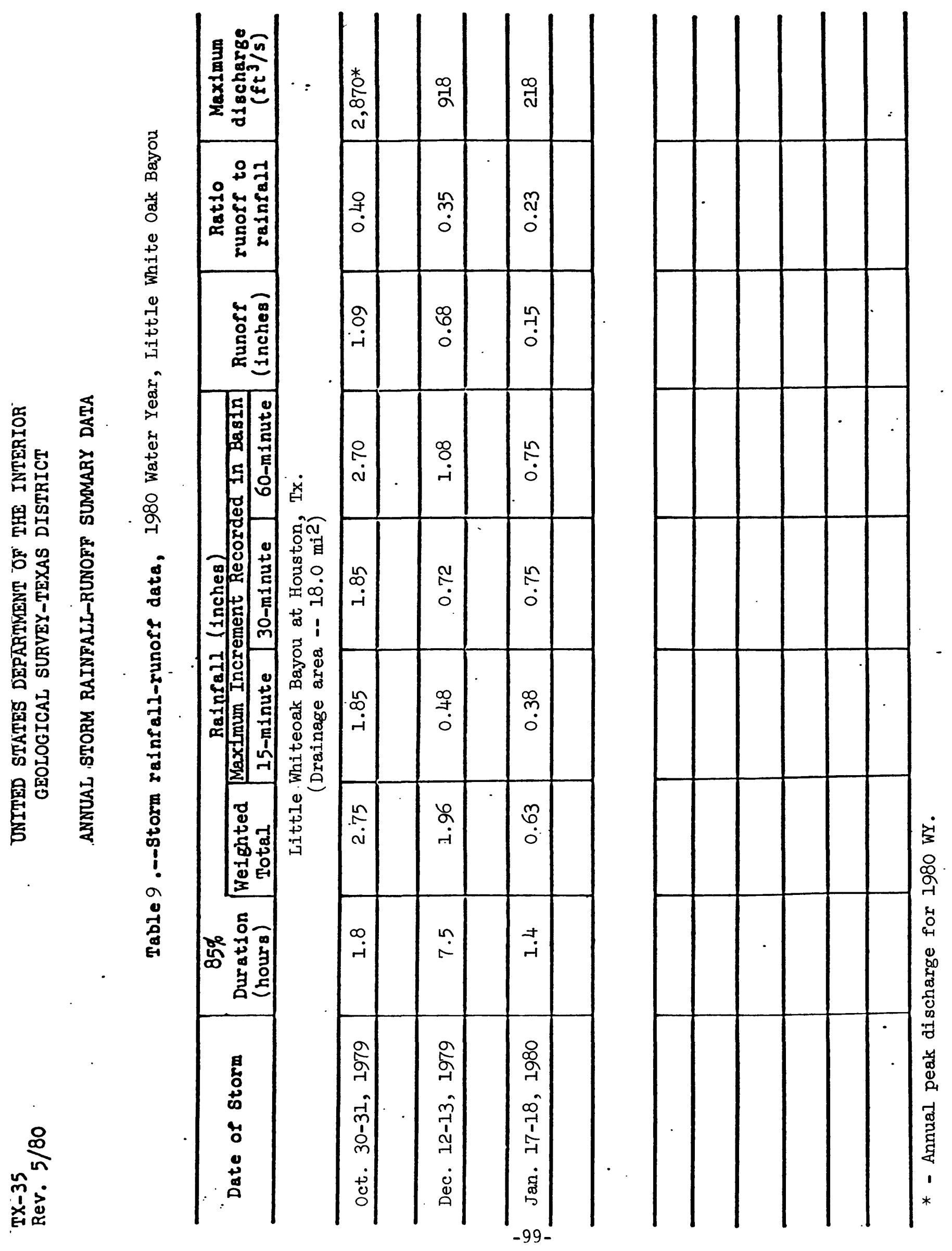




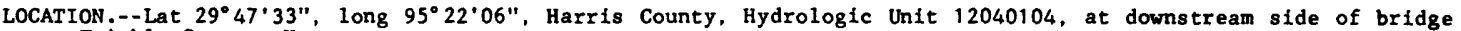
at Trimble Street, Houston.

DRAINAGE AREA. $--18.0 \mathrm{mi}^{2}\left(46.6 \mathrm{~km}^{2}\right)$.

PERIOD OF RECORD.--June 1979 to current year. June to September 1979 published as Little Whiteoak Bayou at Houston $(08074550)$.

GAGE.--Flood-hydrograph and rafnfall recorder and crest-stage gage. Datum of gage is National Geodetic Vertical Datum of 1929 . 1973 adjustment. Prior to June 1979 occasional discharge measurements to arbitrary datum and water-quality samples were obtained at site $6,200 \mathrm{ft}(1,890 \mathrm{~m})$ downstream at North Main Street bridge (station 08074550 , Little Whiteoak Bayou at Houston).

REMARKS.--Additional storm rainfall-runoff data for this site can be obtalned from the report "Hydrologic Data for Urban Studies in the Houston, Texas Metropolltan Area, 1980". The record for June to September 1979 was published in the 1979 edition of this publication as station Little Whiteoak Bayou at Houston (08074550).

EXTREMES FOR PERIOD OF RECORD.--Maximum discharge, 4,750 $\mathrm{ft}^{3} / \mathrm{s}\left(135 \mathrm{~m}^{3} / \mathrm{s}\right)$ Sept. 19, 1979, elevation, $37.76 \mathrm{ft}$ $(11.509 \mathrm{~m})$.

EXTREMES FOR CURRENT YEAR.--Peak discharges for period June to September above base of $1,000 \mathrm{ft}^{3} / \mathrm{s}\left(28.3 \mathrm{~m}^{3} / \mathrm{s}\right)$ and maximum $(*)$ :

\begin{tabular}{|c|c|c|c|c|c|c|c|c|c|c|c|}
\hline Date & Time & $\begin{array}{c}\text { Discl } \\
\left(\mathrm{ft}^{3} / \mathrm{s}\right)\end{array}$ & $\underset{\left(m^{3} / 8\right)}{\operatorname{arge}}$ & $\begin{array}{l}\text { Gage } \\
(\mathrm{ft})\end{array}$ & $\begin{array}{r}\text { helght } \\
(m)\end{array}$ & Date & Time & \multicolumn{2}{|c|}{$\left(\mathrm{ft}^{\mathrm{D} / \mathrm{s})} \begin{array}{c}\text { Discharge } \\
\left(\mathrm{m}^{3} / \mathrm{s}\right)\end{array}\right.$} & \multicolumn{2}{|c|}{$\begin{array}{l}\text { Gage height } \\
\text { (ft) }(\mathrm{m})\end{array}$} \\
\hline $\begin{array}{rr}\text { Oct. } & 30 \\
\text { Nov: } & 21 \\
\text { aDec. } & 12 \\
\text { a Jan. } 17 \\
\text { Jan. } 22\end{array}$ & $\begin{array}{c}1815 \\
\text { unknown } \\
1230 \\
1915 \\
1115\end{array}$ & $\begin{array}{r}\star 2,870 \\
1,470 \\
918 \\
218 \\
2,370\end{array}$ & $\begin{array}{c}81.3 \\
41.6 \\
26.0 \\
6.17 \\
67.1\end{array}$ & $\begin{array}{l}33.76 \\
29.42 \\
27.51 \\
23.76 \\
32.41\end{array}$ & $\begin{array}{r}10.290 \\
8.967 \\
8.385 \\
7.242 \\
9.879\end{array}$ & $\begin{array}{rr}\text { Mar. } & 27 \\
\text { Mar. } & 29 \\
\text { aMay } & 7 \\
\text { Sept. } 5\end{array}$ & $\begin{array}{c}1715 \\
1200 \\
\text { unknown } \\
2115\end{array}$ & $\begin{array}{r}1,940 \\
1,340 \\
b 50 \\
807\end{array}$ & $\begin{array}{c}54.9 \\
37.9 \\
1.42 \\
22.9\end{array}$ & $\begin{array}{r}31.12 \\
29.15 \\
\mathrm{~b} 22.10 \\
27.53\end{array}$ & $\begin{array}{l}9.485 \\
8.885 \\
6.736 \\
8.391\end{array}$ \\
\hline
\end{tabular}

a Water-quality samples were obtained during this runoff event.

b About.

WATER-QUALITY RECORDS

PERIOD OF RECORD.--Chemical, biochemical, and pesticide analyses: June 1979 to current year.

WATER QUALITY DATA, WATER YEAR OCTOBER 1979 TO SEPTEMBER 1980

\begin{tabular}{|c|c|c|c|c|c|c|c|c|c|c|}
\hline DATE & TIME & $\begin{array}{l}\text { STREAM- } \\
\text { FLOW, } \\
\text { INSTAN- } \\
\text { TANEOUS } \\
\text { (CFS) }\end{array}$ & $\begin{array}{l}\text { SPE- } \\
\text { CIFIC } \\
\text { CON- } \\
\text { DUCT- } \\
\text { ANCE } \\
\text { (MICRO- } \\
\text { MHOS) }\end{array}$ & $\begin{array}{c}\text { PH } \\
\text { FIELD } \\
\text { (UNITS) }\end{array}$ & $\begin{array}{c}\text { TEMPER- } \\
\text { ATURE, } \\
\text { WATER } \\
\text { (DEG C) }\end{array}$ & $\begin{array}{l}\text { COLOR } \\
\text { (PLAT- } \\
\text { INUM } \\
\text { COBALT } \\
\text { UNITS) }\end{array}$ & $\begin{array}{l}\text { TUR- } \\
\text { BID- } \\
\text { ITY } \\
\text { (NTU) }\end{array}$ & $\begin{array}{c}\text { OXYGEN, } \\
\text { DIS- } \\
\text { SOLVED } \\
\text { (MG/L) }\end{array}$ & $\begin{array}{c}\text { OXYGEN, } \\
\text { DIS- } \\
\text { SOLVED } \\
\text { (PER- } \\
\text { CENT } \\
\text { SATUR- } \\
\text { ATION) }\end{array}$ & $\begin{array}{c}\text { OXYGEN } \\
\text { DEMAND, } \\
\text { BIOCHEM } \\
\text { UN INH I B } \\
5 \text { DAY } \\
\text { (MG/L) }\end{array}$ \\
\hline $\begin{array}{l}\text { DEC } \\
11 \ldots \\
12 \ldots \\
12 \ldots \\
13 \ldots \\
\text { JAN }\end{array}$ & $\begin{array}{l}1120 \\
1140 \\
1600 \\
1330\end{array}$ & $\begin{array}{l}843^{9.0} \\
391 \\
28\end{array}$ & $\begin{array}{l}762 \\
211 \\
190 \\
349\end{array}$ & $\begin{array}{l}7.0 \\
7.4 \\
7.3 \\
6.9\end{array}$ & $\begin{array}{l}18.0 \\
17.5 \\
15.0 \\
13.5\end{array}$ & $\begin{array}{l}10 \\
30 \\
50 \\
--\end{array}$ & $\begin{array}{l}4.4 \\
350 \\
270 \\
46\end{array}$ & $\begin{array}{l}5.7 \\
8.7 \\
8.4 \\
8.1\end{array}$ & $\begin{array}{l}59 \\
89 \\
82 \\
76\end{array}$ & $\begin{array}{l}9.6 \\
25 \\
17 \\
17\end{array}$ \\
\hline $\begin{array}{r}17 \ldots \\
17 \ldots \\
17 \ldots \\
18 \ldots \\
\text { MAY }\end{array}$ & $\begin{array}{l}1435 \\
1610 \\
1815 \\
1105\end{array}$ & $\begin{array}{r}102 \\
189 \\
156 \\
13\end{array}$ & $\begin{array}{l}470 \\
433 \\
301 \\
353\end{array}$ & $\begin{array}{l}6.9 \\
6.8 \\
7.0 \\
6.9\end{array}$ & $\begin{array}{l}19.0 \\
19.5 \\
18.5 \\
17.5\end{array}$ & $\begin{array}{l}40 \\
30 \\
50 \\
50\end{array}$ & $\begin{array}{r}140 \\
96 \\
310 \\
84\end{array}$ & $\begin{array}{l}5.4 \\
6.3 \\
6.4 \\
5.1\end{array}$ & $\begin{array}{l}57 \\
68 \\
67 \\
52\end{array}$ & $\begin{array}{l}55 \\
18 \\
13 \\
14\end{array}$ \\
\hline${ }_{\text {JUL }}^{07} \cdots$ & 1050 & 40 & 420 & 6.7 & 22.5 & 30 & 33 & 4.5 & 52 & 15 \\
\hline $\begin{array}{l}14 \ldots \\
\operatorname{SEP}\end{array}$ & 1330 & 7.9 & 660 & 7.9 & 30.5 & 40 & 8.0 & 6.5 & 86 & 35 \\
\hline $\begin{array}{l}06 \ldots \\
08 \ldots\end{array}$ & $\begin{array}{l}1300 \\
1050\end{array}$ & $\begin{array}{r}144 \\
11\end{array}$ & $\begin{array}{l}196 \\
320\end{array}$ & $\begin{array}{l}7.5 \\
7.4\end{array}$ & $\begin{array}{l}26.0 \\
26.5\end{array}$ & $\begin{array}{l}50 \\
45\end{array}$ & $\begin{array}{l}74 \\
16\end{array}$ & $\begin{array}{l}5.6 \\
2.1\end{array}$ & $\begin{array}{l}68 \\
26\end{array}$ & ${ }^{13} 5.0$ \\
\hline
\end{tabular}

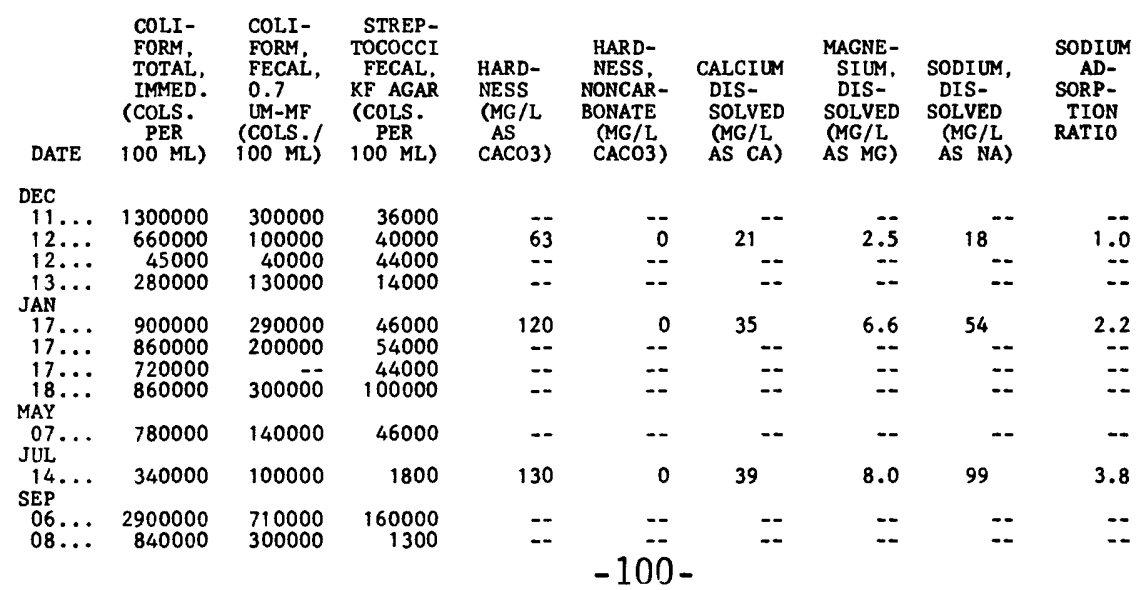


SAN JACINTO RIVER BASIN

08074540 LITTLE WHITEOAK BAYOU AT TRIMBLE STREET AT HOUSTON, TX--Continued

WATER QUALITY DATA, WATER YEAR OCTOBER 1979 TO SEPTEMBER 1980

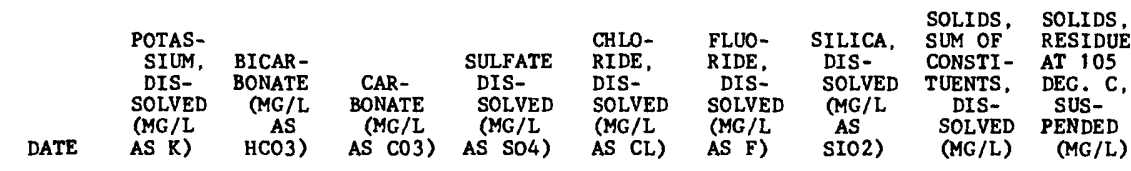

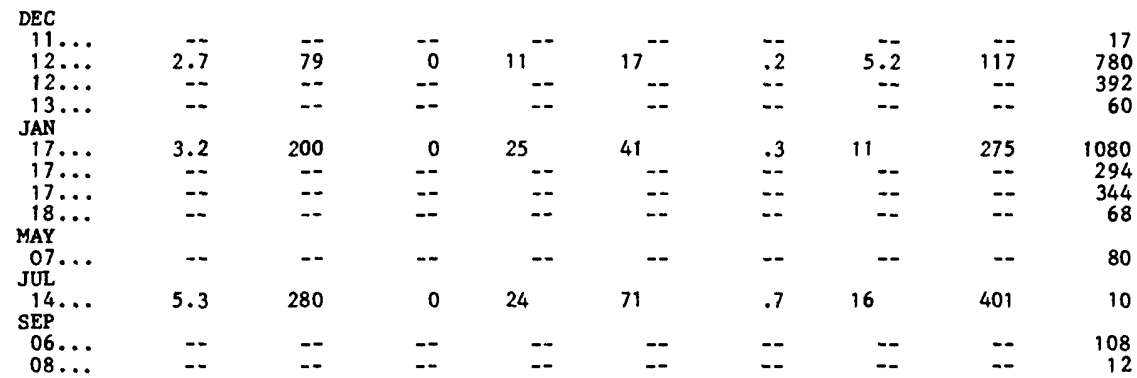

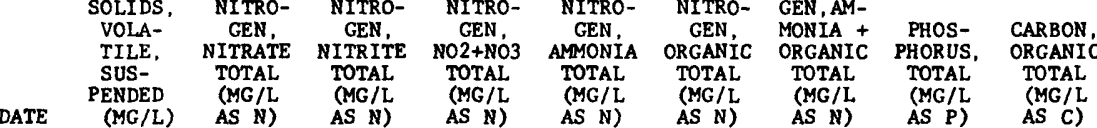

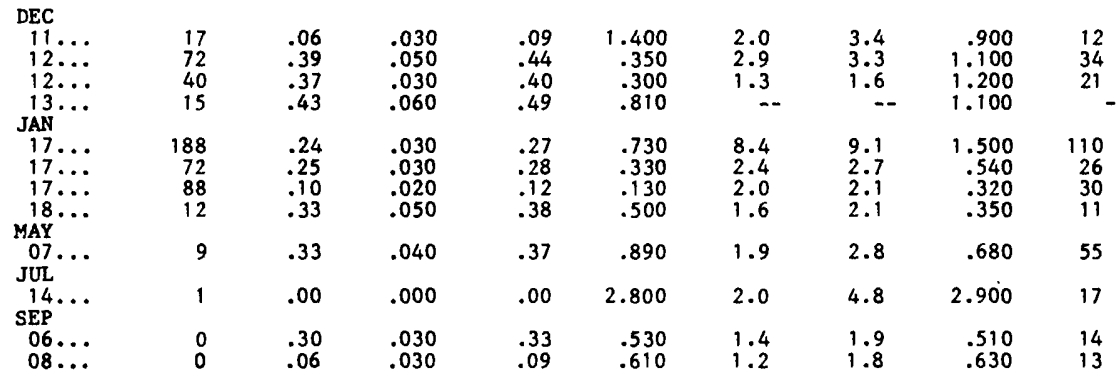

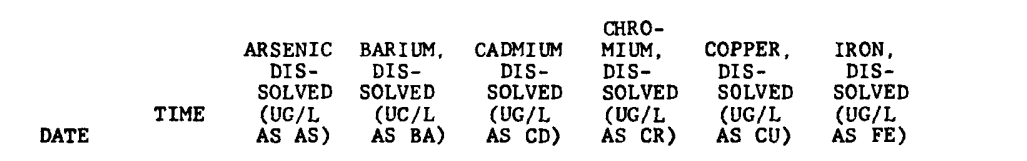

\begin{tabular}{|c|c|c|c|c|c|c|c|}
\hline & 1140 & 3 & 60 & $<1$ & 0 & 0 & 50 \\
\hline & 1435 & 2 & 100 & 1 & 0 & 0 & 200 \\
\hline $14 \ldots$ & 1330 & 6 & 200 & $<1$ & 0 & 1 & $<10$ \\
\hline
\end{tabular}

\begin{tabular}{|c|c|c|c|c|c|c|}
\hline DATE & $\begin{array}{l}\text { LEAD, } \\
\text { DIS- } \\
\text { SOLVED } \\
\text { (UG/L } \\
\text { AS PB) }\end{array}$ & $\begin{array}{l}\text { MANGA- } \\
\text { NESE, } \\
\text { DIS- } \\
\text { SOLVED } \\
\text { (UG/L } \\
\text { AS MN) }\end{array}$ & $\begin{array}{l}\text { MERCURY } \\
\text { DIS- } \\
\text { SOLVED } \\
\text { (UG } / L \\
\text { AS HG) }\end{array}$ & $\begin{array}{l}\text { SELE- } \\
\text { NIUM, } \\
\text { DIS- } \\
\text { SOLVED } \\
\text { (UG/L } \\
\text { AS SE) }\end{array}$ & $\begin{array}{c}\text { SILVER, } \\
\text { DIS- } \\
\text { SOLVED } \\
\text { (UG/L } \\
\text { AS AG) }\end{array}$ & $\begin{array}{l}\text { ZINC, } \\
\text { DIS- } \\
\text { SOLVED } \\
\text { (UG/L } \\
\text { AS } 2 N \text { ) }\end{array}$ \\
\hline $\begin{array}{c}\text { DEC } \\
12 \ldots \\
\text { JAN }\end{array}$ & 5 & 5 & .0 & 0 & 0 & 5 \\
\hline $\begin{array}{l}17 \\
\text { JuL }\end{array}$ & 4 & 260 & .0 & 1 & 0 & 7 \\
\hline & 0 & 1 & .1 & 1 & 0 & 8 \\
\hline
\end{tabular}


SAN JACINTO RIVER BASIN

08074540 LITTLE WHITEOAK BAYOU AT TRIMBLE STREET AT HOUSTON, TX--Continued

WATER QUALITY DATA, WATER YEAR OCTOBER 1979 TO SEPTEMBER 1980

\begin{tabular}{|c|c|c|c|c|c|c|c|c|c|c|}
\hline DATE & TIME & $\begin{array}{c}\text { PCB } \\
\text { TOTAL } \\
\text { (UG/L) }\end{array}$ & $\begin{array}{l}\text { NAPH- } \\
\text { THA- } \\
\text { LENES, } \\
\text { POLY- } \\
\text { CHLOR. } \\
\text { TOTAL } \\
\text { (UC/L) }\end{array}$ & $\begin{array}{c}\text { ALDRIN, } \\
\text { TOTAL } \\
\text { (UG/L) }\end{array}$ & $\begin{array}{l}\text { CHLOR- } \\
\text { DANE, } \\
\text { TOTAL } \\
\text { (UG/L) }\end{array}$ & $\begin{array}{l}\text { DDD, } \\
\text { TOTAL } \\
\text { (UG/L) }\end{array}$ & $\begin{array}{c}\text { DDE, } \\
\text { TOTAL } \\
\text { (UG /L) }\end{array}$ & $\begin{array}{l}\text { DDT } \\
\text { TOTAL } \\
\text { (UG/L) }\end{array}$ & $\begin{array}{l}\text { DI- } \\
\text { AZ INON, } \\
\text { TOTAL } \\
\text { (UG/L) }\end{array}$ & $\begin{array}{l}\text { DI- } \\
\text { ELDRIN } \\
\text { TOTAL } \\
\text { (UG/L) }\end{array}$ \\
\hline $\begin{array}{c}\text { DEC } \\
12 \ldots \\
\text { JAN }\end{array}$ & 1140 & .00 & .00 & .00 & .6 & .00 & .01 & .00 & .55 & .01 \\
\hline $17 \ldots$ & 1435 & .90 & - & .00 & .8 & .03 & .03 & .00 & .18 & .03 \\
\hline
\end{tabular}

\begin{tabular}{|c|c|c|c|c|c|c|c|c|c|}
\hline DATE & $\begin{array}{l}\text { ENDO- } \\
\text { SULFAN, } \\
\text { TOTAL } \\
\text { (UG/L) }\end{array}$ & $\begin{array}{l}\text { ENDR IN, } \\
\text { TOTAL } \\
\text { (UG/L) }\end{array}$ & $\begin{array}{l}\text { ETH ION, } \\
\text { TOTAL } \\
\text { (UG/L) }\end{array}$ & $\begin{array}{l}\text { HEPTA- } \\
\text { CHLOR, } \\
\text { TOTAL } \\
\text { (UG/L) }\end{array}$ & $\begin{array}{l}\text { HEPTA- } \\
\text { CHLOR } \\
\text { EPOXIDE } \\
\text { TOTAL } \\
\text { (UG/L) }\end{array}$ & $\begin{array}{c}\text { HEPTA- } \\
\text { CHLOR } \\
\text { EPOXIDE } \\
\text { TOT. IN } \\
\text { BOTTOM } \\
\text { MATL. } \\
\text { (UG/KG) }\end{array}$ & $\begin{array}{c}\text { LINDANE } \\
\text { TOTAL } \\
(\text { UG/L) }\end{array}$ & $\begin{array}{l}\text { MALA- } \\
\text { THION, } \\
\text { TOTAL } \\
\text { (UG/L) }\end{array}$ & $\begin{array}{c}\text { METH - } \\
\text { OXY- } \\
\text { CHLOR, } \\
\text { TOTAL } \\
\text { (UG/L) }\end{array}$ \\
\hline $\begin{array}{l}\text { DEC } \\
12 \ldots \\
\text { JAN }\end{array}$ & .00 & .00 & .00 & .04 & .00 & -- & .00 & .34 & .00 \\
\hline $17 \ldots$ & .00 & .00 & .00 & .02 & -- & .0 & .00 & .13 & .00 \\
\hline DATE & $\begin{array}{l}\text { METHYL } \\
\text { PARA- } \\
\text { TH ION, } \\
\text { TOTAL } \\
\text { (UG/L) }\end{array}$ & $\begin{array}{l}\text { METHYL } \\
\text { TRI- } \\
\text { THION, } \\
\text { TOTAL } \\
\text { (UG/L) }\end{array}$ & $\begin{array}{l}\text { MIREX, } \\
\text { TOTAL } \\
\text { (UG/L) }\end{array}$ & $\begin{array}{l}\text { PARA- } \\
\text { IHION, } \\
\text { TOTAL } \\
\text { (UG /L) }\end{array}$ & $\begin{array}{l}\text { TOX- } \\
\text { APHENE, } \\
\text { TOTAL } \\
\text { (UG/L) }\end{array}$ & $\begin{array}{l}\text { TOTAL } \\
\text { TRI- } \\
\text { THION } \\
\text { (UG/L) }\end{array}$ & $\begin{array}{l}2,4-D \text {, } \\
\text { TOTAL } \\
\text { (UG/L) }\end{array}$ & $\begin{array}{c}2,4,5-\mathrm{T} \\
\text { TOTAL } \\
\text { (UG/L) }\end{array}$ & $\begin{array}{c}\text { SILVEX, } \\
\text { TOTAL } \\
\text { (UG/L) }\end{array}$ \\
\hline $\begin{array}{l}\text { DEC } \\
12 \ldots \\
\text { JAN }\end{array}$ & .00 & .00 & .02 & .00 & 0 & .00 & .08 & .04 & .06 \\
\hline $17 \ldots$ & .00 & .00 & .00 & .00 & 0 & .00 & .06 & .02 & .00 \\
\hline
\end{tabular}




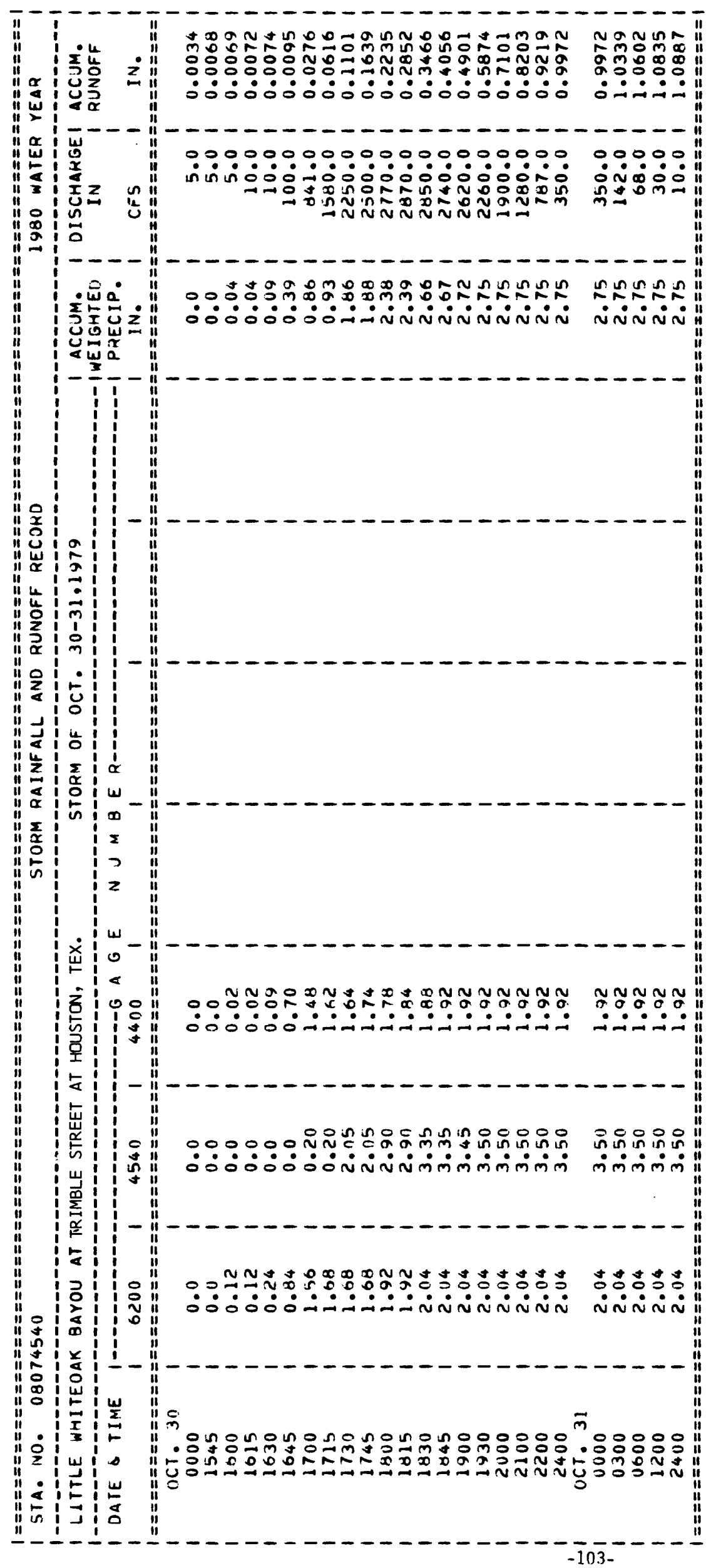




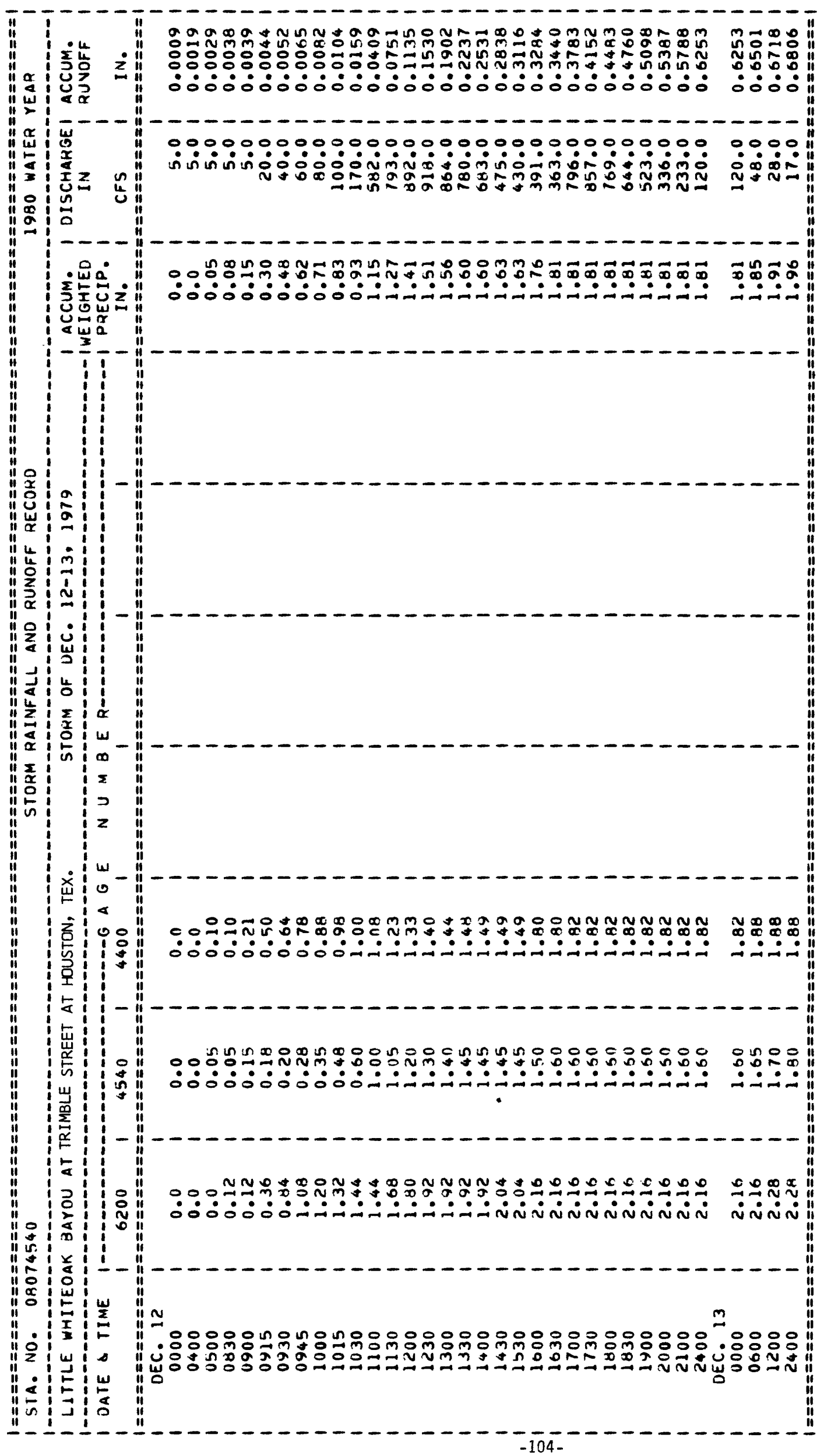




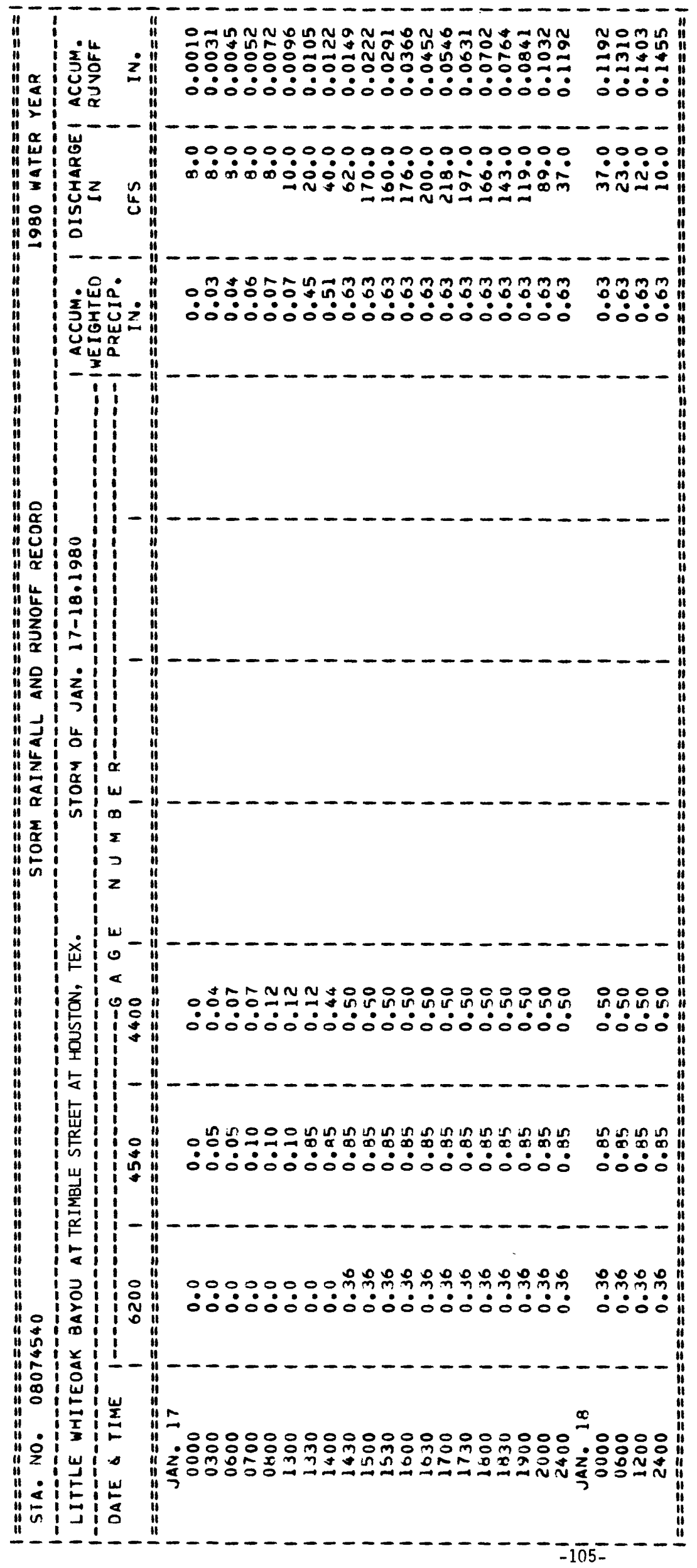




\section{BRAYS BAYOU DRAINAGE BASIN}

The locations of data-collection sites in and near the Brays Bayou drainage basin are shown in figure 11.

Keeqans Bayou, Bintliff Ditch, and Hummingbird Street Ditch are shown on separate drainage basins within the Brays Bayou section.

Weighted-mean rainfall in the drainaqe basin for the 1980 water year hased on eleven rain gaqes was 36.21 inches or 11.93 inches less than the 30year (1941-70) averaqe of 48.19 inches for Houston.

The storms of Oct. 30-Nov. 1, Dec. 1?-14, and Jan. 20-24 were selected for analysis for the 1980 water year at station 08074760, Brays Bayou at Alief, Tex. The storm of Jan. 20-25 was selected for analysis at station 08074810 , Brays Bayou at Gessner Drive, Houston, Tex., and the storms of Dec. 12-14 and Jan. 20-25 were selected for analysis at station 08075000 , Brays Bayou at Houston. 


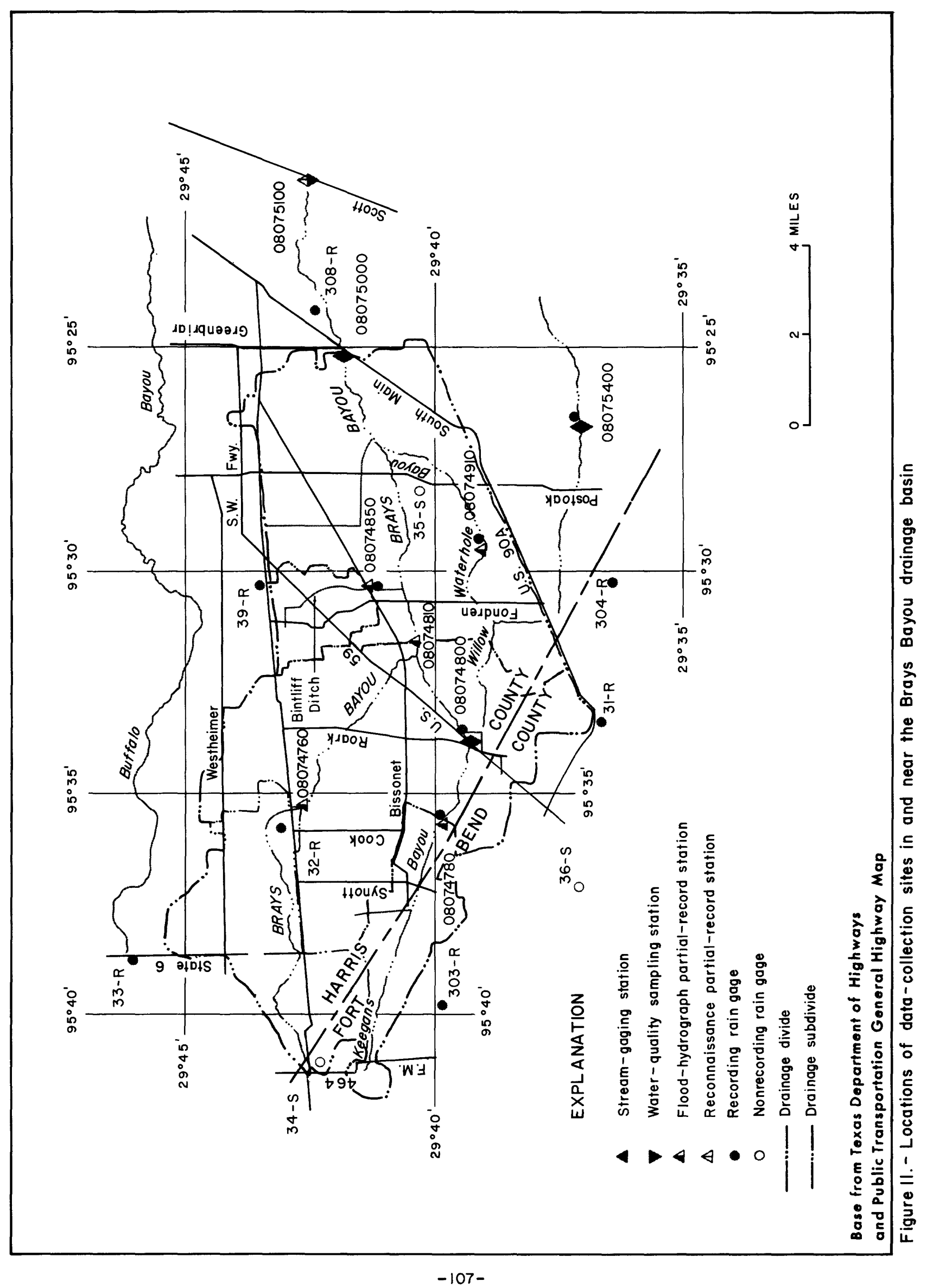




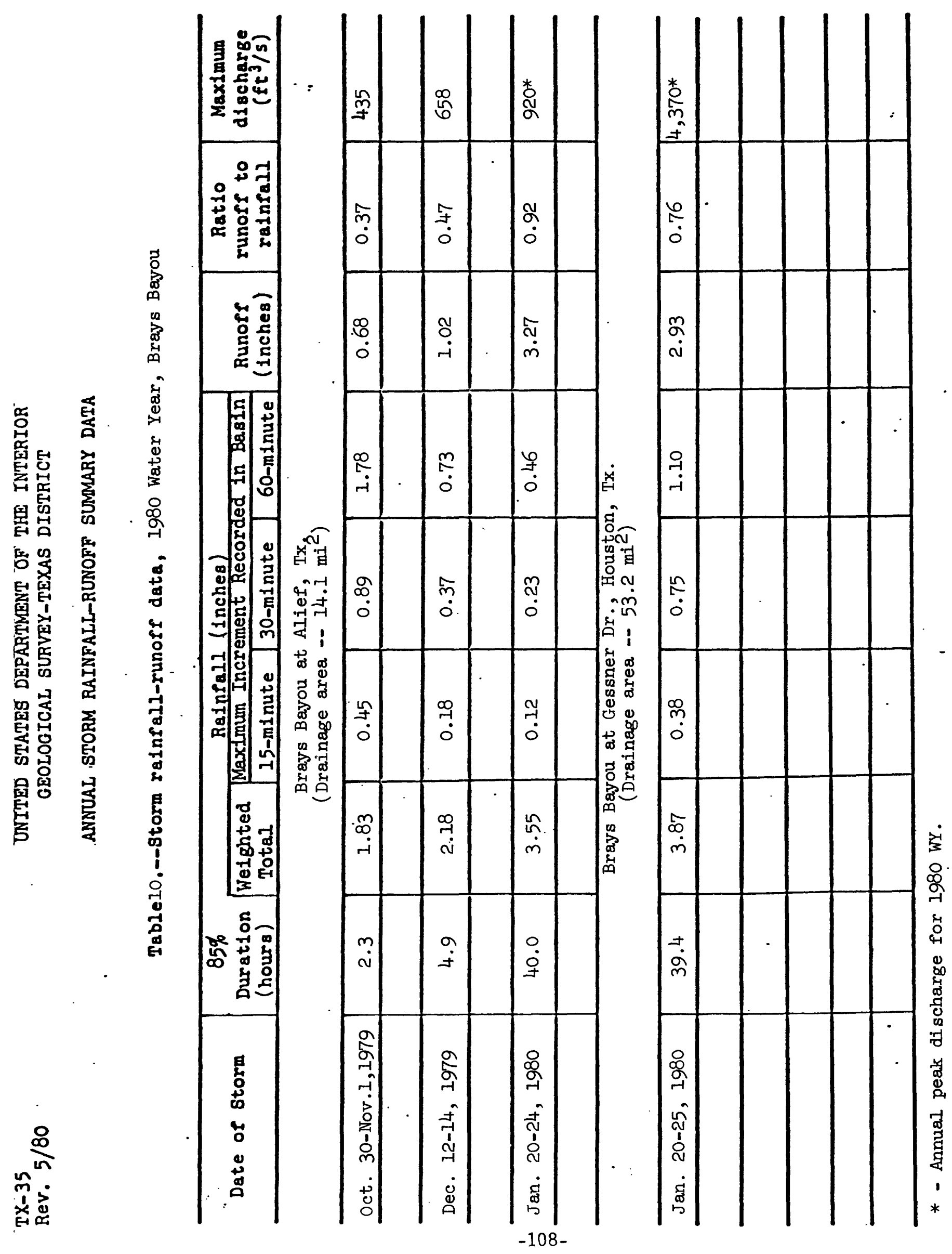




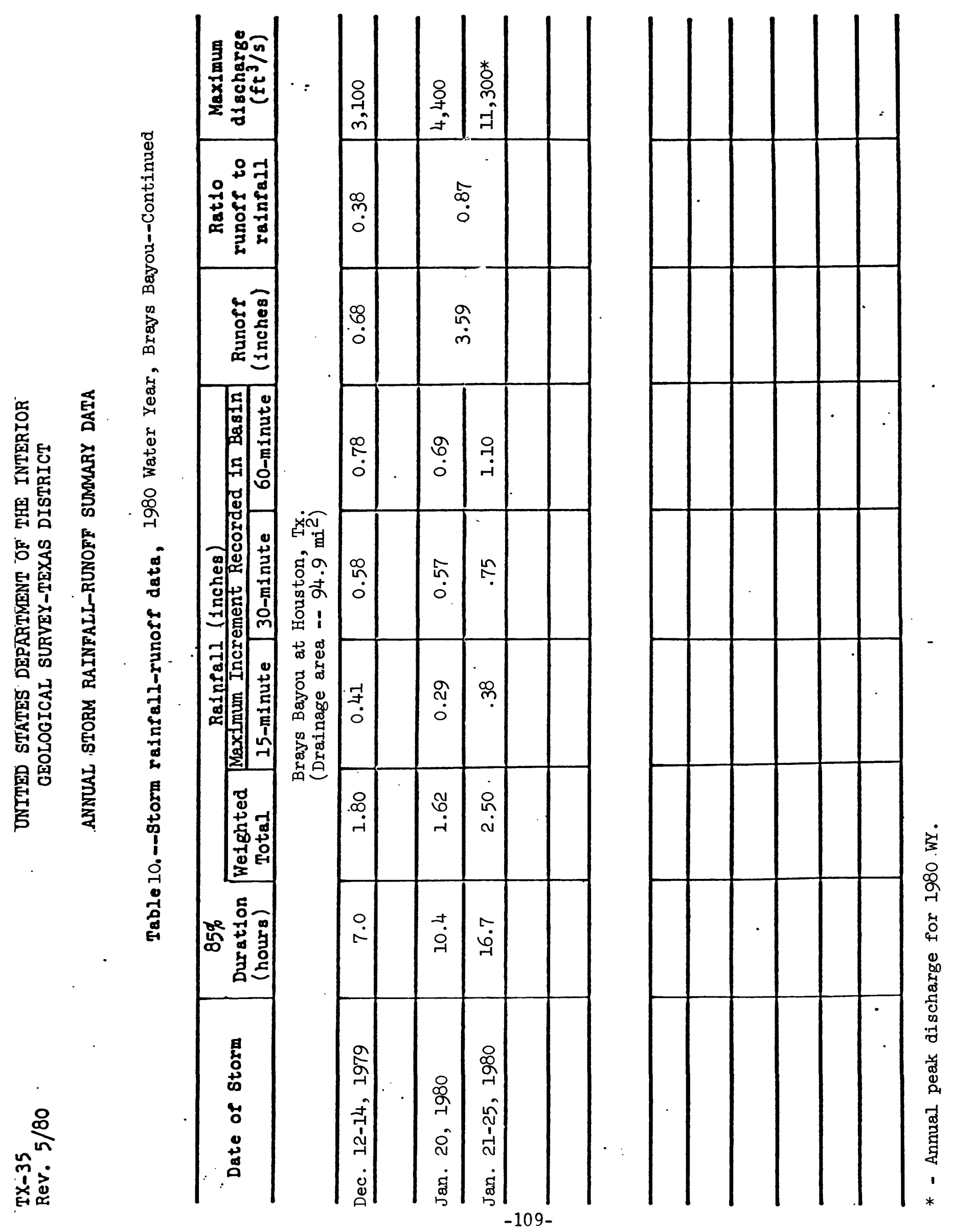


08074760 BRAYS BAYOU AT ALIEF, TEX.

(Flood-hydrograph partial-record station)

LOCATION.--Lat $29^{\circ} 42^{\prime} 39^{\prime \prime}$, long $95^{\circ} 35^{\prime} 13^{\prime \prime}$, Harris County, Hydrologic unit 12040104, near center of channel on downstream side of bridge on High Star Street in Al ief, Tex.

DRAINAGE AREA (revised).--14.1 $\mathrm{mi}^{2}$ effective Jan. $1,1978.12 .9 \mathrm{mi}^{2}$ effective Feb. 3, 1977 to Dec. 31, 1977.

PERIOD OF RECORD.--Feb. 3, 1977 to present.

GAGE.--Digital flood-hydrograph recorder and crest-stage gage. Datum of gage is $55.88 \mathrm{ft}$ National Geodetic Vertical Datum of 1929, 1957 adjustment; unadjusted for land-surface subsidence.

REMARKS.--Records fair.

EXTREMES FOR PERIOD OF RECORD.--Maximum discharge $3,270 \mathrm{ft}^{3} / \mathrm{s}$, Sept. $19,1979$. (Gage-height $17.15 \mathrm{ft}$ ). Minimum discharge not determined.

EXTREMES FOR CURRENT YEAR.--Peak discharge above base of $300 \mathrm{ft}^{3} / \mathrm{s}$ or maximum $(*):$

DATE

0ct. 30

Dec. 12

Jan. 21

Jan. 21

Jan. 22

Mar. 27

Mar. 29

Sep. 6
TIME

2300

1700

0200

2230

1200

2000

1500

0730
DISCHARGE

$\left(\mathrm{ft}^{3} / \mathrm{s}\right)$

\section{5}

658

719

483

*920

551

417

772
GAGE HEIGHT

$(\mathrm{ft})$

9.62

10.38

10.42

9.73

10.95

10.04

9.55

11.21

Minimum discharge not determined. 


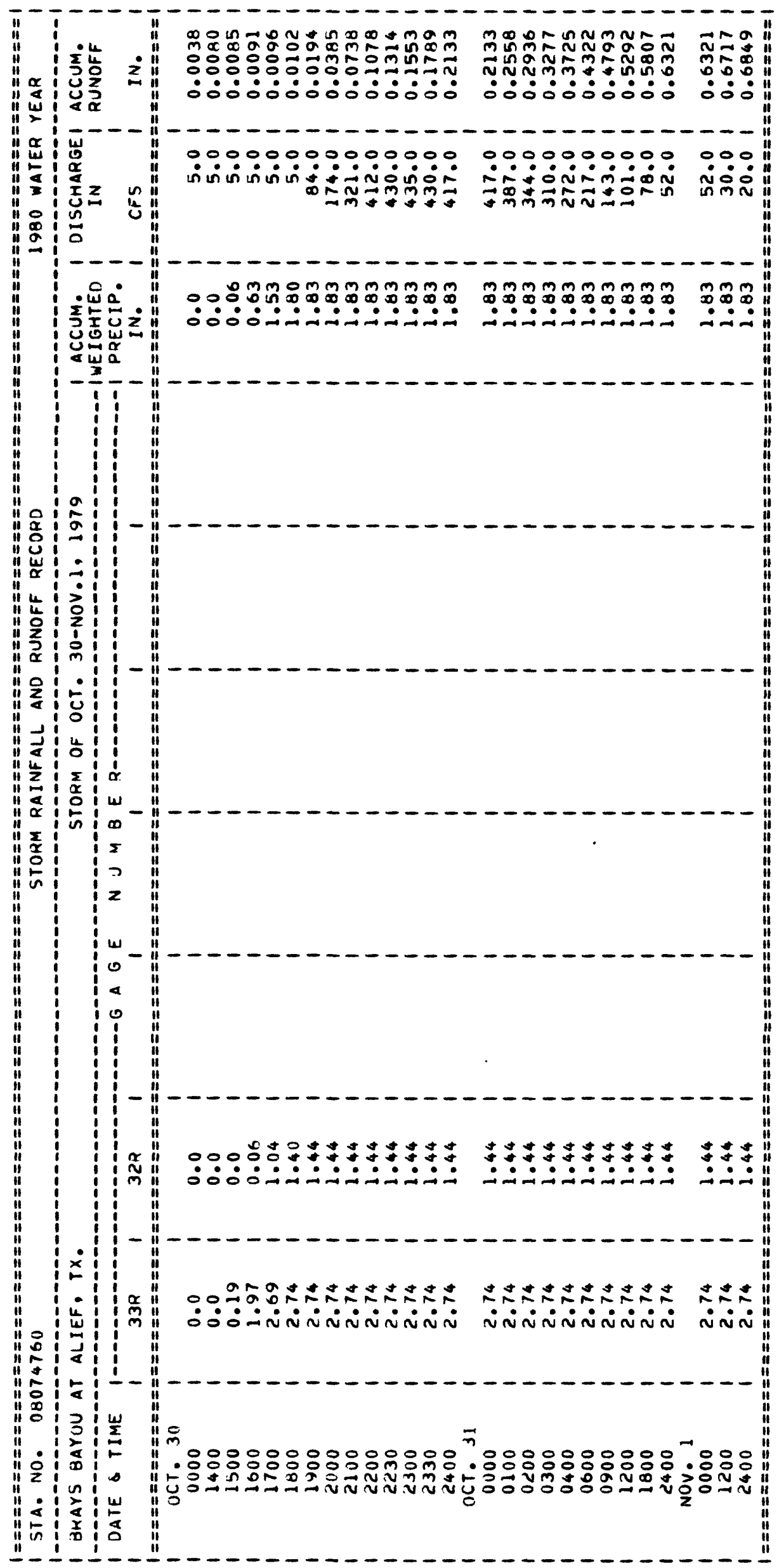

$-111-$ 


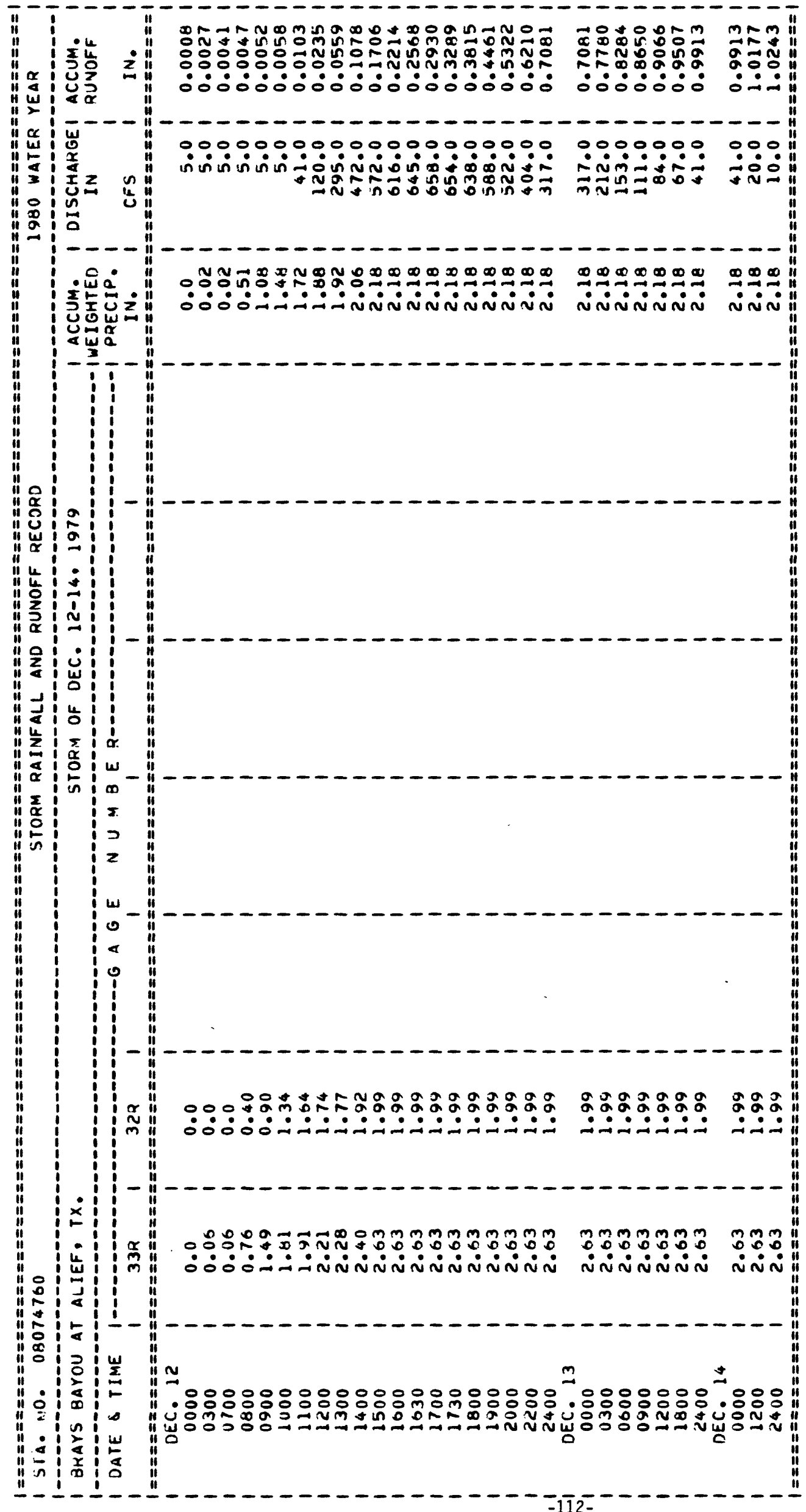




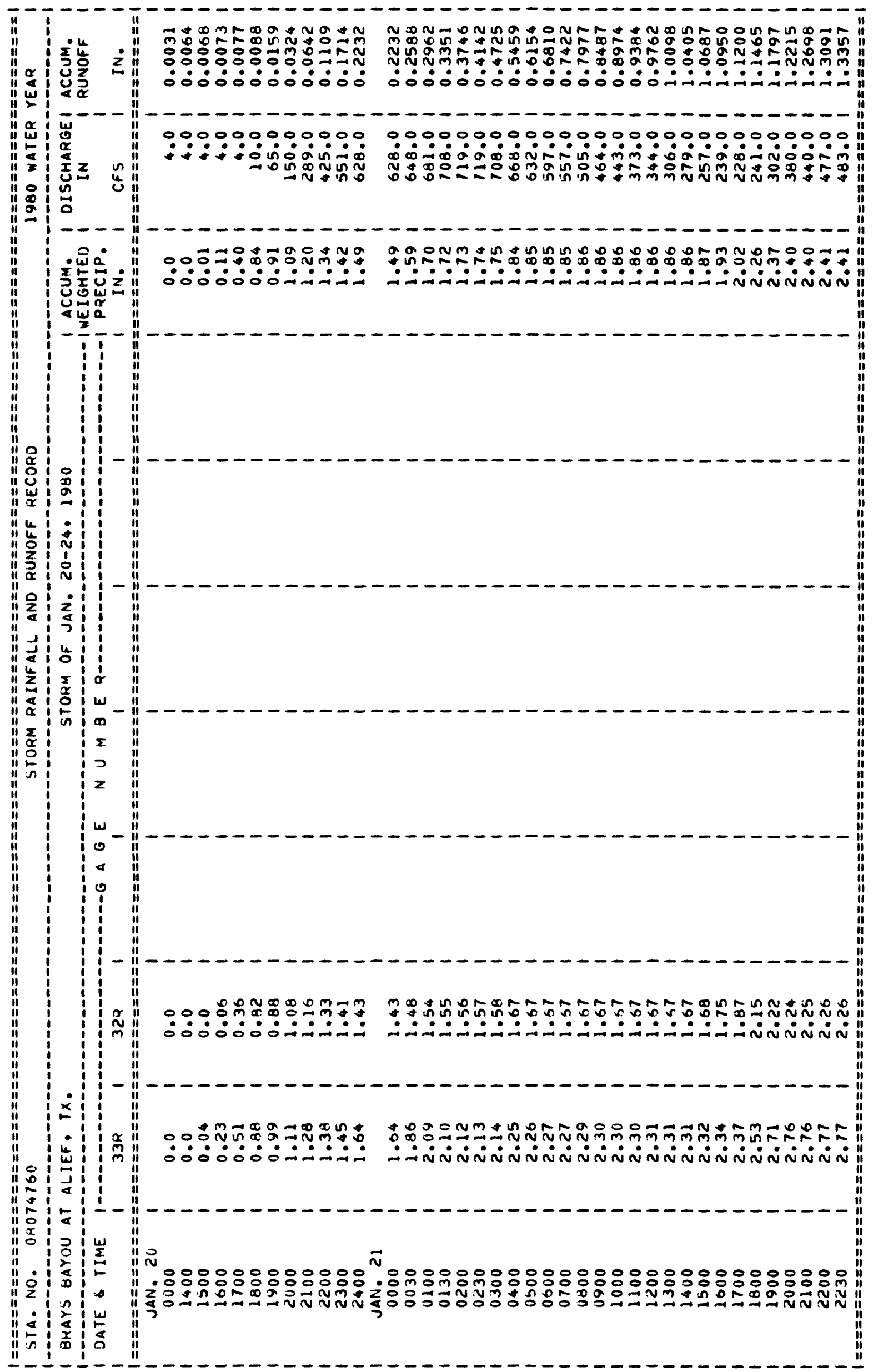




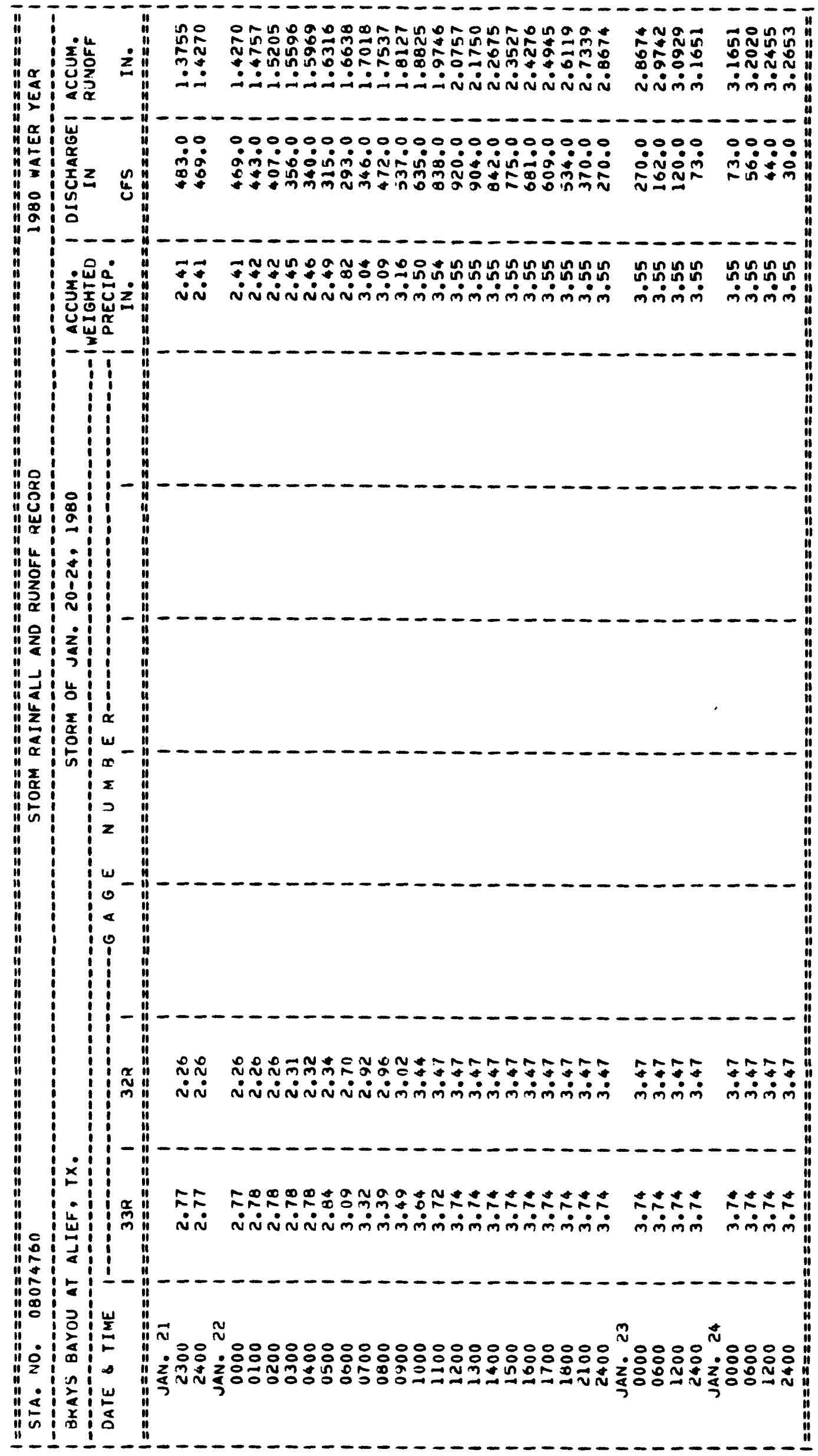




\section{KEEGANS BAYOU DRAINAGE BASIN}

The locations of data-collection sites in and near the Keegans Bayou drainage basin are shown in figure 12 .

Weighted-mean rainfall in the drainage basin, based on four rain aages for the 1980 water year was 35.73 inches or 12.46 inches 1 ess than the 30 -year (1941-70) average of 48.19 inches for Houston.

No storms were analyzed for station 08074780, Keegans Bayou at Keegan Road near Houston. The storms of Dec. 12-14 and Jan. 20-25 were selected for analysis at station 08074800, Keegans Bayou at Roark Road near Houston. 


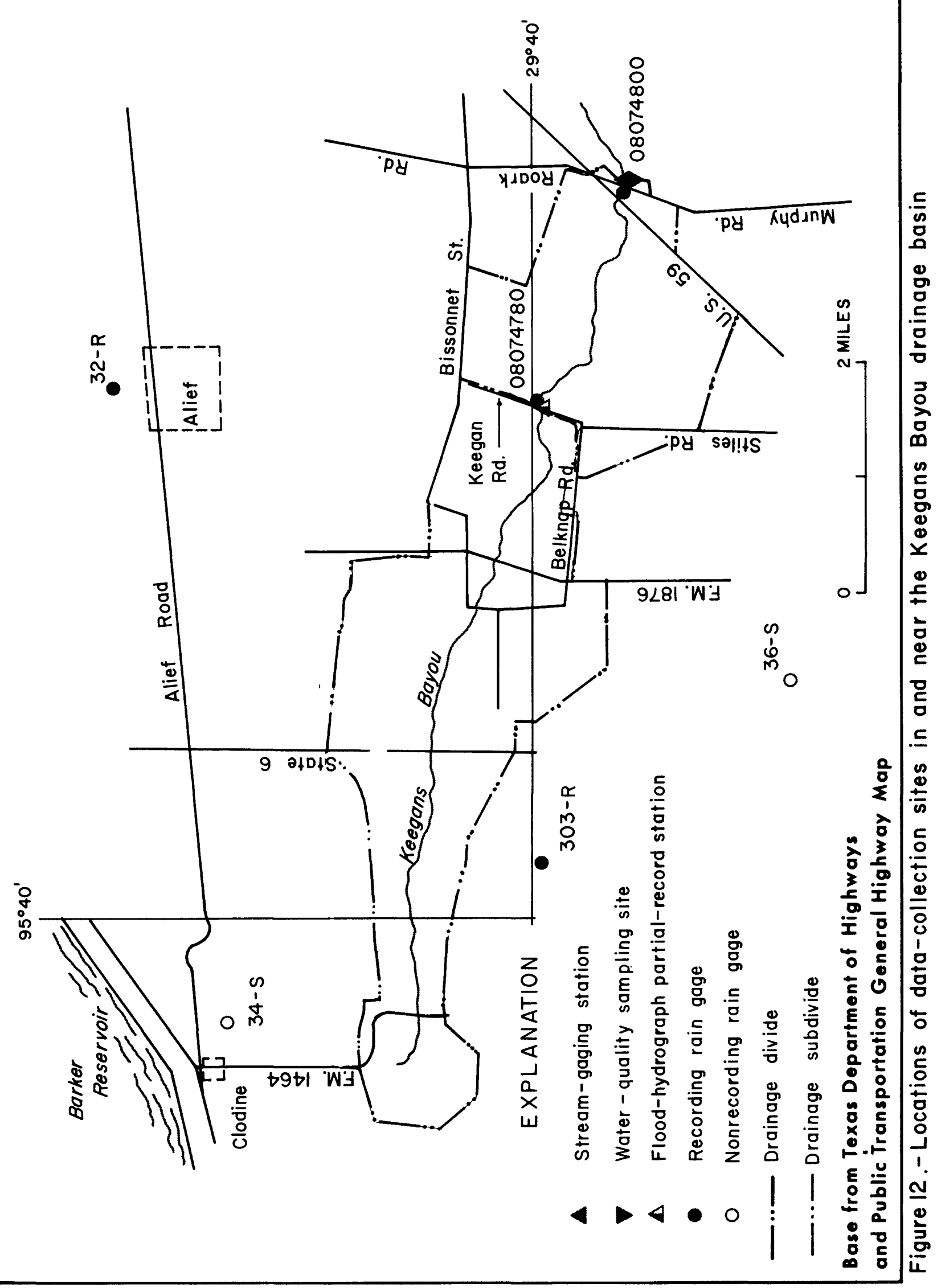




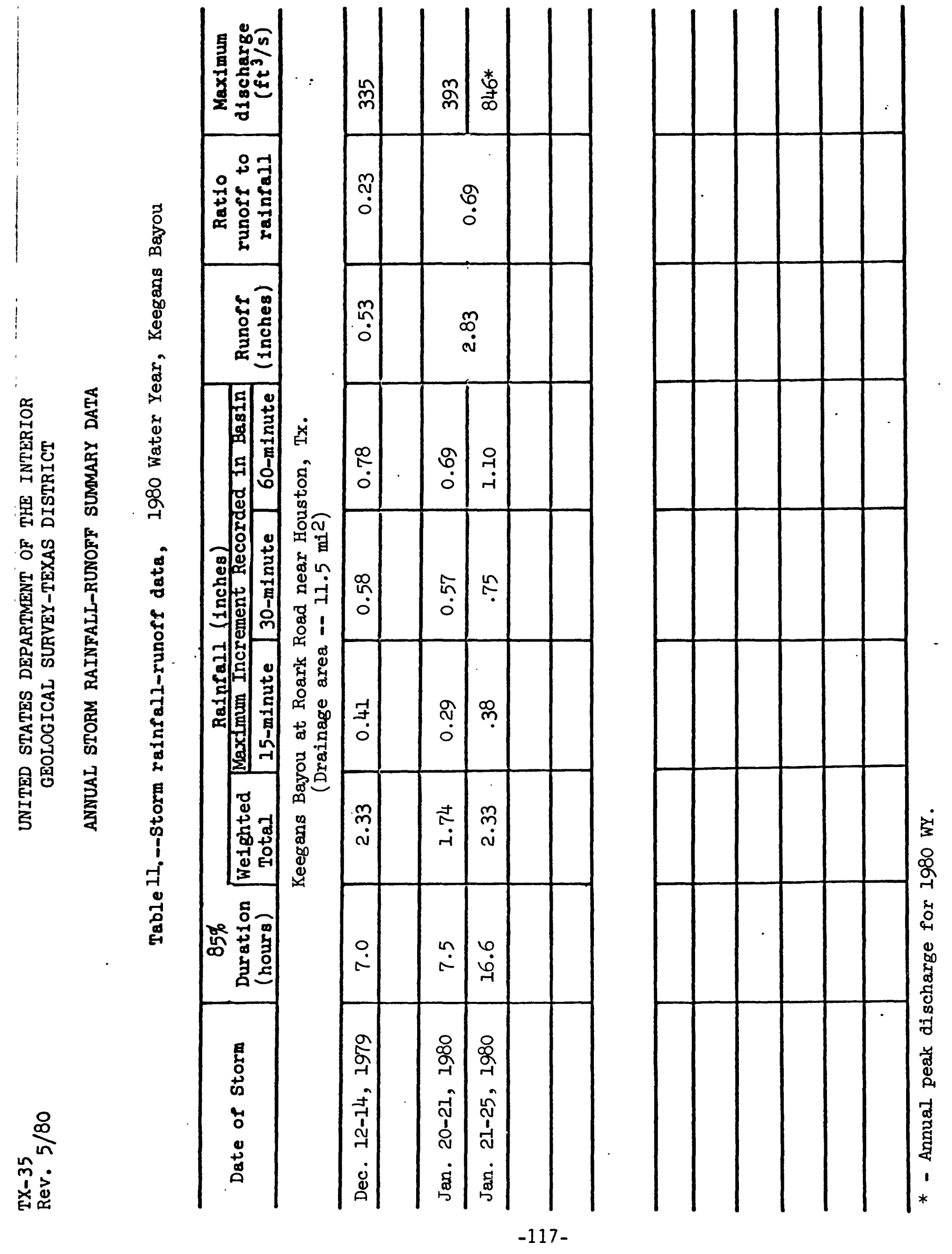


08074780 KEEGANS BAYOU AT KEEGAN ROAD NEAR HOUSTON, TEX.

(Flood-hydrograph partial-record station)

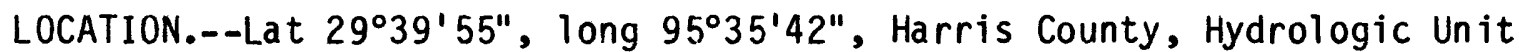
12040104 on downstream side of bridge on Keegan Road, 2.35 miles upstream from station, Keegans Bayou at Roark Road, and about 16 miles southwest of Houston.

DRAINAGE AREA.--7.47 $\mathrm{mi}^{2}$. Prior to Jan. 1, 1978, $7.87 \mathrm{mi}^{2}$. Prior to Oct. 1, $1973,6.93 \mathrm{mi}^{2}$.

PERIOD OF RECORD.--August 1964 to September 1971, August 5, 1974 to current year.

GAGE.--Digital flood-hydrograph and rainfall recorders and crest-stage gage. Prior to April 25, 1978 a flood-hydrograph and rainfall recorder (type SR) and crest-stage gage. Datum of gage is National Geodetic Vertical Datum of 1929,1973 adjustment, unadjusted for land-surface subsidence.

REMARKS.--Records fair.

EXTREMES FOR PERIOD OF RECORD.--Maximum discharge $748 \mathrm{ft}^{3} / \mathrm{s}$, Sept. 19, 1979. (Gage height $78.97 \mathrm{ft}$ ).

Maximum elevation 83.55 ft April 14, 1966, (prior to channel improvement). Minimum discharge not determined.

EXTREMES FOR CURRENT YEAR.--Peak discharge above base of $250 \mathrm{ft}^{3} / \mathrm{s}$, and maximum $(*)$ :

$\begin{array}{cccc}\text { DATE } & \text { TIME } & \begin{array}{c}\text { DISCHARGE } \\ \left(\mathrm{ft}^{3} / \mathrm{s}\right)\end{array} & \begin{array}{c}\text { GAGE HEIGHT } \\ (\mathrm{ft})\end{array} \\ \text { Jan. } 22 & 0730 & * 330 & 74.10\end{array}$

Minimum discharge not determined. 
SAN JACINTO RIVER BASIN

08074800 KEEGANS BAYOU AT ROARK ROAD NEAR HOUSTON, TX

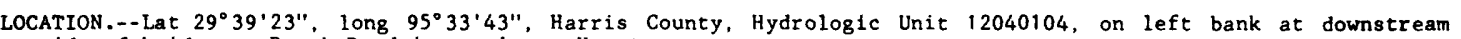
side of bridge on Roark Road in southwest Houston.

DRAINAGE AREA. $-11.5 \mathrm{mi}^{2}\left(29.8 \mathrm{~km}^{2}\right)$. Oct. 1, 1976, to Dec. 31, 1977, $12.0 \mathrm{mi}^{2}\left(31.1 \mathrm{~km}^{2}\right)$; August $1964 \mathrm{ko}^{2}$ Sept. 30, 1976, $11.6 \mathrm{~mL}^{2}\left(30.0 \mathrm{~km}^{2}\right)$. Drainage area changes were the result of ditch relocations or extensions.

WATER-DISCHARGE RECORDS

PERIOD OF RECORD.--August 1964 to current year.

REVISED RECORDS.--WRD TX-74-1: Drainage area. WDR TX-77-2: Drainage area.

GAGE.-Water-stage recorder and crest-stage gage. Datum of gage is National Geodetic Vertical Datum of 1929 . 1957 adjustment; unadjusted for land-surface subsidence.

RDMARKS.--Water-discharge records fair. Recording rain gage at station.

AVERAGE DISCHARGE. --16 years, $12.0 \mathrm{ft}^{3} / \mathrm{s}\left(0.340 \mathrm{~m}^{3} / \mathrm{s}\right), 8.690 \mathrm{acre}-\mathrm{ft} / \mathrm{yr}\left(10.7 \mathrm{hm}^{3} / \mathrm{yr}\right)$.

EXTREMES FOR PERIOD OF RECORD.--Maximum discharge, $1.640 \mathrm{ft}^{3} / \mathrm{s}\left(46.4 \mathrm{~m}^{3} / \mathrm{s}\right)$ Sept. 19 , 1979 (corrected), elevation, $74.54 \mathrm{ft}(22.720 \mathrm{~m})$; no $\mathrm{flow}$ for many days.

EXTREMES FOR CURRENT YEAR.--Peak discharges above base of $400 \mathrm{ft}^{3} / \mathrm{s}\left(11.3 \mathrm{~m}^{3} / \mathrm{s}\right)$ and maximum (*):

\begin{tabular}{|c|c|c|c|c|c|}
\hline & Time & \multicolumn{2}{|c|}{$\begin{array}{c}\text { Discharge } \\
\left(\mathrm{ft}^{3} / \mathrm{s}\right)\left(\mathrm{m}^{3} / \mathrm{s}\right)\end{array}$} & \multicolumn{2}{|c|}{$\underset{(\mathrm{ft})}{\text { Elevation }}(\mathrm{m})$} \\
\hline $\begin{array}{rr}\text { aDec. } & 12 \\
\text { Jan. } & 22 \\
\text { aMay } & 8\end{array}$ & $\begin{array}{l}1330 \\
0800 \\
1530\end{array}$ & $\begin{array}{r}335 \\
\star 846 \\
21\end{array}$ & $\begin{array}{r}9.49 \\
24.0 \\
.59\end{array}$ & $\begin{array}{l}67.01 \\
70.46 \\
62.53\end{array}$ & $\begin{array}{l}20.425 \\
21.476 \\
19.059\end{array}$ \\
\hline
\end{tabular}

Minimum daily discharge, $3.3 \mathrm{ft}^{3} / \mathrm{s}\left(0.093 \mathrm{~m}^{3} / \mathrm{s}\right)$ May 30 .

DISCHARGE, IN CUBIC FEET PER SECOND, WATER YEAR OCTOBER 1979 TO SEPTEMBER 1980

\begin{tabular}{|c|c|c|c|c|c|c|c|c|c|c|c|c|}
\hline DAY & OCT & NOV & DEC & JAN & FEB & MAR & APR & MAY & JUN & JUL & AUG & SEP \\
\hline $\begin{array}{l}1 \\
2 \\
3 \\
4 \\
5\end{array}$ & $\begin{array}{l}6.8 \\
6.3 \\
6.3 \\
5.9 \\
6.2\end{array}$ & $\begin{array}{l}5.9 \\
4.0 \\
4.4 \\
4.9 \\
5.1\end{array}$ & $\begin{array}{l}4.4 \\
4.4 \\
4.3 \\
4.2 \\
4.2\end{array}$ & $\begin{array}{l}4.8 \\
4.5 \\
46 \\
11 \\
6.8\end{array}$ & $\begin{array}{c}6.6 \\
16 \\
7.2 \\
6.5 \\
7.2\end{array}$ & $\begin{array}{l}7.3 \\
5.2 \\
5.2 \\
6.3 \\
5.3\end{array}$ & $\begin{array}{l}7.0 \\
6.1 \\
5.9 \\
4.7 \\
4.9\end{array}$ & $\begin{array}{l}23 \\
7.5 \\
4.6 \\
4.6 \\
4.3\end{array}$ & $\begin{array}{l}4.0 \\
4.1 \\
3.8 \\
3.8 \\
3.7\end{array}$ & $\begin{array}{l}5.2 \\
5.5 \\
5.1 \\
4.9 \\
4.9\end{array}$ & $\begin{array}{l}5.2 \\
4.9 \\
5.0 \\
5.1 \\
5.3\end{array}$ & $\begin{array}{r}5.4 \\
5.9 \\
6.0 \\
7.7 \\
33\end{array}$ \\
\hline $\begin{array}{r}6 \\
7 \\
8 \\
9 \\
10\end{array}$ & $\begin{array}{l}6.0 \\
6.0 \\
6.2 \\
6.3 \\
5.8\end{array}$ & $\begin{array}{l}4.4 \\
4.7 \\
4.7 \\
5.0 \\
4.7\end{array}$ & $\begin{array}{l}4.1 \\
4.1 \\
4.1 \\
4.3 \\
4.4\end{array}$ & $\begin{array}{l}5.8 \\
5.0 \\
4.6 \\
4.5 \\
4.5\end{array}$ & $\begin{array}{l}6.3 \\
5.6 \\
49 \\
24 \\
11\end{array}$ & $\begin{array}{l}4.8 \\
4.7 \\
4.6 \\
4.6 \\
4.8\end{array}$ & $\begin{array}{l}5.2 \\
5.2 \\
4.8 \\
4.4 \\
4.7\end{array}$ & $\begin{array}{l}4.1 \\
6.0 \\
6.8 \\
5.6 \\
4.1\end{array}$ & $\begin{array}{r}3.9 \\
4.0 \\
3.8 \\
130 \\
15\end{array}$ & $\begin{array}{l}4.9 \\
4.8 \\
4.9 \\
5.1 \\
4.9\end{array}$ & $\begin{array}{l}5.6 \\
5.4 \\
5.2 \\
5.3 \\
5.3\end{array}$ & $\begin{array}{r}238 \\
71 \\
44 \\
27 \\
13\end{array}$ \\
\hline $\begin{array}{l}11 \\
12 \\
13 \\
14 \\
15\end{array}$ & $\begin{array}{l}5.6 \\
5.1 \\
5.6 \\
5.7 \\
5.9\end{array}$ & $\begin{array}{l}4.7 \\
4.7 \\
4.7 \\
4.9 \\
4.9\end{array}$ & $\begin{array}{c}4.3 \\
123 \\
30 \\
10 \\
6.9\end{array}$ & $\begin{array}{l}4.5 \\
4.6 \\
4.6 \\
4.6 \\
4.4\end{array}$ & $\begin{array}{l}8.9 \\
8.0 \\
6.8 \\
15 \\
13\end{array}$ & $\begin{array}{l}4.6 \\
4.8 \\
4.3 \\
4.6 \\
6.8\end{array}$ & $\begin{array}{l}4.6 \\
4.8 \\
8.4 \\
5.6 \\
4.8\end{array}$ & $\begin{array}{r}3.9 \\
3.9 \\
5.7 \\
14 \\
4.6\end{array}$ & $\begin{array}{l}6.4 \\
5.5 \\
5.5 \\
5.3 \\
5.4\end{array}$ & $\begin{array}{l}5.0 \\
5.0 \\
6.6 \\
5.3 \\
5.1\end{array}$ & $\begin{array}{r}5.2 \\
5.4 \\
6.0 \\
5.3 \\
23\end{array}$ & $\begin{array}{l}9.0 \\
7.2 \\
6.4 \\
5.3 \\
5.9\end{array}$ \\
\hline $\begin{array}{l}16 \\
17 \\
18 \\
19 \\
20\end{array}$ & $\begin{array}{l}5.7 \\
5.7 \\
5.2 \\
5.2 \\
5.6\end{array}$ & $\begin{array}{l}4.5 \\
4.5 \\
4.7 \\
5.0 \\
5.1\end{array}$ & $\begin{array}{l}5.3 \\
5.0 \\
5.5 \\
4.8 \\
4.3\end{array}$ & $\begin{array}{l}4.8 \\
41 \\
14 \\
8.4 \\
89\end{array}$ & $\begin{array}{l}13 \\
9.5 \\
7.9 \\
6.6 \\
5.9\end{array}$ & $\begin{array}{l}6.6 \\
14.6 \\
5.6 \\
5.8 \\
5.0\end{array}$ & $\begin{array}{l}4.1 \\
4.3 \\
4.2 \\
4.1 \\
4.1\end{array}$ & $\begin{array}{r}6.5 \\
6.4 \\
4.3 \\
93 \\
9.9\end{array}$ & $\begin{array}{l}5.7 \\
6.3 \\
6.3 \\
6.2 \\
6.3\end{array}$ & $\begin{array}{l}4.9 \\
4.7 \\
4.7 \\
4.9 \\
5.2\end{array}$ & $\begin{array}{l}7.4 \\
5.8 \\
5.6 \\
5.5 \\
5.4\end{array}$ & $\begin{array}{l}5.6 \\
5.5 \\
5.5 \\
6.0 \\
5.6\end{array}$ \\
\hline $\begin{array}{l}21 \\
22 \\
23 \\
24 \\
25\end{array}$ & $\begin{array}{l}5.7 \\
7.3 \\
4.8 \\
4.9 \\
4.0\end{array}$ & $\begin{array}{l}55 \\
11 \\
7.2 \\
4.7 \\
4.5\end{array}$ & $\begin{array}{l}4.0 \\
3.9 \\
5.5 \\
6.3 \\
5.1\end{array}$ & $\begin{array}{r}183 \\
403 \\
132 \\
48 \\
16\end{array}$ & $\begin{array}{l}5.8 \\
5.7 \\
5.3 \\
5.2 \\
4.9\end{array}$ & $\begin{array}{l}4.2 \\
4.1 \\
4.8 \\
4.6 \\
4.2\end{array}$ & $\begin{array}{r}4.6 \\
4.6 \\
4.2 \\
3.5 \\
62\end{array}$ & $\begin{array}{l}5.2 \\
4.2 \\
4.8 \\
4.7 \\
4.1\end{array}$ & $\begin{array}{l}5.9 \\
5.7 \\
5.7 \\
5.5 \\
5.4\end{array}$ & $\begin{array}{l}6.5 \\
5.6 \\
5.1 \\
5.0 \\
5.1\end{array}$ & $\begin{array}{l}5.3 \\
5.5 \\
5.8 \\
9.0 \\
6.9\end{array}$ & $\begin{array}{l}7.7 \\
6.3 \\
5.7 \\
5.8 \\
5.6\end{array}$ \\
\hline $\begin{array}{l}26 \\
27 \\
28 \\
29 \\
30 \\
31\end{array}$ & $\begin{array}{l}5.5 \\
4.4 \\
4.5 \\
4.6 \\
37 \\
14\end{array}$ & $\begin{array}{l}4.5 \\
4.3 \\
4.1 \\
4.2 \\
4.3 \\
-\cdot-\end{array}$ & $\begin{array}{r}4.3 \\
4.8 \\
4.8 \\
48 \\
8.0 \\
5.9\end{array}$ & $\begin{array}{l}14 \\
12 \\
8.8 \\
8.0 \\
7.3 \\
7.8\end{array}$ & $\begin{array}{l}4.6 \\
4.7 \\
4.7 \\
4.7 \\
-. . \\
-.-\end{array}$ & $\begin{array}{c}6.4 \\
126 \\
34 \\
110 \\
28 \\
12\end{array}$ & $\begin{array}{l}8.4 \\
5.0 \\
4.9 \\
5.5 \\
6.0 \\
. .-\end{array}$ & $\begin{array}{l}4.1 \\
4.1 \\
4.0 \\
4.0 \\
3.3 \\
3.7\end{array}$ & $\begin{array}{l}5.5 \\
5.2 \\
5.3 \\
5.2 \\
5.1 \\
-.-\end{array}$ & $\begin{array}{c}5.2 \\
5.8 \\
16 \\
7.1 \\
6.1 \\
5.6\end{array}$ & $\begin{array}{l}6.3 \\
5.7 \\
5.5 \\
5.9 \\
5.5 \\
5.3\end{array}$ & $\begin{array}{l}20 \\
36 \\
14 \\
11 \\
84 \\
\ldots\end{array}$ \\
\hline $\begin{array}{l}\text { TOTAL } \\
\text { MEAN } \\
\text { MAX } \\
\text { MIN } \\
\text { AC-FT } \\
(t \dagger)\end{array}$ & $\begin{array}{r}213.8 \\
6.90 \\
37 \\
4.0 \\
424 \\
2.08\end{array}$ & $\begin{array}{r}199.3 \\
6.64 \\
55 \\
4.0 \\
395 \\
1.45\end{array}$ & $\begin{array}{r}342.2 \\
11.0 \\
123 \\
3.9 \\
679 \\
3.62\end{array}$ & $\begin{array}{r}1117.3 \\
36.0 \\
403 \\
4.4 \\
2220 \\
5.80\end{array}$ & $\begin{array}{r}279.6 \\
9.64 \\
49 \\
4.6 \\
555 \\
1.64\end{array}$ & $\begin{array}{r}453.2 \\
14.6 \\
126 \\
4.1 \\
899 \\
3.53\end{array}$ & $\begin{array}{r}210.6 \\
7.02 \\
62 \\
3.5 \\
418 \\
1.39\end{array}$ & $\begin{array}{r}269.0 \\
8.68 \\
93 \\
3.3 \\
534 \\
3.10\end{array}$ & $\begin{array}{r}289.5 \\
9.65 \\
130 \\
3.7 \\
574 \\
1.86\end{array}$ & $\begin{array}{r}174.7 \\
5.64 \\
16 \\
4.7 \\
347 \\
1.10\end{array}$ & $\begin{array}{r}193.6 \\
6.25 \\
23 \\
4.9 \\
384 \\
1.89\end{array}$ & $\begin{array}{r}709.1 \\
23.6 \\
238 \\
5.3 \\
1410 \\
8.27\end{array}$ \\
\hline $\begin{array}{l}\text { CAL YR } \\
\text { WTR YR }\end{array}$ & $\begin{array}{ll}79 & 1 \\
80 & 1\end{array}$ & $\begin{array}{r}1095 \\
445\end{array}$ & $\begin{array}{l}\text { MEAN } \\
\text { MEAN }\end{array}$ & $\begin{array}{l}30.0 \\
12.2\end{array}$ & $\begin{array}{rr}\text { IX } & 1100 \\
\text { ix } & 403\end{array}$ & $\begin{array}{l}\text { MIN } 2.3 \\
\text { MIN } 3.3\end{array}$ & $\begin{array}{l}A C-F T \\
A C-F T\end{array}$ & $\begin{array}{r}21730 \\
8830\end{array}$ & $\begin{array}{ll}t t & 61.3 \\
t+ & 35.7\end{array}$ & & & \\
\hline
\end{tabular}

tt Weighted-mean rainfall, in inches based on four rain gages. 


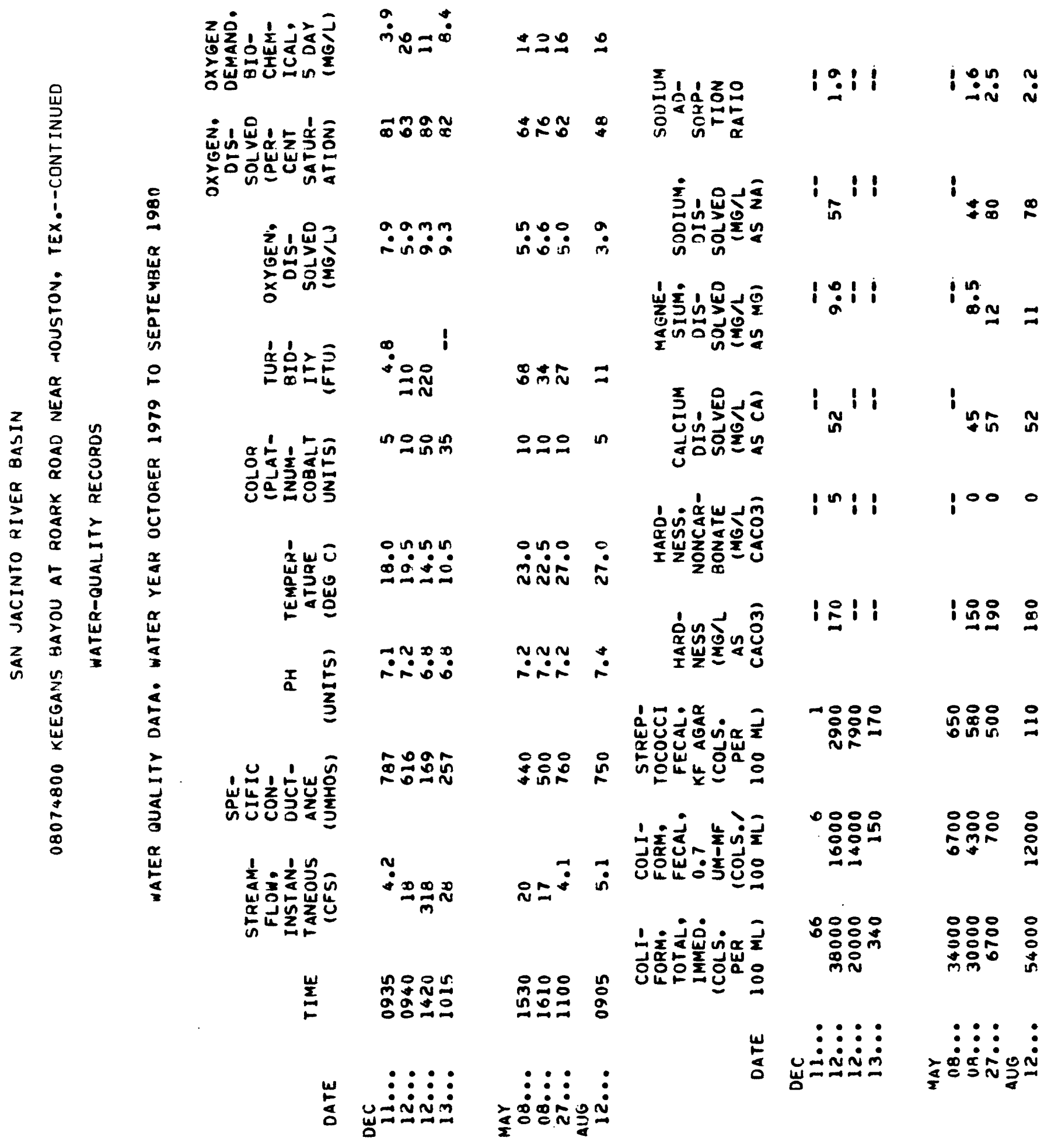




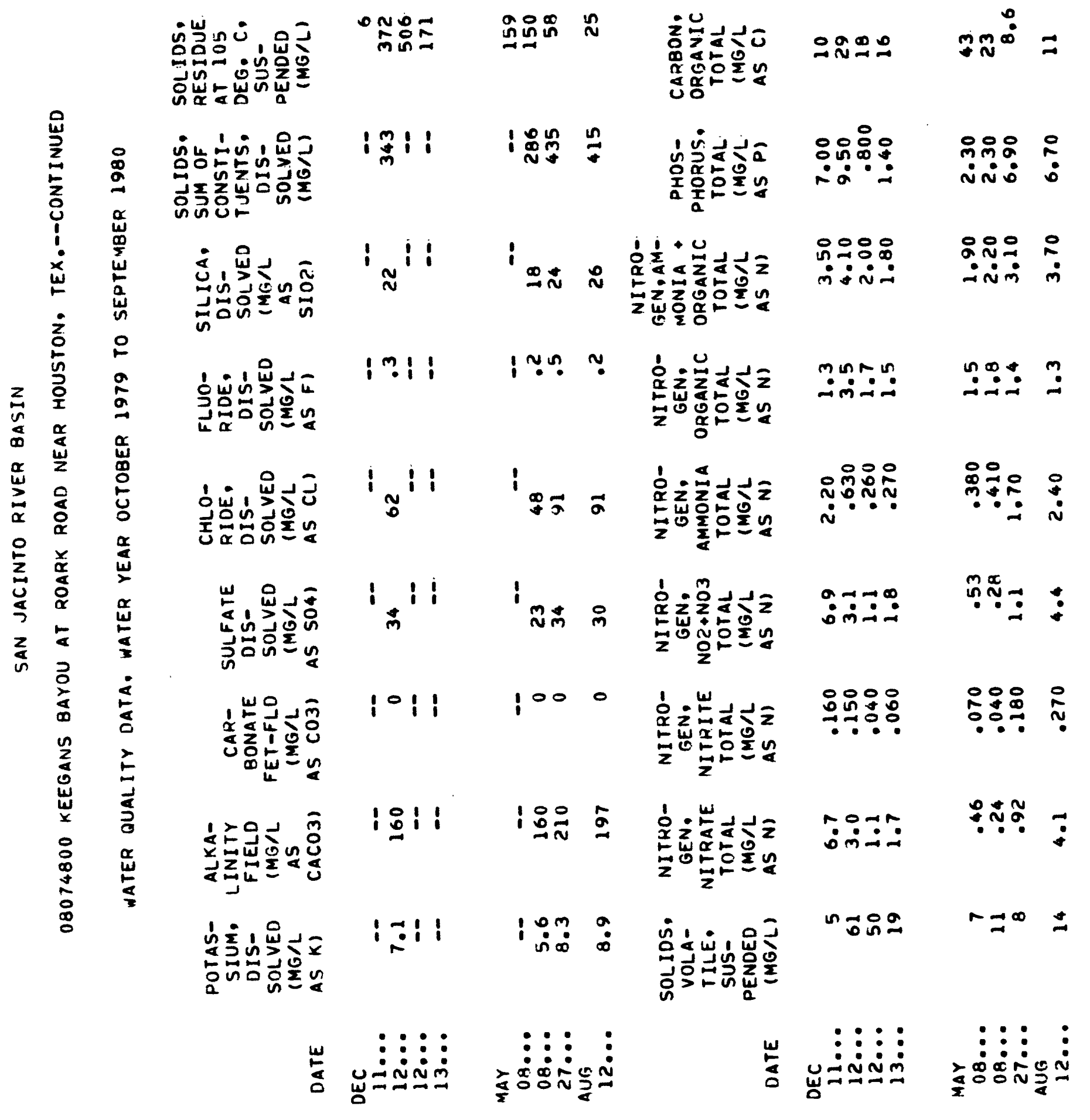




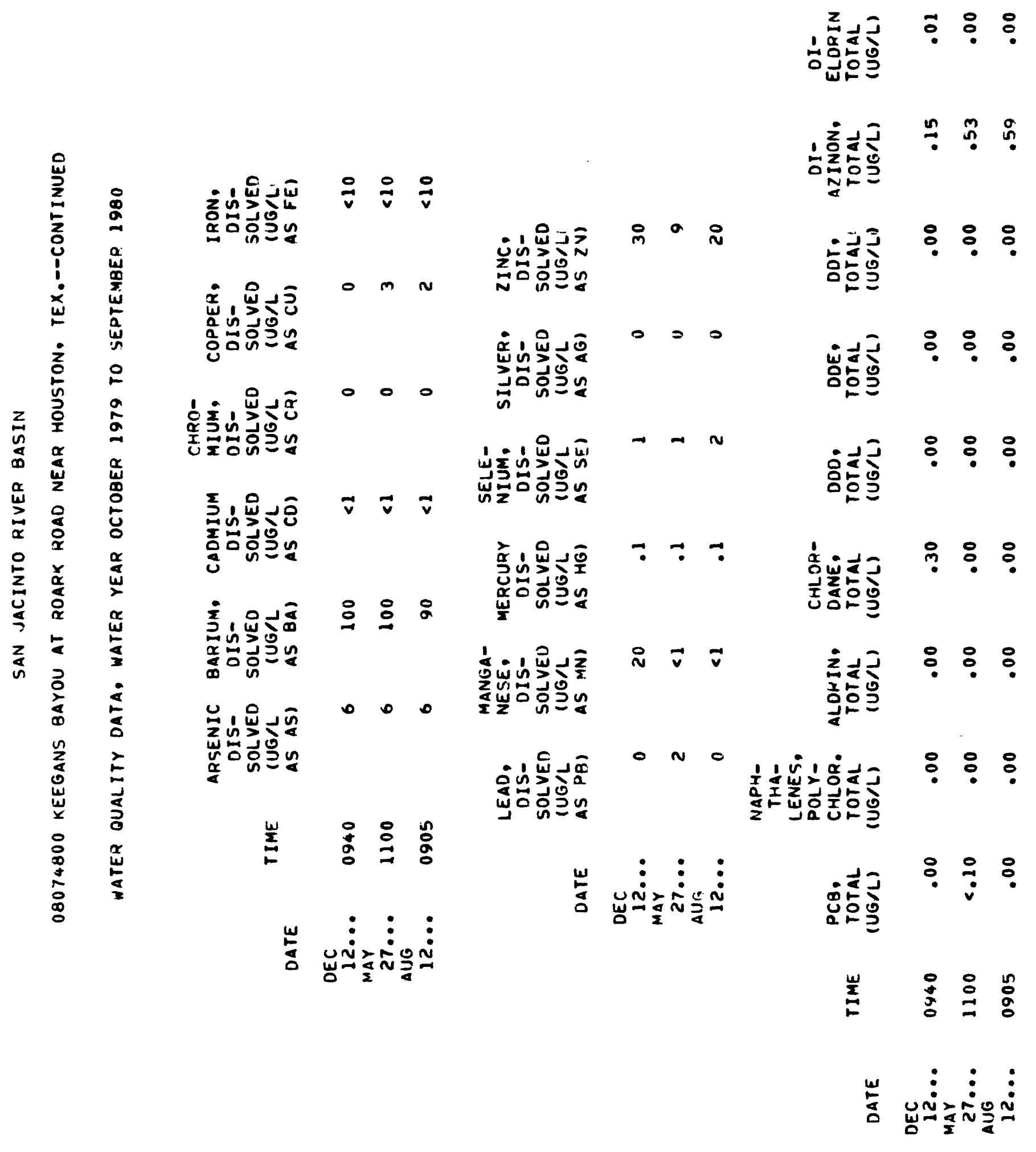




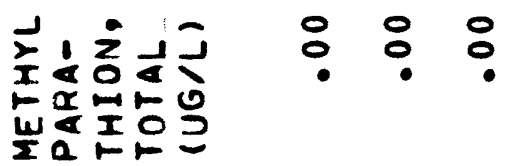

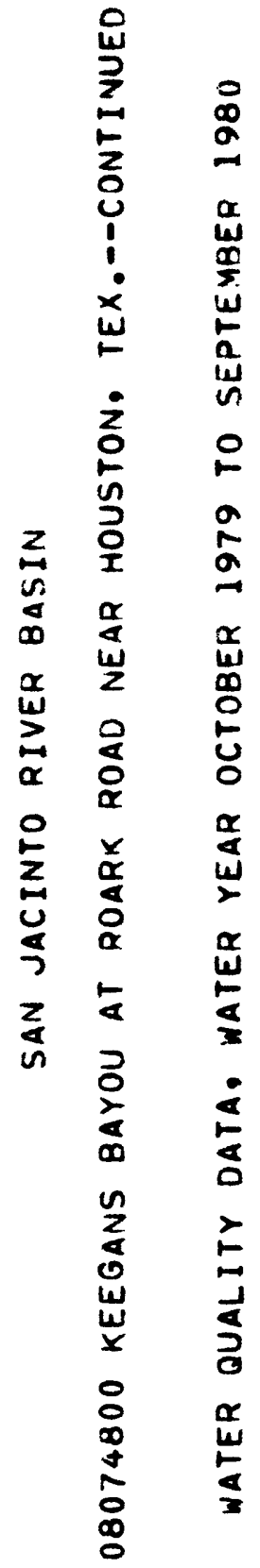

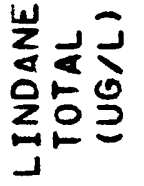
$\underset{0}{0} 0$

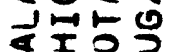

다는

웅 Fx WOISO

岁

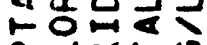
品至员乌

w元00

00 a $-1-0$ 岌宁包引

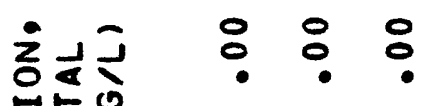
I응

단

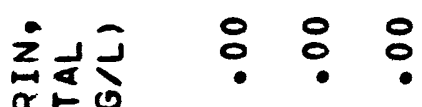

은

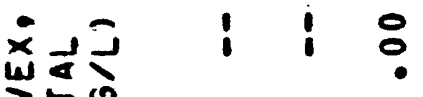

$\underset{n=0}{n=0}$

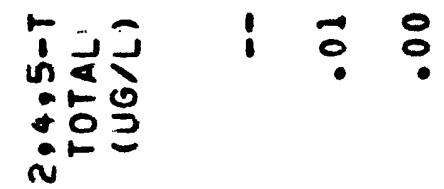

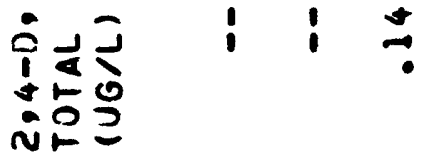
<

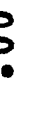
宸过 인ㄴㅇㅇ 立正j

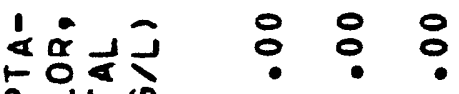

1213

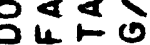

$2 \pm 0=$

$\omega \vec{n}$
1 $\frac{1}{2}$ u $\leq 0$

aIO

121

$\alpha 0<1$

닌

$x \geqslant 1$

w

뚱ㅇ

$\Sigma$ I

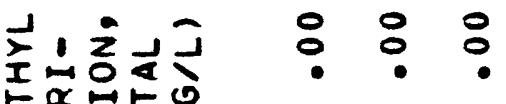
WFIO

00

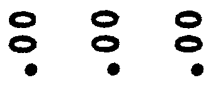$$
\text { 웅 }
$$$$
00
$$

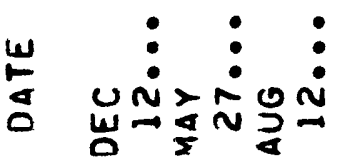

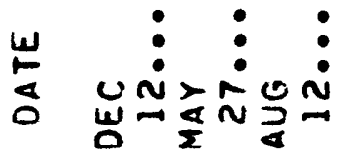




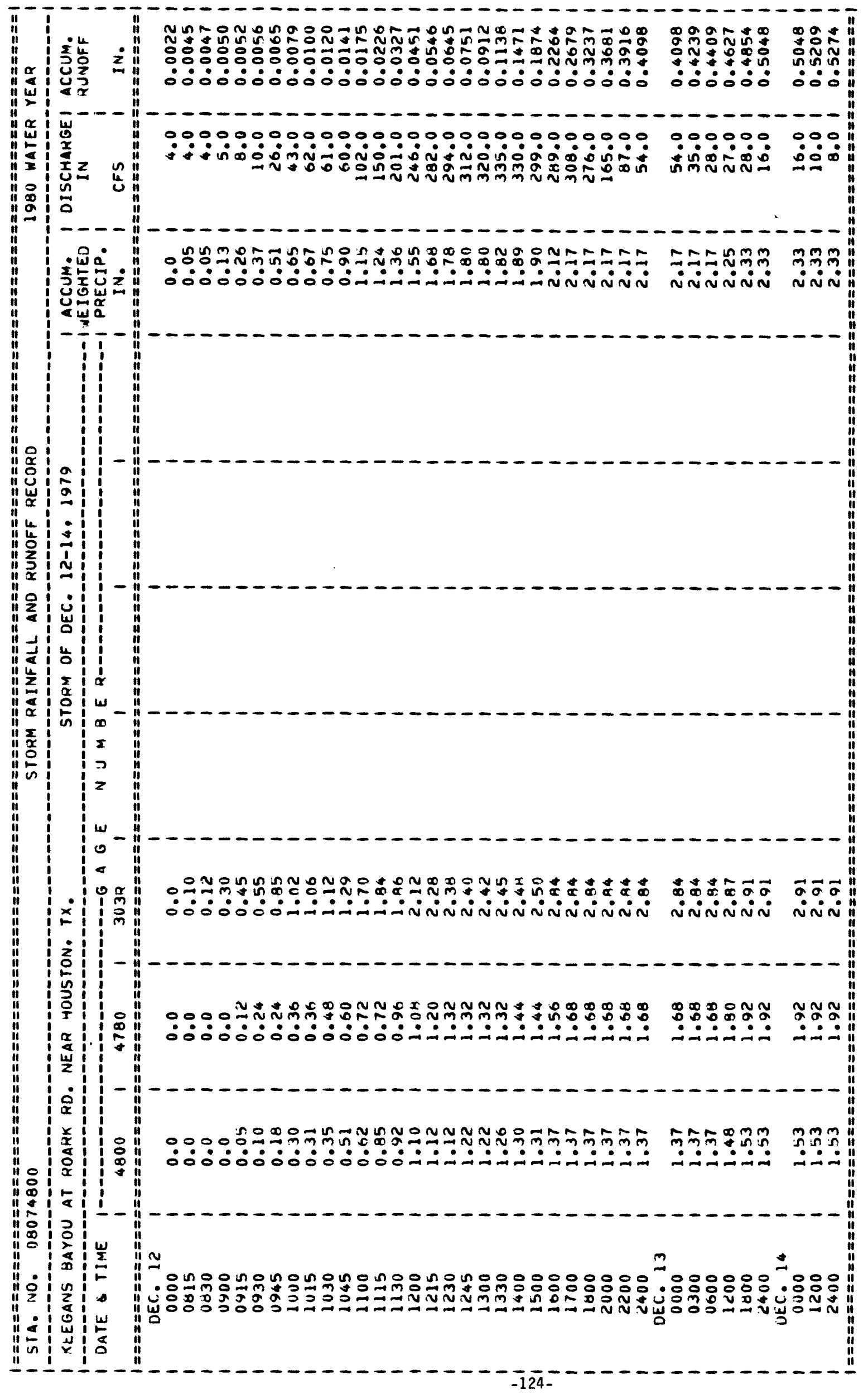




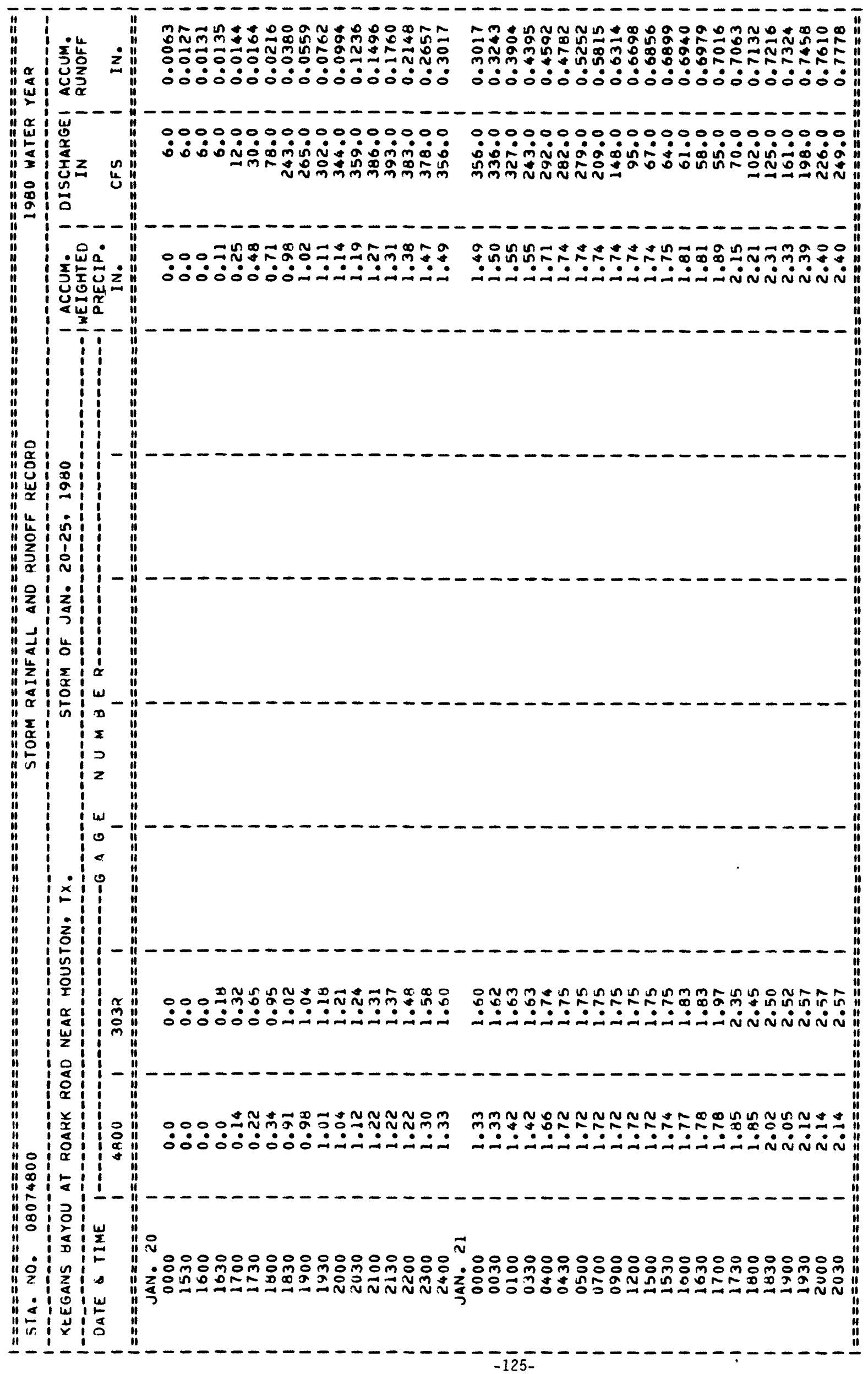




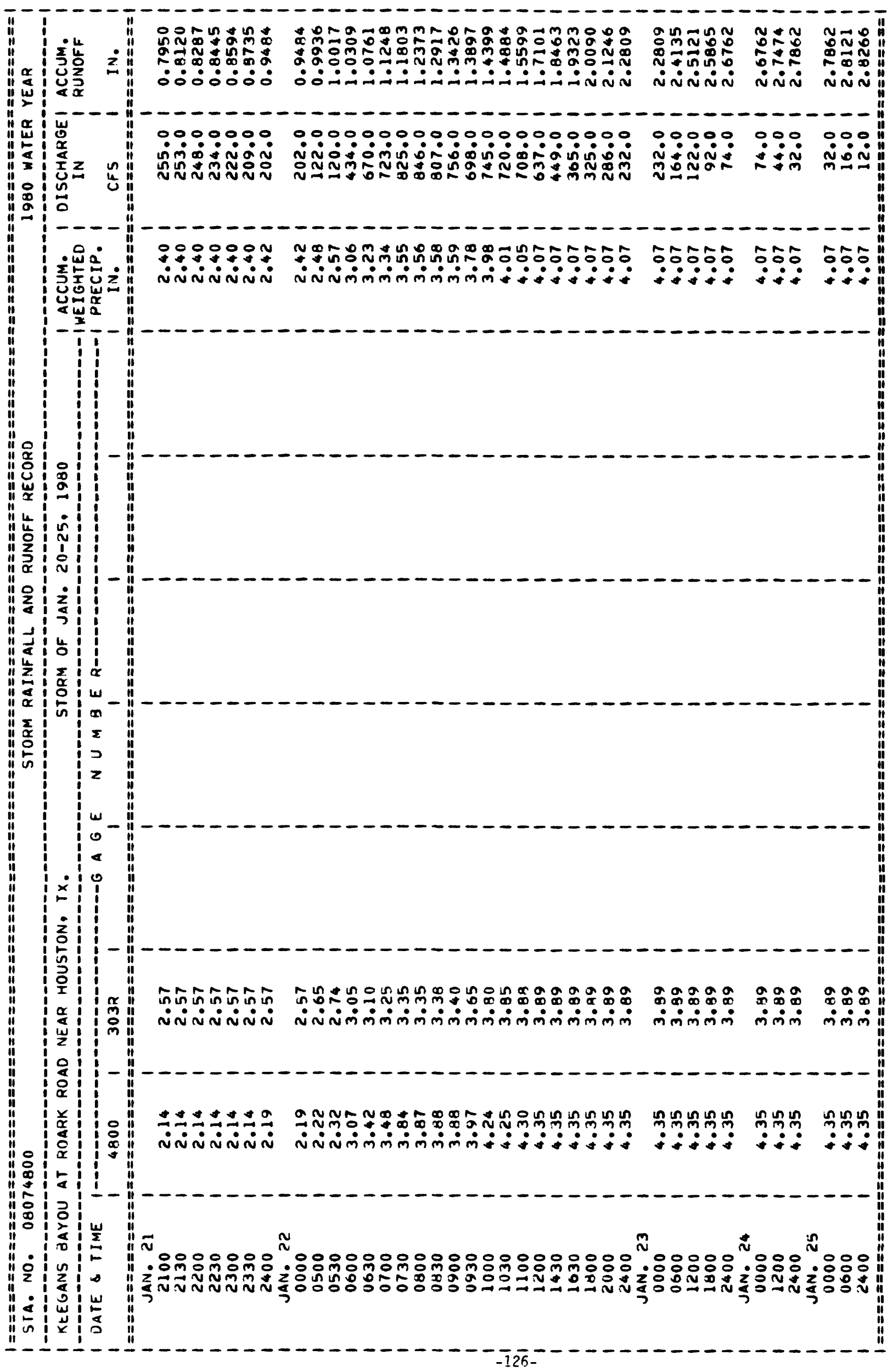


08074810 BRAYS BAYOU AT GESSNER DRIVE, HOUSTON, TEX.

(Flood-hydrograph partial-record station)

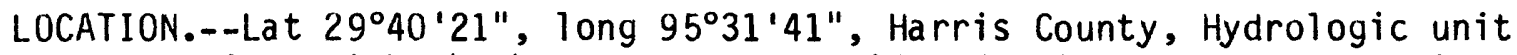
12040104 on right bank on downstream side of bridge at Gessner Drive in southwest Houston.

DRAINAGE AREA (revised).--53.2 $\mathrm{mi}^{2}$. Prior to Jan. 1, 1978, $51.7 \mathrm{mi}^{2}$.

PERIOD OF RECORD.--Feb. 1, 1977 to current year.

GAGE.--Digital flood-hydrograph recorder and crest-stage gage. Datum of gages is National Geodetic Vertical Datum of 1929, 1964 adjustment, unadjusted for 1 and-surface subsidence.

REMARKS.--Records good.

EXTREMES FOR PERIOD OF RECORD.--Maxinum discharge $11,300 \mathrm{ft}^{3} / \mathrm{s}$, Sept. 19, 1979 (elevation $59.21 \mathrm{ft}$ ); minimum discharge not determined.

EXTREMES FOR CURRENT YEAR.--Peak discharge above base of $2,200 \mathrm{ft}^{3} / \mathrm{s}$ and maximum $(*)$ :

DATE TIME DISCHARGE

Jan. 22

0845

Minimum dischrge not determined.

$$
\left(\mathrm{ft}^{3} / \mathrm{s}\right)
$$

GAGE HEIGHT

53.22

$\star_{4370}$ 


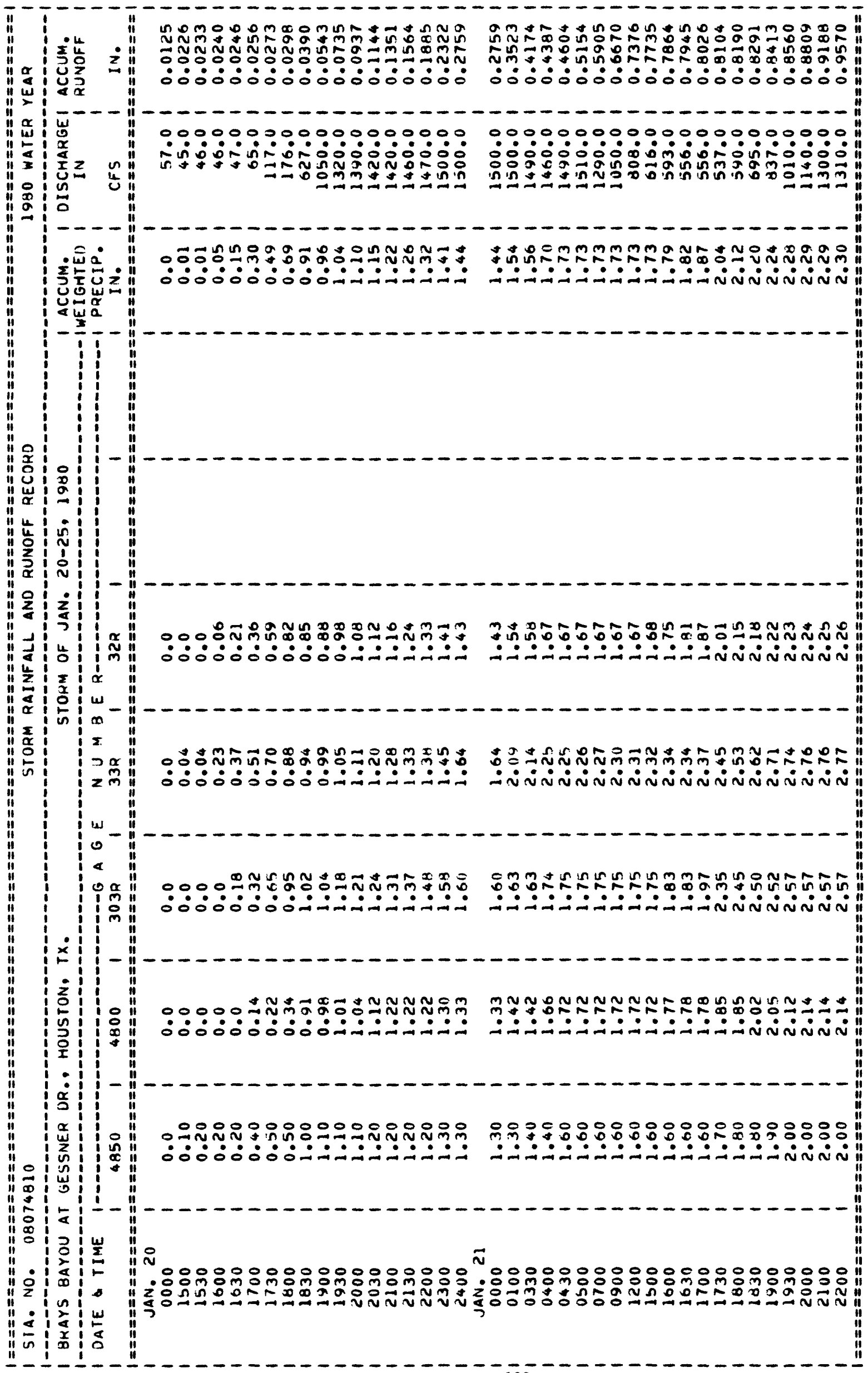

$-128-$ 


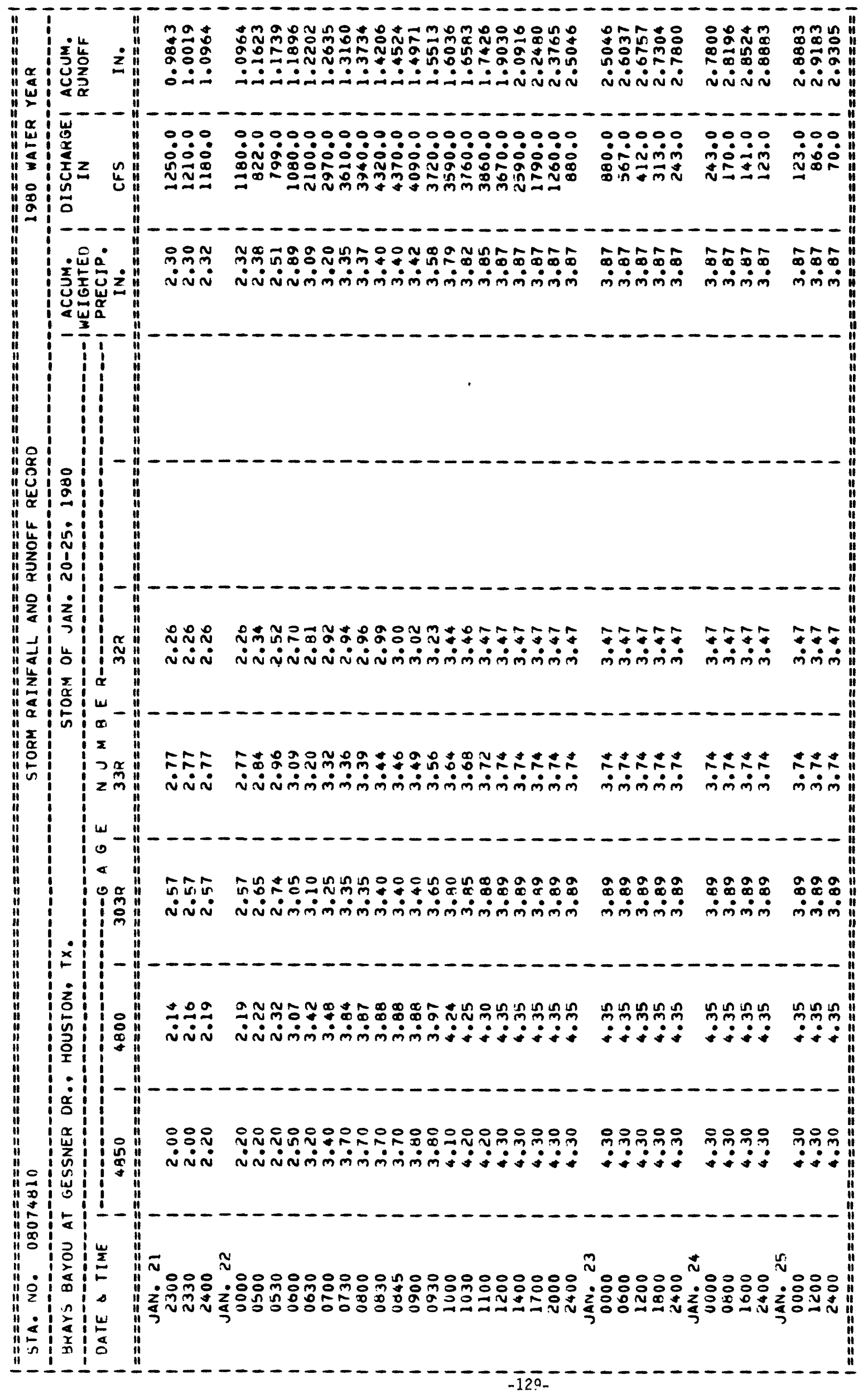




\section{BINTLIFF DITCH DRAINAGE BASIN}

The location of data-collection sites in and near the Bintliff Ditch drainage basin are shown in figure 13. The gage was removed Jan. 31, 1980 to allow for construction of a new bridge.

Weighted-mean rainfall for the 1980 water year was not determined.

The storm of Jan. 20-22 was selected for analysis at station 08074850 , Bintliff Ditch at Bissonnet Street, Houston, Tex. 


\section{EXPLANATION}

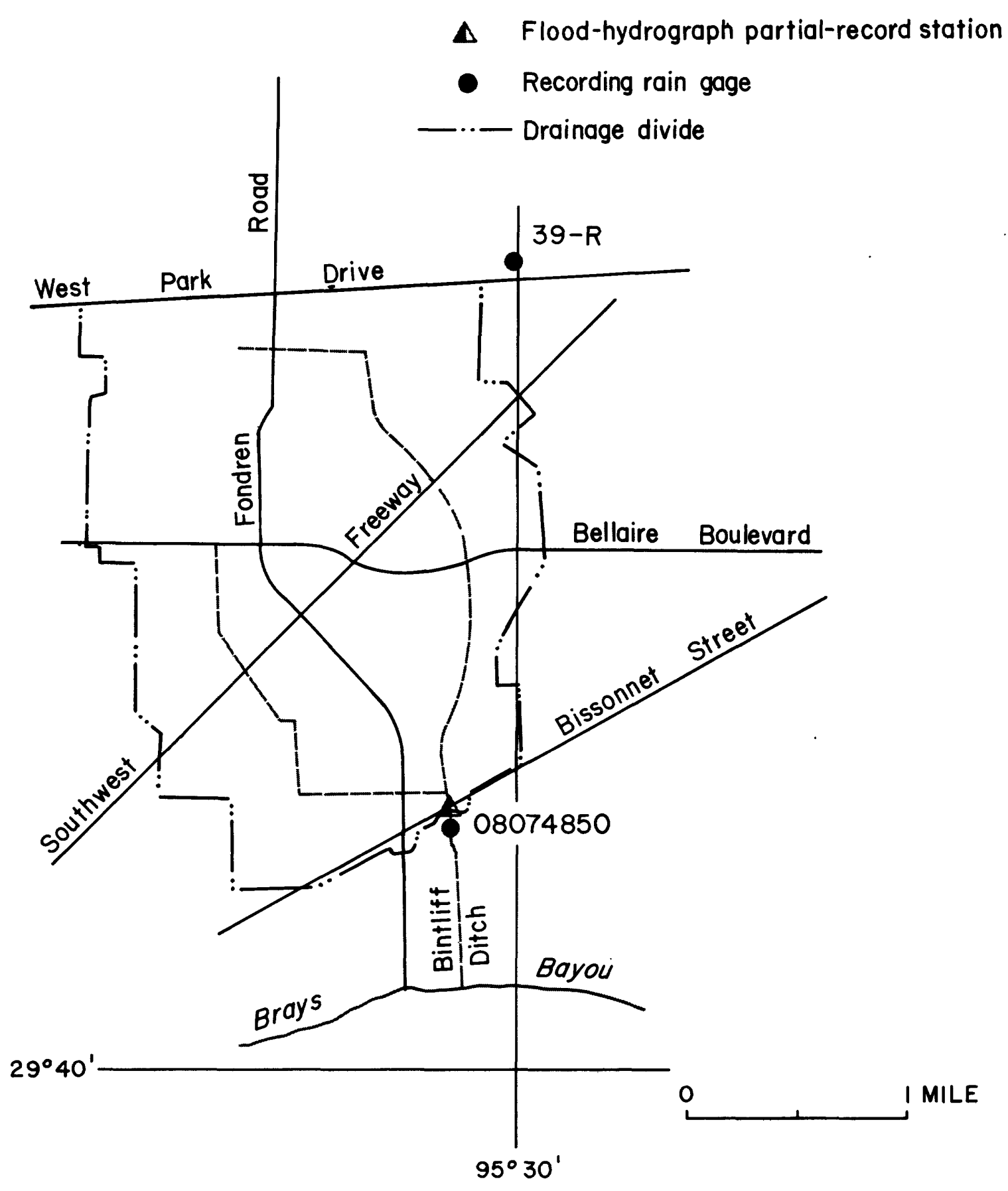

Base from Enco Street map of Houston

Figure 13. - Locations of data-collection sites in and near the Bintliff Ditch drainage basin 


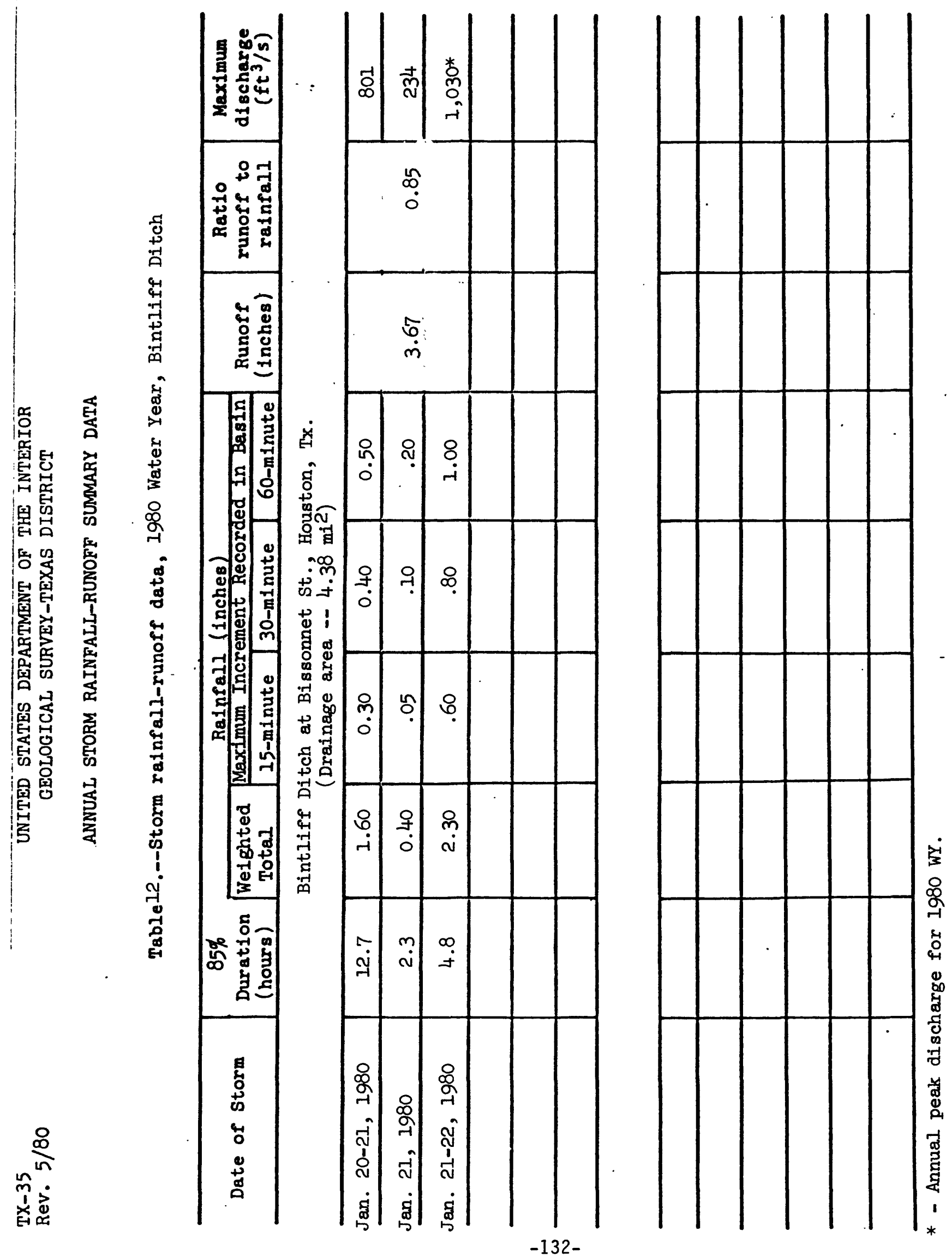


08074850 Bintliff Ditch at Bissonnet Street, Houston, Tex. (Flood-hydrograph partial-record station)

LOCATION.--Lat $29^{\circ} 41^{\prime} 16^{\prime \prime}$, long $95^{\circ} 30^{\prime} 20^{\prime \prime}$, Harris County, Hydrologic Unit 12040104 , downstream side of bridge on Bissonnet Street, in southwest Houston, Tex.

DRAINAGE AREA.--4.38 $\mathrm{mi}^{2}$. Prior to October $1,1973,4.29 \mathrm{mi}^{2}$.

PERIOD OF RECORD.--August 1968 to January 30, 1980. Ga ged was removed January 30,1980 to al low new bridge to be constructed.

GAGE.--Digital flood-hydrograph and rainfall recorders and crest-stage gage. Prior to Mar. 29, 1978 flood-hydrograph and rainfall recorder (type SR) and crest-stage gage. Daturn of gage is National Geodetic Vertical Datum of 1929, 1964 adjustment, unadjusted for land-surface subsidence.

REMARKS.--Records fair.

EXTREMES FOR PERIOD OF RECORD.--Maximum discharge $1,280 \mathrm{ft}^{3} / \mathrm{s}$, (revised), June 15, 1976 (elevation $63.19 \mathrm{ft}$ ). Minimum not determined.

EXTREMES FOR CURRENT YEAR.--Peak discharge above base of $900 \mathrm{ft}^{3} / \mathrm{s}$ (revised) and maximum $(*)$ :

DATE TIME DISCHARGE

$\left(\mathrm{ft}^{3} / \mathrm{s}\right)$

Jan. 22

0635

$\star 1,030$

Minimum discharge not determined.
GAGE HEIGHT

61.50 


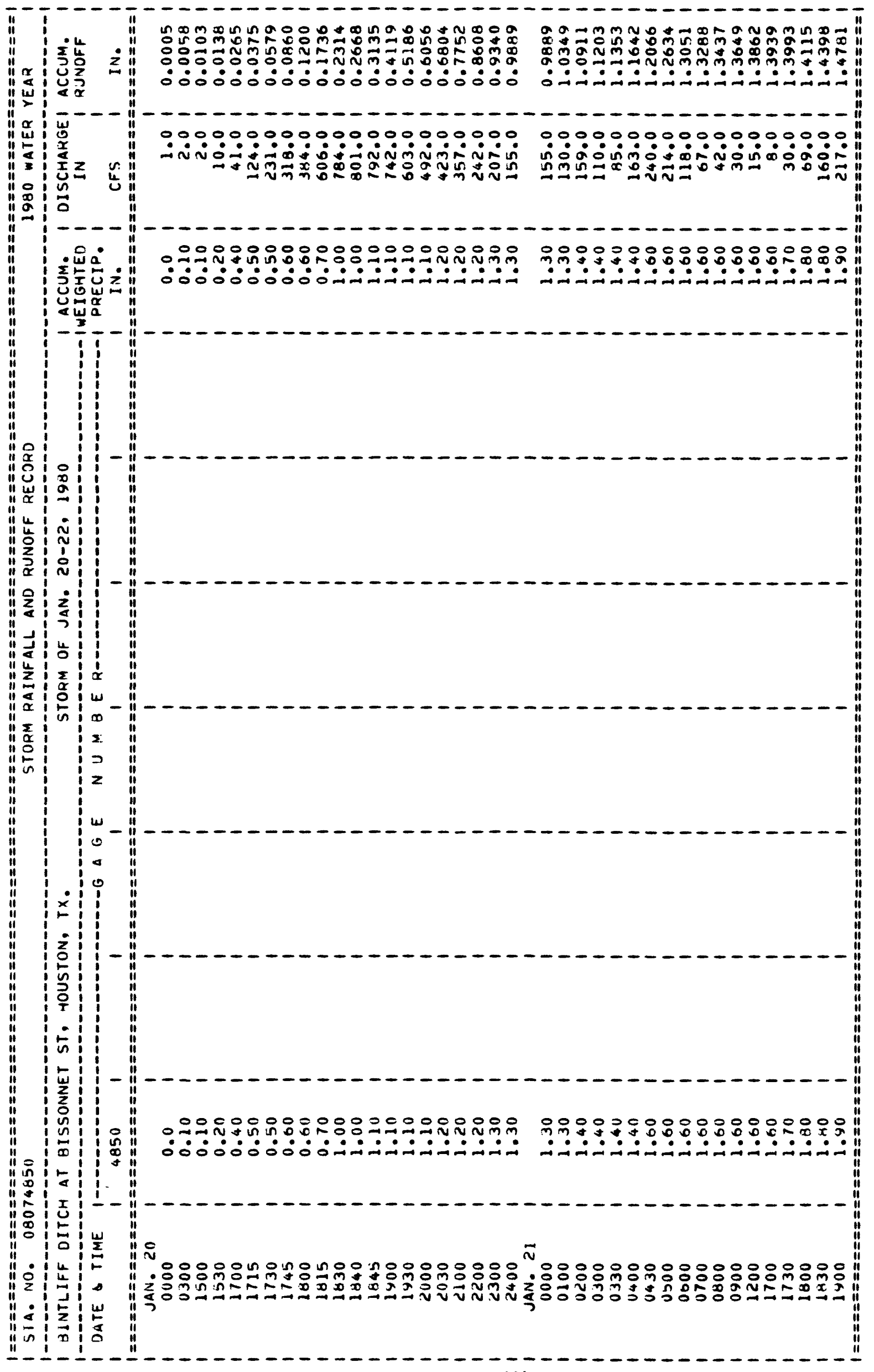

$-134$. 


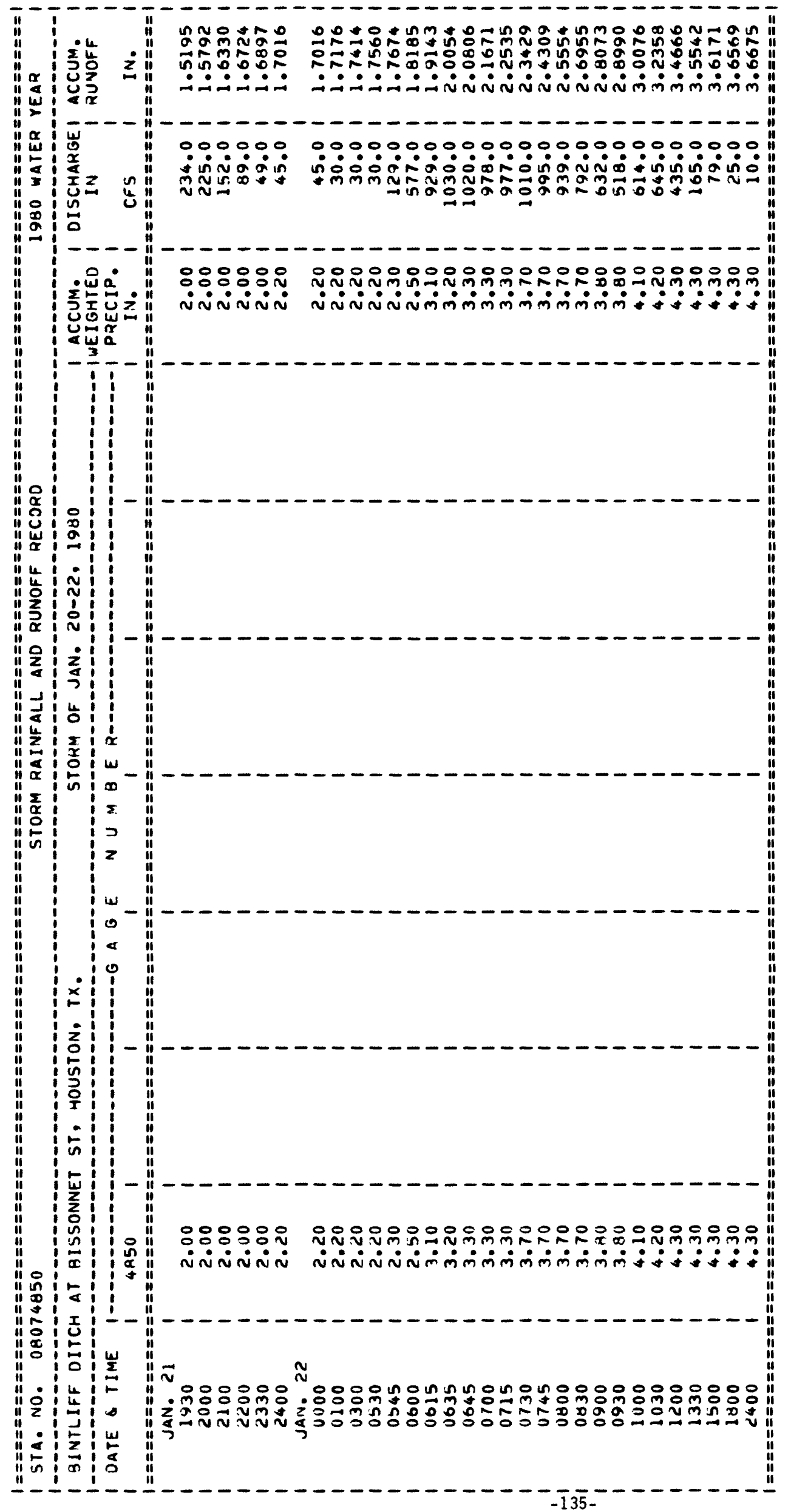




\section{HUMMINGBIRD STREET DITCH DRAINAGE BASIN}

The location of data-collection sites in the Hummingbird Street Ditch drainage basin are shown in figure 14.

Weighted-mean rainfall for the 1980 water year was not determined.

No storms were analyzed for the 1980 water year. 


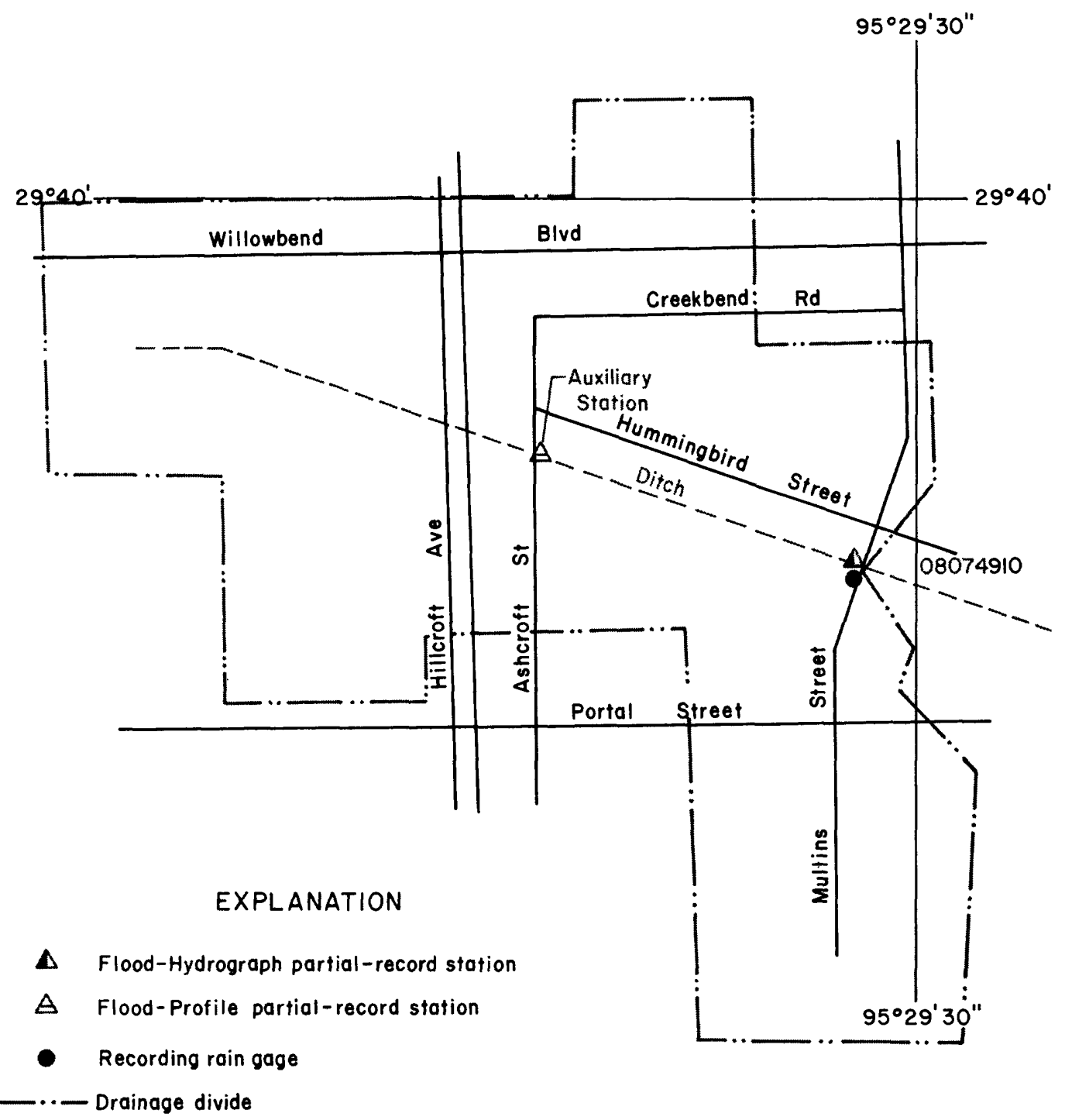

0 .25 .5 MILE

Figure 14.-Locations of data-collection sites in and near the Hummingbird Street Ditch drainage basin 


\section{HUMMINGBIRD STREET DITCH AT HOUSTON, TEX. \\ (Flood-hydrograph partial-record station)}

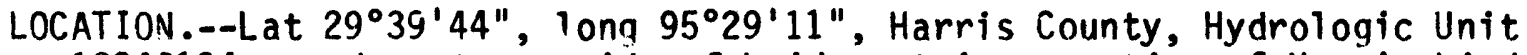
12040104 , at downstream side of bridge at intersection of Humminabird Street Ditch and Mullins Street in southwest Houston.

DRAINAGE AREA.--0.32 $\mathrm{mi}^{2}$.

PERIOD OF RECORD.--Nov. 3, 1978 to current year.

GAGE.--Digital flood-hydrograph and rainfall recorders and crest-stage qage. Datum of gage is National Geodetic Vertical Datum of 1924, 1973 adjustment, unadjusted for land-surface subsidence.

REMARKS.--Records poor. Heavy vegetal growth makes a stage-discharae relationship difficult to define.

EXTREMES FOR PERIOD OF RECORD.--Maximum discharge $149 \mathrm{ft}^{3} / \mathrm{s}$ Apr. 19, 1979; maximum gage-height, $59.31 \mathrm{ft}$, June 2, 1979; no flow for many days.

EXTREMES FOR CURRENT YEAR.--Peak discharge above base of $75 \mathrm{ft}^{3} / \mathrm{s}$ and maximum $(*):$

DATE

TIME

DISCHARGE

$\left(\mathrm{ft}^{3} / \mathrm{s}\right)$

GAGE HEIGHT

$(\mathrm{ft})$

Jan. 22

unknown

$\star 98$

57.35

No flow for many days. 
SAN JACINTO RIVER BASIN

08075000 BRAYS BAYOU AT HOUSTON, TX

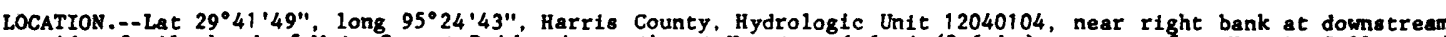
side of pile bend of Main Street Bridge in southwest Houston, $1.6 \mathrm{mi}$ (2.6 km) upstream from Harris Gully, and $11.6 \mathrm{mi}(18.7 \mathrm{~km})$ upstream from Buffalo Bayou.

DRAINAGE AREA.--94.9 $\mathrm{ms}^{2}\left(245.8 \mathrm{~km}^{2}\right)$. Prior to October $1976,88.4 \mathrm{mi}^{2}\left(229.0 \mathrm{~km}^{2}\right)$. Changes due to drainage ditch relocations.

WATER-DISCHARGE RECORDS

PERIOD OF RECORD.--May 1936 to current year.

REVISED RECORDS.--WSP 1732: Drainage area.

GAGE.--Water-stage recorder. Datum of gage $1 \mathrm{~s} 7.16 \mathrm{ft}(2.182 \mathrm{~m})$ National Geodetic Vertical Datum of 1929 , 197 adjustment; unadjusted for land-surface subsidence. Prior to June 20, 1936, nonrecording gage, and June 20, 1936, to Nov. 25, 1959, water-stage recorder at site $0.8 \mathrm{mi}(1.3 \mathrm{~km})$ downstream at same datum.

RDMARKS.-Water-discharge records good. No diversion above station. Low flow is mostly sewage effluent from Houston suburbs.

AVERAGE DISCHARGE, --44 years, $116 \mathrm{ft}^{3} / \mathrm{s}\left(3.285 \mathrm{~m}^{3} / \mathrm{s}\right), 84,040 \mathrm{acre}-\mathrm{ft} / \mathrm{yr}(104 \mathrm{hm} / \mathrm{yr})$.

EXTREMES FOR PERIOD OF RECORD.--Maximum discharge, 29,000 $\mathrm{ft} / \mathrm{s}\left(821 \mathrm{~m}^{3} / \mathrm{s}\right)$ June 15, 1976 , gage height, 52.13 ft

$(15.889 \mathrm{~m})$; minimum dally, $0.1 \mathrm{ft} / \mathrm{s}\left(0.003 \mathrm{~m}^{3} / \mathrm{s}\right)$ Oct. 11, 12, 1937, Mar. 14, Apr. 1. 1958. Maximum discharge, that of June 15 , 1976 .

EXTRDMES OUTSIDE PERIOD OF RECORD.--Maximum stage since $1911,56.0 \mathrm{ft}(17.07 \mathrm{~m})$ in June $1919 \mathrm{before}$ channel rectification, former site, from information by engineer for city of Houston.

EXTREMES FOR CURRENT YEAR,--Peak discharges above base of $6.000 \mathrm{ft}^{3} / \mathrm{s}\left(170 \mathrm{~m}^{3} / \mathrm{s}^{\circ} \text { and maximum ( }\right)^{\circ}$ :

\begin{tabular}{|c|c|c|c|c|c|}
\hline Date & Time & $\begin{array}{c}\text { D1 } 8 \mathrm{cl} \\
\left(\mathrm{ft}^{3} / 8\right)\end{array}$ & $\underset{\left(\mathrm{m}^{3} / 8\right)}{\operatorname{sige}}$ & $\begin{array}{c}\text { Gage } \\
(\mathrm{ft})\end{array}$ & $\begin{array}{l}\text { height } \\
\text { (m) }\end{array}$ \\
\hline $\begin{aligned} & \text { Oct. } 30 \\
& \text { Dec. } 12 \\
& \text { Jan. } 22\end{aligned}$ & $\begin{array}{l}1815 \\
1315 \\
1045\end{array}$ & $\begin{array}{r}6,390 \\
3,100 \\
* 11,300\end{array}$ & $\begin{array}{l}181 \\
87.8 \\
320\end{array}$ & $\begin{array}{l}36.04 \\
32.13 \\
40.66\end{array}$ & $\begin{array}{r}10.985 \\
9.793 \\
12.393\end{array}$ \\
\hline
\end{tabular}

$$
\begin{aligned}
& \text { a Water-quality samples were obtained during } \\
& \text { this runoff event. }
\end{aligned}
$$

Minimum datjy discharge, $78 \mathrm{ft}^{\prime} / \mathrm{s}\left(2.21 \mathrm{~m}^{3} / \mathrm{s}\right)$ June 14 .

DISCHARGE, IN CUBIC FEET PER SECOND, WATER YEAR OCTOBER 1979 TO SEPTEMBER 1980

\begin{tabular}{|c|c|c|c|c|c|c|c|c|c|c|c|c|}
\hline DAY & OCT & Nov & DEC & JAN & FEB & MAR & APR & MAY & JUN & JUL & AUG & SEP \\
\hline $\begin{array}{l}1 \\
2 \\
3 \\
4 \\
5\end{array}$ & $\begin{array}{l}119 \\
118 \\
117 \\
115 \\
110\end{array}$ & $\begin{array}{r}127 \\
103 \\
100 \\
97 \\
97\end{array}$ & $\begin{array}{r}99 \\
100 \\
99 \\
98 \\
93\end{array}$ & $\begin{array}{l}120 \\
100 \\
600 \\
200 \\
150\end{array}$ & $\begin{array}{l}100 \\
140 \\
120 \\
105 \\
120\end{array}$ & $\begin{array}{r}195 \\
97 \\
94 \\
115 \\
96\end{array}$ & $\begin{array}{r}125 \\
107 \\
105 \\
96 \\
91\end{array}$ & $\begin{array}{r}244 \\
192 \\
101 \\
88 \\
81\end{array}$ & $\begin{array}{l}84 \\
85 \\
84 \\
82 \\
82\end{array}$ & $\begin{array}{l}87 \\
91 \\
92 \\
92 \\
96\end{array}$ & $\begin{array}{l}111 \\
109 \\
105 \\
109 \\
248\end{array}$ & $\begin{array}{l}119 \\
198 \\
122 \\
110 \\
424\end{array}$ \\
\hline $\begin{array}{r}6 \\
7 \\
8 \\
9 \\
10\end{array}$ & $\begin{array}{l}110 \\
108 \\
105 \\
101 \\
100\end{array}$ & $\begin{array}{r}97 \\
91 \\
92 \\
103 \\
92\end{array}$ & $\begin{array}{l}89 \\
88 \\
93 \\
92 \\
88\end{array}$ & $\begin{array}{r}130 \\
120 \\
110 \\
105 \\
98\end{array}$ & $\begin{array}{l}110 \\
100 \\
405 \\
528 \\
193\end{array}$ & $\begin{array}{l}88 \\
89 \\
90 \\
88 \\
91\end{array}$ & $\begin{array}{r}99 \\
107 \\
93 \\
84 \\
83\end{array}$ & $\begin{array}{r}82 \\
129 \\
155 \\
117 \\
85\end{array}$ & $\begin{array}{r}83 \\
83 \\
83 \\
750 \\
232\end{array}$ & $\begin{array}{r}99 \\
104 \\
103 \\
102 \\
102\end{array}$ & $\begin{array}{l}114 \\
209 \\
141 \\
115 \\
102\end{array}$ & $\begin{array}{r}1810 \\
517 \\
579 \\
240 \\
137\end{array}$ \\
\hline $\begin{array}{l}11 \\
12 \\
13 \\
14 \\
15\end{array}$ & $\begin{array}{l}98 \\
93 \\
90 \\
88 \\
88\end{array}$ & $\begin{array}{l}90 \\
90 \\
91 \\
90 \\
93\end{array}$ & $\begin{array}{r}88 \\
1070 \\
475 \\
180 \\
135\end{array}$ & $\begin{array}{l}94 \\
92 \\
90 \\
95 \\
90\end{array}$ & $\begin{array}{l}127 \\
109 \\
103 \\
243 \\
309\end{array}$ & $\begin{array}{l}94 \\
92 \\
79 \\
81 \\
98\end{array}$ & $\begin{array}{r}85 \\
94 \\
264 \\
116 \\
97\end{array}$ & $\begin{array}{r}80 \\
81 \\
117 \\
258 \\
103\end{array}$ & $\begin{array}{r}102 \\
90 \\
84 \\
78 \\
80\end{array}$ & $\begin{array}{l}100 \\
102 \\
102 \\
106 \\
104\end{array}$ & $\begin{array}{r}109 \\
106 \\
102 \\
99 \\
290\end{array}$ & $\begin{array}{r}112 \\
106 \\
102 \\
99 \\
107\end{array}$ \\
\hline $\begin{array}{l}16 \\
17 \\
18 \\
19 \\
20\end{array}$ & $\begin{array}{r}89 \\
89 \\
89 \\
89 \\
101\end{array}$ & $\begin{array}{l}93 \\
91 \\
91 \\
92 \\
93\end{array}$ & $\begin{array}{r}108 \\
95 \\
90 \\
86 \\
84\end{array}$ & $\begin{array}{r}85 \\
742 \\
292 \\
143 \\
882\end{array}$ & $\begin{array}{l}245 \\
160 \\
130 \\
120 \\
110\end{array}$ & $\begin{array}{r}114 \\
227 \\
98 \\
90 \\
97\end{array}$ & $\begin{array}{l}92 \\
83 \\
84 \\
81 \\
82\end{array}$ & $\begin{array}{l}189 \\
206 \\
122 \\
842 \\
184\end{array}$ & $\begin{array}{l}85 \\
80 \\
81 \\
82 \\
81\end{array}$ & $\begin{array}{l}107 \\
103 \\
102 \\
103 \\
110\end{array}$ & $\begin{array}{l}220 \\
115 \\
107 \\
102 \\
106\end{array}$ & $\begin{array}{r}104 \\
100 \\
97 \\
110 \\
105\end{array}$ \\
\hline $\begin{array}{l}21 \\
22 \\
23 \\
24 \\
25\end{array}$ & $\begin{array}{r}108 \\
147 \\
96 \\
91 \\
89\end{array}$ & $\begin{array}{r}725 \\
257 \\
188 \\
103 \\
97\end{array}$ & $\begin{array}{r}82 \\
80 \\
120 \\
250 \\
120\end{array}$ & $\begin{array}{r}2150 \\
4880 \\
803 \\
300 \\
250\end{array}$ & $\begin{array}{l}105 \\
100 \\
100 \\
100 \\
133\end{array}$ & $\begin{array}{r}88 \\
85 \\
105 \\
97 \\
83\end{array}$ & $\begin{array}{r}82 \\
85 \\
83 \\
82 \\
723\end{array}$ & $\begin{array}{r}109 \\
91 \\
87 \\
90 \\
82\end{array}$ & $\begin{array}{r}121 \\
143 \\
142 \\
90 \\
84\end{array}$ & $\begin{array}{l}342 \\
156 \\
122 \\
110 \\
110\end{array}$ & $\begin{array}{r}98 \\
98 \\
97 \\
130 \\
122\end{array}$ & $\begin{array}{r}105 \\
99 \\
95 \\
91 \\
98\end{array}$ \\
\hline $\begin{array}{l}26 \\
27 \\
28 \\
29 \\
30 \\
31\end{array}$ & $\begin{array}{r}93 \\
92 \\
92 \\
102 \\
998 \\
456\end{array}$ & $\begin{array}{r}97 \\
97 \\
94 \\
92 \\
90 \\
- \\
-\end{array}$ & $\begin{array}{r}90 \\
87 \\
85 \\
650 \\
200 \\
140\end{array}$ & $\begin{array}{l}195 \\
160 \\
140 \\
125 \\
115 \\
105\end{array}$ & $\begin{array}{r}106 \\
95 \\
95 \\
107 \\
- \\
-\end{array}$ & $\begin{array}{r}145 \\
1610 \\
658 \\
1030 \\
383 \\
174\end{array}$ & $\begin{array}{r}151 \\
93 \\
90 \\
85 \\
89 \\
--\end{array}$ & $\begin{array}{l}92 \\
85 \\
86 \\
92 \\
86 \\
85\end{array}$ & $\begin{array}{r}84 \\
83 \\
83 \\
84 \\
89 \\
--\end{array}$ & $\begin{array}{l}110 \\
263 \\
452 \\
173 \\
125 \\
116\end{array}$ & $\begin{array}{l}110 \\
117 \\
142 \\
145 \\
168 \\
135\end{array}$ & $\begin{array}{l}346 \\
582 \\
244 \\
140 \\
900 \\
---\end{array}$ \\
\hline $\begin{array}{l}\text { TOTAL } \\
\text { MEAN } \\
\text { MAX } \\
\text { MIN } \\
\text { AC-FT } \\
(\dagger+)\end{array}$ & $\begin{array}{r}4381 \\
141 \\
998 \\
88 \\
8690 \\
2.11\end{array}$ & $\begin{array}{r}3753 \\
125 \\
725 \\
90 \\
7440 \\
1.47\end{array}$ & $\begin{array}{r}5254 \\
169 \\
1070 \\
80 \\
10420 \\
3.13\end{array}$ & $\begin{array}{r}13561 \\
437 \\
4880 \\
85 \\
26900 \\
5.84\end{array}$ & $\begin{array}{r}4518 \\
156 \\
528 \\
95 \\
8960 \\
1.73\end{array}$ & $\begin{array}{r}6571 \\
212 \\
1610 \\
79 \\
13030 \\
3.78\end{array}$ & $\begin{array}{r}3631 \\
121 \\
723 \\
81 \\
7200 \\
1.57\end{array}$ & $\begin{array}{r}4441 \\
143 \\
842 \\
80 \\
8810 \\
3.31\end{array}$ & $\begin{array}{r}3494 \\
116 \\
750 \\
78 \\
6930 \\
1.99\end{array}$ & $\begin{array}{r}4086 \\
132 \\
452 \\
87 \\
8100 \\
1.64\end{array}$ & $\begin{array}{r}4081 \\
132 \\
290 \\
97 \\
8090 \\
2.01\end{array}$ & $\begin{array}{r}7998 \\
267 \\
1810 \\
91 \\
15860 \\
7.63\end{array}$ \\
\hline
\end{tabular}

$\begin{array}{llllllllllll}\text { CAL YR } 1979 & \text { TOTAL } & 116683 & \text { MEAN } 320 & \text { MAX } & 9620 & \text { MIN 65 } & \text { AC-FT } & 231400 & \dagger \dagger & 59.41 \\ \text { WTR YR } & 1980 & \text { TOTAL } & 65769 & \text { MEAN } 180 & \text { MAX } & 4880 & \text { MIN 78 } & \text { AC-FT } & 130500 & \dagger \dagger & 36.21\end{array}$

t† Weighted-mean rainfall, in inches, based on eleven rain gages. 


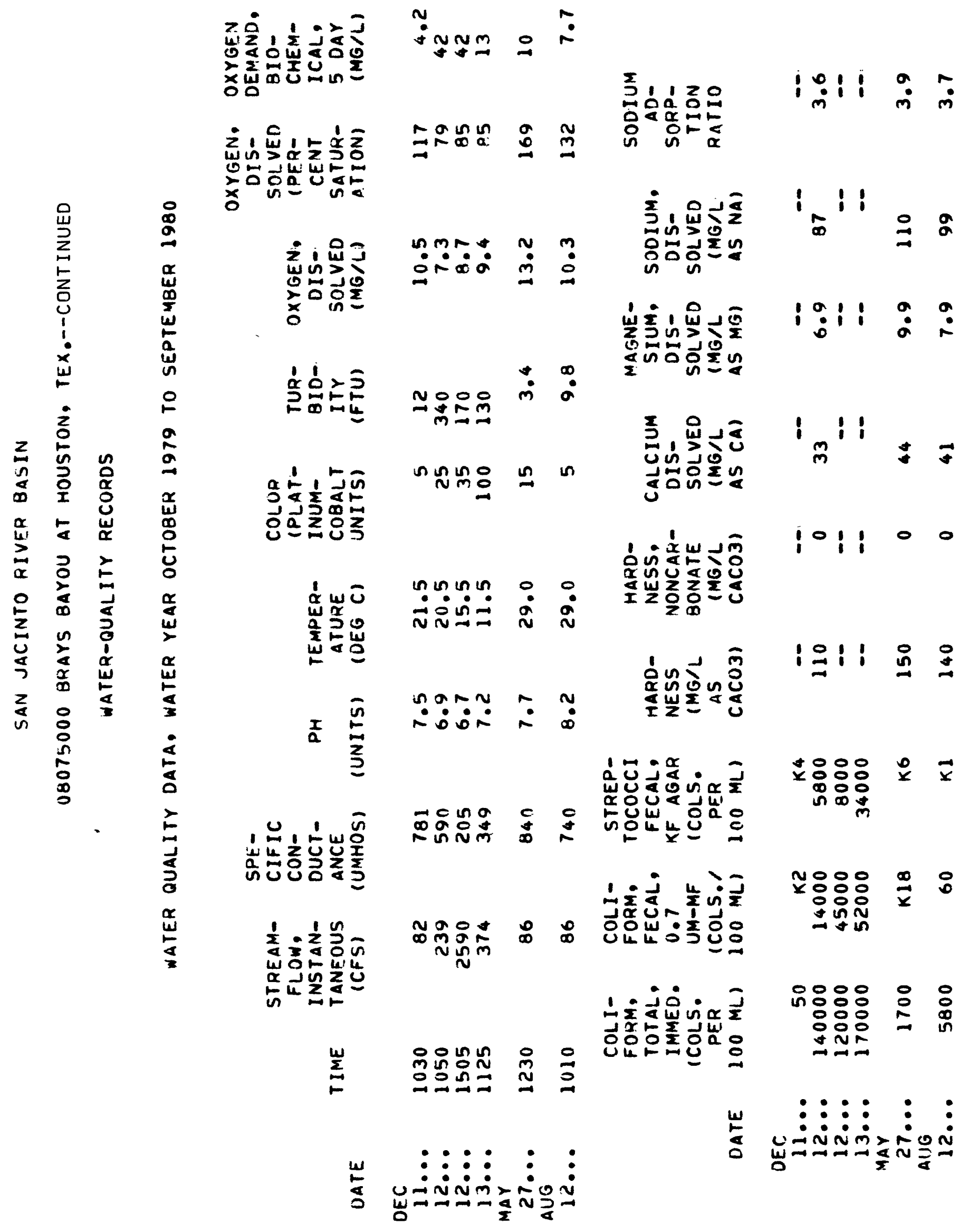




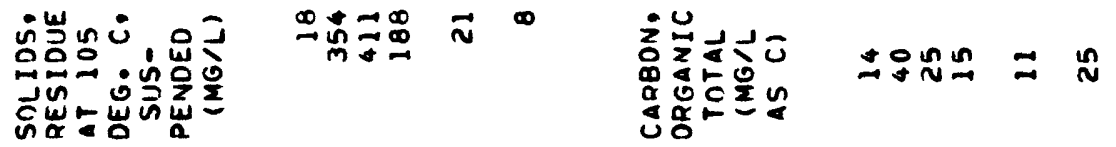

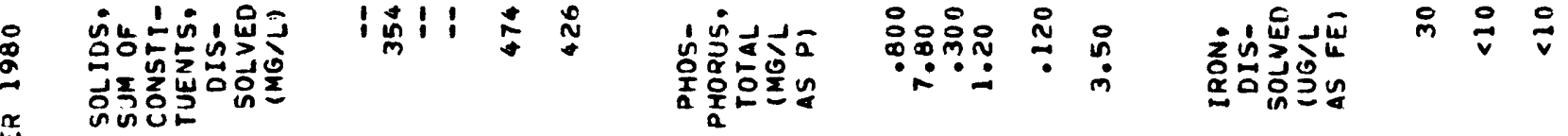

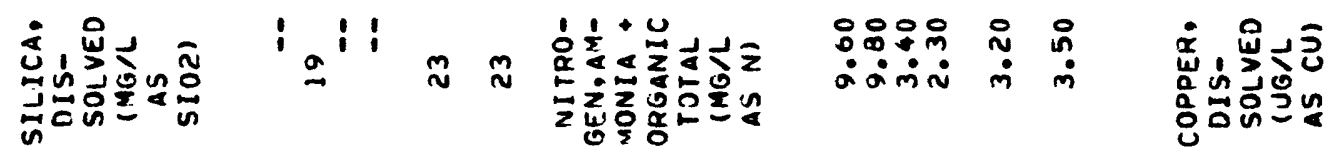

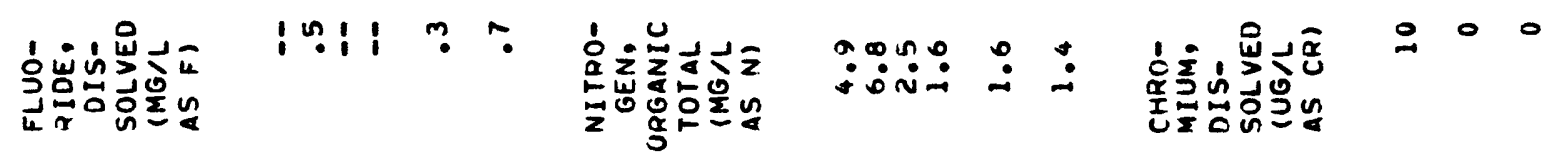

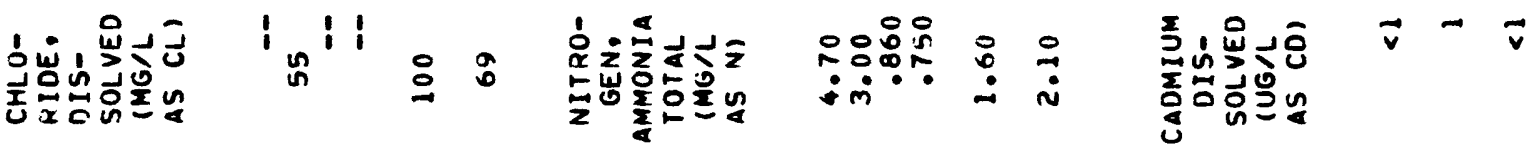

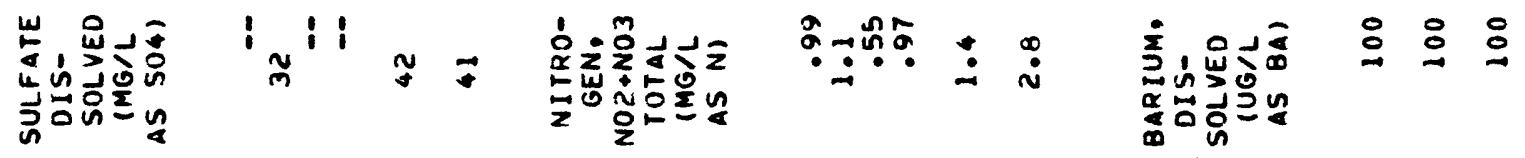

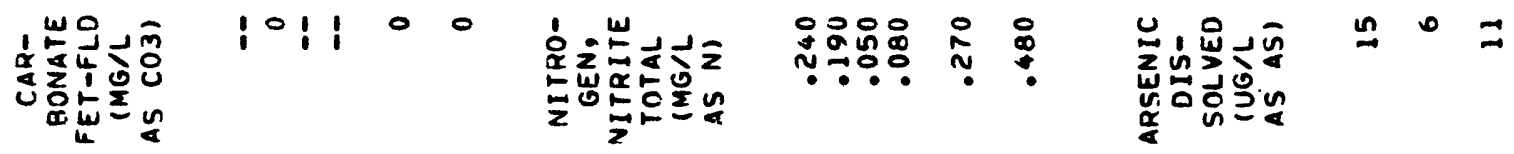

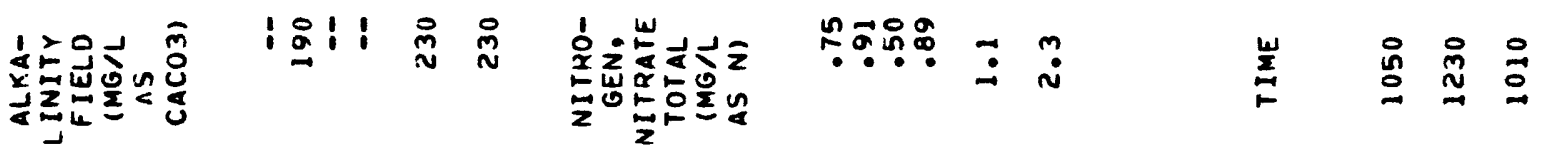

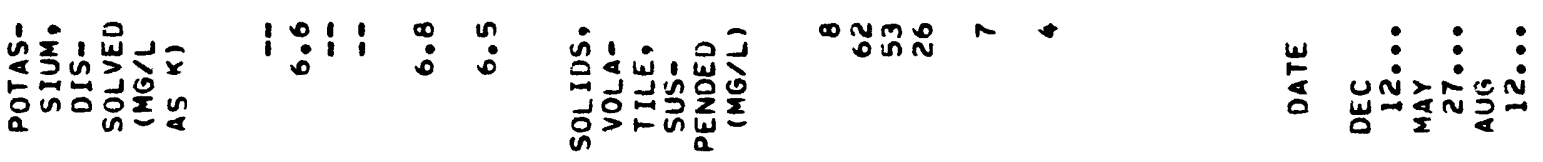

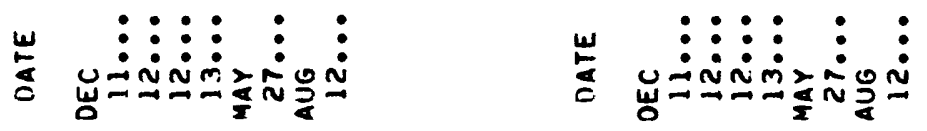




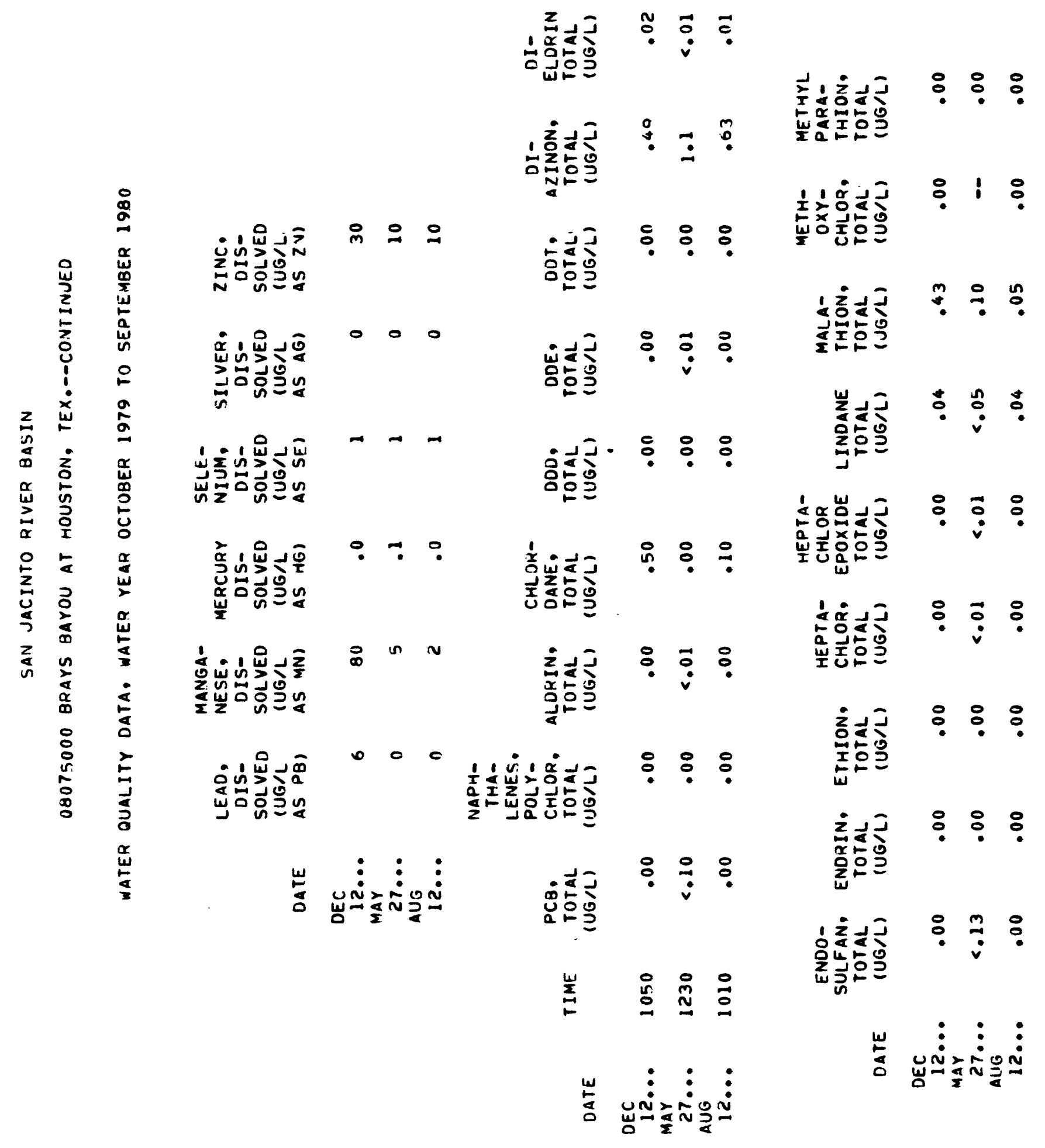




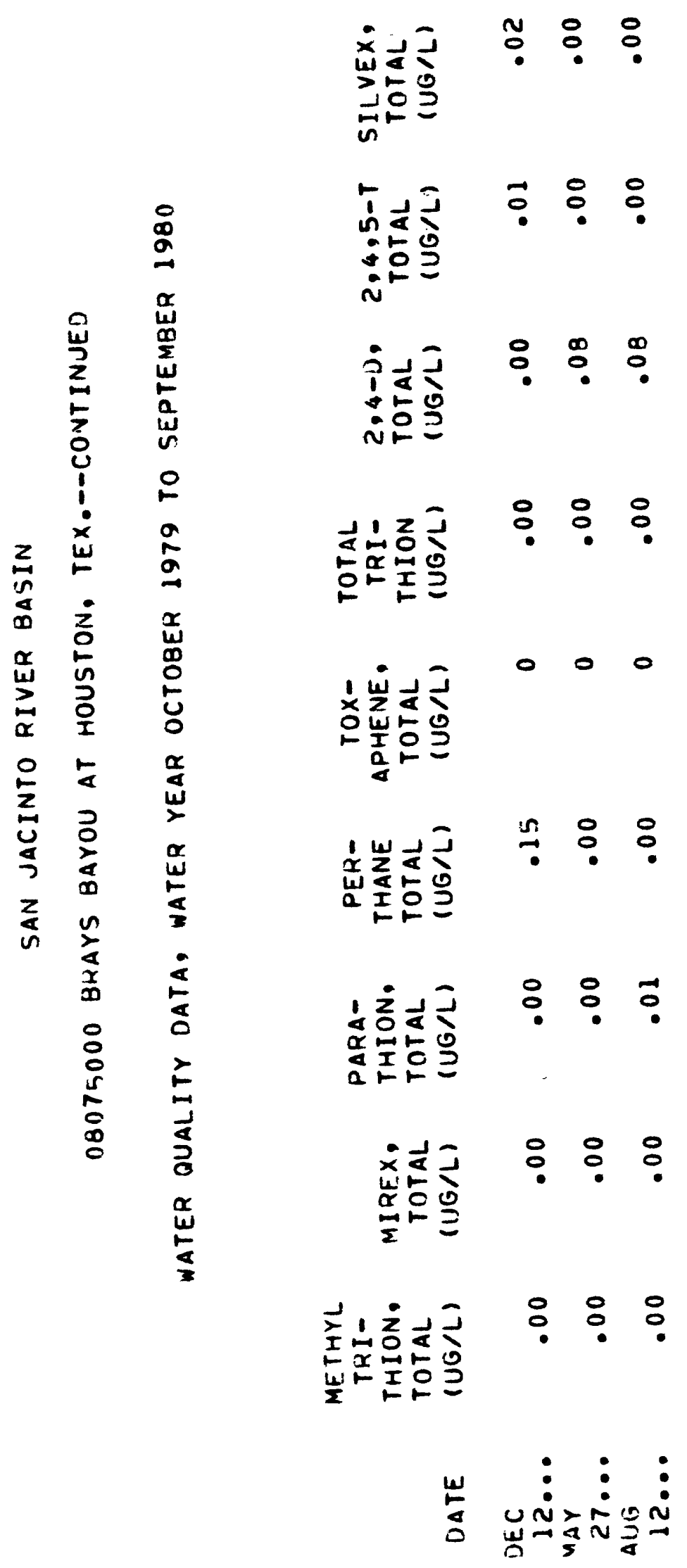




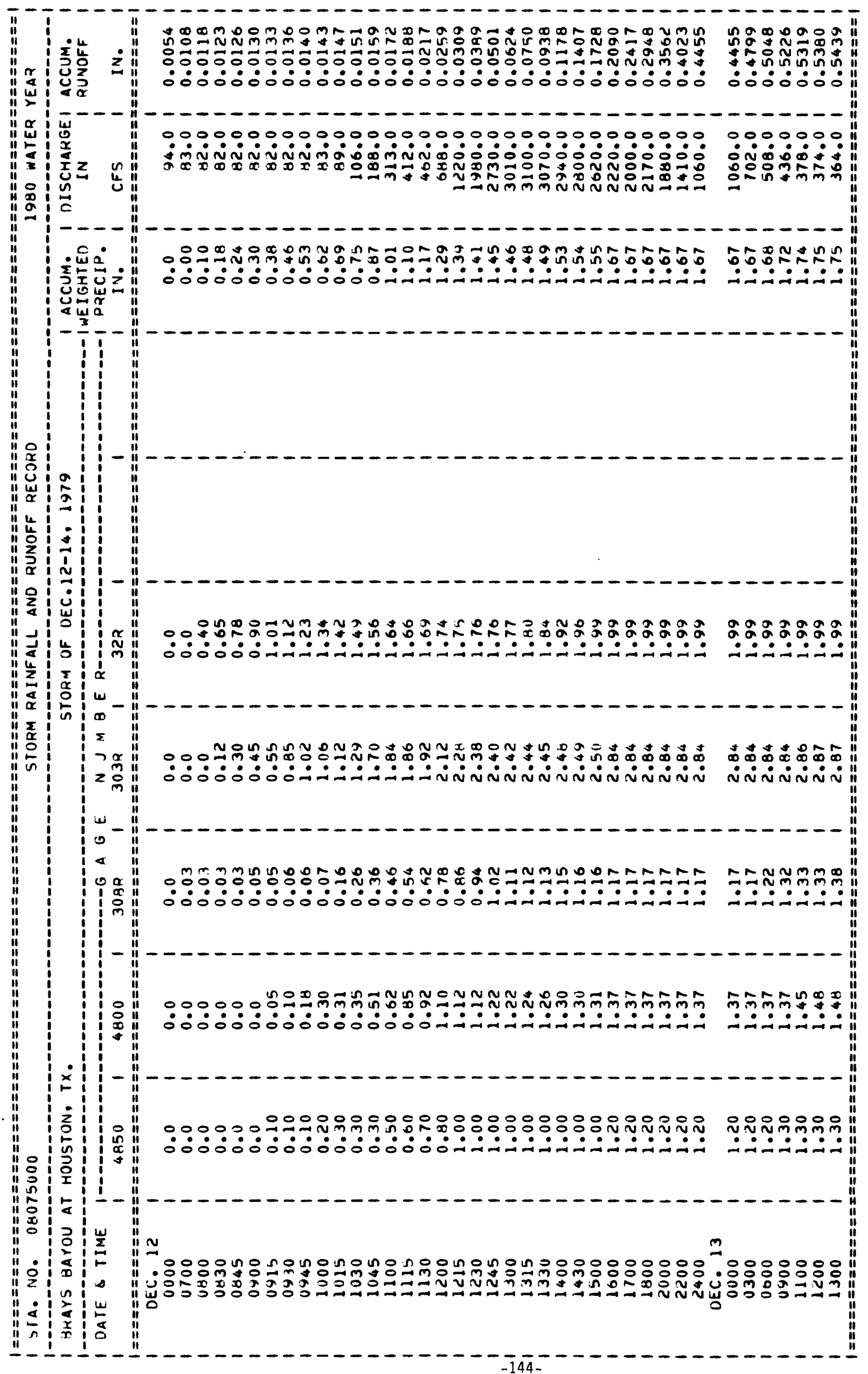




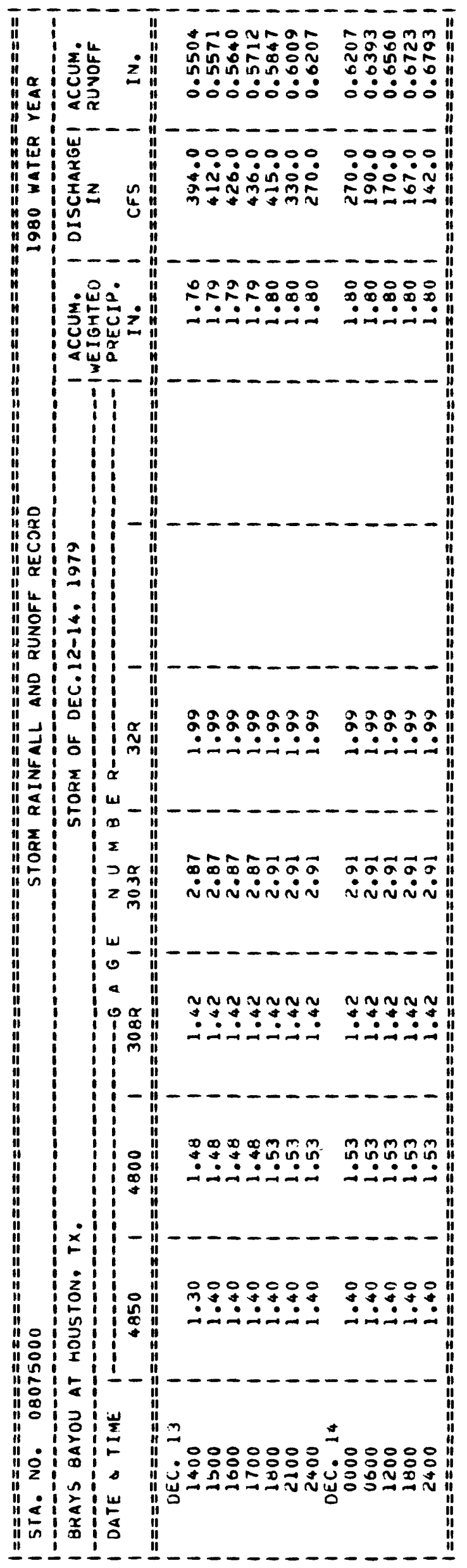




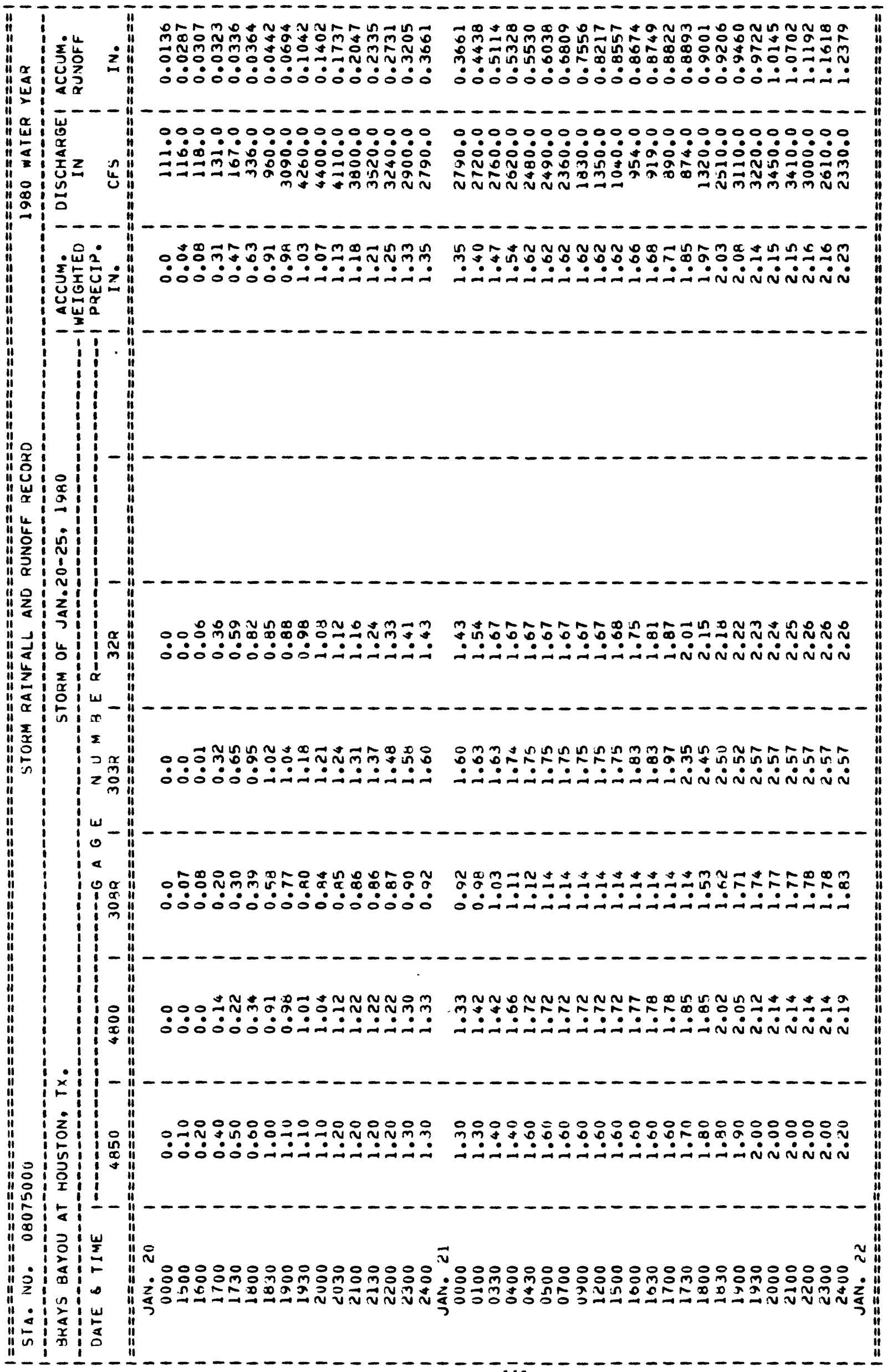

$-146-$ 


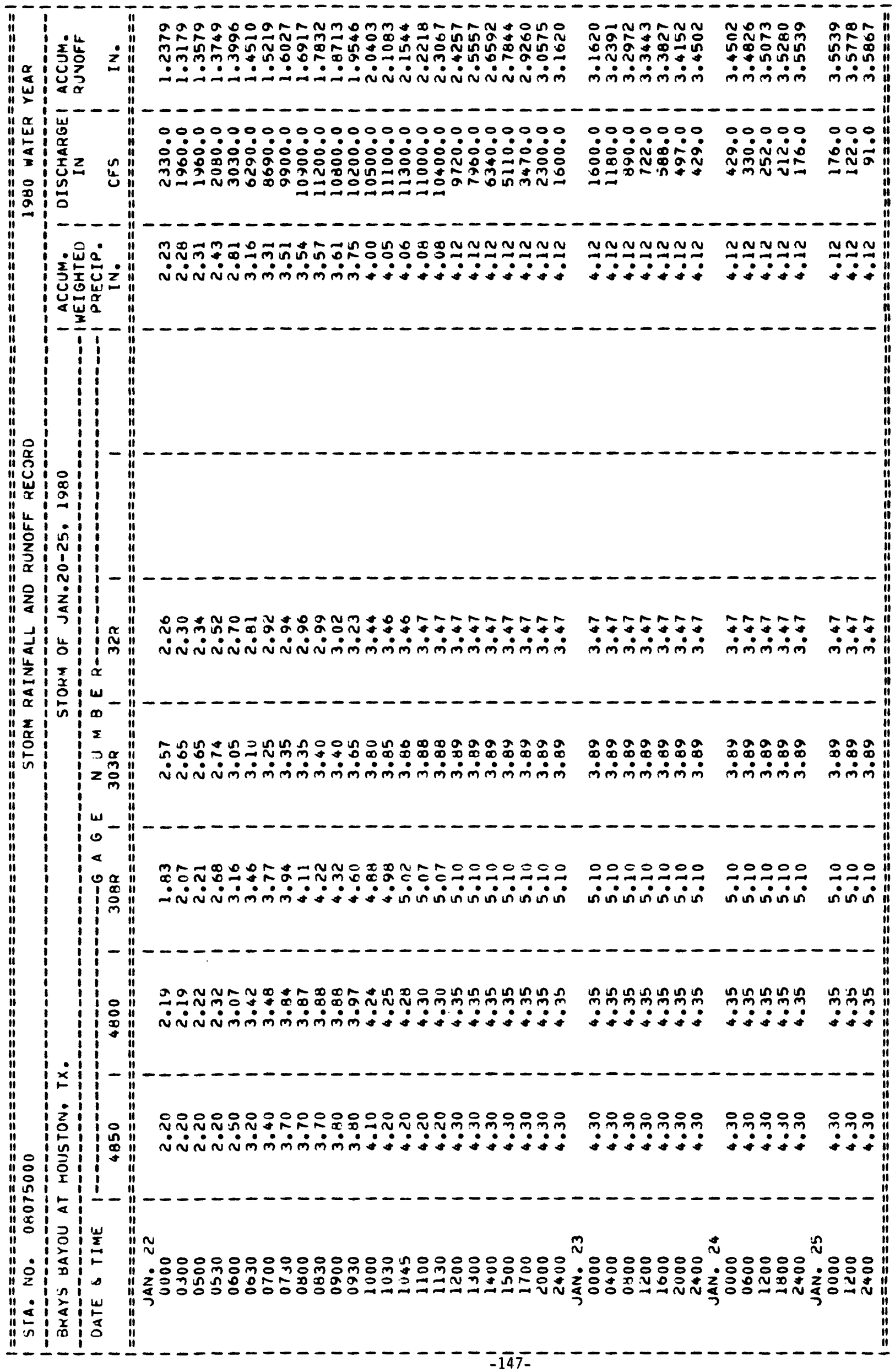


SAN JACINTO RIVER BASIN

08075100 BRAYS BAYOU AT SCOTT STREET, HOUSTON, TX (Low-flow partial-record station)

LOCATION.--Lat $29^{\circ} 42^{\prime} 35^{\prime \prime}$, long $95^{\circ} 21^{\prime} 23^{\prime \prime}$, Harris County, Hydrologic Unit 12040104, at bridge on Scott Street in Houston.

DRAINAGE AREA. $--106 \mathrm{mi}^{2}\left(275 \mathrm{~km}^{2}\right)$.

PERIOD OF RECORD.--Occasional discharge measurements and water-quality data: May 1971 to current year.

WATER QUALITY DATA, WATER YEAR OCTOBER 1979 TO SEPTEMBER 1980

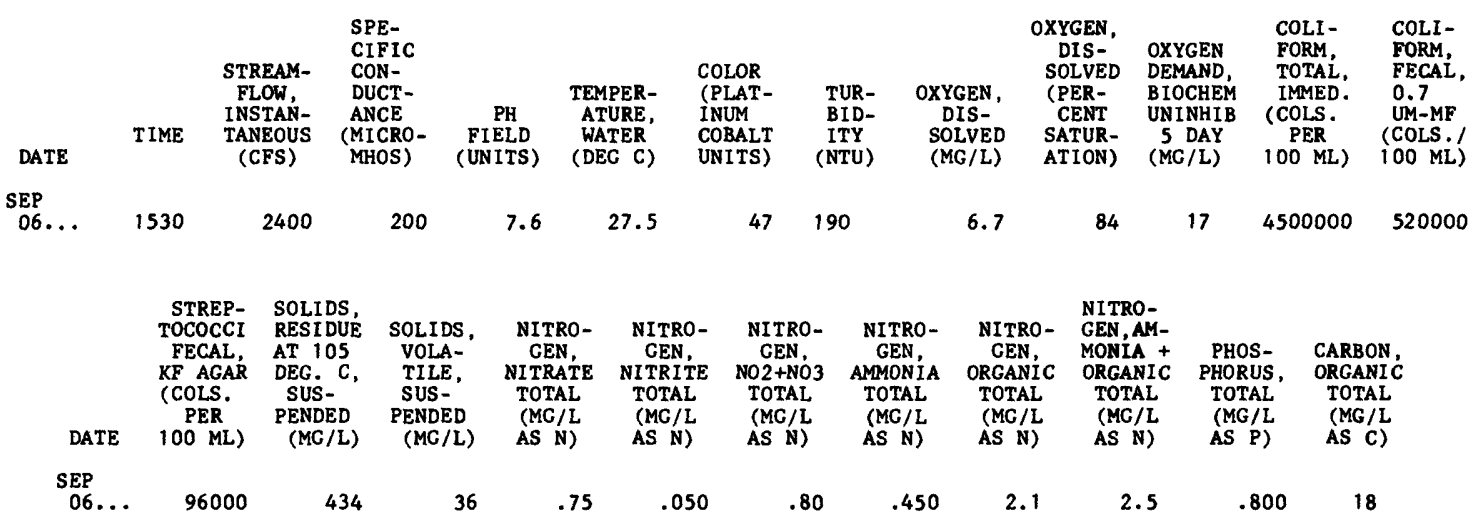




\section{SIMS BAYOU DRAINAGE BASIN}

The locations of data-collection sites in and near the Sims Bayou drainage basin are shown in fiqure 15 . section.

Berry Bayou is shown as a separate drainage basin within the Sims Bayou

Weighted-mean rainfall for the upper portion of the drainaqe basin above the Hiram Clarke Street station, based on two rain gages for the 1980 water year was 36.61 inches, or 11.58 inches less than the 30-year $(1941-70)$ average of 48.19 inches for Houston.

Weighted-mean rainfall in the drainaqe basin above the Telephone Road station, based on six rain gages, for the 1980 water year was 37.66 inches or 10.53 inches less than the 30-year (1941-70) average of 48.19 inches for Houston.

No storms were analyzed for the 1980 water year at station 08075470 , Sims Bayou at Martin Luther King Blvd.

The storms of 0ct. 30-31 and Jan. 20-27 were selected for analysis at station 08075400, Sims Bayou at Hiram Clarke Street, and station 08075500, Sims Bayou at Houston. 

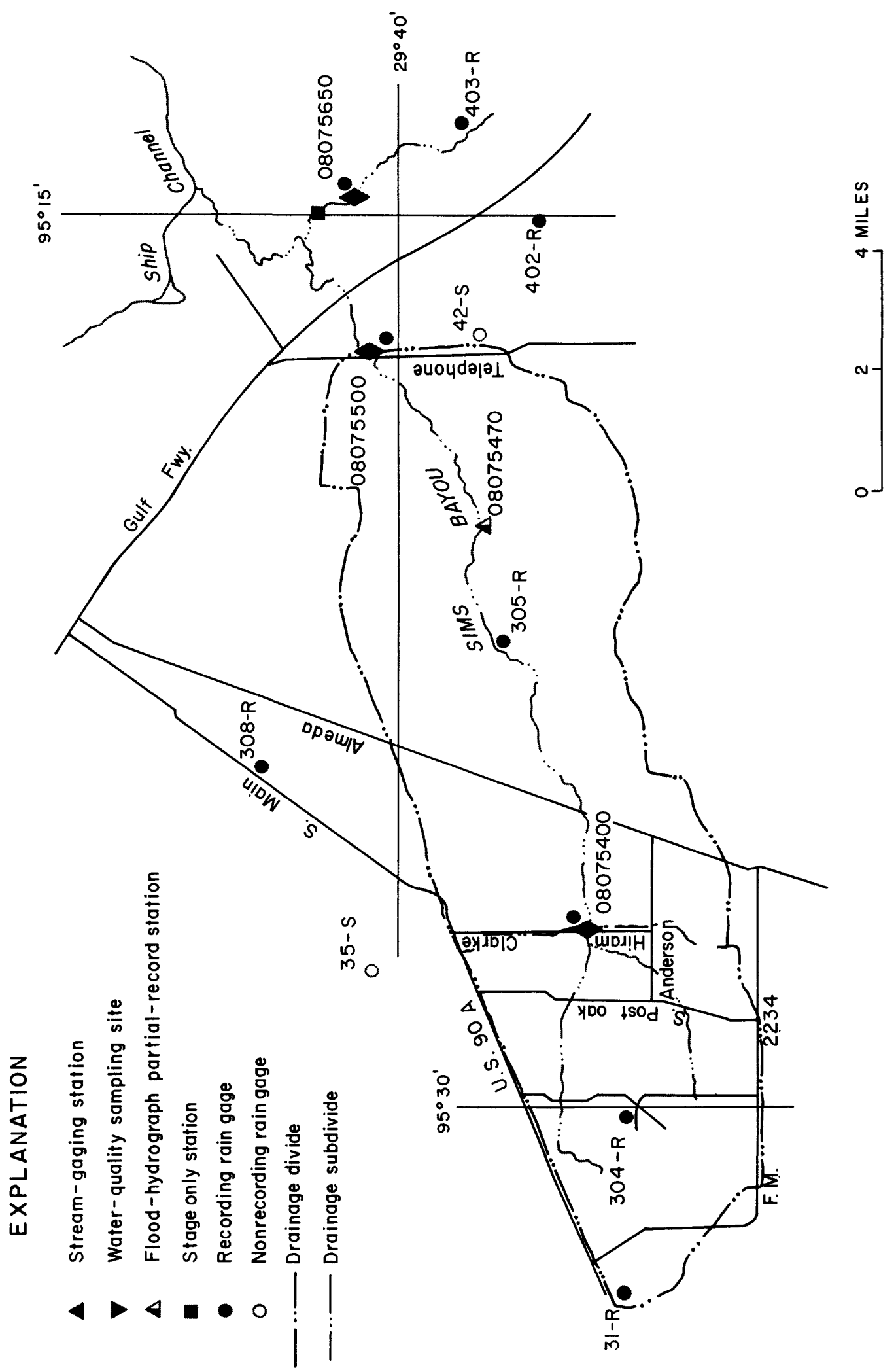

동 


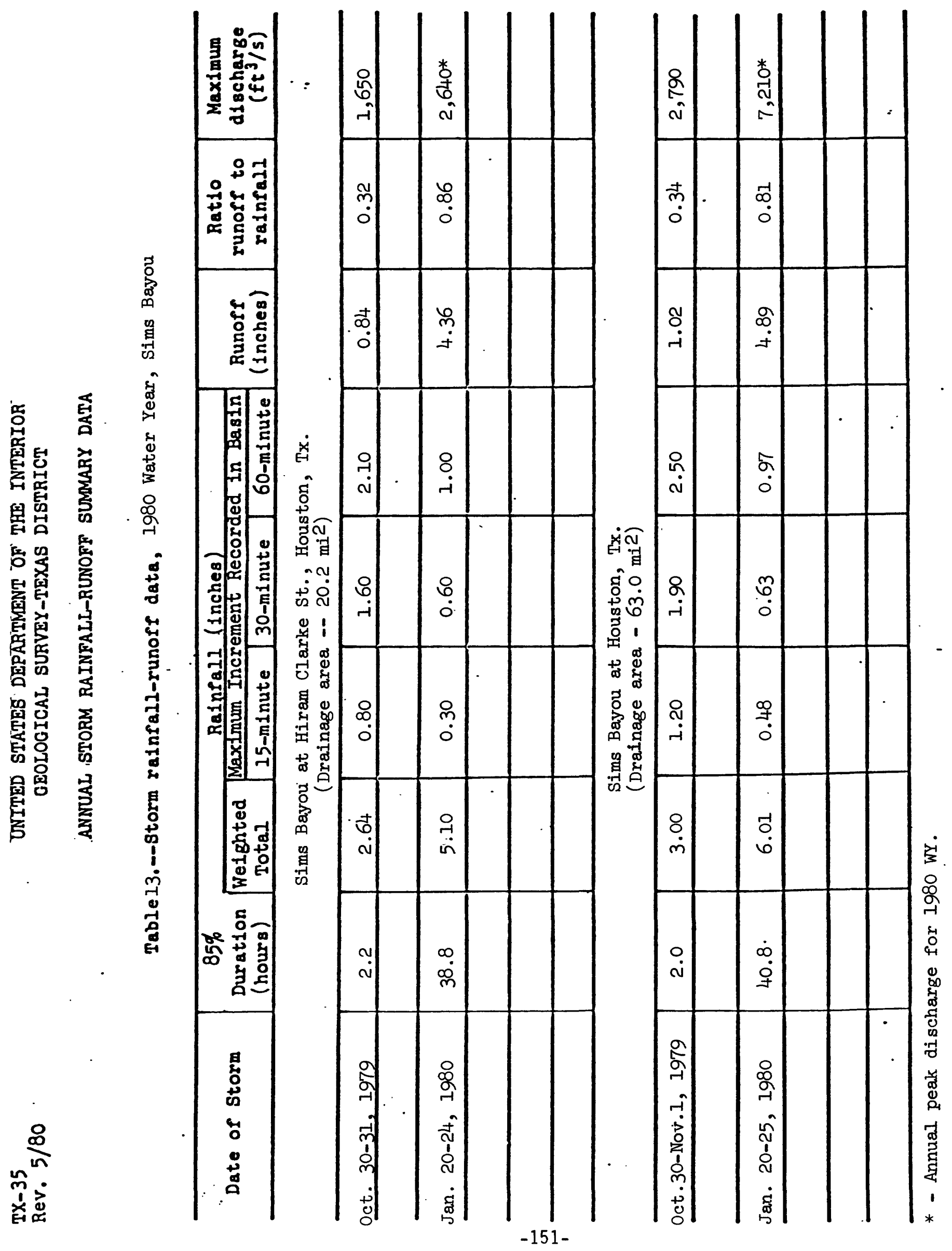


SAN JACINTO RIVER BASIN

08075400 SIMS BAYOU AT HIRAM CLARKE STREET, HOUSTON, TX

LOCATION.--Lat 29 $37^{\prime} 07^{\prime \prime}$. Iong $95^{\circ} 26^{\prime} 45^{\prime \prime}$, Harris County, Hydrologic Unit 12040104, on right bank at downstream side of bridge on Hiram Clarke Street in southwest Houston, $12.7 \mathrm{mi}(20.4 \mathrm{~km})$ upstream from gage Sims Bayou at Houston, and $19.7 \mathrm{mi}(31.7 \mathrm{~km})$ upstream from mouth.

DRAINAGE AREA.-- $-20.2 \mathrm{mi}^{2}\left(52.3 \mathrm{~km}^{2}\right)$.

WATER-DISCHARGE RECORDS

PERIOD OF RECORD.--August 1964 to current year (discharge measurements and supplemental peak discharges only Dec. 6, 1978, to Aug. 31, 1979).

GAGE.--Water-stage recorder. Datum of gage is National Geodetic Vertical Datum of 1929 , 1959 adjustment; unadjusted for land-surface subsidence.

REMARKS.--Water-discharge records fair. Channel bed was lowered 5 to 6 ft (1.5 to $1.8 \mathrm{~m})$ during rectification of 1978. No known diversion above station. Low flow is partly sustained by sewage effluent from Houston suburbs. Records furnished by Houston Lighting and Power Co. show that during the current year, about 630 acre-ft $\left(777,000 \mathrm{~m}^{3}\right)$ of ground water was used for cooling purposes then released to the bayou about 300 ft $(90 \mathrm{~m})$ upstream from gage. Recording rain gage located at station.

AVERAGE DISCHARGE.--15 years (water years $1965-78,1980), 27.1 \mathrm{ft}^{3} / \mathrm{s}\left(0.767 \mathrm{~m}^{3} / \mathrm{s}\right), 19,630 \mathrm{acre}-\mathrm{ft} / \mathrm{yr}(24.2 \mathrm{hm} / \mathrm{yr})$. EXTREMES FOR PERIOD OF RECORD.--Maximum discharge, about $4,500 \mathrm{ft}^{3} / \mathrm{s}\left(127 \mathrm{~m}^{3} / \mathrm{s}\right) \mathrm{June} 15$, 1976, elevation, $57.12 \mathrm{ft}(17.410 \mathrm{~m})$; minimum dally, $1.5 \mathrm{ft}^{3} / \mathrm{s}\left(0.042 \mathrm{~m}^{3} / \mathrm{s}\right) \mathrm{July} 26,1965$.

EXTREMES FOR CURRENT YEAR.--Peak discharges above base of $650 \mathrm{ft}^{3} / \mathrm{s}\left(18.4 \mathrm{~m}^{3} / \mathrm{s}\right)$, and maximum (*):

\begin{tabular}{|c|c|c|c|c|c|c|c|c|c|c|c|c|}
\hline Date & Time & \multicolumn{2}{|c|}{$\begin{array}{c}\text { Discharge } \\
\left(\mathrm{ft}^{3} / \mathrm{s}\right)\left(\mathrm{m}^{3} / \mathrm{s}\right)\end{array}$} & \multicolumn{2}{|c|}{$\underset{(\mathrm{ft})}{\text { Elevation }}(\mathrm{m})$} & \multicolumn{2}{|l|}{ Date } & Time & \multicolumn{2}{|c|}{$\begin{array}{c}\text { Discharge } \\
\left(\mathrm{ft}^{3} / \mathrm{s}\right)\left(\mathrm{m}^{3} / \mathrm{s}\right)\end{array}$} & \multicolumn{2}{|c|}{$\underset{(\mathrm{ft})}{\text { Elevation }}(\mathrm{m})$} \\
\hline $\begin{array}{rr}\text { Oct. } & 30 \\
\text { aJan. } & 3 \\
\text { Jan. } & 22 \\
\text { Feb. } & 8\end{array}$ & $\begin{array}{l}1945 \\
0730 \\
1045 \\
1900\end{array}$ & $\begin{array}{r}1,650 \\
153 \\
\star 2,640 \\
890\end{array}$ & $\begin{array}{c}46.7 \\
4.33 \\
74.8 \\
25.2\end{array}$ & $\begin{array}{l}45.84 \\
38.83 \\
48.97 \\
42.95\end{array}$ & $\begin{array}{l}13.972 \\
11.835 \\
14.926 \\
13.091\end{array}$ & $\begin{array}{l}\text { Apr. } \\
\text { aJuly } \\
\text { Sept. }\end{array}$ & $\begin{array}{l}25 \\
21 \\
27\end{array}$ & $\begin{array}{l}1300 \\
1445 \\
1945\end{array}$ & $\begin{array}{l}678 \\
197 \\
792\end{array}$ & $\begin{array}{c}19.2 \\
5.58 \\
22.4\end{array}$ & $\begin{array}{l}42.47 \\
39.55 \\
43.01\end{array}$ & $\begin{array}{l}12.945 \\
12.055 \\
13.109\end{array}$ \\
\hline
\end{tabular}

a Water-quality samples were obtained during this runoff event.

Minimum daily discharge $7.7 \mathrm{ft}^{3} / \mathrm{s}(0.22 \mathrm{~m} / \mathrm{s})$ July 5 .

DISCHARGE, IN CUBIC FEET PER SECOND, WATER YEAR OCTOBER 1979 TO SEPTEMBER 1980

\begin{tabular}{|c|c|c|c|c|c|c|c|c|c|c|c|c|}
\hline DAY & OCT & Nov & DEC & JAN & FEB & MAR & APR & MAY & JUN & JUL. & AUG & SEP \\
\hline $\begin{array}{l}1 \\
2 \\
3 \\
4 \\
5\end{array}$ & $\begin{array}{l}12 \\
11 \\
12 \\
11 \\
9.2\end{array}$ & $\begin{array}{l}23 \\
16 \\
17 \\
15 \\
17\end{array}$ & $\begin{array}{l}11 \\
13 \\
11 \\
11 \\
10\end{array}$ & $\begin{array}{l}16 \\
15 \\
57 \\
23 \\
16\end{array}$ & $\begin{array}{l}32 \\
33 \\
29 \\
26 \\
26\end{array}$ & $\begin{array}{l}22 \\
17 \\
19 \\
16 \\
14\end{array}$ & $\begin{array}{l}30 \\
26 \\
32 \\
26 \\
18\end{array}$ & $\begin{array}{r}175 \\
139 \\
31 \\
23 \\
18\end{array}$ & $\begin{array}{l}9.9 \\
9.8 \\
12 \\
15 \\
14\end{array}$ & $\begin{array}{l}10 \\
11 \\
10 \\
10 \\
7.7\end{array}$ & $\begin{array}{l}15 \\
13 \\
13 \\
13 \\
39\end{array}$ & $\begin{array}{l}15 \\
19 \\
15 \\
18 \\
49\end{array}$ \\
\hline $\begin{array}{r}6 \\
7 \\
8 \\
9 \\
10\end{array}$ & $\begin{array}{l}9.2 \\
9.6 \\
9.2 \\
9.6 \\
9.6\end{array}$ & $\begin{array}{l}16 \\
13 \\
14 \\
16 \\
16\end{array}$ & $\begin{array}{l}10 \\
11 \\
11 \\
10 \\
11\end{array}$ & $\begin{array}{l}15 \\
13 \\
13 \\
13 \\
14\end{array}$ & $\begin{array}{r}17 \\
16 \\
226 \\
196 \\
56\end{array}$ & $\begin{array}{l}17 \\
16 \\
18 \\
16 \\
15\end{array}$ & $\begin{array}{l}20 \\
20 \\
18 \\
16 \\
19\end{array}$ & $\begin{array}{l}15 \\
22 \\
25 \\
18 \\
14\end{array}$ & $\begin{array}{l}12 \\
14 \\
14 \\
58 \\
23\end{array}$ & $\begin{array}{l}8.1 \\
8.4 \\
11 \\
11 \\
11\end{array}$ & $\begin{array}{l}20 \\
15 \\
14 \\
14 \\
12\end{array}$ & $\begin{array}{r}366 \\
253 \\
316 \\
83 \\
27\end{array}$ \\
\hline $\begin{array}{l}11 \\
12 \\
13 \\
14 \\
15\end{array}$ & $\begin{array}{l}8.8 \\
8.8 \\
8.4 \\
8.8 \\
8.8\end{array}$ & $\begin{array}{l}17 \\
15 \\
14 \\
16 \\
16\end{array}$ & $\begin{array}{l}10 \\
97 \\
59 \\
30 \\
18\end{array}$ & $\begin{array}{l}13 \\
13 \\
12 \\
11 \\
12\end{array}$ & $\begin{array}{l}28 \\
22 \\
20 \\
24 \\
33\end{array}$ & $\begin{array}{l}15 \\
16 \\
15 \\
14 \\
15\end{array}$ & $\begin{array}{l}21 \\
19 \\
44 \\
23 \\
17\end{array}$ & $\begin{array}{l}14 \\
14 \\
17 \\
36 \\
17\end{array}$ & $\begin{array}{r}11 \\
9.9 \\
9.2 \\
9.0 \\
9.7\end{array}$ & $\begin{array}{l}11 \\
12 \\
10 \\
10 \\
11\end{array}$ & $\begin{array}{l}13 \\
15 \\
15 \\
13 \\
29\end{array}$ & $\begin{array}{l}13 \\
12 \\
16 \\
17 \\
16\end{array}$ \\
\hline $\begin{array}{l}16 \\
17 \\
18 \\
19 \\
20\end{array}$ & $\begin{array}{r}9.2 \\
9.2 \\
10 \\
9.6 \\
8.8\end{array}$ & $\begin{array}{l}13 \\
12 \\
13 \\
13 \\
13\end{array}$ & $\begin{array}{l}16 \\
14 \\
14 \\
14 \\
12\end{array}$ & $\begin{array}{r}14 \\
50 \\
33 \\
18 \\
154\end{array}$ & $\begin{array}{l}32 \\
25 \\
19 \\
20 \\
17\end{array}$ & $\begin{array}{l}16 \\
26 \\
18 \\
14 \\
14\end{array}$ & $\begin{array}{l}19 \\
26 \\
28 \\
20 \\
16\end{array}$ & $\begin{array}{r}28 \\
23 \\
16 \\
197 \\
51\end{array}$ & $\begin{array}{l}9.6 \\
10 \\
10 \\
11 \\
9.3\end{array}$ & $\begin{array}{l}12 \\
12 \\
11 \\
11 \\
12\end{array}$ & $\begin{array}{l}25 \\
16 \\
15 \\
15 \\
15\end{array}$ & $\begin{array}{l}17 \\
13 \\
11 \\
10 \\
11\end{array}$ \\
\hline $\begin{array}{l}21 \\
22 \\
23 \\
24 \\
25\end{array}$ & $\begin{array}{l}9.6 \\
14 \\
10 \\
9.6 \\
9.2\end{array}$ & $\begin{array}{l}34 \\
38 \\
24 \\
12 \\
13\end{array}$ & $\begin{array}{l}12 \\
12 \\
53 \\
45 \\
16\end{array}$ & $\begin{array}{r}485 \\
1430 \\
222 \\
56 \\
34\end{array}$ & $\begin{array}{l}17 \\
16 \\
16 \\
17 \\
17\end{array}$ & $\begin{array}{l}13 \\
13 \\
17 \\
16 \\
12\end{array}$ & $\begin{array}{r}16 \\
14 \\
14 \\
17 \\
258\end{array}$ & $\begin{array}{l}17 \\
15 \\
13 \\
10 \\
11\end{array}$ & $\begin{array}{l}11 \\
9.4 \\
12 \\
11 \\
11\end{array}$ & $\begin{array}{l}63 \\
20 \\
20 \\
12 \\
11\end{array}$ & $\begin{array}{l}15 \\
14 \\
14 \\
11 \\
12\end{array}$ & $\begin{array}{l}12 \\
10 \\
11 \\
9.6 \\
8.7\end{array}$ \\
\hline $\begin{array}{l}26 \\
27 \\
28 \\
29 \\
30 \\
31\end{array}$ & $\begin{array}{c}9.6 \\
12.6 \\
9.6 \\
10 \\
326 \\
128\end{array}$ & $\begin{array}{c}11 \\
12 \\
14 \\
10 \\
11 \\
--\end{array}$ & $\begin{array}{r}13 \\
13 \\
14 \\
109 \\
29 \\
18\end{array}$ & $\begin{array}{l}28 \\
24 \\
21 \\
24 \\
30 \\
33\end{array}$ & $\begin{array}{r}16 \\
16 \\
14 \\
15 \\
-- \\
--\end{array}$ & $\begin{array}{r}19 \\
301 \\
182 \\
214 \\
94 \\
39\end{array}$ & $\begin{array}{r}53 \\
19 \\
18 \\
18 \\
17 \\
-7\end{array}$ & $\begin{array}{l}10 \\
10 \\
11 \\
11 \\
10 \\
10\end{array}$ & $\begin{array}{l}10 \\
11 \\
17 \\
16 \\
13 \\
-\ldots\end{array}$ & $\begin{array}{r}12 \\
12 \\
103 \\
55 \\
14 \\
14\end{array}$ & $\begin{array}{l}12 \\
12 \\
13 \\
13 \\
14 \\
14\end{array}$ & $\begin{array}{r}66 \\
250 \\
185 \\
36 \\
271 \\
-\ldots\end{array}$ \\
\hline $\begin{array}{l}\text { TOTAL } \\
\text { MEAN } \\
\text { MAX } \\
\text { MIN } \\
\text { AC-FT } \\
(+\dagger)\end{array}$ & $\begin{array}{r}740.4 \\
23.9 \\
326 \\
8.4 \\
1470 \\
2.83\end{array}$ & $\begin{array}{r}484 \\
16.1 \\
38 \\
10 \\
960 \\
1.22\end{array}$ & $\begin{array}{r}727 \\
23.5 \\
109 \\
10 \\
1440 \\
2.99\end{array}$ & $\begin{array}{r}2922 \\
94.3 \\
1430 \\
11 \\
5800 \\
6.08\end{array}$ & $\begin{array}{r}1041 \\
35.9 \\
226 \\
14 \\
2060 \\
2.04\end{array}$ & $\begin{array}{r}1253 \\
40.4 \\
301 \\
12 \\
2490 \\
3.29\end{array}$ & $\begin{array}{r}902 \\
30.1 \\
258 \\
14 \\
1790 \\
2.32\end{array}$ & $\begin{array}{r}1021 \\
32.9 \\
197 \\
10 \\
2030 \\
3.81\end{array}$ & $\begin{array}{r}401.8 \\
13.4 \\
58 \\
9.0 \\
797 \\
1.19\end{array}$ & $\begin{array}{r}546.2 \\
17.6 \\
103 \\
7.7 \\
1080 \\
1.64\end{array}$ & $\begin{array}{r}483 \\
15.6 \\
39 \\
11 \\
958 \\
1.25\end{array}$ & $\begin{array}{r}2156.3 \\
71.9 \\
366 \\
8.7 \\
4280 \\
7.95\end{array}$ \\
\hline
\end{tabular}

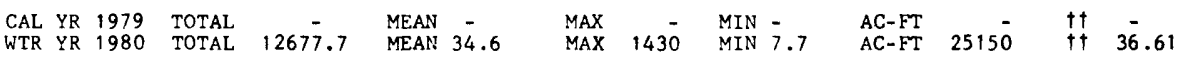

$\dagger \dagger$ Weighted-mean rainfall, in inches, based on two rain gages.

NOTE.--No elevation record Dec. 6 to Sept. 18. 


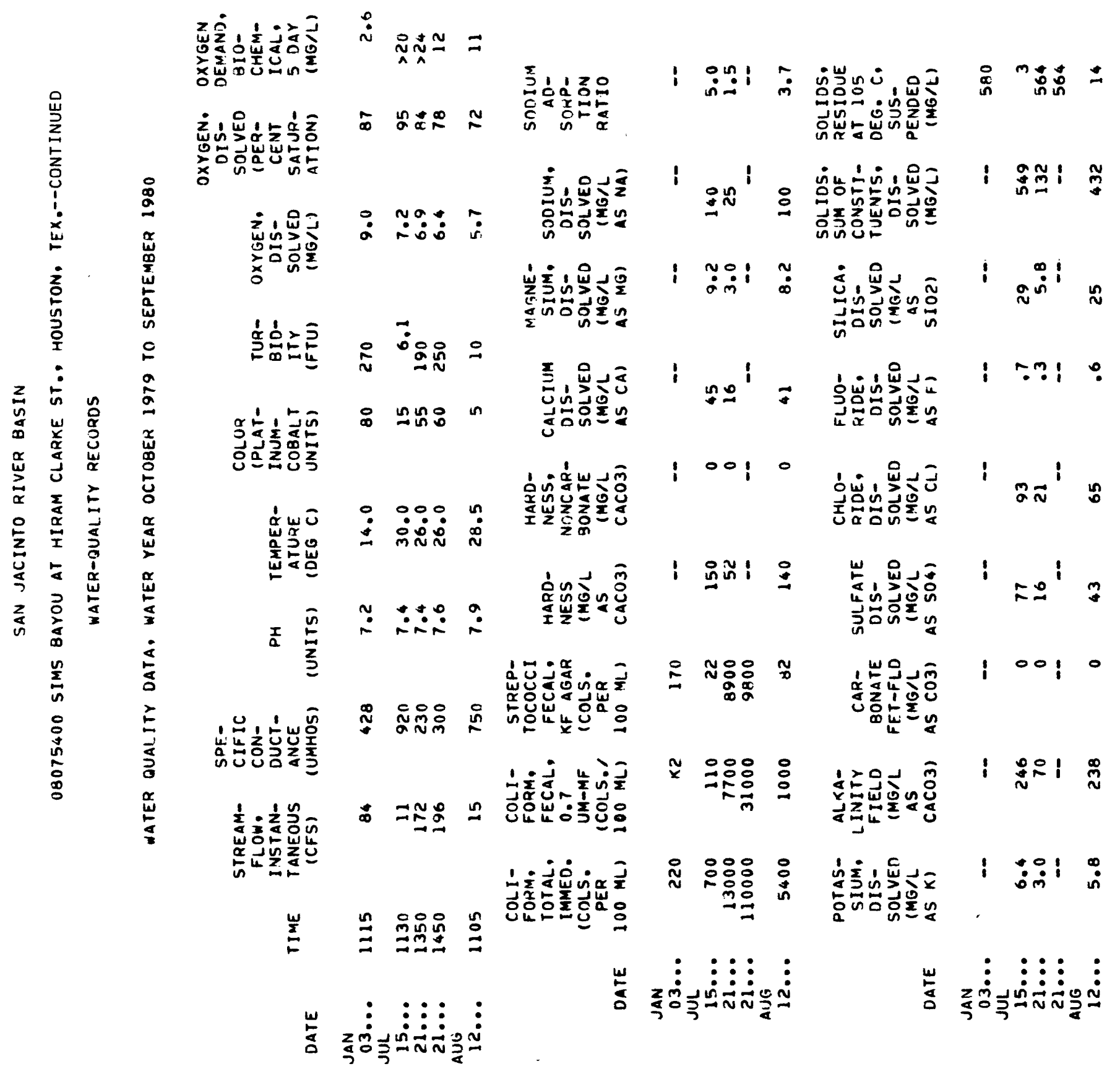




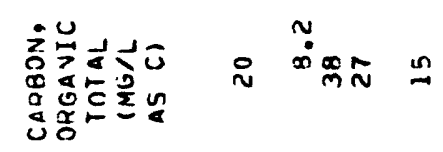

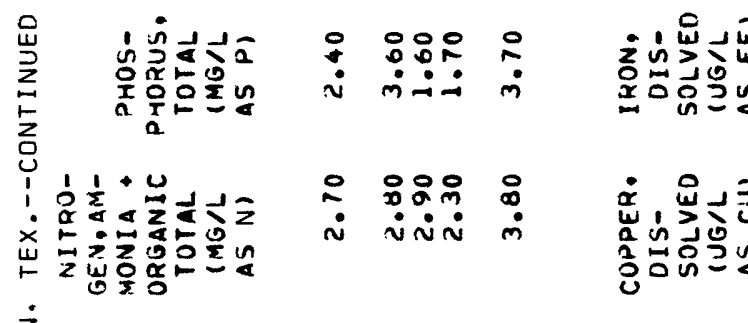

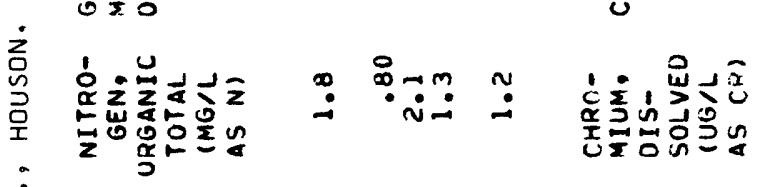

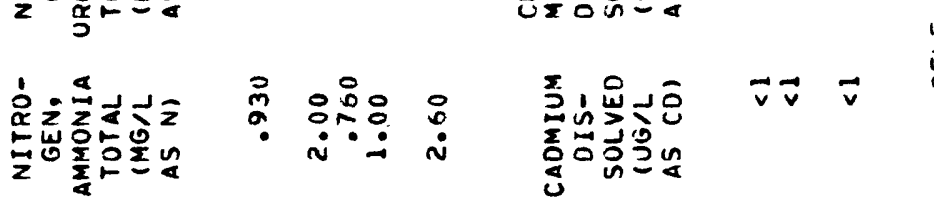

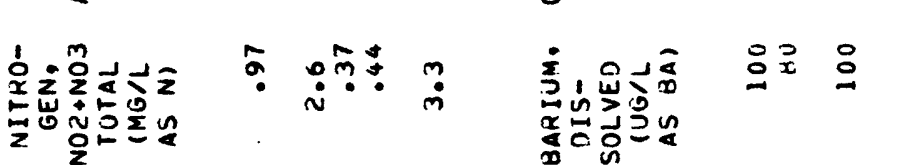

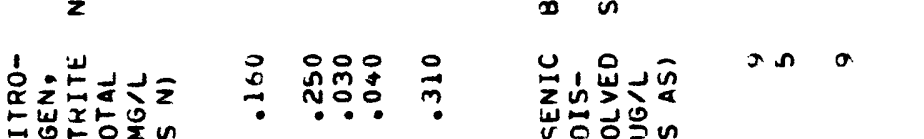

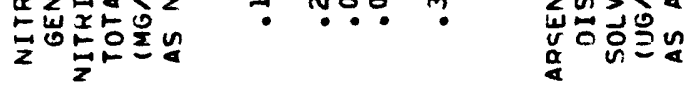

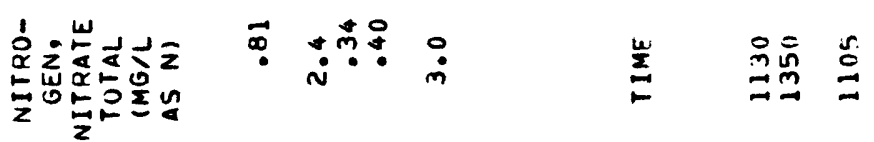

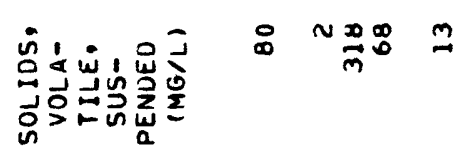

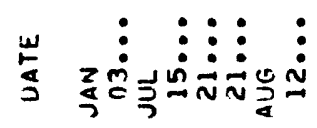

崖

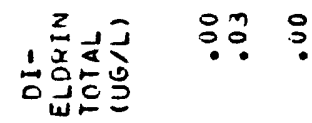

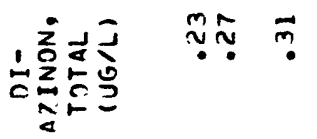

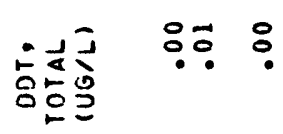

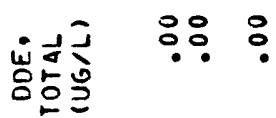

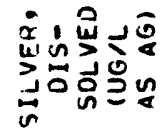

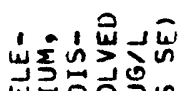

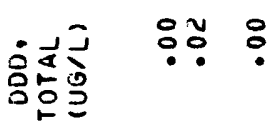

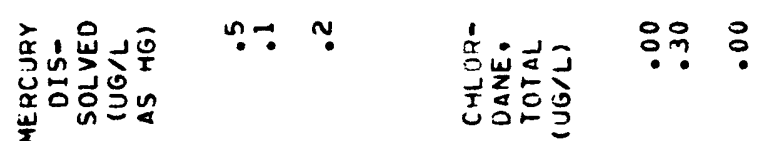

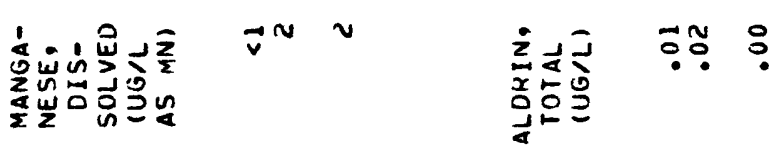

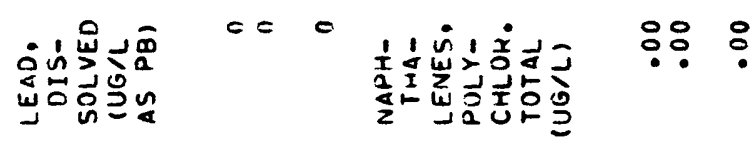

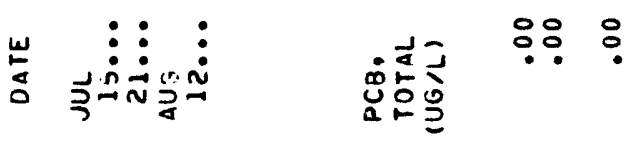

崖 


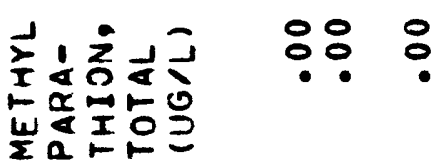

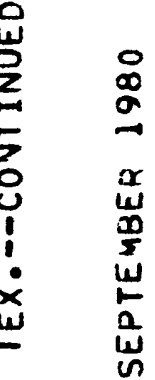

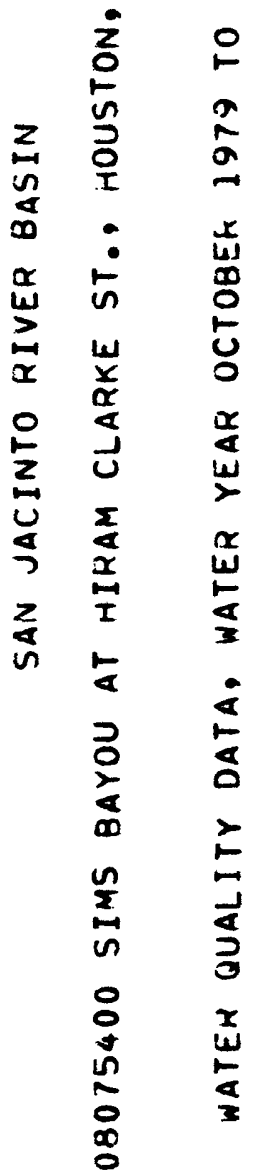

IIOS

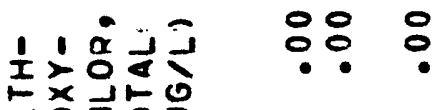

능전인

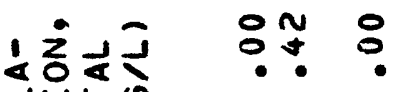

J4

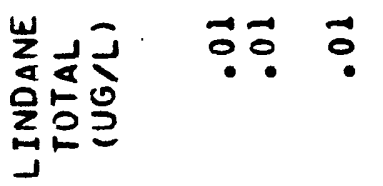

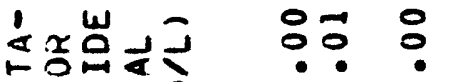

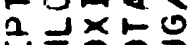

w둔은?

w

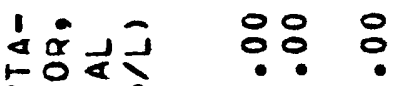

a 150

岌U른

我

등워

딴므

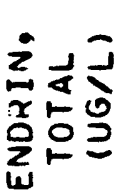

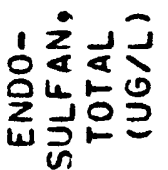

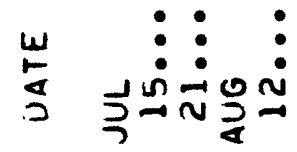

希落吕: $>$ ㄴㅇㅇ

도을

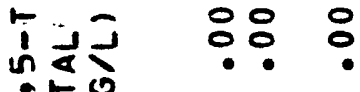

\&5응

15

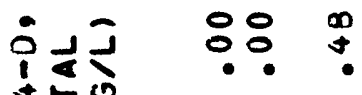
$+15$

$N-2$

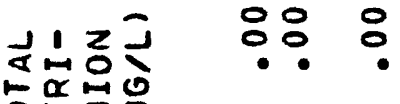

안프

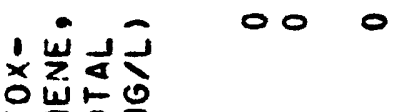

$\frac{1}{a} 0 ?$

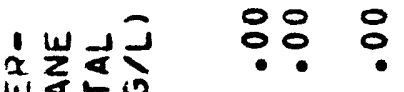

w 150

IN

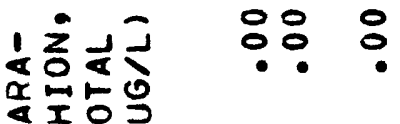

a

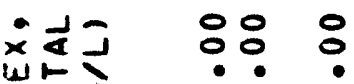

प्山ि0

는

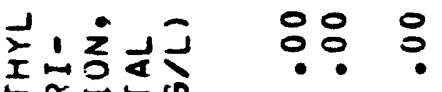
$x \mapsto \leftarrow 0$

WrI은?
崖: 


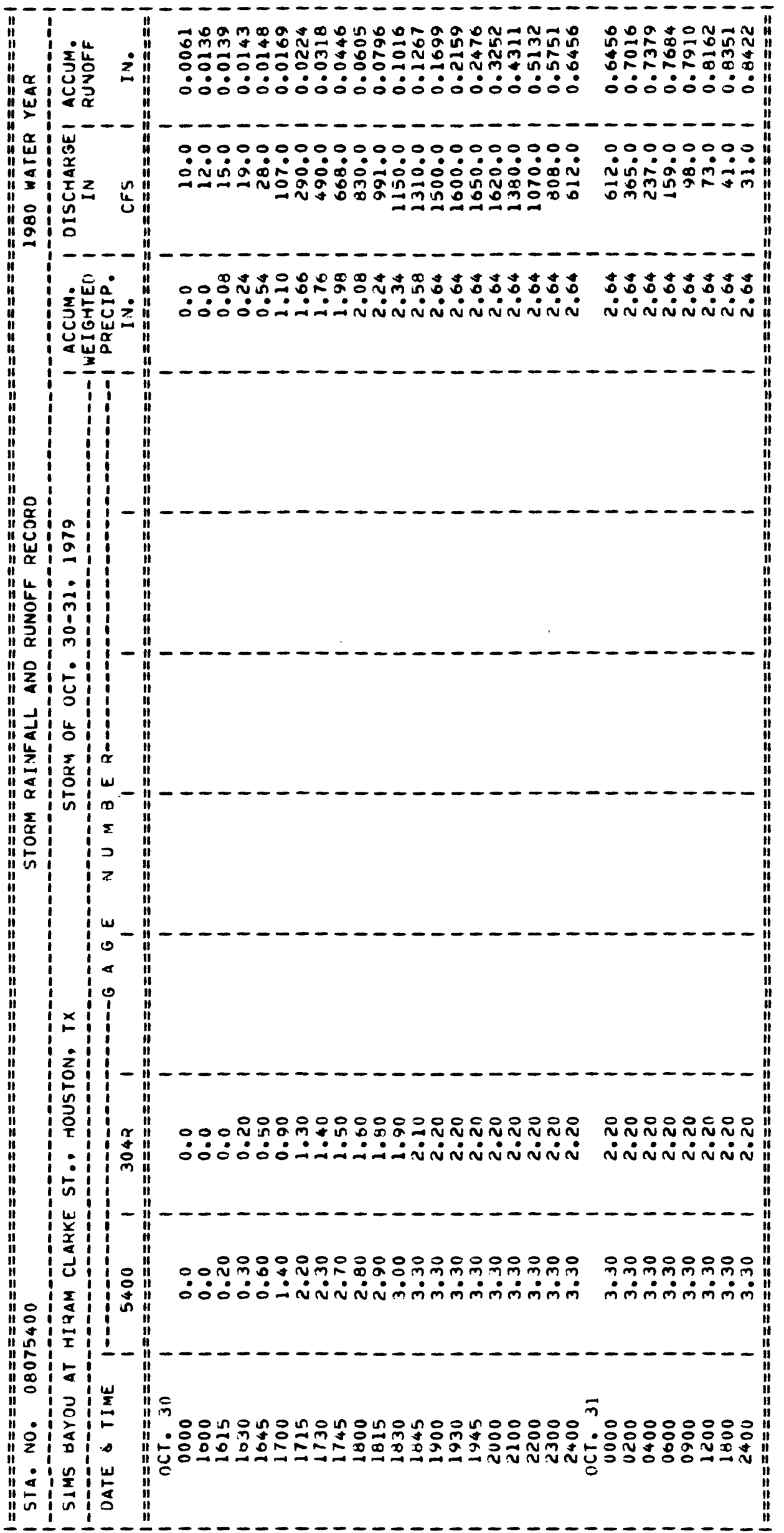

$-156-$ 


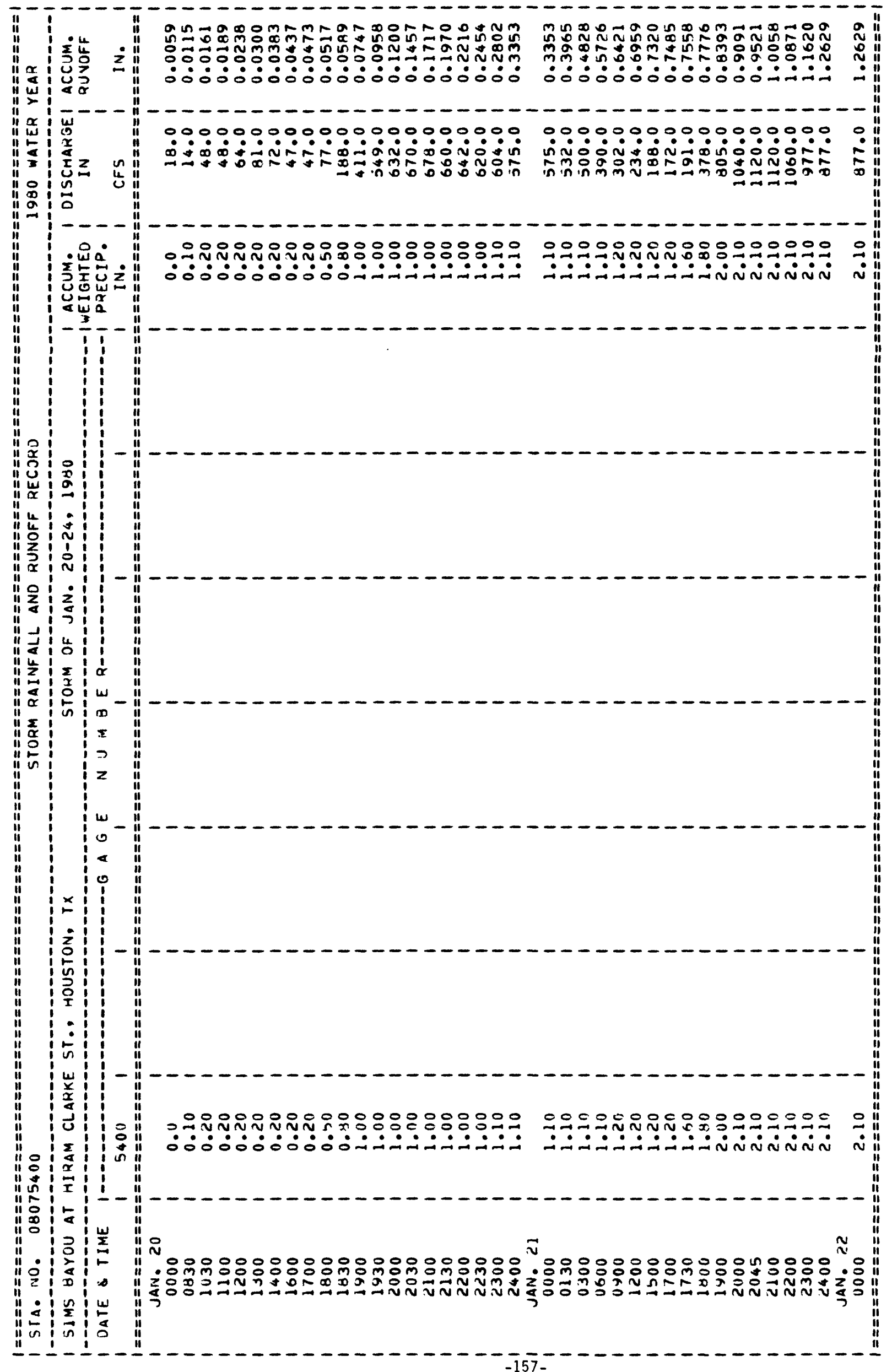




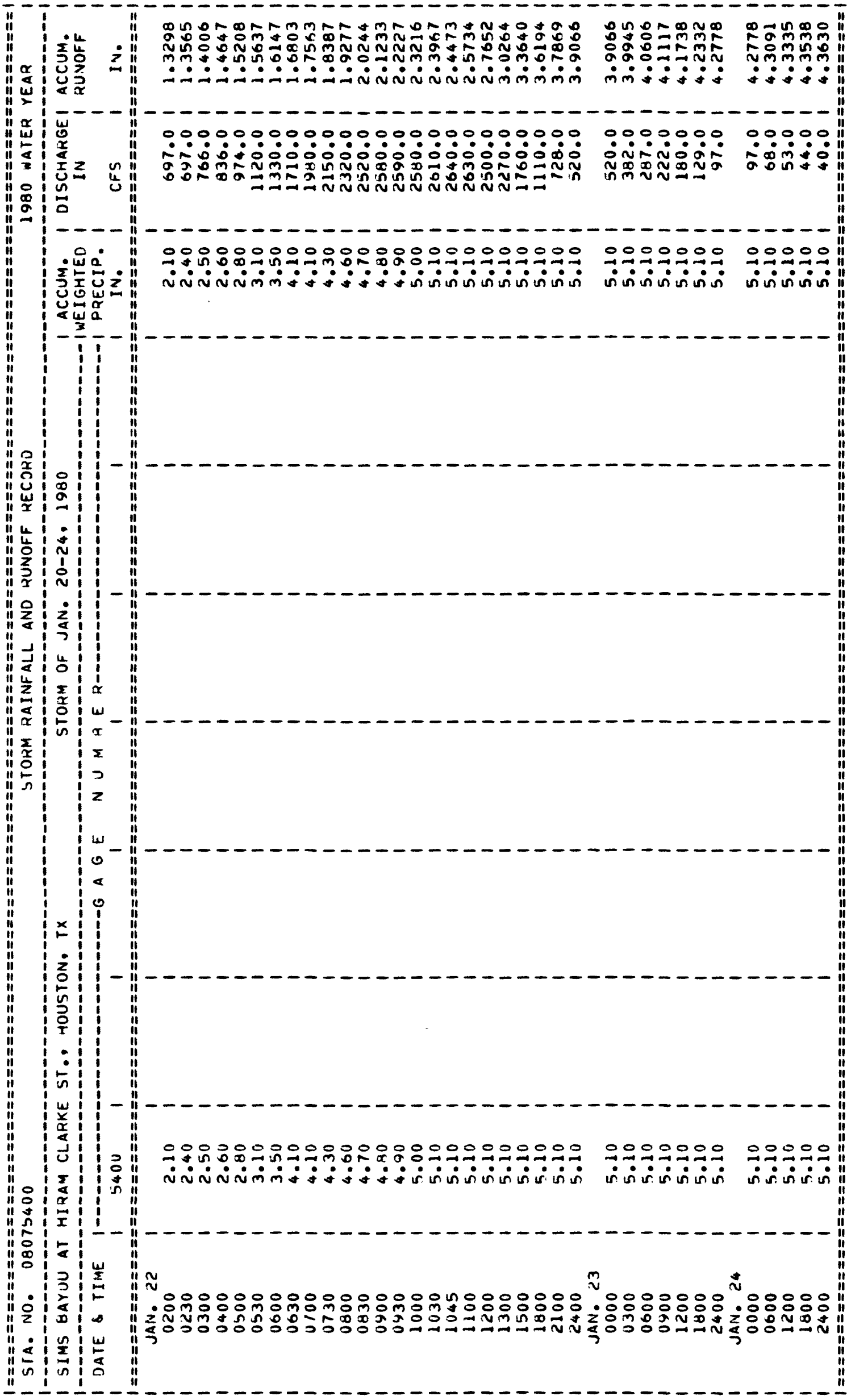


D8075470 SIMS BAYOU AT MARTIN LUTHER KING BLVD., HOUSTON, TEX.

(Flood-hydrograph partial-record station)

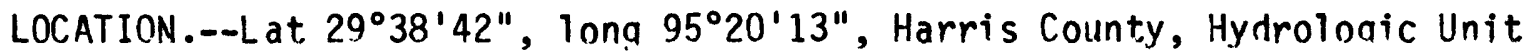
12040104 , at downstream side of upstream bridge on Martin Luther King Boulevard (formerly South Park Boulevard), $1 . \overline{6}$ miles upstream from Atchison, Topeka, and Santa Fe Railway Co. bridge in south Houston.

DRAINAGE. $--48.4 \mathrm{mi}^{2}$.

PERIOD OF RECORD.--October 1977 to current year.

GAGE.--Digital flood-hydrograph and rainfall recorders and crest-stage gage. Datum of gage is National Geodetic Vertical Datum of 1929, 1973 adjustment, unadjusted for land-surface subsidence.

REMARKS.--Gage-height records good. Peak discharges were not computed at this time because an adequate stage-discharge relationship has not been determined.

EXTREMES FOR PERIOD OF RECORD.--Maximum discharae (est.) 1,500 $\mathrm{ft}^{3} / \mathrm{s} \mathrm{Jan.} \mathrm{19,}$ 1978 (elevation unknown); minimum not determined.

EXTREMES OUTSIDE PERIOD OF RECORD.--Peak stage of $38.28 \mathrm{ft}$ (discharqe unknown) on June 15,1976 . This same storm produced the largest peak for the period of record (1952-79) at the aaging station Sims Bayou at Houston (08075500).

EXTREMES FOR CURRENT YEAR.--nnly peak gage height published this year The five highest peaks and maximum $(*)$ :

$\begin{array}{cc}\text { DATE TIME } & \left(\mathrm{ft}^{3} / \mathrm{s}\right)\end{array}$

GAGE HEIGHT

$(f t)$

oct. 30

2300

Jan. 22

Feb. 8

Mar. 27

1115

2215

2030

Mar. 29 unk nown

do.

to.

do.

do.
25.26

$\star 34.10$

23.48

21.24

20.73

Minimum discharge not determined. 
SAN JACINTO RIVER BASIN

08075500 SIMS BAYOU AT HOUSTON, TX

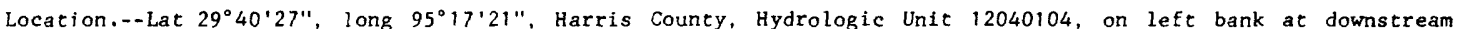
side of bridge on State Highway 35 in southeast Houston and $7.0 \mathrm{mi}(11.3 \mathrm{~km})$ upstream from mouth.

DRAINAGE AREA. $--63.0 \mathrm{mi}^{2}\left(163.2 \mathrm{~km}^{2}\right)$. Prior to Oct. 1, 1976, $64.0 \mathrm{mi}^{2}\left(165.8 \mathrm{~km}^{2}\right)$.

WATER-DISCHARGE RECORDS

PERIOD OF RECORD.--October 1952 to current year.

REVISED RECORDS.--WSP 1922: 1960. WDR TX-76-2: 1975 (M).

GAGE.--Water-stage recorder and crest-stage gage. Datum of gage is $3.09 \mathrm{ft}(0.942 \mathrm{~m})$ below National Geodetic Vertical Datum of 1929, 1973 adjustment; unadjusted for land-surface subsidence.

REMARKS.--Water-discharge records fair. Low flow is largely sustained by sewage effluent from Houston suburbs and industrial wastes. Harris County Flood Control District rainfall and gage height telemetry at station.

AVERAGE DISCHARGE. - -28 years, $79.4 \mathrm{ft}^{3} / \mathrm{s}\left(2.249 \mathrm{~m}^{3} / \mathrm{s}\right), 57,530 \mathrm{acre}-\mathrm{ft} / \mathrm{yr}\left(70.9 \mathrm{hm}^{3} / \mathrm{yr}\right)$.

EXTREMES FOR PERIOD OF RECORD.--Maximum discharge, $11,200 \mathrm{ft} 3 / \mathrm{s}\left(317 \mathrm{~m}^{3} / \mathrm{s}\right)$ June 9, 1975, and June 16 , 1976 ; maximum gage height, $33.17 \mathrm{ft}(10.110 \mathrm{~m})$ June 9,$1975 ;$ minimum daily, $0.9 \mathrm{ft}^{3} / \mathrm{s}\left(0.025 \mathrm{~m}^{3} / \mathrm{s}\right) \mathrm{Aug}$. 7 , 1955 . EXTREMES FOR CURRENT YEAR.--Peak discharges above base of $2,200 \mathrm{ft}^{3} / \mathrm{s}\left(62.3 \mathrm{~m}^{3} / \mathrm{s}\right.$ ) and maximum (*):

\begin{tabular}{cccccc} 
Date & Time & \multicolumn{2}{c}{$\begin{array}{c}\text { Discharge } \\
\left(\mathrm{ft}^{3} / \mathrm{s}\right)\end{array}$} & $\begin{array}{c}\text { Gage height } \\
\left(\mathrm{m}^{3} / \mathrm{s}\right)\end{array}$ & $\begin{array}{c}\text { (ft) } \\
(\mathrm{m})\end{array}$ \\
Oct. 30 & 2115 & 2.790 & 79.0 & 21.92 & 6.681 \\
Jan. 22 & 1245 & $* 7.210$ & 204 & 29.53 & 9.001 \\
aJuly 21 & 1315 & 271 & 7.67 & 11.86 & 3.615
\end{tabular}

a Water-quality samples were obtained during this runoff event.

Minimum daily discharge, $34 \mathrm{ft}^{3} / \mathrm{s}\left(0.96 \mathrm{~m}^{3} / \mathrm{s}\right)$ for many days .

DISCHARGE, IN CUBIC FEET PER SECOND, WATER YEAR OCTOBER 1979 TO SEPTEMBER 1980

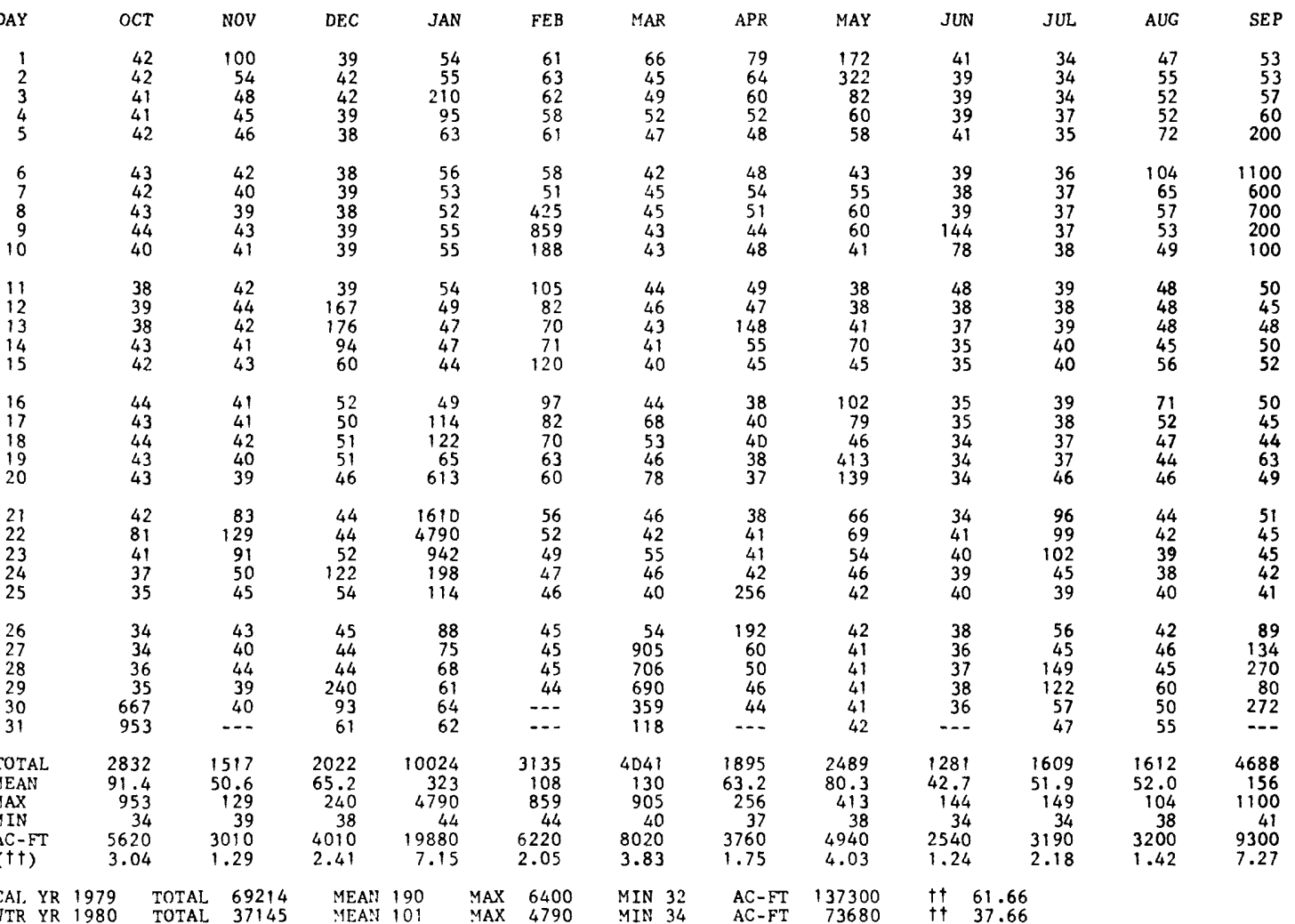

i† Weighted-mean rainfall, in inches, based on six rain gages. 
SAN JACINTO RIVER BASIN

08075500 SIMS BAYOU AT HOUSTON, TX--Continued

WATER-QUALITY RECORDS

PERIOD OF RECORD.--Chemical, biochemical, and pesticide analyses: Dctober 1968 to current year.

WATER QUALITY DATA, WATER YEAR OCTOBER 1979 TO SEPTEMBER 1980

\begin{tabular}{|c|c|c|c|c|c|c|c|c|c|c|}
\hline DATE & TIME & $\begin{array}{l}\text { STREAM- } \\
\text { FLOW, } \\
\text { INSTAN- } \\
\text { TANEOUS } \\
\text { (CFS) }\end{array}$ & $\begin{array}{l}\text { SPE- } \\
\text { CIFIC } \\
\text { CON- } \\
\text { DUCT- } \\
\text { ANCE } \\
\text { (MICRO- } \\
\text { MHOS) }\end{array}$ & $\begin{array}{c}\text { PH } \\
\text { FIELD } \\
\text { (UNITS) }\end{array}$ & $\begin{array}{c}\text { TEMPER- } \\
\text { ATURE, } \\
\text { WATER } \\
\text { (DEG C) }\end{array}$ & $\begin{array}{l}\text { COLOR } \\
\text { (PLAT- } \\
\text { INUM } \\
\text { COBALT } \\
\text { UNITS) }\end{array}$ & $\begin{array}{c}\text { TUR- } \\
\text { BID- } \\
\text { ITY } \\
\text { (NTU) }\end{array}$ & $\begin{array}{c}\text { OXYGEN, } \\
\text { DIS - } \\
\text { SOLVED } \\
\text { (MG/L) }\end{array}$ & $\begin{array}{c}\text { OXYGEN, } \\
\text { OIS- } \\
\text { SOLVED } \\
\text { (PER- } \\
\text { CENI } \\
\text { SATUR- } \\
\text { ATION) }\end{array}$ & $\begin{array}{c}\text { OXYGEN } \\
\text { DEMAND, } \\
\text { B IOCHEM } \\
\text { UN I NHI } \\
5 \text { DAY } \\
(M G / L)\end{array}$ \\
\hline AN & & & & & & & & & & \\
\hline$\underset{\text { JUL }}{03} \ldots$ & 1310 & 323 & 894 & 7.1 & 14.5 & 40 & 180 & 9.0 & 88 & 60 \\
\hline $\begin{array}{r}15 \ldots \\
21 \ldots \\
\text { AUG }\end{array}$ & $\begin{array}{l}1300 \\
1615\end{array}$ & $\begin{array}{r}40 \\
142\end{array}$ & $\begin{array}{r}1040 \\
381\end{array}$ & $\begin{array}{l}7.5 \\
7.5\end{array}$ & $\begin{array}{l}31.0 \\
27.0\end{array}$ & $\begin{array}{l}25 \\
60\end{array}$ & $70^{2.4}$ & $\begin{array}{l}7.6 \\
4.9\end{array}$ & $\begin{array}{r}100 \\
60\end{array}$ & $\begin{array}{l}30 \\
14\end{array}$ \\
\hline $12 \ldots$ & 1210 & 49 & 840 & 7.8 & 29.5 & 10 & 17 & 3.4 & 44 & 6. \\
\hline
\end{tabular}

\begin{tabular}{|c|c|c|c|c|c|c|c|c|c|}
\hline DATE & $\begin{array}{l}\text { COLI- } \\
\text { FORM, } \\
\text { TOTAL, } \\
\text { IMMED. } \\
\text { (COLS. } \\
\text { PER } \\
\text { 100 ML) }\end{array}$ & $\begin{array}{l}\text { COLI- } \\
\text { FORM, } \\
\text { FECAL, } \\
0.7 \\
\text { UM-MF } \\
\text { (COLS./ } \\
100 \mathrm{ML} \text { ) }\end{array}$ & $\begin{array}{c}\text { STREP- } \\
\text { TOCOCCI } \\
\text { FECAL, } \\
\text { KF AGAR } \\
\text { (COLS. } \\
\text { PER } \\
100 \mathrm{ML} \text { ) }\end{array}$ & $\begin{array}{l}\text { HARD- } \\
\text { NESS } \\
\text { (NG/L } \\
\text { AS } \\
\text { CACO3) }\end{array}$ & $\begin{array}{c}\text { HARD- } \\
\text { NESS, } \\
\text { NONCAR- } \\
\text { BONATE } \\
(\text { MG } / L \\
\text { CACO3) }\end{array}$ & $\begin{array}{l}\text { CALCIUM } \\
\text { DIS- } \\
\text { SOLVED } \\
\text { (MG/L } \\
\text { AS CA) }\end{array}$ & $\begin{array}{l}\text { MAGNE- } \\
\text { SIUM, } \\
\text { DIS- } \\
\text { SOLVED } \\
\text { (MG/L } \\
\text { AS MG) }\end{array}$ & $\begin{array}{l}\text { SODIUM, } \\
\text { DIS- } \\
\text { SOLVED } \\
\text { (MG/L } \\
\text { AS NA) }\end{array}$ & $\begin{array}{l}\text { SODIUM } \\
\text { AD- } \\
\text { SORP- } \\
\text { TION } \\
\text { RATIO }\end{array}$ \\
\hline JAN & & & & & & & & & \\
\hline$\underset{\text { JUL }}{03} \cdots$ & 460000 & 60000 & 14000 & - & - & - & -- & -- & \\
\hline $\begin{array}{r}15 \ldots \\
21 \ldots \\
\text { AUG }\end{array}$ & $\begin{array}{l}140000 \\
140000\end{array}$ & $\begin{array}{r}39000 \\
110000\end{array}$ & $\begin{array}{r}2300 \\
41000\end{array}$ & $\begin{array}{r}130 \\
70\end{array}$ & $\begin{array}{l}0 \\
0\end{array}$ & $\begin{array}{l}37 \\
22\end{array}$ & $\begin{array}{l}8.0 \\
3.6\end{array}$ & $\begin{array}{r}180 \\
50\end{array}$ & $\begin{array}{l}7.0 \\
2.6\end{array}$ \\
\hline $12 \ldots$ & 340000 & 70000 & 520 & 120 & 0 & 37 & 7.1 & 130 & 5.1 \\
\hline DATE & $\begin{array}{l}\text { POTAS- } \\
\text { SIUM, } \\
\text { DIS- } \\
\text { SOLVED } \\
\text { (MG/L } \\
\text { AS K) }\end{array}$ & $\begin{array}{c}\text { BICAR - } \\
\text { BONATE } \\
\text { (MG/L } \\
\text { AS } \\
\text { HC03) }\end{array}$ & $\begin{array}{c}\text { CAR- } \\
\text { BONATE } \\
\text { (MG/L } \\
\text { AS C03) }\end{array}$ & $\begin{array}{l}\text { SULFATE } \\
\text { DIS- } \\
\text { SOLVED } \\
\text { (MG/L } \\
\text { AS S04) }\end{array}$ & $\begin{array}{l}\text { CHLO- } \\
\text { RIDE, } \\
\text { DIS- } \\
\text { SOLVED } \\
\text { (MG /L } \\
\text { AS CL) }\end{array}$ & $\begin{array}{l}\text { FLUD- } \\
\text { RIDE, } \\
\text { DIS- } \\
\text { SOLVED } \\
\text { (MG/L } \\
\text { AS F) }\end{array}$ & $\begin{array}{l}\text { SILICA, } \\
\text { DIS- } \\
\text { SOLVED } \\
\text { (MG/L } \\
\text { AS } \\
\text { SIO2) }\end{array}$ & $\begin{array}{l}\text { SOLIDS, } \\
\text { SUM OF } \\
\text { CONSTI - } \\
\text { TUENTS, } \\
\text { DIS- } \\
\text { SOLVED } \\
\text { (MG /L) }\end{array}$ & $\begin{array}{l}\text { SOLIDS, } \\
\text { RESIDUE } \\
\text { AT } 105 \\
\text { DEG. C. } \\
\text { SUS- } \\
\text { PENDED } \\
\text { (MG/L) }\end{array}$ \\
\hline JAN & & & & & & & & & \\
\hline$\underset{\text { JUL }}{03 \ldots}$ & -- & -- & -- & -- & -- & -- & -- & -- & \\
\hline $\begin{array}{r}15 \ldots \\
21 \ldots\end{array}$ & $\begin{array}{l}6.0 \\
3.8\end{array}$ & $\begin{array}{l}240 \\
110\end{array}$ & $\begin{array}{l}0 \\
0\end{array}$ & $\begin{array}{r}160 \\
24\end{array}$ & $\begin{array}{r}100 \\
46\end{array}$ & $\begin{array}{l}.6 \\
.3\end{array}$ & $\begin{array}{l}16 \\
6.4\end{array}$ & $\begin{array}{l}626 \\
210\end{array}$ & \\
\hline $12 \ldots$ & 4.7 & 220 & 0 & 120 & 83 & .5 & 12 & 503 & 4 \\
\hline DATE & $\begin{array}{l}\text { SOLIDS, } \\
\text { VOLA- } \\
\text { TILE. } \\
\text { SUS- } \\
\text { PENDED } \\
\text { (MG/L) }\end{array}$ & $\begin{array}{l}\text { NITRO- } \\
\text { GEN, } \\
\text { NITRATE } \\
\text { TOTAL } \\
(\text { MG/L } \\
\text { AS N) }\end{array}$ & $\begin{array}{l}\text { WITRO- } \\
\text { GHN } \\
\text { NITRITE } \\
\text { TOTAL } \\
\text { (MG/L } \\
\text { AS N) }\end{array}$ & $\begin{array}{c}\text { NITRO- } \\
\text { GEN } \\
\text { NO2+NO3 } \\
\text { TOTAL } \\
(\text { MG / } \\
\text { AS N) }\end{array}$ & $\begin{array}{l}\text { NITRO- } \\
\text { GEN, } \\
\text { AMMONIA } \\
\text { TOTAL } \\
\text { (MG /L } \\
\text { AS N) }\end{array}$ & $\begin{array}{l}\text { NITRO- } \\
\text { GEN, } \\
\text { ORGANIC } \\
\text { TOTAL } \\
\text { (MG/L } \\
\text { AS N) }\end{array}$ & $\begin{array}{l}\text { NITRO- } \\
\text { GEN, AM- } \\
\text { MONIA + } \\
\text { ORGANIC } \\
\text { TOTAL } \\
\text { (MG /L } \\
\text { AS N) }\end{array}$ & $\begin{array}{l}\text { PHOS- } \\
\text { PHORUS. } \\
\text { TOTAL } \\
\text { (MG /L } \\
\text { AS P) }\end{array}$ & $\begin{array}{l}\text { CARBON, } \\
\text { ORGANIC } \\
\text { TOTAL } \\
\text { (MG/L } \\
\text { AS C) }\end{array}$ \\
\hline JAN & & & & & & & & & \\
\hline$\underset{\text { JUL }}{03} \cdots$ & 74 & 1.3 & .310 & 1.6 & 1.300 & 3.0 & 4.3 & 1.600 & 26 \\
\hline $\begin{array}{r}15 \ldots \\
21 \ldots \\
\text { AUG }\end{array}$ & $\begin{array}{r}0 \\
18\end{array}$ & $\begin{array}{l}1.1 \\
.76\end{array}$ & $\begin{array}{l}.220 \\
.070\end{array}$ & $\begin{array}{c}1.3 \\
.83\end{array}$ & $\begin{array}{r}2.000 \\
.930\end{array}$ & $\begin{array}{l}1.1 \\
1.6\end{array}$ & $\begin{array}{l}3.1 \\
2.5\end{array}$ & $\begin{array}{l}3.000 \\
1.300\end{array}$ & $\begin{array}{l}11 \\
18\end{array}$ \\
\hline 12. & 18 & 1.3 & .510 & 1.8 & 1.700 & 1.8 & 3.5 & 2.000 & 18 \\
\hline
\end{tabular}

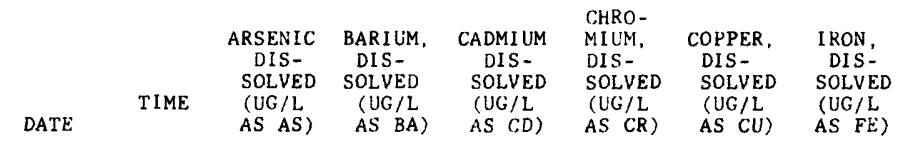

$\begin{array}{rrrrrrrr}\text { JUL } & & & & & & & \\ 15 \ldots & 1300 & 12 & 100 & <1 & 10 & 3 & <10 \\ 21 \ldots & 1615 & 11 & 50 & <1 & 10 & 4 & 30 \\ \text { AUG } & & 11 & 90 & <1 & 0 & 2 & <10 \\ 12 \ldots & 1210 & 11 & & & & & \end{array}$

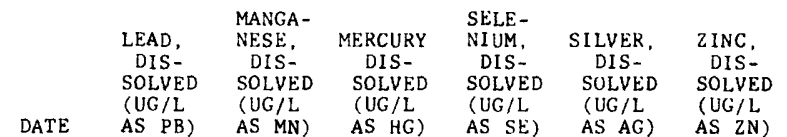


SAN JACINTO RIVER BASIN

08075500 SIMS BAYOU AT HOUSTON, TX--Continued

WATER QUALITY DATA, WATER YEAR OCTOBER 1979 TO SEPTEMBER 1980

\begin{tabular}{|c|c|c|c|c|c|c|c|c|c|}
\hline DATE & TIME & $\begin{array}{l}\text { PCB, } \\
\text { TOTAL } \\
\text { (UG/L) }\end{array}$ & $\begin{array}{l}\text { NAPH- } \\
\text { THA- } \\
\text { LENES, } \\
\text { POLY- } \\
\text { CHLOR. } \\
\text { TOTAL } \\
\text { (UG/L) }\end{array}$ & $\begin{array}{l}\text { ALDRIN, } \\
\text { TOTAL } \\
\text { (UG/L) }\end{array}$ & $\begin{array}{l}\text { CHLOR- } \\
\text { DANE, } \\
\text { TOTAL } \\
\text { (UG/L) }\end{array}$ & $\begin{array}{l}\text { DDD, } \\
\text { TOTAL } \\
\text { (UG/L) }\end{array}$ & $\begin{array}{l}\text { DDE, } \\
\text { TOTAL } \\
\text { (UG/L) }\end{array}$ & $\begin{array}{l}\text { DDT, } \\
\text { TOTAL } \\
\text { (UG/L) }\end{array}$ & $\begin{array}{c}\text { DI- } \\
\text { AZ INON, } \\
\text { TOTAL } \\
\text { (UG/L) }\end{array}$ \\
\hline $\begin{array}{l}\text { JUL } \\
15 \ldots \\
21 \ldots \\
\text { AUG }\end{array}$ & $\begin{array}{l}1300 \\
1615\end{array}$ & $\begin{array}{l}.0 \\
.0\end{array}$ & $\begin{array}{l}.00 \\
.00\end{array}$ & .00 & .0 & $\begin{array}{l}.00 \\
.00\end{array}$ & $\begin{array}{l}.00 \\
.00\end{array}$ & $\begin{array}{l}.00 \\
.02\end{array}$ & 1.04 \\
\hline $12 \ldots$ & 1210 & .0 & .00 & .00 & .0 & .00 & .00 & .00 & .07 \\
\hline DATE & $\begin{array}{l}\text { DI- } \\
\text { ELDRIN } \\
\text { TOTAL } \\
\text { (UG/L) }\end{array}$ & $\begin{array}{l}\text { ENDO- } \\
\text { SULFAN, } \\
\text { TOTAL } \\
\text { (UG/L) }\end{array}$ & $\begin{array}{l}\text { ENDRIN, } \\
\text { TOTAL } \\
\text { (UG/L) }\end{array}$ & $\begin{array}{l}\text { ETHION, } \\
\text { TOTAL } \\
\text { (UG/L) }\end{array}$ & $\begin{array}{l}\text { HEPTA- } \\
\text { CHLOR, } \\
\text { TOTAL } \\
\text { (UG/L) }\end{array}$ & $\begin{array}{c}\text { HEPTA- } \\
\text { CHLOR } \\
\text { EPOXIDE } \\
\text { TOTAL } \\
\text { (UG/L) }\end{array}$ & $\begin{array}{c}\text { LINDANE } \\
\text { TOTAL } \\
\text { (UG/L) }\end{array}$ & $\begin{array}{l}\text { MALA- } \\
\text { THION, } \\
\text { TOTAL } \\
\text { (UG/L) }\end{array}$ & $\begin{array}{c}\text { METH- } \\
\text { OXY- } \\
\text { CHLOR, } \\
\text { TOTAL } \\
\text { (UG/L) }\end{array}$ \\
\hline $\begin{array}{l}\text { JUL } \\
15 \ldots \\
21 \ldots \\
\text { AUG }\end{array}$ & $\begin{array}{l}.00 \\
.01\end{array}$ & $\begin{array}{l}.00 \\
.00\end{array}$ & $\begin{array}{l}.00 \\
.00\end{array}$ & $\begin{array}{l}.00 \\
.00\end{array}$ & $\begin{array}{l}.00 \\
.00\end{array}$ & $\begin{array}{l}.00 \\
.00\end{array}$ & $\begin{array}{l}.01 \\
.01\end{array}$ & $\begin{array}{l}.01 \\
.25\end{array}$ & $\begin{array}{l}.00 \\
.00\end{array}$ \\
\hline $12 \ldots$ & .00 & .00 & .00 & .00 & .00 & .00 & .01 & .00 & .00 \\
\hline DATE & $\begin{array}{l}\text { METHYL } \\
\text { PARA- } \\
\text { THION, } \\
\text { TOTAL } \\
\text { (UG/L) }\end{array}$ & $\begin{array}{l}\text { METHYL } \\
\text { TRI- } \\
\text { THION, } \\
\text { TOTAL } \\
\text { (UG/L) }\end{array}$ & $\begin{array}{l}\text { MIREX, } \\
\text { TOTAL } \\
\text { (UG/L) }\end{array}$ & $\begin{array}{l}\text { PARA- } \\
\text { THION, } \\
\text { TOTAL } \\
\text { (UG/L) }\end{array}$ & $\begin{array}{l}\text { TOX- } \\
\text { APHENE, } \\
\text { TOTAL } \\
\text { (UG /L) }\end{array}$ & $\begin{array}{l}\text { TOTAL } \\
\text { TRI- } \\
\text { THION } \\
\text { (UG/L) }\end{array}$ & $\begin{array}{l}2,4-D \text {, } \\
\text { TOTAL } \\
\text { (UG/L) }\end{array}$ & $\begin{array}{l}2,4,5-T \\
\text { TOTAL } \\
\text { (UG/L) }\end{array}$ & $\begin{array}{l}\text { S ILVEX, } \\
\text { TOTAL } \\
\text { (UG/L) }\end{array}$ \\
\hline $\begin{array}{l}\text { JUL } \\
15 \ldots \\
21 \ldots \\
\text { AUG }\end{array}$ & $\begin{array}{l}.00 \\
.00\end{array}$ & $\begin{array}{l}.00 \\
.00\end{array}$ & $\begin{array}{l}.00 \\
.00\end{array}$ & $\begin{array}{l}.00 \\
.00\end{array}$ & $\begin{array}{l}0 \\
0\end{array}$ & $\begin{array}{l}.00 \\
.00\end{array}$ & $\begin{array}{l}.00 \\
.23\end{array}$ & $\begin{array}{l}.00 \\
.01\end{array}$ & $\begin{array}{l}.00 \\
.0^{\circ}\end{array}$ \\
\hline 12. & .00 & .00 & .00 & .00 & 0 & .00 & .07 & .00 & .00 \\
\hline
\end{tabular}




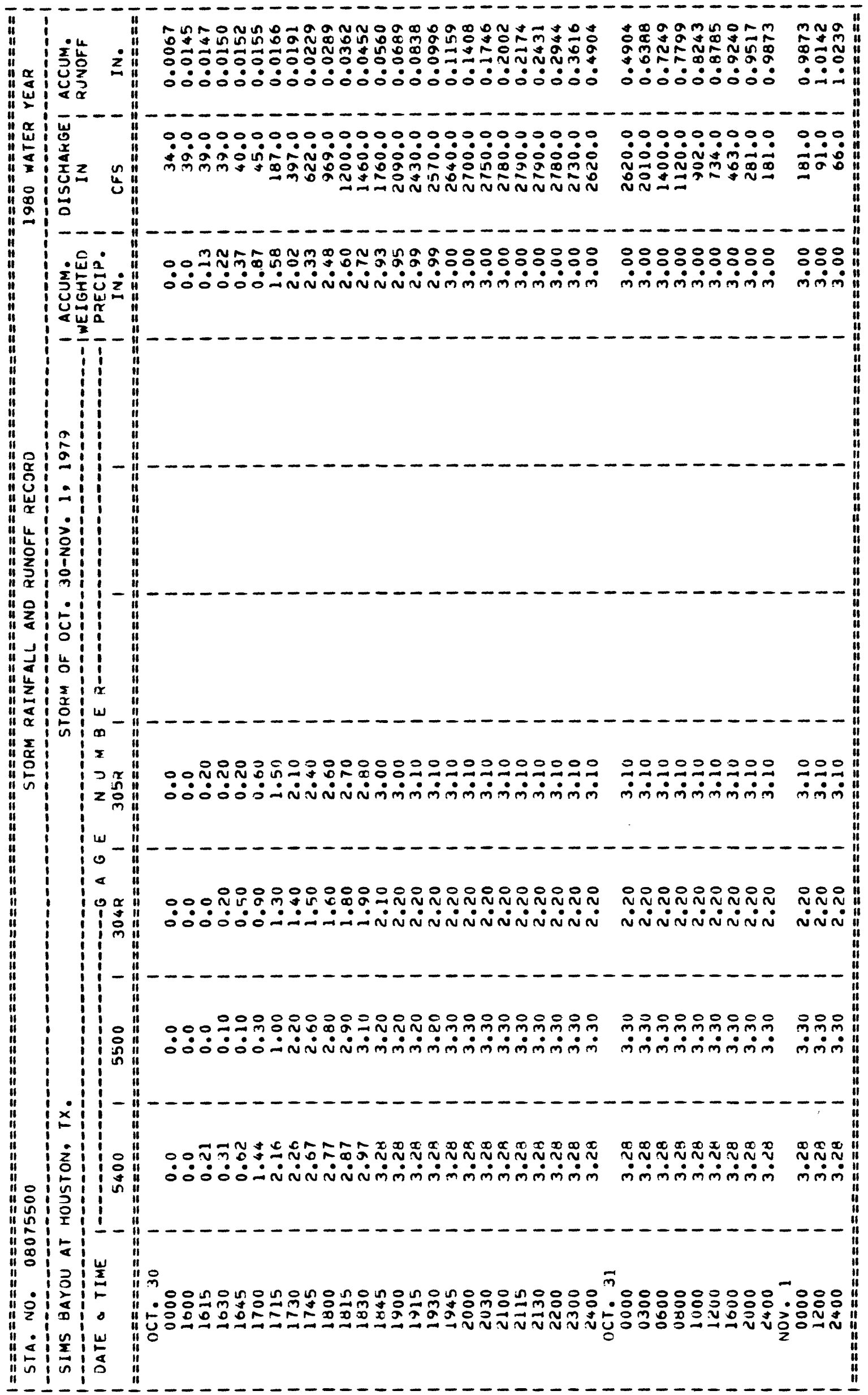

$-163-$ 


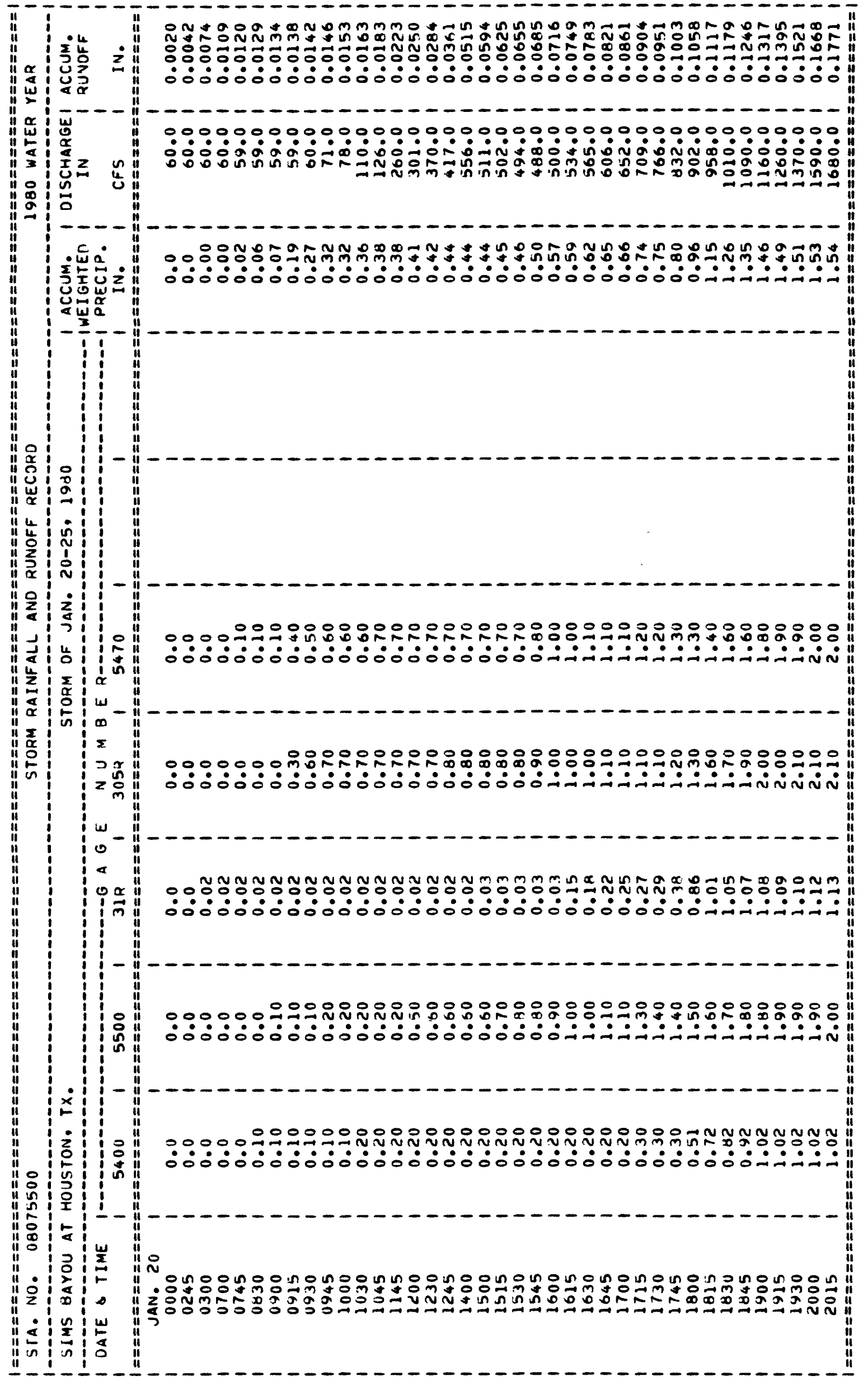

$-164-$ 


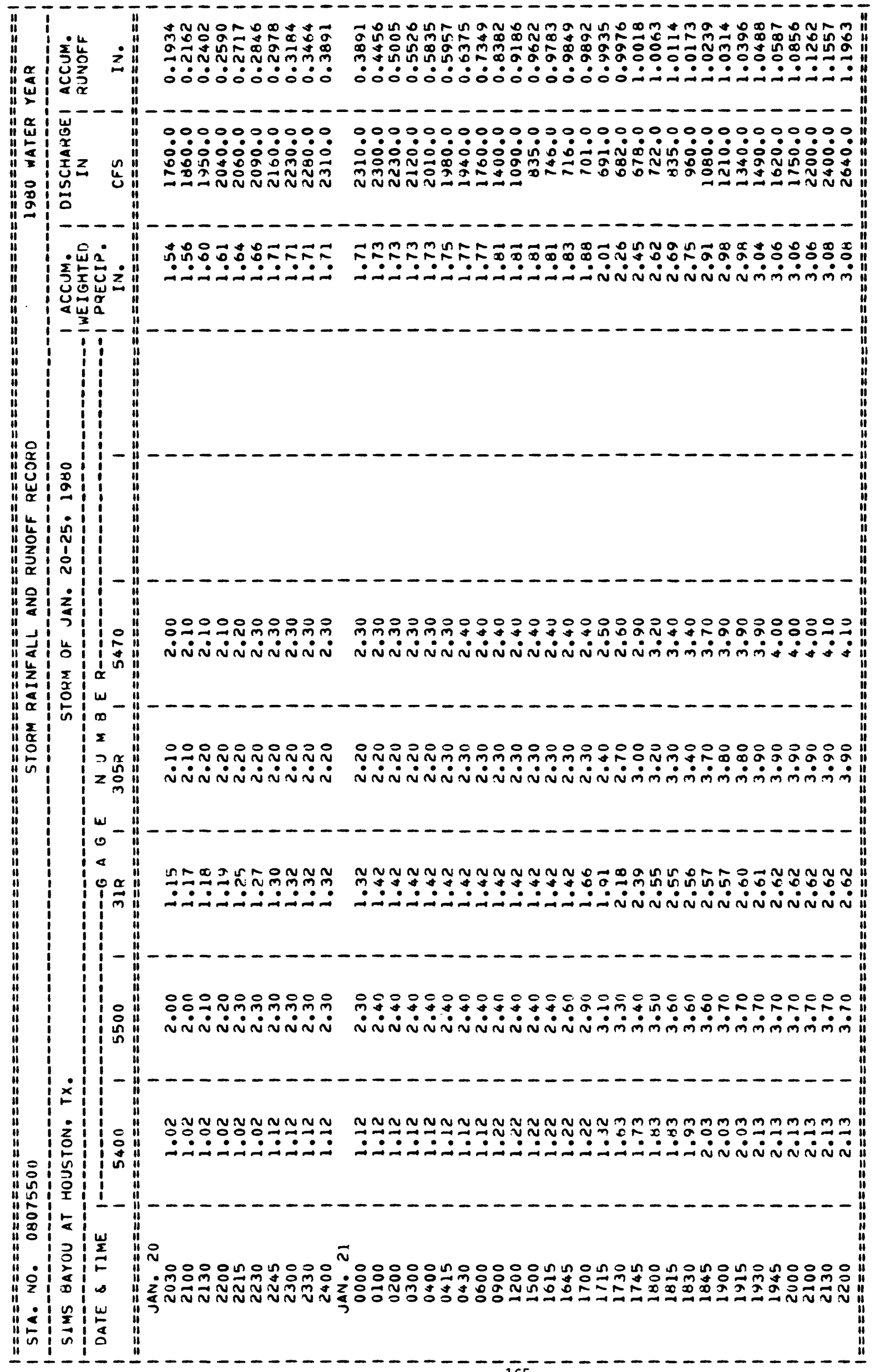

$-165-$ 


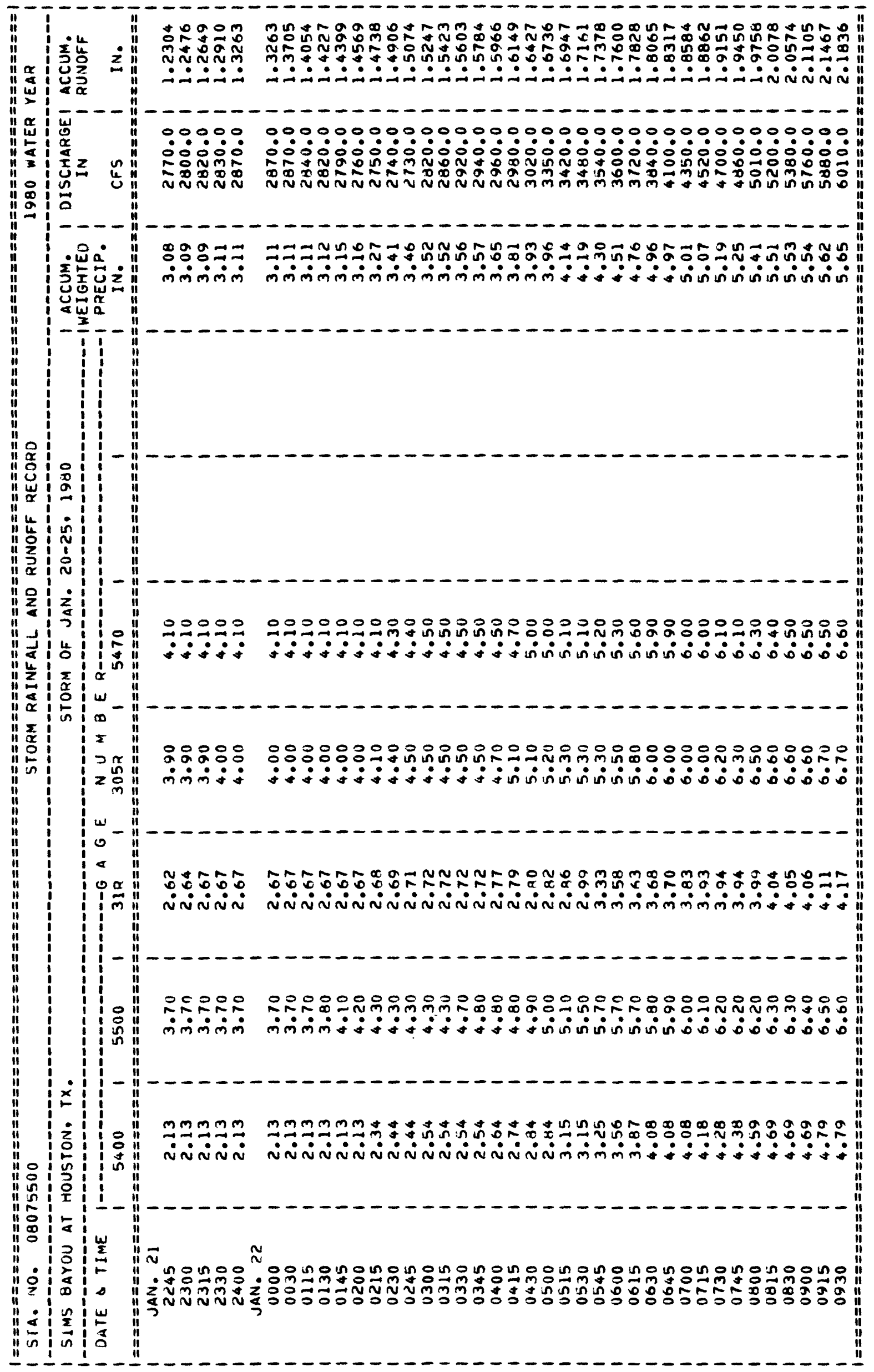

$-166-$ 


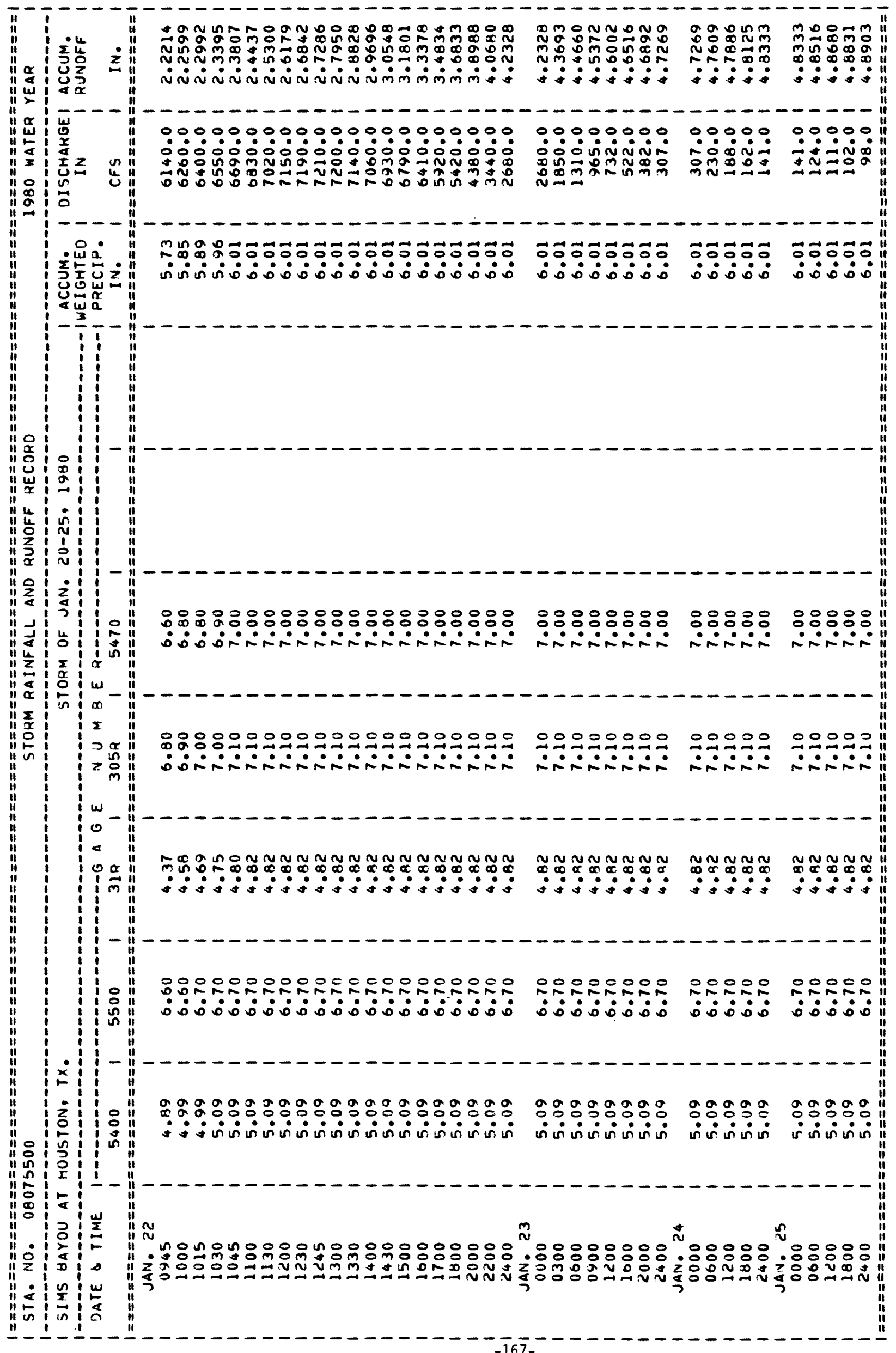




\section{BERRY BAYOU DRAINAGE BASIN}

The locations of data-collection sites in and near the Berry Bayou drainage basin are shown in figure 16.

Weighted-mean rainfall over the drainage basin for the 1980 water year was not determined.

The storm of Jan. 20-22 was selected for analysis at gaging station 08075550 , Berry Bayou at Gilpin Street. The storm of Oct. 30-31 was selected for analysis at station 08075650 , Berry Bayou at Forest 0aks Street. 


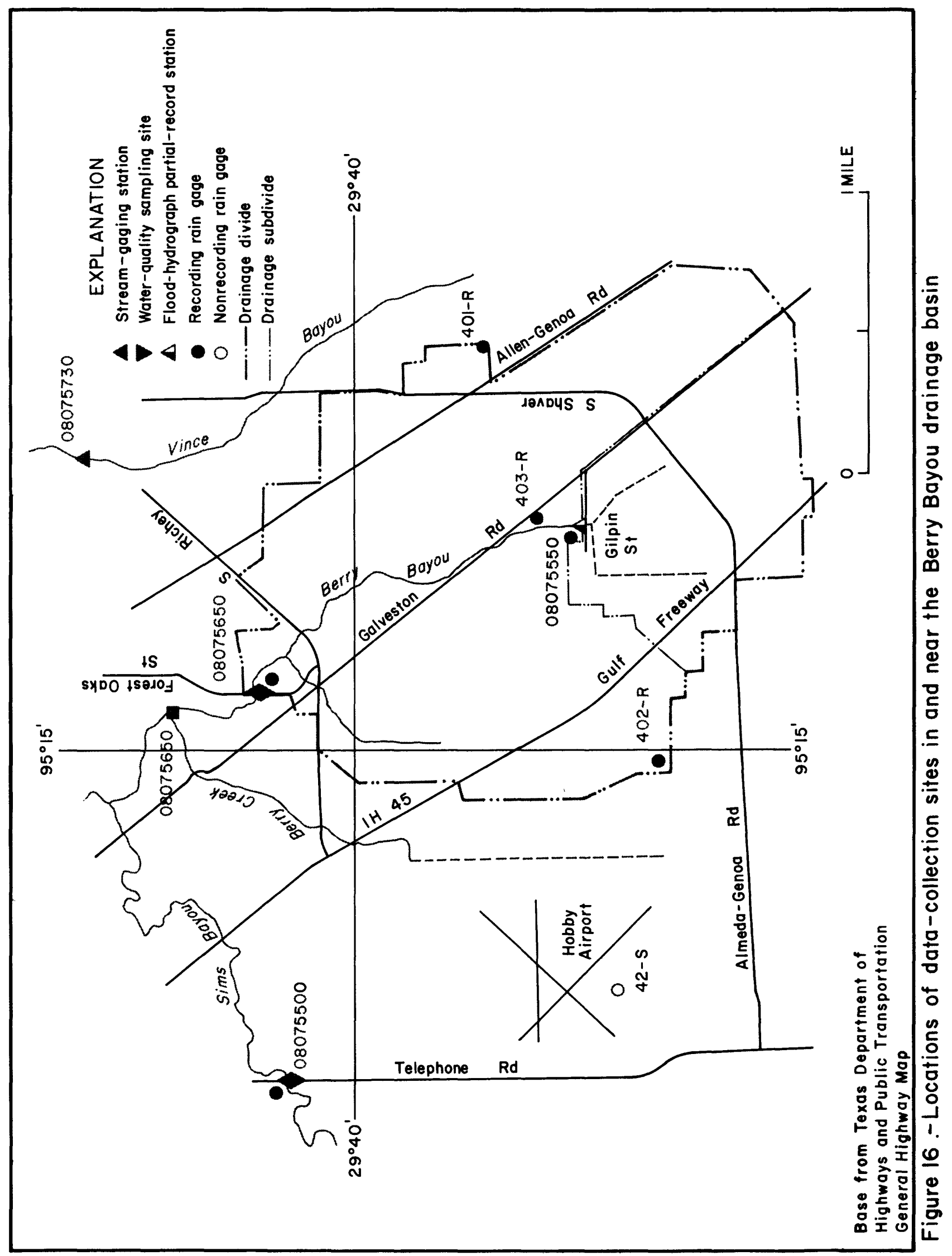




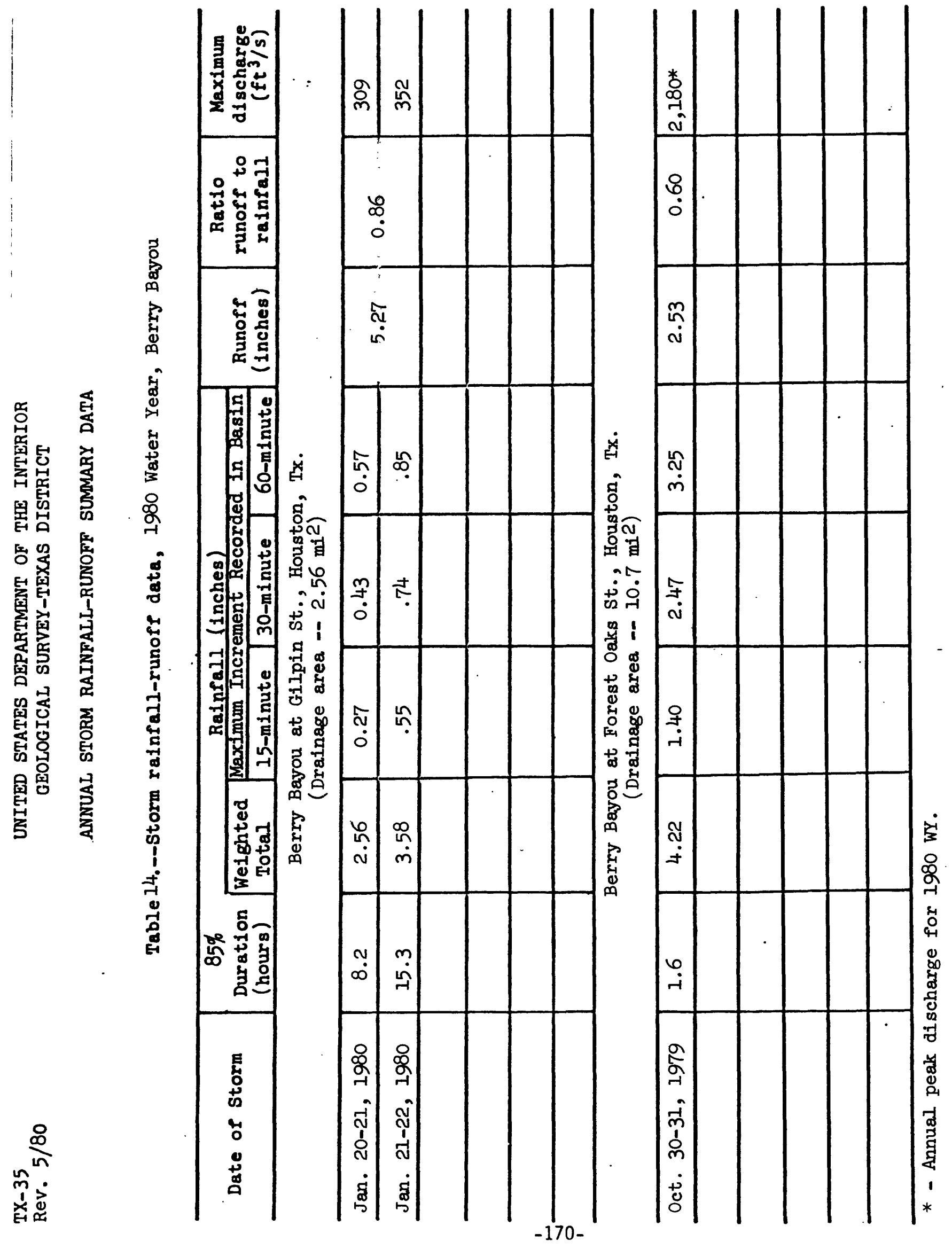


08075550 BERRY BAYOU AT GILPIN STREET, HOUSTON, TEX. (Flood-hydrograph partial-record station)

LOCATION.--Lat $29^{\circ} 38^{\prime} 32^{\prime \prime}$, long $95^{\circ} 13^{\prime} 22^{\prime \prime}$, Harris County, Hydrologic Unit 12040104 , at bridge on Gilpin Street in southeast Houston.

DRAINAGE AREA.--2.56 $\mathrm{mi}^{2}$. Oct. 1, 1973 to Oct. 1, 1978, $2.87 \mathrm{mi}^{2}$. Prior to Oct. $1,1973,3.26 \mathrm{mi}^{2}$.

PERIOD OF RECORD.--April 1964 to current year.

GAGE.--Digital flood-hydrograph and rainfall recorders and crest-stage gage. Prior to April 26, 1978 a flood hydrograph and rainfall recorder (type SR) and a crest-stage gage. Datum of gage is National Geodetic Vertical Datum of 1929,1959 adjustment, unadjusted for land surface subsidence.

REMARKS.--Records fair.

EXTREMES FOR PERIOD OF RECORD.--Maximum discharge, $738 \mathrm{ft}^{3} / \mathrm{s}$ May 10, 1968: Maximum elevation, $37.07 \mathrm{ft}$, July 26, 1979 . Minimum not determined.

EXTREMES FOR CURRENT YEAR.--Peak discharges above base of $300 \mathrm{ft}^{3} / \mathrm{s}$ and maximum (*):

DATE

Oct. 30

Jan. 20

Jan. 21

Jan. 22
TIME

unknown

2015

2000

0715
DISCHARGE

$\left(\mathrm{ft}^{3} / \mathrm{s}\right)$

*394

309

308

352
GAGE HEIGHT

35.39

33.58

33.57

34.10

Minimum discharge not determined. 


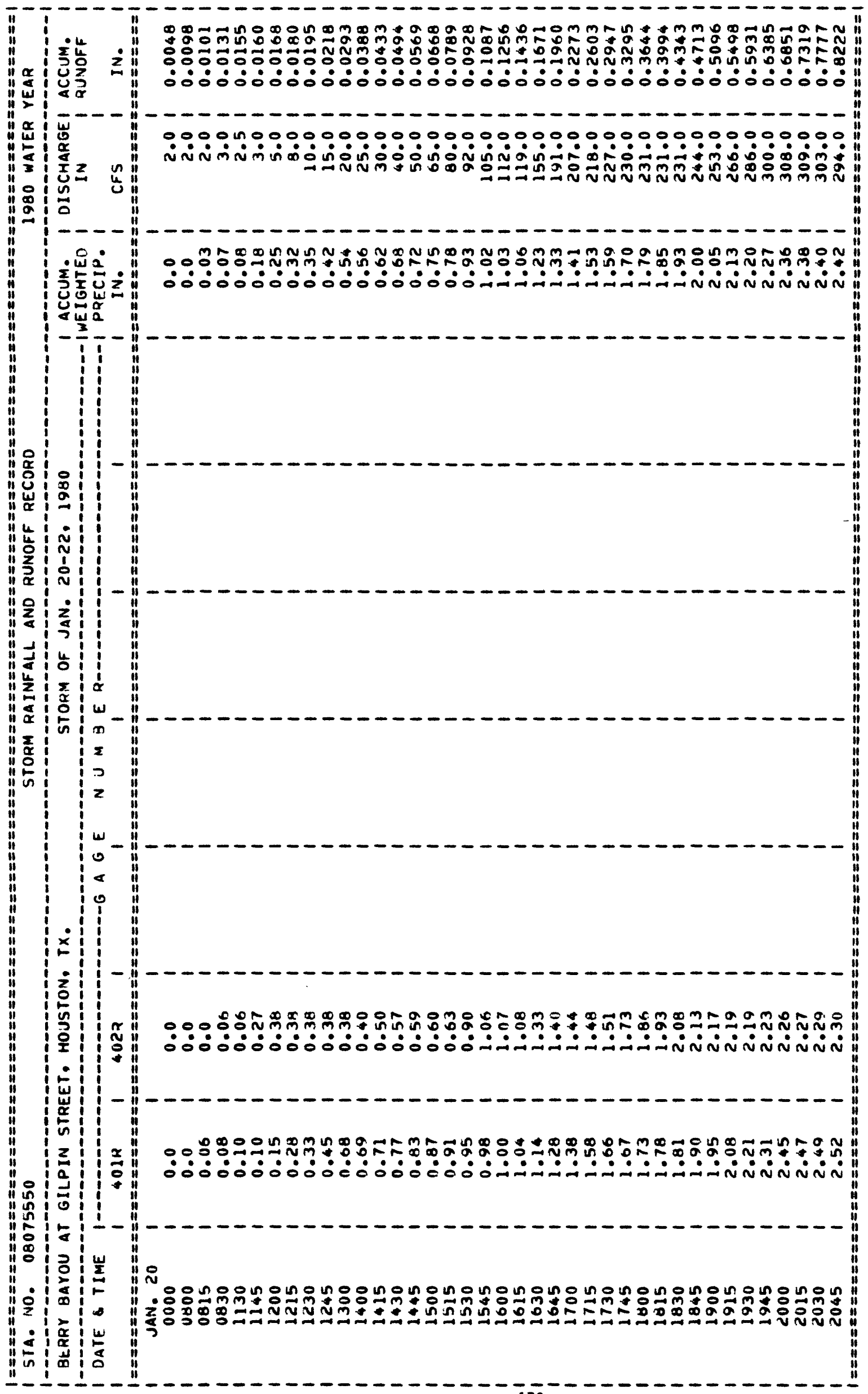




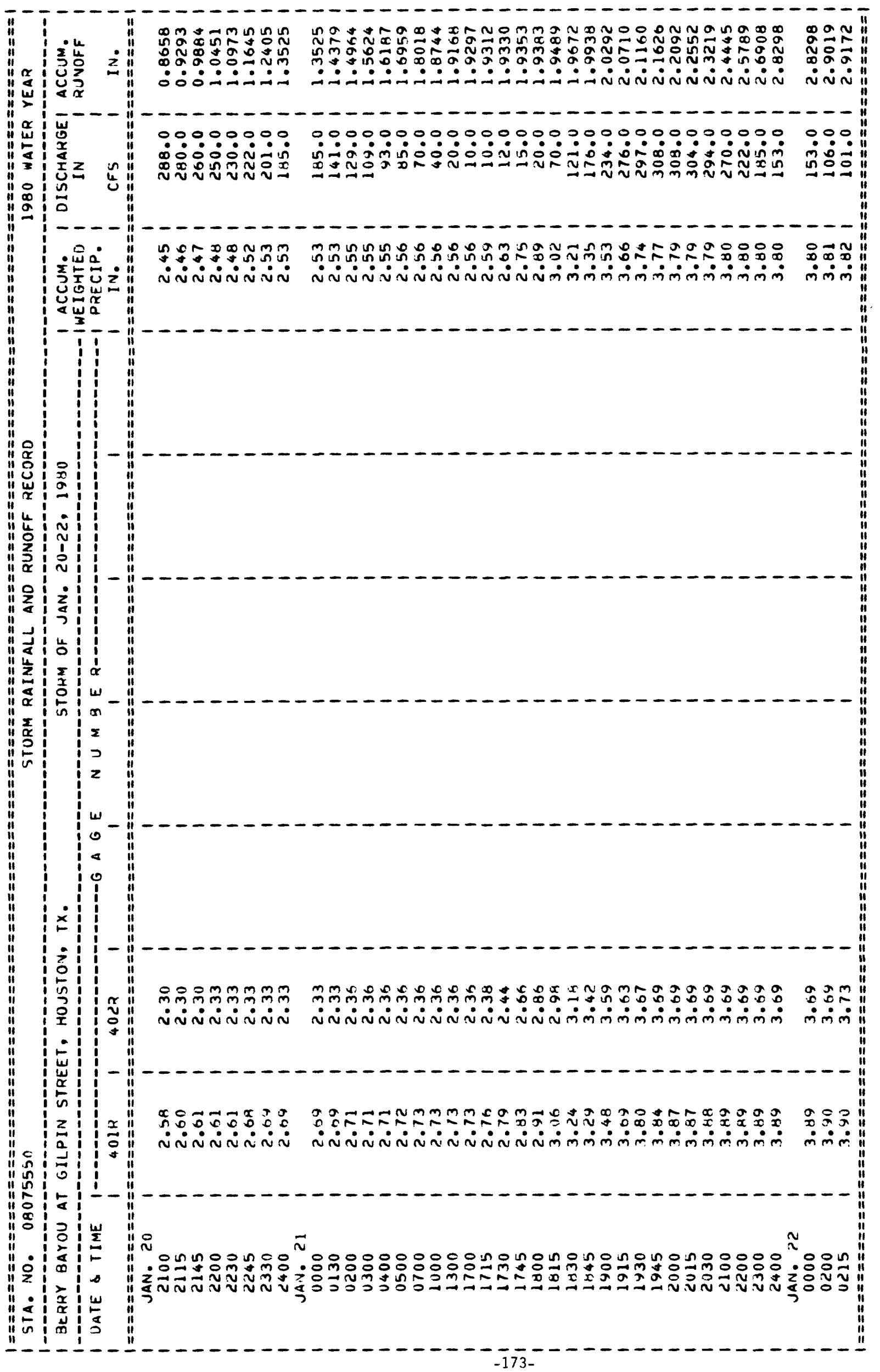




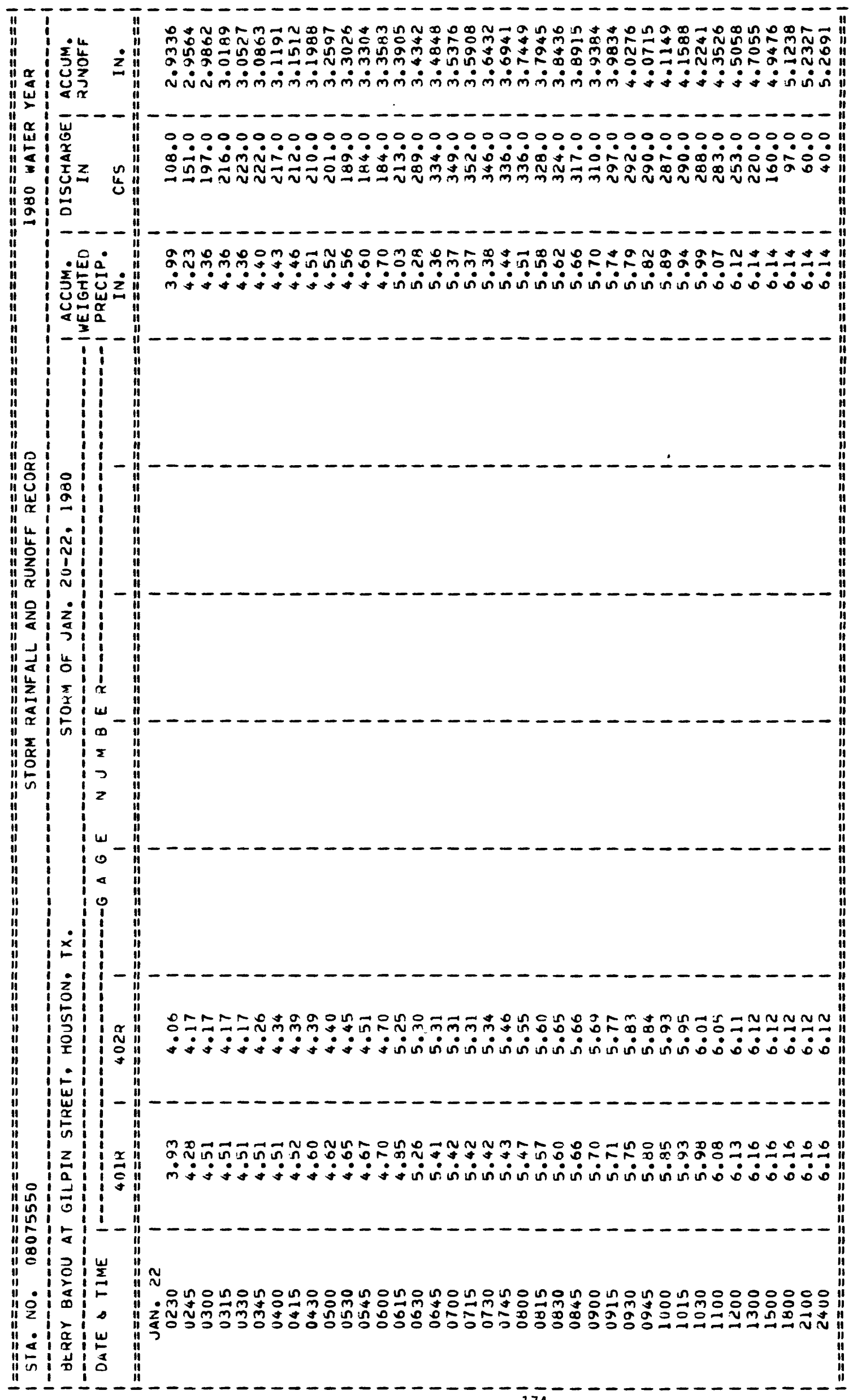


08075650 BERRY BAYOU AT FOREST OAKS STREET, HOUSTON, TX (Flood-hydrograph partial-record station)

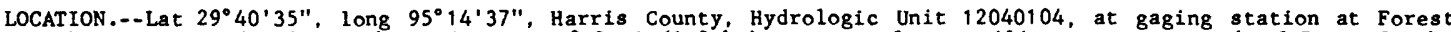
Oaks Street Bridge in southeast Houston, $0.8 \mathrm{mi}(1.3 \mathrm{~km})$ upstream from auxiliary gage at mouth of Berry Creek. and $1.7 \mathrm{mi}(2.7 \mathrm{~km})$ upstream from Sims Bayou.

DRAINAGE AREA. $-10.7 \mathrm{mi}^{2}\left(27.7 \mathrm{~km}^{2}\right)$. Prior to Oct. 1, 1973, $11.1 \mathrm{mi}^{2}\left(28.7 \mathrm{~km}^{2}\right)$. Oct. 1, 1976, to Dec. 31 , 1977, $10.1 \mathrm{mi}^{2}\left(26.2 \mathrm{~km}^{2}\right)$. Drainage ditch relocationa resulted in drainage area changes. WATER-DISCHARGE RECORDS

PERIOD OF RECORD.--October 1967 to current year. April 1964 to September 1966 operated as a daily discharge station.

GAGE.--Water-stage recorder and crest-stage gages. Datum of gage is $2.72 \mathrm{ft}(0.829 \mathrm{~m})$ below National Geodetic Vertical Datum of 1929. 1973 adjustment. Auxiliary water-stage recorder $0.8 \mathrm{mi}(1.3 \mathrm{~km})$ downstream at same datum. June 25, 1964, to Jan. 11, 1965, auxiliary nonrecording gage $0.8 \mathrm{mi}(1.3 \mathrm{~km})$ downstream at same datum.

REMARRS.--Additional storm rainfall-runoff data for this site can be obtained from the report "Hydrologic Data for Urban Studies in the Houston, Texas Metropolitan Area, 1980."

EXTREMES FOR PERIOD OF RECORD.--Maximum discharge, 4,650 $\mathrm{ft}^{3} / \mathrm{s}\left(132 \mathrm{~m}^{3} / \mathrm{s}\right)$, revised, July 26 , 1979; maximum gage height, $23.85 \mathrm{ft}(7.269 \mathrm{~m})$ Sept. 20, 1979.

EXTREMES FOR CURRENT YEAR.--Peak discharges above base of $900 \mathrm{ft}^{3} / \mathrm{s}\left(25.5 \mathrm{~m}^{3} / \mathrm{s}\right)$ and maximum ( $(*)$ :

\begin{tabular}{cccccc} 
Date & Time & \multicolumn{2}{c}{$\begin{array}{c}\text { Discharge } \\
\left(\mathrm{ft}^{3} / \mathrm{s}\right)\end{array}$} & $\begin{array}{c}\text { Gage height } \\
\left(\mathrm{m}^{3} / \mathrm{s}\right)\end{array}$ & $\begin{array}{c}(\mathrm{ft}) \\
(\mathrm{m})\end{array}$ \\
Oct. 30 & 1945 & $* 2,180$ & 61.7 & b16.74 & 5.102 \\
aJan. 22 & 1000 & 1,280 & 36.2 & b17.92 & 5.462 \\
aJuly 28 & 1615 & 128 & 3.62 & b 5.67 & 1.728 \\
a Water-quality samples were obtained during this runoff event. \\
b Not at same time as peak discharge.
\end{tabular}

REVISIONS.--The maximum discharge for the water year 1979 has been revised to $4,650 \mathrm{ft}^{3} / \mathrm{s}(132 \mathrm{~m} / \mathrm{s})$, July 26 , 1979, superseding figure published in the report for 1979. Peak discharge of Mar. 19 (2000 hours) has been ris superseding figure published in the report for 979 . Peak discharge of Mar. 19 (2000 hours) has been (0130 hours) has been revised to $4,350 \mathrm{ft}^{3} / \mathrm{s}\left(123 \mathrm{~m}^{3} / \mathrm{s}\right)$. The instantaneous flow value for the water-quality sample collected on July 26 (1255 hours) has been revised to $2,380 \mathrm{ft} / \mathrm{s}\left(6.74 \mathrm{~m}^{3} / \mathrm{s}\right)$.

\section{WATER-QUALITY RECORDS}

PERIOD OF RECORD.--Chemical, biochemical, and pesticide analyses. October 1968 to current year. Water temperatures: April 1964 to current year.

WATER QUALITY DATA, WATER YEAR OCTOBER 1979 TO SEPTEMBER 1980

\begin{tabular}{|c|c|c|c|c|c|c|c|c|c|c|}
\hline DATE & TIME & $\begin{array}{l}\text { STREAM- } \\
\text { FLOW, } \\
\text { INSTAN- } \\
\text { TANEOUS } \\
\text { (CFS) }\end{array}$ & $\begin{array}{l}\text { SPE- } \\
\text { CIFIC } \\
\text { CON- } \\
\text { DUCT- } \\
\text { ANCE } \\
\text { (MICRO- } \\
\text { MHOS) }\end{array}$ & $\begin{array}{c}\text { Pll } \\
\text { FIELD } \\
\text { (UNITS) }\end{array}$ & $\begin{array}{c}\text { TEMPER- } \\
\text { ATURE, } \\
\text { WATER } \\
\text { (DEG C) }\end{array}$ & $\begin{array}{l}\text { COLOR } \\
\text { (PLAT- } \\
\text { INUM } \\
\text { COBALT } \\
\text { UNITS) }\end{array}$ & $\begin{array}{c}\text { TUR- } \\
\text { BID- } \\
\text { ITY } \\
\text { (NTU) }\end{array}$ & $\begin{array}{c}\text { OXYGEN, } \\
\text { DIS- } \\
\text { SOLVED } \\
\text { (MG /L) }\end{array}$ & $\begin{array}{c}\text { OXYGEN, } \\
\text { DIS- } \\
\text { SOLVED } \\
\text { (PER- } \\
\text { CENT } \\
\text { SATUR- } \\
\text { ATION) }\end{array}$ & $\begin{array}{c}\text { OXYGEN } \\
\text { DEMAND, } \\
\text { BIOCHEM } \\
\text { UNINH I B } \\
5 \mathrm{DAY} \\
(\mathrm{MG} / \mathrm{L})\end{array}$ \\
\hline $\begin{array}{l}\text { JAN } \\
22 \ldots \\
\text { JUL }\end{array}$ & 1010 & 1270 & 119 & 7.0 & 16.0 & 120 & 110 & 7.9 & 79 & 6.9 \\
\hline $28 \ldots$ & 1350 & 8.4 & 1360 & 7.3 & 26.0 & 25 & 150 & 3.6 & 44 & 65 \\
\hline
\end{tabular}

\begin{tabular}{|c|c|c|c|c|c|c|c|c|c|}
\hline DATE & $\begin{array}{l}\text { COLI- } \\
\text { FORM, } \\
\text { TOTAL, } \\
\text { IMMED. } \\
\text { (COLS. } \\
\text { PER } \\
100 \mathrm{ML} \text { ) }\end{array}$ & $\begin{array}{l}\text { COLI- } \\
\text { FORM, } \\
\text { FECAL, } \\
0.7 \\
\text { UM-MF } \\
\text { (COLS./ } \\
100 \mathrm{ML} \text { ) }\end{array}$ & $\begin{array}{l}\text { STREP- } \\
\text { TOCOCCI } \\
\text { FECAL, } \\
\text { KF AGAR } \\
\text { (COLS. } \\
\text { PER } \\
100 \mathrm{ML} \text { ) }\end{array}$ & $\begin{array}{l}\text { HARD- } \\
\text { IIESS } \\
\text { (MC /L } \\
\text { AS } \\
\text { CACO3) }\end{array}$ & $\begin{array}{c}\text { HARD- } \\
\text { NESS } \\
\text { NONCAR- } \\
\text { BONATE } \\
\text { (MG/L } \\
\text { CACO3) }\end{array}$ & $\begin{array}{l}\text { CALCIUM } \\
\text { DIS- } \\
\text { SOLVED } \\
\text { (MG/L } \\
\text { AS CA) }\end{array}$ & $\begin{array}{l}\text { MAGNE- } \\
\text { SIUM, } \\
\text { DIS- } \\
\text { SOLVED } \\
\text { (MG/L } \\
\text { AS MG) }\end{array}$ & $\begin{array}{c}\text { SODIUM, } \\
\text { DIS- } \\
\text { SOLVED } \\
\text { (MG/L } \\
\text { AS NA) }\end{array}$ & $\begin{array}{c}\text { SODIUM } \\
\text { AD- } \\
\text { SORP- } \\
\text { TION } \\
\text { RATIO }\end{array}$ \\
\hline $\begin{array}{l}\text { JAN } \\
22 \\
\text { JUL }\end{array}$ & 190000 & 140000 & 45000 & 44 & 0 & 14 & 2.2 & 5.3 & .3 \\
\hline $28 \ldots$ & 920000 & 240000 & 56000 & 120 & 0 & 37 & 7.5 & 220 & 8.6 \\
\hline DATE & $\begin{array}{l}\text { POTAS- } \\
\text { SIUM, } \\
\text { DIS- } \\
\text { SOLVED } \\
\text { (MG/L } \\
\text { AS K) }\end{array}$ & $\begin{array}{c}\text { BICAR- } \\
\text { BONATE } \\
\text { (MG / L } \\
\text { AS } \\
\text { HCO3) }\end{array}$ & $\begin{array}{c}\text { CAR- } \\
\text { BONATE } \\
(M C / L \\
\text { AS CO3) }\end{array}$ & $\begin{array}{c}\text { SULFATE. } \\
\text { DIS- } \\
\text { SOLVED } \\
\text { (MG/L } \\
\text { AS S04) }\end{array}$ & $\begin{array}{l}\text { CHLO- } \\
\text { RIDE, } \\
\text { DIS- } \\
\text { SOLVED } \\
(M G / L \\
\text { AS CL) }\end{array}$ & $\begin{array}{l}\text { FLUO- } \\
\text { RIDE, } \\
\text { DIS- } \\
\text { SOLVED } \\
\text { (MG/L } \\
\text { AS F) }\end{array}$ & $\begin{array}{l}\text { SILICA. } \\
\text { DIS- } \\
\text { SOLVED } \\
\text { (MC/L } \\
\text { AS } \\
\text { SIO2) }\end{array}$ & $\begin{array}{c}\text { SOLIDS, } \\
\text { SUM OF } \\
\text { CONSTI- } \\
\text { TUENTS, } \\
\text { DIS- } \\
\text { SOLVED } \\
\text { (MG/L) }\end{array}$ & $\begin{array}{l}\text { SOLIDS, } \\
\text { RESIDUE } \\
\text { AT } 105 \\
\text { DEG. C, } \\
\text { SUS- } \\
\text { PENDED } \\
\text { (MG/L) }\end{array}$ \\
\hline $\begin{array}{l}\text { JAN } \\
22 \ldots \\
\text { JUL }\end{array}$ & 2.2 & 54 & 0 & 4.6 & 4.7 & .1 & 5.1 & 65 & 186 \\
\hline & 5.4 & 150 & 0 & 29 & 340 & .6 & 6.6 & 721 & 406 \\
\hline DA & $\begin{array}{l}\text { SOLIDS, } \\
\text { VOLA- } \\
\text { TILE, } \\
\text { SUS- } \\
\text { PENDED } \\
\text { (MG/L) }\end{array}$ & $\begin{array}{c}\text { NITRO- } \\
\text { GEN, } \\
\text { NITRATE } \\
\text { TOTAL } \\
\text { (MG/L } \\
\text { AS N) }\end{array}$ & $\begin{array}{c}\text { NITRO- } \\
\text { GEN, } \\
\text { NITR ITE } \\
\text { TOTAL } \\
\text { (MG/L } \\
\text { AS II) }\end{array}$ & $\begin{array}{c}\text { NITRO- } \\
\text { GEN, } \\
\text { NO2+NO3 } \\
\text { TOTAL } \\
\text { (MG/L } \\
\text { AS Ni) }\end{array}$ & $\begin{array}{l}\text { NITRO- } \\
\text { GEN } \\
\text { AMMONIA } \\
\text { TOTAL } \\
\text { (MG/L } \\
\text { AS N) }\end{array}$ & $\begin{array}{l}\text { IITRO- } \\
\text { GEN, } \\
\text { ORGANIC } \\
\text { TOTAL } \\
\text { (MG/L } \\
\text { AS N) }\end{array}$ & $\begin{array}{l}\text { NITRO- } \\
\text { GEN, AM- } \\
\text { MONIA + } \\
\text { ORGANIC } \\
\text { TOTAL } \\
\text { (MG/L } \\
\text { AS N) }\end{array}$ & $\begin{array}{c}\text { PHOS- } \\
\text { PHORUS, } \\
\text { TOTAL } \\
\text { (MG/L } \\
\text { AS P) }\end{array}$ & $\begin{array}{c}\text { CARBON, } \\
\text { ORGANIC } \\
\text { TOTAL } \\
\text { (MC/L } \\
\text { AS C) }\end{array}$ \\
\hline
\end{tabular}

\begin{tabular}{|c|c|c|c|c|c|c|c|c|c|}
\hline $\begin{array}{l}\text { JAN } \\
22 \ldots\end{array}$ & 44 & .09 & .010 & .10 & .080 & 1.0 & 1.1 & .180 & 11 \\
\hline $28 \ldots$ & 138 & .59 & .160 & .75 & 2.800 & 13 & 16 & 4.100 & 48 \\
\hline
\end{tabular}


SAN JACINTO RIVER BASIN

08075650 BERRY BAYOU AT FOREST OAKS STREET, HOUSTON, TX--Continued

WATER QUALITY DATA, WATER YEAR OCTOBER 1979 TO SEPTEMBER 1980

\begin{tabular}{|c|c|c|c|c|c|c|c|}
\hline DATE & TIME & $\begin{array}{c}\text { ARSENIC } \\
\text { DIS- } \\
\text { SOLVED } \\
\text { (UG/L } \\
\text { AS AS) }\end{array}$ & $\begin{array}{l}\text { BARIUM, } \\
\text { DIS- } \\
\text { SOLVED } \\
\text { (UG/L } \\
\text { AS BA) }\end{array}$ & $\begin{array}{c}\text { CADMIUM } \\
\text { DIS- } \\
\text { SOLVED } \\
\text { (UG/L } \\
\text { AS CD) }\end{array}$ & $\begin{array}{l}\text { CHRO- } \\
\text { MIUM, } \\
\text { DIS- } \\
\text { SOLVED } \\
\text { (UG/L } \\
\text { AS CR) }\end{array}$ & $\begin{array}{l}\text { COPPER, } \\
\text { DIS- } \\
\text { SOLVED } \\
\text { (UG/L } \\
\text { AS CU) }\end{array}$ & $\begin{array}{l}\text { IRON, } \\
\text { DIS- } \\
\text { SOLVED } \\
\text { (UG/L } \\
\text { AS FE) }\end{array}$ \\
\hline & & & & & & & \\
\hline $\begin{array}{l}22 . . . \\
\text { IUL }\end{array}$ & 1010 & 2 & 30 & $<1$ & 0 & 1 & 80 \\
\hline & 1350 & 11 & 300 & $<1$ & 10 & 2 & 60 \\
\hline
\end{tabular}

\begin{tabular}{|c|c|c|c|c|c|c|}
\hline DATE & $\begin{array}{l}\text { LEAD, } \\
\text { DIS- } \\
\text { SOLVED } \\
\text { (UG/L } \\
\text { AS PB) }\end{array}$ & $\begin{array}{l}\text { MANGA- } \\
\text { NESE, } \\
\text { DIS- } \\
\text { SOLVED } \\
\text { (UG/L } \\
\text { AS MN) }\end{array}$ & $\begin{array}{c}\text { MERCURY } \\
\text { DIS- } \\
\text { SOLVED } \\
\text { (UG/L } \\
\text { AS HG) }\end{array}$ & $\begin{array}{l}\text { SELE- } \\
\text { NIUM, } \\
\text { DIS- } \\
\text { SOLVED' } \\
\text { (UG/L } \\
\text { AS SE) }\end{array}$ & $\begin{array}{c}\text { SILVER, } \\
\text { DIS- } \\
\text { SOLVED } \\
\text { (UG/L } \\
\text { AS AG) }\end{array}$ & $\begin{array}{l}\text { ZINC, } \\
\text { DIS- } \\
\text { SOLVED } \\
\text { (UG/L } \\
\text { AS ZN) }\end{array}$ \\
\hline $\begin{array}{l}\text { JAN } \\
22 \\
\text { JUL }\end{array}$ & 2 & 1 & .1 & 0 & 0 & 7 \\
\hline 30 & 1 & 160 & .0 & 0 & 0 & 10 \\
\hline
\end{tabular}

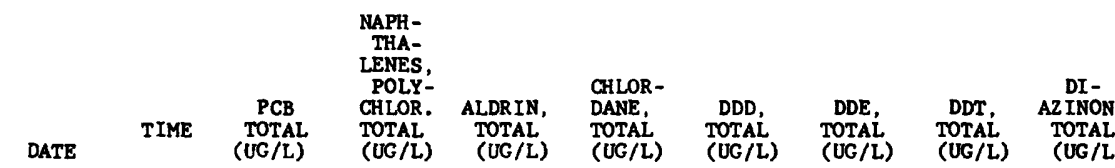

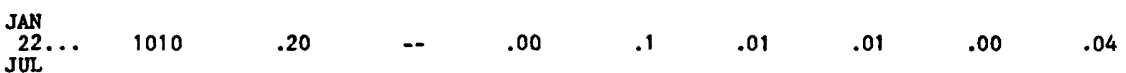

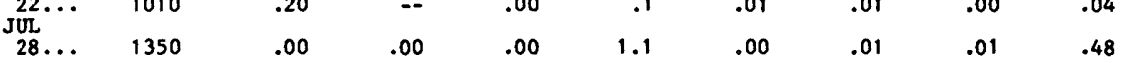

DI- ENDO-

ELDRIN SULFAN, ENDRIN, ETHION, HEPTA- CHLOR

EPOXIDE LINDANE THION, CHLOR,

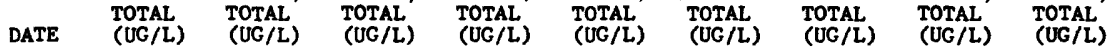

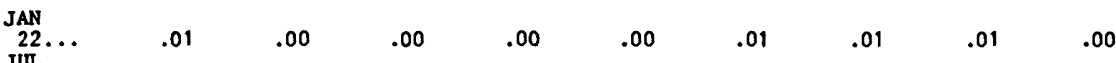

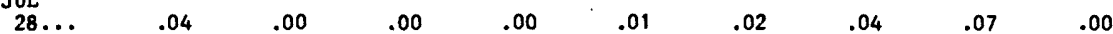

\begin{tabular}{|c|c|c|c|c|c|c|c|c|c|}
\hline DATE & $\begin{array}{l}\text { METHYL } \\
\text { PARA- } \\
\text { THION, } \\
\text { TOTAL, } \\
\text { (UG /L) }\end{array}$ & $\begin{array}{l}\text { METHYL } \\
\text { TR I- } \\
\text { TH ION, } \\
\text { TOTAL } \\
\text { (UG /L) }\end{array}$ & $\begin{array}{l}\text { MIREX, } \\
\text { TOTAL } \\
\text { (UG/L) }\end{array}$ & $\begin{array}{l}\text { PARA- } \\
\text { THION, } \\
\text { TOTAL } \\
\text { (UG /L) }\end{array}$ & $\begin{array}{l}\text { TOX- } \\
\text { APHENE, } \\
\text { TOTAL } \\
\text { (UG /L) }\end{array}$ & $\begin{array}{l}\text { TOTAL } \\
\text { TRI- } \\
\text { THION } \\
\text { (UG/L) }\end{array}$ & $\begin{array}{l}2,4-D, \\
\text { TOTAL } \\
\text { (UG/L) }\end{array}$ & $\begin{array}{l}2,4,5-\mathrm{T} \\
\text { TOTAL } \\
\text { (UG /L) }\end{array}$ & $\begin{array}{l}\text { SILVEX, } \\
\text { TOTAL, } \\
\text { (UG/L) }\end{array}$ \\
\hline $\begin{array}{r}\text { JAN } \\
22 \ldots \\
\text { JUL }\end{array}$ & .00 & .00 & .00 & .00 & 0 & .00 & .07 & .01 & .00 \\
\hline & .00 & .00 & .00 & .01 & 0 & .00 & .20 & .00 & .00 \\
\hline
\end{tabular}




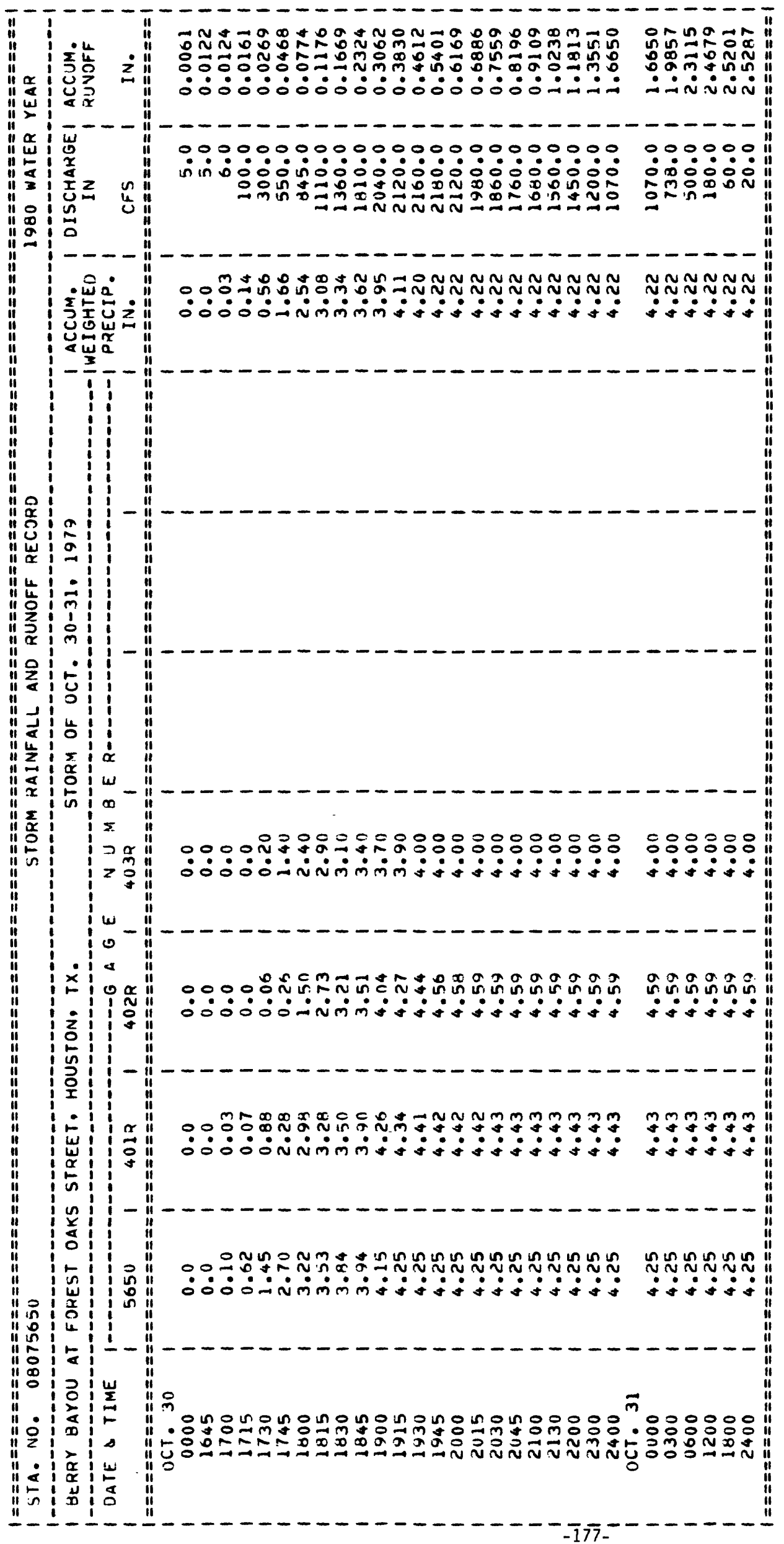




\section{VINCE BAYOU DRAINAGE BASIN}

The locations of data-collection sites in and near the drainage basin are shown in fiqure 17.

Weighted-mean rainfall in the drainage basin based on two rain gages for the 1980 water year was 36.02 inches or 12.17 inches less than the 30-year (1941-70) average of 48.19 inches for Houston.

No storms were analyzed for the 1980 water year. 


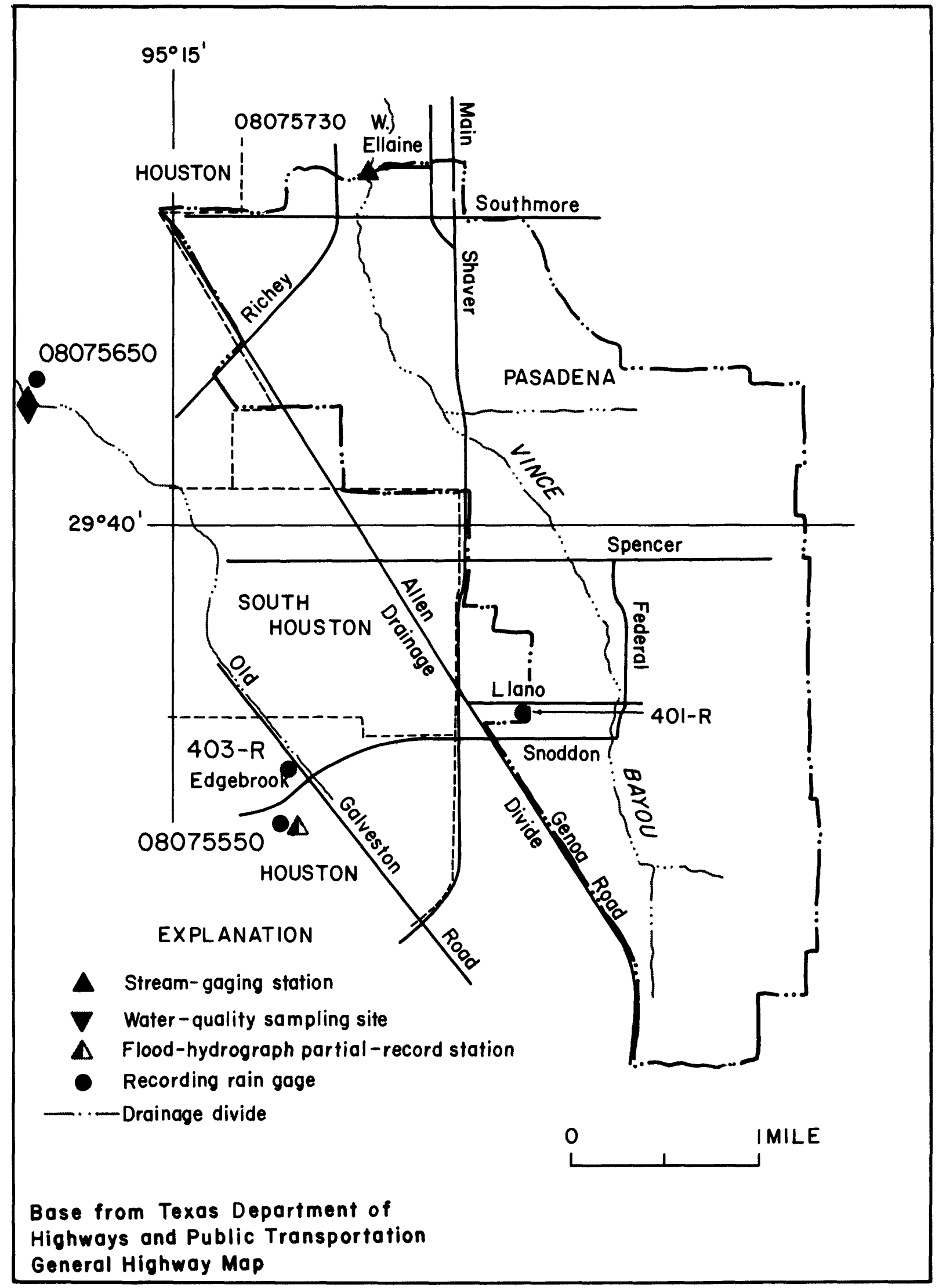

Figure 17.- Locations of data-collection sites in and near the Vince Bayou drainage basin 


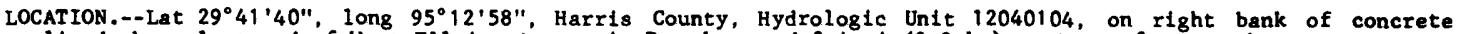
Iined channel at end of West Ellaine Avenue in Pasadena and $2.4 \mathrm{mI}(3.9 \mathrm{~km})$ upstream from mouth.

DRAINAGE AREA. $-7.32 \mathrm{mi}^{2}\left(18.96 \mathrm{~km}^{2}\right)$. Prior to Jan. 1, 1978, 8.21 $\mathrm{mi}^{2}\left(21.26 \mathrm{~km}^{2}\right)$. Jan. $1 \mathrm{to}$ Sept. 30 , 1978, $7.61 \mathrm{mi}^{2}\left(19.71 \mathrm{~km}^{2}\right)$. Drainage area revisions due to drainage ditch changes.

PERIOD OF RECORD.--October 1971 to current year.

GAGE.--Water-stage recorder and crest-stage gage. Datum of gage is $2.54 \mathrm{ft}(0.774 \mathrm{~m})$ below National Geodetic Vertical Datum of 1929, 1973 adjustment; unadjusted for land-surface subsidence (levels by Corps of Engineers). REMARKS.--Records fair. Low flow is sustained by sewage effluent.

AVERAGE DISCHARGE.--9 years, $17.5 \mathrm{ft}^{3} / \mathrm{s}\left(0.496 \mathrm{~m}^{3} / \mathrm{s}\right), 12,680 \mathrm{scre}-\mathrm{ft} / \mathrm{yr}\left(15.6 \mathrm{hm}^{3} / \mathrm{yr}^{2}\right)$. EXTREMES FOR PERIOD OF RECORD.--Maximum discharge, $3,940 \mathrm{ft}^{3} / \mathrm{s}\left(112 \mathrm{~m}^{3} / \mathrm{s}\right)$ July 26,1979 , gage helght, $16.93 \mathrm{ft}$
$(5.160 \mathrm{~m})$; no flow Aug. 5, 6, 18, 1972 .

EXTREMES FOR CURRENT YEAR.--Peak discharges above base of $1,400 \mathrm{ft}^{3} / \mathrm{s}\left(39.6 \mathrm{~m}^{3} / \mathrm{s}\right)$ and maximum (*):

\begin{tabular}{|c|c|c|c|c|c|}
\hline Date & Time & $\begin{array}{r}\text { Disc } \\
\left(\mathrm{ft}^{3} / \mathrm{s}\right)\end{array}$ & $\left(\mathrm{m}^{3} / \mathrm{s}\right)$ & $\begin{array}{c}\text { Gage } \\
(\mathrm{ft})\end{array}$ & $\begin{array}{l}\text { height } \\
\text { (m) }\end{array}$ \\
\hline $\begin{array}{ll}\operatorname{Tan} . & 20 \\
\operatorname{Tan} . & 22\end{array}$ & $\begin{array}{l}2000 \\
0715\end{array}$ & $\begin{array}{r}1,460 \\
* 1,940\end{array}$ & $\begin{array}{l}41.3 \\
54.9\end{array}$ & $\begin{array}{l}13.03 \\
13.92\end{array}$ & 4.24 \\
\hline
\end{tabular}

Minimum dally discharge, $0.08 \mathrm{ft}^{3} / \mathrm{s}\left(0.002 \mathrm{~m}^{3} / \mathrm{s}\right)$ May $10,11$.

DISCHARGE, IN CUBIC FEET PER SECOND, WATER YEAR OCTOBER 1979 TO SEPTEMBER 1980

\begin{tabular}{|c|c|c|c|c|c|c|c|c|c|c|c|c|}
\hline DAY & OCT & NOV & DEC & JAN & FEB & MAR & APR & MAY & JUN & JUL & AUG & SEP \\
\hline $\begin{array}{l}1 \\
2 \\
3 \\
4 \\
5\end{array}$ & $\begin{array}{l}.39 \\
.23 \\
.30 \\
.56 \\
.22\end{array}$ & $\begin{array}{l}2.7 \\
2.1 \\
.96 \\
.56 \\
.42\end{array}$ & $\begin{array}{l}.32 \\
.25 \\
.39 \\
.41 \\
.49\end{array}$ & $\begin{array}{c}.32 \\
54^{.30} \\
5.6 \\
1.8\end{array}$ & $\begin{array}{l}1.5 \\
3.1 \\
4.8 \\
4.5 \\
15\end{array}$ & $\begin{array}{l}16 \\
1.0 \\
.73 \\
1.9 \\
1.2\end{array}$ & $\begin{array}{l}1.1 \\
1.2 \\
1.7 \\
.82 \\
.40\end{array}$ & $\begin{array}{r}77 \\
5.2 \\
.56 \\
.38 \\
.22\end{array}$ & $\begin{array}{l}.19 \\
.15 \\
.32 \\
.17 \\
.13\end{array}$ & $\begin{array}{l}1.3 \\
1.2 \\
1.3 \\
1.6 \\
1.9\end{array}$ & $\begin{array}{l}1.0 \\
.65 \\
.41 \\
.83 \\
.99\end{array}$ & $\begin{array}{r}.30 \\
.40 \\
.40 \\
.30 \\
50^{.30}\end{array}$ \\
\hline $\begin{array}{r}6 \\
7 \\
8 \\
9 \\
10\end{array}$ & $\begin{array}{l}.19 \\
.11 \\
.15 \\
.38 \\
.78\end{array}$ & $\begin{array}{l}1.3 \\
1.3 \\
1.7 \\
2.7 \\
.41\end{array}$ & $\begin{array}{l}.66 \\
.42 \\
.49 \\
.26 \\
.26\end{array}$ & $\begin{array}{l}1.0 \\
.78 \\
.67 \\
.45 \\
.82\end{array}$ & $\begin{array}{r}7.4 \\
2.0 \\
139 \\
39 \\
6.1\end{array}$ & $\begin{array}{r}.81 \\
.92 \\
1.1 \\
.96 \\
1.1\end{array}$ & $\begin{array}{l}1.7 \\
1.4 \\
.64 \\
.62 \\
.50\end{array}$ & $\begin{array}{l}.19 \\
7.5 \\
5.2 \\
.56 \\
.08\end{array}$ & $\begin{array}{c}.17 \\
1.4 \\
70^{.33} \\
2.9\end{array}$ & $\begin{array}{l}1.0 \\
.68 \\
.82 \\
.81 \\
.66\end{array}$ & $\begin{array}{l}.71 \\
1.9 \\
.51 \\
.26 \\
.64\end{array}$ & $\begin{array}{r}25 \\
5.0 \\
1.5 \\
1.0 \\
.80\end{array}$ \\
\hline $\begin{array}{l}11 \\
12 \\
13 \\
14 \\
15\end{array}$ & $\begin{array}{l}.90 \\
.78 \\
2.7 \\
3.3 \\
2.7\end{array}$ & $\begin{array}{l}.18 \\
.25 \\
.22 \\
.20 \\
.19\end{array}$ & $\begin{array}{l}19^{.26} \\
38 \\
4.6 \\
1.0\end{array}$ & $\begin{array}{l}1.7 \\
.85 \\
.56 \\
.59 \\
1.7\end{array}$ & $\begin{array}{r}3.0 \\
3.0 \\
3.0 \\
6.1 \\
14\end{array}$ & $\begin{array}{l}.98 \\
1.0 \\
.87 \\
.92 \\
.86\end{array}$ & $\begin{array}{c}1.3 \\
1.2 \\
28 \\
.54 \\
1.7\end{array}$ & $\begin{array}{r}.08 \\
.15 \\
.30 \\
1.2 \\
1.9\end{array}$ & $\begin{array}{l}.34 \\
.20 \\
.22 \\
.17 \\
.28\end{array}$ & $\begin{array}{r}.98 \\
.74 \\
.77 \\
.82 \\
1.1\end{array}$ & $\begin{array}{r}.59 \\
.31 \\
.97 \\
.81 \\
1.2\end{array}$ & $\begin{array}{l}.70 \\
.60 \\
.55 \\
.50 \\
.50\end{array}$ \\
\hline $\begin{array}{l}16 \\
17 \\
18 \\
19 \\
20\end{array}$ & $\begin{array}{l}1.2 \\
.78 \\
.66 \\
.56 \\
.46\end{array}$ & $\begin{array}{l}.33 \\
.28 \\
.30 \\
.45 \\
.35\end{array}$ & $\begin{array}{l}.56 \\
.46 \\
.27 \\
.61 \\
.27\end{array}$ & $\begin{array}{r}17 \\
131 \\
25 \\
12 \\
455\end{array}$ & $\begin{array}{l}8.5 \\
6.6 \\
5.3 \\
3.3 \\
2.4\end{array}$ & $\begin{array}{l}2.7 \\
17 \\
23^{.94}\end{array}$ & $\begin{array}{l}2.6 \\
2.1 \\
2.8 \\
1.1 \\
.71\end{array}$ & $\begin{array}{c}52 \\
15 \\
122.66 \\
2.7\end{array}$ & $\begin{array}{l}.50 \\
.50 \\
.46 \\
.17 \\
.11\end{array}$ & $\begin{array}{l}.85 \\
.78 \\
.87 \\
.75 \\
2.2\end{array}$ & $\begin{array}{l}2.9 \\
.80 \\
1.0 \\
.97 \\
.83\end{array}$ & $\begin{array}{c}1.0 \\
1.2 \\
12^{.38} \\
1.2\end{array}$ \\
\hline $\begin{array}{l}21 \\
22 \\
23 \\
24 \\
25\end{array}$ & $\begin{array}{c}32.56 \\
1.4 \\
1.0 \\
.73\end{array}$ & $\begin{array}{l}23 \\
15 \\
7.1 \\
.38 \\
.28\end{array}$ & $\begin{array}{l}.43 \\
.46 \\
1.5 \\
3.0 \\
1.2\end{array}$ & $\begin{array}{r}344 \\
642 \\
38 \\
16 \\
15\end{array}$ & $\begin{array}{l}1.2 \\
1.1 \\
1.2 \\
1.3 \\
1.2\end{array}$ & $\begin{array}{r}.99 \\
.36 \\
2.0 \\
1.1 \\
.33\end{array}$ & $\begin{array}{r}.55 \\
.38 \\
.26 \\
19^{.56}\end{array}$ & $\begin{array}{c}1.2 \\
.46 \\
.26 \\
.38 \\
1.0\end{array}$ & $\begin{array}{l}.52 \\
.64 \\
.24 \\
.21 \\
.20\end{array}$ & $\begin{array}{l}2.9 \\
3.0 \\
1.6 \\
.70 \\
.54\end{array}$ & $\begin{array}{l}.85 \\
.70 \\
.21 \\
.59 \\
.26\end{array}$ & $\begin{array}{l}.66 \\
.78 \\
.78 \\
.56 \\
.46\end{array}$ \\
\hline $\begin{array}{l}26 \\
27 \\
28 \\
29 \\
30 \\
31\end{array}$ & $\begin{array}{r}1.56 \\
.66 \\
168 \\
41\end{array}$ & $\begin{array}{l}.26 \\
.24 \\
.46 \\
.65 \\
.48 \\
.-.\end{array}$ & $\begin{array}{c}.81 \\
.89 \\
2.2 \\
43 \\
1.2 \\
.54\end{array}$ & $\begin{array}{l}9.0 \\
5.8 \\
2.3 \\
2.4 \\
1.5 \\
1.7\end{array}$ & $\begin{array}{l}.94 \\
.75 \\
1.1 \\
3.0 \\
.0 . \\
.--\end{array}$ & $\begin{array}{c}7.1 \\
238 \\
46 \\
170 \\
12 \\
1.9\end{array}$ & $\begin{array}{l}.66 \\
.38 \\
.26 \\
.30 \\
.19 \\
-.--\end{array}$ & $\begin{array}{l}.78 \\
.46 \\
.78 \\
.38 \\
.26 \\
.22\end{array}$ & $\begin{array}{l}.26 \\
.38 \\
.56 \\
.66 \\
.90 \\
-.--\end{array}$ & $\begin{array}{c}1.76 \\
27.4 \\
4.3 \\
1.8 \\
1.4\end{array}$ & $\begin{array}{l}.57 \\
8.3 \\
1.0 \\
8.4 \\
1.0 \\
.30\end{array}$ & $\begin{array}{c}8.1 \\
8.1 \\
1.7 \\
44^{.56} \\
-.--\end{array}$ \\
\hline $\begin{array}{l}\text { TOTAL } \\
\text { MEAN } \\
\text { MAX } \\
\text { MIN } \\
\text { AC-FT } \\
(\dagger \dagger)\end{array}$ & $\begin{array}{r}265.20 \\
8.55 \\
168 \\
.11 \\
526 \\
4.99\end{array}$ & $\begin{array}{r}64.75 \\
2.16 \\
23 \\
.18 \\
i 28 \\
1.14\end{array}$ & $\begin{array}{r}124.21 \\
4.01 \\
43 \\
.25 \\
246 \\
1.98\end{array}$ & $\begin{array}{r}1788.84 \\
57.7 \\
642 \\
.30 \\
3550 \\
7.81\end{array}$ & $\begin{array}{r}289.39 \\
9.98 \\
139 \\
.75 \\
574 \\
1.83\end{array}$ & $\begin{array}{r}554.55 \\
17.9 \\
238 \\
.33 \\
1100 \\
4.29\end{array}$ & $\begin{array}{r}74.67 \\
2.49 \\
28 \\
.19 \\
148 \\
1.15\end{array}$ & $\begin{array}{r}299.06 \\
9.65 \\
122 \\
.08 \\
593 \\
3.92\end{array}$ & $\begin{array}{r}83.28 \\
2.78 \\
70 \\
111 \\
165 \\
1.15\end{array}$ & $\begin{array}{r}66.53 \\
2.15 \\
27 \\
.54 \\
i 32 \\
1.35\end{array}$ & $\begin{array}{r}40.46 \\
1.31 \\
8.4 \\
.21 \\
80 \\
1.17\end{array}$ & $\begin{array}{r}169.03 \\
5.63 \\
50 \\
.30 \\
335 \\
5.24\end{array}$ \\
\hline $\begin{array}{l}\text { CAL YR } \\
\text { WTR YR }\end{array}$ & $\begin{array}{l}1979 \\
1980\end{array}$ & $\begin{array}{rr}11 & 115 \\
L & 38\end{array}$ & .01 & $\begin{array}{ll}\text { MEAN } & 31.7 \\
\text { MEAN } & 10.4\end{array}$ & $\begin{array}{l}\text { MAX } \\
\text { MAX }\end{array}$ & $\begin{array}{r}1520 \\
642\end{array}$ & $\begin{array}{l}.11 \\
.08\end{array}$ & $\begin{array}{l}A C-F T \\
A C-F T\end{array}$ & $\begin{array}{r}22940 \\
7580\end{array}$ & $\begin{array}{l}72.14 \\
36.02\end{array}$ & & \\
\hline
\end{tabular}

t† Weighted-mean rainfall, in Inches, based on two rain gages. 


\section{HUNTING BAYOU DRAINAGE BASIN}

The locations of data-collection sites in and near the Hunting Bayou drainage basin are shown in figure 18.

Weighted-mean rainfall in the drainage basin based on three rain gages for the 1980 water year was 41.76 inches, or 6.43 inches less than the 30 -year (1941-70) average of 48.19 inches for Houston.

The storms of Jan. 20-23 and April 25-26 were selected for analysis at station 08075760, Hunting Bayou at Falls Street. The storms of Jan. 20-24 and April 25-27 were selected for analysis at station 08075770, Hunting Bayou at Interstate Highway 610. 


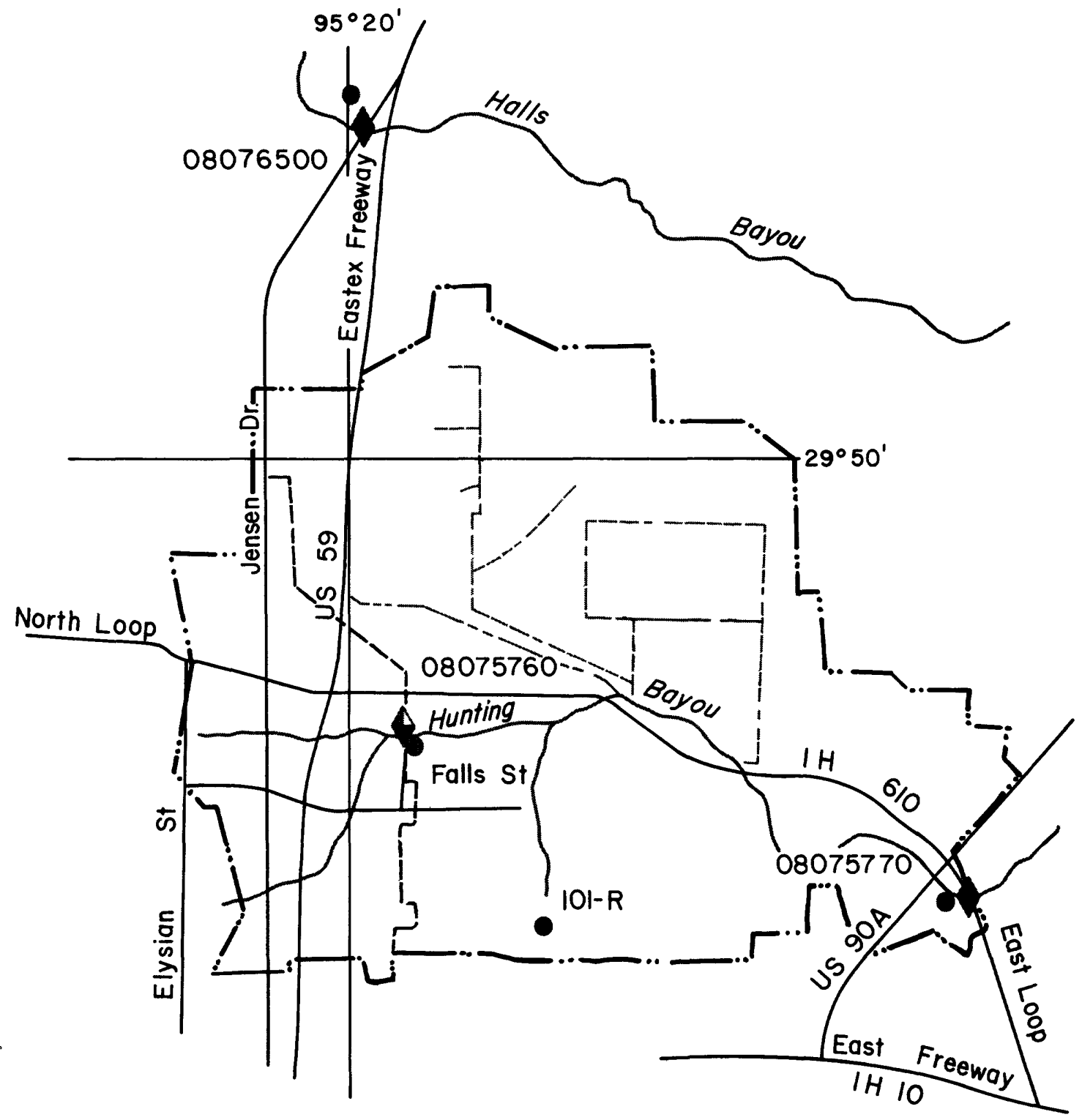

12-R

EXPLANATION

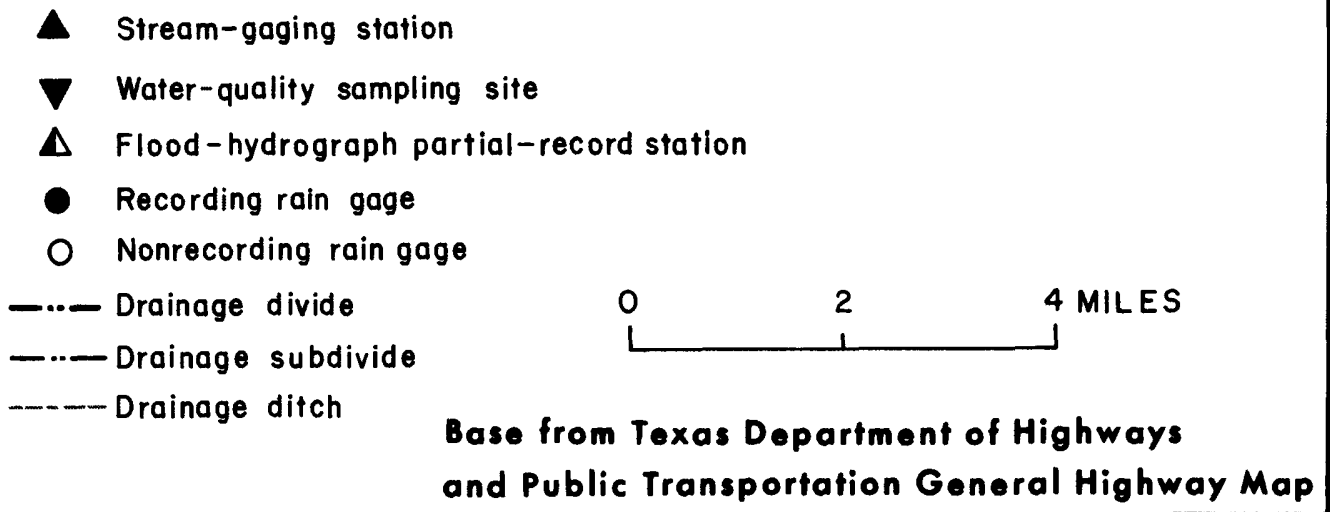

Figure 18.-Locations of data-collection sites in and near the Hunting Bayou drainage basin 


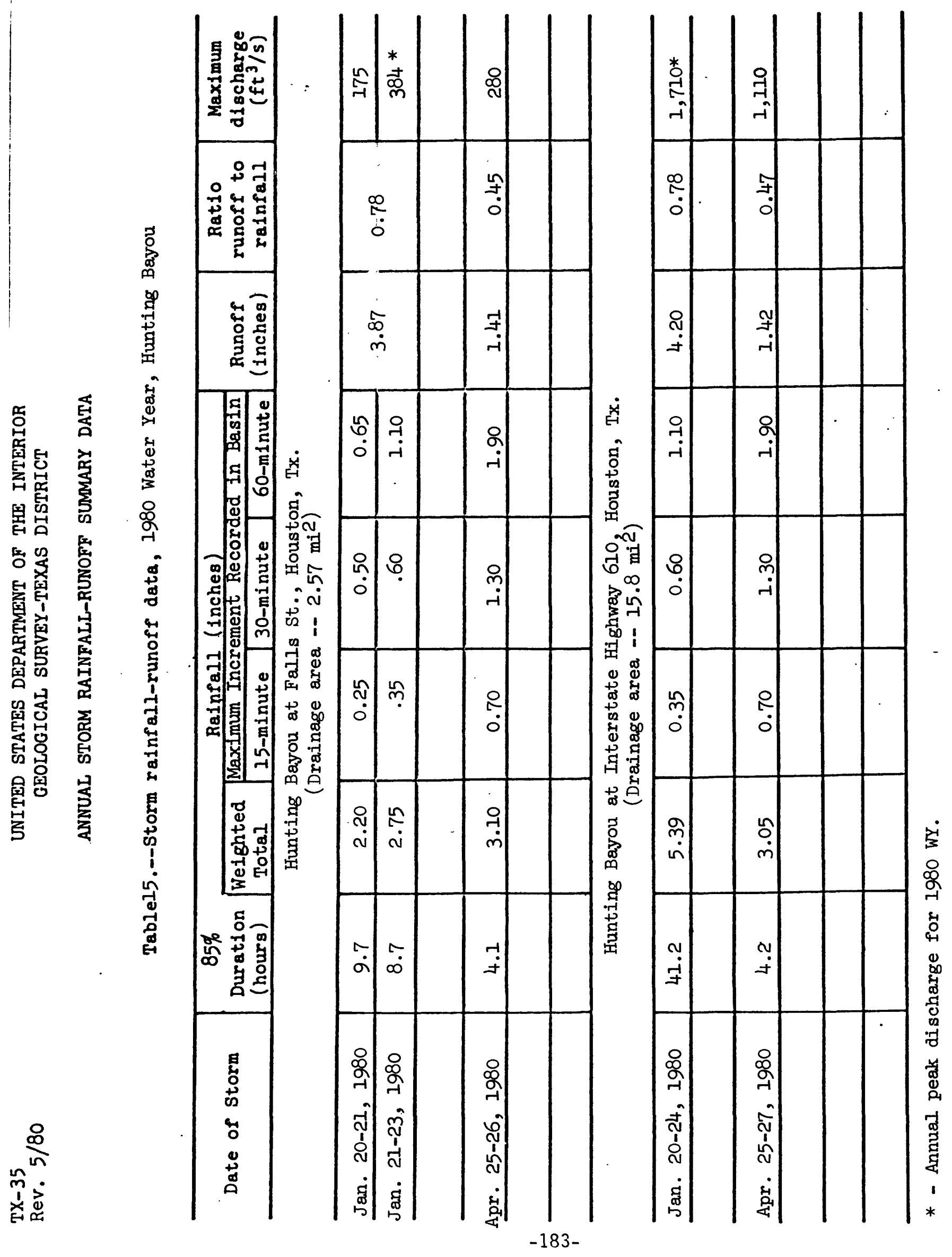


SAN JACINTO RIVER BASIN

08075760 HUNTING BAYOU AT FALLS STREET, HOUSTON, TX (Flood-hydrograph partial-record station)

LOCATION.--Lat $29^{\circ} 48^{\prime} 22^{\prime \prime}$, Iong $95^{\circ} 19^{\prime} 50^{\prime \prime}$, Harris County, Hydrologic Unit 12040104, at downstream side of bridge on Falls Street in northeast Houston.

DRAINAGE AREA. $-2.57 \mathrm{mi}^{2}\left(6.66 \mathrm{~km}^{2}\right)$, Oct. 1, 1973, to Sept. 30, 1978, $2.75 \mathrm{mi}^{2}$ (7.12 $\left.\mathrm{km}^{2}\right)$. Prior to Oct. 1,

$1973,3.50 \mathrm{mi}^{2}\left(9.07 \mathrm{~km}^{2}\right)$. Drainage area changes due to changes in storm sewers.

WATER-DISCHARGE RECORDS

PERIOD OF RECORD.--Apr 111964 to current year.

GAGE.--Flood-hydrograph and rainfall recorder and crest-stage gage. Datum of gage is National Geodetic Vertical Datum of 1929 .

REMARKS.--Records poor. Additional storm rainfall-runoff data for this site can be obtained from the report "Hydrologic Data for Urban Studies in the Houston, Texas Metropolitan Area, 1980."

EXTREMES FOR PERIOD OF RECORD.--Maximum discharge, $778 \mathrm{ft}^{3} / \mathrm{s}\left(22.0 \mathrm{~m}^{3} / \mathrm{s}\right)$ June 13, 1973, elevation, 46.70 ft $(14.234 \mathrm{~m})$; maximum elevation, $47.35 \mathrm{ft}(14.432 \mathrm{~m})$ Sept. $1,1979$.

EXTREMES FOR CURRENT YEAR.--Peak discharges above base of $250 \mathrm{ft}^{3} / \mathrm{s}\left(7.08 \mathrm{~m}^{3} / \mathrm{s}\right)$ and maximum $(*)$ :

\begin{tabular}{|c|c|c|c|c|c|c|c|c|c|c|c|}
\hline Date & Time & \multicolumn{2}{|c|}{$\begin{array}{c}\text { Discharge } \\
\left(\mathrm{ft}^{3} / \mathrm{s}\right)\left(\mathrm{m}^{3} / \mathrm{s}\right)\end{array}$} & \multicolumn{2}{|c|}{$\underset{(f t)}{\text { Elevation }}(\mathrm{m})$} & Date & Time & \multicolumn{2}{|c|}{$\begin{array}{c}\text { Discharge } \\
\left(\mathrm{ft}^{3 / \mathrm{s}}\right)\left(\mathrm{m}^{3} / \mathrm{s}\right)\end{array}$} & \multicolumn{2}{|c|}{$\underset{(f t)}{\text { Elevation }}(\mathrm{m})$} \\
\hline $\begin{aligned} & \text { Oct. } 30 \\
& \text { Jan. } 22 \\
& \text { aJan. } 28 \\
& \text { aMar. } 27\end{aligned}$ & $\begin{array}{c}\text { unknown } \\
0800 \\
\text { unknown } \\
1630\end{array}$ & $\begin{array}{r}292 \\
\times 384 \\
\text { b3 } \\
208\end{array}$ & $\begin{array}{c}8.27 \\
10.9 \\
.085 \\
5.89\end{array}$ & $\begin{array}{c}44.18 \\
43.12 \\
\text { unknown } \\
41.42\end{array}$ & $\begin{array}{c}13.466 \\
13.143 \\
- \\
12.625\end{array}$ & $\begin{array}{lr}\text { aApr. } & 25 \\
\text { aMay } & 28 \\
\text { aAug. } & 6\end{array}$ & $\begin{array}{l}\text { b1 } 215 \\
\text { unknown } \\
\text { unknown }\end{array}$ & $\begin{array}{r}280 \\
\text { b1 } \\
\text { b1 }\end{array}$ & $\begin{array}{l}7.93 \\
.028 \\
.028\end{array}$ & $\begin{array}{c}42.49 \\
\text { unknown } \\
\text { unknown }\end{array}$ & $\begin{array}{c}12.951 \\
- \\
-\end{array}$ \\
\hline
\end{tabular}

a Water-quality samples were obtained during this runoff event.

b About.

WATER-QUALITY RECORDS

FERIOD OF RECORD.--Chemical, biochemical, and pesticide analyses: October 1970 to current year. Water temperatures: April 1964 to current year.

WATER QUALITY DATA, WATER YEAR OCTOBER 1979 TO SEPTEMBER 1980

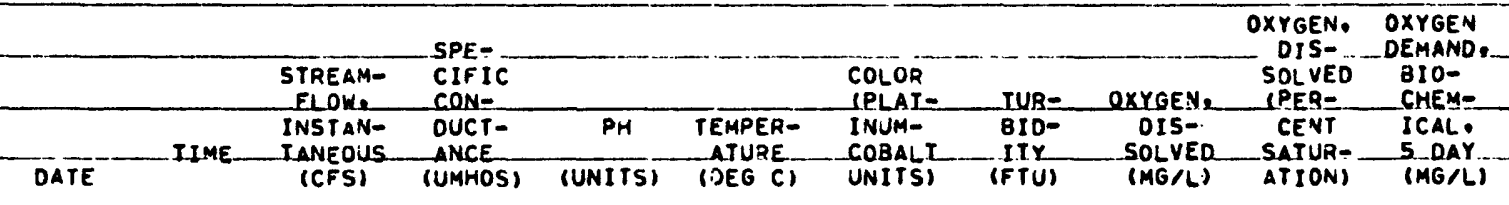

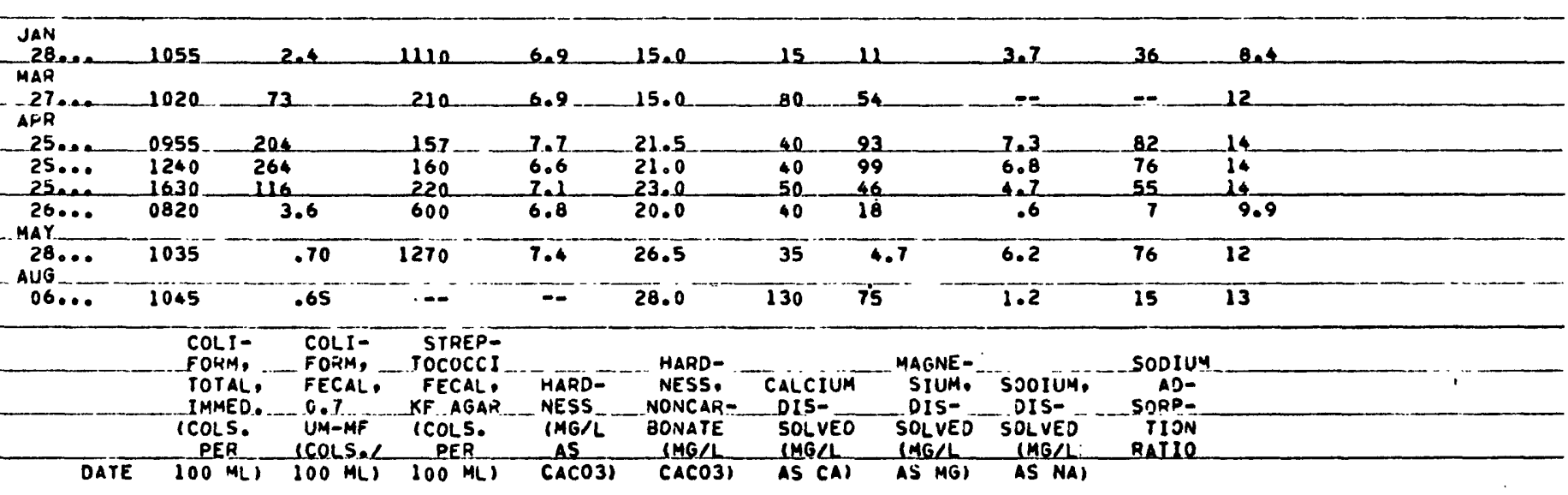

JAN

$28 \ldots \ldots 520000 \ldots 150000 \ldots 11000 \ldots$

MAR $27.0 .540000 \quad 190000 \quad 180000 \ldots$

APR $25.230000 \quad 100000 \ldots 74000 \ldots$

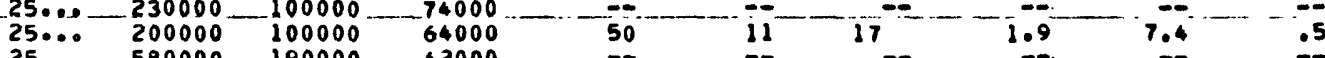

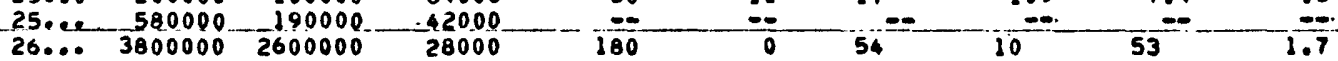

MAY

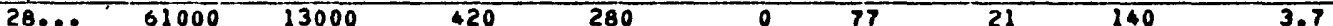

AUG

$06 \ldots 860000210000-3000-\infty \ldots$ 


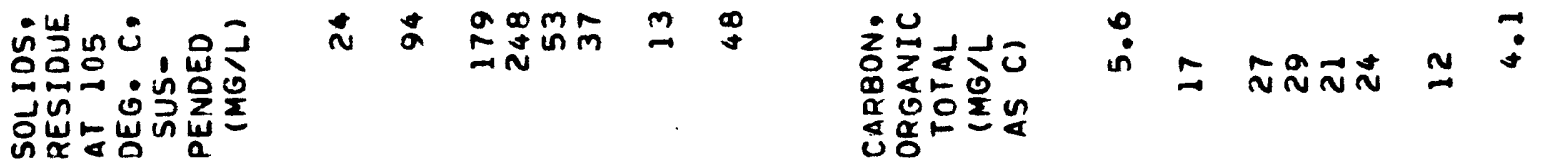

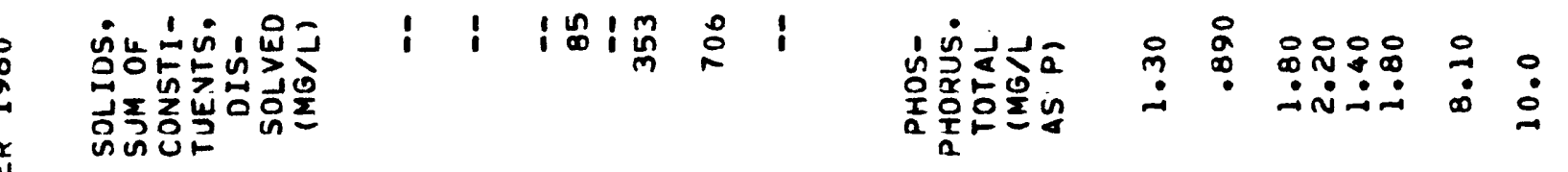

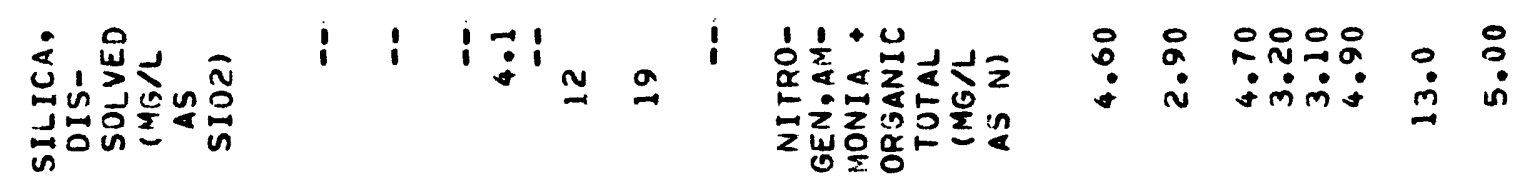

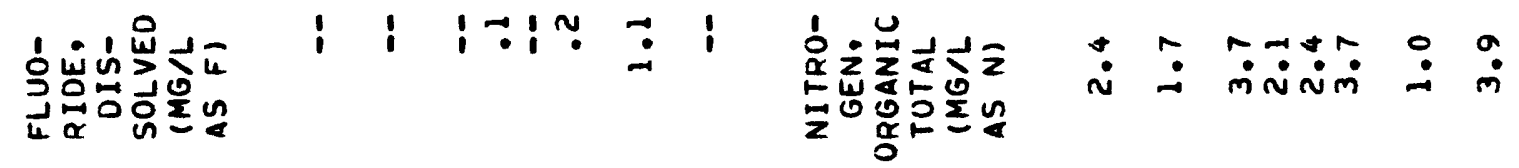

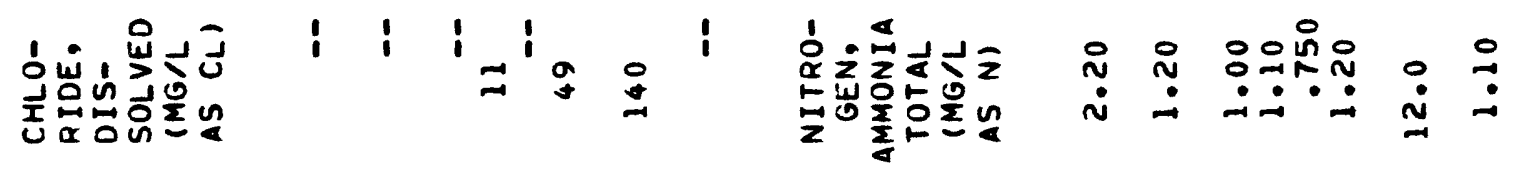

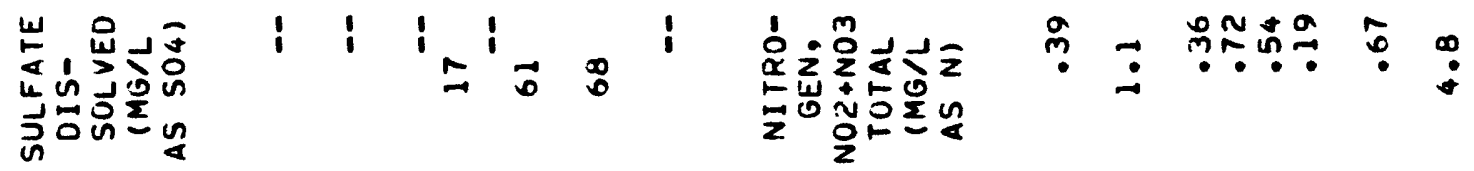

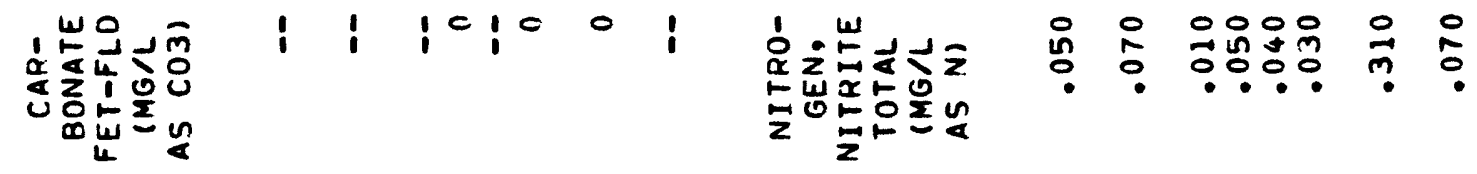

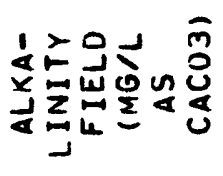

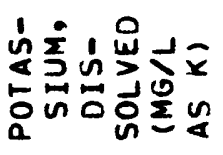

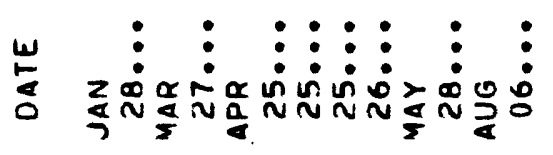

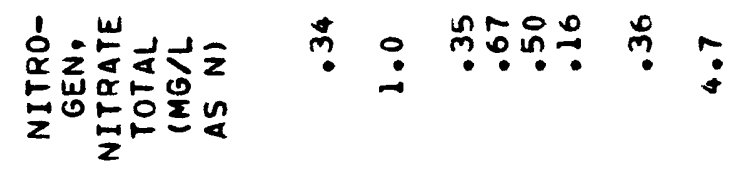

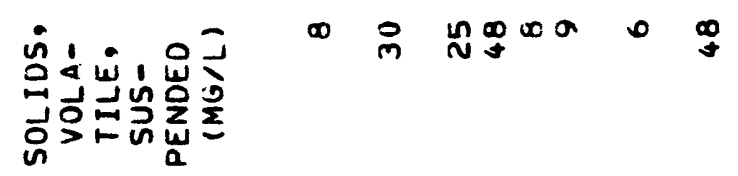

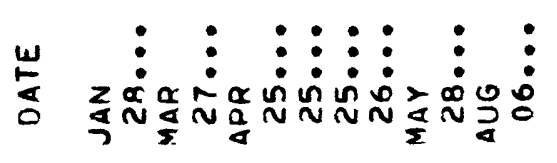




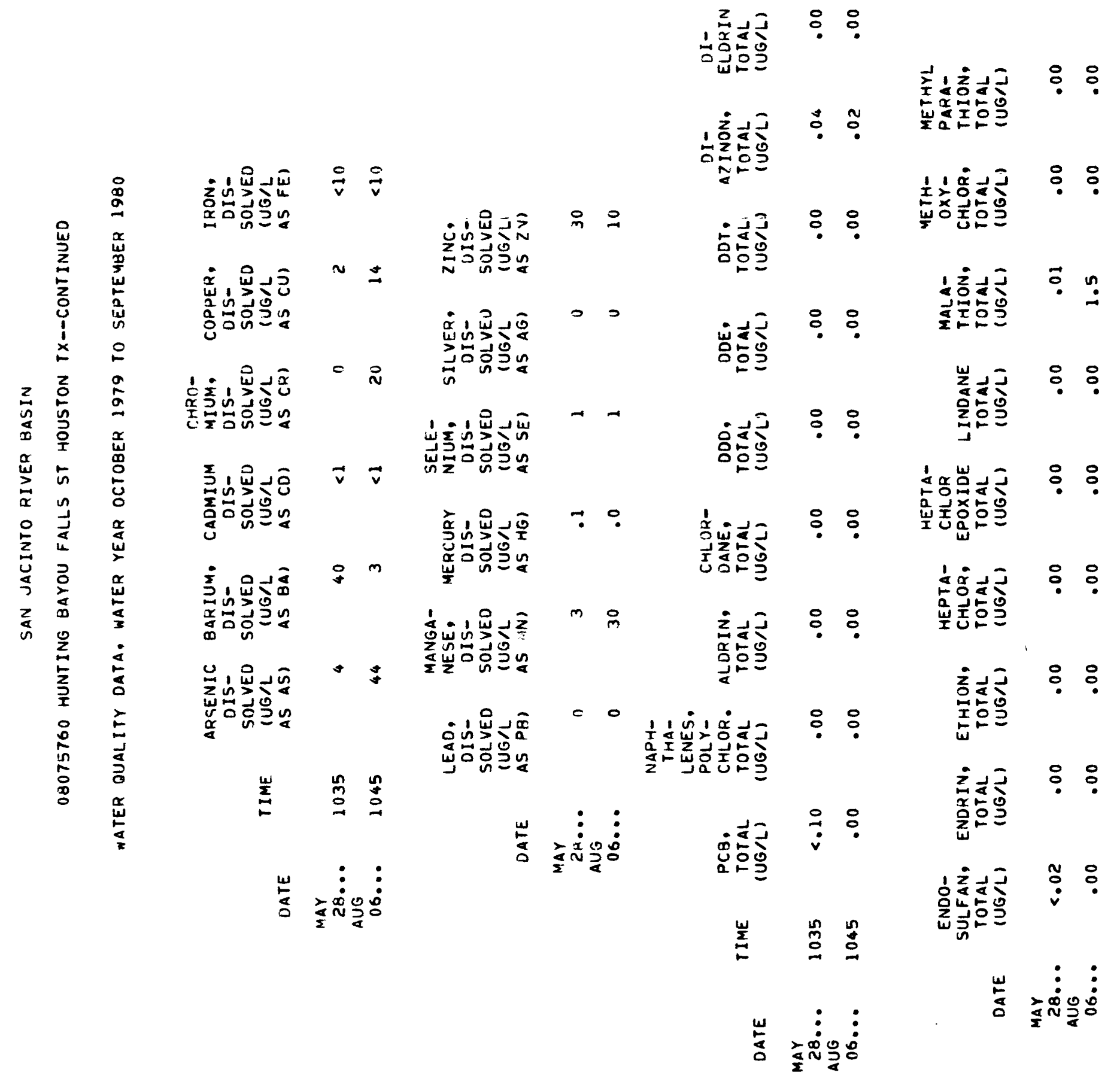




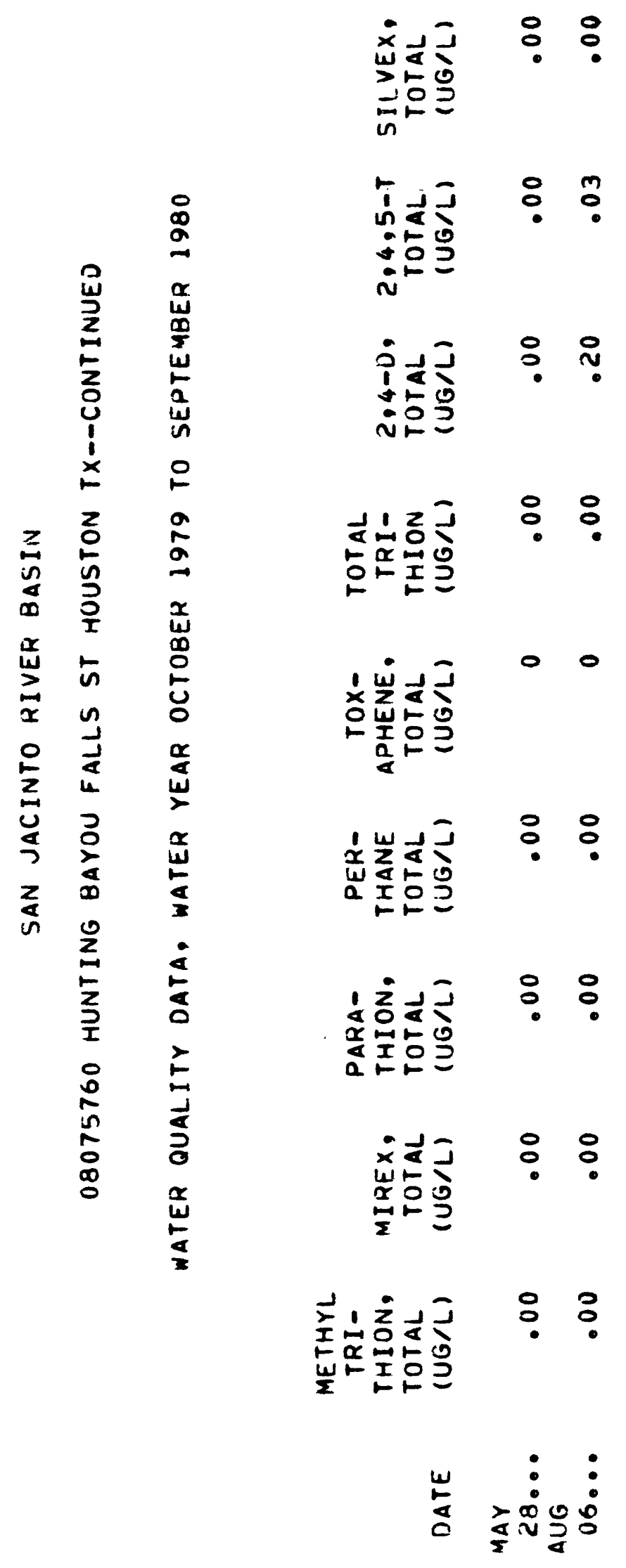




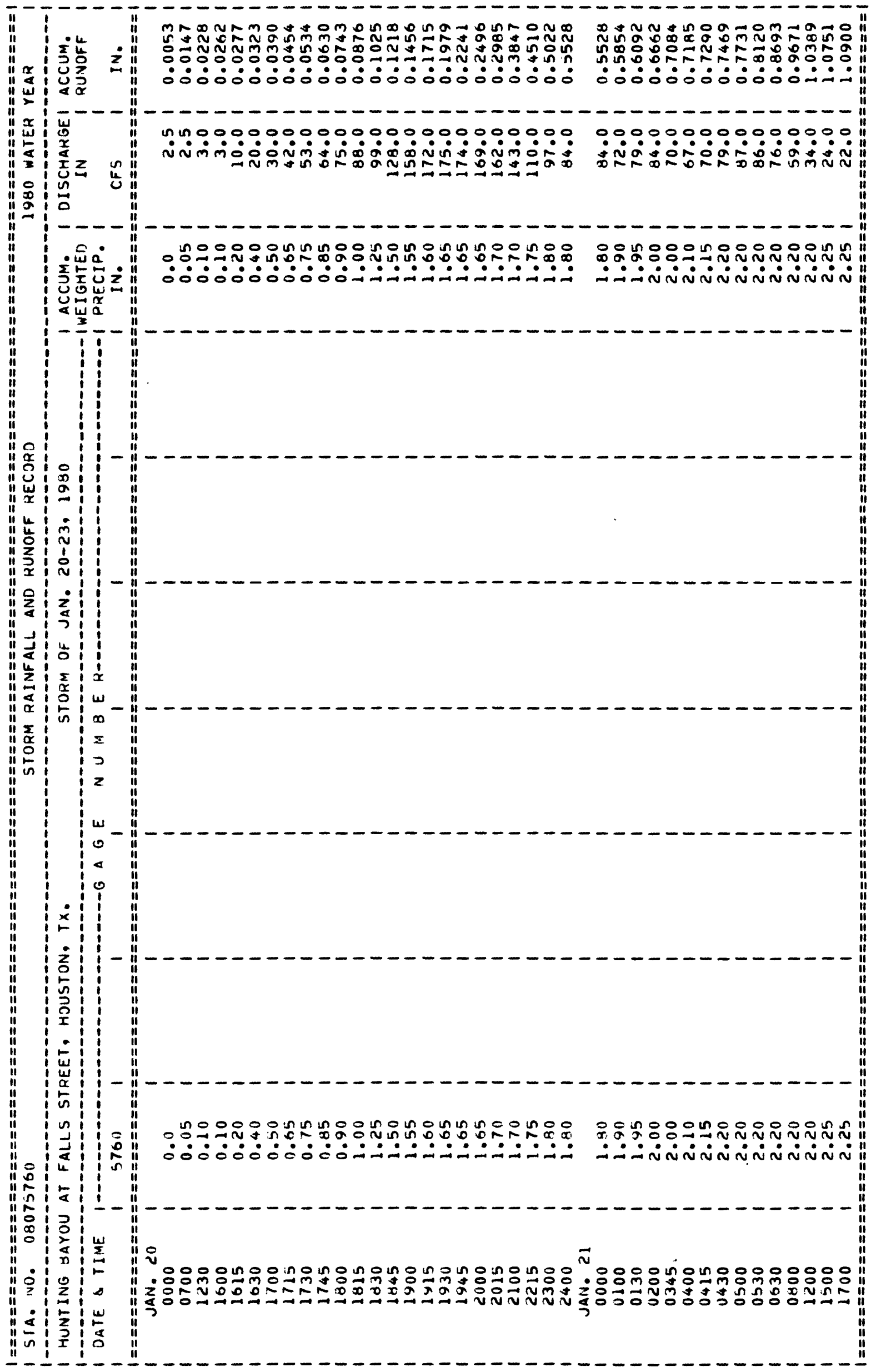




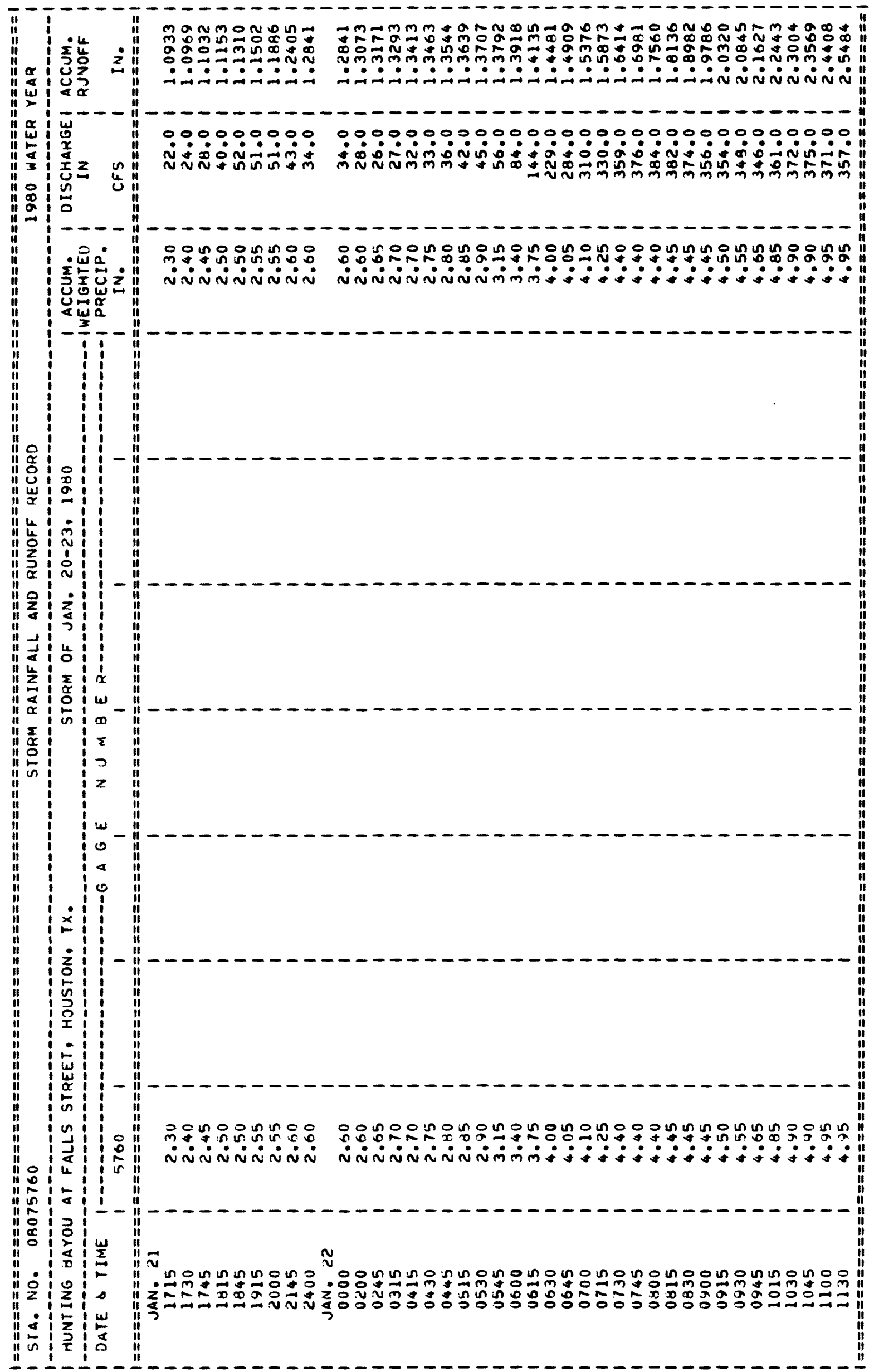




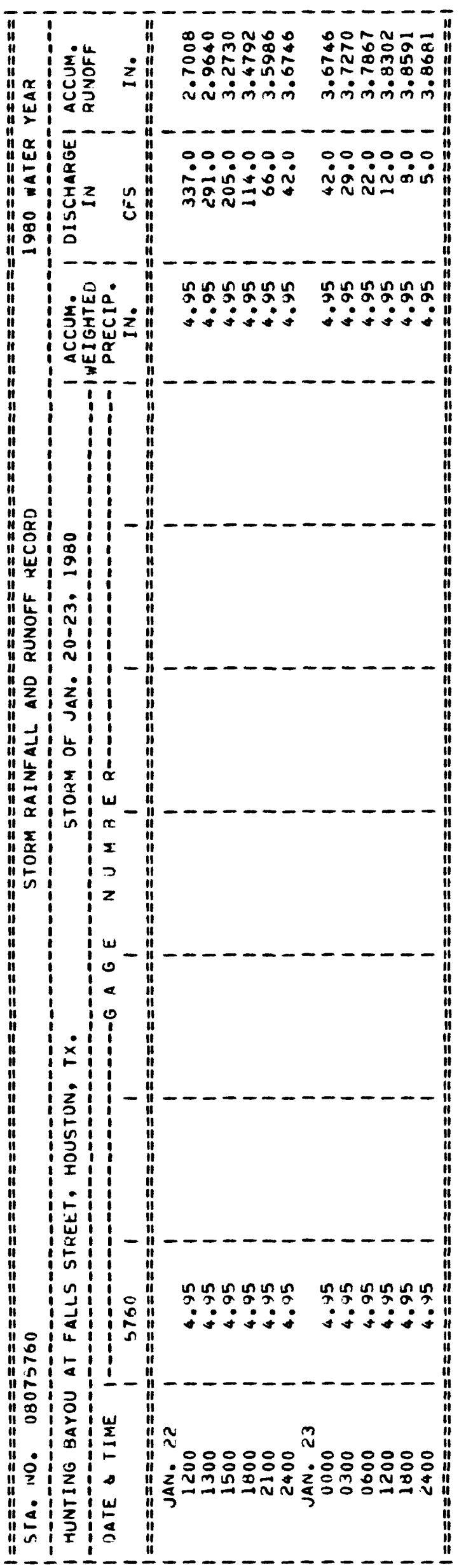




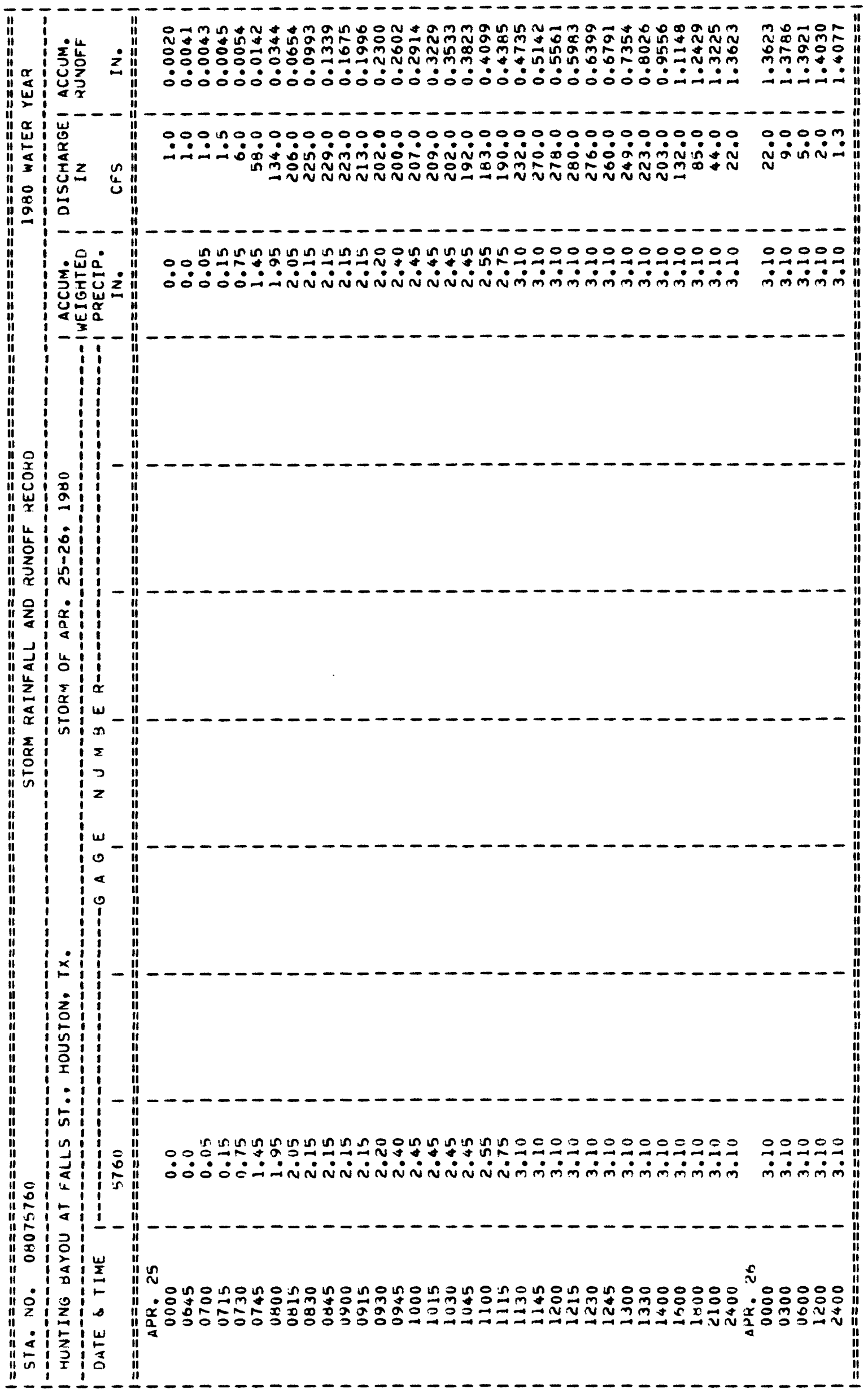


SAN JACINTO RIVER - BASIN

08075770 HUNTING BAYOU AT INTERSTATE HIGHWAY 610, HOUSTON, TX

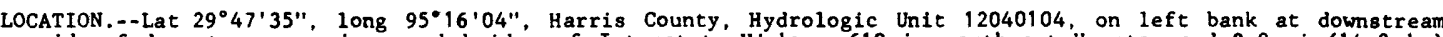
side of downstream service road bridge of Interstate Highway 610 in northeast Houston and $8.8 \mathrm{mi}(14.2 \mathrm{~km})$ upstream from mouth.

DRAINAGE AREA. $--15.8 \mathrm{mi}^{2}\left(40.9 \mathrm{~km}^{2}\right)$. Prior to Oct. 1, 1973, $16.8 \mathrm{mi}^{2}\left(43.5 \mathrm{~km}^{2}\right)$. 0ct. 1, 1973, to Sept. 30 , $1978,14.7 \mathrm{mi}^{2}\left(38.1 \mathrm{~km}^{2}\right)$. Changes due to storm sewer relocations.

WATER-DISCHARGE RECORDS

PERIOD OF RECORD.--April 1964 to current year. Prior to October 1973, published as "U.S. Highway 90-A, Houston". REVISED RECORDS.--WRD TX-74-1: Drainage area.

GAGE.--Water-stage recorder and crest-stage gage. Datum of gage is National Geodetic Vertical Datum of 1929 1959 adjustment; unadjusted for land-surface subsidence. Prior to Oct. 1, 1972, water-stage recorder at site $1,800 \mathrm{ft}(549 \mathrm{~m})$ upstream at same datum.

REMARKS.-Water-discharge records fair except for period of no gage-height, which are poor. Low flow is largely maintained by sewage and industrial effluent. Recording rain gage at station.

AVERAGE DISCHARGE.--16 years, $23.0 \mathrm{ft}^{3} / \mathrm{s}\left(0.651 \mathrm{~m}^{3} / \mathrm{s}\right), 16,660 \mathrm{acre}-\mathrm{ft} / \mathrm{yr}\left(20.5 \mathrm{hm}^{3} / \mathrm{yr}^{2}\right)$.

EXTREMES FOR PERIOD OF RECORD.--Maximum discharge, 3,380 $\mathrm{ft}^{3} / \mathrm{s}\left(95.7 \mathrm{~m}^{3} / \mathrm{s}\right)$ June 13, 1973, elevation, 38.11 ft $(11.616 \mathrm{~m})$; maximum gage height, $39.28 \mathrm{ft}(11.973 \mathrm{~m})$ June 15,1976 ; minimum daily, $0.88 \mathrm{ft} / \mathrm{s}\left(0.025 \mathrm{~m}^{3} / \mathrm{s}\right)$ Aug. 24, 1971 .

EXTREMES FOR CURRENT YEAR.--Peak discharges above base of $700 \mathrm{ft}^{3} / \mathrm{s}\left(19.8 \mathrm{~m}^{3} / \mathrm{s}\right)$ and maximum (*):

\begin{tabular}{|c|c|c|c|c|c|c|c|c|c|c|c|}
\hline Date & Time & $\begin{array}{r}\text { Disc } \\
\left(\mathrm{ft}^{3 / 8} / \mathrm{s}\right)\end{array}$ & $\left(\mathrm{m}^{3} / \mathrm{s}\right)$ & $\begin{array}{l}\text { Elev } \\
\text { (ft) }\end{array}$ & $\begin{array}{l}\text { (m) } \\
\text { (mion }\end{array}$ & Date & Time & $\begin{array}{r}\text { Dis } \\
\left(\mathrm{ft}^{3} / \mathrm{s}\right)\end{array}$ & $\begin{array}{l}\operatorname{arge} \\
\left(\mathrm{m}^{3} / 8\right)\end{array}$ & $\begin{array}{l}\text { Eleva } \\
(\mathrm{ft})\end{array}$ & tion \\
\hline $\begin{array}{ll}\text { Oct. } & 31 \\
\text { Jan. } & 22\end{array}$ & $\begin{array}{l}2200 \\
1130\end{array}$ & $\begin{array}{r}1,140 \\
* 1,710\end{array}$ & $\begin{array}{l}32.3 \\
48.4\end{array}$ & $\begin{array}{l}33.89 \\
35.30\end{array}$ & $\begin{array}{l}10.330 \\
10.759\end{array}$ & $\begin{array}{ll}\text { aMar. } 27 \\
\text { aApr. } 25\end{array}$ & $\begin{array}{l}1900 \\
1400\end{array}$ & $\begin{array}{r}927 \\
1,110\end{array}$ & $\begin{array}{l}26.3 \\
31.4\end{array}$ & $\begin{array}{l}31.45 \\
32.44\end{array}$ & $\begin{array}{l}9.586 \\
9.888\end{array}$ \\
\hline
\end{tabular}

a Water-quality samples were obtained during this runoff event.

Minimum daily discharge, $2.8 \mathrm{ft}^{3} / \mathrm{s}\left(0.079 \mathrm{~m}^{3} / \mathrm{s}\right) \mathrm{July} 5-7$.

DISCHARGE, IN CUBIC FEET PER SECOND, WATER YEAR OCTOBER 1979 TO SEPTEMBER 1980

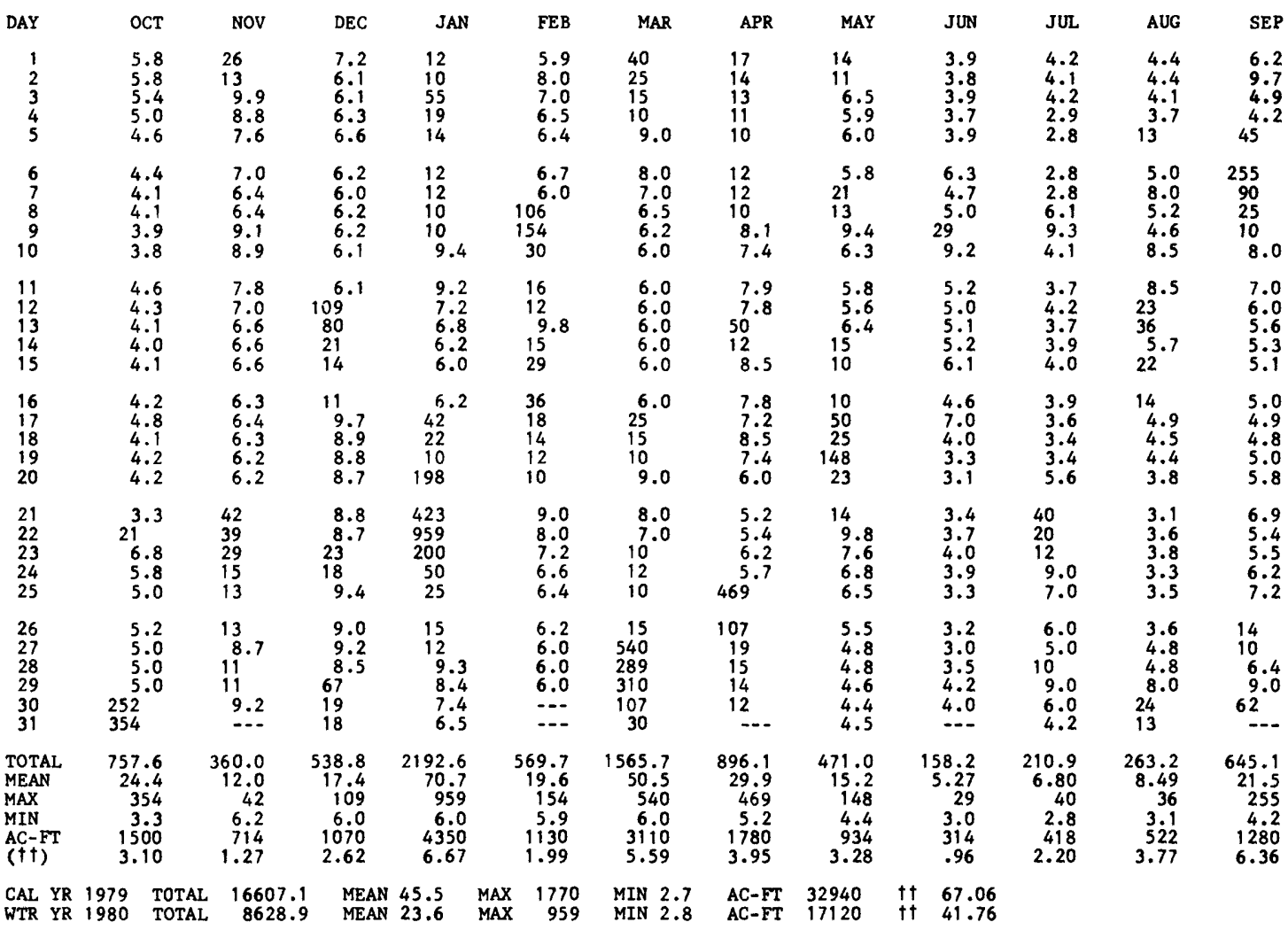

t† Weighted-mean rainfall, in inches, based on three rain gages.

NOTE.--No gage-height record Feb. 20 to Mar. 26. 


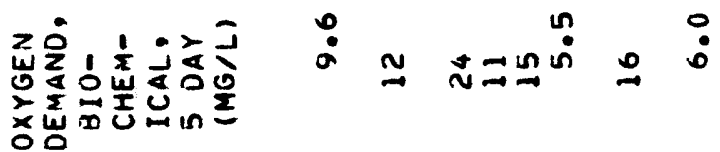

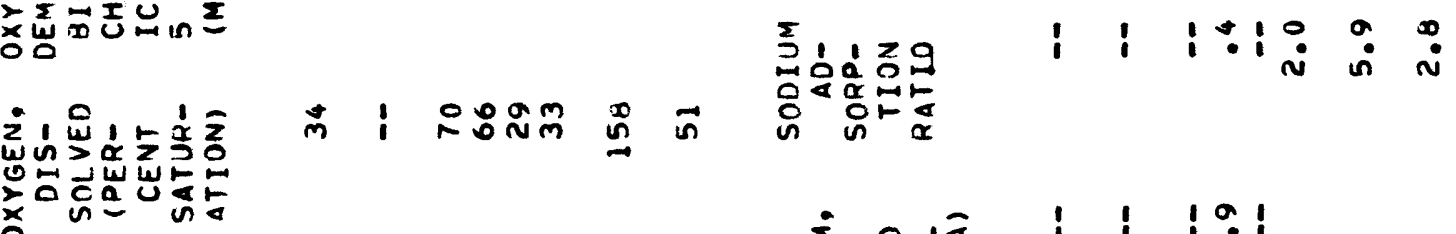

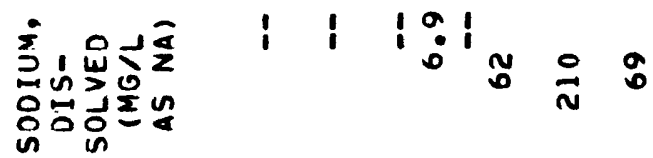

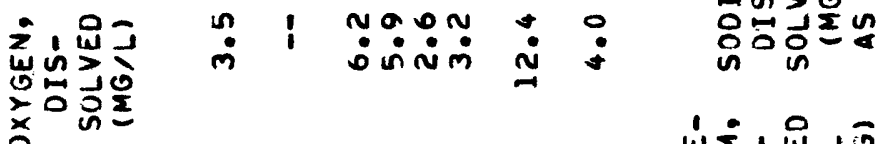

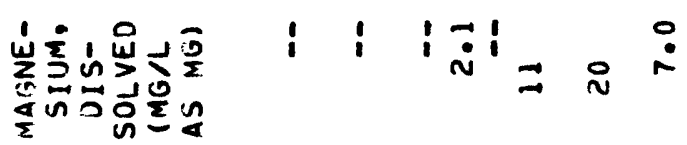

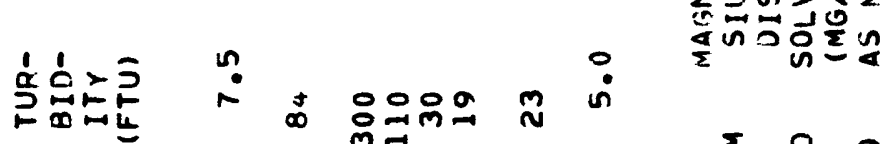

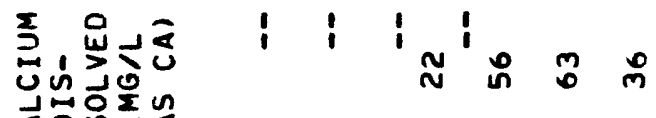

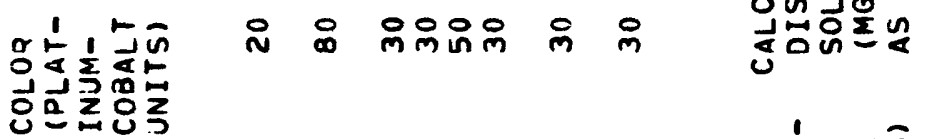

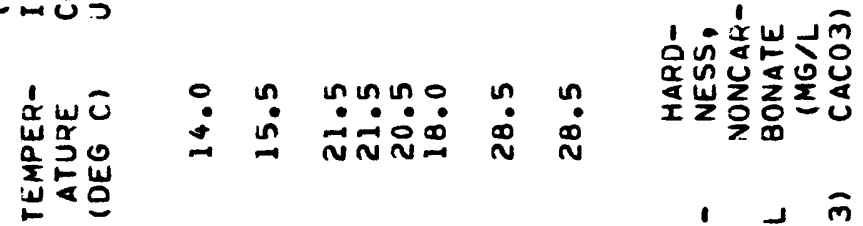

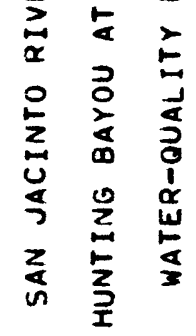

$$
\begin{aligned}
& \begin{array}{l}
0 \\
\hat{n} \\
\\
0 \\
0
\end{array}
\end{aligned}
$$

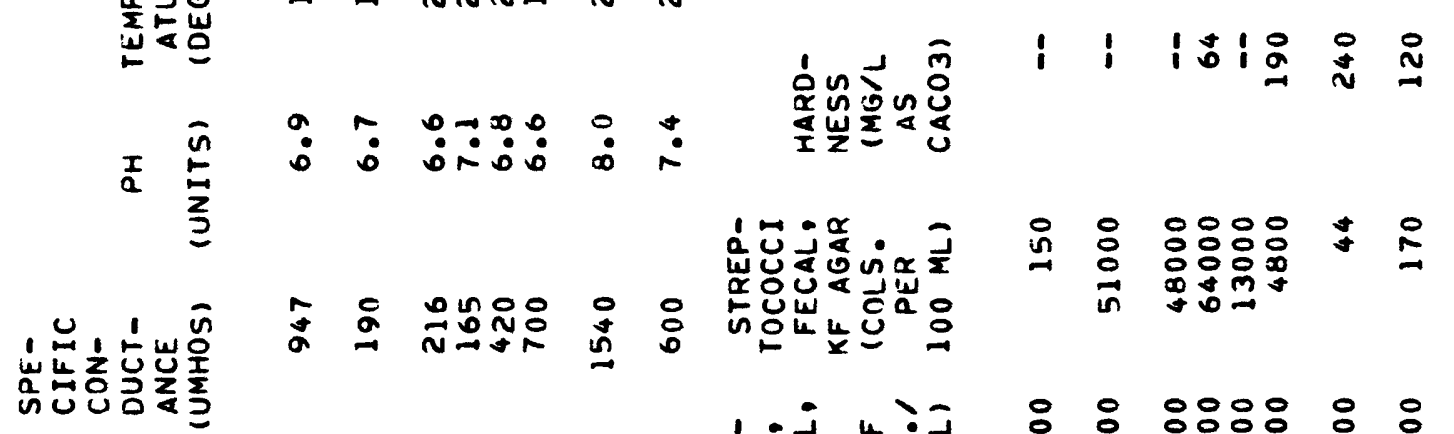

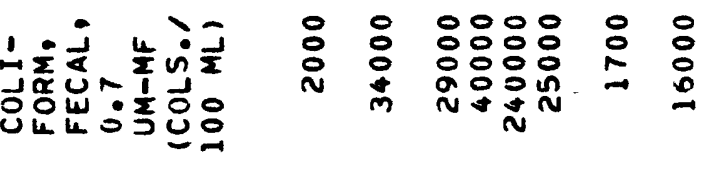

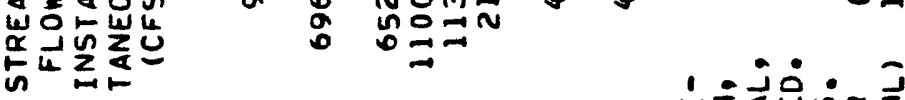

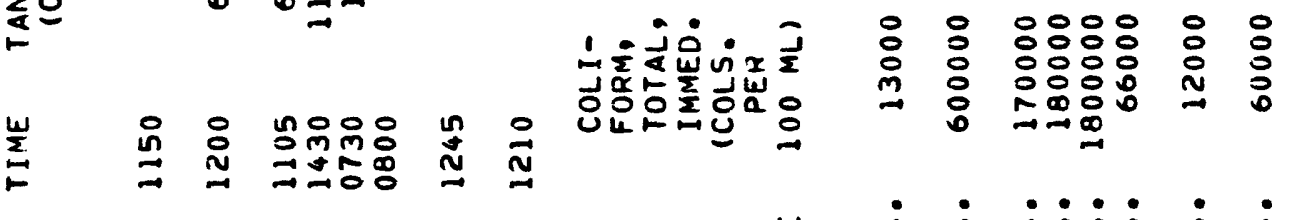

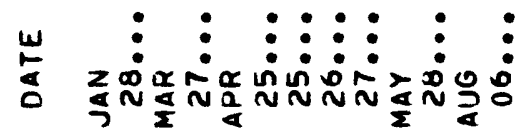

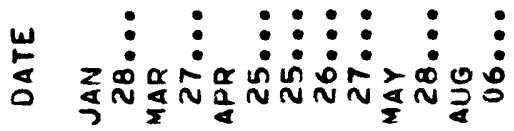




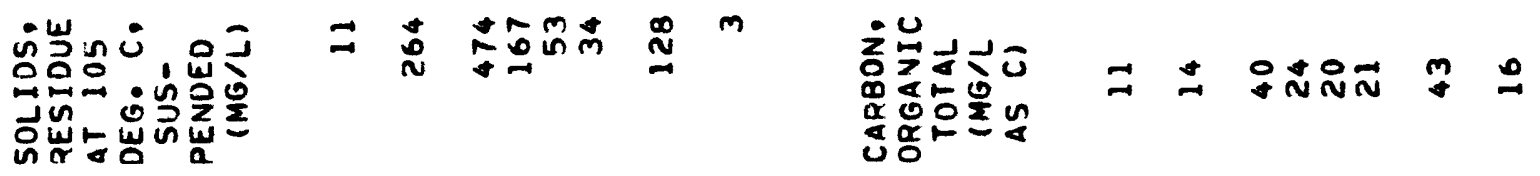
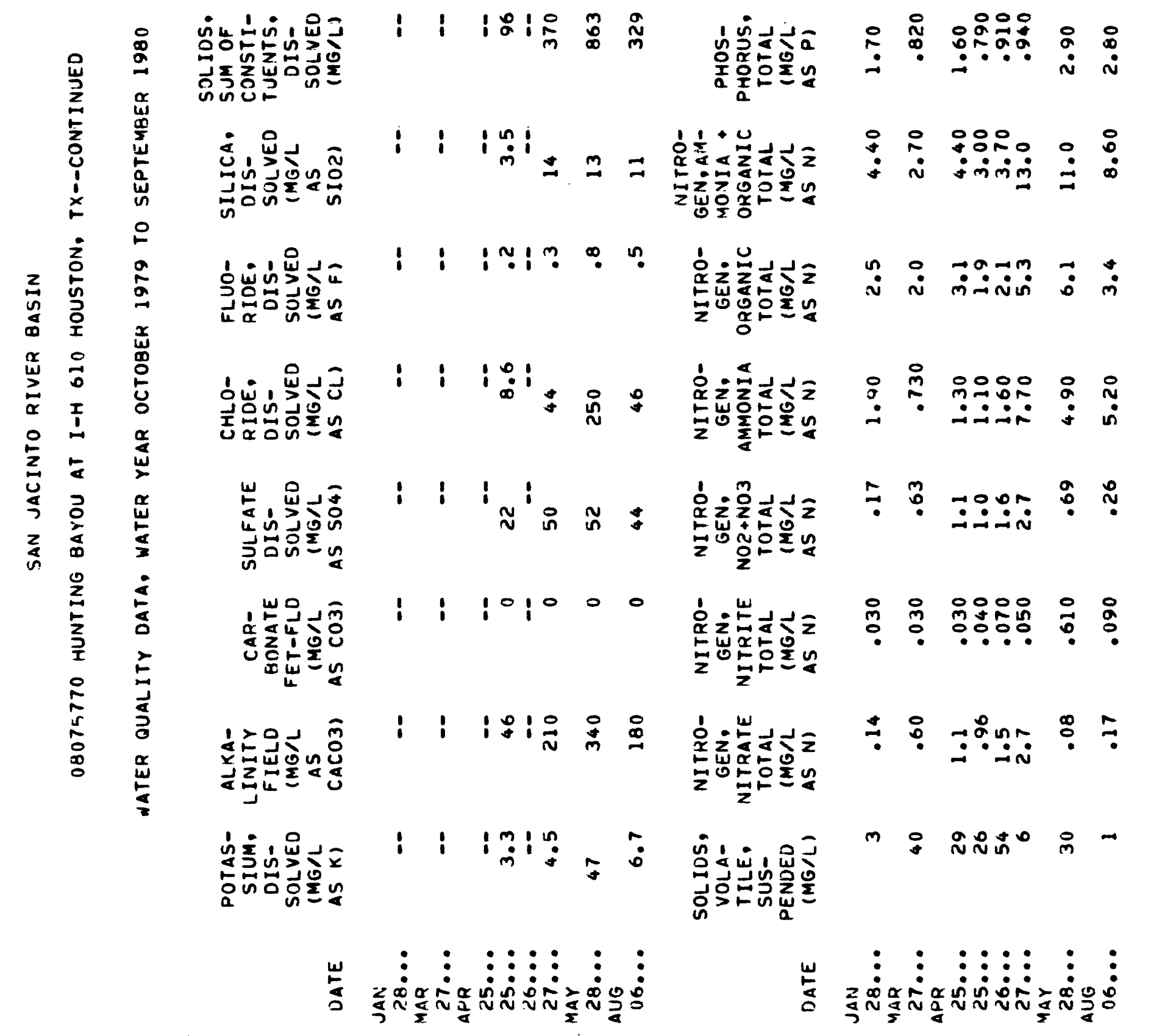


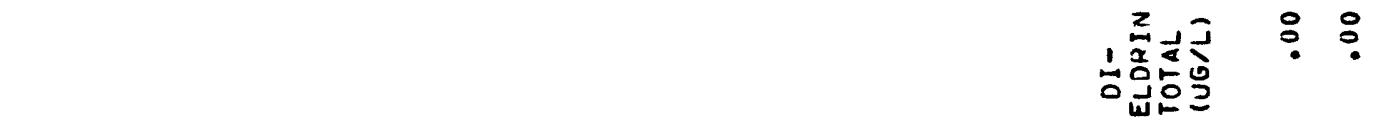

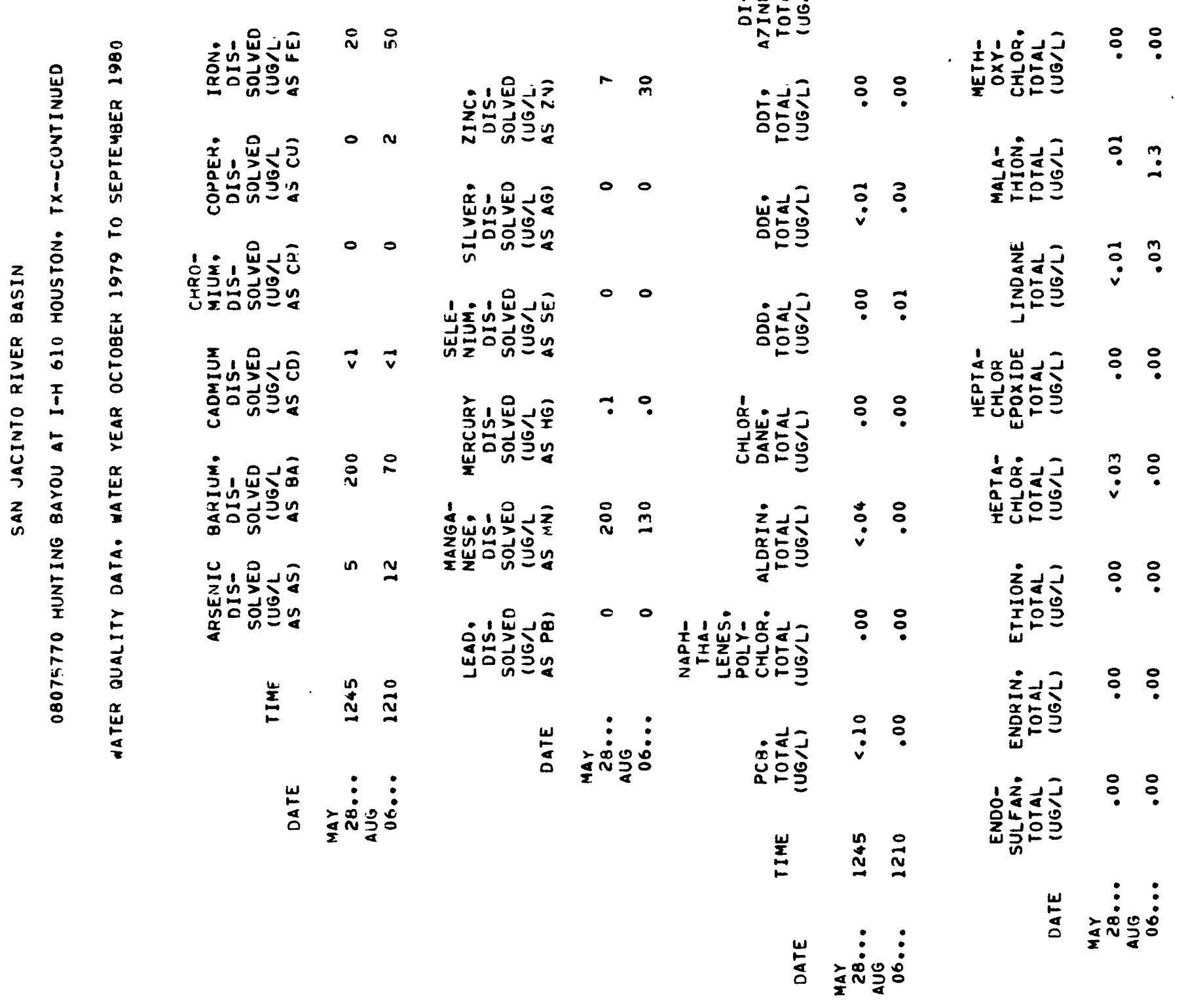




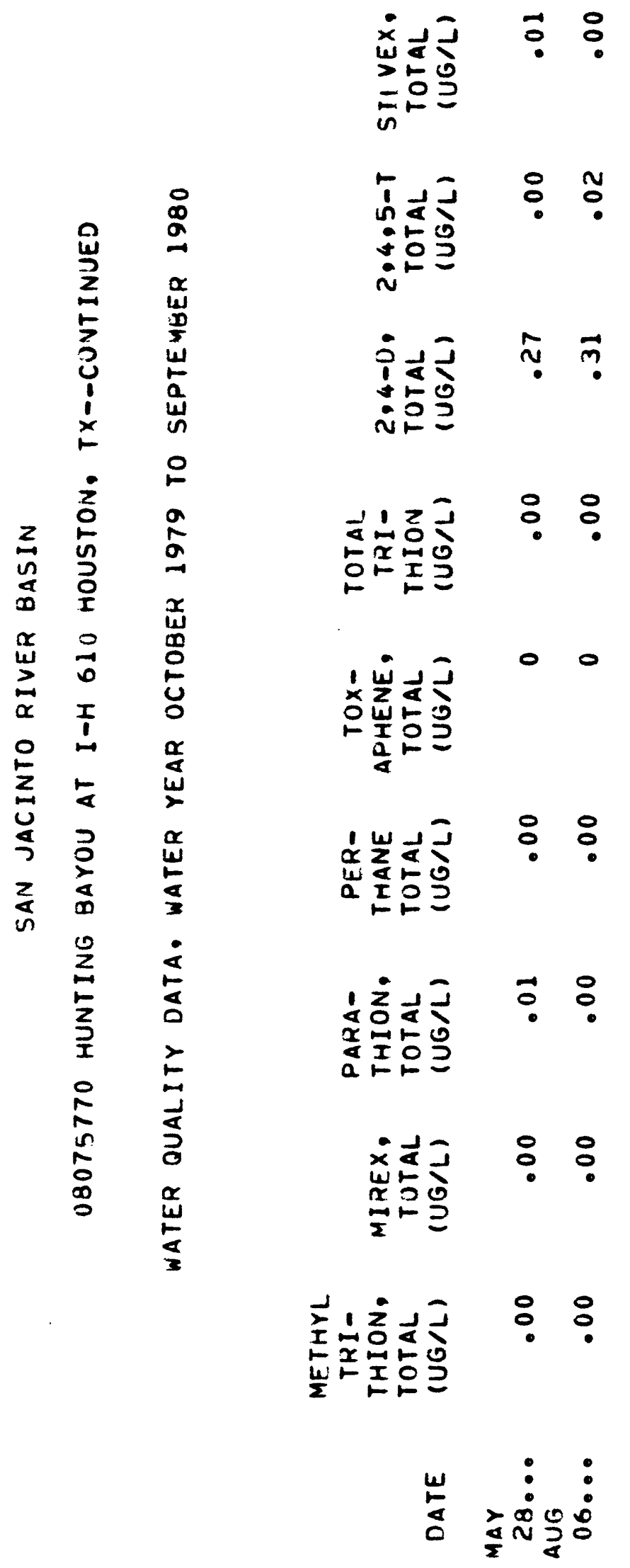




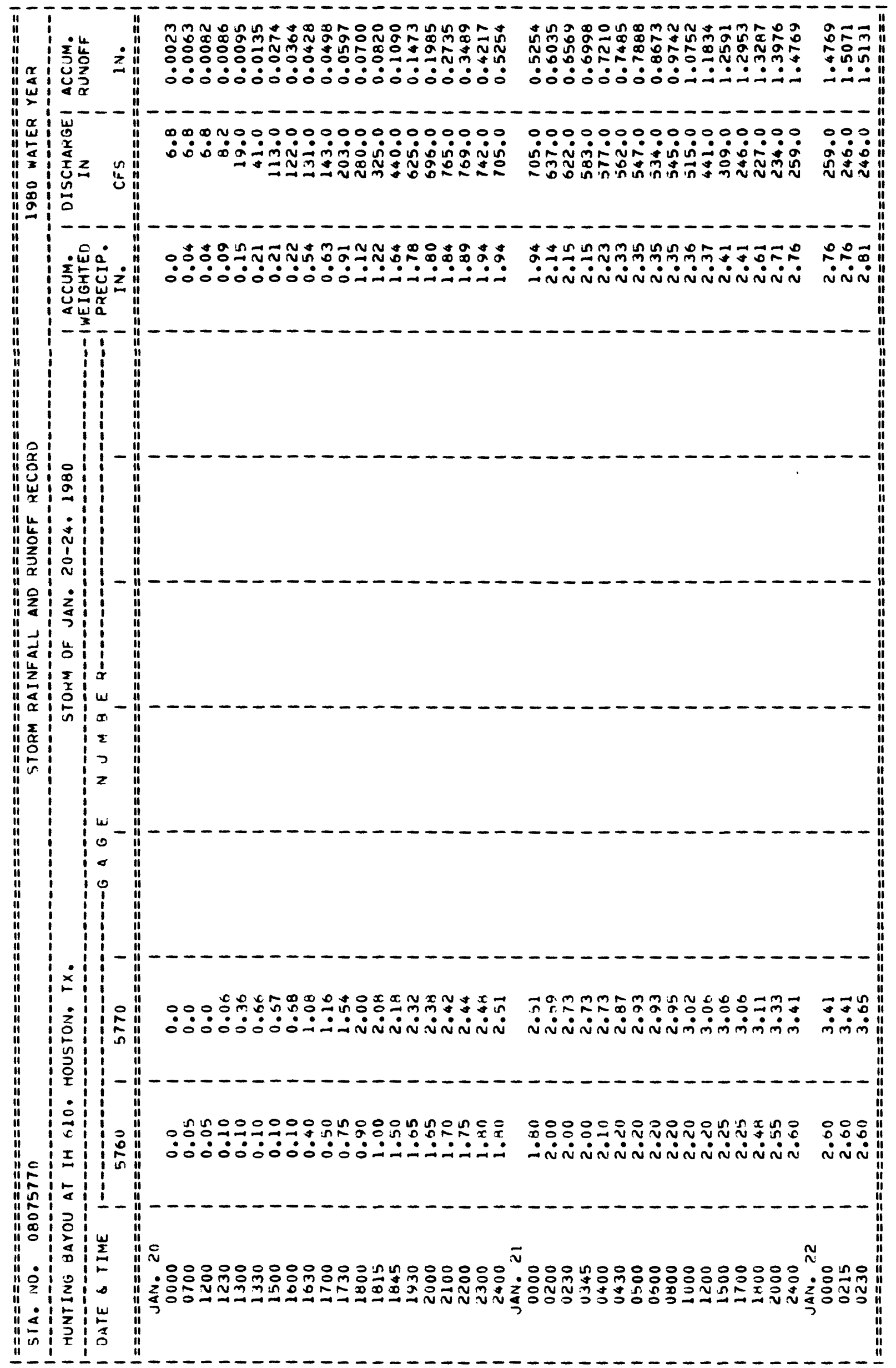

-197- 


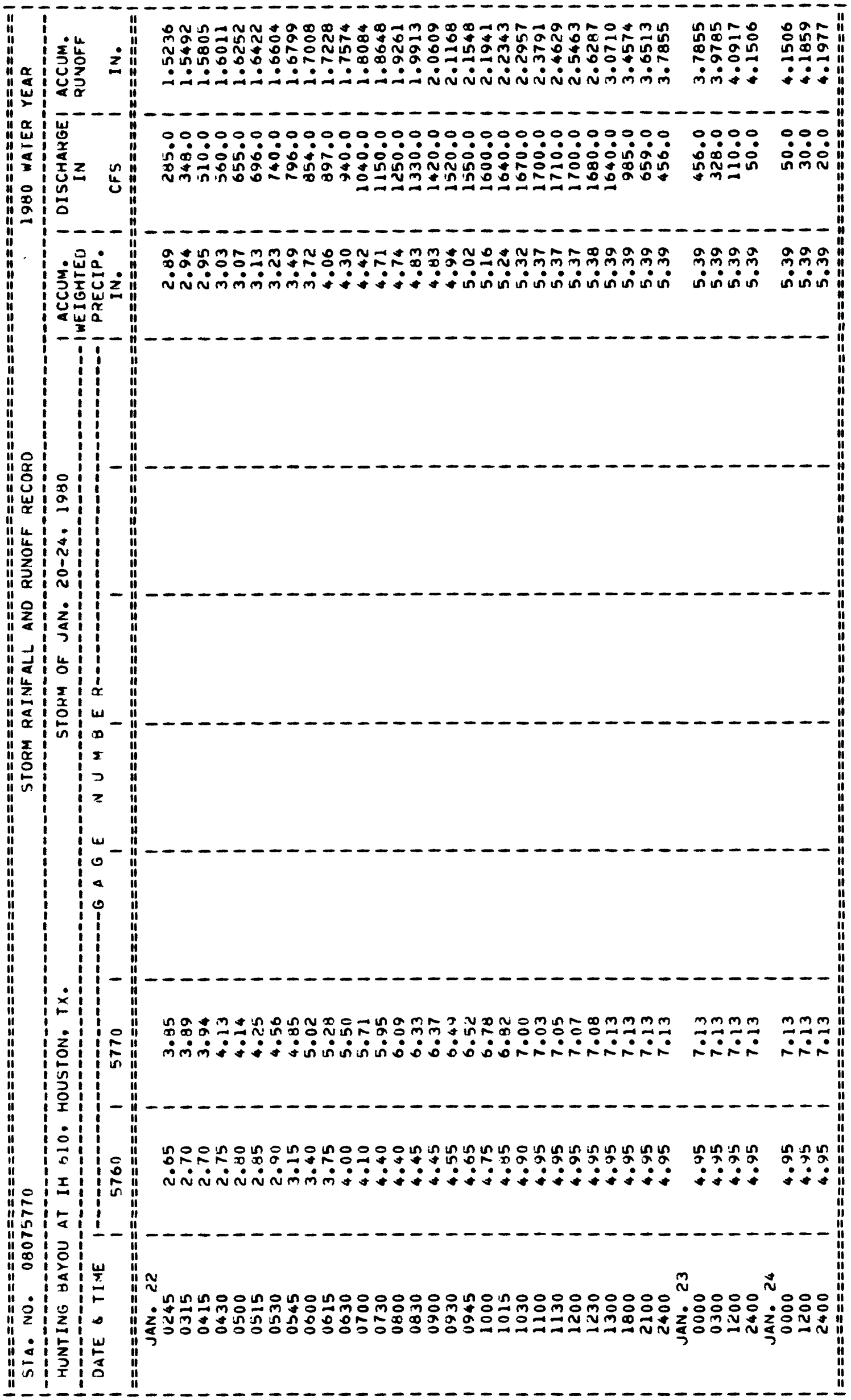

$-198-$ 


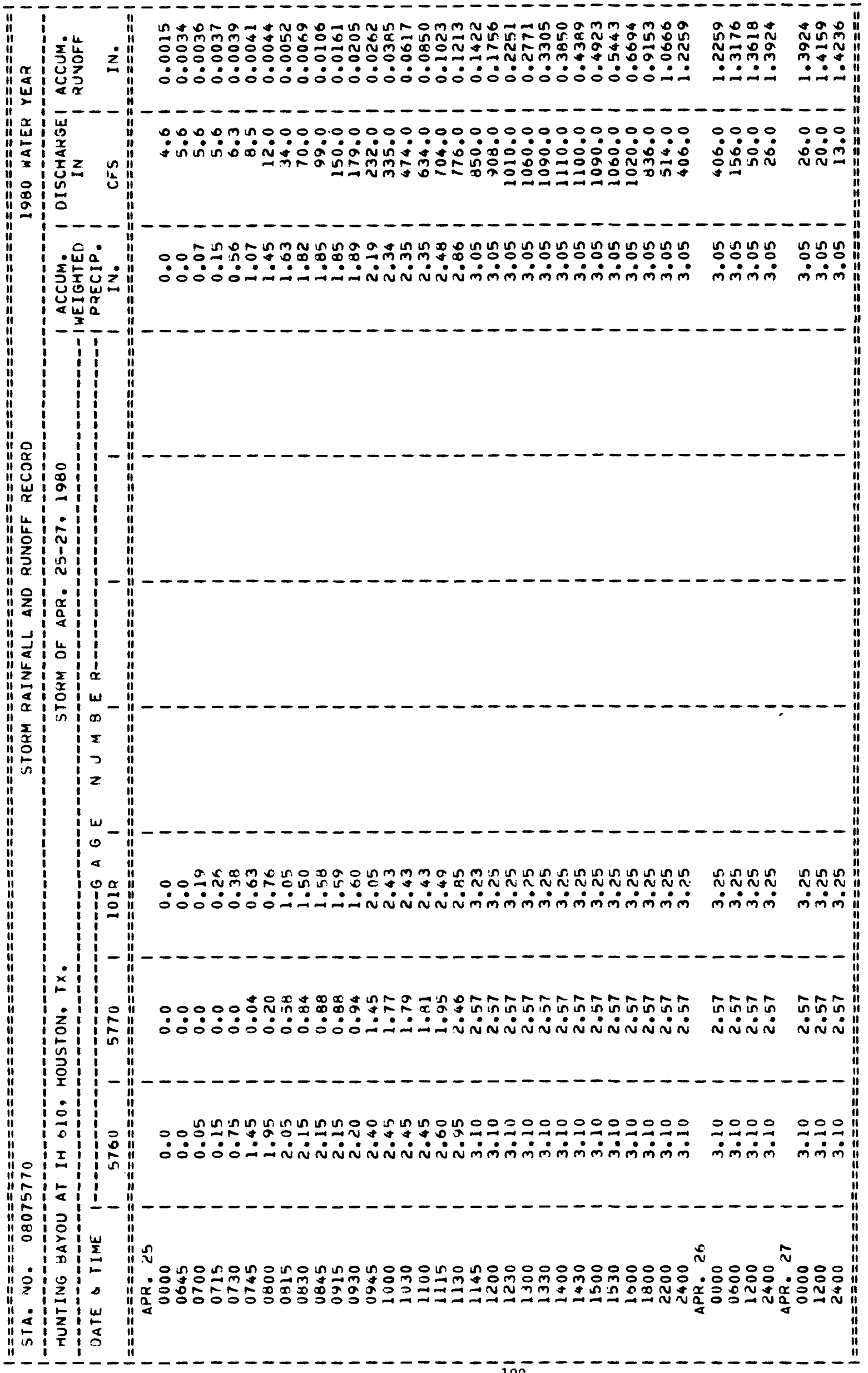




\section{GREENS BAYOU DRAINAGE BASIN}

The locations of aata-collection sites in and near the Greens Bayou drainage basin above U.S. Highway 59 are shown in figure 19. Data-collection sites in the lower portion of the drainage basin are shown in figure 1.

Halls Bayou, which is a part of the Greens Bayou drainage basin, is shown as a separate drainage basin within the Greens Bayou section.

Weighted-mean rainfall for the orainage basin, above the U.S. Highway 75 station, based on five rain gages, for the 1980 water year was 35.91 inches or 12.28 inches less than the 30-year (1941-70) average of 48.19 inches for Houston.

Weighted-mean rainfall for the drainage basin above the U.S. Highway 59 station, based on seven rain gages, for the 1980 water year was 36.08 inches or 12.11 inches less than the 30-year (1941-70) average of 48.19 inches for Houston.

The storm of Feb. 8-11 was selected for analys is at station 08075780, Greens Bayou at Cutten Road near Houston. The storms of Jan. 20-24 and Mar. 27-31 were selected for analysis at station 08075900, Greens Bayou at U.S. Highway 75 near Houston. The storms of Jan. 20-26 and Mar. 27-April 2 were selected for analys is at station 08076000, Greens Bayou near Houston (U.S. Highway 59) and at station 08076700, Greens Bayou at Ley Road, Houston. 


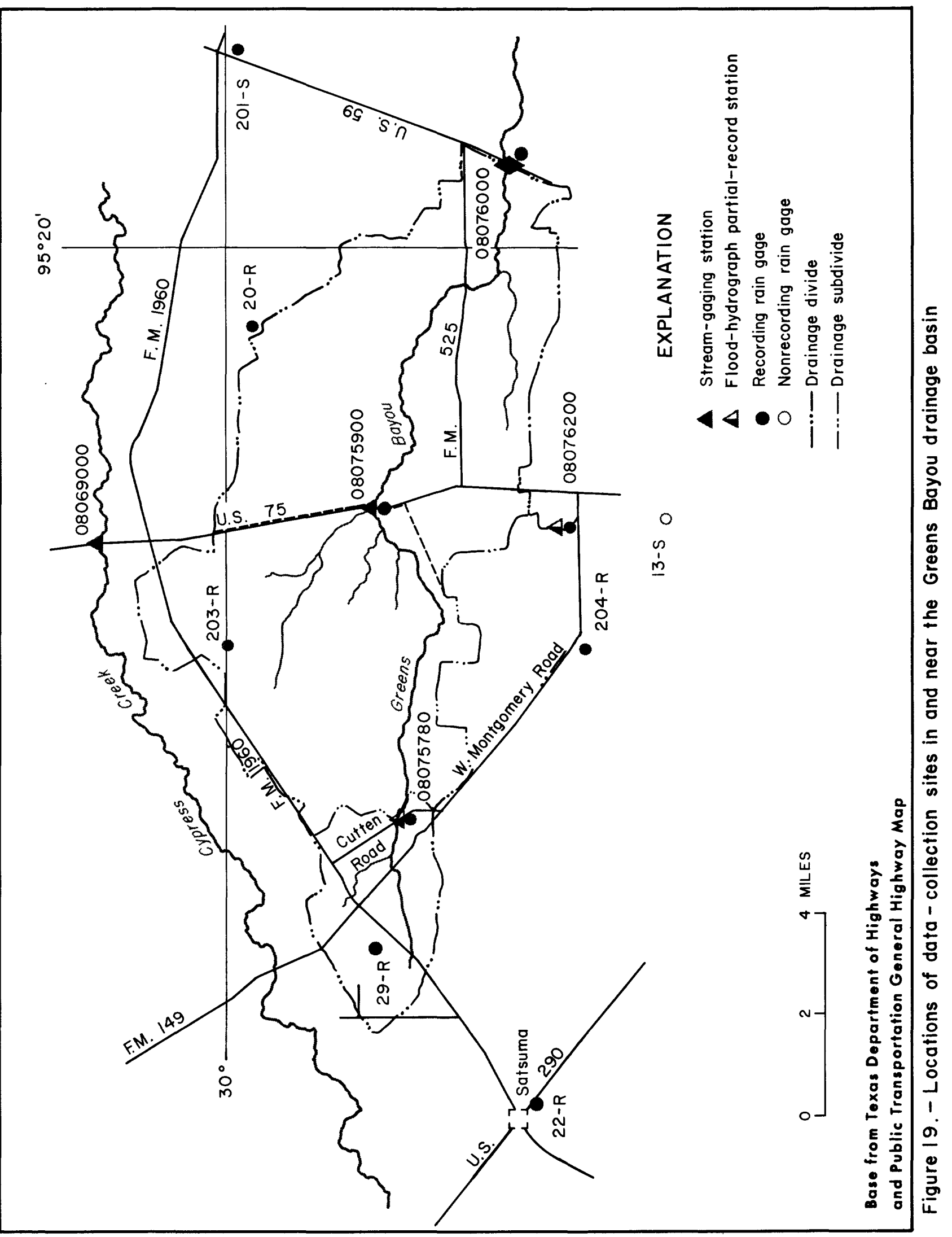




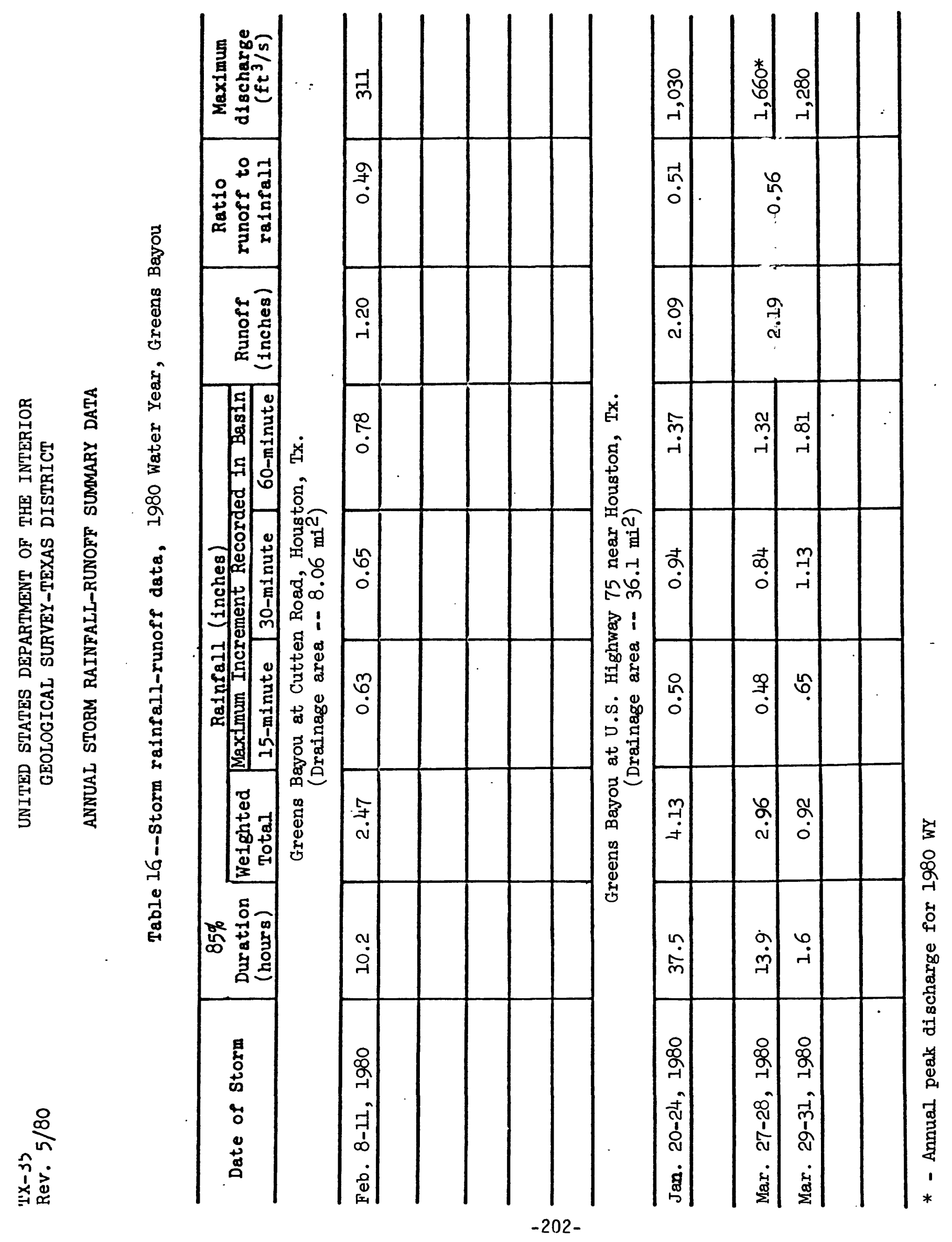




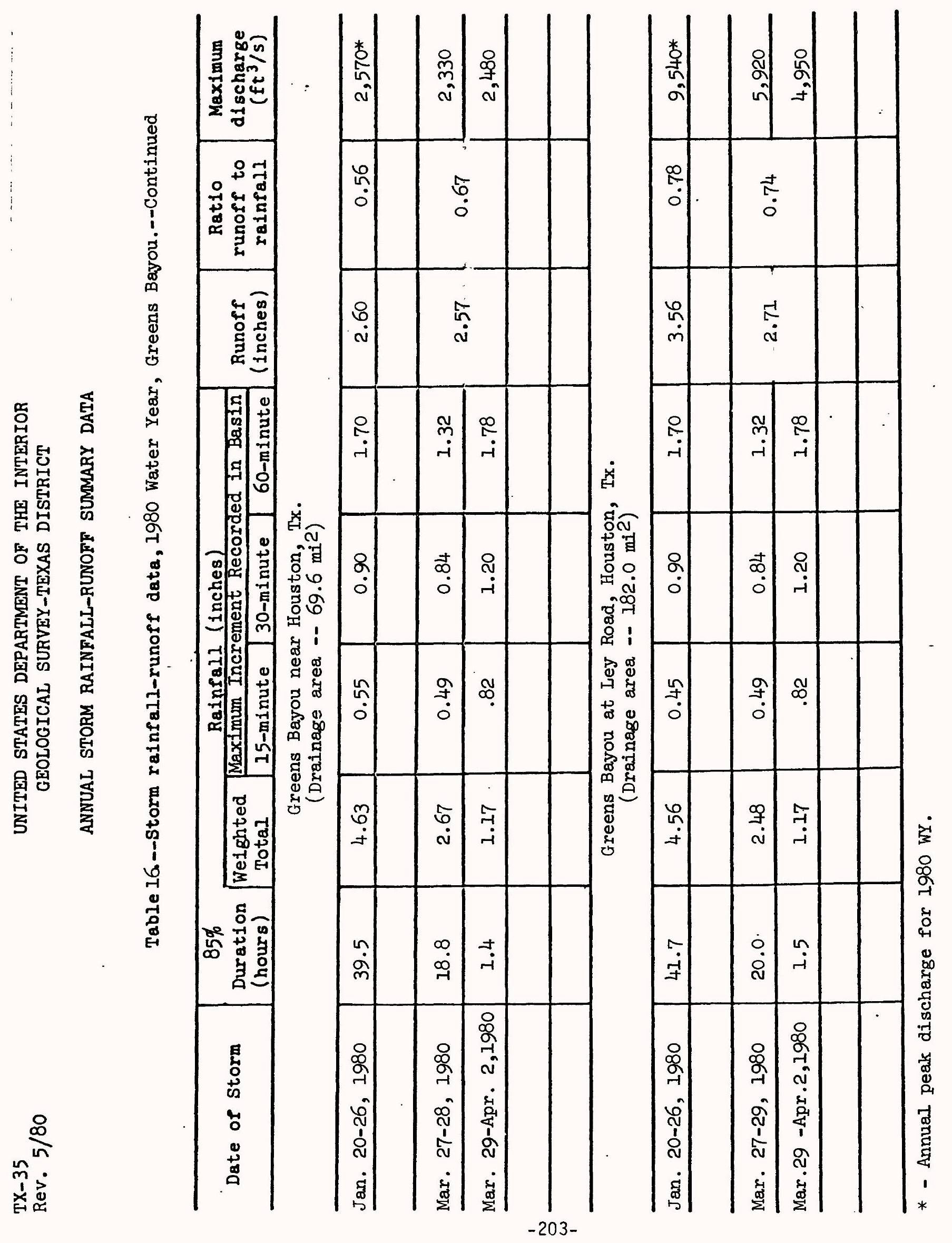


08075780 GREENS BAYOU AT CUTTEN ROAD NEAR HOUSTON, TEX. (Flood-hydrograph partial-record station)

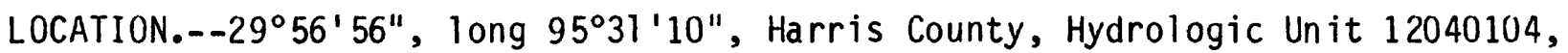
at dowmstream side of bridge on Cutten Road, 16.2 miles upstream from station 08076000 , Greens Bayou near Houston, and 16.5 miles northwest of the main post office in downtown Houston.

DRAINAGE AREA.--8.06 $\mathrm{mi}^{2}$. Prior to Oct. 1, 1973, $8.73 \mathrm{mi}^{2}$."

PERIOD OF RECORD.--Aug. 1964 to Nov. 1977, April 20, 1978 to current year.

GAGE.--Digital flood-hydrograph and rainfall recorders and crest-stage gage. Prior to Nov. 1977 a flood-hydrograph recorder (type SR) and crest-stage gage. Datum of gage is National Geodetic Vertical Datum of 1929, 1957 adjustment, unadjusted for land-surface subsidence.

REMARKS.--Records fair.

EXTREMES FOR PERIOD OF RECORD.--Maximum discharge, $576 \mathrm{ft}^{3} / \mathrm{s}$, Sept. 19, 1979 (elevation $113.16 \mathrm{ft}$ ) after channel rectification. Maximum discharge, 520 $\mathrm{ft}^{3} / \mathrm{s}$, June 13, 1973 (elevation 118.27), prior to channel rectification; minimum not determined.

EXTREMES FOR CURRENT YEAR.--Peak discharge above base of $150 \mathrm{ft}^{3} / \mathrm{s}$ and maximum $(*)$ :

DATE

Oct. 30

Dec. 12

Jan. 21

Jan. 21

Feb. 8

Mar. 27

Sept. 6
TIME

unknown

1100

0645

2115

2115

1730

1445
DISCHARGE

$\left(\mathrm{ft}^{3} / \mathrm{s}\right)$

192

184

177

183

311

*409

186
ELEVATION

109.15

109.10

109.00

109.04

110.10

110.64

110.11

Minimum discharge not determined. 


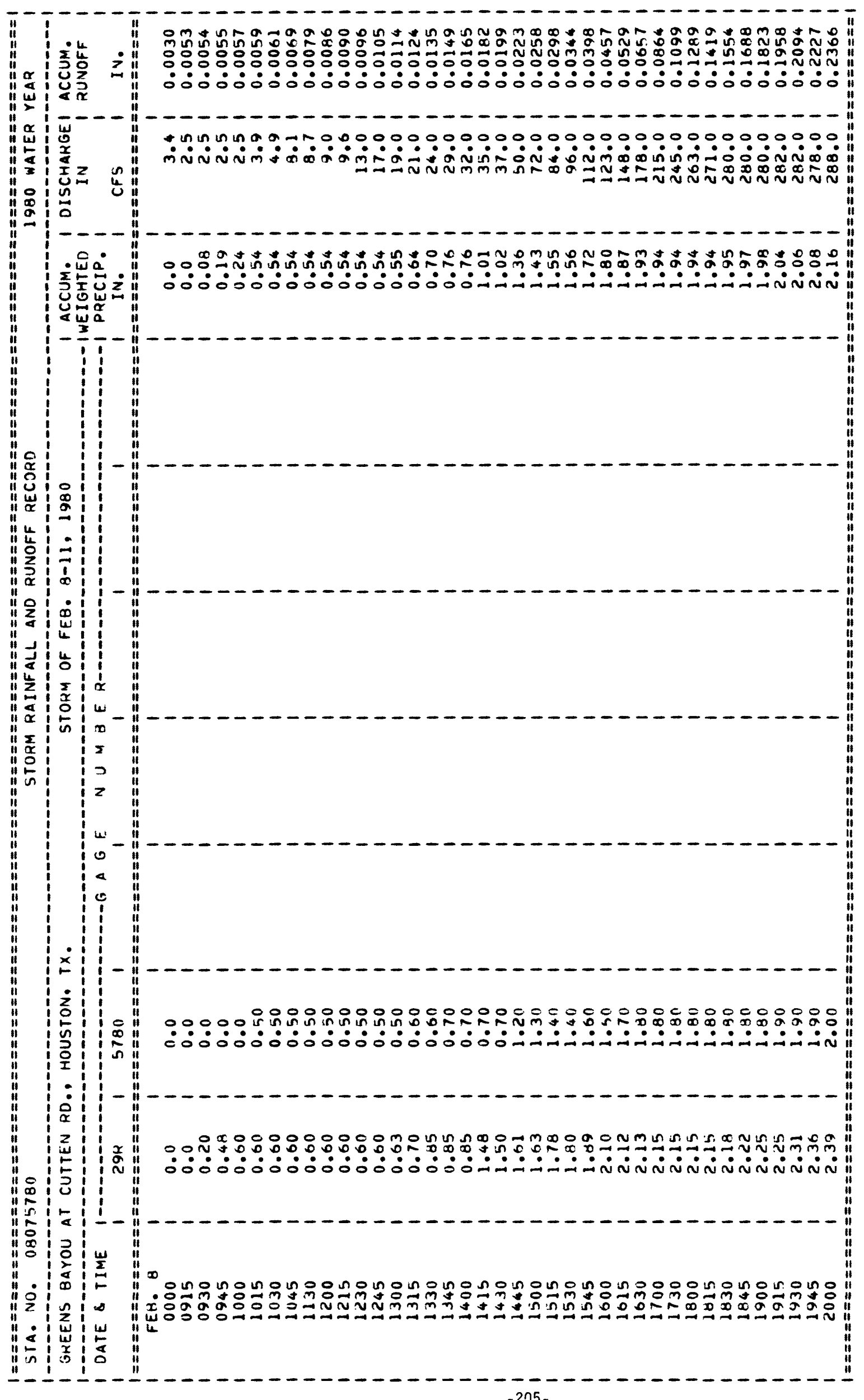

$-2.25-$ 


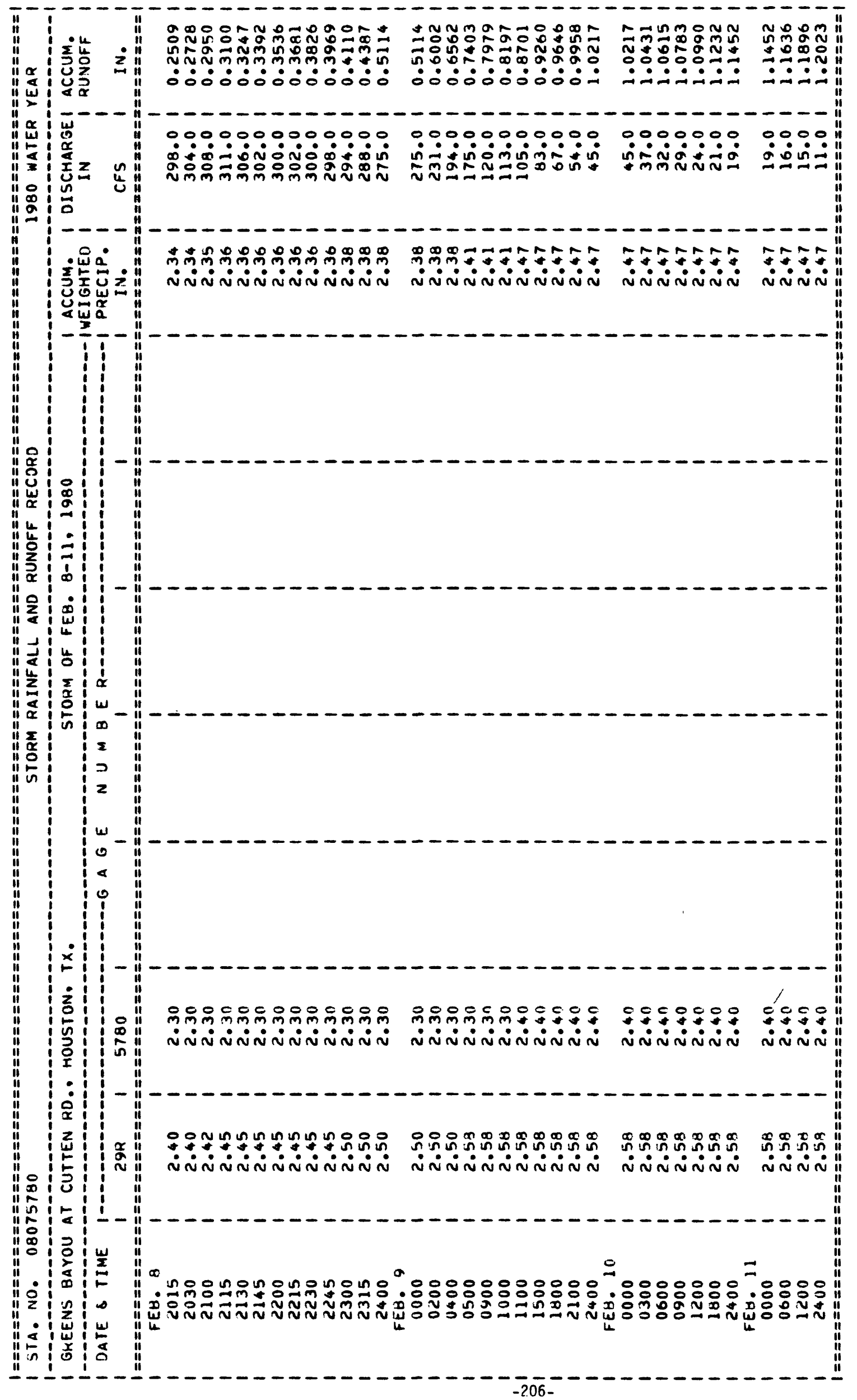




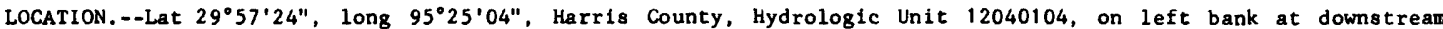
side of U.S. Highway 75 bridge, $9.0 \mathrm{mi}(14.5 \mathrm{~km})$ upstream from station 08076000 , and $21 \mathrm{mi}(34 \mathrm{~km})$ upstream from Halls Bayou.

DRAinage AREa. --36.1 $\mathrm{mi}^{2}\left(93.5 \mathrm{~km}^{2}\right)$. Prior to October $1973,34.8 \mathrm{mi}^{2}\left(90.1 \mathrm{~km}^{2}\right)$.

PERIOD OF RECORD.--August 1965 to current year.

REVISED RECORDS.--WDR TX-76-1: Drainage area.

GAGE.--Water-stage recorder and crest-stage gage, Datum of gage is National Geodetic Datum of 1929 . 1959 adjustment; unadjusted for land-surface subsidence.

REMARKS.--Records fair. Records furnished by Houston Lighting and Power Co. show that about 2,640 acre-ft (3.26 $\mathrm{hm}^{3}$ ) of ground water used for cooling purposes was released to bayou about $8 \mathrm{mi}(13 \mathrm{~km})$ upstream from gage during the current year. No know diversion above station. Recording rain gage at station. Several observations of water temperature were made during the year.

AVERAGE DISCHARGE. -15 years, $30.8 \mathrm{ft}^{3} / \mathrm{s}\left(0.872 \mathrm{~m}^{3} / \mathrm{s}\right), 22,310 \mathrm{acre}-\mathrm{ft} / \mathrm{yr}\left(27.5 \mathrm{hm}^{3} / \mathrm{yr}^{2}\right)$.

EXTREMES FOR PERIOD OF RECORD.--Maximum discharge, $2,950 \mathrm{ft}^{3} / \mathrm{s}\left(83.5 \mathrm{~m}^{3} / \mathrm{s}\right)$ Sept. 20, 1979, elevation, $90.46 \mathrm{ft}$ $(27.572 \mathrm{~m})$; maximum elevation, $91.09 \mathrm{ft}(27.764 \mathrm{~m})$ Feb. 21, 1969; minimum daily discharge, $0.16 \mathrm{ft}^{3} / \mathrm{s}(0.004$ $\left.\mathrm{m}^{3} / \mathrm{s}\right)$ Oct. $21,22,1969$.

EXTREMES FOR CURRENT YEAR.--Peak discharges above base of $800 \mathrm{ft}^{3} / \mathrm{s}\left(22.7 \mathrm{~m}^{3} / \mathrm{s}\right.$ ) and maximum (*):

\begin{tabular}{|c|c|c|c|c|c|}
\hline Date & Time & $\begin{array}{r}\text { Disc } \\
\left(\mathrm{ft}^{3} / \mathrm{s}\right)\end{array}$ & $\begin{array}{l}r g e \\
\left(m^{3} / s\right)\end{array}$ & $\begin{array}{l}\text { Gage } \\
(\mathrm{ft})\end{array}$ & $\begin{array}{r}\text { height } \\
\text { (I) }\end{array}$ \\
\hline $\begin{array}{l}\text { Jan. } 21 \\
\text { Feb. } \\
\text { Mar. } 27\end{array}$ & $\begin{array}{l}0430 \\
0030 \\
2000\end{array}$ & $\begin{array}{r}1,030 \\
962 \\
* 1,660\end{array}$ & $\begin{array}{l}29.2 \\
27.2 \\
47.0\end{array}$ & $\begin{array}{l}83.90 \\
83.60 \\
86.13\end{array}$ & $\begin{array}{l}25.573 \\
25.481 \\
26.252\end{array}$ \\
\hline
\end{tabular}

Minimum daily discharge, $6.1 \mathrm{ft}^{3} / \mathrm{s}\left(0.17 \mathrm{~m}^{3} / \mathrm{s}\right)$ Aug. 21 .

DISCHARGE, IN CUBIC FEET PER SECOND, WATER YEAR OCTOBER 1979 TO SEPTEMBER 1980

\begin{tabular}{|c|c|c|c|c|c|c|c|c|c|c|c|c|}
\hline DAY & OCT & NOV & DEC & JAN & FE'B & MAR & $A P R$ & MAY & JUN & JUL & AUG & SEP \\
\hline $\begin{array}{l}1 \\
2 \\
3 \\
4 \\
5\end{array}$ & $\begin{array}{c}13 \\
11 \\
11 \\
11 \\
9.4\end{array}$ & $\begin{array}{l}21 \\
13 \\
12 \\
11 \\
12\end{array}$ & $\begin{array}{r}8.6 \\
8.8 \\
9.4 \\
9.2 \\
10\end{array}$ & $\begin{array}{l}13 \\
12 \\
24 \\
15 \\
13\end{array}$ & $\begin{array}{l}14 \\
15 \\
15 \\
14 \\
15\end{array}$ & $\begin{array}{l}33 \\
14 \\
15 \\
17 \\
16\end{array}$ & $\begin{array}{l}40 \\
32 \\
28 \\
21 \\
17\end{array}$ & $\begin{array}{l}10 \\
10 \\
10 \\
9.6 \\
9.5\end{array}$ & $\begin{array}{l}11 \\
11 \\
9.9 \\
11 \\
11\end{array}$ & $\begin{array}{l}8.2 \\
8.6 \\
8.5 \\
8.6 \\
8.4\end{array}$ & $\begin{array}{c}9.5 \\
10 \\
8.0 \\
7.0 \\
23\end{array}$ & $\begin{array}{l}22 \\
18 \\
13 \\
13 \\
24\end{array}$ \\
\hline $\begin{array}{r}6 \\
7 \\
8 \\
9 \\
10\end{array}$ & $\begin{array}{r}8.5 \\
8.5 \\
9.5 \\
11 \\
9.6\end{array}$ & $\begin{array}{c}10 \\
9.0 \\
8.2 \\
9.6 \\
11\end{array}$ & $\begin{array}{l}9.1 \\
8.8 \\
7.9 \\
8.9 \\
9.2\end{array}$ & $\begin{array}{l}11 \\
11 \\
11 \\
10 \\
11\end{array}$ & $\begin{array}{r}13 \\
12 \\
264 \\
524 \\
107\end{array}$ & $\begin{array}{l}13 \\
12 \\
11 \\
11 \\
12\end{array}$ & $\begin{array}{l}15 \\
17 \\
15 \\
12 \\
12\end{array}$ & $\begin{array}{l}9.1 \\
13 \\
11 \\
13 \\
9.6\end{array}$ & $\begin{array}{l}13 \\
11 \\
11 \\
87 \\
35\end{array}$ & $\begin{array}{l}8.1 \\
7.7 \\
8.3 \\
8.6 \\
9.4\end{array}$ & $\begin{array}{r}11 \\
7.9 \\
8.6 \\
7.2 \\
6.2\end{array}$ & $\begin{array}{r}378 \\
185 \\
64 \\
29 \\
16\end{array}$ \\
\hline $\begin{array}{l}11 \\
12 \\
13 \\
14 \\
15\end{array}$ & $\begin{array}{l}9.4 \\
9.5 \\
9.1 \\
8.5 \\
9.5\end{array}$ & $\begin{array}{l}8.5 \\
8.5 \\
8.4 \\
8.3 \\
8.1\end{array}$ & $\begin{array}{c}8.5 \\
283 \\
98 \\
23 \\
16\end{array}$ & $\begin{array}{l}10 \\
9.8 \\
11 \\
13 \\
11\end{array}$ & $\begin{array}{l}53 \\
35 \\
26 \\
27 \\
37\end{array}$ & $\begin{array}{l}12 \\
14 \\
12 \\
13 \\
13\end{array}$ & $\begin{array}{l}12 \\
13 \\
34 \\
16 \\
15\end{array}$ & $\begin{array}{c}10 \\
9.7 \\
11 \\
58 \\
101\end{array}$ & $\begin{array}{l}16 \\
12 \\
11 \\
11 \\
9.2\end{array}$ & $\begin{array}{c}9.1 \\
10 \\
8.7 \\
9.6 \\
9.4\end{array}$ & $\begin{array}{r}6.3 \\
7.1 \\
7.2 \\
7.4 \\
14\end{array}$ & $\begin{array}{r}11 \\
9.6 \\
8.3 \\
9.6 \\
8.6\end{array}$ \\
\hline $\begin{array}{l}16 \\
17 \\
18 \\
19 \\
20\end{array}$ & $\begin{array}{l}8.6 \\
9.2 \\
12 \\
12 \\
11\end{array}$ & $\begin{array}{l}10 \\
9.9 \\
8.2 \\
8.1 \\
8.2\end{array}$ & $\begin{array}{l}13 \\
12 \\
11 \\
10 \\
10\end{array}$ & $\begin{array}{r}13 \\
17 \\
14 \\
12 \\
236\end{array}$ & $\begin{array}{l}66 \\
35 \\
25 \\
23 \\
23\end{array}$ & $\begin{array}{l}14 \\
15 \\
11 \\
12 \\
81\end{array}$ & $\begin{array}{l}14 \\
13 \\
14 \\
14 \\
12\end{array}$ & $\begin{array}{r}145 \\
210 \\
48 \\
270 \\
91\end{array}$ & $\begin{array}{l}8.9 \\
11 \\
12 \\
11 \\
8.9\end{array}$ & $\begin{array}{l}9.3 \\
9.0 \\
9.3 \\
8.6 \\
8.7\end{array}$ & $\begin{array}{r}34 \\
9.2 \\
7.2 \\
6.7 \\
6.3\end{array}$ & $\begin{array}{l}10 \\
10 \\
10 \\
8.1 \\
7.9\end{array}$ \\
\hline $\begin{array}{l}21 \\
22 \\
23 \\
24 \\
25\end{array}$ & $\begin{array}{l}11 \\
20 \\
13 \\
10 \\
9.7\end{array}$ & $\begin{array}{l}39 \\
32 \\
24 \\
9.6 \\
9.3\end{array}$ & $\begin{array}{l}11 \\
12 \\
58 \\
33 \\
14\end{array}$ & $\begin{array}{r}794 \\
736 \\
216 \\
77 \\
45\end{array}$ & $\begin{array}{l}18 \\
20 \\
21 \\
14 \\
17\end{array}$ & $\begin{array}{l}25 \\
12 \\
14 \\
14 \\
12\end{array}$ & $\begin{array}{c}12 \\
10 \\
9.0 \\
8.8 \\
124\end{array}$ & $\begin{array}{l}36 \\
21 \\
17 \\
13 \\
12\end{array}$ & $\begin{array}{l}9.3 \\
61 \\
43 \\
12 \\
9.7\end{array}$ & $\begin{array}{l}10 \\
7.9 \\
8.4 \\
9.0 \\
8.9\end{array}$ & $\begin{array}{l}6.1 \\
8.6 \\
12 \\
14 \\
12\end{array}$ & $\begin{array}{l}8.7 \\
7.7 \\
7.5 \\
8.0 \\
8.0\end{array}$ \\
\hline $\begin{array}{l}26 \\
27 \\
28 \\
29 \\
30 \\
31\end{array}$ & $\begin{array}{c}13 \\
10 \\
10 \\
9.3 \\
118 \\
188\end{array}$ & $\begin{array}{l}10 \\
9.2 \\
8.4 \\
7.9 \\
8.3 \\
-. .\end{array}$ & $\begin{array}{l}12 \\
12 \\
11 \\
81 \\
22 \\
15\end{array}$ & $\begin{array}{l}33 \\
22 \\
17 \\
16 \\
17 \\
14\end{array}$ & $\begin{array}{r}14 \\
13 \\
14 \\
13 \\
-- \\
-\cdots\end{array}$ & $\begin{array}{r}21 \\
558 \\
767 \\
567 \\
174 \\
66\end{array}$ & $\begin{array}{l}26 \\
10 \\
8.8 \\
9.5 \\
9.2 \\
---\end{array}$ & $\begin{array}{l}13 \\
13 \\
13 \\
25 \\
14 \\
12\end{array}$ & $\begin{array}{l}9.8 \\
8.5 \\
8.4 \\
7.8 \\
8.6 \\
-.-\end{array}$ & $\begin{array}{l}8.1 \\
9.0 \\
76 \\
44 \\
11 \\
9.4\end{array}$ & $\begin{array}{l}16 \\
20 \\
17 \\
21 \\
25 \\
25\end{array}$ & $\begin{array}{r}8.0 \\
7.5 \\
7.5 \\
10 \\
200 \\
-\end{array}$ \\
\hline $\begin{array}{l}\text { TOTAL } \\
\text { MEAN } \\
\text { MAX } \\
\text { MIN } \\
\text { AC-FT } \\
(+t)\end{array}$ & $\begin{array}{r}613.3 \\
19.8 \\
188 \\
8.5 \\
1220 \\
2.46\end{array}$ & $\begin{array}{r}360.7 \\
12.0 \\
39 \\
7.9 \\
715 \\
1.33\end{array}$ & $\begin{array}{r}855.4 \\
27.6 \\
283 \\
7.9 \\
1700 \\
3.20\end{array}$ & $\begin{array}{r}2462.8 \\
79.4 \\
794 \\
9.8 \\
4880 \\
4.42\end{array}$ & $\begin{array}{r}1487 \\
51.3 \\
524 \\
12 \\
2950 \\
2.38\end{array}$ & $\begin{array}{r}2581 \\
83.3 \\
767 \\
11 \\
5120 \\
5.41\end{array}$ & $\begin{array}{r}593.3 \\
19.8 \\
124 \\
8.8 \\
1180 \\
1.28\end{array}$ & $\begin{array}{r}1247.5 \\
40.2 \\
270 \\
9.1 \\
2470 \\
4.82\end{array}$ & $\begin{array}{r}500.0 \\
16.7 \\
87 \\
7.8 \\
992 \\
1.73\end{array}$ & $\begin{array}{r}377.8 \\
12.2 \\
76 \\
7.7 \\
749 \\
1.21\end{array}$ & $\begin{array}{r}380.5 \\
12.3 \\
34 \\
6.1 \\
755 \\
2.21\end{array}$ & $\begin{array}{r}1128.0 \\
37.6 \\
378 \\
7.5 \\
2240 \\
5.46\end{array}$ \\
\hline
\end{tabular}

$\begin{array}{lllllllllllll}\text { CAL YR } 1979 & \text { TOTAL } & 20475.5 & \text { MEAN } & 56.1 & \text { MAX } & 1870 & \text { MIN } 6.4 & \text { AC-FT } & 40610 & \dagger \dagger & 54.50 \\ \text { WTR YR } 1980 & \text { TOTAL } & 12587.3 & \text { MEAN } & 34.4 & \text { MAX } & 794 & \text { MIN } & 6.1 & \text { AC-FT } & 24970 & \dagger & 35.91\end{array}$

tt Weighted-mean ralnfall, in inches, based on five rain gages. 


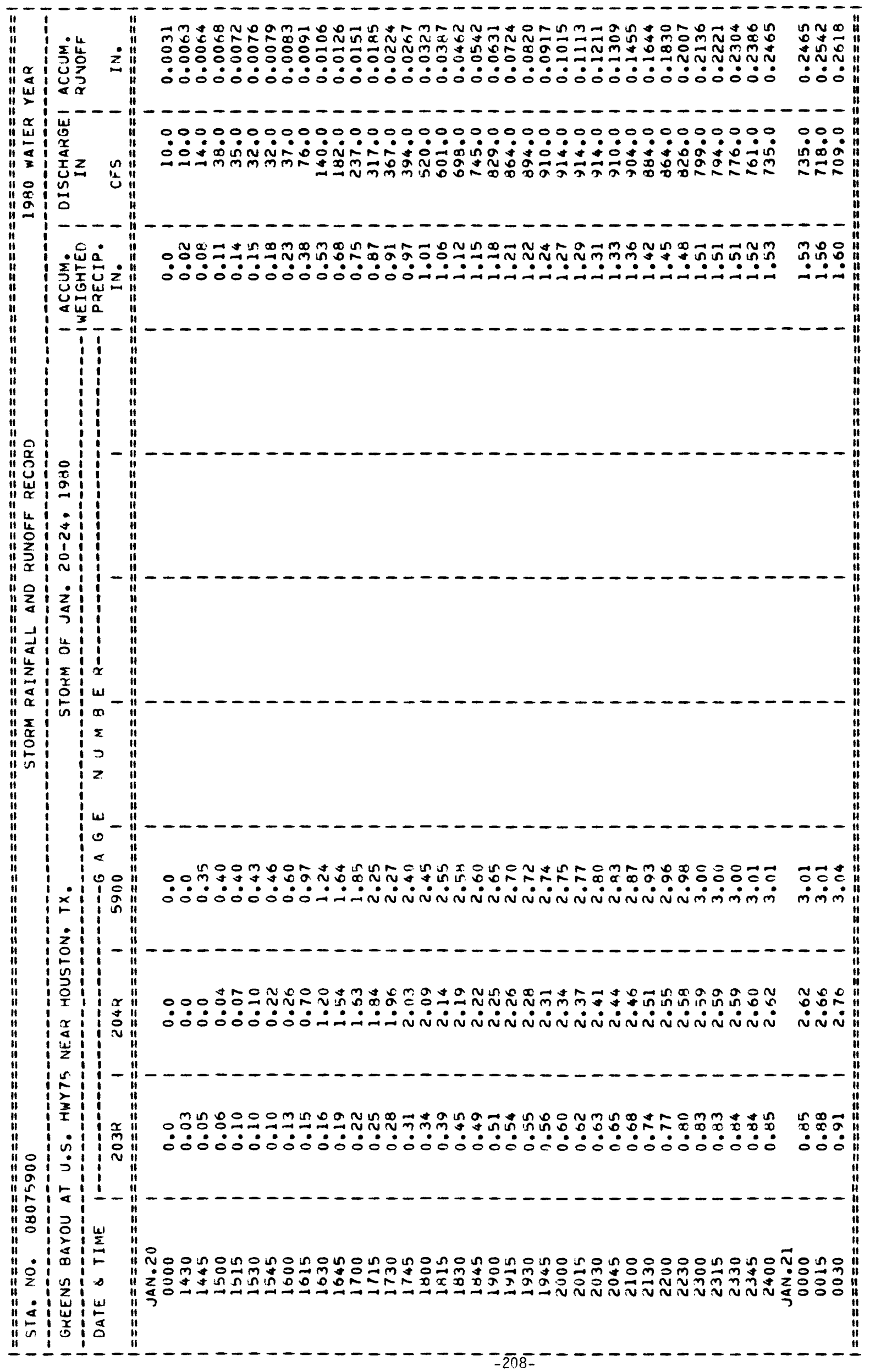




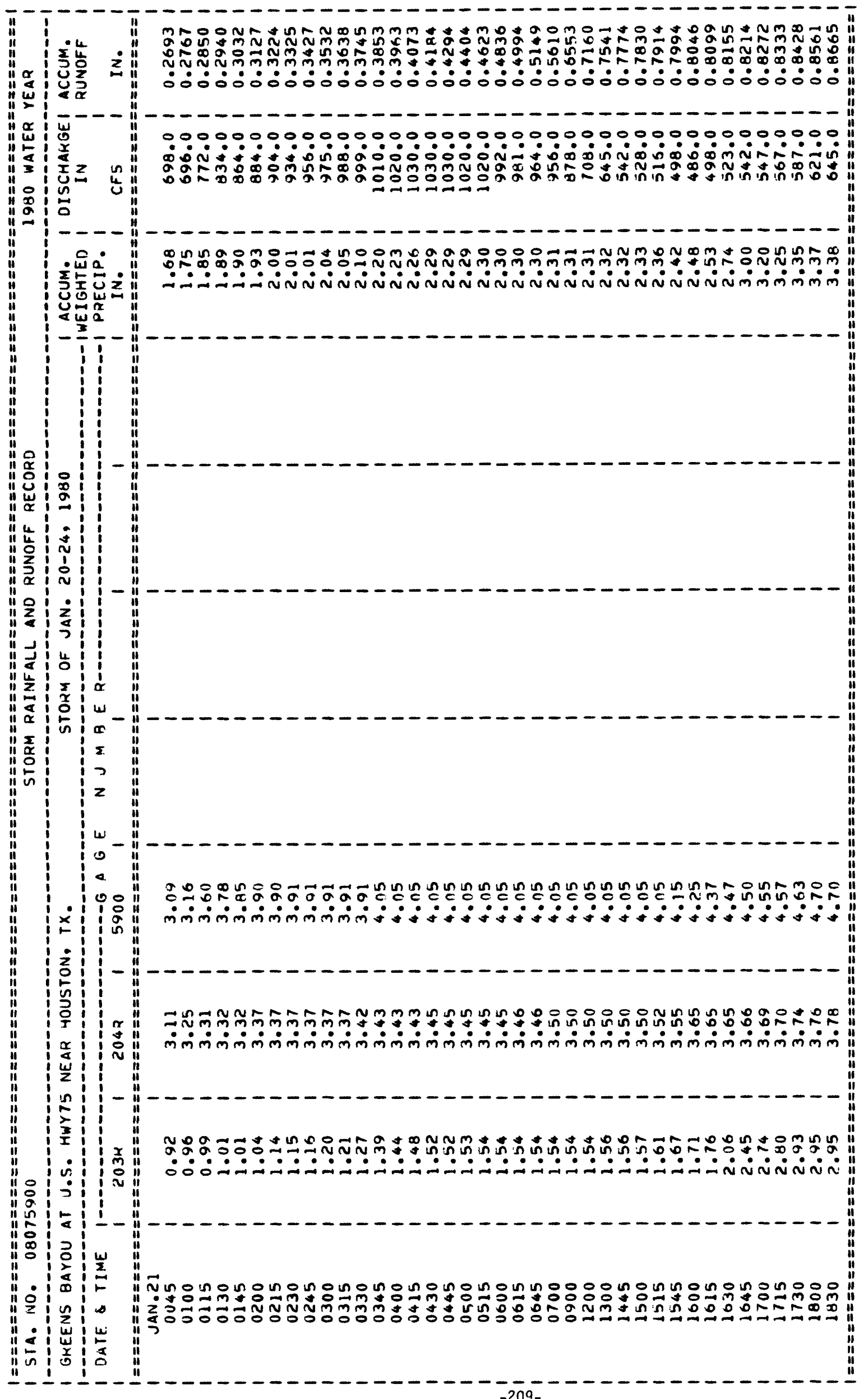




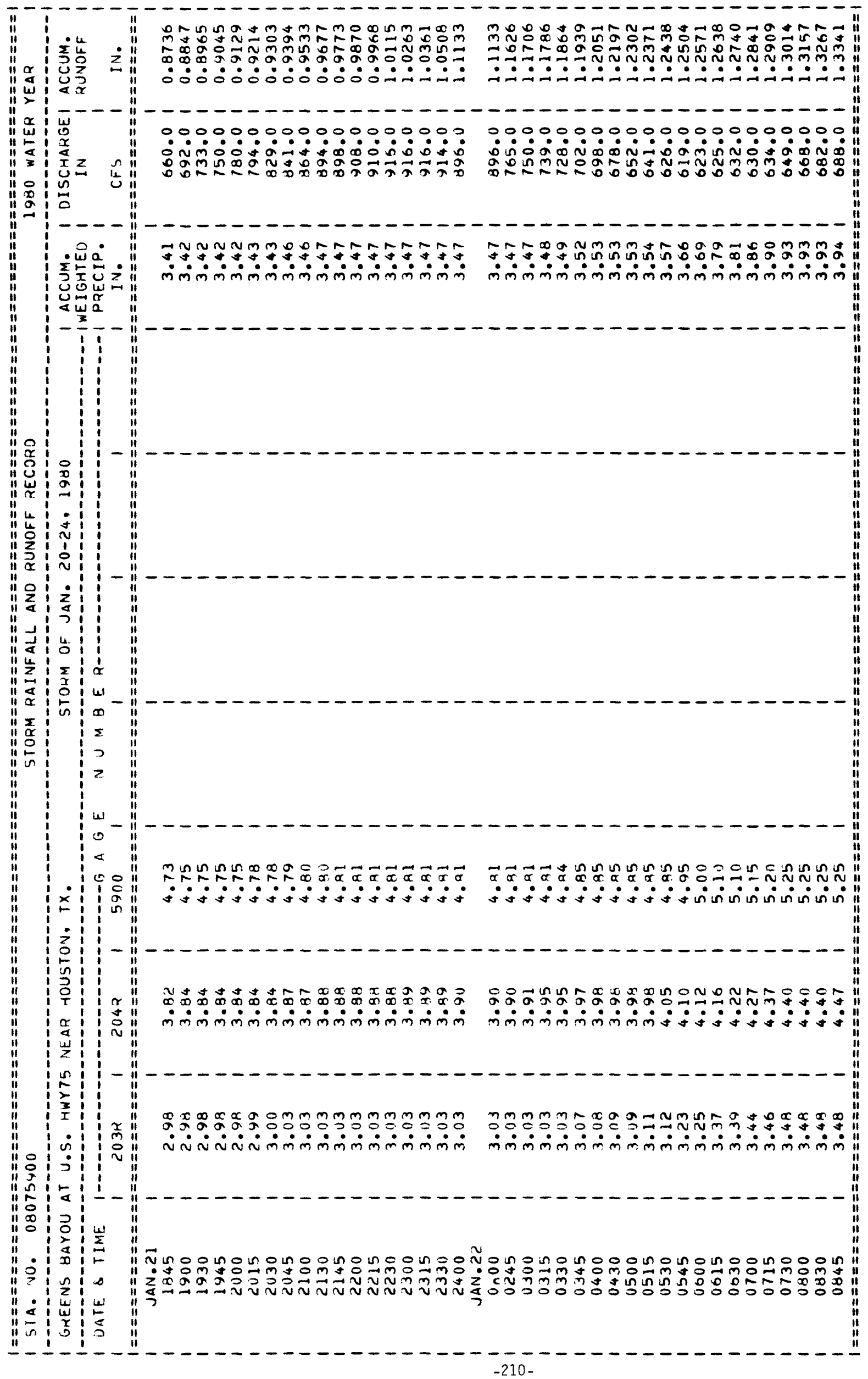




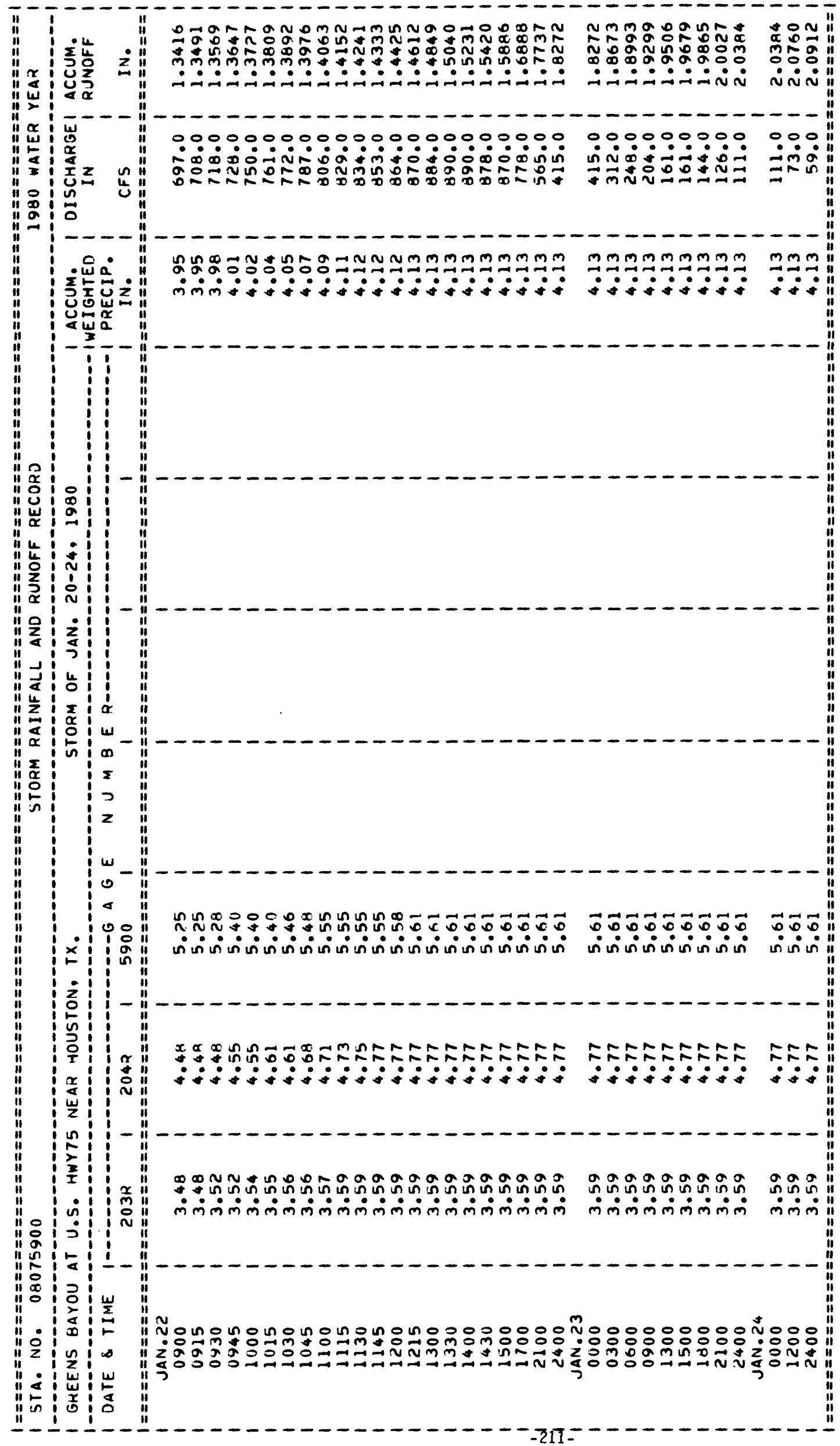




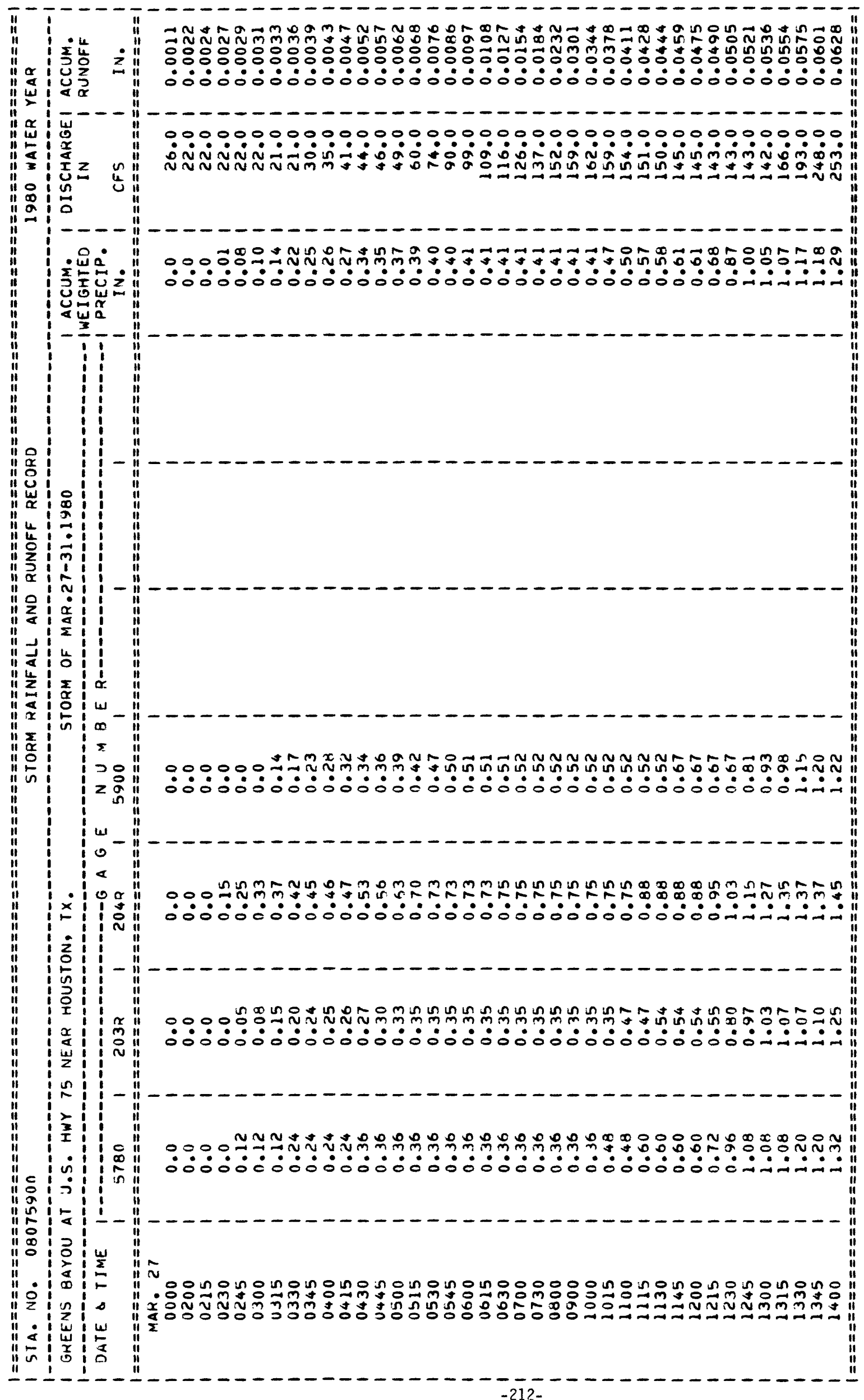




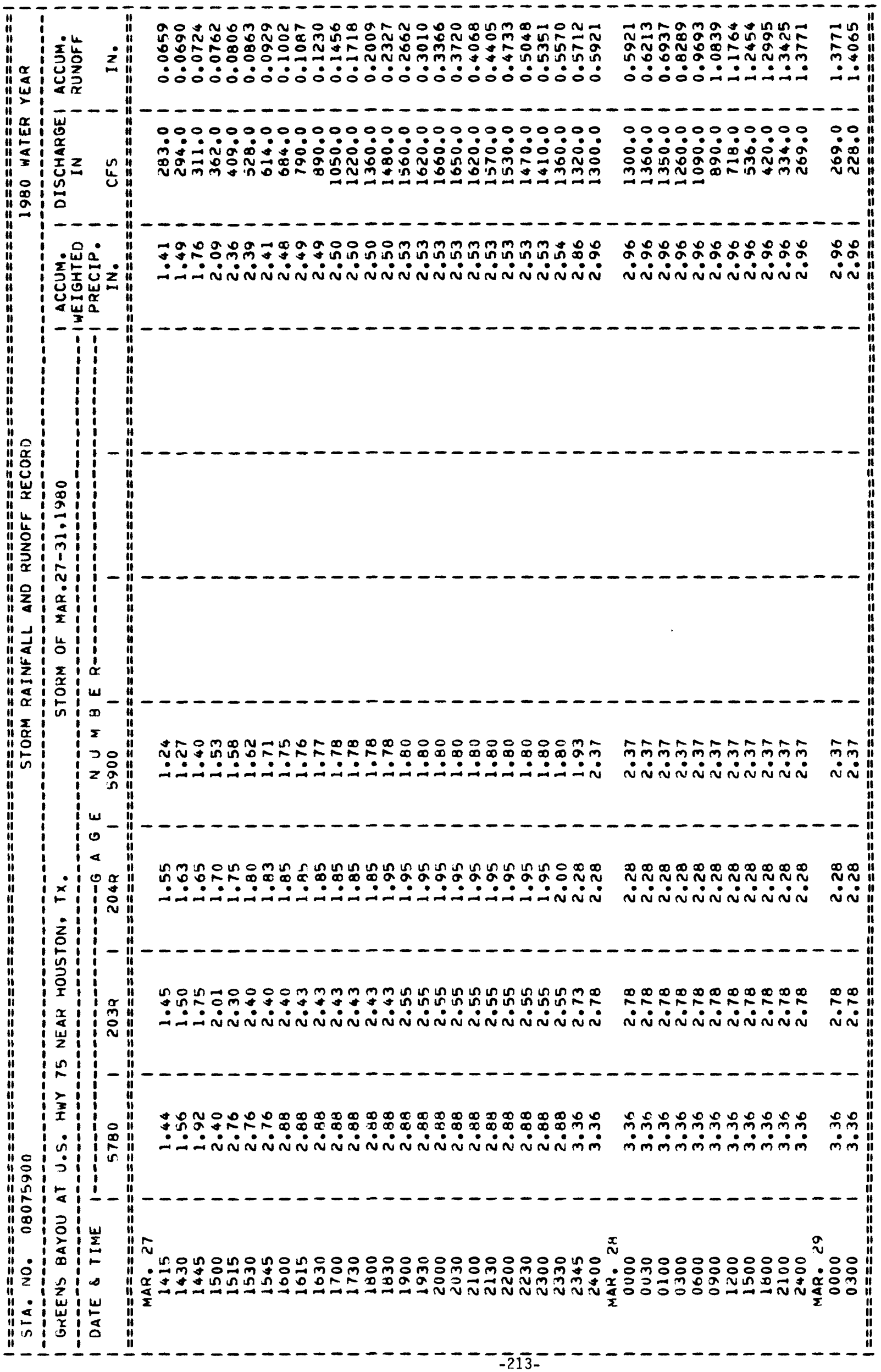




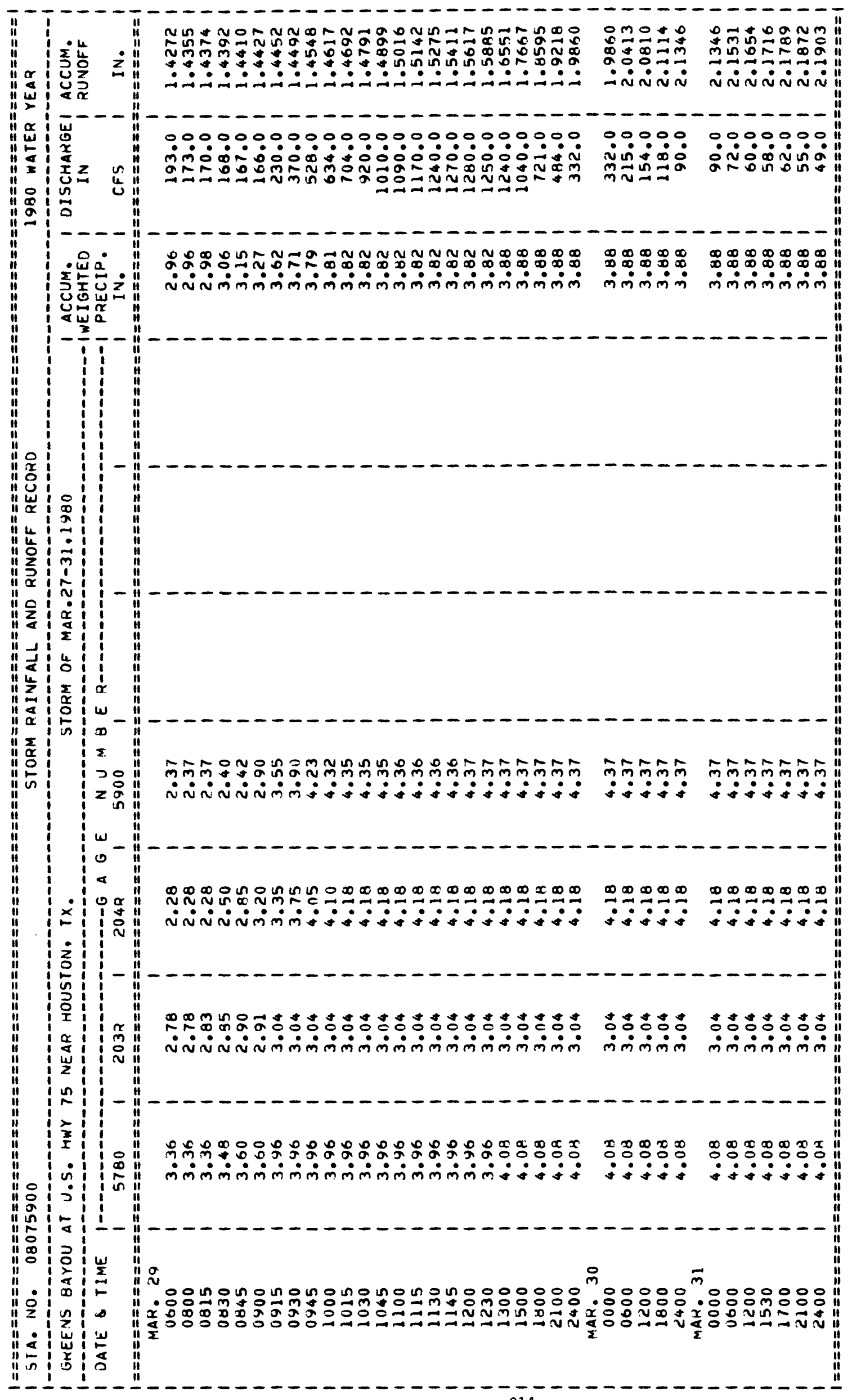


SAN JACINTO RIVER BASIN

08076000 GREENS BAYOU NEAR HOUSTON, TX

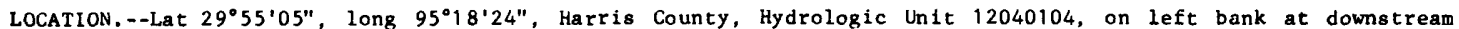
ide of bridge on U.S. Highway $59.10 .5 \mathrm{mi}(16.9 \mathrm{~km})$ northeast of Houston, $12.0 \mathrm{mi}(19.3 \mathrm{~km}) \mathrm{ups} \mathrm{tream} \mathrm{from}$ side of bridge on U.S. Highway $59,10.5 \mathrm{mi}(16.9 \mathrm{~km})$

DRAINAGE AREA. $=69.6 \mathrm{mi}^{2}\left(180.3 \mathrm{~km}^{2}\right)$. Prior to Oct. $1,1973,72.7 \mathrm{mi}^{2}\left(188.3 \mathrm{~km}^{2}\right)$.

\section{WATER-DISCHARGE RECORDS}

PERIOD OF RECORD. - - October 1952 to current year.

REVISED RECORDS.--WSP 1732: Drainage area.

GAGE. - Water-stage recorder. Datum of gage is $0.66 \mathrm{ft}(0.201 \mathrm{~m})$ below National Geodetic Vertical Datum of 1929 , 1957 adjustment; unadjusted for land-surface subsidence.

REMARKS,--Water-discharge records fafr. Channel rectified during the water years 1974-75. No known diversion above station. Low flow is sustained by Houston Light and Power Co. effluent, which is obtained from groundwater sources. Recording rain gage at station.

AVERAGE DISCHARGE. --28 years, $56.4 \mathrm{ft}^{3} / \mathrm{s}\left(1.597 \mathrm{~m}^{3} / \mathrm{s}\right), 40,860 \mathrm{acre}-\mathrm{ft} / \mathrm{yr}\left(50.4 \mathrm{hm}^{3} / \mathrm{yr}^{2}\right)$.

EXTREMES FOR PERIOD OF RECORD.--Maximum discharge, $7,730 \mathrm{ft}^{3} / \mathrm{s}\left(219 \mathrm{~m}^{3} / \mathrm{s}\right)$ Apr. 18, 1976, gage height, 61.92 ft $(18.873 \mathrm{~m})$; maximum gage height. $65.75 \mathrm{ft}(20.041 \mathrm{~m})$ Sept. 12, 1961 (prior to channel rectification); no flow at times.

EXTREMES FOR CURRENT YEAR.--Peak discharges above baae of $1,700 \mathrm{ft} / \mathrm{s}\left(48.1 \mathrm{~m}^{3} / \mathrm{s}\right)$ and maximum (*):

\begin{tabular}{|c|c|c|c|c|c|c|c|c|c|c|c|}
\hline Date & Time & $\begin{array}{r}\text { Disc } \\
\left(\mathrm{ft}^{3 / \mathrm{s}}\right)\end{array}$ & $\left(\mathrm{m}^{3} / \mathrm{s}\right)$ & $\begin{array}{l}\text { Gage } \\
(f t)\end{array}$ & $\begin{array}{r}\text { height } \\
\text { (m) }\end{array}$ & Date & Time & $\begin{array}{r}\text { Disc } \\
\left(\mathrm{ft}^{3} / \mathrm{s}\right)\end{array}$ & $\begin{array}{l}\mathrm{rge} \\
\left(\mathrm{m}^{3} / 8\right)\end{array}$ & $\begin{array}{l}\text { Gage } \\
(\mathrm{ft} t)\end{array}$ & $\begin{array}{l}\text { height } \\
\text { (m) }\end{array}$ \\
\hline $\begin{array}{ll}\text { aOct. } & 22 \\
\text { aNov. } & 21 \\
\text { aJan. } 21\end{array}$ & $\begin{array}{l}1230 \\
2230 \\
0500\end{array}$ & $\begin{array}{r}119 \\
587 \\
2,040\end{array}$ & $\begin{array}{l}3.37 \\
16.6 \\
57.8\end{array}$ & $\begin{array}{l}46.71 \\
50.14 \\
57.44\end{array}$ & $\begin{array}{l}14.237 \\
15.283 \\
17.508\end{array}$ & $\begin{array}{c}\text { aJan. } 22 \\
\text { Mar. } 29\end{array}$ & $\begin{array}{l}1200 \\
1500\end{array}$ & $\begin{array}{r}\star 2,570 \\
2,480\end{array}$ & $\begin{array}{l}72.8 \\
70.2\end{array}$ & $\begin{array}{l}58.38 \\
58.18\end{array}$ & $\begin{array}{l}17.794 \\
17.733\end{array}$ \\
\hline
\end{tabular}

a Water-quality samples were obtained during this runoff event.

Minimum daily discharge, $13 \mathrm{ft}^{3} / \mathrm{s}\left(0.37 \mathrm{~m}^{3} / \mathrm{s}\right)$ Aug. 11 . DISCHARGE, IN CUBIC FEET PER SECOND, WATER YEAR OCTOBER 1979 TO SEPTEMBER 1980

\begin{tabular}{|c|c|c|c|c|c|c|c|c|c|c|c|c|}
\hline DAY & OCT & Nov & $D E C$ & JAN & FEB & MAR & APR & MAY & JUN & JUL & AUG & SEP \\
\hline $\begin{array}{l}1 \\
2 \\
3 \\
4 \\
5\end{array}$ & $\begin{array}{l}25 \\
20 \\
20 \\
19 \\
17\end{array}$ & $\begin{array}{l}51 \\
27 \\
23 \\
23 \\
21\end{array}$ & $\begin{array}{l}20 \\
17 \\
17 \\
17 \\
19\end{array}$ & $\begin{array}{l}24 \\
22 \\
65 \\
34 \\
25\end{array}$ & $\begin{array}{l}31 \\
36 \\
36 \\
31 \\
38\end{array}$ & $\begin{array}{l}82 \\
32 \\
28 \\
32 \\
32\end{array}$ & $\begin{array}{l}99 \\
70 \\
60 \\
46 \\
39\end{array}$ & $\begin{array}{r}133 \\
71 \\
29 \\
24 \\
24\end{array}$ & $\begin{array}{l}24 \\
24 \\
21 \\
23 \\
23\end{array}$ & $\begin{array}{l}22 \\
20 \\
20 \\
20 \\
20\end{array}$ & $\begin{array}{l}18 \\
18 \\
17 \\
15 \\
39\end{array}$ & $\begin{array}{l}23 \\
19 \\
16 \\
16 \\
27\end{array}$ \\
\hline $\begin{array}{r}6 \\
7 \\
8 \\
9 \\
10\end{array}$ & $\begin{array}{l}16 \\
15 \\
16 \\
17 \\
16\end{array}$ & $\begin{array}{l}20 \\
19 \\
16 \\
19 \\
20\end{array}$ & $\begin{array}{l}17 \\
15 \\
16 \\
16 \\
17\end{array}$ & $\begin{array}{l}22 \\
20 \\
22 \\
23 \\
21\end{array}$ & $\begin{array}{r}34 \\
29 \\
381 \\
906 \\
255\end{array}$ & $\begin{array}{l}28 \\
26 \\
26 \\
23 \\
23\end{array}$ & $\begin{array}{l}37 \\
41 \\
34 \\
29 \\
24\end{array}$ & $\begin{array}{l}24 \\
36 \\
32 \\
31 \\
26\end{array}$ & $\begin{array}{r}24 \\
24 \\
24 \\
163 \\
88\end{array}$ & $\begin{array}{l}20 \\
19 \\
21 \\
22 \\
21\end{array}$ & $\begin{array}{l}33 \\
17 \\
18 \\
16 \\
14\end{array}$ & $\begin{array}{r}1000 \\
505 \\
165 \\
54 \\
28\end{array}$ \\
\hline $\begin{array}{l}11 \\
12 \\
13 \\
14 \\
15\end{array}$ & $\begin{array}{l}16 \\
16 \\
16 \\
15 \\
16\end{array}$ & $\begin{array}{l}21 \\
16 \\
17 \\
16 \\
15\end{array}$ & $\begin{array}{r}18 \\
666 \\
367 \\
57 \\
32\end{array}$ & $\begin{array}{l}23 \\
18 \\
19 \\
20 \\
24\end{array}$ & $\begin{array}{r}111 \\
72 \\
54 \\
48 \\
73\end{array}$ & $\begin{array}{l}26 \\
29 \\
24 \\
24 \\
23\end{array}$ & $\begin{array}{l}25 \\
34 \\
76 \\
39 \\
31\end{array}$ & $\begin{array}{r}26 \\
25 \\
55 \\
147 \\
128\end{array}$ & $\begin{array}{l}32 \\
25 \\
23 \\
21 \\
20\end{array}$ & $\begin{array}{l}20 \\
20 \\
21 \\
21 \\
20\end{array}$ & $\begin{array}{l}13 \\
15 \\
17 \\
16 \\
30\end{array}$ & $\begin{array}{l}21 \\
17 \\
16 \\
15 \\
15\end{array}$ \\
\hline $\begin{array}{l}16 \\
17 \\
18 \\
19 \\
20\end{array}$ & $\begin{array}{l}17 \\
15 \\
17 \\
19 \\
19\end{array}$ & $\begin{array}{l}17 \\
20 \\
16 \\
16 \\
17\end{array}$ & $\begin{array}{l}25 \\
22 \\
20 \\
19 \\
17\end{array}$ & $\begin{array}{r}24 \\
59 \\
39 \\
23 \\
391\end{array}$ & $\begin{array}{r}135 \\
77 \\
52 \\
44 \\
43\end{array}$ & $\begin{array}{r}32 \\
37 \\
24 \\
25 \\
139\end{array}$ & $\begin{array}{l}29 \\
26 \\
26 \\
29 \\
26\end{array}$ & $\begin{array}{l}448 \\
566 \\
130 \\
651 \\
249\end{array}$ & $\begin{array}{l}19 \\
22 \\
25 \\
25 \\
23\end{array}$ & $\begin{array}{l}18 \\
19 \\
18 \\
18 \\
19\end{array}$ & $\begin{array}{l}53 \\
23 \\
16 \\
16 \\
15\end{array}$ & $\begin{array}{l}16 \\
16 \\
16 \\
23 \\
16\end{array}$ \\
\hline $\begin{array}{l}21 \\
22 \\
23 \\
24 \\
25\end{array}$ & $\begin{array}{l}19 \\
48 \\
26 \\
16 \\
15\end{array}$ & $\begin{array}{r}184 \\
163 \\
71 \\
26 \\
19\end{array}$ & $\begin{array}{r}19 \\
20 \\
118 \\
142 \\
31\end{array}$ & $\begin{array}{r}1600 \\
1840 \\
632 \\
213 \\
113\end{array}$ & $\begin{array}{l}37 \\
33 \\
38 \\
31 \\
26\end{array}$ & $\begin{array}{l}59 \\
25 \\
26 \\
30 \\
22\end{array}$ & $\begin{array}{r}26 \\
24 \\
22 \\
22 \\
343\end{array}$ & $\begin{array}{l}79 \\
45 \\
36 \\
29 \\
25\end{array}$ & $\begin{array}{r}22 \\
43 \\
135 \\
32 \\
22\end{array}$ & $\begin{array}{l}52 \\
23 \\
20 \\
18 \\
18\end{array}$ & $\begin{array}{l}14 \\
15 \\
14 \\
15 \\
16\end{array}$ & $\begin{array}{l}18 \\
15 \\
15 \\
16 \\
16\end{array}$ \\
\hline $\begin{array}{l}26 \\
27 \\
28 \\
29 \\
30 \\
31\end{array}$ & $\begin{array}{r}16 \\
17 \\
15 \\
15 \\
210 \\
639\end{array}$ & $\begin{array}{r}22 \\
21 \\
20 \\
19 \\
18 \\
--\end{array}$ & $\begin{array}{r}23 \\
23 \\
20 \\
208 \\
51 \\
30\end{array}$ & $\begin{array}{l}79 \\
56 \\
45 \\
43 \\
39 \\
34\end{array}$ & $\begin{array}{r}29 \\
25 \\
26 \\
25 \\
--- \\
---\end{array}$ & $\begin{array}{r}37 \\
924 \\
1560 \\
1320 \\
645 \\
192\end{array}$ & $\begin{array}{r}91 \\
28 \\
23 \\
23 \\
22 \\
- \\
-\end{array}$ & $\begin{array}{l}27 \\
28 \\
27 \\
37 \\
29 \\
25\end{array}$ & $\begin{array}{r}25 \\
20 \\
19 \\
18 \\
19 \\
-\end{array}$ & $\begin{array}{r}18 \\
24 \\
192 \\
155 \\
25 \\
18\end{array}$ & $\begin{array}{l}15 \\
21 \\
19 \\
16 \\
27 \\
22\end{array}$ & $\begin{array}{r}16 \\
19 \\
18 \\
18 \\
456 \\
---\end{array}$ \\
\hline $\begin{array}{l}\text { TOTAL } \\
\text { MEAN } \\
\text { MAX } \\
\text { MIN } \\
\text { AC-FT } \\
(\dagger+)\end{array}$ & $\begin{array}{r}1383 \\
44.6 \\
639 \\
15 \\
2740 \\
2.51\end{array}$ & $\begin{array}{r}973 \\
32.4 \\
184 \\
15 \\
1930 \\
1.50\end{array}$ & $\begin{array}{r}2099 \\
67.7 \\
666 \\
15 \\
4160 \\
3.21\end{array}$ & $\begin{array}{r}5612 \\
181 \\
1840 \\
18 \\
11130 \\
5.00\end{array}$ & $\begin{array}{r}2756 \\
95.0 \\
906 \\
25 \\
5470 \\
2.22\end{array}$ & $\begin{array}{r}5555 \\
179 \\
1560 \\
22 \\
11020 \\
5.36\end{array}$ & $\begin{array}{r}1444 \\
48.1 \\
343 \\
22 \\
2860 \\
1.55\end{array}$ & $\begin{array}{r}3242 \\
105 \\
651 \\
24 \\
6430 \\
4.65\end{array}$ & $\begin{array}{r}1028 \\
34.3 \\
163 \\
18 \\
2040 \\
1.74\end{array}$ & $\begin{array}{r}962 \\
31.0 \\
192 \\
18 \\
1910 \\
1.40\end{array}$ & $\begin{array}{r}613 \\
19.8 \\
53 \\
13 \\
1220 \\
1.55\end{array}$ & $\begin{array}{r}2631 \\
87.7 \\
1000 \\
15 \\
5220 \\
5.39\end{array}$ \\
\hline
\end{tabular}

$\begin{array}{lllllllllllll}\text { CAL YR } 1979 & \text { TOTAL } & 44022 & \text { MEAN } & 121 & \text { MAX } & 4280 & \text { MIN } 13 & \text { AC-FT } & 87320 & +\dagger & 55.01 \\ \text { WTR YR } & 1980 & \text { TOTAL } & 28298 & \text { MEAN } & 77.3 & \text { MAX } & 1840 & \text { MIN } 13 & \text { AC-FT } & 56130 & +\dagger & 36.08\end{array}$

$\dagger$ Weighted-mean rainfall, in inches, based on seven rain gages. 


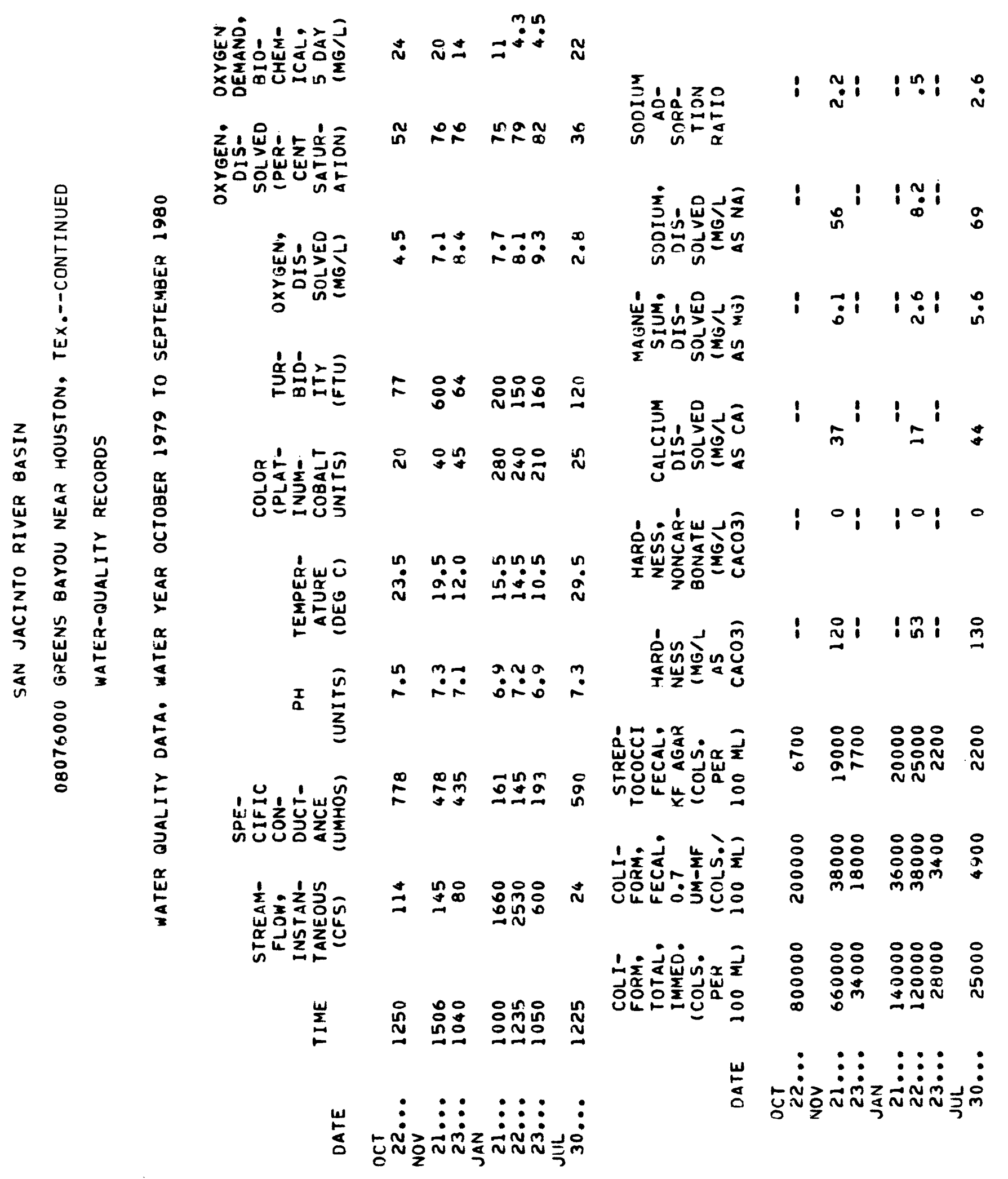




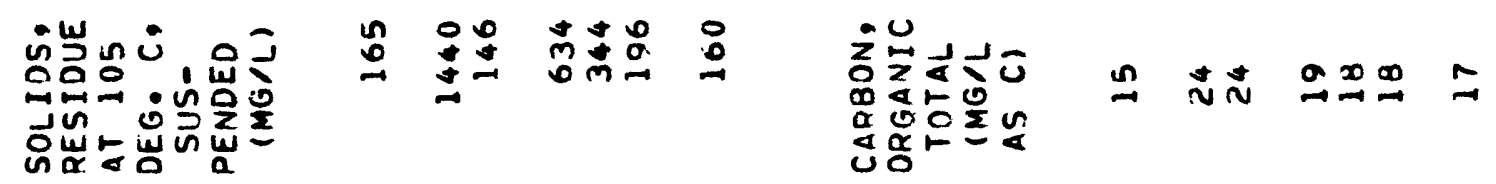

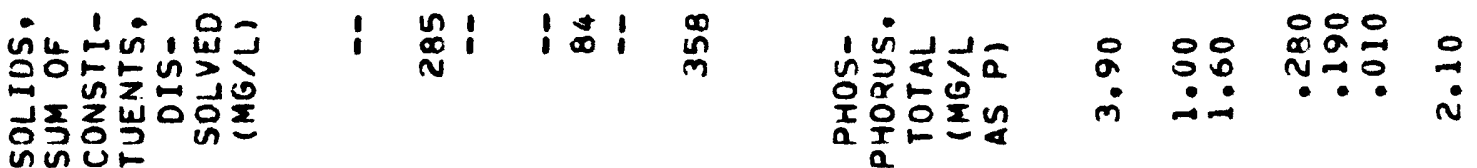

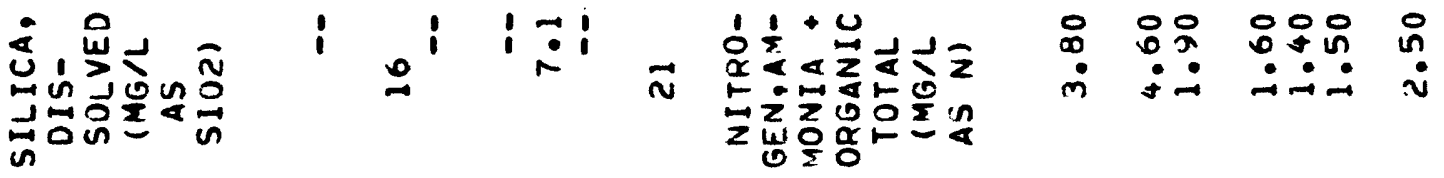

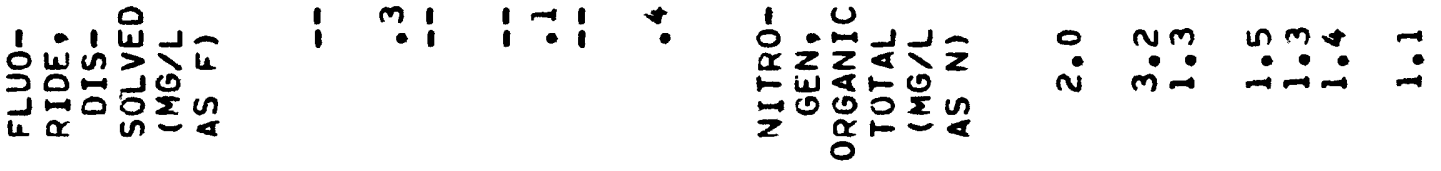

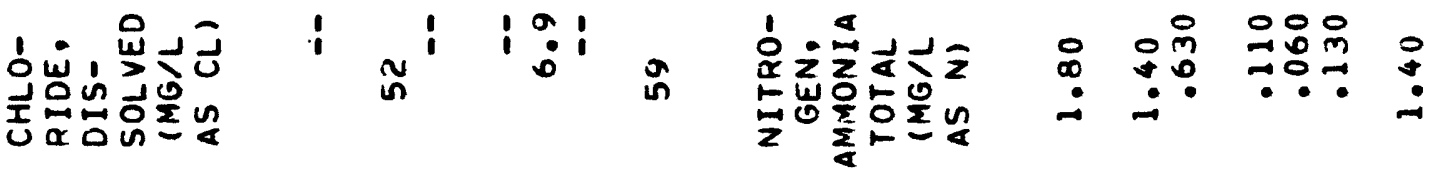

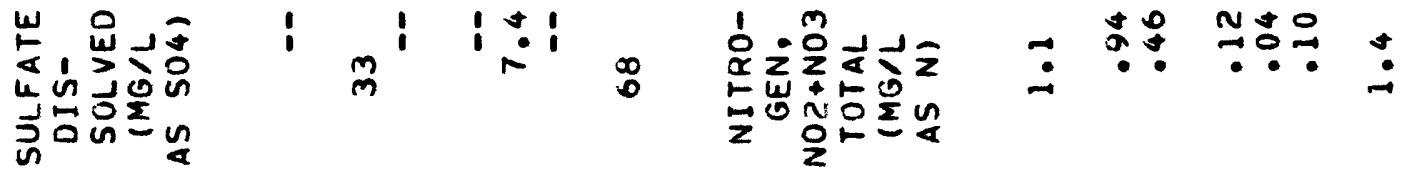

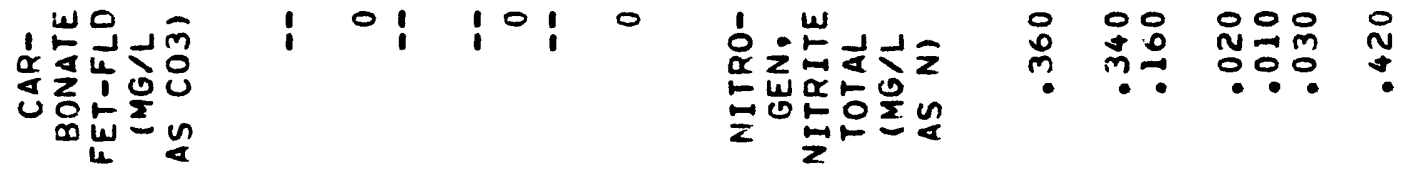

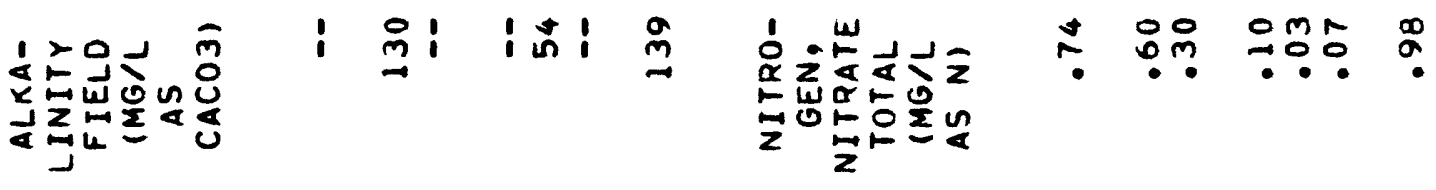

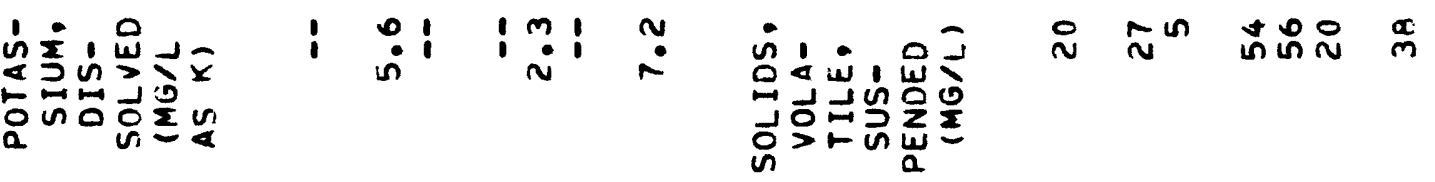

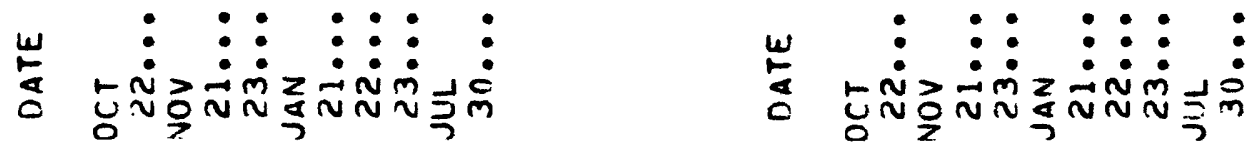




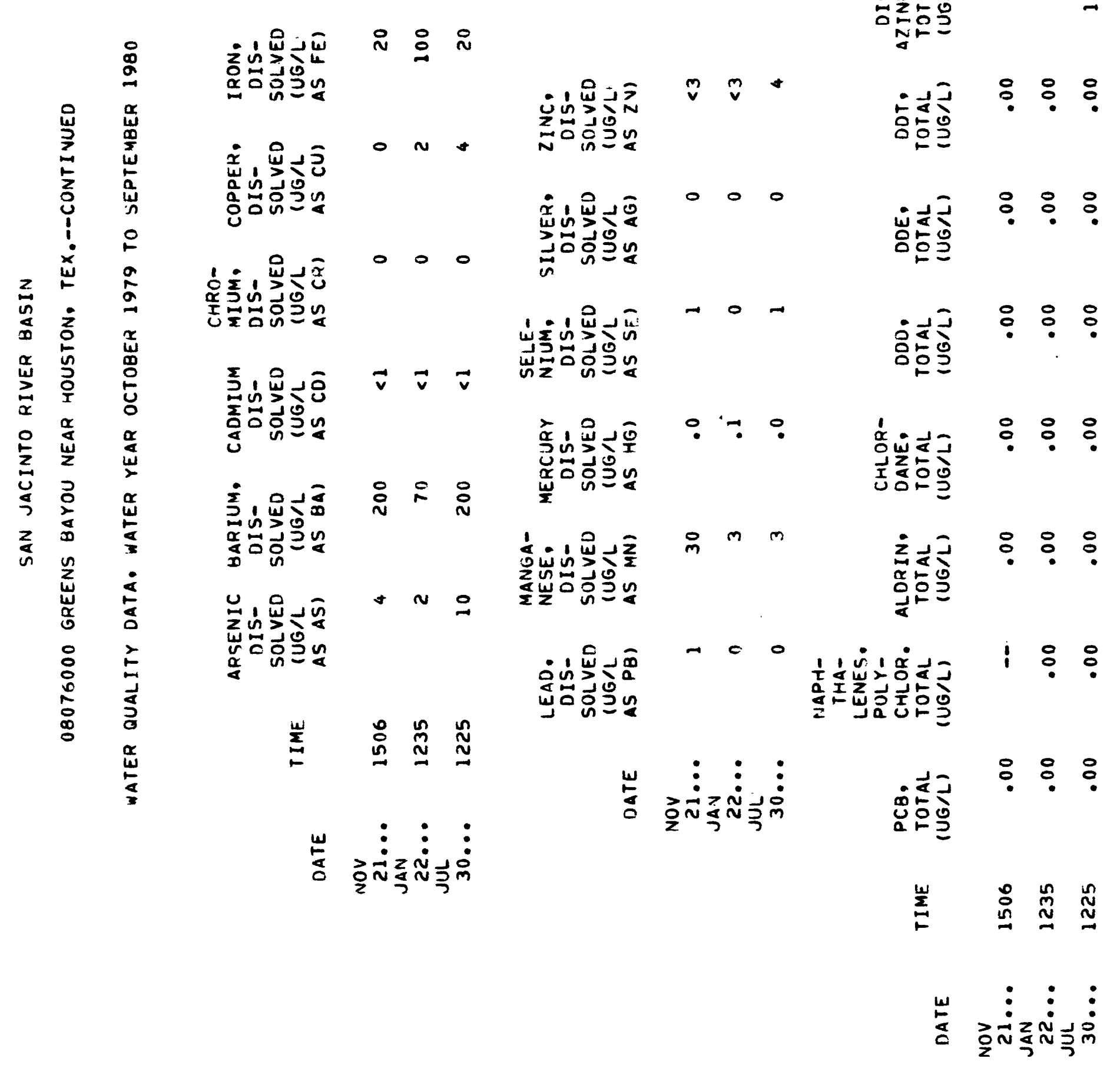




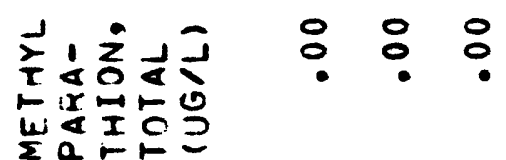

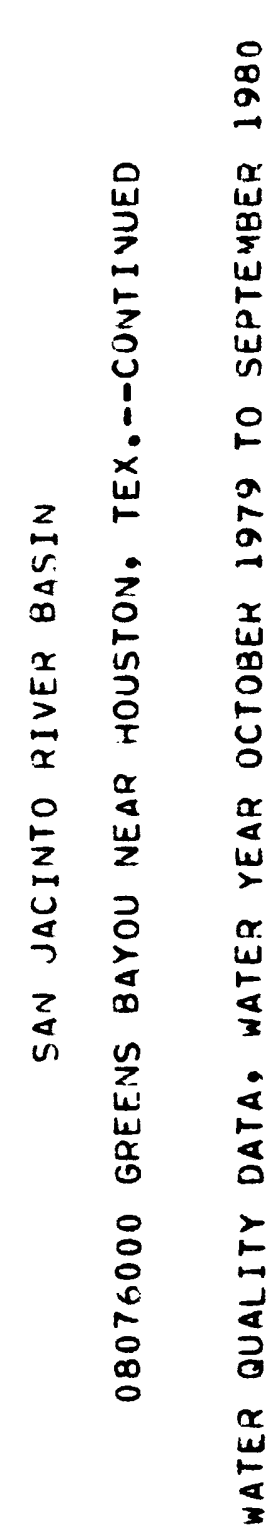

$\begin{array}{llll}1 & 0 & 0 & 0\end{array}$

ㅇㅇㅇ

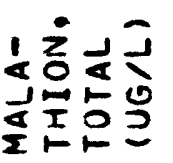

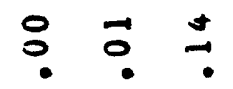
IID

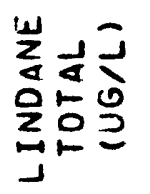

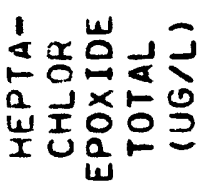

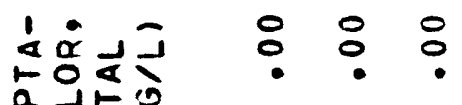
u $=0$

$$
\text { - } 0 \text { ? }
$$

000

00

$\tau \omega$

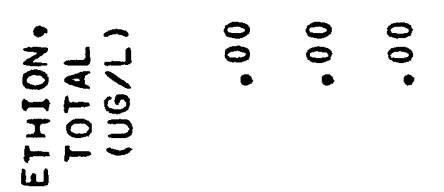

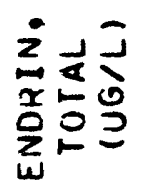

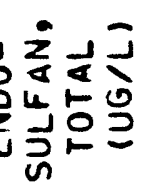

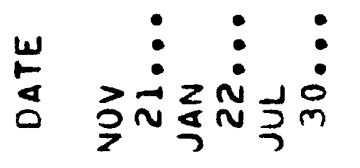

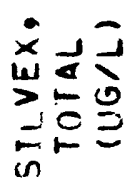

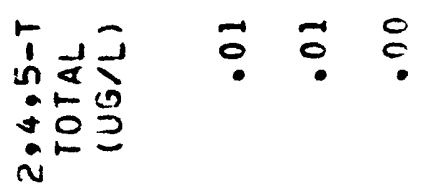

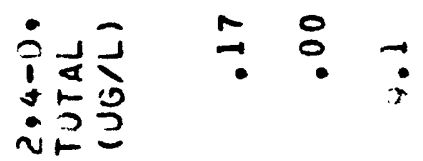

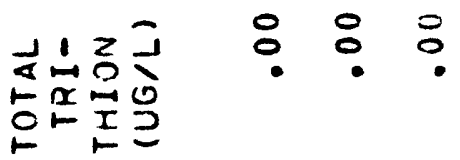

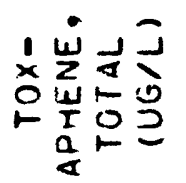

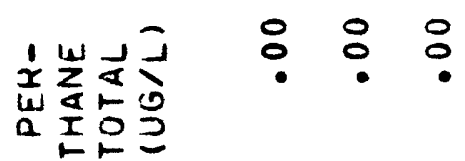

立牙 : : : 눙엉

aㄴㄷㄴ

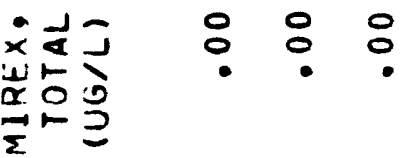

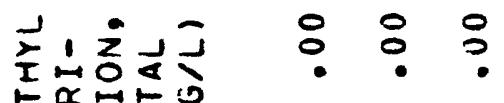

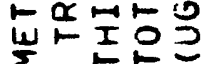

崖 


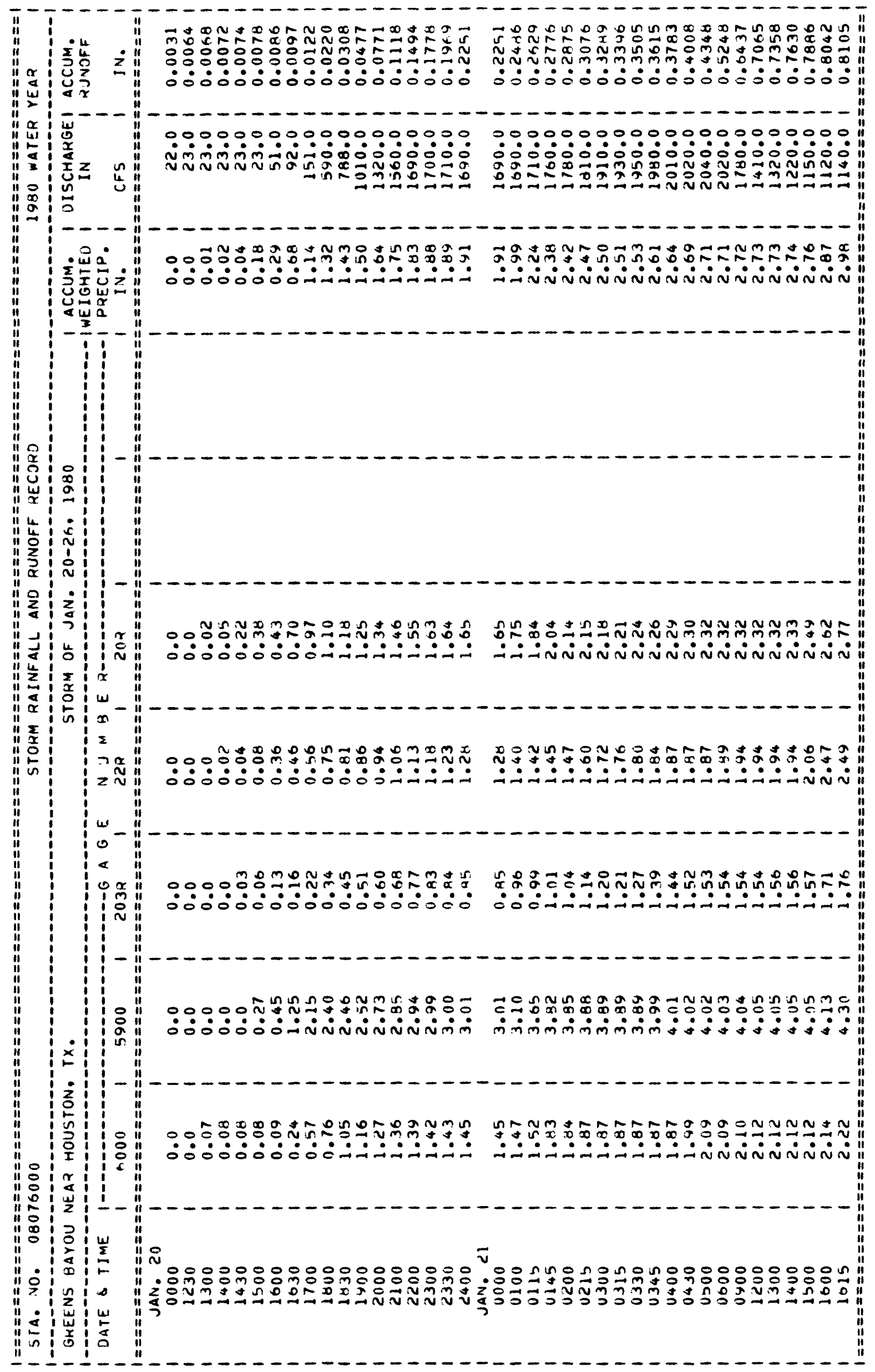

$-220-$ 


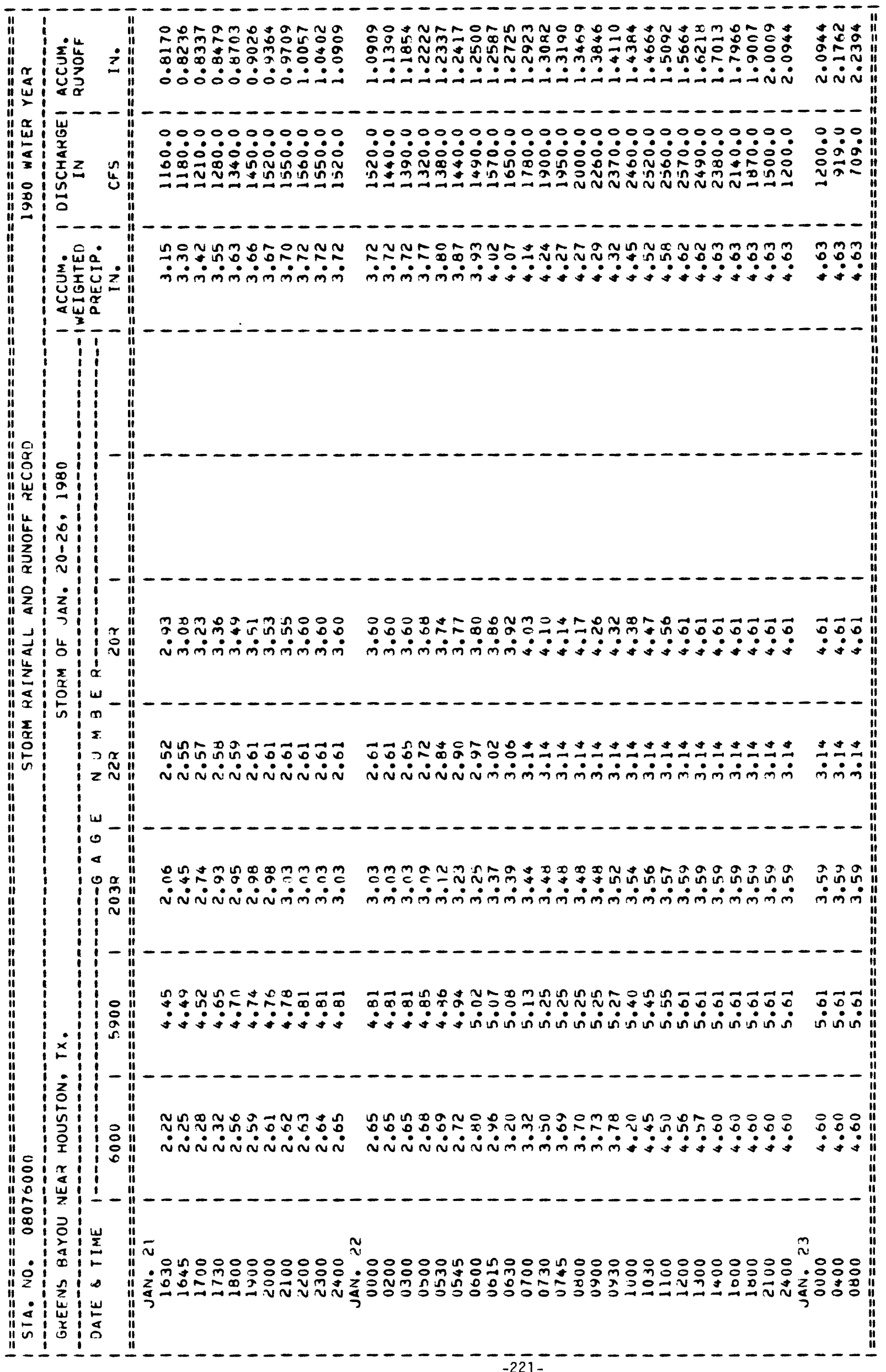




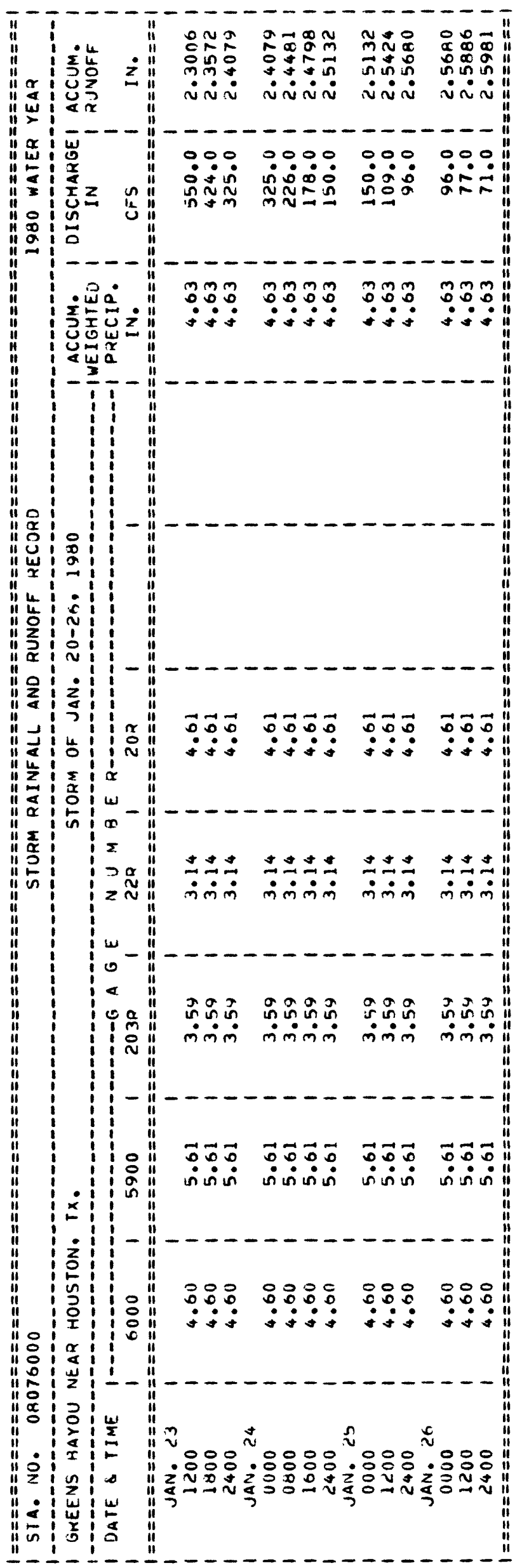




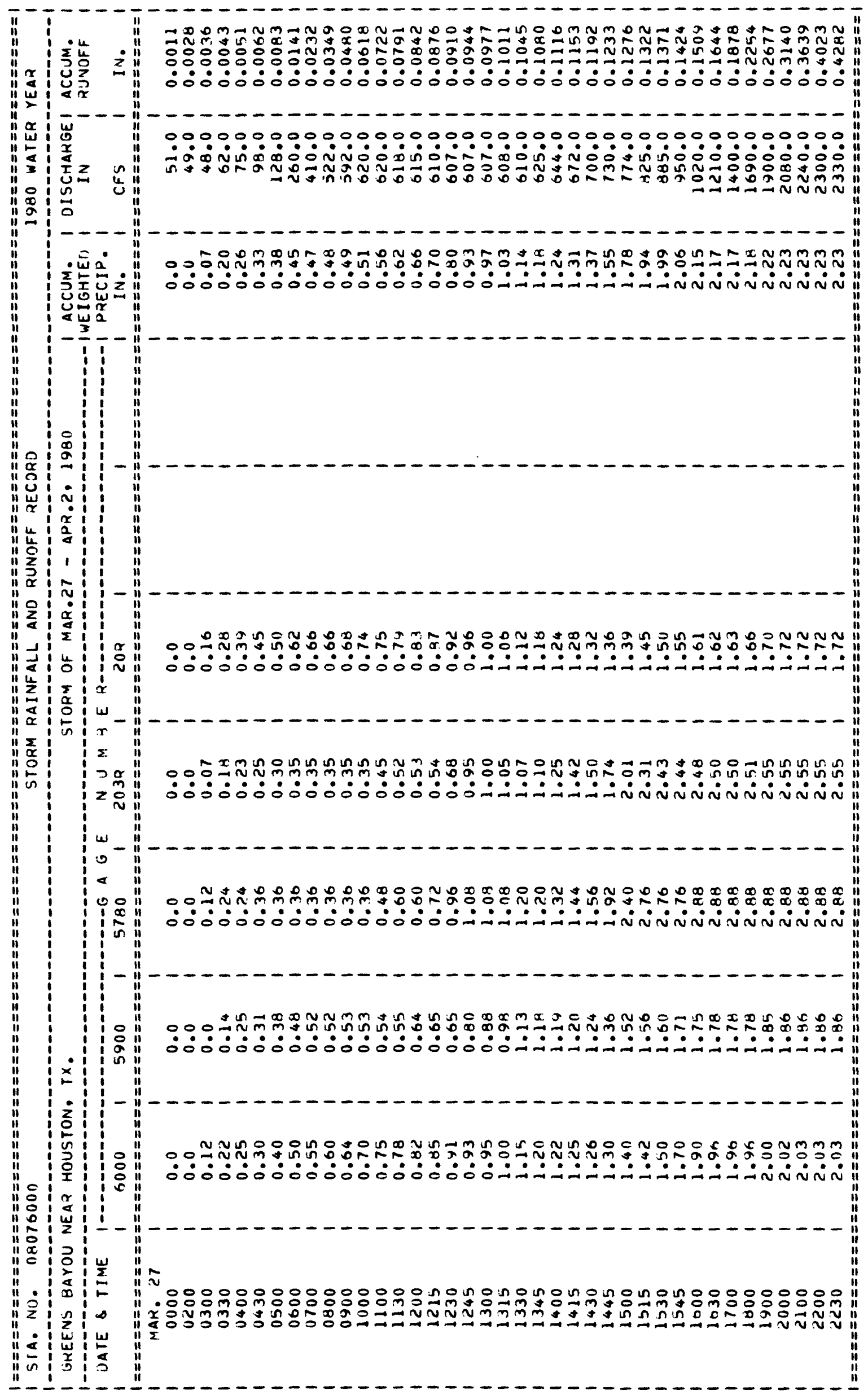




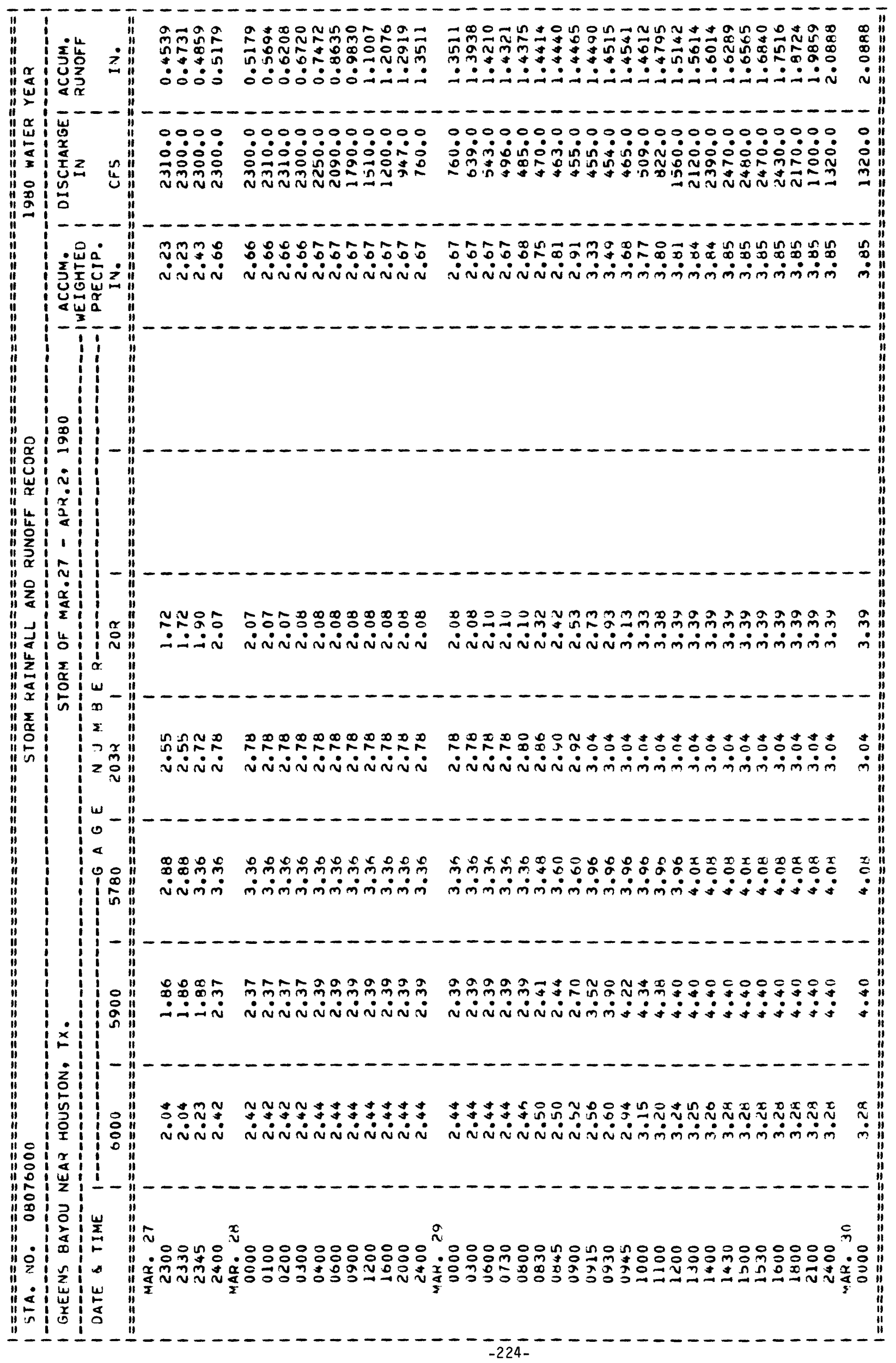




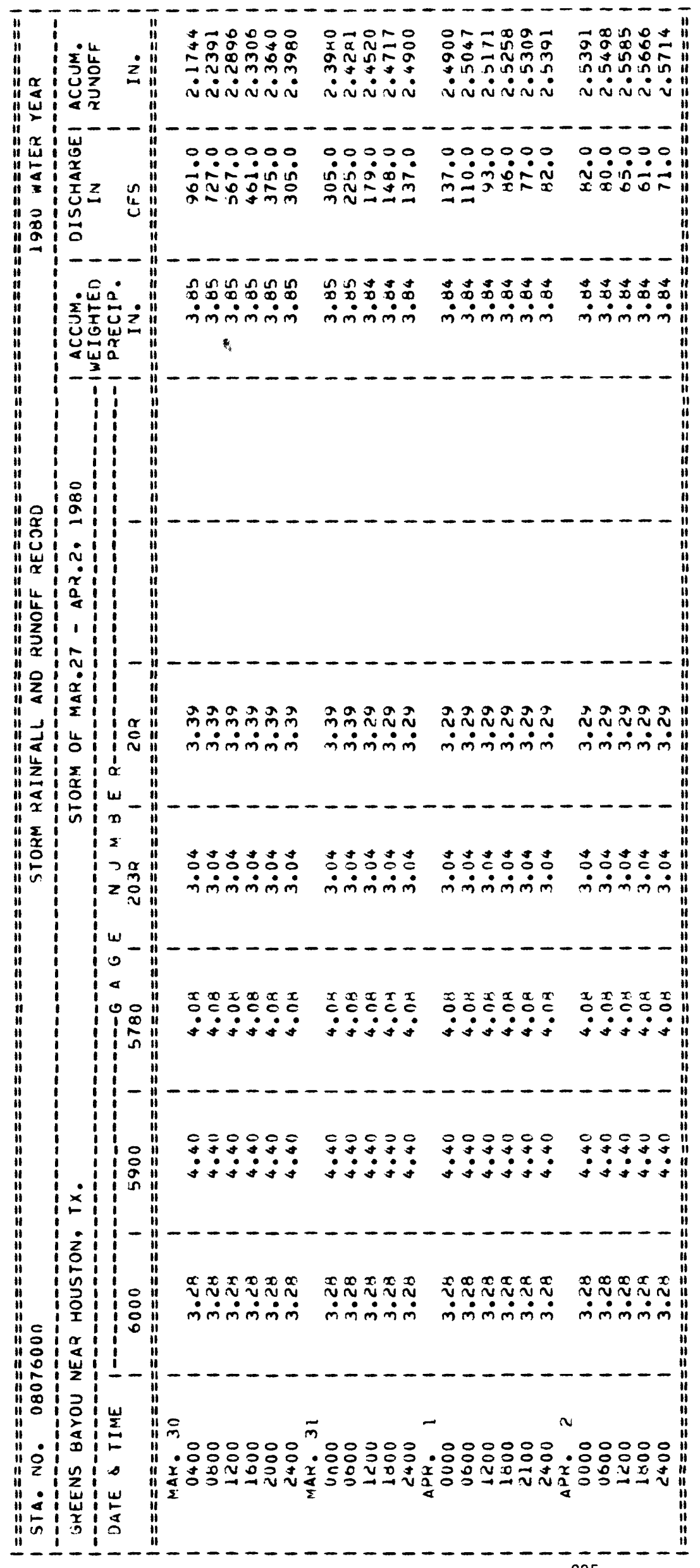




\section{HALLS BAYOU DRAINAGE BASIN}

The locations of data-collection sites in and near the Yalls Bayou drainage basin are shown in figure 20 .

Weighted-mean rainfall for the drainaqe basin, hased on five rain qages above the Jensen Drive station for the 1980 water year was 35.53 inches, or 12.66 inches less than the 30-year (1941-70) average of 48.19 inches for Houston.

The storms of Jan. 20-24, and Sept. 5-7 were selected for analysis at station 03076200, Halls Bayou at Deertrail Street near Houston. The storms of Nov. 11-24 and Jan. 20-24 were selected for analys is at station 08076500 , Halls Bayou at Houston (Jensen Drive). 


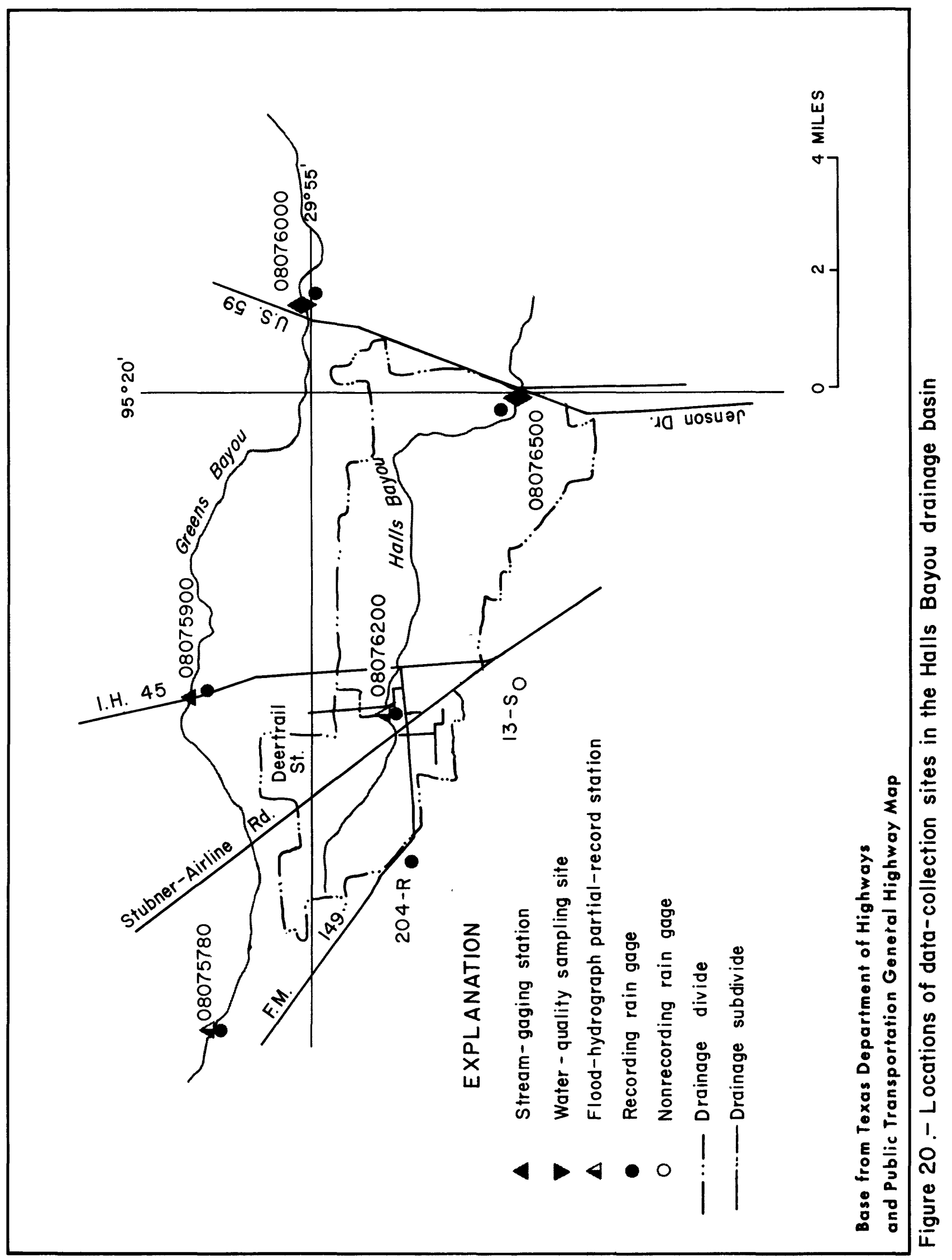




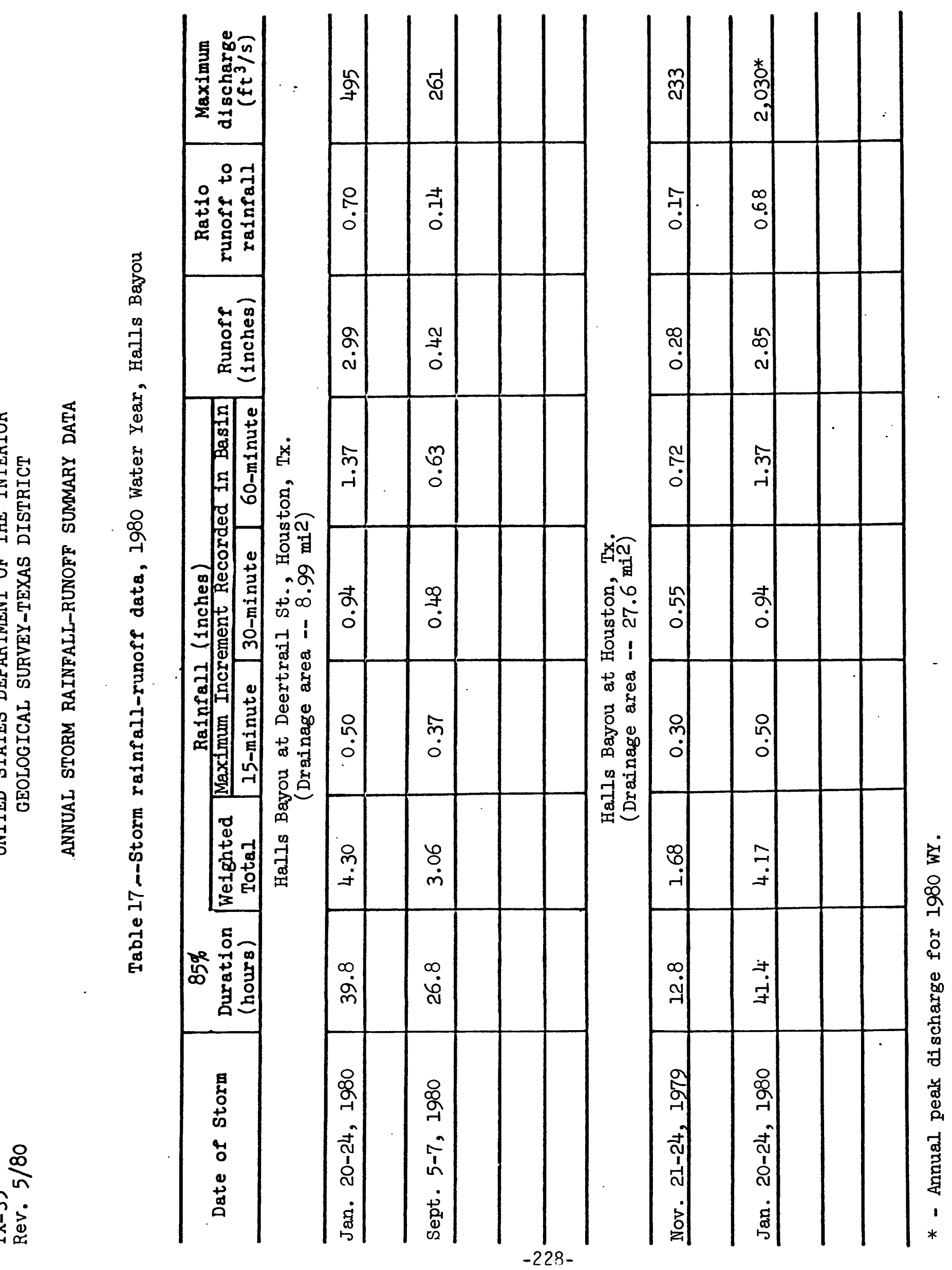




\section{HALLS BAYOU AT DEERTRAIL STREET NEAR HOUSTON, TEX. (Flood-hydrograph partial-record station)}

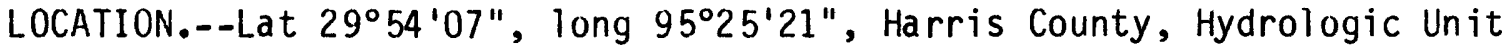
12040104 , at downstream side of bridge on Deertrail Street, 0.6 mile west of U.S. Highway $75,3.0$ miles north of city limits of Houston, and 7.7 miles upstream from station 08076500, Halls Bayou at Houston.

DRAINAGE AREA (revised).--8.99 $\mathrm{mi}^{2}$. For period 0ct. 1, 1973 to Sept. 30, $1977,8.69 \mathrm{mi}^{2}$. Prior to Oct. 1, 1973, $6.31 \mathrm{mi}^{2}$.

PERIOD OF RECORD.--Aug. 1964 to current year.

GAGE.--Digital flood-hydrograph and rainfall recorders and crest-stage gage. Prior to April 27, 1978 a flood-hydrograph and rainfall recorder (type SR) and crest-stage gage. Datum of gage is National Geodetic Vertical Datum of 1929, 1961 adjustment, unadjusted for land-surface subsidence.

REMARKS.--Records fair.

EXTREMES FOR PERIOD OF RECORD.--Maximum discharge, 1,180 $\mathrm{ft}^{3} / \mathrm{s}$, Mar. 20 , 1972; maximum gage height, $86.07 \mathrm{ft}$, April 18, 1976. Minimum not determined.

EXTREMES FOR CURRENT YEAR.--Peak discharge above base of $400 \mathrm{ft}^{3} / \mathrm{s}$ (revised) and maximum $(*)$ :

DATE TIME DISCHARGE

$(\mathrm{ft} 3 / \mathrm{s})$

Jan. 21

Mar. 27

Mar. 29
0300

1900

unknown
495

370

*649
ELEVATION

81.89

81.17

83.15

Minimum discharge not determined. 


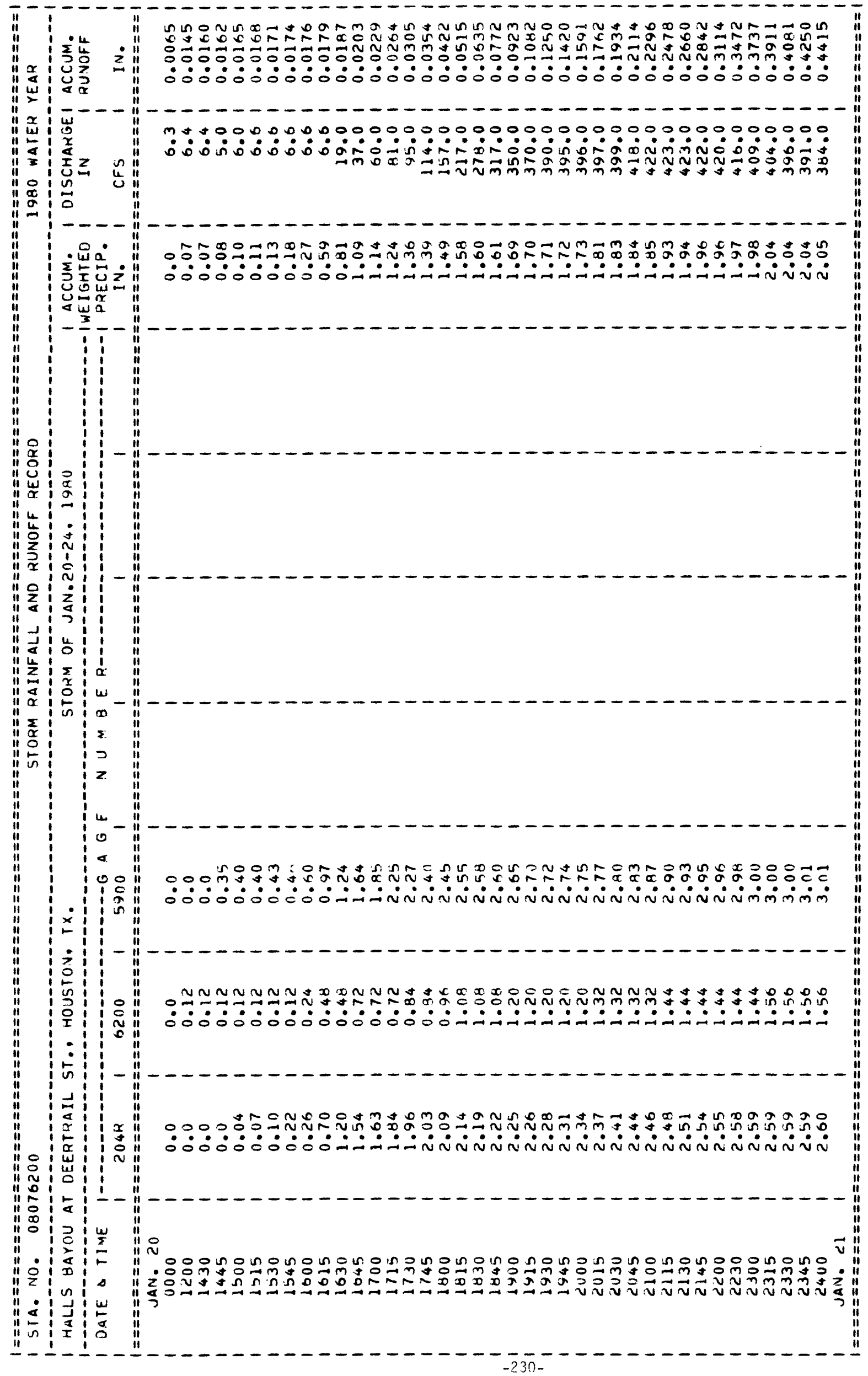




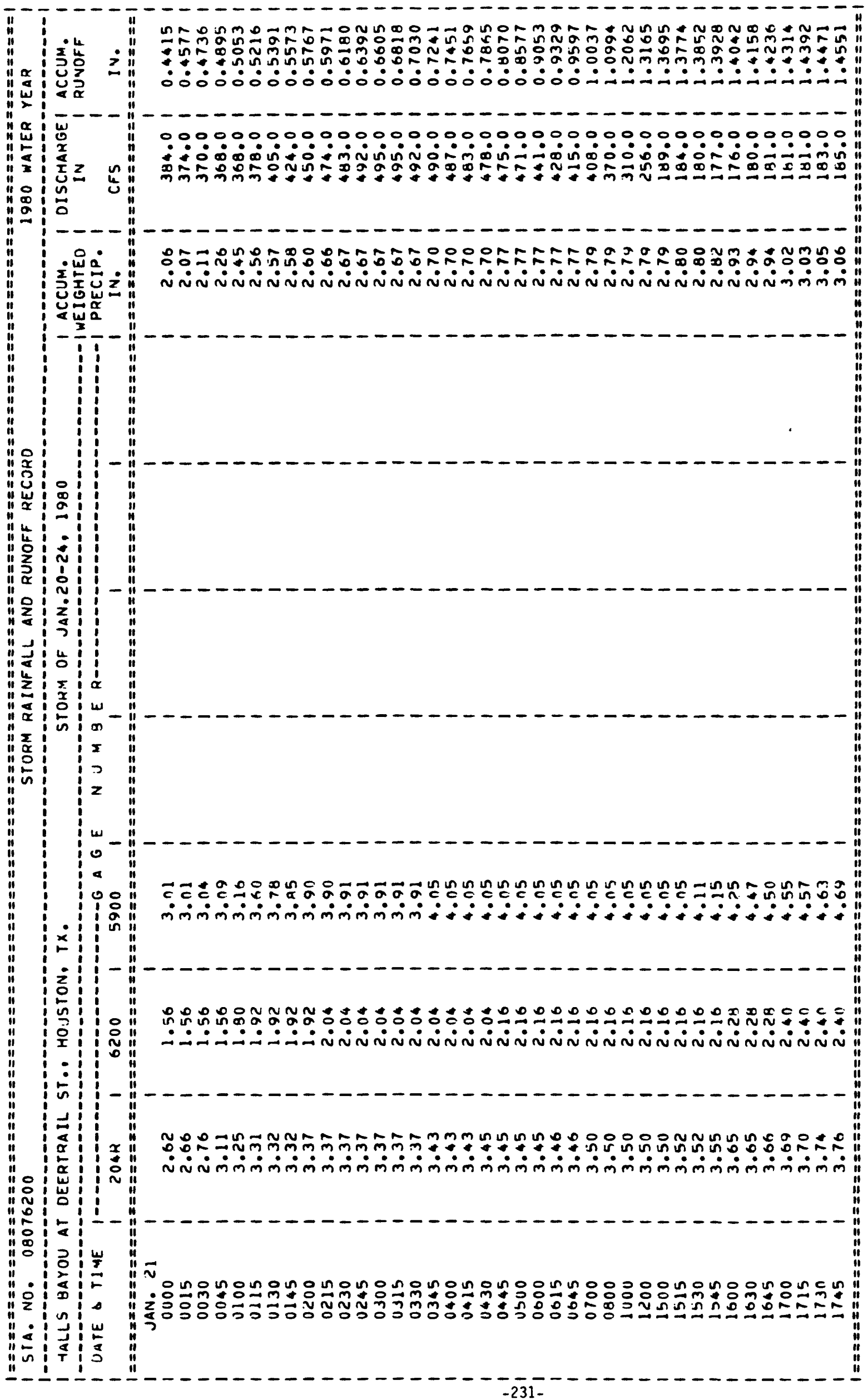




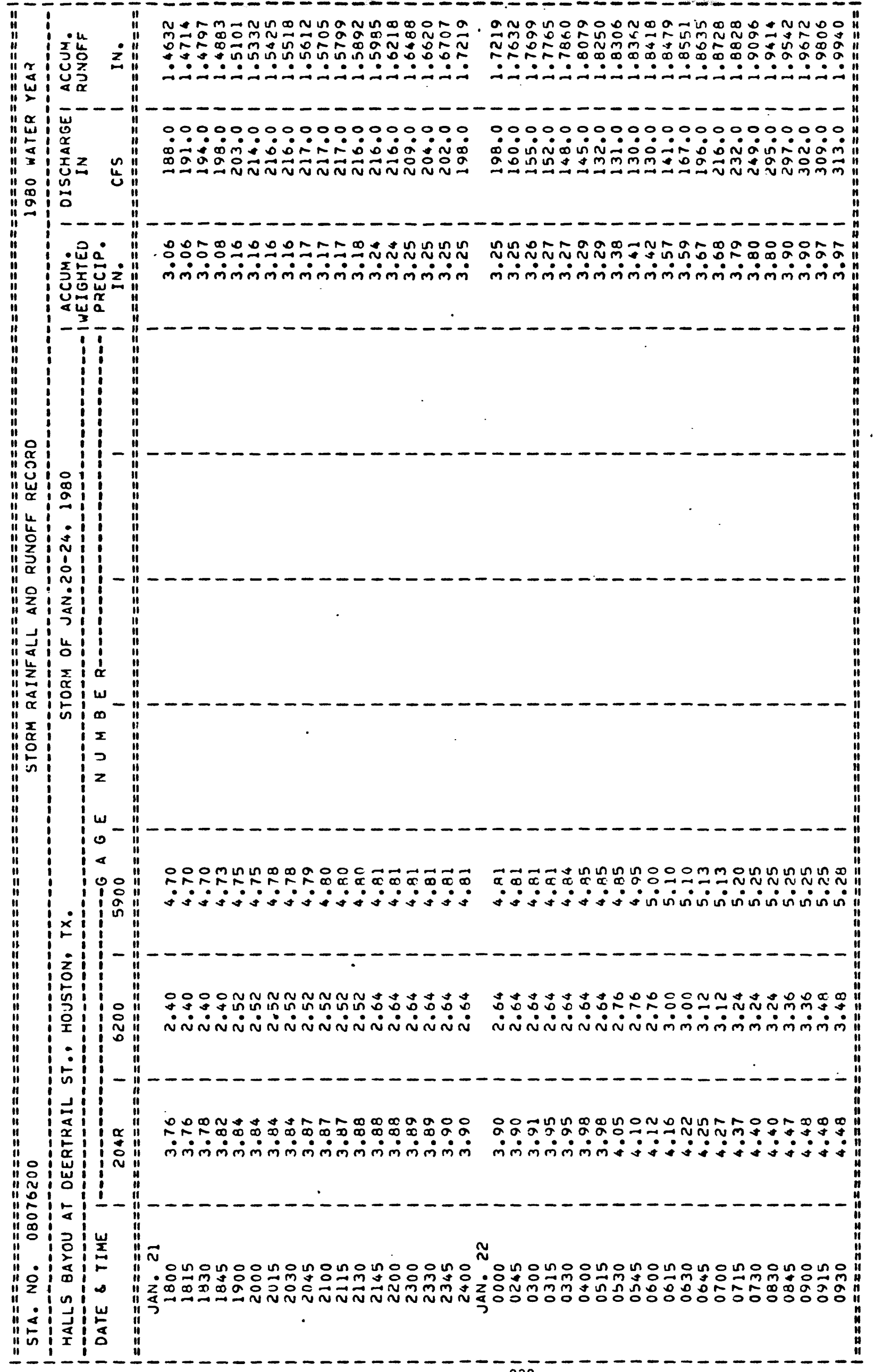




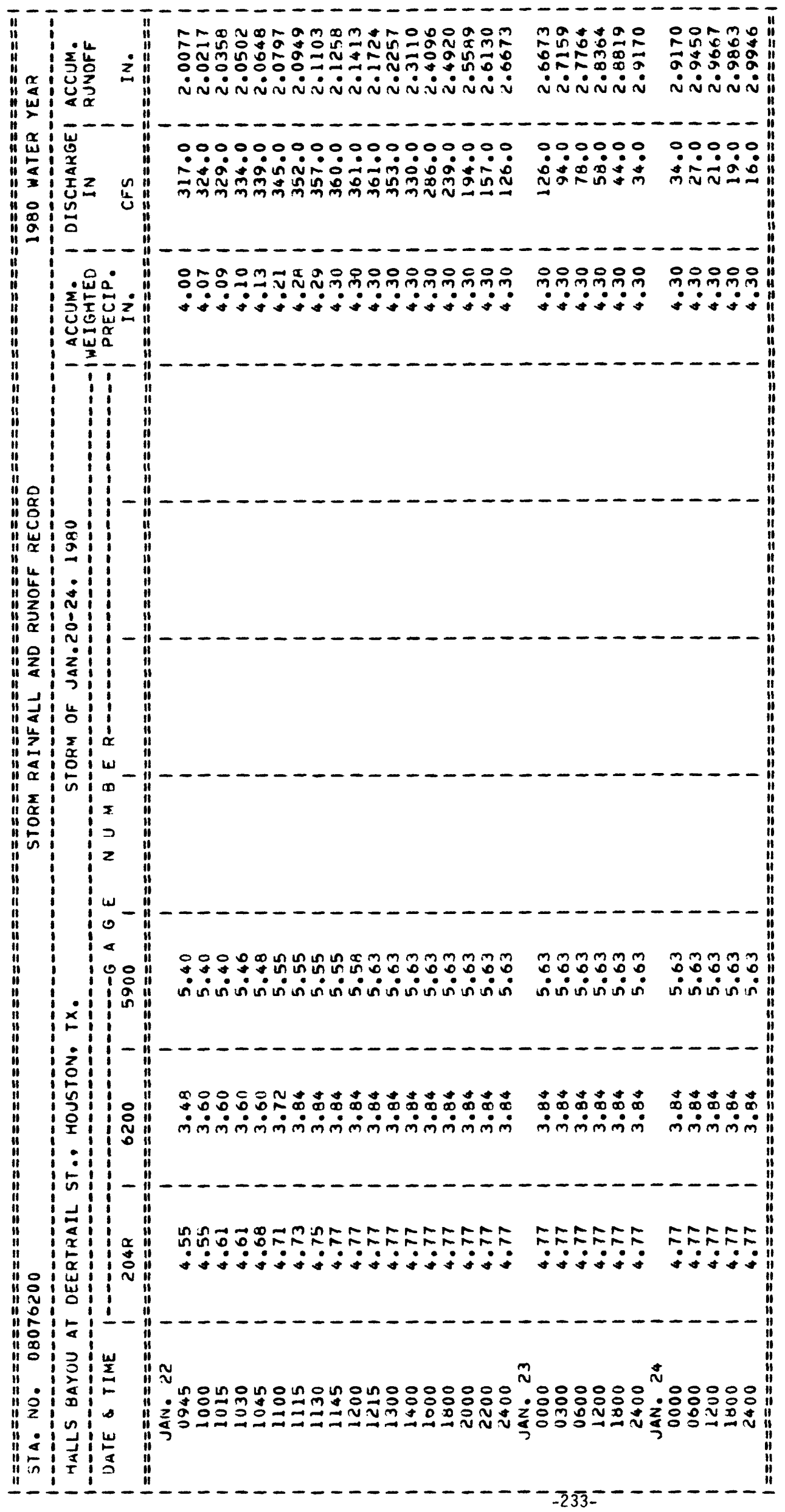




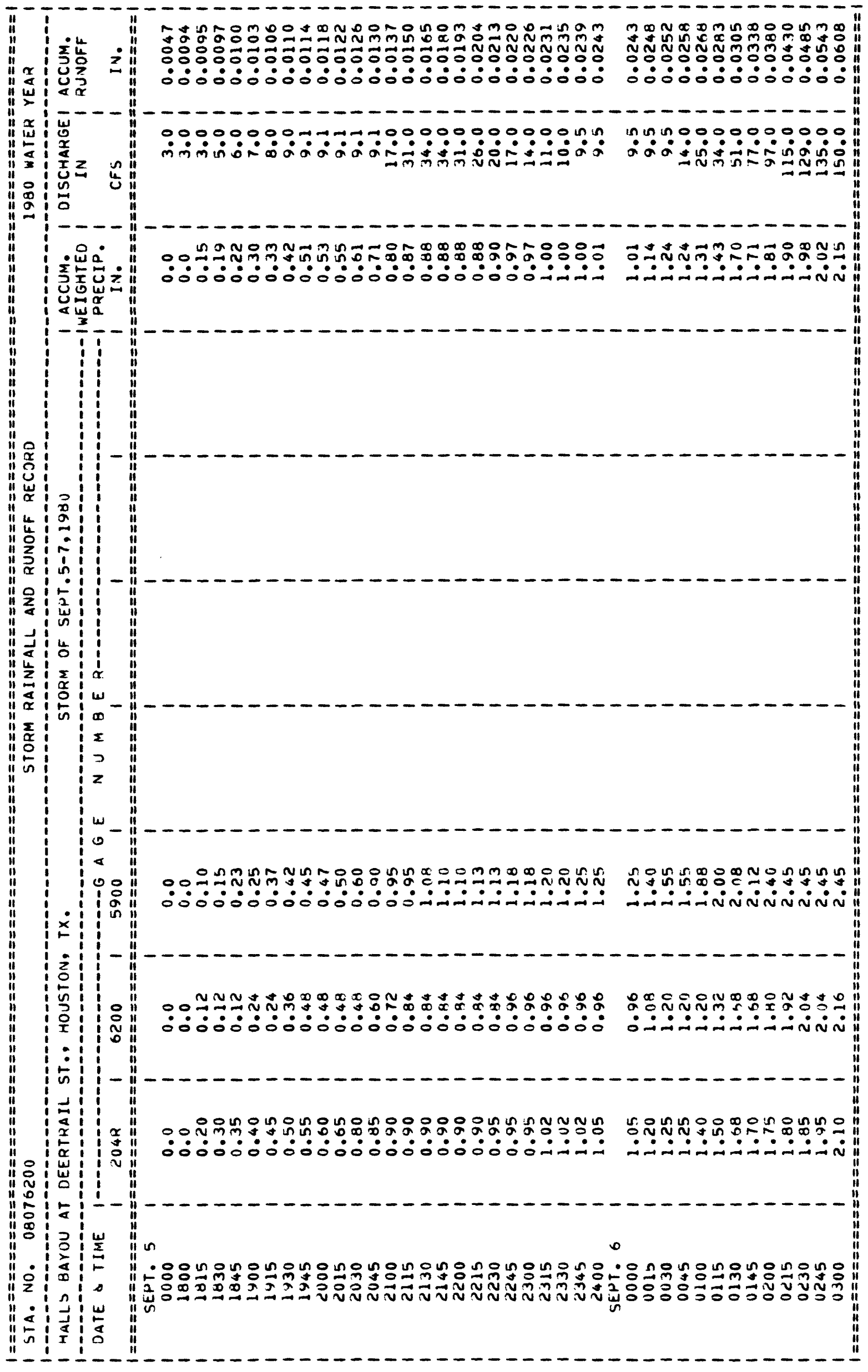

-234 - 


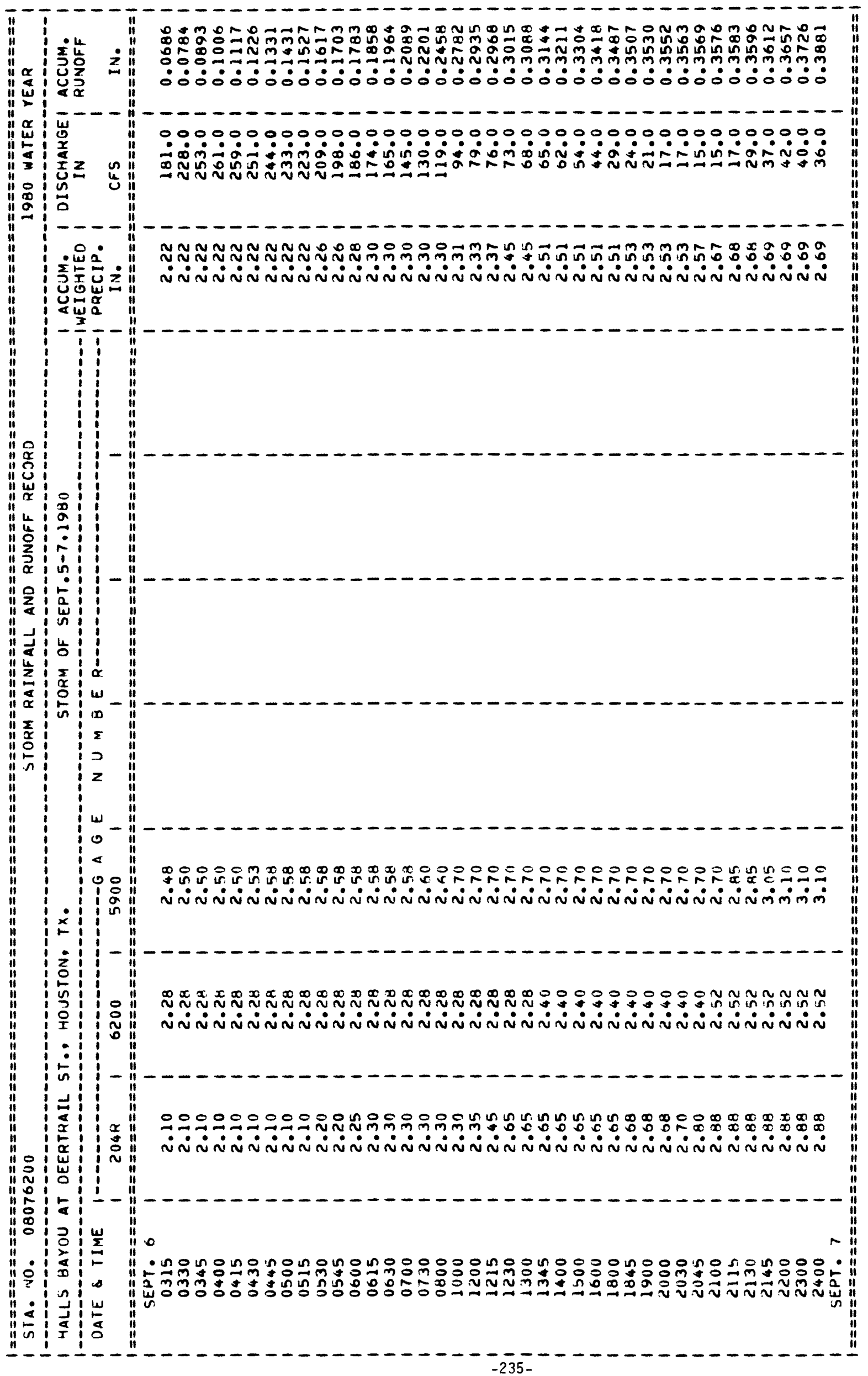




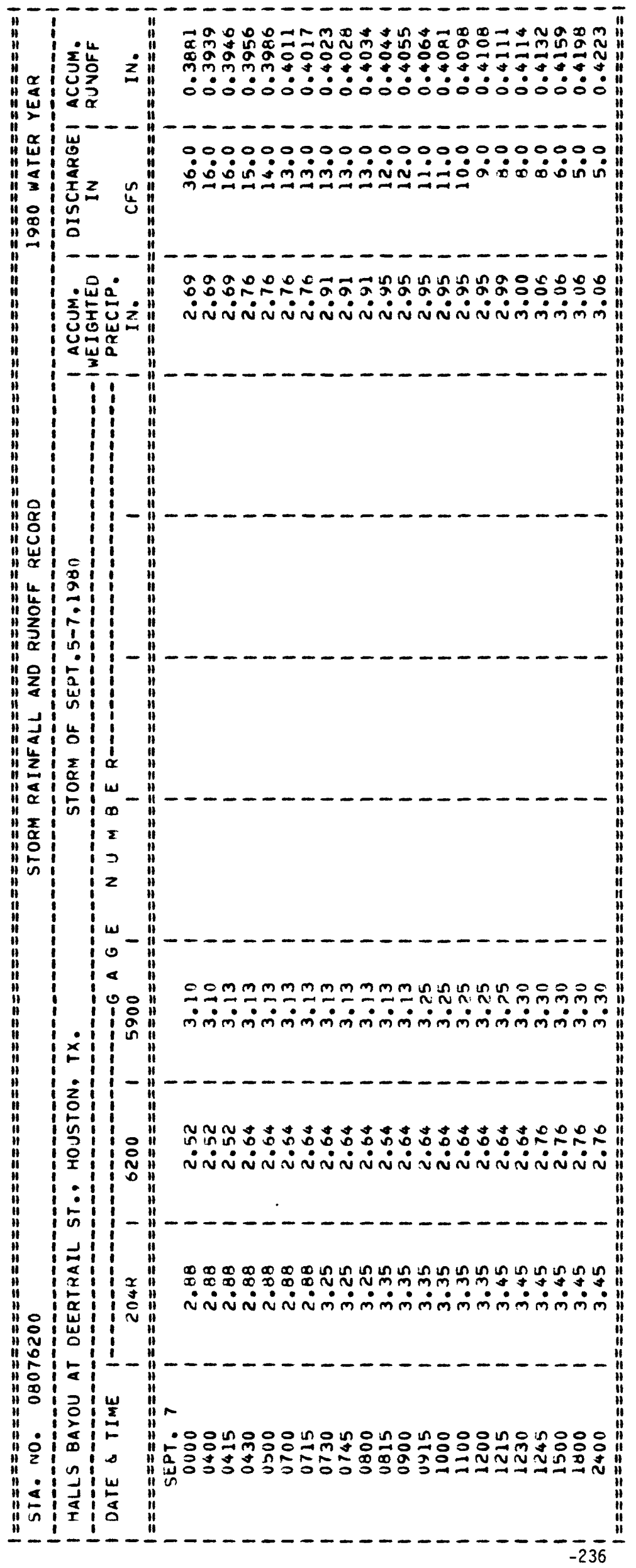


SAN JACINTO RIVER BASIN

08076500 HALLS BAYOU AT HOUSTON, TX

LOCATION.--Lat $29^{\circ} 51^{\prime} 42^{\prime \prime}$, long $95^{\circ} 20^{\prime} 05^{\prime \prime}$, Harris County, Hydrologic Unit 12040104, on right bank at downstream side of bridge on Jensen Drive in northeast section of Houston and $11.0 \mathrm{mi}$ (17.7 km) upstream from mouth.

DRAINAGE AREA. $-27.6 \mathrm{mi}^{2}\left(71.5 \mathrm{~km}^{2}\right)$. Oct. 1, 1973, to Sept. 30, 1977, $28.3 \mathrm{mi}^{2}$ (73.3 $\left.\mathrm{km}^{2}\right)$. Prior to Oct. 1 , 1973, $24.7 \mathrm{mi}^{2}\left(64.0 \mathrm{~km}^{2}\right)$. Changes were result of drainage ditch extensions or relocations.

WATER-DISCHARGE RECORDS

PERIOD OF RECORD.--October 1952 to current year.

REVISED RECORDS.--WSP 1732: Drainage area. WDR TX-76-2: Drainage area.

GAGE.--Water-stage recorder. Datum of gage is $0.66 \mathrm{ft}(0.201 \mathrm{~m})$ below National Geodetic Vertical Datum of 1929 , 1957 adfustment; unadjusted for land-surface subsidence.

REMARKS.--Water-discharge records fair except those for April, May, and September, which are poor. No known diversion above station. Low flow is sustained by sewage effluent from Houston suburbs.

AVERAGE DISCHARGE.--28 years, $27.4 \mathrm{ft}^{3} / \mathrm{s}\left(0.776 \mathrm{~m}^{3} / \mathrm{s}\right), 19.850 \mathrm{acre}-\mathrm{ft} / \mathrm{yr}\left(24.5 \mathrm{hm}^{3}\right)$.

EXTRDMES FOR PERIOD OF RECORD.--Maximum discharge, 3,780 $\mathrm{ft}^{3} / \mathrm{s}\left(107 \mathrm{~m}^{3} / \mathrm{s}\right)$ Mar. 21, 1972, gage height, $60.70 \mathrm{ft}$

$(18.501 \mathrm{~m})$; maximum gage height, $60.75 \mathrm{ft}(18.517 \mathrm{~m})$ June 13, 1973; no flow at times prior to 1956.

EXTREMES FOR CURRENT YEAR.--Peak discharges above base of $1,200 \mathrm{ft}^{3} / \mathrm{s}\left(34.0 \mathrm{~m}^{3} / \mathrm{s}\right)$ and maximum ( $(*)$ :

\begin{tabular}{|c|c|c|c|c|c|c|c|c|c|c|c|}
\hline Date & Time & $\begin{array}{l}\text { Disch } \\
\left(\mathrm{ft}^{3} / \mathrm{s}\right)\end{array}$ & $\begin{array}{l}\text { rge } \\
\left(\mathrm{m}^{3} / \mathrm{s}\right)\end{array}$ & $\begin{array}{l}\text { Gage } \\
(\mathrm{ft})\end{array}$ & $\begin{array}{r}\text { height } \\
\text { (m) }\end{array}$ & Date & Time & $\begin{array}{r}\text { Discl } \\
\left(\mathrm{ft}^{3} / \mathrm{s}\right)\end{array}$ & $\begin{array}{l}\operatorname{rgge} \\
\left(\mathrm{m}^{3} / \mathrm{s}\right)\end{array}$ & $\begin{array}{l}\text { Cage } \\
(f t)\end{array}$ & $\begin{array}{r}\text { helght } \\
\text { (m) }\end{array}$ \\
\hline $\begin{array}{ll}\text { aOct. } 22 \\
\text { aNov. } 21 \\
\text { aJan. } 21\end{array}$ & $\begin{array}{c}\text { unknown } \\
2030 \\
0630\end{array}$ & $\begin{array}{r}60 \\
233 \\
1.060\end{array}$ & $\begin{array}{l}1.70 \\
6.60 \\
30.0\end{array}$ & $\begin{array}{c}\text { unknown } \\
49.50 \\
54.72\end{array}$ & $\begin{array}{l}15.088 \\
16.679\end{array}$ & $\begin{array}{r}\text { aJan. } 22 \\
\text { Mar. } 27\end{array}$ & $\begin{array}{l}1300 \\
2000\end{array}$ & $\begin{array}{r}* 2,030 \\
1,280\end{array}$ & $\begin{array}{l}57.5 \\
36.2\end{array}$ & $\begin{array}{l}57.79 \\
56.06\end{array}$ & $\begin{array}{l}17.614 \\
17.087\end{array}$ \\
\hline
\end{tabular}

a Water-quality samples were obtained during this runoff event.

Minimum dally discharge, $6.4 \mathrm{ft}^{3} / \mathrm{s}\left(0.18 \mathrm{~m}^{3} / \mathrm{s}\right)$ Aug. 25 .

DISCHARGE, IN CUBIC FEET PER SECOND, WATER YEAR OCTOBER 1979 TO SEPTEMBER 1980 MEAN VALUES

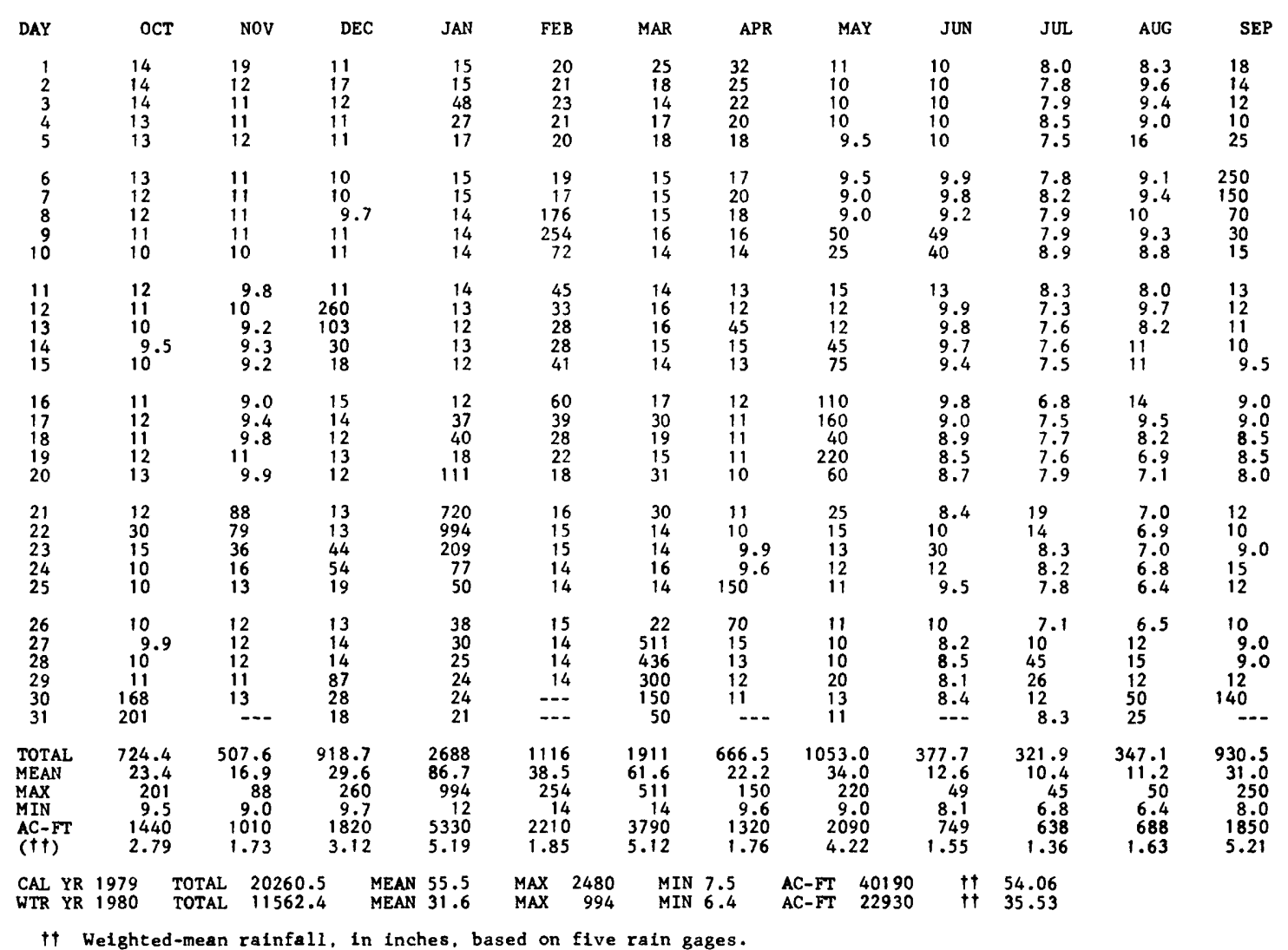




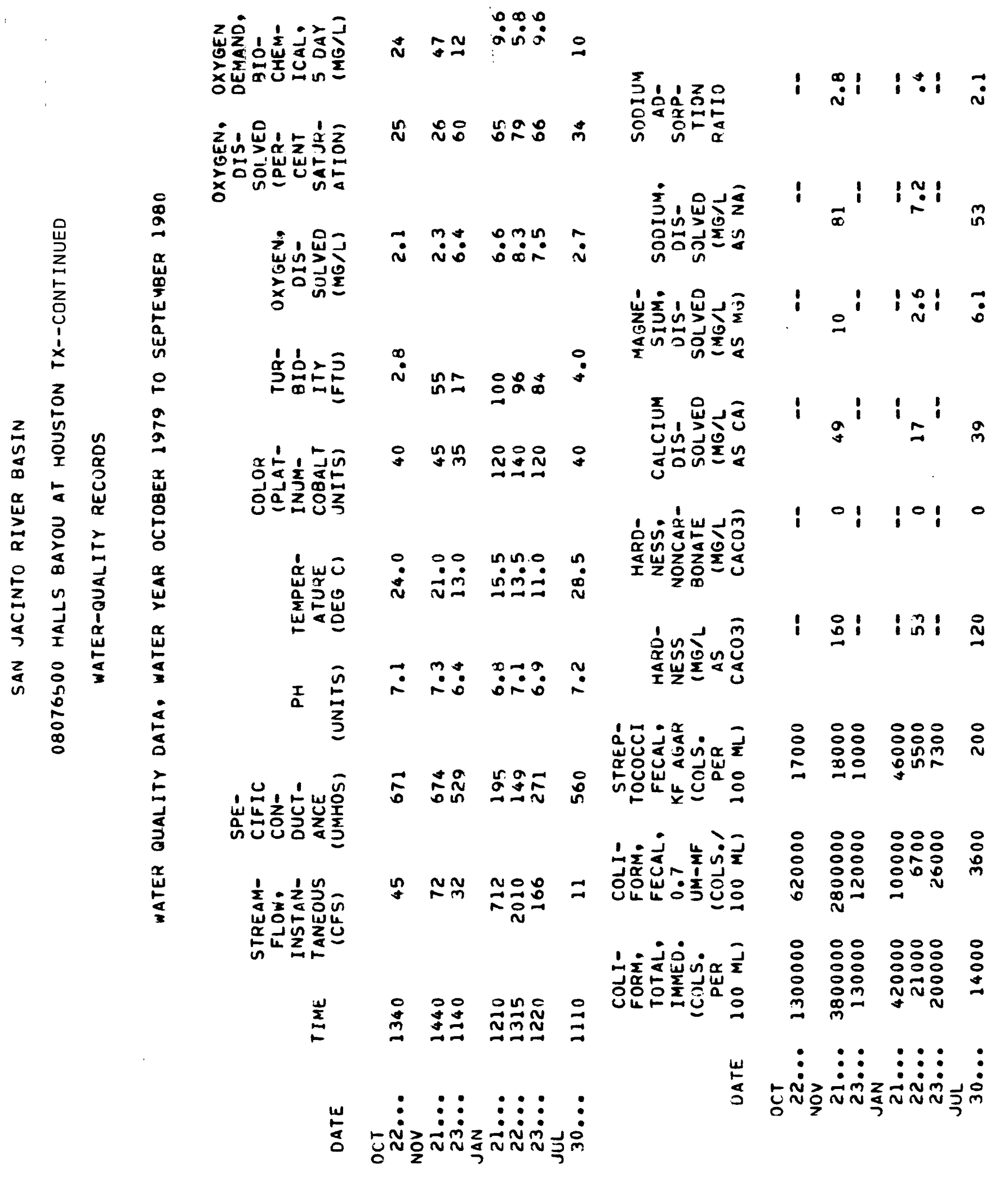




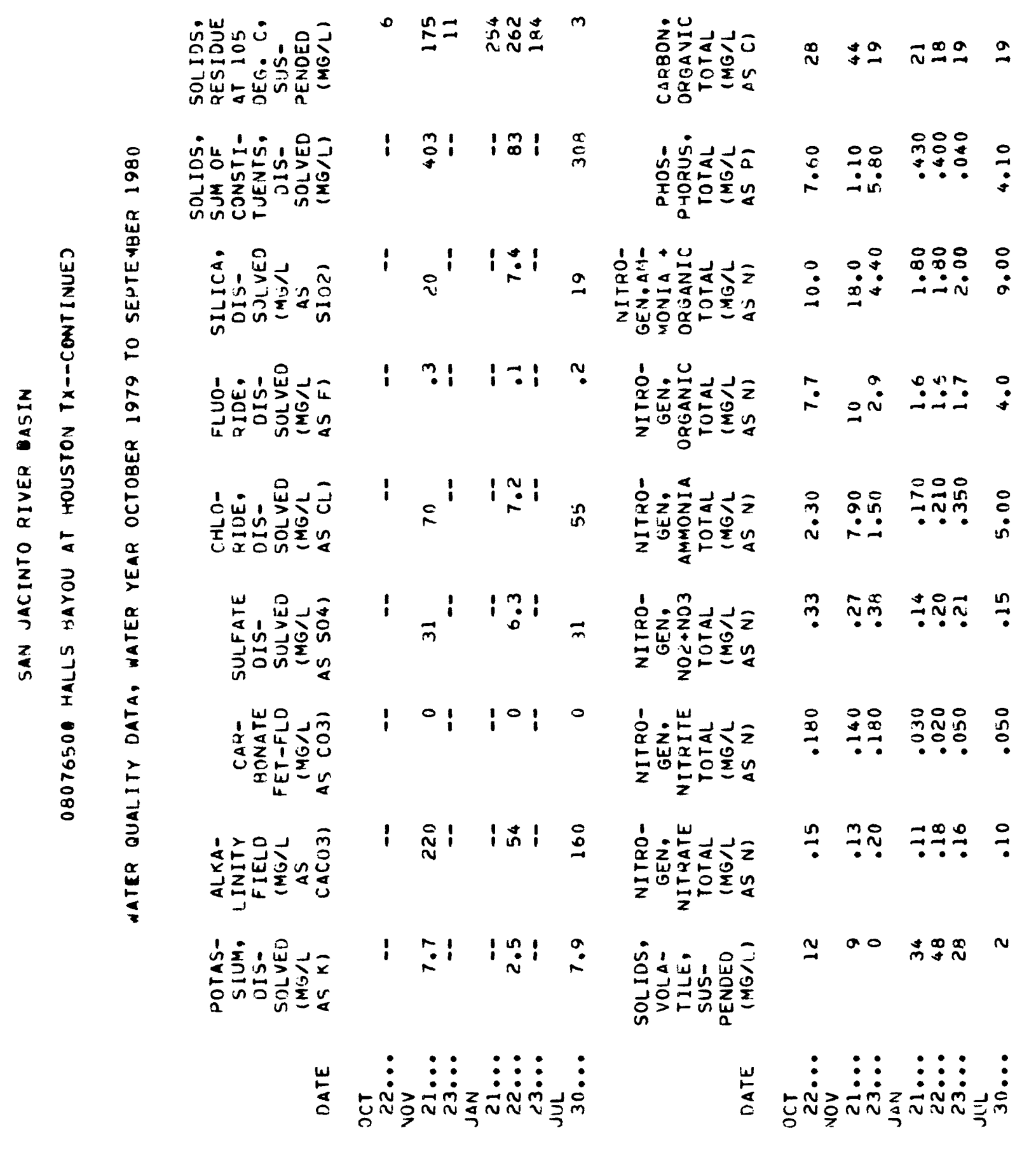




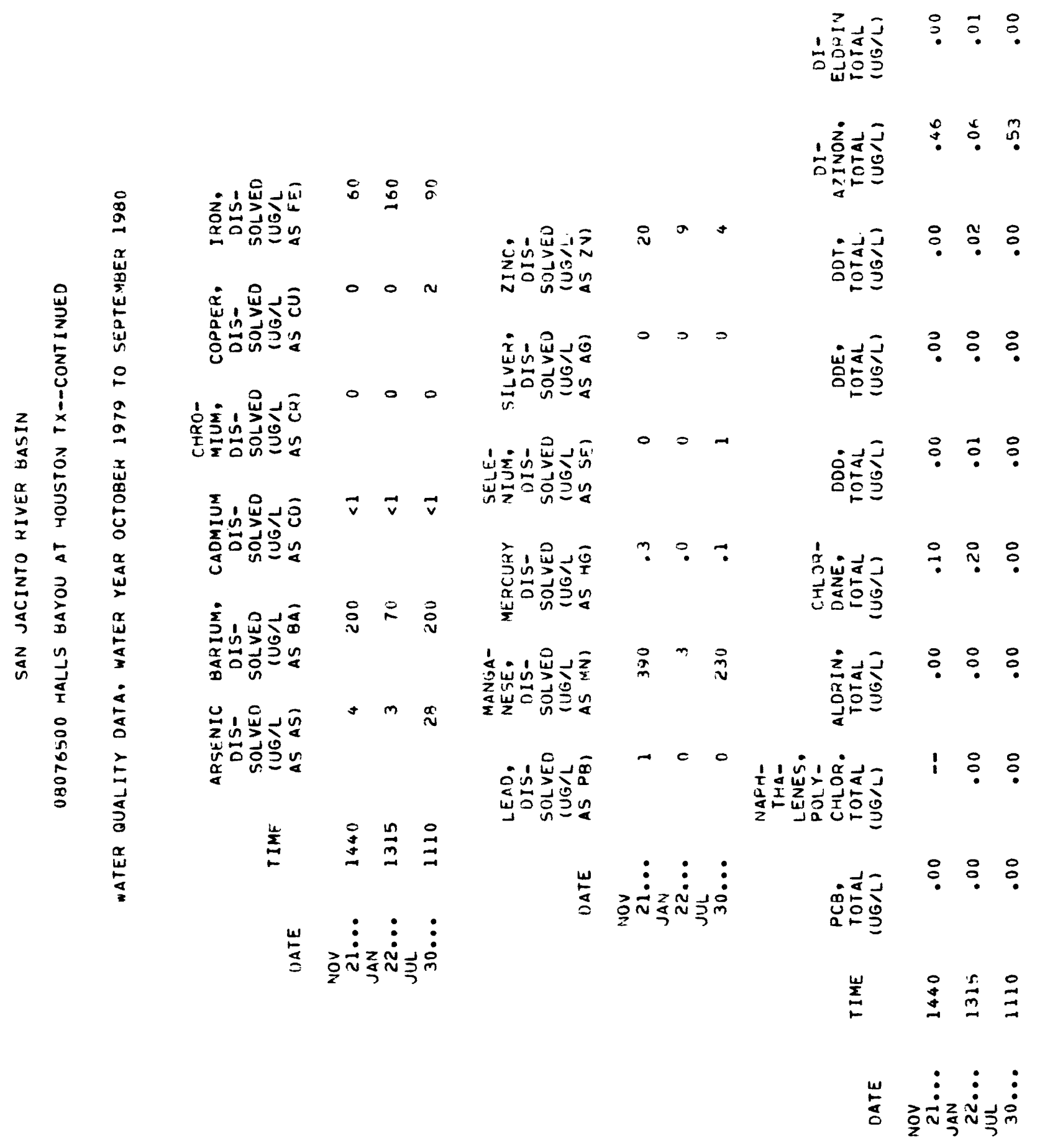




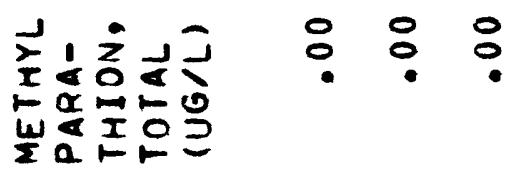

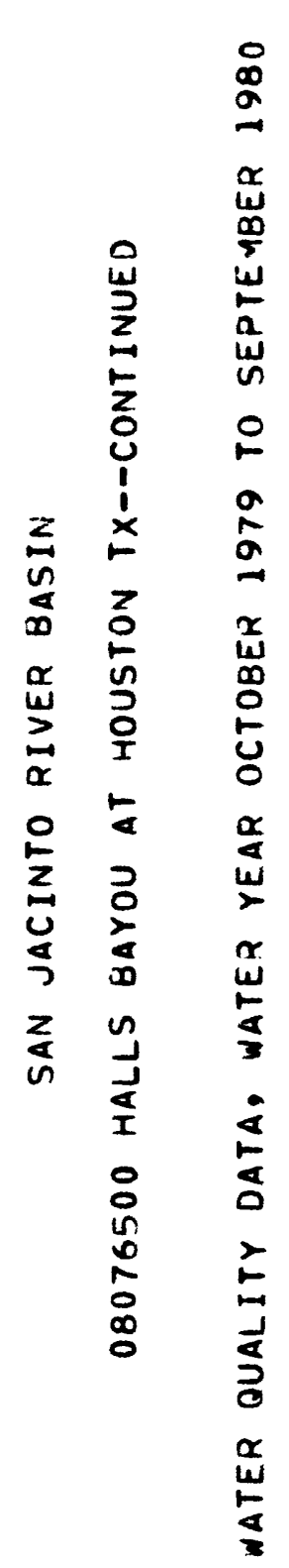

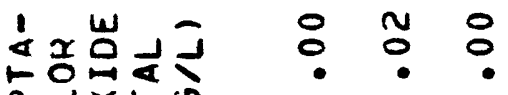

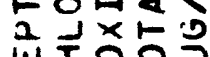

똥은?

in

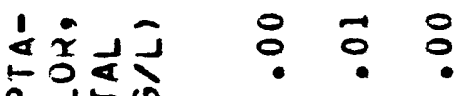

a

岌矛은?

낭엉

I윰ำ

00

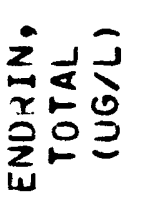

요

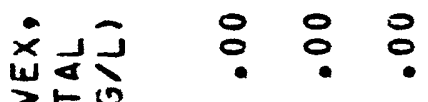

元它包 ที่

$\div 0 ?$ 家家

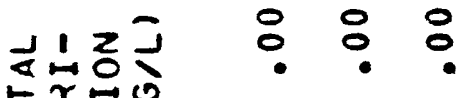

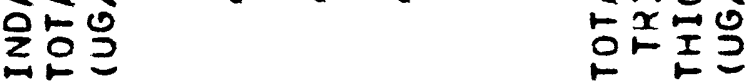

000

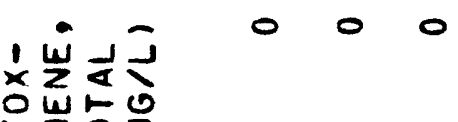

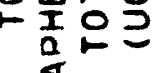

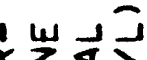

यूट⿺辶

a

$\therefore: 0$

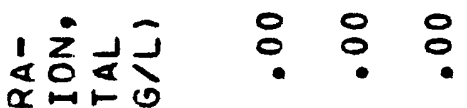
붕옹

도은

$\therefore \frac{1}{4}=$

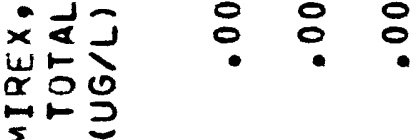

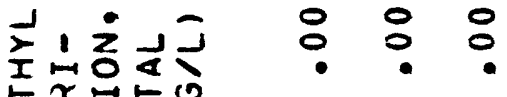

넌도은

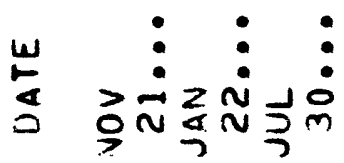




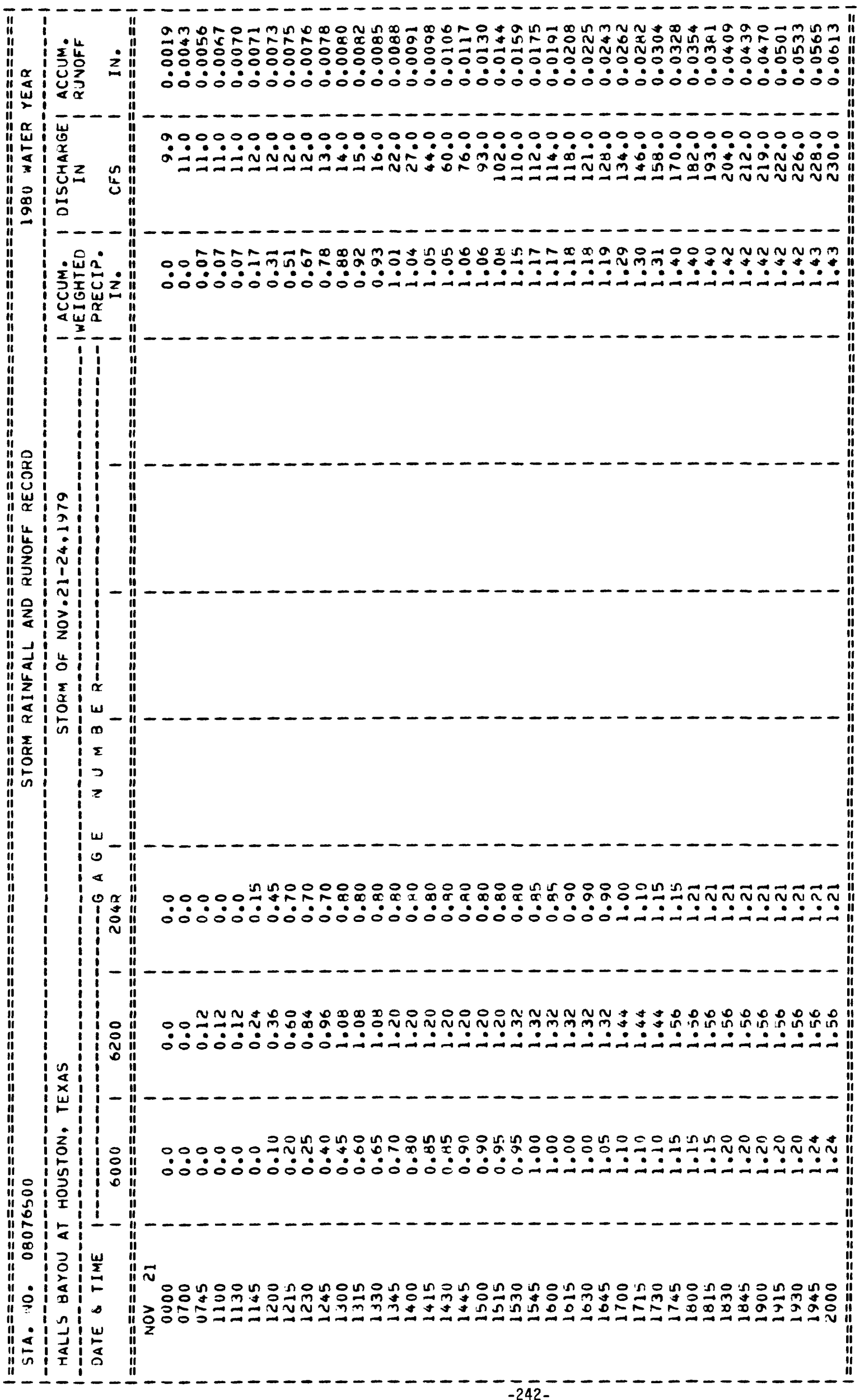




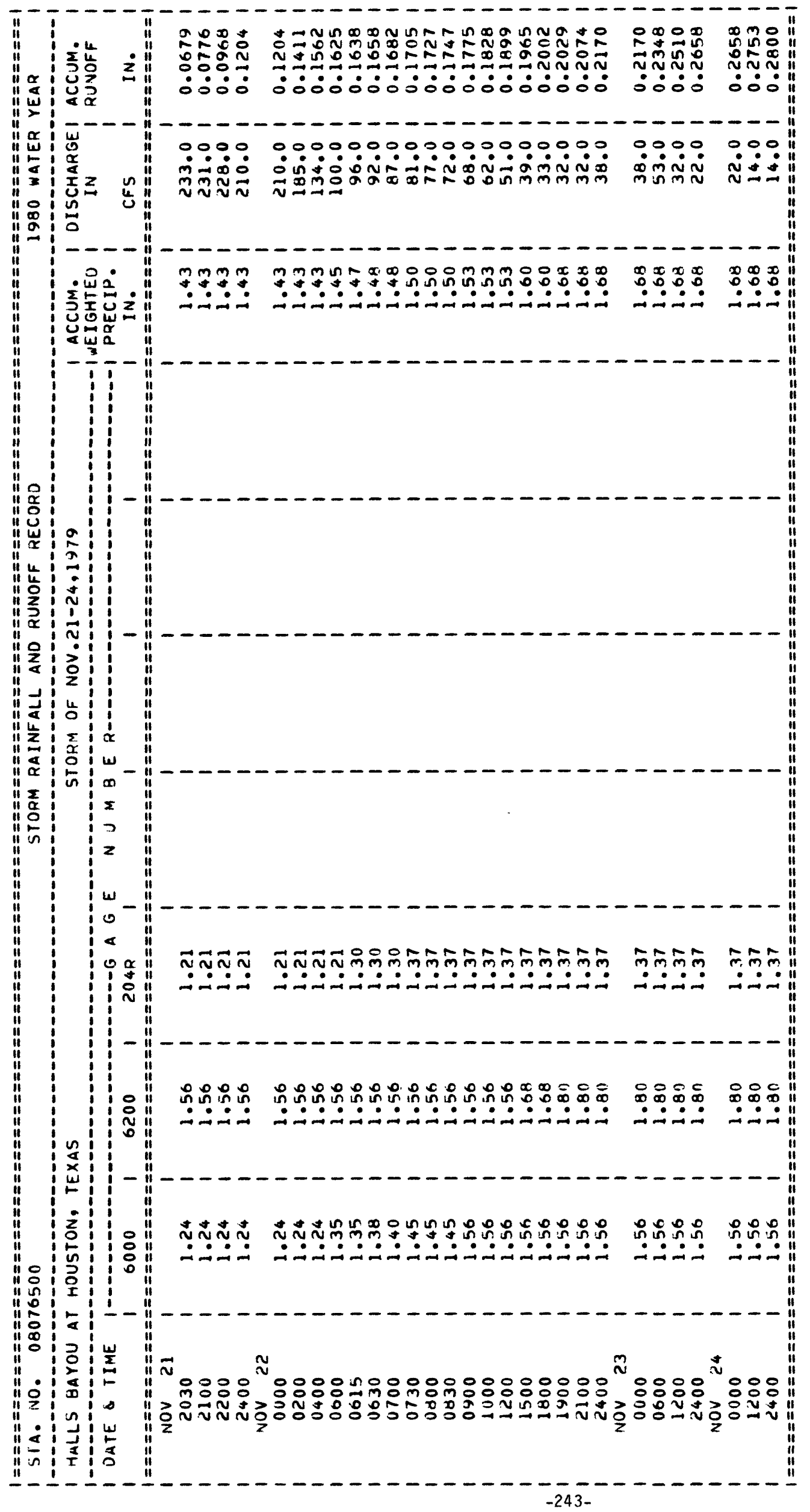




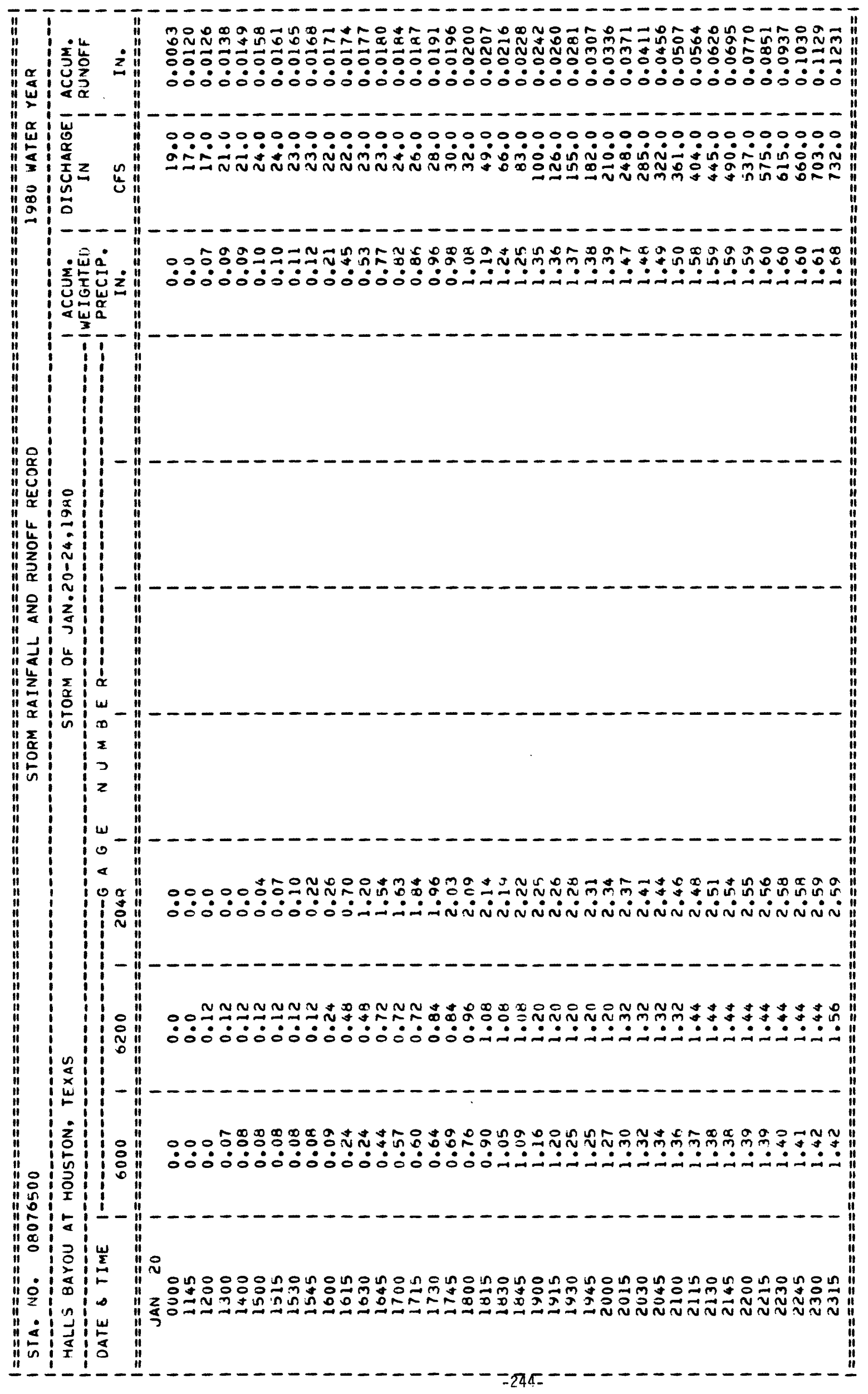




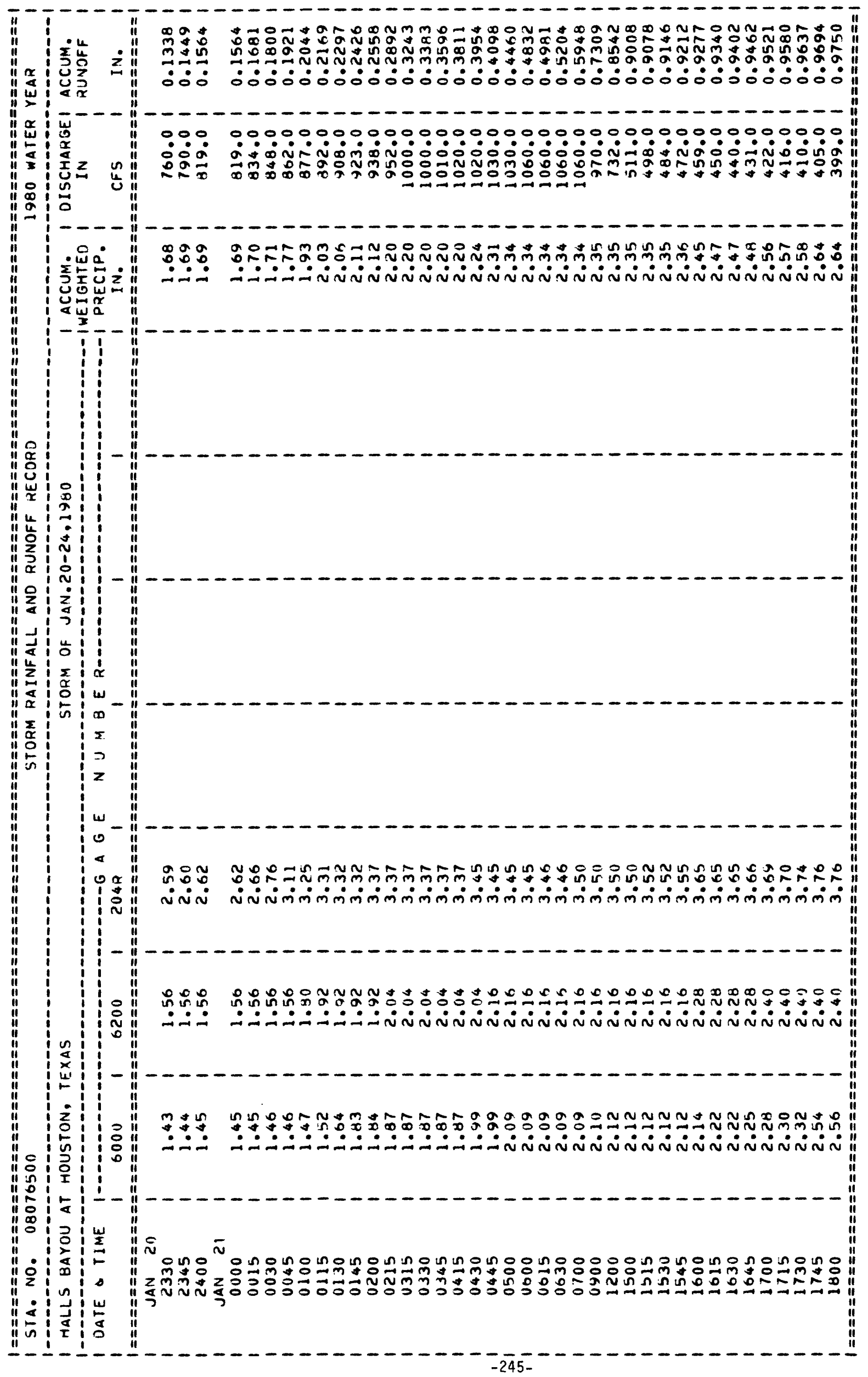




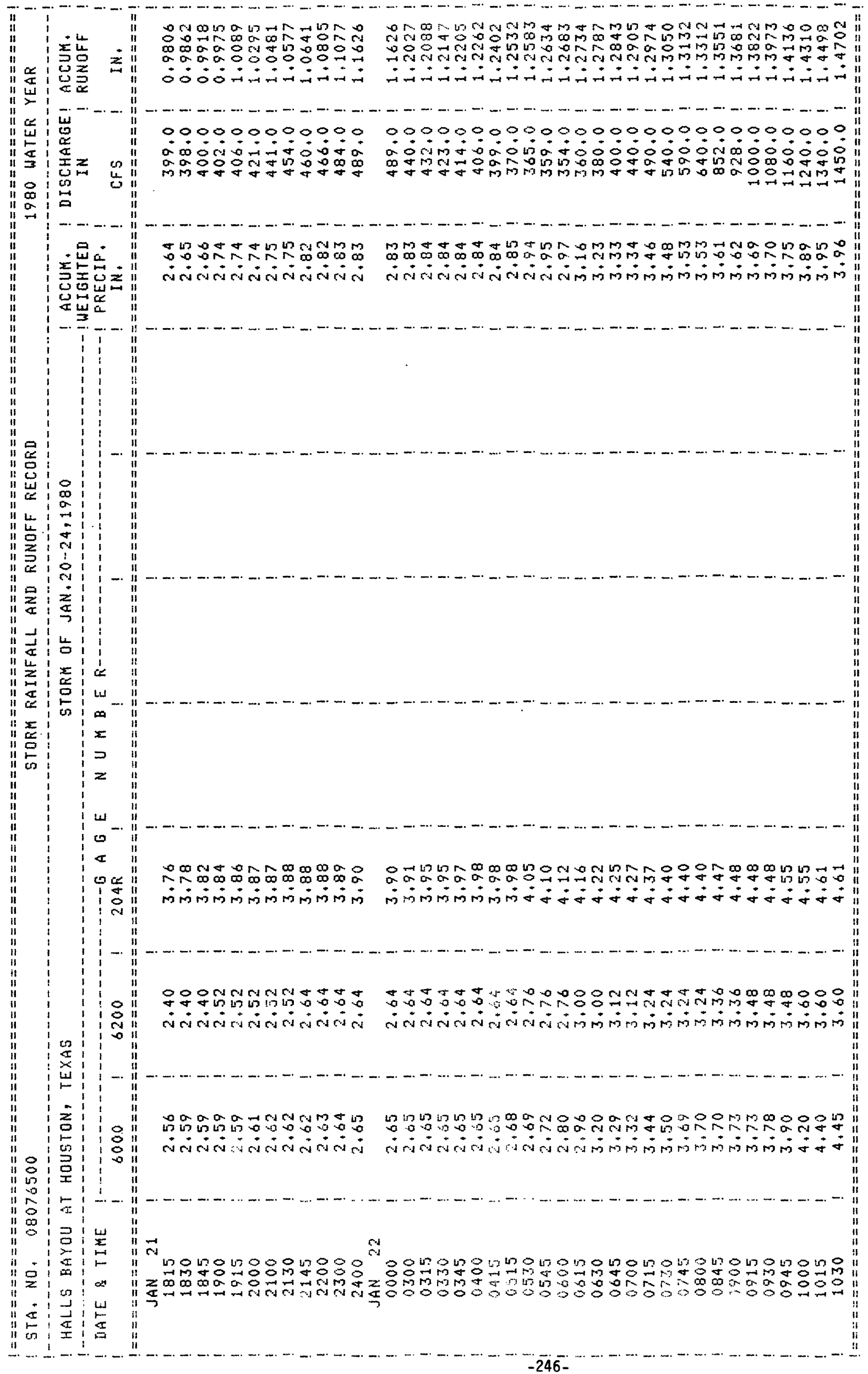




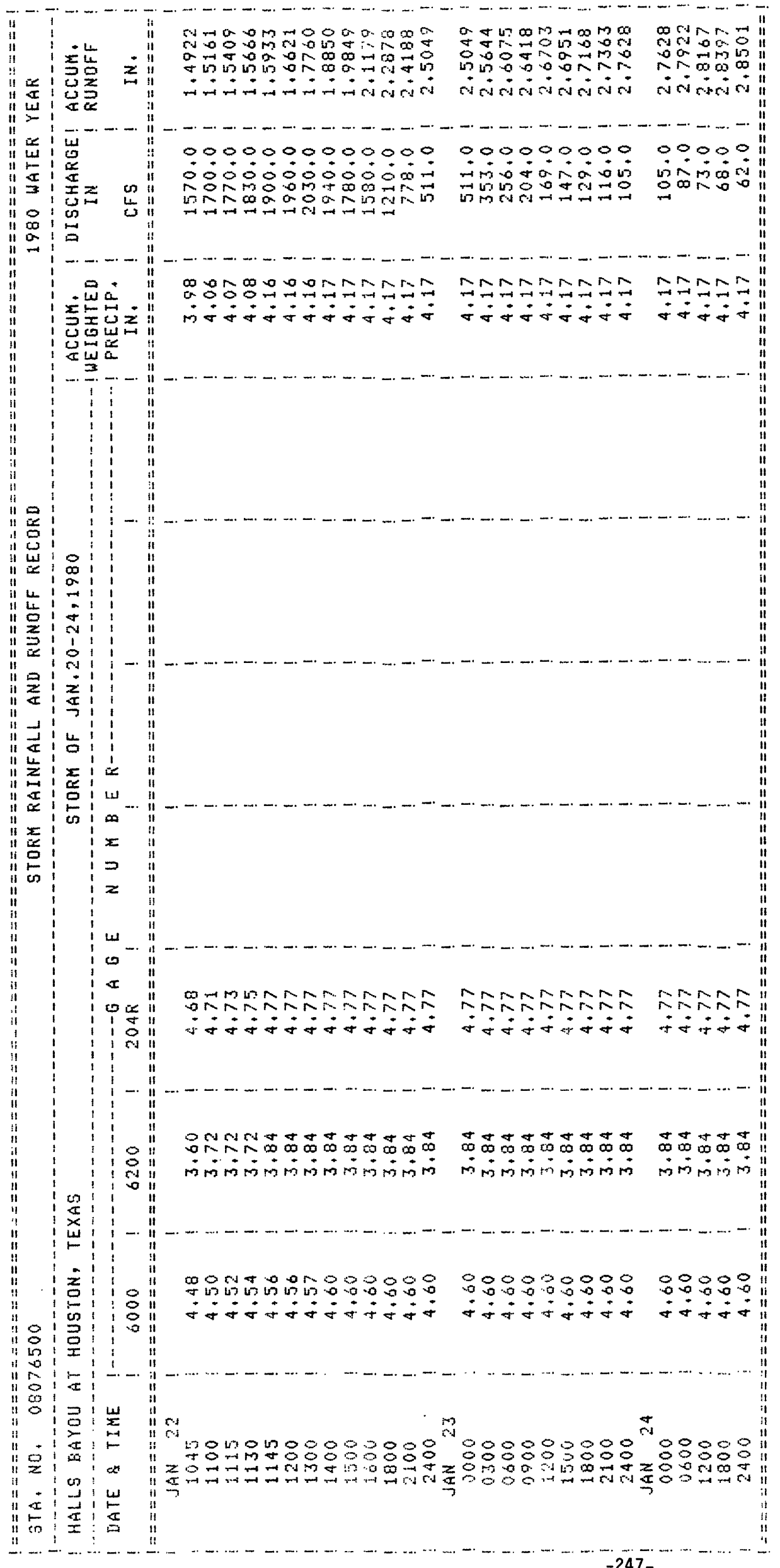

$-247-$ 
SAN JACINTO RIVER BASIN

08076700 GREENS BAYOU AT LEY ROAD, HOUSTON, TX

LOCATION,--Lat $29^{\circ} 50^{\prime} 13^{\prime \prime}$, long 95 $13^{\prime} 59^{\prime \prime}$, Harris County, Hydrologic Unit 12040104, on right bank at downstream side of Ley Road Bridge in northeast Houston and $300 \mathrm{ft}(91 \mathrm{~m})$ downstream from mouth of Halls Bayou.

DRAINAGE AREA.-- $-182 \mathrm{mi}^{2}\left(471 \mathrm{~km}^{2}\right)$.

WATER-DISCHARGE RECORDS

PERIOD OF RECORD.--November 1962 to December 1964, May to September 1971 (discharge measurements only), October 1971 to current year.

GAGE.--Water-stage recorder. Datum of gage is $2.13 \mathrm{ft}(0.649 \mathrm{~m})$ below National Geodetic Vertical Datum of 1929 , 1973 adjustment.

REMARKS.--Water-discharge records falr except those below $1,000 \mathrm{ft}^{3} / \mathrm{s}\left(28.3 \mathrm{~m}^{3} / \mathrm{s}\right)$, which are poor. Discharge $1 \mathrm{~s}$ computed for all storms which produce peak discharges over $1,000 \mathrm{ft}^{3} / \mathrm{s}\left(28.3 \mathrm{~m}^{3} / \mathrm{s}\right)$. Tidal influences on the stage-discharge relationship affect discharge below about $500 \mathrm{ft}^{3} / \mathrm{s}\left(14.2 \mathrm{~m}^{3} / \mathrm{s}\right)$. Discharge below $1,000 \mathrm{ft} / \mathrm{s}$ $\left(28.3 \mathrm{~m}^{3} / \mathrm{s}\right)$ is estimated following designated storm periods only.

EXTREMES FOR PERIOD OF RECORD.--Maximum discharge, $16,700 \mathrm{ft}^{3} / \mathrm{s}\left(473 \mathrm{~m}^{3} / \mathrm{s}\right)$ June 13, 1973, gage height, $34.27 \mathrm{ft}$ $(10.445 \mathrm{~m})$; minimum not determined (affected by tides).

EXTREMES FOR CURRENT YEAR.--Peak discharges above base of $4,200 \mathrm{ft}^{3} / \mathrm{s}\left(119 \mathrm{~m}^{3} / \mathrm{s}\right)$ and maximum (*):

\begin{tabular}{|c|c|c|c|c|c|}
\hline Date & Time & $\begin{array}{r}\text { Disc } \\
\left(\mathrm{ft}^{3} / \mathrm{s}\right)\end{array}$ & $\underset{\left(\mathrm{m}^{3} / \mathrm{s}\right)}{\operatorname{rrge}}$ & $\begin{array}{l}\text { Gage } \\
(\mathrm{ft})\end{array}$ & $\begin{array}{c}\text { height } \\
\text { (m) }\end{array}$ \\
\hline $\begin{aligned} \text { aJan. } & 22 \\
\text { Mar. } & 28\end{aligned}$ & $\begin{array}{l}1900 \\
0600\end{array}$ & $\begin{array}{r}* 9,540 \\
5,920\end{array}$ & $\begin{array}{l}270 \\
168\end{array}$ & $\begin{array}{l}26.15 \\
21.75\end{array}$ & $\begin{array}{l}7.971 \\
6.629\end{array}$ \\
\hline
\end{tabular}

Minimum discharge not determined (affected by tides).

DISCHARGE, IN CUBIC FEET PER SECOND, WATER YEAR OCTOBER 1979 TO SEPTEMBER 1980

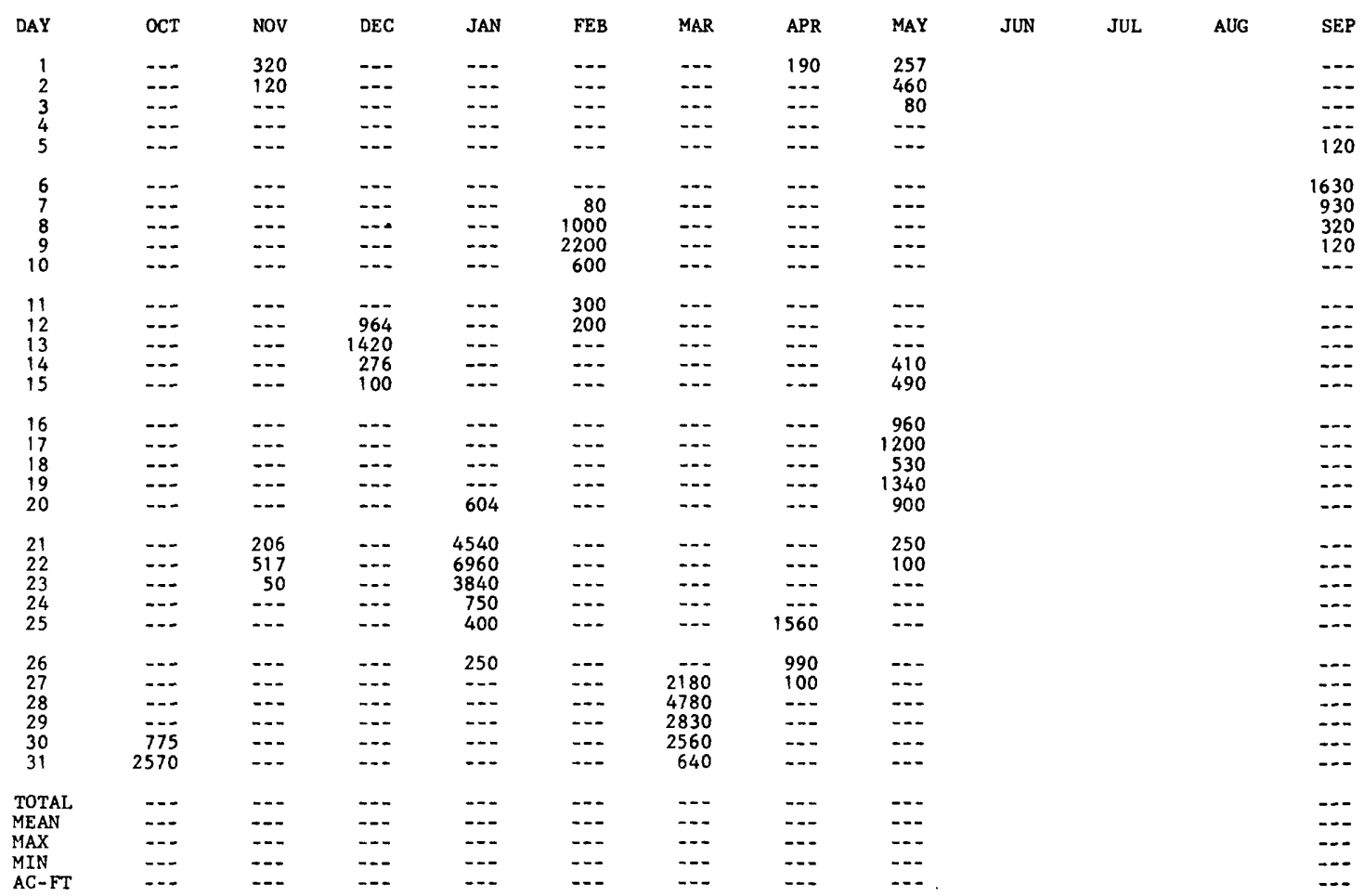

$\begin{array}{lllll}\text { CAL YR } 1979 & \text { TOTAL - } & \text { MEAN - } & \text { MAX - } & \text { MIN - } \\ \text { WTR YR } 1980 & \text { TOTAL - FT - } & \text { MEAN - } & \text { MAX - } & \text { MIN - }\end{array}$ 


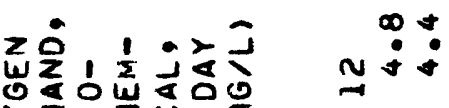

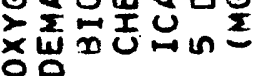

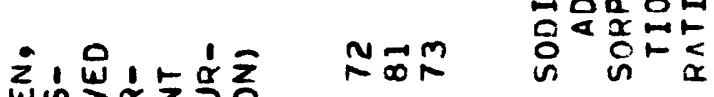

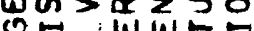

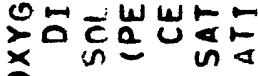

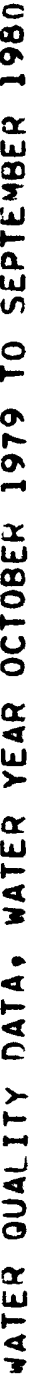

N N

$$
\begin{aligned}
& \sum_{0} \lim _{0}^{0} \quad m 0 \% \\
& \text { 용 }
\end{aligned}
$$

$\frac{1}{0} 05$ 테난

ót

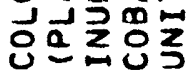

ò w

w

눈

$\sum_{1}=\underline{0}$

$i_{a}$

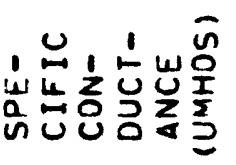

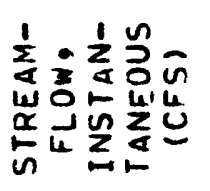

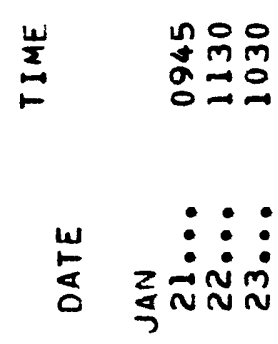

. 0 !

$\operatorname{lom}_{0 \rightarrow \infty}=$

0 in

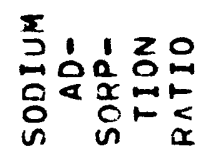

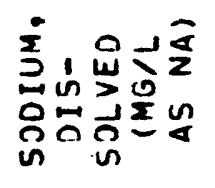

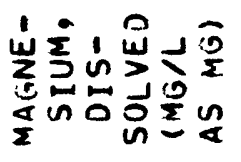

$\infty \div$ :

$\mathbf{N}$

$\stackrel{\infty}{\infty} \underset{\infty}{\infty}$

in 0 Un

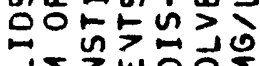

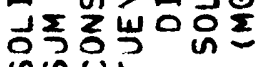

जUU⿺

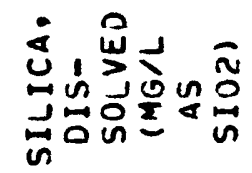

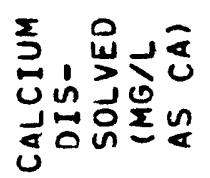

$\underset{n}{\infty} \underbrace{i}$

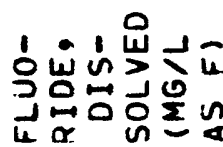

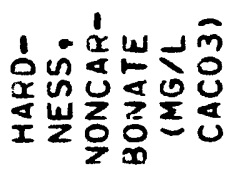

$\rightarrow$

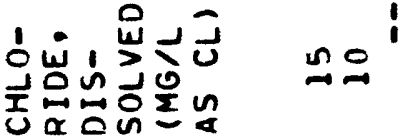

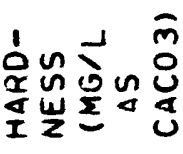

พัะ

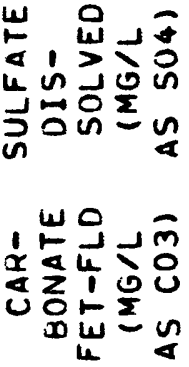

î́

țニ

$\begin{array}{lll}\circ & 0 & 0 \\ 0 & 0 & 0 \\ \circ & 0 & 0\end{array}$

m

$1 \div j 4: 5$

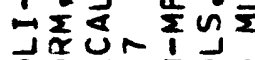

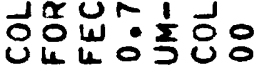
m요

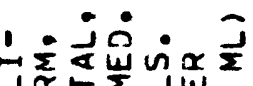

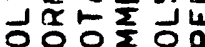
인인ㅇㅇㅇ
옹ㅇㅇㅇ 웅요

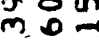

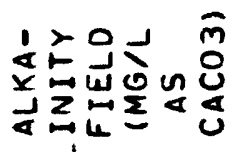

웅ㅇㅇㅇㅇㅇ 웅요 401

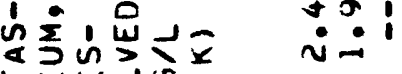

$$
\begin{aligned}
& \text { 比它造 }
\end{aligned}
$$

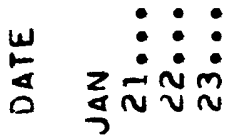

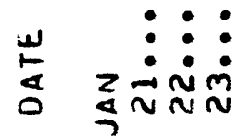




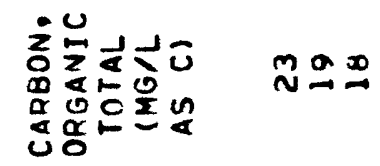

总古引

,

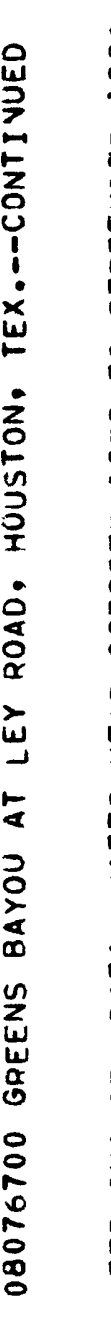

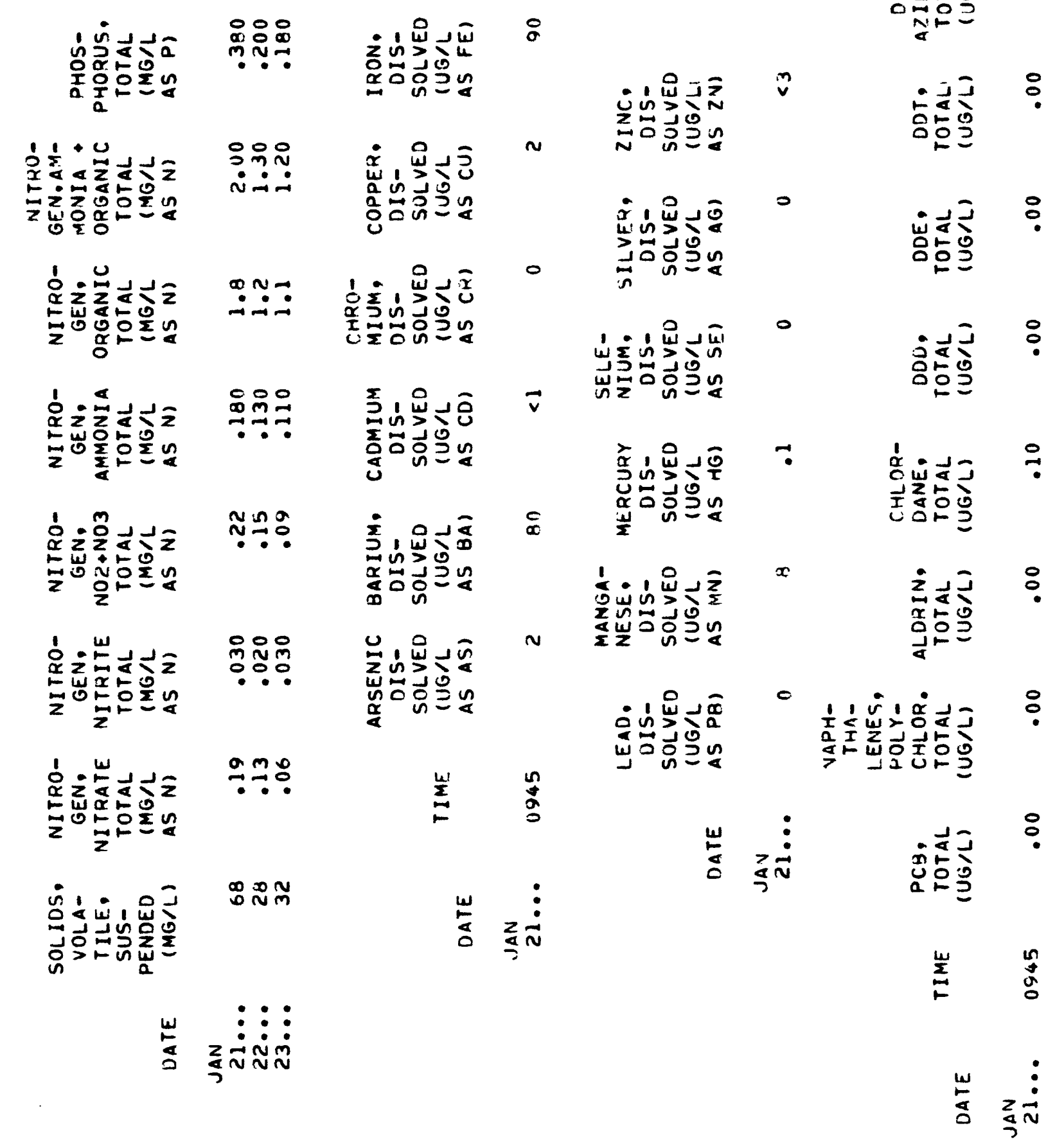




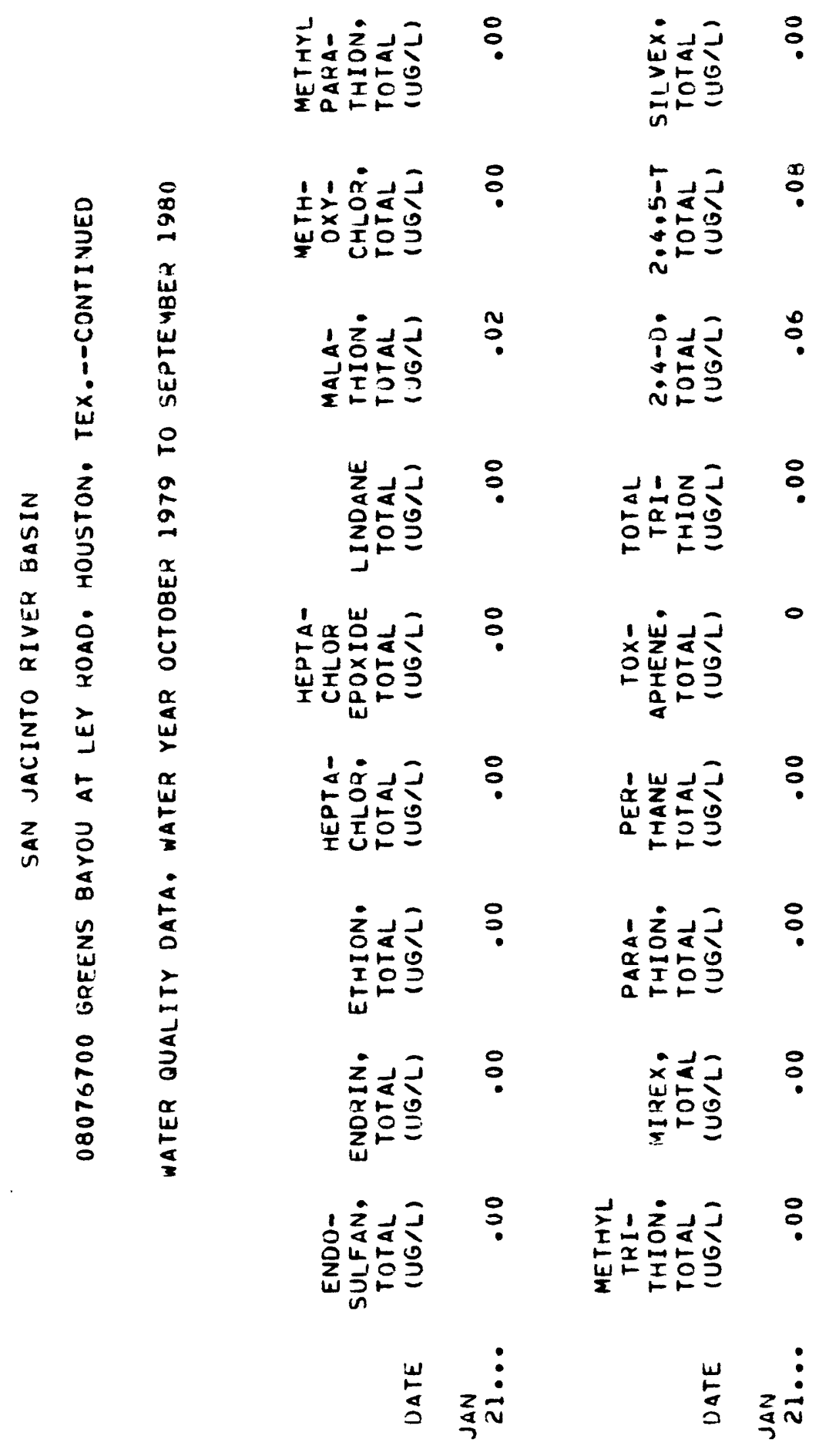




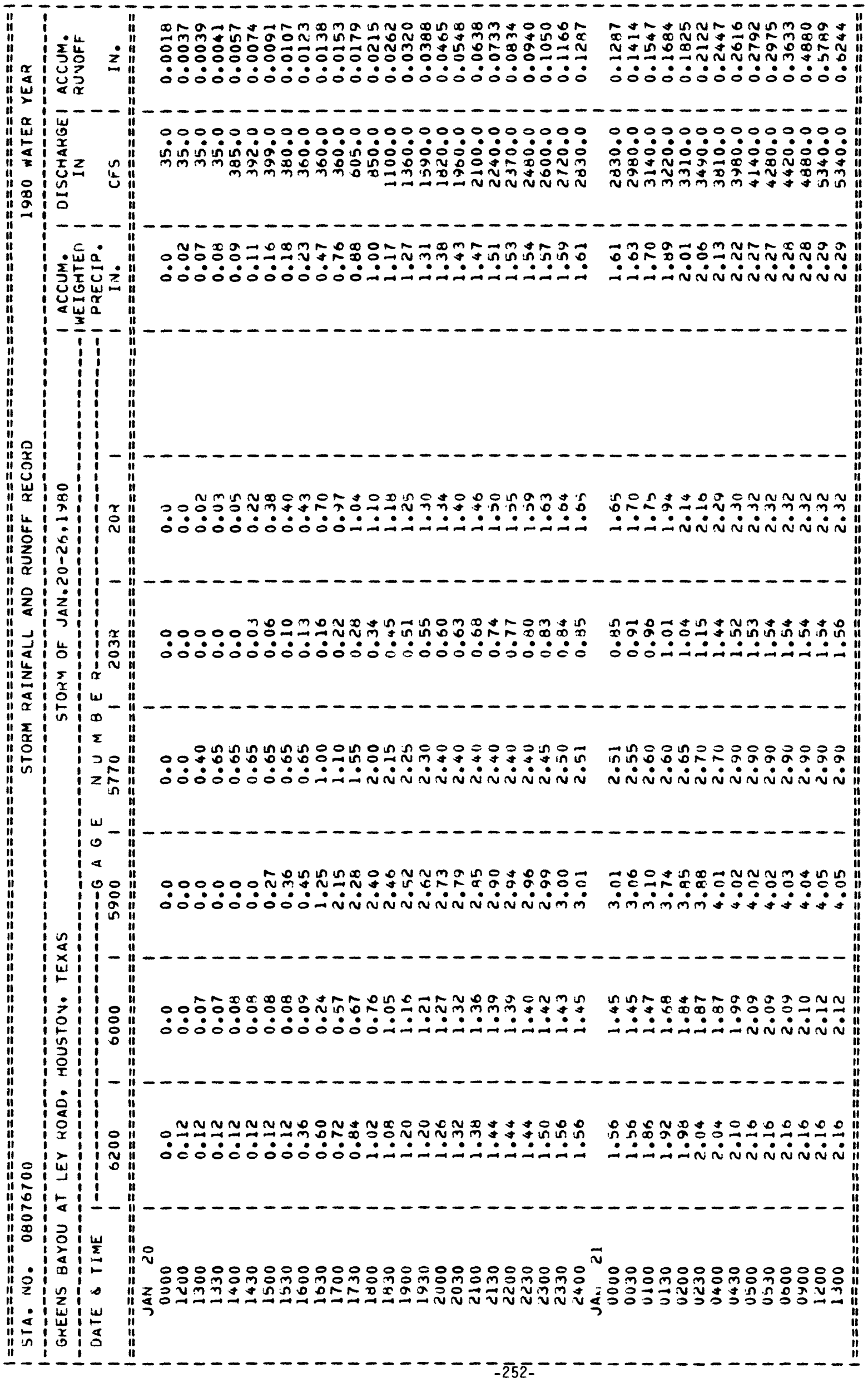




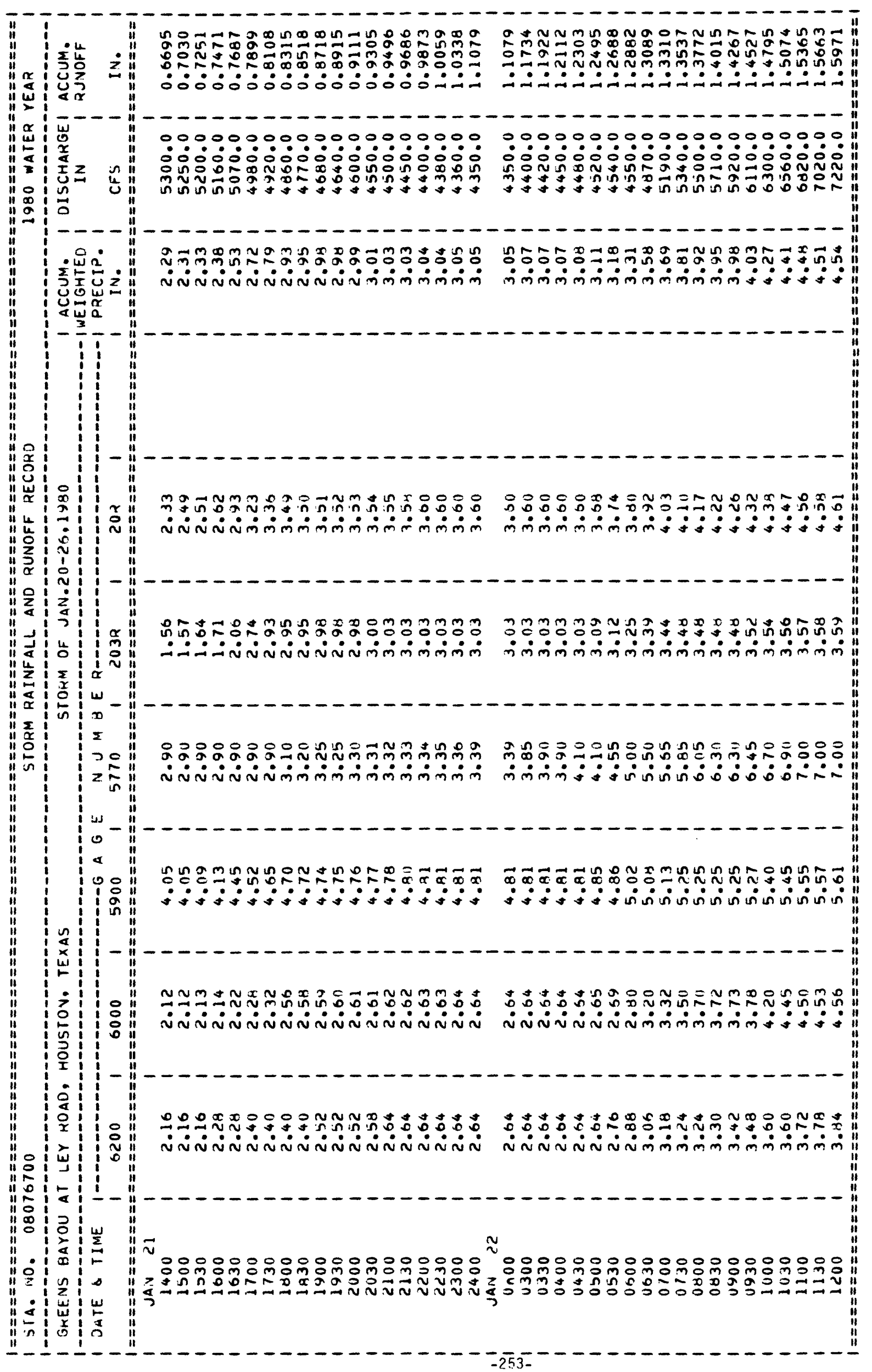




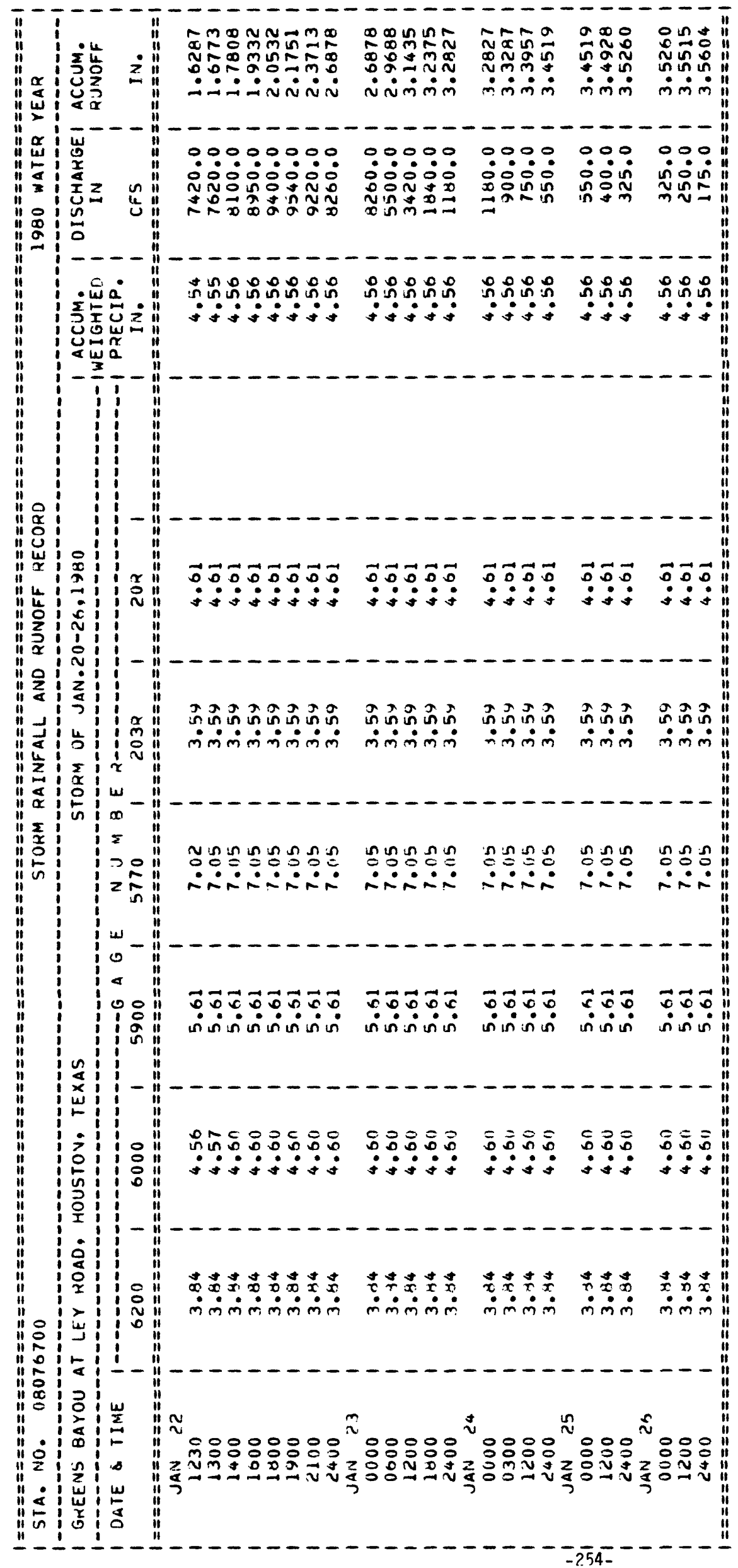




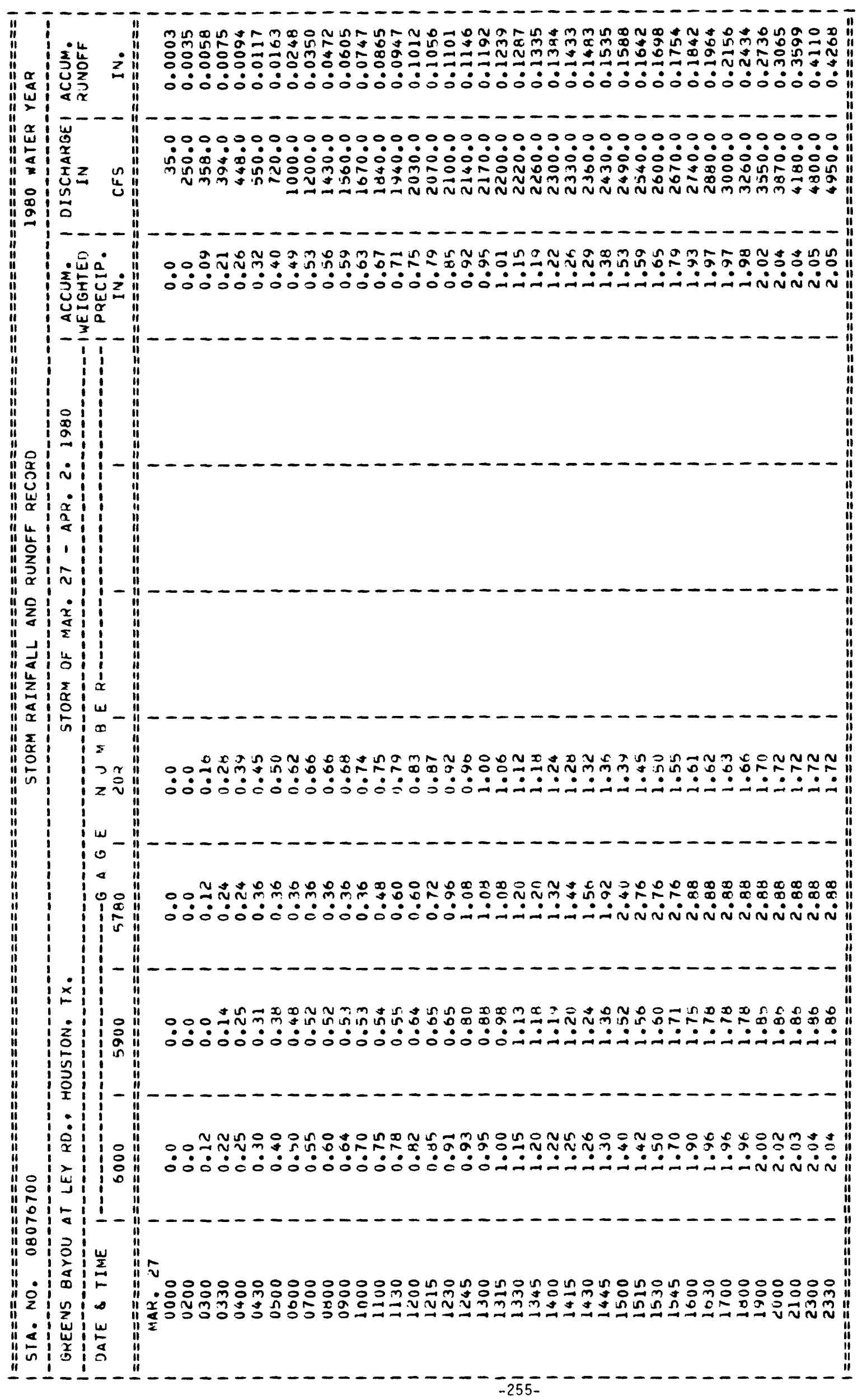




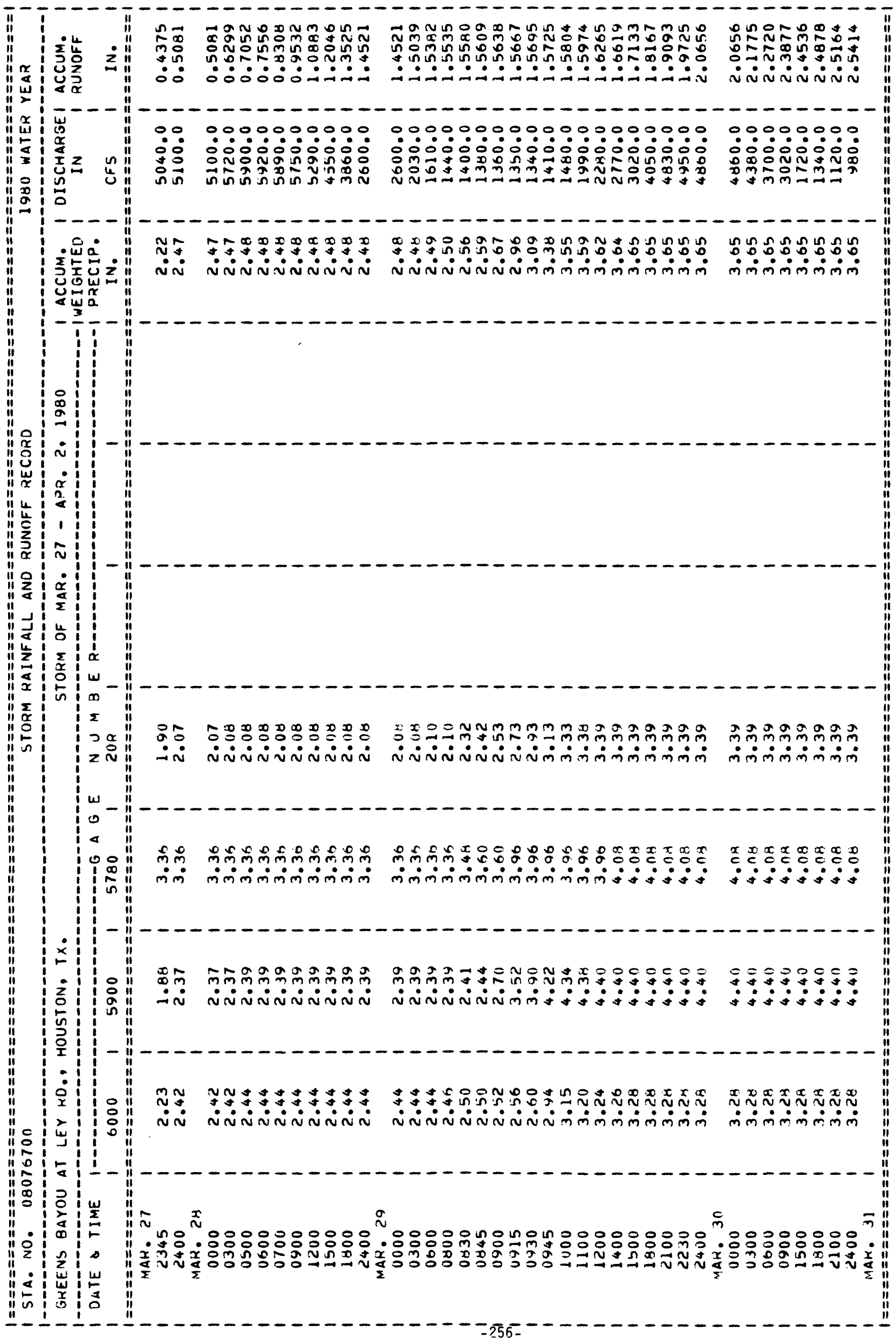




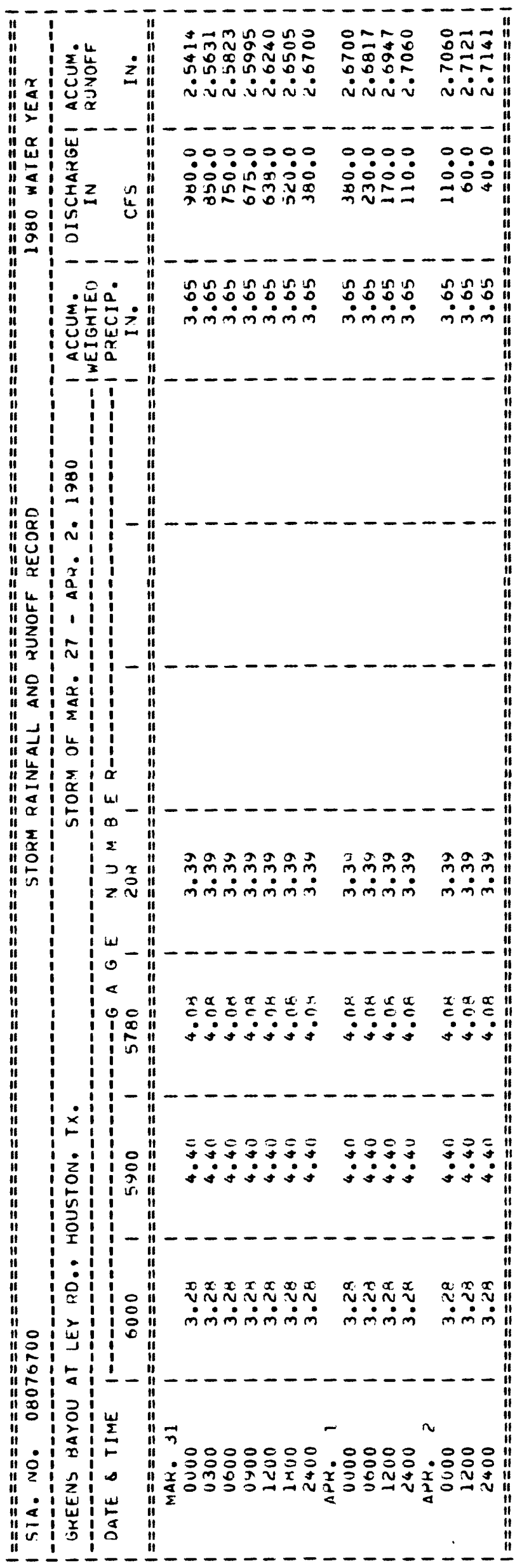


CLEAR CREEK BASIN

08077000 CLEAR CREEK NEAR PEARLAND, TX

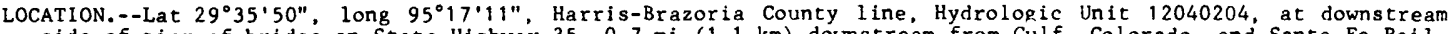
side of pier of bridge on State Highway $35,0.7 \mathrm{mi}(1.1 \mathrm{~km})$ downstream from Gulf, Colorado, and Santa Fe Railway Co. bridge, $1.2 \mathrm{mi}(1.9 \mathrm{~km})$ upstream from Hickory Slough, $2.3 \mathrm{mi}(3.7 \mathrm{~km})$ north of Pearland, and about $30 \mathrm{mi}(48 \mathrm{~km})$ upstream from head of Clear Lake.

DRAINAGE AREA. $--38.8 \mathrm{mi}^{2}\left(100.5 \mathrm{~km}^{2}\right)$.

PERIOD OF RECORD.--July to October 1944, March to October 1946, April 1947 to December 1959, March 1963 to current year. Discharge for some high-water periods in 1944 and 1946 published in WSP 1392.

REVISED RECORDS.--WSP 1392: $1947(\mathrm{M})$.

GAGE.--Water-stage recorder. Datum of gage is $26.58 \mathrm{ft}(8.102 \mathrm{~m})$ National Geodetic Vertical Datum of 1929 , 1973 adjustment; prior records unadjusted for land-surface subsidence. Prior to June 9, 1948, nonrecoraing gage, adjustment; prior records unadjusted for land-surface subsidence. Prior to June 9, 1948, nonrecording gage,
and June 9, 1948, to Apr. 22, 1952, water-stage recorder at same site and datum $5.80 \mathrm{ft}$ (1.768 $\mathrm{m}$ ) higher.

REMARKS.--Records good except those for period of no gage-height record, which are fair. Large area of riceland above station is irrigated with water from the Brazos River. Low flow from April to October is largely drainabove station is irrigated with water from the Brazos River. Low flow from April to October is largely drainage from irrigated lands. Many dive

AVERAGE DISCHARGE.--29 years (water years $1948-59,1964-80), 36.7 \mathrm{ft} \mathrm{t}^{3} / \mathrm{s}\left(1.039 \mathrm{~m}^{3} / \mathrm{s}\right), 26,590 \mathrm{acre-ft} / \mathrm{yr}(32.8$ $\mathrm{hm}^{3} / \mathrm{yr}$ ).

EXTREMES FOR PERIOD OF RECORD.--Maximum discharge, 2,170 $\mathrm{ft}^{3} / \mathrm{s}\left(61.5 \mathrm{~m}^{3} / \mathrm{s}\right)$ Mar. 18, 1957; maximum gage height, $18.57 \mathrm{ft}(5.660 \mathrm{~m})$ July 26,1979 ; no flow at times.

EXTREMES OUTSIDE PERIOD OF RECORD.--Flood of June 26, 1960 (stage and discharge unknown), may have exceeded that of Mar. 18, 1957. Channel was rectified in 1933,1952, 1968, and 1978 .

EXTREMES FOR CURRENT YEAR.--Peak discharges above base of $600 \mathrm{ft}^{3} / \mathrm{s}\left(17.0 \mathrm{~m}^{3} / \mathrm{s}\right)$ and maximum (*):

\begin{tabular}{|c|c|c|c|c|c|}
\hline Date & Time & $\begin{array}{r}\text { Disc } \\
\left(\mathrm{ft}^{3} / \mathrm{s}\right)\end{array}$ & $\stackrel{\left(\mathrm{m}^{3} / \mathrm{s}\right)}{\mathrm{rge}}$ & $\begin{array}{l}\text { Gage } \\
(\mathrm{ft})\end{array}$ & $\begin{array}{r}\text { height } \\
(m)\end{array}$ \\
\hline Oct. 31 & $\begin{array}{r}0100 \\
\text { about }\end{array}$ & 697 & 19.7 & 10.62 & 3.237 \\
\hline $\begin{array}{ll}\text { Jan. } & 22 \\
\text { Mar. } & 29\end{array}$ & $\begin{array}{l}2400 \\
2200\end{array}$ & $\begin{array}{r}* 1,800 \\
614\end{array}$ & $\begin{array}{l}51.0 \\
17.4\end{array}$ & $\begin{array}{r}\text { a1 } 7.89 \\
9.90\end{array}$ & $\begin{array}{l}5.453 \\
3.018\end{array}$ \\
\hline
\end{tabular}

Minimum daily discharge, $0.74 \mathrm{ft}^{3} / \mathrm{s}\left(0.021 \mathrm{~m}^{3} / \mathrm{s}\right)$ Oct. 29 .

DISCHARGE, IN CUBIC FEET PER SECOND, WATER YLAR OCTOBER 1979 TO SEPTEMBER 1980

\begin{tabular}{|c|c|c|c|c|c|c|c|c|c|c|c|c|}
\hline DAY & OCT & NOV & DEC & JAN & FEB & MAR & APR & MAY & JUN & $\mathrm{JUL}$ & AUG & SEP \\
\hline $\begin{array}{l}1 \\
2 \\
3 \\
4 \\
5\end{array}$ & $\begin{array}{l}10 \\
8.0 \\
7.0 \\
6.0 \\
5.0\end{array}$ & $\begin{array}{r}109 \\
40 \\
21 \\
15 \\
11\end{array}$ & $\begin{array}{l}2.0 \\
2.0 \\
2.1 \\
5.6 \\
7.6\end{array}$ & $\begin{array}{l}6.6 \\
4.5 \\
4.3 \\
4.4 \\
4.4\end{array}$ & $\begin{array}{l}9.8 \\
8.2 \\
8.3 \\
7.7 \\
6.7\end{array}$ & $\begin{array}{l}4.5 \\
3.5 \\
3.1 \\
3.5 \\
3.5\end{array}$ & $\begin{array}{c}61 \\
33 \\
24 \\
14 \\
8.0\end{array}$ & $\begin{array}{r}30 \\
160 \\
95 \\
29 \\
11\end{array}$ & $\begin{array}{l}15 \\
15 \\
17 \\
15 \\
14\end{array}$ & $\begin{array}{l}11 \\
9.7 \\
9.3 \\
8.9 \\
9.3\end{array}$ & $\begin{array}{l}9.6 \\
7.0 \\
5.1 \\
3.8 \\
3.4\end{array}$ & $\begin{array}{l}2.2 \\
1.9 \\
2.5 \\
1.8 \\
3.5\end{array}$ \\
\hline $\begin{array}{r}6 \\
7 \\
8 \\
9 \\
10\end{array}$ & $\begin{array}{l}4.5 \\
4.0 \\
3.5 \\
3.0 \\
2.7\end{array}$ & $\begin{array}{l}8.0 \\
6.0 \\
5.0 \\
4.0 \\
3.0\end{array}$ & $\begin{array}{l}5.7 \\
3.8 \\
2.6 \\
1.9 \\
1.5\end{array}$ & $\begin{array}{l}4.4 \\
4.4 \\
4.0 \\
3.5 \\
3.1\end{array}$ & $\begin{array}{r}6.0 \\
5.1 \\
40 \\
314 \\
168\end{array}$ & $\begin{array}{l}3.1 \\
3.1 \\
3.1 \\
3.0 \\
2.9\end{array}$ & $\begin{array}{l}5.6 \\
5.4 \\
4.8 \\
3.8 \\
3.0\end{array}$ & $\begin{array}{l}5.2 \\
3.9 \\
4.5 \\
6.0 \\
4.6\end{array}$ & $\begin{array}{l}13 \\
13 \\
8.9 \\
11 \\
17\end{array}$ & $\begin{array}{r}8.4 \\
9.1 \\
9.3 \\
9.6 \\
11\end{array}$ & $\begin{array}{l}4.6 \\
3.2 \\
2.7 \\
2.8 \\
2.6\end{array}$ & $\begin{array}{l}40 \\
32 \\
59 \\
40 \\
18\end{array}$ \\
\hline $\begin{array}{l}11 \\
12 \\
13 \\
14 \\
15\end{array}$ & $\begin{array}{l}2.3 \\
2.0 \\
1.8 \\
1.6 \\
1.4\end{array}$ & $\begin{array}{l}2.5 \\
2.0 \\
1.5 \\
1.3 \\
1.2\end{array}$ & $\begin{array}{c}1.3 \\
1.8 \\
14 \\
15 \\
9.7\end{array}$ & $\begin{array}{l}3.0 \\
2.6 \\
2.2 \\
2.0 \\
2.0\end{array}$ & $\begin{array}{l}75 \\
42 \\
26 \\
20 \\
59\end{array}$ & $\begin{array}{l}2.9 \\
2.9 \\
2.6 \\
2.3 \\
2.1\end{array}$ & $\begin{array}{c}2.8 \\
2.8 \\
27 \\
11 \\
5.6\end{array}$ & $\begin{array}{l}3.2 \\
2.7 \\
3.0 \\
3.6 \\
4.3\end{array}$ & $\begin{array}{l}25 \\
14 \\
9.8 \\
8.4 \\
8.4\end{array}$ & $\begin{array}{l}12 \\
12 \\
11 \\
12 \\
13\end{array}$ & $\begin{array}{l}2.3 \\
1.9 \\
1.6 \\
4.5 \\
4.5\end{array}$ & $\begin{array}{l}12 \\
10 \\
6.5 \\
4.4 \\
3.2\end{array}$ \\
\hline $\begin{array}{l}16 \\
17 \\
18 \\
19 \\
20\end{array}$ & $\begin{array}{l}1.2 \\
1.1 \\
1.0 \\
.90 \\
.90\end{array}$ & $\begin{array}{l}1.1 \\
1.1 \\
1.1 \\
1.8 \\
2.2\end{array}$ & $\begin{array}{l}7.6 \\
5.5 \\
4.0 \\
3.2 \\
2.9\end{array}$ & $\begin{array}{r}2.0 \\
3.0 \\
6.0 \\
7.0 \\
10\end{array}$ & $\begin{array}{l}60 \\
46 \\
28 \\
20 \\
15\end{array}$ & $\begin{array}{l}2.3 \\
4.6 \\
3.1 \\
3.1 \\
6.8\end{array}$ & $\begin{array}{r}3.8 \\
2.9 \\
6.6 \\
21 \\
8.3\end{array}$ & $\begin{array}{c}8.6 \\
13 \\
9.9 \\
120 \\
85\end{array}$ & $\begin{array}{l}11 \\
11 \\
10 \\
9.2 \\
8.9\end{array}$ & $\begin{array}{l}13 \\
14 \\
14 \\
14 \\
12\end{array}$ & $\begin{array}{l}3.4 \\
6.8 \\
6.0 \\
4.2 \\
3.0\end{array}$ & $\begin{array}{l}2.2 \\
1.8 \\
1.4 \\
1.4 \\
1.3\end{array}$ \\
\hline $\begin{array}{l}21 \\
22 \\
23 \\
24 \\
25\end{array}$ & $\begin{array}{l}1.5 \\
1.4 \\
1.2 \\
1.1 \\
1.0\end{array}$ & $\begin{array}{l}3.0 \\
8.5 \\
9.5 \\
6.6 \\
4.6\end{array}$ & $\begin{array}{l}2.8 \\
2.8 \\
2.8 \\
2.8 \\
3.1\end{array}$ & $\begin{array}{r}100 \\
1150 \\
1500 \\
800 \\
250\end{array}$ & $\begin{array}{l}12 \\
10 \\
8.1 \\
6.7 \\
5.3\end{array}$ & $\begin{array}{c}11 \\
7.8 \\
6.7 \\
5.7 \\
3.9\end{array}$ & $\begin{array}{l}16 \\
15 \\
16 \\
15 \\
25\end{array}$ & $\begin{array}{l}4 ! \\
32 \\
19 \\
14 \\
13\end{array}$ & $\begin{array}{l}11 \\
12 \\
17 \\
13 \\
18\end{array}$ & $\begin{array}{l}13 \\
19 \\
23 \\
34 \\
23\end{array}$ & $\begin{array}{l}2.8 \\
2.5 \\
2.4 \\
2.3 \\
2.2\end{array}$ & $\begin{array}{l}1.4 \\
1.3 \\
1.1 \\
1.0 \\
.92\end{array}$ \\
\hline $\begin{array}{l}26 \\
27 \\
28 \\
29 \\
30 \\
31\end{array}$ & $\begin{array}{r}.90 \\
.80 \\
.75 \\
128^{.74} \\
449\end{array}$ & $\begin{array}{l}3.7 \\
3.2 \\
2.9 \\
2.4 \\
2.1 \\
-. .\end{array}$ & $\begin{array}{l}3.2 \\
2.9 \\
2.4 \\
16 \\
16 \\
10\end{array}$ & $\begin{array}{r}130 \\
65 \\
35 \\
23 \\
17 \\
13\end{array}$ & $\begin{array}{l}4.2 \\
3.7 \\
3.5 \\
4.1 \\
-. . \\
-.-\end{array}$ & $\begin{array}{l}4^{4.0} \\
408 \\
373 \\
421 \\
149\end{array}$ & $\begin{array}{l}28 \\
26 \\
23 \\
20 \\
7.0 \\
---\end{array}$ & $\begin{array}{l}15 \\
18 \\
18 \\
16 \\
17 \\
17\end{array}$ & $\begin{array}{l}15 \\
13 \\
12 \\
11 \\
11 \\
\ldots\end{array}$ & $\begin{array}{l}24 \\
22 \\
38 \\
53 \\
35 \\
16\end{array}$ & $\begin{array}{l}2.1 \\
2.1 \\
2.2 \\
4.3 \\
4.0 \\
3.2\end{array}$ & $\begin{array}{r}2.9 \\
5.9 \\
48 \\
40 \\
39\end{array}$ \\
\hline $\begin{array}{l}\text { TOTAL } \\
\text { MEAN } \\
\text { MAX } \\
\text { MIN } \\
\text { AC-F"I }\end{array}$ & $\begin{array}{r}654.29 \\
21.1 \\
449 \\
.74 \\
1300\end{array}$ & $\begin{array}{r}284.3 \\
9.48 \\
109 \\
1.1 \\
564\end{array}$ & $\begin{array}{r}164.6 \\
5.31 \\
16 \\
1.3 \\
326\end{array}$ & $\begin{array}{r}4166.4 \\
134 \\
1500 \\
2.0 \\
8260\end{array}$ & $\begin{array}{r}1022.4 \\
35.3 \\
314 \\
3.5 \\
2030\end{array}$ & $\begin{array}{r}1640.1 \\
52.9 \\
421 \\
2.1 \\
3250\end{array}$ & $\begin{array}{r}445.4 \\
14.8 \\
61 \\
2.8 \\
883\end{array}$ & $\begin{array}{r}822.5 \\
26.5 \\
160 \\
2.7 \\
1630\end{array}$ & $\begin{array}{r}387.6 \\
12.9 \\
25 \\
8.4 \\
769\end{array}$ & $\begin{array}{r}522.6 \\
16.9 \\
53 \\
8.4 \\
1040\end{array}$ & $\begin{array}{r}113.1 \\
3.65 \\
9.6 \\
1.6 \\
224\end{array}$ & $\begin{array}{r}386.62 \\
12.9 \\
59 \\
.92 \\
767\end{array}$ \\
\hline
\end{tabular}

$\begin{array}{llllllllll}\text { CAL YR } 1979 & \text { TOTAL } & 31717.49 & \text { MEAN } 86.9 & \text { MAX } & 1910 & \text { MI: } & .74 & \text { AC-FT } & 62910 \\ \text { WTR YR } 1980 & \text { TOTAL } & 10609.91 & \text { MEAN } 29.0 & \text { MAX } & 1500 & \text { MI: } & .74 & \text { AC-FT } & 21040\end{array}$

NoTE.--No gage-height record Jan. 17-28. 
Table 18.--Recording and nonrecording rain gages in the Houston area at sites other than stream-gaging stations

\begin{tabular}{|c|c|c|c|}
\hline $\begin{array}{l}\text { Station } \\
\text { no. } 1 /\end{array}$ & Station name & Location & Period of record $2 /$ \\
\hline $10-5$ & Houston Heights & $\begin{array}{l}\text { Lat } 29^{\circ} 47^{\prime} \text {, long } 95^{\circ} 26^{\prime} \\
\text { near Houston. }\end{array}$ & -- \\
\hline $12-R$ & $\begin{array}{l}\text { Houston-WB, } \\
\text { City }\end{array}$ & $\begin{array}{l}\text { Lat } 29^{\circ} 46^{\prime} \text {, long } 95^{\circ} 22^{\prime} \\
\text { at old Federal Building } \\
\text { in downtown Houston. }\end{array}$ & - \\
\hline $13-5$ & $\begin{array}{l}\text { Houston- } \\
\text { Independent } \\
\text { Heights }\end{array}$ & $\begin{array}{l}\text { Lat } 29^{\circ} 52^{\prime}, \text { long } 95^{\circ} 25^{\prime} \\
\text { in northern section of } \\
\text { Houston. }\end{array}$ & -- \\
\hline $20-R$ & $\begin{array}{l}\text { Houston WSO } \\
\text { Airport }\end{array}$ & $\begin{array}{l}\text { Lat } 29^{\circ} 59^{\prime} \text {, long } 95^{\circ} 22^{\prime} \\
\text { at Houston Intercontinental } \\
\text { Airport in north Houston. }\end{array}$ & - \\
\hline $21-R$ & Brittmore & $\begin{array}{l}\text { Lat } 29^{\circ} 51^{\prime} 02^{\prime \prime} \text {, long } \\
95^{\circ} 33^{\prime} 46^{\prime \prime} \text {, behind home of } \\
\text { Mrs. Annie A. Joseph, } \\
10610 \text { Tanner Road, in } \\
\text { northwest Houston. }\end{array}$ & Miay 6,1964 to date \\
\hline $22-R$ & Houston-Satsuma & $\begin{array}{l}\text { Lat } 29^{\circ} 54^{\prime}, \text { long } 95^{\circ} 37^{\prime} \\
\text { at Satsuma community } \\
\text { northwest of Houston. }\end{array}$ & -- \\
\hline $23-5$ & $\begin{array}{l}\text { Houston-North } \\
\text { Houston }\end{array}$ & $\begin{array}{l}\text { Lat } 29^{\circ} 53^{\prime}, \text { long } 95^{\circ} 31^{\prime} \\
\text { near Fairbanks-North } \\
\text { Houston Road, Houston. }\end{array}$ & -- \\
\hline $24-5$ & $\begin{array}{l}\text { Houston-Spring } \\
\text { Branch }\end{array}$ & $\begin{array}{l}\text { Lat } 29^{\circ} 48^{\prime}, \text { long } 95^{\circ} 30^{\prime} \\
\text { on Ridgecrest Street, } \\
\text { Houston. }\end{array}$ & -- \\
\hline $29-R$ & Mills Road & $\begin{array}{l}\text { Lat } 29^{\circ} 57^{\prime} 29^{\prime \prime} \text {, long } \\
95^{\circ} 33^{\prime} 40^{\prime \prime} \text {, at home of } \\
\text { Frances L. Farquhar, } \\
9502 \text { Milis Road, north- } \\
\text { west Harris County, } \\
\text { Houston }\end{array}$ & July 30,1970 to date \\
\hline
\end{tabular}

See footnotes at end of table. 
Table 18.--Recording and nonrecording rain gages in the Houston area at sites other than stream-gaging stations--Continued

\begin{tabular}{|c|c|c|c|}
\hline $\begin{array}{l}\text { Station } \\
\text { no. } 1 /\end{array}$ & Station name & Location & Period of record $2 /$ \\
\hline $31-R$ & Stafford & $\begin{array}{l}\text { Lat } 29^{\circ} 36^{\prime} 43^{\prime \prime} \text {, long } \\
95^{\circ} 32^{\prime} 58^{\prime \prime} \text {, at Ft. Bend } \\
\text { County Water Control and } \\
\text { Improvement District } \\
\text { No. 2, Stafford. }\end{array}$ & May 9, 1964 to date \\
\hline $32-R$ & Houston-Alief & $\begin{array}{l}\text { Lat } 29^{\circ} 43^{\prime} \text {, long } 95^{\circ} 36^{\prime} \\
\text { at Al ief. }\end{array}$ & - \\
\hline $33-R$ & Houston-Addicks & $\begin{array}{l}\text { Lat } 29^{\circ} 46^{\prime} \text {, long } 95^{\circ} 39^{\prime} \\
\text { at U.S. Army Corps of } \\
\text { Engineers office, Addicks }\end{array}$ & -- \\
\hline $34-5$ & Clodine & $\begin{array}{l}\text { Lat } 29^{\circ} 43^{\prime} \text {, long } 95^{\circ} 41^{\prime} \\
\text { at Clodine. }\end{array}$ & -- \\
\hline $35-5$ & $\begin{array}{l}\text { Houston- } \\
\text { Westbury }\end{array}$ & $\begin{array}{l}\text { Lat } 29^{\circ} 40^{\prime} \text {, long } 95^{\circ} 28^{\prime} \\
\text { in Westbury Subdivision, } \\
\text { Houston. }\end{array}$ & -- \\
\hline $36-5$ & Sugar Land & $\begin{array}{l}\text { Lat } 29^{\circ} 37^{\prime} \text {, long } 95^{\circ} 38^{\prime} \\
\text { at Sugar Land. }\end{array}$ & - \\
\hline $39-R$ & KHTV & $\begin{array}{l}\text { Lat } 29^{\circ} 43^{\prime} 25^{\prime \prime} \text {, long } \\
95^{\circ} 30^{\prime} 06^{\prime \prime} \text {, at station } \\
\text { KHTV-TV at Hillcroft } \\
\text { and West Park Drive, } \\
\text { Houston. }\end{array}$ & $\begin{array}{l}\text { Aug. } 22,1967 \text { to } \\
\text { Sept. 30, 1970; } \\
\text { Oct. 1, } 1971 \text { to date }\end{array}$ \\
\hline $42-5$ & $\begin{array}{l}\text { Houston FAA } \\
\text { Airport }\end{array}$ & $\begin{array}{l}\text { Lat } 29^{\circ} 39^{\prime} \text {, lony } 95^{\circ} 17^{\prime} \\
\text { at old Terminal Building, } \\
\text { William P. Hobby Airport, } \\
\text { Houston. }\end{array}$ & $\cdots$ \\
\hline $101-R$ & Liberty Road & $\begin{array}{l}\text { Lat } 29^{\circ} 47^{\prime} 19^{\prime \prime} \text {, long } \\
95^{\circ} 18^{\prime} 50^{\prime \prime} \text {, near intersec- } \\
\text { tion of Liberty Road and } \\
\text { Sakowitz Street, Houston. }\end{array}$ & Aug. 23, 1972 to date \\
\hline $201-S$ & Humble & $\begin{array}{l}\text { Lat } 30^{\circ} 00^{\prime} \text {, long } 95^{\circ} 15^{\prime} \\
\text { at Humble. }\end{array}$ & - \\
\hline
\end{tabular}

See footnotes at end of table 
Table 18.--Recording and nonrecording rain gages in the Houston area at sites other than stream-gaging stations--Continued

\begin{tabular}{|c|c|c|c|}
\hline $\begin{array}{l}\text { Station } \\
\text { no. } 1 /\end{array}$ & Station name & Location & Period of record 21 \\
\hline $202-S$ & $\begin{array}{l}\text { Houston-San } \\
\text { Jacinto Dam }\end{array}$ & $\begin{array}{l}\text { Lat } 29^{\circ} 55^{\prime} \text {, long } 95^{\circ} 09^{\prime} \\
\text { on west bank of Lake } \\
\text { Houston at San Jacinto } \\
\text { River Dam, Houston. }\end{array}$ & -- \\
\hline $203-R$ & Mintz Lane & $\begin{array}{l}\text { Lat } 29^{\circ} 59^{\prime} 53^{\prime \prime} \text {, long } \\
95^{\circ} 28^{\prime} 39^{\prime \prime} \text {, at home of } \\
\text { Mr. Draper D. Mintz, in } \\
\text { northwest Harris County, } \\
\text { Houston. }\end{array}$ & Aug. 23, 1972 to date \\
\hline $204-R$ & Breen Street & $\begin{array}{l}\text { Lat } 29^{\circ} 53^{\prime} 57^{\prime \prime} \text {, long } \\
95^{\circ} 27^{\prime} 38^{\prime \prime} \text {, at home of } \\
\text { Mr. Joseph 0. Eiland, } \\
4909 \text { Breen, in north- } \\
\text { west Harris County, } \\
\text { Houston. }\end{array}$ & Aug. 23, 1972 to date \\
\hline $205-R$ & Frontier Street & $\begin{array}{l}\text { Lat } 29^{\circ} 50^{\prime} 08^{\prime \prime}, \text { long } \\
95^{\circ} 31^{\prime} 22^{\prime \prime}, \text { at home of } \\
\text { Mrs. Eva S. Murphree } \\
\text { near intersection of } \\
\text { Frontier Street and } \\
\text { Outpost Street in north- } \\
\text { west Harris County, } \\
\text { Houston. }\end{array}$ & Nov. 9, 1972 to date \\
\hline $303-R$ & Four Corners & $\begin{array}{l}\text { Lat } 29^{\circ} 40^{\prime} 07^{\prime \prime} \text {, long } \\
95^{\circ} 39^{\prime} 36^{\prime \prime} \text {, Fort Bend } \\
\text { County behind home of } \\
\text { Mr. Richard Wright, } \\
900 \text { feet west of inter- } \\
\text { section of Gaston Road } \\
\text { and Gains Road at Four } \\
\text { Corners community. }\end{array}$ & Sept. 24, 1975 to date \\
\hline $304-R$ & Chasewood & $\begin{array}{l}\text { Lat } 29^{\circ} 36^{\prime} 32^{\prime \prime}, \text { long } \\
95^{\circ} 29^{\prime} 57^{\prime \prime} \text {, Fort Bend } \\
\text { County inside water- } \\
\text { treatment plant at } 1700 \\
\text { Chasewood Street. }\end{array}$ & Oct. 29,1975 to date \\
\hline
\end{tabular}

See footnotes at end of table. 
Table 18.--Recording and nonrecording rain gages in the Houston area at sites other than stream-gaging stations--Continued

\begin{tabular}{|c|c|c|c|}
\hline $\begin{array}{r}\text { Station } \\
\text { no. } 1 /\end{array}$ & Station name & Location & Period of record $2 /$ \\
\hline $305-R$ & Furman & $\begin{array}{l}\text { Lat } 29^{\circ} 37^{\prime} 45^{\prime \prime} \text {, long } \\
95^{\circ} 22^{\prime} 45^{\prime \prime} \text {, Harris County } \\
\text { on extreme right side of } \\
\text { floodway for Sims Bayou } \\
\text { at } 14201 \text { Furman Street. }\end{array}$ & Sept. 24,1975 to date \\
\hline $308-R$ & Public Health & $\begin{array}{l}115 \mathrm{~N} \text {. MacGregor, } \\
\text { Houston. }\end{array}$ & - \\
\hline $401-R$ & Llano Street & $\begin{array}{l}\text { Lat } 29^{\circ} 39^{\prime} 11^{\prime \prime}, \text { long } \\
95^{\circ} 12^{\prime} 07^{\prime \prime} \text {, behind home } \\
\text { of Mrs. Lana H. Sims, } \\
702 \text { Llano, Pasadena, in } \\
\text { Southeast Harris County. }\end{array}$ & Nov. 9, 1972 to date \\
\hline $402-R$ & Klondike & $\begin{array}{l}\text { Lat } 29^{\circ} 38^{\prime} 06^{\prime \prime} \text {, long } \\
95^{\circ} 15^{\prime} 04^{\prime \prime} \text {, behind home } \\
\text { of H. F. Reams, } 9302 \\
\text { Klondike, } 10.9 \text { miles } \\
\text { southeast of Harris } \\
\text { County Courthouse, } \\
\text { Houston. }\end{array}$ & Nov. 11,1973 to date \\
\hline $403-R$ & Ed gebrook & $\begin{array}{l}\text { Lat } 29^{\circ} 38^{\prime} 55^{\prime \prime} \text {, long } \\
95^{\circ} 12^{\prime} 55^{\prime \prime} \text {, southeast } \\
\text { Harris County, in } \\
\text { Sewage Treatment Plant } \\
\text { near the intersection } \\
\text { of 0ld Galveston Road } \\
\text { and Edgebrook Street. }\end{array}$ & Sept: 19,1975 to date \\
\hline
\end{tabular}

See footnotes at end of table. 
Table 18.--Recording and nonrecording rain gages in the Houston area at sites other than stream-gaging stations--Continued

\begin{tabular}{llll}
\hline $\begin{array}{c}\text { Station } \\
\text { no. } 1 \text { f }\end{array}$ & Station name & Location & Period of record 2/ \\
\hline $404-5$ & Deer Park & $\begin{array}{l}\text { Lat } 29^{\circ} 43^{\prime}, \text { long } 95^{\circ} 08^{\prime} \\
\text { Harris County near Houston. }\end{array}$ & - \\
\hline
\end{tabular}

1/ Station numbers are arbitrarily assigned for use in this project as follows: $R$, recording rain gage; $S$, nonrecording rain gage.

2) Period of record is given only for those stations operated and maintained by the U.S. Geological Survey for this project. 


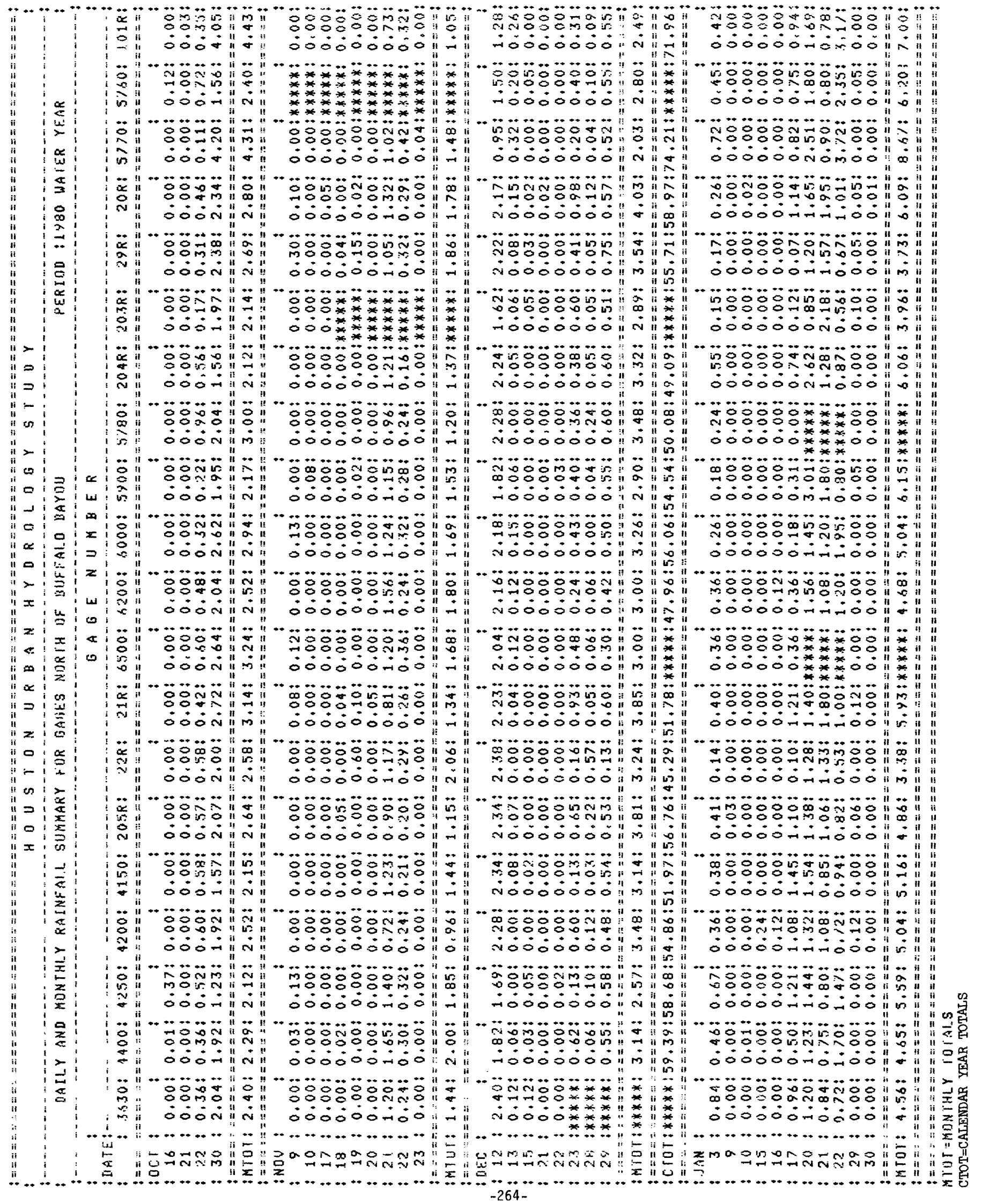




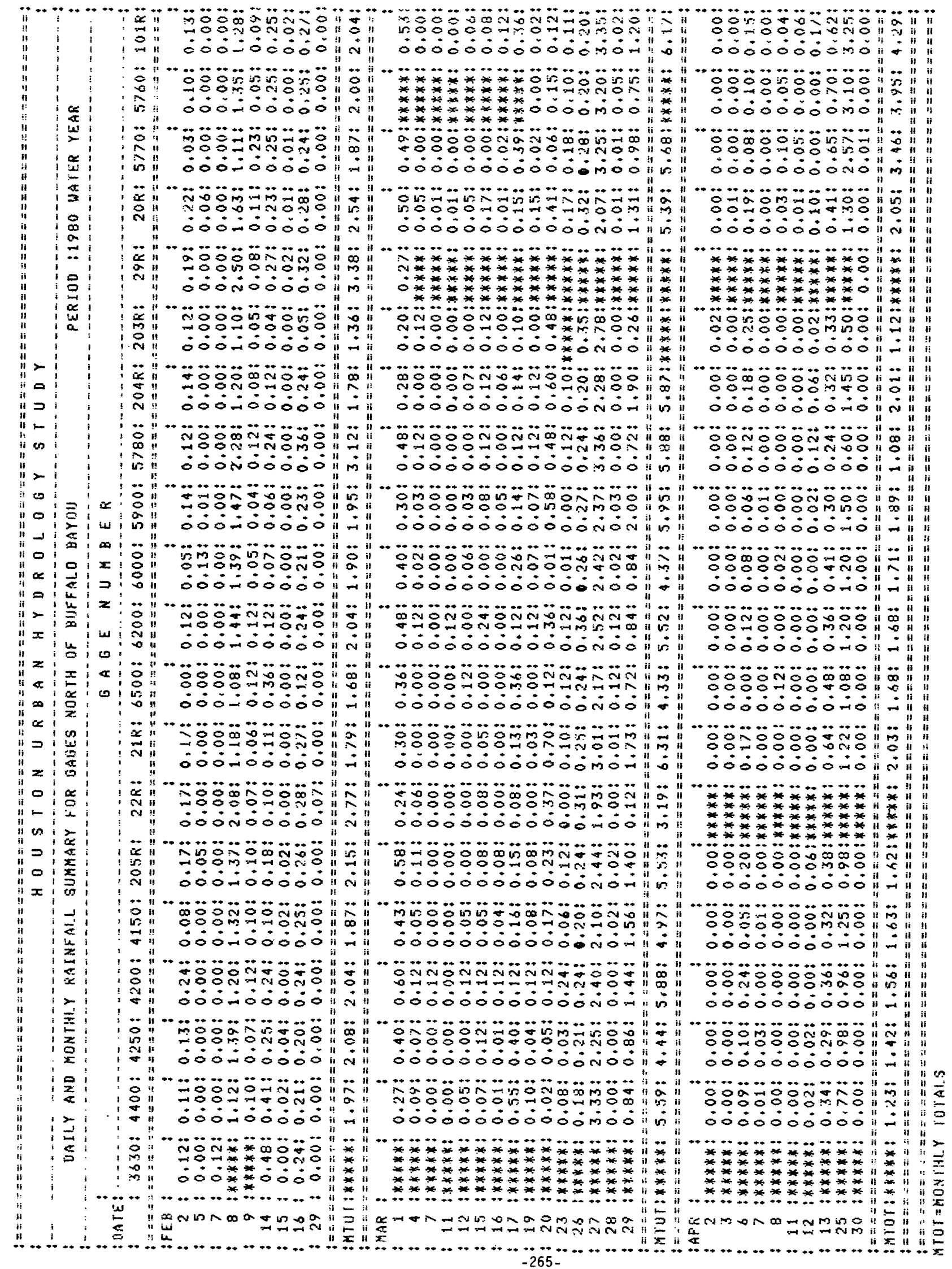




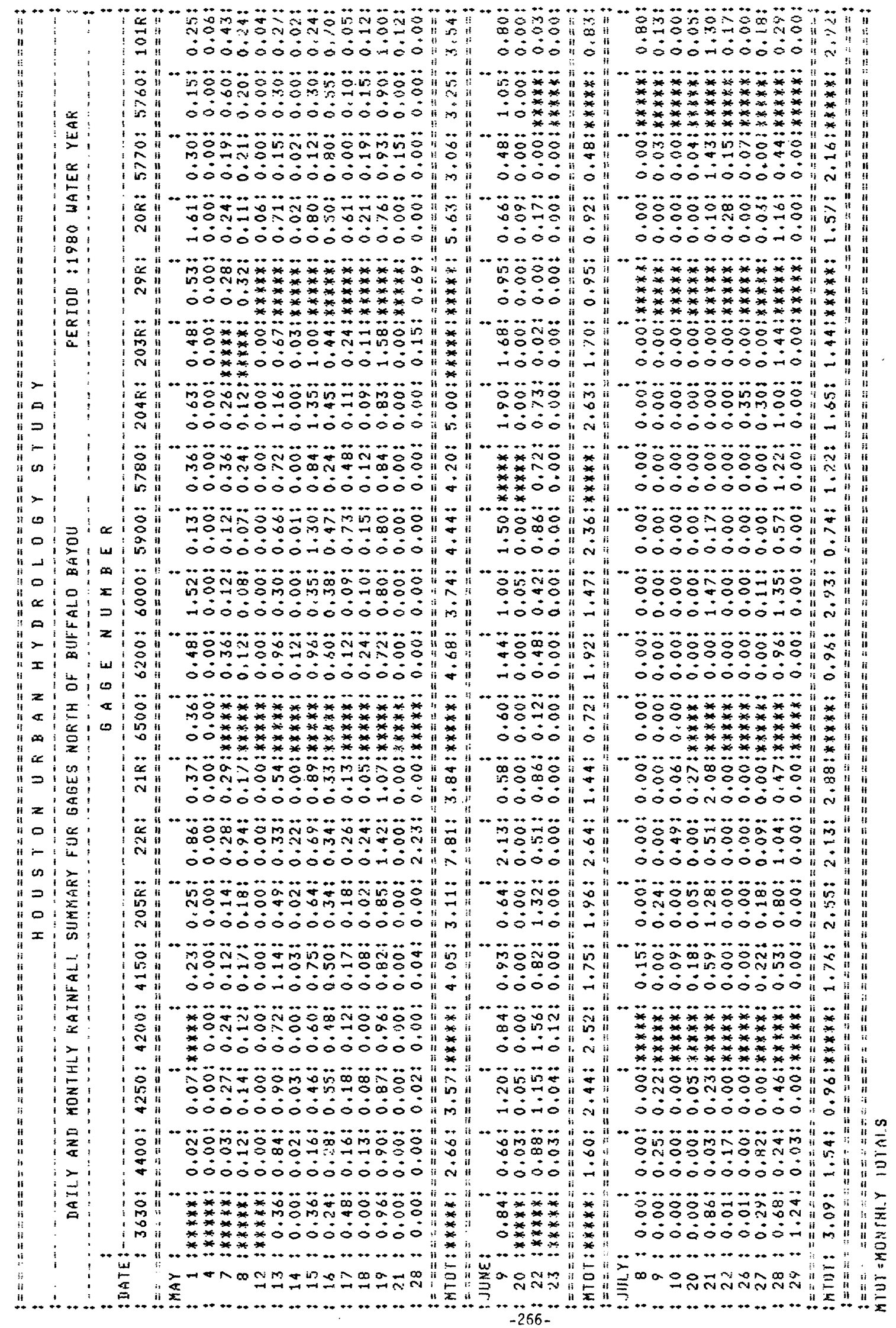




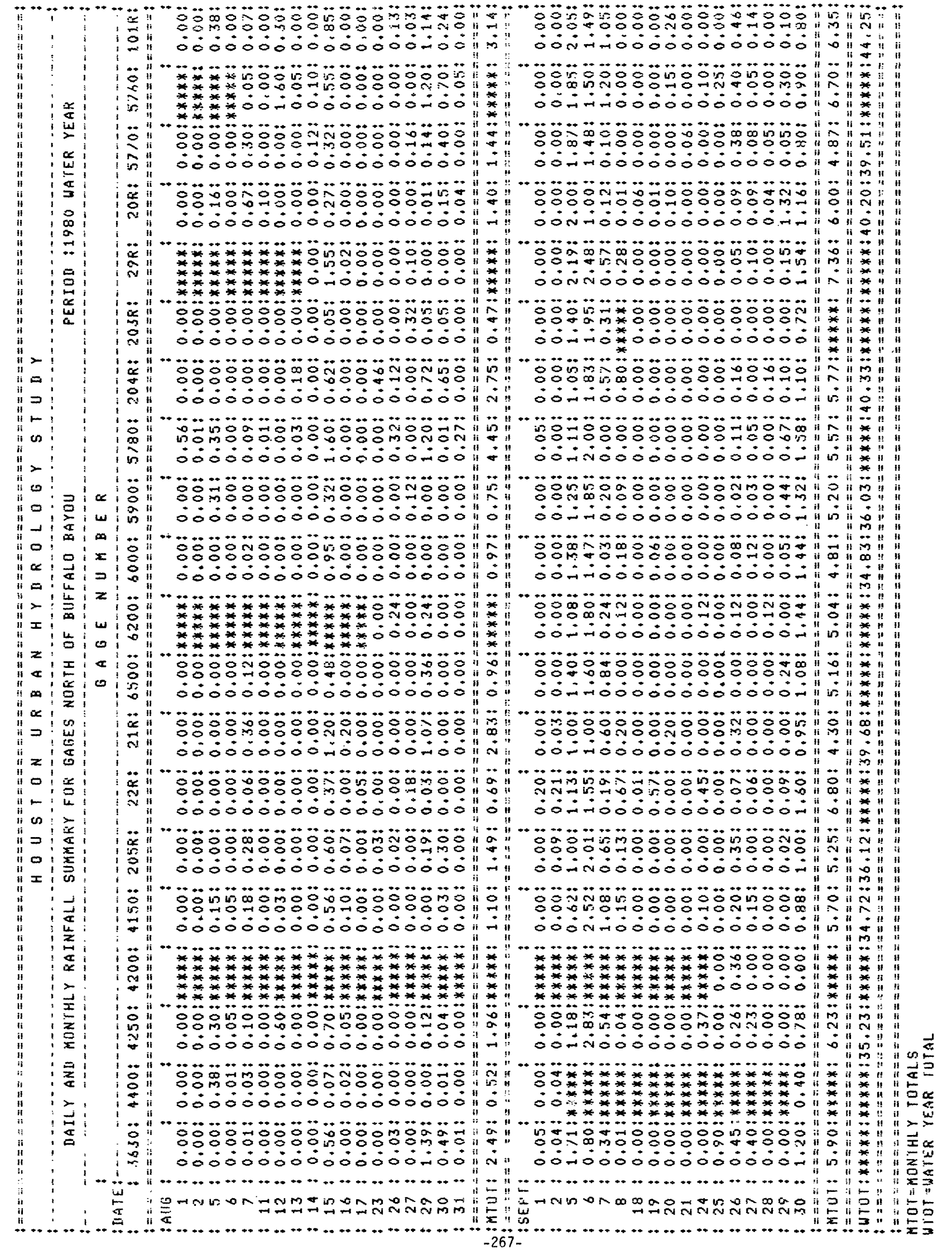




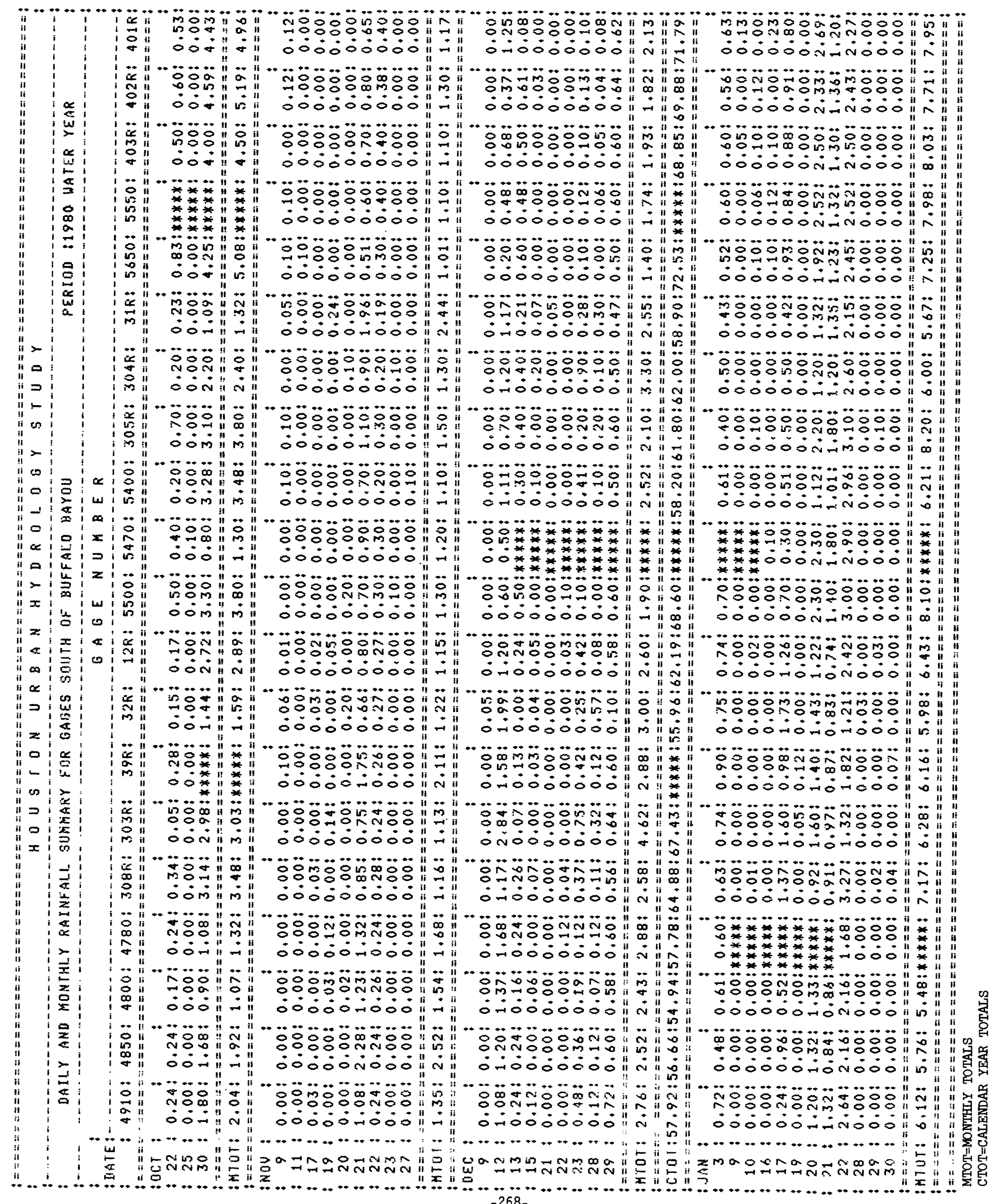
$-258-$ 


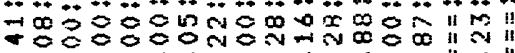
000000000000-0.

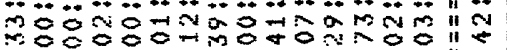

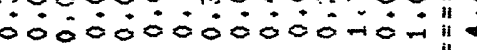

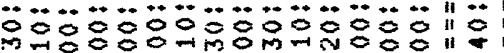
-00000000000்

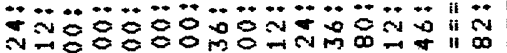
000000000005-100

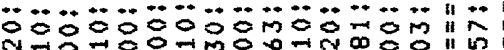

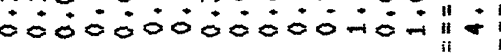

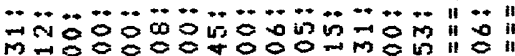

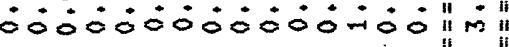

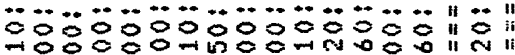

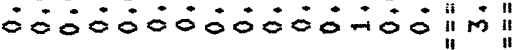

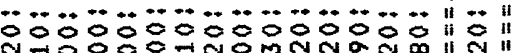

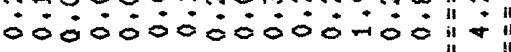

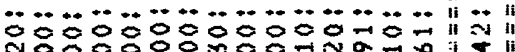
000000000ல்0்

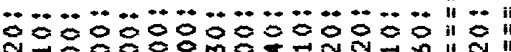
000000000000

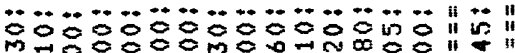

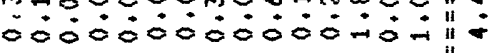

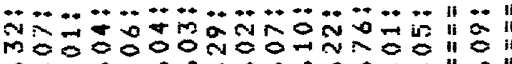

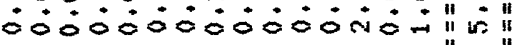

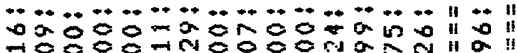

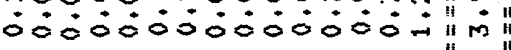

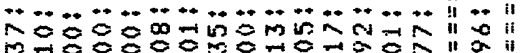

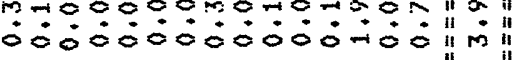

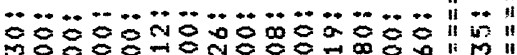

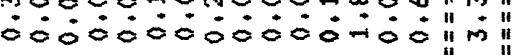

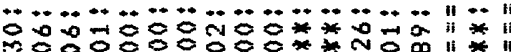

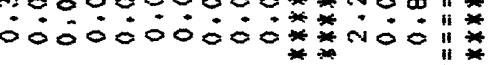

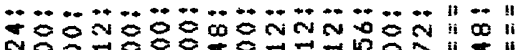

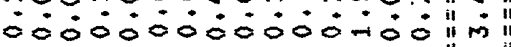

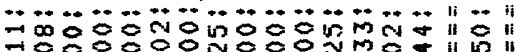
000000000000்-

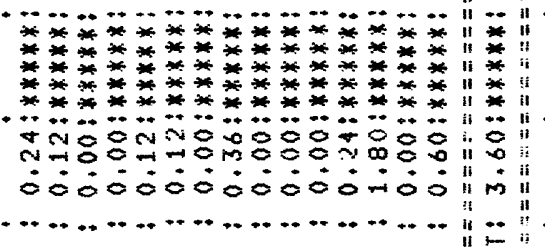

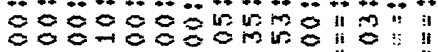
00000000000

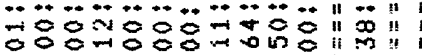
0000000000: "1"

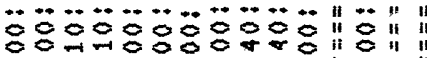
000000000்: "

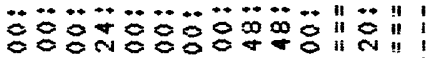
00000000000

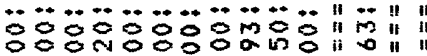
¿0000000000"1"

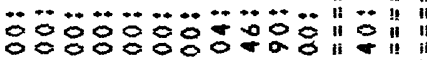
000000000000"

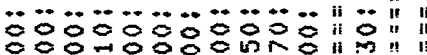
05005000:-0."

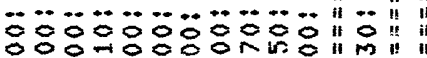
¿000000000: "

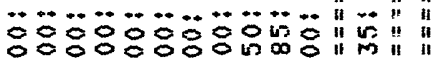
ธ00000000-1"

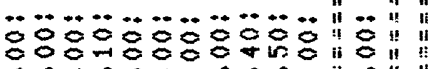
00000000000"11"

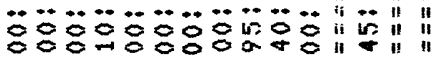
$00000000000\|"\| " \|$

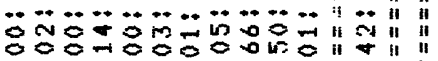

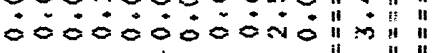

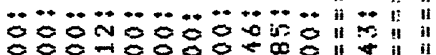
:00000000000

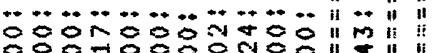
¿000000000-0"

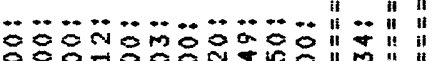
¿00000000000 "

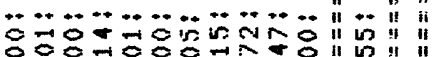

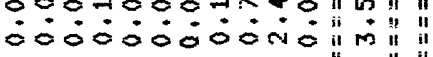

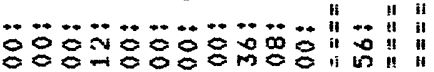

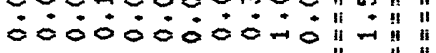

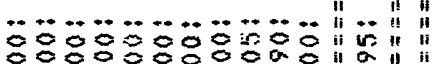
0000000000000

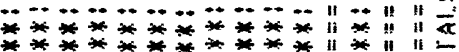

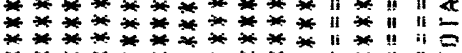

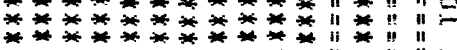

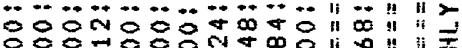
00000000000 "i 


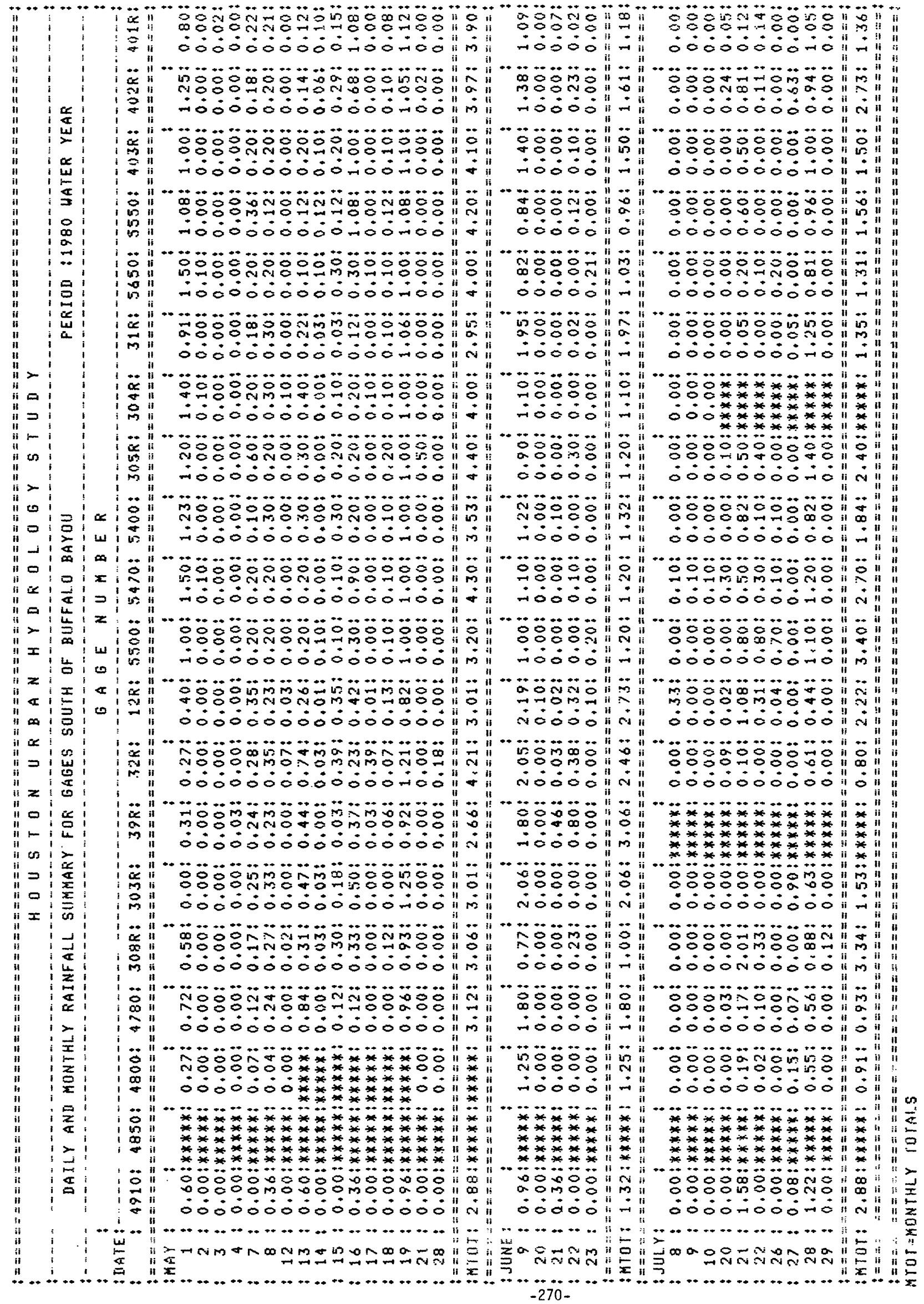




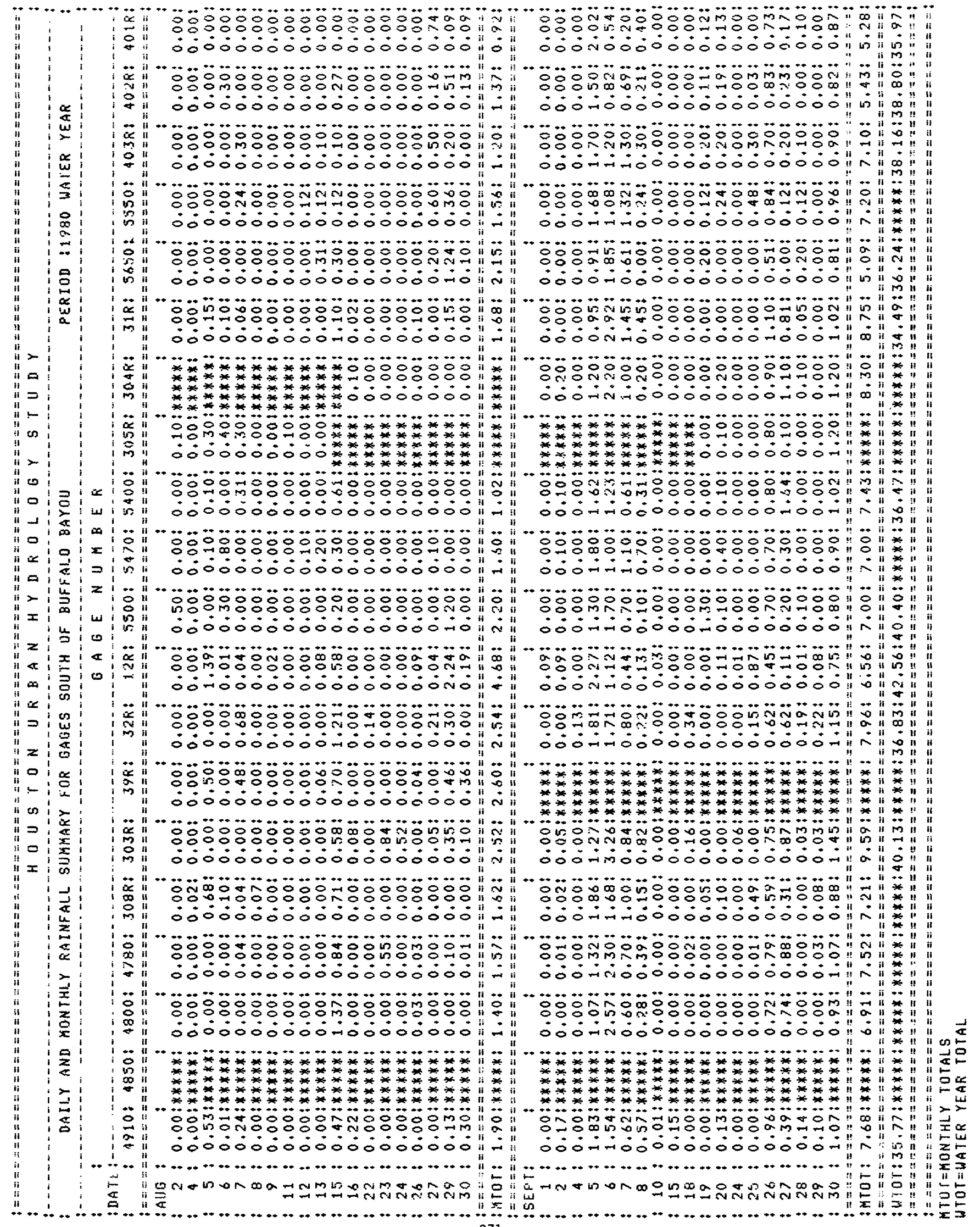




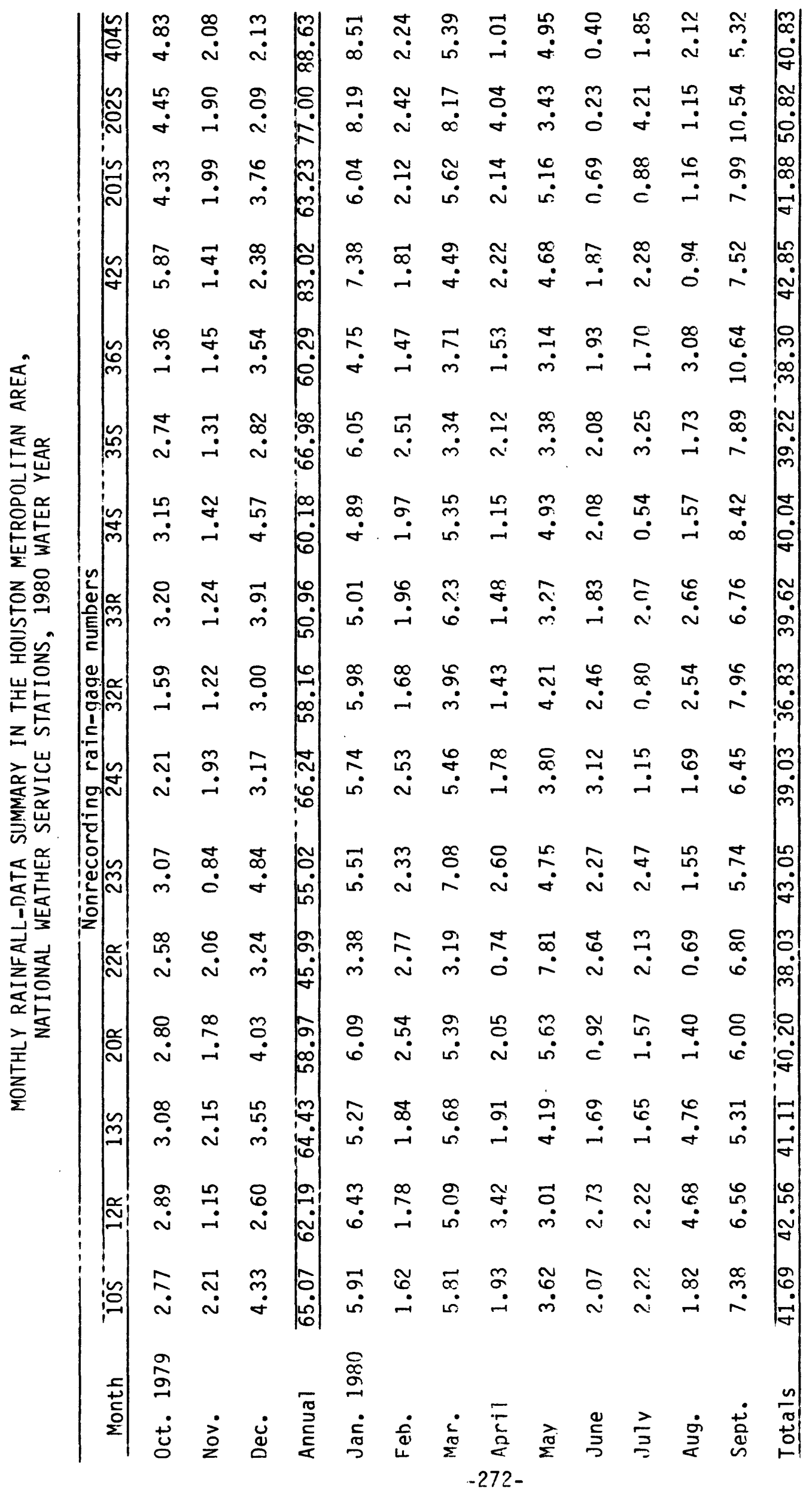

